\title{
Copper-Catalyzed Amino Lactonization and Amino Oxygenation of Alkenes Using $O$-Benzoylhydroxylamines
}

\author{
Brett N. Hemric, Kun Shen, and Qiu Wang* \\ Department of Chemistry, Duke University, Durham, NC 27708 \\ Email: qiu.wang@duke.edu
}

SUPPORTING INFORMATION

Table of Contents

1.1 General Methods $\quad$ S1

1.2 Condition Optimization for Amino Lactonization Reaction $\quad$ S2

1.3 Synthesis of Starting Materials $\quad$ S6

1.4 Amino Lactonization Protocols and Characterization Data of Synthesized Compounds S

1.5 Mechanistic Study Protocols and Characterization Data of Synthesized Compounds $\quad$ S26

1.6 Intermolecular Amino Oxygenation Protocols and Characterization Data of Synthesized Compounds S30

$\begin{array}{lll}1.7 & \text { References } & \text { S31 }\end{array}$

$\begin{array}{lll}\text { Spectra } & \text { S32 }\end{array}$

1.9 X-Ray Crystallography Information $\quad$ S213

\subsection{General Methods}

\section{General Procedures}

Glassware and stir bars were dried either with a propane torch or in an oven at $140{ }^{\circ} \mathrm{C}$ overnight and cooled/stored in a dessicator filled with Drierite. Optimization and substrate screens were performed in 1 Dram glass vials with Tefloncoated micro stir bar. All other reactions were performed in round-bottom flasks with rubber septa. Plastic syringes were used for the transfer of pure solvents, while glass pipets were used for transfer of crude reaction solutions. Analytical thinlayer chromatography (TLC) was performed using aluminum plates coated with a $0.25 \mathrm{~mm}$ layer of $230-400$ mesh silica gel with fluorescent indicator $(254 \mathrm{~nm})$. TLC plates were visualized by exposure to ultraviolet light and treatment with either vanillin or $\mathrm{KMnO}_{4}$ stain. Organic solutions were concentrated under reduced pressure using a rotary evaporator and flash chromatography performed using $60 \AA$ silica gel and HPLC-grade solvents.

\section{Materials}

Commercial reagents and solvents were used as received. Anhydrous solvents $\left(\mathrm{Et}_{2} \mathrm{O}, \mathrm{CH}_{2} \mathrm{Cl}_{2}\right.$, Toluene, Dioxane, and THF) were obtained from a departmentally-maintained Innovative Technologies solvent purification system. Activated, neutral, Brockmann Grade I (58-60^̊ mesh powder). 


\section{Instrumentation}

Nuclear magnetic resonance spectra were recorded on $400 \mathrm{MHz}$ or $500 \mathrm{MHz}$ spectrophotometer at room temperature unless otherwise noted. Chemical shifts for ${ }^{1} \mathrm{H}$ NMR are reported in parts per million (ppm, $\delta$ ) and referenced to residual protium in $\mathrm{CDCl}_{3}\left(\delta\right.$ 7.26). Chemical shifts for ${ }^{13} \mathrm{C} \mathrm{NMR}$ are reported as total carbons in parts per million (ppm, $\left.\delta\right)$ and referenced to the carbon resonances of $\mathrm{CDCl}_{3}(\delta 77.0)$. NMR values are reported as follows: chemical shift, multiplicity (s = singlet, $\mathrm{d}=$ doublet, $\mathrm{t}=$ triplet, $\mathrm{q}=$ quartet, quin = quintet, $\mathrm{m}=$ multiplet, $\mathrm{br}=$ broad $)$, coupling constant $(\mathrm{Hz})$, and integration. Infrared spectroscopic data are reported in wavenumbers $\left(\mathrm{cm}^{-1}\right)$. High-resolution mass spectra were obtained through the Duke University Mass Spectrometry Facility using a liquid chromatography-electrospray ionization mass spectrometer.

\subsection{Condition Optimization for Amino Lactonization Reaction}

\section{General Optimization Screening Conditions}

In an oven-dried 1 Dram vial, 2-(1-methylvinyl)benzoic acid 1a (32.4 mg, $0.2 \mathrm{mmol})$, 4-benzoyloxymorpholine $\mathbf{2 a}$ (82.9 $\mathrm{mg}, 0.4 \mathrm{mmol})$, copper (II) trifluoromethanesulfonate $(7.2 \mathrm{mg}, 0.02 \mathrm{mmol})$, and bathocuproine $(7.2 \mathrm{mg}, 0.02 \mathrm{mmol}) \mathrm{were}$ combined and $1.0 \mathrm{~mL}$ of anhydrous 1,2-dichloroethane was added. The vial was capped and stirred with Teflon-coated stir bar at $80{ }^{\circ} \mathrm{C}$, monitoring consumption of $\mathbf{2} \mathbf{a}$ by TLC. The resulting solution was filtered through activated, neutral $\mathrm{Al}_{2} \mathrm{O}_{3}$ and condensed by rotary evaporation to yield the crude product. To determine yields by ${ }^{1} \mathrm{H}$ NMR spectroscopy, $0.75 \mathrm{~mL}$ $\mathrm{CDCl}_{3}$ was added to the crude reaction mixture, upon which $7 \mu \mathrm{L}$ of dibromomethane was added by $10 \mu \mathrm{L}$ microsyringe. The resulting solution was analyzed by ${ }^{1} \mathrm{H}$ NMR with a 1 second relaxation time and a minimum of 12 scans.

Table S1. Reactant Equivalents Screen for Methylated 2-Vinylbenzoic Acid.a

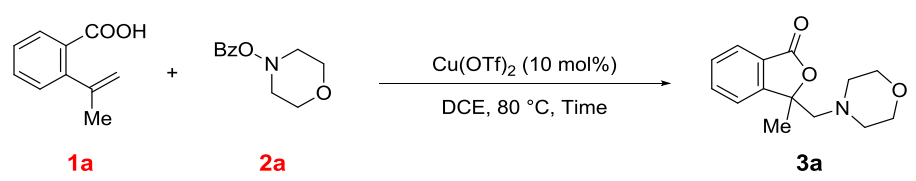

\begin{tabular}{ccccc}
\hline Entry & $\mathbf{1}(\mathrm{eq})$ & $\mathbf{2}(\mathrm{eq})$ & Time $(\mathrm{h})$ & $\mathbf{3 a}^{\boldsymbol{b}}(\%)$ \\
\hline 1 & 1 & 3 & 0.25 & 32 \\
$\mathbf{2}$ & $\mathbf{1}$ & $\mathbf{2}$ & $\mathbf{0 . 2 5}$ & $\mathbf{5 5}$ \\
3 & 1 & 1.5 & 0.25 & 41 \\
4 & 1 & 1.2 & 0.25 & 39 \\
5 & 1 & 1 & 0.25 & 33 \\
6 & 2 & 1 & 0.25 & 47 \\
7 & 3 & 1 & 0.25 & 54 \\
\hline
\end{tabular}

${ }^{\mathrm{a}}$ Reaction Conditions: 1a, 2a, and $\mathrm{Cu}(\mathrm{OTf})_{2}(0.02 \mathrm{mmol})$ in $\mathrm{DCE}(1.0 \mathrm{~mL})$ at $80{ }^{\circ} \mathrm{C}$. ${ }^{\text {b}}$ Yield determined by ${ }^{1} \mathrm{H}$ NMR spectroscopy with dibromomethane as a quantitative internal standard.

Table S2. Catalyst Loading Screen for Methylated 2-Vinylbenzoic Acid. ${ }^{\text {a }}$

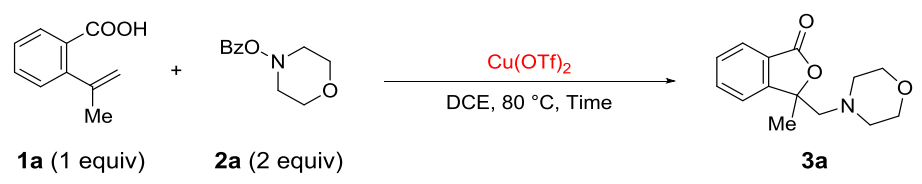

\begin{tabular}{cccc}
\hline Entry & $\mathrm{Cu}(\mathrm{OTf})_{2}(\mathrm{~mol} \%)$ & Time $(\mathrm{h})$ & $\mathbf{3 a}^{b}(\%)$ \\
\hline 1 & $5 \%$ & 1.5 & 40
\end{tabular}




\begin{tabular}{llcl}
$\mathbf{2}$ & $\mathbf{1 0 \%}$ & $\mathbf{1 . 5}$ & $\mathbf{4 5}$ \\
3 & $15 \%$ & 1.5 & 50 \\
4 & $20 \%$ & 1.5 & 52 \\
5 & $30 \%$ & 0.25 & 50 \\
\hline
\end{tabular}

${ }^{\text {a}}$ Reaction Conditions: 1a $(0.2 \mathrm{mmol}), \mathbf{2 a}(0.4 \mathrm{mmol})$, and $\mathrm{Cu}(\mathrm{OTf})_{2}$ in DCE $(1.0 \mathrm{~mL})$ at $80{ }^{\circ} \mathrm{C}$. ${ }^{\text {b }}$ Yield determined by ${ }^{1} \mathrm{H}$ NMR spectroscopy with dibromomethane as a quantitative internal standard.

Table S3. Temperature Screen for Methylated 2-Vinylbenzoic Acid. ${ }^{\mathrm{a}}$

\begin{tabular}{lccc} 
1a (1 equiv) & 2a (2 equiv) & Time $(\mathrm{h})$ & $\mathbf{3 a}^{\boldsymbol{b}}(\%)$ \\
\hline Entry & $\mathrm{Temp}\left({ }^{\circ} \mathrm{C}\right)$ & 0.5 & 64 \\
\hline 1 & 100 & $\mathbf{0 . 5}$ & $\mathbf{6 2}$ \\
$\mathbf{2}$ & $\mathbf{8 0}$ & 4.0 & 58 \\
3 & 60 & 17.5 & 44 \\
5 & 40 & 40 & 7 \\
\hline
\end{tabular}

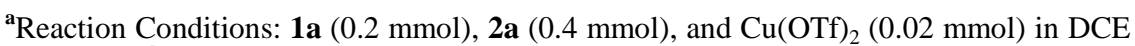
$(1.0 \mathrm{~mL})$. ${ }^{\mathbf{b}}$ Yield determined by ${ }^{1} \mathrm{H}$ NMR spectroscopy with dibromomethane as a quantitative internal standard.

Table S4. Solvent Screen for Methylated 2-Vinylbenzoic Acid. ${ }^{a}$

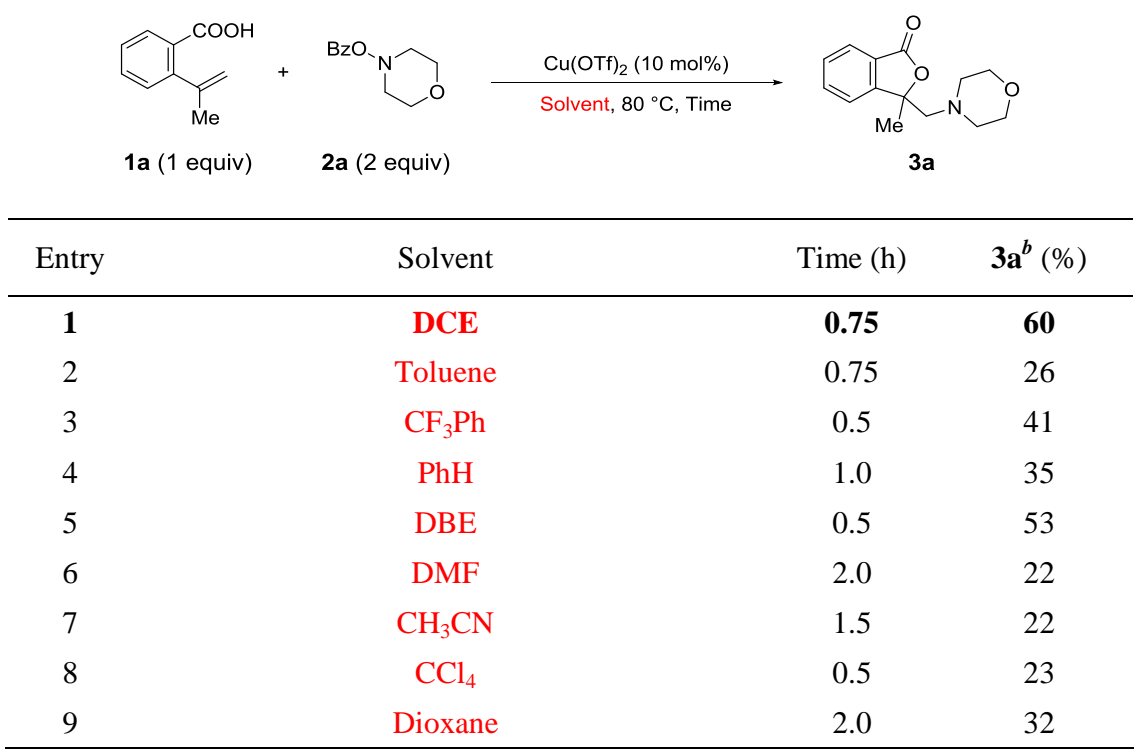

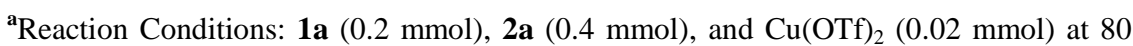
${ }^{\circ} \mathrm{C}$. ${ }^{\text {b }}$ Yield determined by ${ }^{1} \mathrm{H}$ NMR spectroscopy with dibromomethane as a quantitative internal standard. 
Table S5. Catalyst Screen for Methylated 2-Vinylbenzoic Acid. ${ }^{a}$

\begin{tabular}{|c|c|c|c|}
\hline Entry & $\mathrm{Cu}$ catalyst & Time (h) & $3 \mathbf{a}^{b}(\%)$ \\
\hline 1 & $\mathrm{Cu}(\mathrm{OTf})_{2}$ & 0.5 & 57 \\
\hline 2 & $\mathrm{Cu}(\mathrm{OAc})_{2}$ & 1.0 & 39 \\
\hline 3 & $\mathrm{CuCl}_{2}$ & 1.5 & 40 \\
\hline 4 & $\mathrm{Cu}(\mathrm{eh})_{2}$ & 1.0 & 38 \\
\hline 5 & $\mathrm{Cu}(\mathrm{acac})_{2}$ & 1.5 & 21 \\
\hline 6 & {$[\mathrm{Cu}(\mathrm{OTf})]_{2} \cdot$ tol } & 0.5 & 60 \\
\hline 7 & $\mathrm{CuOAc}$ & 1.0 & 36 \\
\hline 8 & {$\left[\mathrm{Cu}\left(\mathrm{CH}_{3} \mathrm{CN}\right)_{4}\right] \mathrm{PF}_{6}$} & 0.5 & 56 \\
\hline 9 & $\mathrm{CuCl}$ & 1.5 & 39 \\
\hline 10 & $\mathrm{CuCN}$ & 1.0 & 38 \\
\hline
\end{tabular}

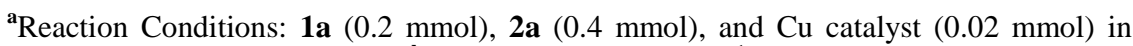
DCE $(1.0 \mathrm{~mL})$ at $80{ }^{\circ} \mathrm{C}$. ${ }^{\mathbf{b}}$ Yield determined by ${ }^{1} \mathrm{H}$ NMR spectroscopy with dibromomethane as a quantitative internal standard.

Table S6. Broad Ligand Screen for Methylated 2-Vinylbenzoic Acid. ${ }^{a}$

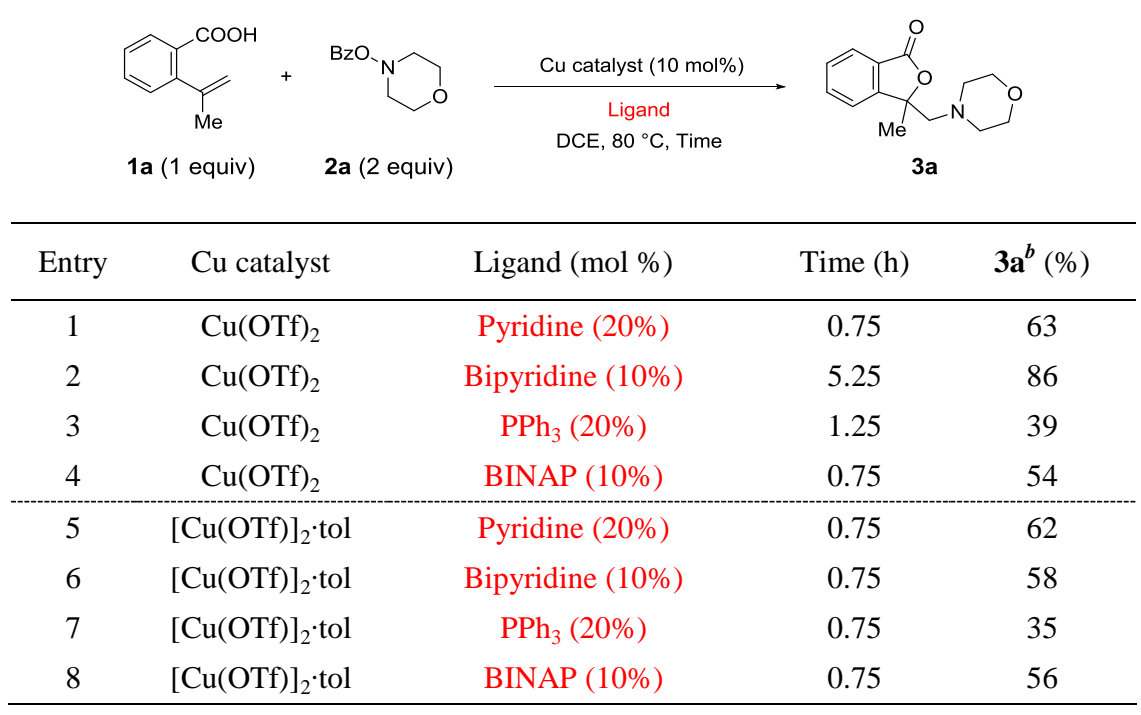

aa Reaction Conditions: 1a $(0.2 \mathrm{mmol}), \mathbf{2 a}(0.4 \mathrm{mmol}), \mathrm{Cu}$ catalyst $(0.02 \mathrm{mmol})$ and Ligand in DCE $(1.0 \mathrm{~mL})$ at $80{ }^{\circ} \mathrm{C}$. b Yield determined by ${ }^{1} \mathrm{H}$ NMR spectroscopy with dibromomethane as a quantitative internal standard. 
Table S7. Nitrogen Ligand Screen. ${ }^{a}$

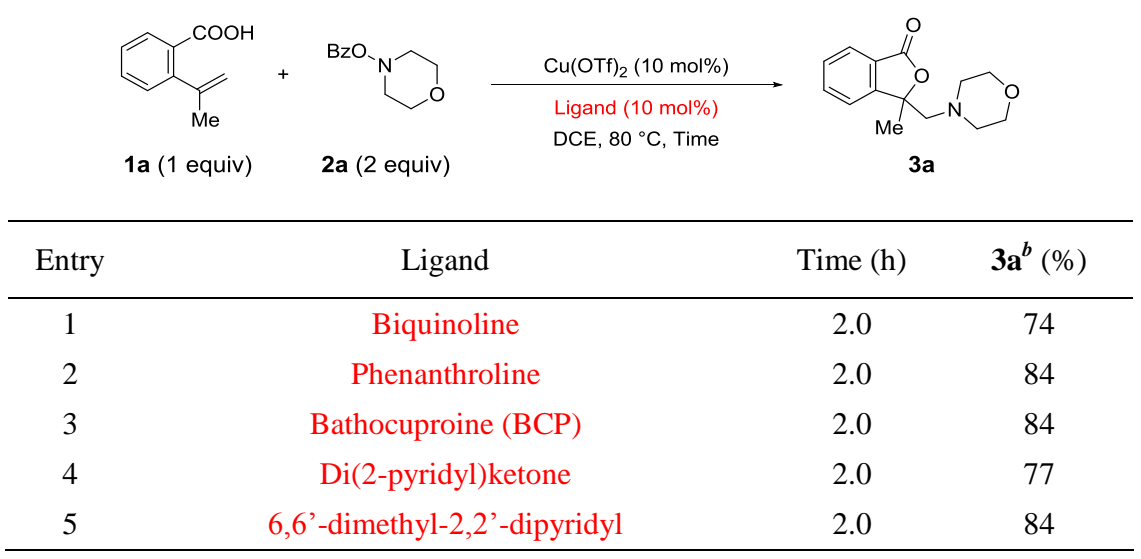

${ }^{a}$ Reaction Conditions: 1a $(0.2 \mathrm{mmol}), \mathbf{2 a}(0.4 \mathrm{mmol}), \mathrm{Cu}(\mathrm{OTf})_{2}(0.02 \mathrm{mmol})$ and Ligand $(0.02 \mathrm{mmol})$ in DCE $(1.0 \mathrm{~mL})$ at $80{ }^{\circ} \mathrm{C}$. ${ }^{\mathbf{b}}$ Yield determined by ${ }^{1} \mathrm{H}$ NMR spectroscopy with dibromomethane as a quantitative internal standard.

Table S8. Alternate Substrate Ligand Screen. ${ }^{a}$

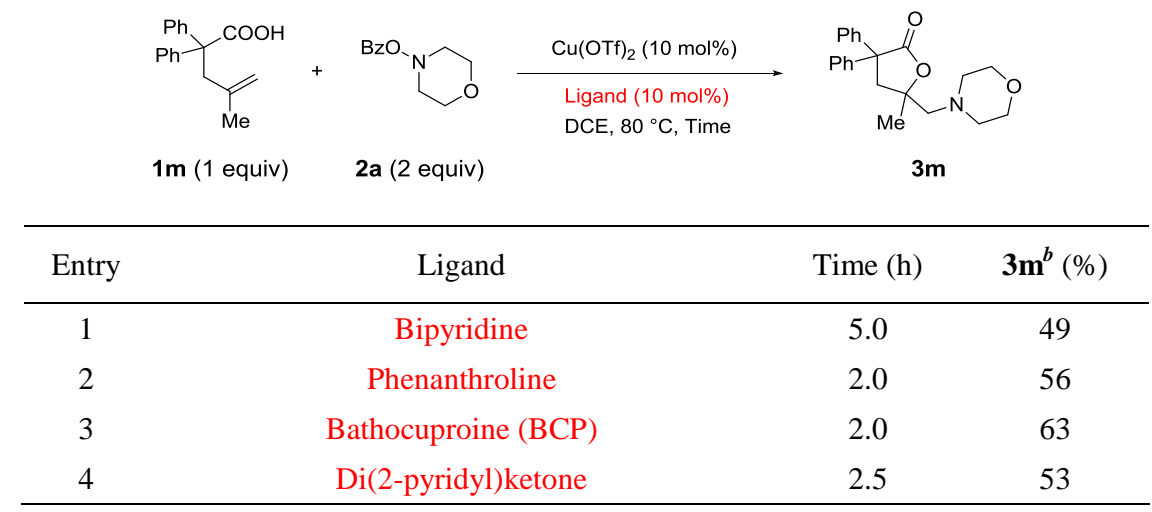

${ }^{\mathrm{a}}$ Reaction Conditions: $\mathbf{1 m}(0.2 \mathrm{mmol}), \mathbf{2 a}(0.4 \mathrm{mmol}), \mathrm{Cu}(\mathrm{OTf})_{2}(0.02 \mathrm{mmol})$ and Ligand $(0.02 \mathrm{mmol})$ in DCE $(1.0 \mathrm{~mL})$ at $80{ }^{\circ} \mathrm{C}$. ${ }^{\mathbf{b}}$ Yield determined by ${ }^{1} \mathrm{H}$ NMR spectroscopy with dibromomethane as a quantitative internal standard.

Table S9. Catalyst/Ligand Ratio. ${ }^{a}$

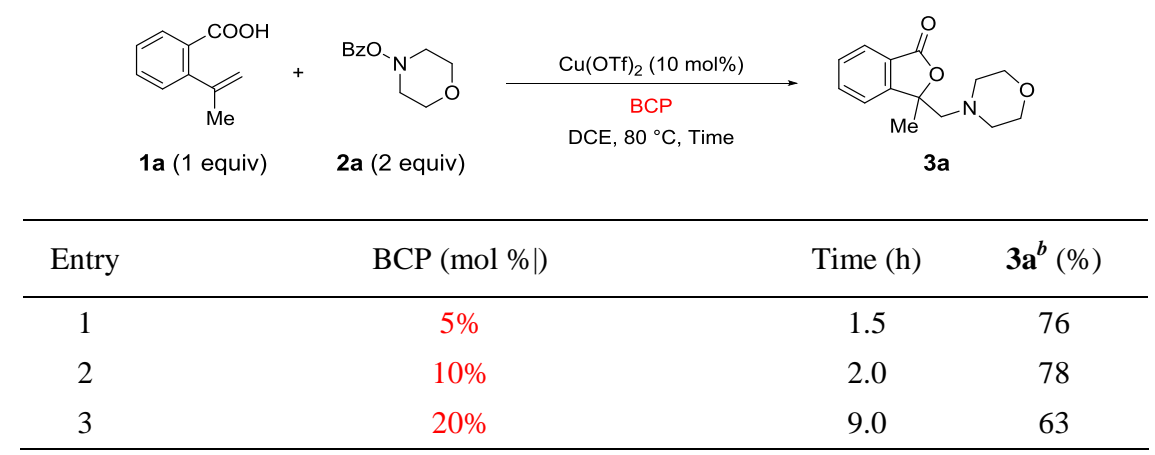

${ }^{\mathrm{a}}$ Reaction Conditions: 1a $(0.2 \mathrm{mmol}), \mathbf{2 a}(0.4 \mathrm{mmol}), \mathrm{Cu}(\mathrm{OTf})_{2}(0.02 \mathrm{mmol})$ and BCP in DCE $(1.0 \mathrm{~mL})$ at $80{ }^{\circ} \mathrm{C}$. ${ }^{\mathbf{b}}$ Yield determined by ${ }^{1} \mathrm{H}$ NMR spectroscopy with dibromomethane as a quantitative internal standard. 


\subsection{Synthesis of Starting Materials}

$\boldsymbol{O}$-Benzoylhydroxylamines (2a-2i) were synthesized according to a previously reported method. ${ }^{1}$

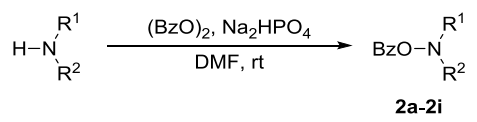

2-(Prop-1-en-2-yl)benzoic acid (1a).

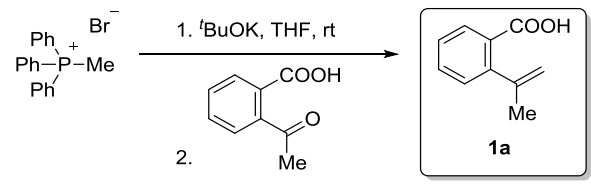

A suspension of potassium tert-butoxide (15.00 g, 2.6 equiv) in THF $(69 \mathrm{~mL})$ was added to a suspension of methyltriphenylphophonium bromide (34.29 g, 1.6 equiv) in THF (138 mL). The resulting yellow solution was stirred at room temperature for $1.5 \mathrm{~h}$, upon which 2-acetylbenzoic acid $(9.85 \mathrm{~g}, 60.0 \mathrm{mmol}, 1.0$ equiv) was added. After the solution was refluxed overnight, the reaction mixture was cooled to room temperature and quenched with acetic acid (3.5 mL), followed by addition of EtOAc $(100 \mathrm{~mL})$. The organic layer was extracted with a saturated aqueous solution of $\mathrm{NaHCO}_{3}$ (200 $\mathrm{mL}$ x 3). The combined aqueous layers were acidified to $\mathrm{pH} 1$ with concentrated $\mathrm{HCl}$ and the organic layer extracted with EtOAc (400 mL x 2). The combined organic layers were washed with water $(200 \mathrm{~mL})$, brine $(200 \mathrm{~mL})$, dried with $\mathrm{Na}_{2} \mathrm{SO}_{4}$, and concentrated in vacuo to afford the crude olefin product. Purification by column chromatography (25\% EtOAc-hexanes) afforded $\mathbf{1 a}$ as a white solid (7.18 g, $44.3 \mathrm{mmol}, 74 \%) . \mathrm{R}_{f}=0.67\left(25 \%\right.$ EtOAc-hexanes); ${ }^{1} \mathrm{H}$ NMR $\left(\mathrm{CDCl}_{3}, 400 \mathrm{MHz}\right): \delta 7.97(\mathrm{dd}, J=7.6,1.3 \mathrm{~Hz}, 1 \mathrm{H}), 7.51(\mathrm{td}, J=7.6,1.3 \mathrm{~Hz}, 1 \mathrm{H}), 7.36(\mathrm{td}, J=7.6,1.3 \mathrm{~Hz}, 1 \mathrm{H}), 7.29-$ $7.26(\mathrm{~m}, 1 \mathrm{H}), 5.15-5.12(\mathrm{~m}, 1 \mathrm{H}), 4.92-3.89(\mathrm{~m}, 1 \mathrm{H}), 2.13(\mathrm{~s}, 3 \mathrm{H}) ;{ }^{13} \mathrm{C} \mathrm{NMR}\left(\mathrm{CDCl}_{3}, 125 \mathrm{MHz}\right): \delta 173.6,146.5,146.3$, 132.6, 130.7, 129.7, 128.0, 127.0, 113.9, 24.3; FTIR (thin film): $\mathrm{cm}^{-1} 2977$ (br), 1689, 1598, 1569, 1406, 1296, 1265, 1075, 895, 766; HRMS-ESI (m/z) Calcd for $\left.\left(\mathrm{C}_{10} \mathrm{H}_{9} \mathrm{O}_{2}\right)([\mathrm{M}-\mathrm{H}]]^{-}\right): 161.0608$; found: 161.0607 .

2-Vinylbenzoic acid (1b) was synthesized by a previously reported method. ${ }^{2}$

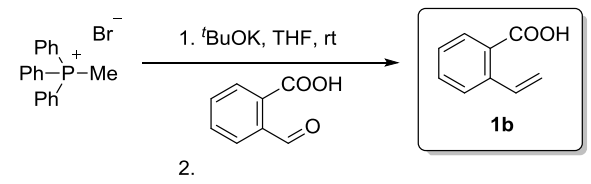

$\mathrm{R}_{f}=0.57$ (25\% EtOAc-hexanes); ${ }^{1} \mathrm{H} \mathrm{NMR}\left(\mathrm{CDCl}_{3}, 400 \mathrm{MHz}\right): \delta 8.04(\mathrm{dd}, J=7.9,1.3 \mathrm{~Hz}, 1 \mathrm{H}), 7.64-7.52(\mathrm{~m}, 3 \mathrm{H}), 7.37$ $(\mathrm{td}, J=7.5,1.3 \mathrm{~Hz}, 1 \mathrm{H}), 5.68(\mathrm{dd}, J=17.4,1.3 \mathrm{~Hz}, 1 \mathrm{H}), 5.39(\mathrm{dd}, J=11.0,1.3 \mathrm{~Hz}, 1 \mathrm{H}) ;{ }^{13} \mathrm{C} \mathrm{NMR}\left(\mathrm{CDCl}_{3}, 125 \mathrm{MHz}\right): \delta$ 173.3, 140.6, 136.0, 133.2, 131.3, 127.6, 127.5, 127.1, 116.8; FTIR (thin film): $\mathrm{cm}^{-1} 2874$ (br), 1674, 1598, 1566, 1484, 1403, 1266, 906, 767; HRMS-ESI (m/z) Calcd for $\left(\mathrm{C}_{9} \mathrm{H}_{7} \mathrm{O}_{2}\right)\left([\mathrm{M}-\mathrm{H}]^{-}\right): 147.0452$; found: 147.0451 .

2-(1-Phenylvinyl)benzoic acid (1c) and 2-(1-(4-Chlorophenyl)vinyl)benzoic acid (1d) were synthesized by a previously reported method. ${ }^{3}$

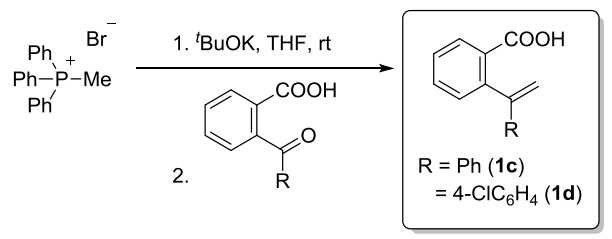

2-(1-Phenylvinyl)benzoic acid (1c). $\mathrm{R}_{f}=0.29\left(25 \%\right.$ EtOAc-hexanes); ${ }^{1} \mathrm{H} \mathrm{NMR}\left(\mathrm{CDCl}_{3}, 400 \mathrm{MHz}\right): \delta 7.96(\mathrm{~d}, J=7.8 \mathrm{~Hz}$, $1 \mathrm{H}), 7.60(\mathrm{tt}, J=7.5,1.3 \mathrm{~Hz}, 1 \mathrm{H}), 7.46(\mathrm{t}, J=7.6 \mathrm{~Hz}, 1 \mathrm{H}), 7.41(\mathrm{~d}, J=7.6 \mathrm{~Hz}, 1 \mathrm{H}), 7.32-7.22(\mathrm{~m}, 5 \mathrm{H}), 5.71(\mathrm{~s}[\mathrm{br}], 1 \mathrm{H})$, $5.26(\mathrm{~s}[\mathrm{br}], 1 \mathrm{H}) ;{ }^{13} \mathrm{C} \mathrm{NMR}\left(\mathrm{CDCl}_{3}, 125 \mathrm{MHz}\right): \delta 172.2,149.5,143.6,140.8,132.4,131.5,129.4,128.0(2 \mathrm{C}), 127.6$, 127.4, 126.7 (2C), 114.3; FTIR (thin film): $\mathrm{cm}^{-1} 3020$ (br), 1696, 1409, 1301, 903, 770, 704; HRMS-ESI (m/z) Calcd for $\left(\mathrm{C}_{15} \mathrm{H}_{11} \mathrm{O}_{2}\right)\left([\mathrm{M}-\mathrm{H}]^{-}\right): 223.0765$; found: 223.0765 . 
2-(1-(4-Chlorophenyl)vinyl)benzoic acid (1d). $\mathrm{R}_{f}=0.58\left(25 \%\right.$ EtOAc-hexanes); ${ }^{1} \mathrm{H}$ NMR $\left(\mathrm{CDCl}_{3}, 400 \mathrm{MHz}\right): \delta 7.96(\mathrm{~d}$, $J=7.6 \mathrm{~Hz}, 1 \mathrm{H}), 7.58(\mathrm{t}, J=7.6 \mathrm{~Hz}, 1 \mathrm{H}), 7.45(\mathrm{t}, J=7.6 \mathrm{~Hz}, 1 \mathrm{H}), 7.36(\mathrm{~d}, J=7.6 \mathrm{~Hz}, 1 \mathrm{H}), 7.22(\mathrm{~d}, J=8.2 \mathrm{~Hz}, 2 \mathrm{H}), 7.14$ $(\mathrm{d}, J=8.2 \mathrm{~Hz}, 2 \mathrm{H}), 5.66(\mathrm{~s}, 1 \mathrm{H}), 5.23(\mathrm{~s}, 1 \mathrm{H}) ;{ }^{13} \mathrm{C} \mathrm{NMR}\left(\left(\mathrm{CD}_{3}\right)_{2} \mathrm{SO}, 125 \mathrm{MHz}\right): \delta 169.0,149.0,142.4,140.4,133.0,132.5$, 132.0, 130.6, 130.6, 129.1 (4C), 128.9, 115.7; FTIR (thin film): $\mathrm{cm}^{-1} 3065,1690,1490,1091,1012,972,835,769$; HRMS-ESI (m/z) Calcd for $\left(\mathrm{C}_{15} \mathrm{H}_{10} \mathrm{ClO}_{3}\right)\left([\mathrm{M}-\mathrm{H}]^{-}\right): 257.0375$; found: 257.0375 .

2-Methyl-6-(prop-1-en-2-yl)benzoic acid (1e).

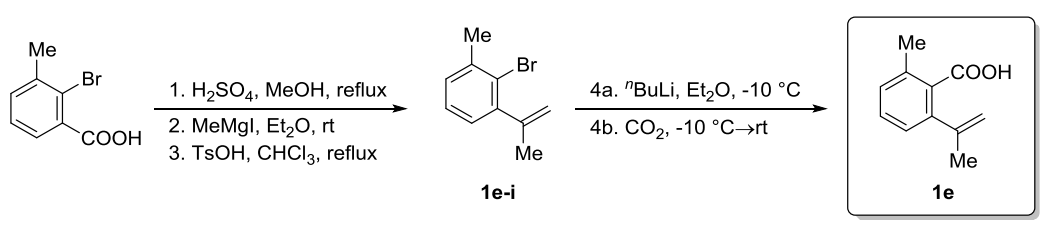

To a solution of 2-bromo-3-methylbenzoic acid (3.66 g, $17.0 \mathrm{mmol}, 1.0$ equiv) in methanol (55 mL) was added concentrated $\mathrm{H}_{2} \mathrm{SO}_{4}(1.9 \mathrm{~mL}, 2.0$ equiv). The resulting solution was stirred at reflux for $16.5 \mathrm{~h}$. The solution was cooled to room temperature and concentrated in vacuo to remove $\sim 50 \%$ of the $\mathrm{MeOH}$. The resulting residue was slowly quenched with a saturated aqueous solution of $\mathrm{NaHCO}_{3}(20 \mathrm{~mL})$. The aqueous layer was extracted with EtOAc (50 mL x 3$)$. The combined organic layers were washed brine $(10 \mathrm{~mL})$, dried with $\mathrm{Na}_{2} \mathrm{SO}_{4}$, and concentrated in vacuo, affording the ester as a clear oil (3.88 g), which was used without further purification.

The above ester ( $3.44 \mathrm{~g}, 15.0 \mathrm{mmol}, 1.0$ equiv) was added to a reaction vessel and vacuum purged three times, backfilling with $\mathrm{N}_{2} . \mathrm{Et}_{2} \mathrm{O}(30 \mathrm{~mL})$ was added and the solution cooled to $0{ }^{\circ} \mathrm{C}$. To this solution was added $\mathrm{MeMgBr}\left(3 \mathrm{M}\right.$ in $\mathrm{Et}_{2} \mathrm{O}$, $15 \mathrm{~mL}, 45.0 \mathrm{mmol}$ ) dropwise over $30 \mathrm{~min}$. The solution was stirred for $17 \mathrm{~h}$ at room temperature. The reaction was slowly quenched with a saturated aqueous solution of $\mathrm{NH}_{4} \mathrm{Cl}(25 \mathrm{~mL})$, followed by the addition of $\mathrm{HCl}(2 \mathrm{M}, 20 \mathrm{~mL})$. The aqueous layer was extracted with EtOAc $(30 \mathrm{~mL}$ x 3). The combined organic layers were washed with brine $(20 \mathrm{~mL})$, dried with $\mathrm{Na}_{2} \mathrm{SO}_{4}$, and concentrated in vacuo, affording the desired tertiary alcohol as a clear oil (3.35 g), which was used without further purification.

A solution of the alcohol (2.29 $\mathrm{g}, 10.0 \mathrm{mmol}, 1.0$ equiv) and toluenesulfonic acid monohydrate (380 mg, $0.2 \mathrm{equiv})$ in chloroform $(27 \mathrm{~mL})$ was refluxed for $3 \mathrm{~h}$. The solution was quenched with a saturated aqueous solution $\mathrm{NaHCO}_{3}(30 \mathrm{~mL})$. The aqueous layer was extracted with EtOAc $(20 \mathrm{~mL}$ x 3). The combined organic layers were washed with brine (35 mL), dried with $\mathrm{Na}_{2} \mathrm{SO}_{4}$, and concentrated in vacuo to afford the crude olefin. Purification by column chromatography (10\% EtOAc-hexanes) afforded olefin 1e-i as a pale oil (1.70 g, $8.0 \mathrm{mmol}, 80 \%)$. $\mathrm{R}_{f}=0.87\left(25 \%\right.$ EtOAc-hexanes); ${ }^{1} \mathrm{H}$ NMR $\left(\mathrm{CDCl}_{3}, 400 \mathrm{MHz}\right): \delta 7.19-7.13(\mathrm{~m}, 2 \mathrm{H}), 7.01(\mathrm{dd}, J=6.3,3.0 \mathrm{~Hz}, 1 \mathrm{H}), 5.23-5.18(\mathrm{~m}, 1 \mathrm{H}), 4.94-4.89(\mathrm{~m}, 1 \mathrm{H}), 2.44(\mathrm{~s}$, 3H), 2.15-2.06 (m, 3H); ${ }^{13} \mathrm{C} \mathrm{NMR}\left(\mathrm{CDCl}_{3}, 125 \mathrm{MHz}\right): \delta 146.7,145.5,138.4,129.2,127.1,126.8,124.1,115.4,23.8,23.6$; FTIR (thin film): $\mathrm{cm}^{-1}$ 2971, 1642, 1463, 1370, 1308, 1182, 1021, 899, 782, 728, 571.

The olefin (1e-i) (1.48 g, $7.0 \mathrm{mmol}, 1.0$ equiv) was added to a reaction vessel and vacuum purged three times, backfilling with $\mathrm{N}_{2}$. $\mathrm{Et}_{2} \mathrm{O}(14 \mathrm{~mL})$ was added and the solution cooled to $-10{ }^{\circ} \mathrm{C}$. $n$-BuLi $(3.4 \mathrm{~mL}, 2.5 \mathrm{M}$ in hexanes, 1.2 equiv) was added dropwise over $7 \mathrm{~min}$ and the resulting bright yellow solution was held at $-10^{\circ} \mathrm{C}$ for $1 \mathrm{~h}$, upon which more $n$-BuLi $\left(2.5 \mathrm{M}\right.$ in hexanes, $0.84 \mathrm{~mL}, 0.30$ equiv) was added. After $30 \mathrm{~min}, \mathrm{CO}_{2}$ was bubbled through the solution for $15 \mathrm{~min}$. at $10{ }^{\circ} \mathrm{C}$. The resulting yellow solution was allowed to reach room temperature over $1.5 \mathrm{~h}$. The solution was quenched with a saturated aqueous solution of $\mathrm{NaHCO}_{3}(20 \mathrm{~mL})$ and the organic layer extracted with aqueous $\mathrm{NaOH}(2 \mathrm{M}, 15 \mathrm{~mL}$ x 4$)$. The combined aqueous layers were acidified with concentrated $\mathrm{HCl}$ to $\mathrm{pH} 1$ and extracted with EtOAc (25 mL x 3$)$. The combined organic layers were washed with brine $(25 \mathrm{~mL})$, dried with $\mathrm{Na}_{2} \mathrm{SO}_{4}$, and concentrated in vacuo to yield the crude acid (1e). Purification by column chromatography (15\% EtOAc-hexanes) afforded the acid (1e) as a white solid (852.1 $\mathrm{mg}, 4.84 \mathrm{mmol}, 69 \%) . \mathrm{R}_{f}=0.18\left(25 \%\right.$ EtOAc-hexanes); ${ }^{1} \mathrm{H} \mathrm{NMR}\left(\mathrm{CDCl}_{3}, 400 \mathrm{MHz}\right): \delta 7.29(\mathrm{t}, J=7.7 \mathrm{~Hz}, 1 \mathrm{H}), 7.14(\mathrm{~d}, J$ $=7.7 \mathrm{~Hz}, 1 \mathrm{H}), 7.11(\mathrm{~d}, J=7.7 \mathrm{~Hz}, 1 \mathrm{H}), 5.16-5.12(\mathrm{~m}, 1 \mathrm{H}), 5.02-4.99(\mathrm{~m}, 1 \mathrm{H}), 2.44(\mathrm{~s}, 3 \mathrm{H}), 2.12(\mathrm{~s}, 3 \mathrm{H}) ;{ }^{13} \mathrm{C} \mathrm{NMR}$ $\left(\mathrm{CDCl}_{3}, 125 \mathrm{MHz}\right): \delta 176.0,144.8,142.2,135.2,131.2,129.5,128.9,125.2,115.3,24.2,19.9$; FTIR (thin film): $\mathrm{cm}^{-1}$ 2969, 1694, 1588, 1436, 1289, 1125, 1073, 902, 798, 776; HRMS-ESI (m/z) Calcd for $\left(\mathrm{C}_{11} \mathrm{H}_{11} \mathrm{O}_{2}\right)\left([\mathrm{M}-\mathrm{H}]^{-}\right): 175.0765$; found: 175.0764 .

3-Methyl-2-(prop-1-en-2-yl)benzoic acid (1f). 


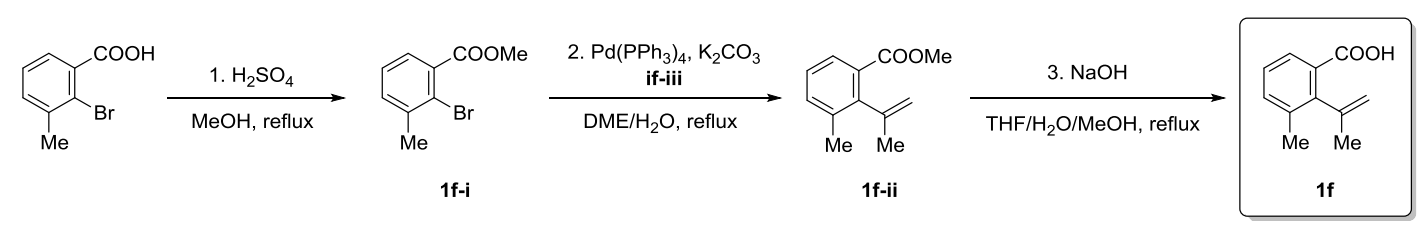

To a solution of 2-bromo-3-methylbenzoic acid (2.15 g, $10.0 \mathrm{mmol}, 1.0$ equiv) in methanol (30 $\mathrm{mL})$ was added concentrated $\mathrm{H}_{2} \mathrm{SO}_{4}(1.1 \mathrm{~mL}, 2.0$ equiv). The resulting solution was refluxed for $14 \mathrm{~h}$. The reaction was cooled to room temperature and concentrated in vacuo to remove $\sim 50 \%$ of the $\mathrm{MeOH}$. The resulting residue was slowly quenched with a saturated solution of $\mathrm{NaHCO}_{3}(20 \mathrm{~mL})$. The aqueous layer was extracted with EtOAc $(30 \mathrm{~mL}$ x 3$)$. The combined organic layers were washed brine $(50 \mathrm{~mL})$, dried with $\mathrm{Na}_{2} \mathrm{SO}_{4}$, and concentrated in vacuo to afford the crude ester. Purification by column chromatography (20\% EtOAc-hexanes) afforded ester $\mathbf{1 f}-\mathbf{i}$ as a clear oil (2.24 g, $9.8 \mathrm{mmol}, 98 \%)$. $\mathbf{R}_{f}=0.77(25 \%$ EtOAc-hexanes); ${ }^{1} \mathrm{H}$ NMR $\left(\mathrm{CDCl}_{3}, 500 \mathrm{MHz}\right): \delta 7.47(\mathrm{~d}, J=7.6 \mathrm{~Hz}, 1 \mathrm{H}), 7.34(\mathrm{~d}, J=7.6 \mathrm{~Hz}, 1 \mathrm{H}), 7.24(\mathrm{t}, J=7.6 \mathrm{~Hz}$, 1H), 3.93 (s, 3H), 2.46 (s, 3H); ${ }^{13} \mathrm{C} \mathrm{NMR}\left(\mathrm{CDCl}_{3}, 125 \mathrm{MHz}\right): \delta 167.7,139.7,133.9,133.1,127.8,126.8,123.0,52.5,23.7$; FTIR (thin film): $\mathrm{cm}^{-1}$ 2951, 1732, 1434, 1297, 1195, 1145, 1030, 754; HRMS-ESI (m/z) Calcd for $\left(\mathrm{C}_{9} \mathrm{H}_{10} \mathrm{BrO}_{2}\right)$ $\left([\mathrm{M}+\mathrm{H}]^{+}\right): 228.9859$; found: 228.9860 .

Following a slight modification of a previously reported procedure, ${ }^{4}$ a solution of ester $\mathbf{1 f - i}$ (1.03 g, $4.5 \mathrm{mmol}, 1.0$ equiv) and tetrakis (triphenylphosphine)palladium $(52.0 \mathrm{mg}, 0.01$ equiv) in DME (36 mL) was stirred at room temperature for 20 min. At this time, $\mathrm{K}_{2} \mathrm{CO}_{3}$ (0.62 g, 1.0 equiv), triisopropenylboroxine (1f-iii) (0.64 g, 0.5 equiv), and DI $\mathrm{H}_{2} \mathrm{O}$ (10.8 mL) were added. The reaction was allowed to reflux for $21.5 \mathrm{~h}$. The reaction was cooled to room temperature and quenched with a saturated solution of $\mathrm{NH}_{4} \mathrm{Cl}(20 \mathrm{~mL})$. The aqueous layer was extracted with $\mathrm{Et}_{2} \mathrm{O}(40 \mathrm{~mL} \times 3)$. The combined organic layers were washed with brine $(50 \mathrm{~mL})$, dried with $\mathrm{Na}_{2} \mathrm{SO}_{4}$, filtered through a pad of silica, and concentrated in vacuo to afford the crude olefin. Purification by column chromatography (10\% EtOAc-hexanes) afforded olefin 1f-ii as a pale oil $(627.9 \mathrm{mg}, 3.3 \mathrm{mmol}, 73 \%) . \mathrm{R}_{f}=0.86\left(25 \%\right.$ EtOAc-hexanes); ${ }^{1} \mathrm{H} \mathrm{NMR}\left(\mathrm{CDCl}_{3}, 400 \mathrm{MHz}\right): \delta 7.61(\mathrm{~d}, J=7.7 \mathrm{~Hz}$, $1 \mathrm{H}), 7.32(\mathrm{~d}, J=7.7 \mathrm{~Hz}, 1 \mathrm{H}), 7.21(\mathrm{t}, J=7.7 \mathrm{~Hz}, 1 \mathrm{H}), 5.19-5.15(\mathrm{~m}, 1 \mathrm{H}), 4.71-4.67(\mathrm{~m}, 1 \mathrm{H}), 3.83(\mathrm{~s}, 3 \mathrm{H}), 2.31(\mathrm{~s}, 3 \mathrm{H})$, 2.11-2.07 (m, 3H); ${ }^{13} \mathrm{C} \mathrm{NMR}\left(\mathrm{CDCl}_{3}, 125 \mathrm{MHz}\right): \delta 168.5,145.3,143.9,135.8,133.2,130.2,126.9,126.4,113.7,51.7$, 23.8, 19.4; FTIR (thin film): $\mathrm{cm}^{-1} 2950,1728,1433,1289,1266,1192,1144,895,762$; HRMS-ESI (m/z) Calcd for $\left(\mathrm{C}_{12} \mathrm{H}_{15} \mathrm{O}_{2}\right)\left([\mathrm{M}+\mathrm{H}]^{+}\right): 191.1067$; found: 191.1066 .

Following a modification from a reported procedure, ${ }^{5}$ olefin $\mathbf{1 f - i i}(570.7 \mathrm{mg}, 3.0 \mathrm{mmol}, 1.0$ equiv) was added to a solution of $\mathrm{NaOH}$ (1.44 g, 12.0 equiv) in THF: $\mathrm{H}_{2} \mathrm{O}: \mathrm{MeOH}(4: 1: 1,18 \mathrm{~mL})$. The reaction allowed to reflux for $12 \mathrm{~h}$. The reaction was cooled to room temperature, quenched with a saturated aqueous solution of $\mathrm{NH}_{4} \mathrm{Cl}(10 \mathrm{~mL})$, and diluted with EtOAc $(30 \mathrm{~mL})$. The organic layer was extracted with aqueous $\mathrm{NaOH}(2 \mathrm{M}, 35 \mathrm{~mL}$ x 3$)$. The combined aqueous layers were acidified with concentrated $\mathrm{HCl}$ to $\mathrm{pH} 1$ and extracted with EtOAc $(40 \mathrm{~mL}$ x 3 ). The combined organic layers were washed with brine $(50 \mathrm{~mL})$, dried with $\mathrm{Na}_{2} \mathrm{SO}_{4}$, and concentrated in vacuo to afford the crude olefin. Purification by column chromatography (10\% EtOAc-hexanes) afforded olefin $\mathbf{1 f}$ as a white solid $(510.5 \mathrm{mg}, 2.9 \mathrm{mmol}, 97 \%) . \mathrm{R}_{f}=0.65$ (25\% EtOAc-hexanes); ${ }^{1} \mathrm{H}$ NMR $\left(\mathrm{CDCl}_{3}, 400 \mathrm{MHz}\right): \delta 7.79(\mathrm{~d}, J=7.7 \mathrm{~Hz}, 1 \mathrm{H}), 7.38(\mathrm{~d}, J=7.7 \mathrm{~Hz}, 1 \mathrm{H}), 7.24(\mathrm{t}, J=7.7$ $\mathrm{Hz}, 1 \mathrm{H}), 5.22-5.19(\mathrm{~m}, 1 \mathrm{H}), 4.76-4.73(\mathrm{~m}, 1 \mathrm{H}), 2.32(\mathrm{~s}, 3 \mathrm{H}), 2.11-2.09(\mathrm{~m}, 3 \mathrm{H}) ;{ }^{13} \mathrm{C} \mathrm{NMR}\left(\mathrm{CDCl}_{3}, 125 \mathrm{MHz}\right): \delta 173.8$, $145.0,144.8,136.1,134.2,128.9,128.0,126.5,113.9,23.9,19.5$; FTIR (thin film): $\mathrm{cm}^{-1} 2970$ (br), 1689, 1406, 1295, 1271, 894, 761; HRMS-ESI (m/z) Calcd for $\left(\mathrm{C}_{11} \mathrm{H}_{11} \mathrm{O}_{2}\right)\left([\mathrm{M}-\mathrm{H}]^{-}\right)$: 175.0765; found: 175.0764 .

\section{Triisopropenylboroxine (1f-iii).}

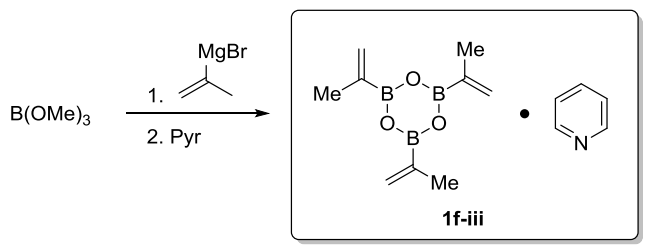

Following a slight modification of a previously reported procedure, ${ }^{4}$ isopropenylmagnesium bromide $(0.32 \mathrm{M}$ in THF, 50 $\mathrm{mL}, 16 \mathrm{mmol}, 1.0$ equiv) was added dropwise over $1 \mathrm{~h}$ to a solution of trimethyl borate (3.2 $\mathrm{mL}, 1.8 \mathrm{equiv})$ in THF (30 $\mathrm{mL})$ at $-78{ }^{\circ} \mathrm{C}$. The reaction was allowed to stir at $-78{ }^{\circ} \mathrm{C}$ for $1 \mathrm{~h}$. The reaction was quenched with aqueous $\mathrm{HCl}(2 \mathrm{M}, 5$ $\mathrm{mL})$ and allowed to warm to room temperature. The reaction was diluted with brine (10 $\mathrm{mL})$ and the aqueous layer extracted with $\mathrm{Et}_{2} \mathrm{O}\left(20 \mathrm{~mL}\right.$ x 4). The combined organic layers were washed with $\mathrm{DI}_{2} \mathrm{O}(30 \mathrm{~mL})$ and brine $(30 \mathrm{~mL})$, dried with $\mathrm{Na}_{2} \mathrm{SO}_{4}$, and concentrated in vacuo to afford the crude boronic acid residue ( $\left.25 \mathrm{~mL}\right)$. Pyridine (10 mL) was added to this residue and the solution stirred at room temperature overnight. The solution was concentrated in vacuo to 
afford the crude boroxine. Purification by kugelrohr distillation afforded boroxine $\mathbf{1 f}$-iii as a white solid (1.41 g, 5.0 mmol, 94\%). $\mathrm{R}_{f}=0.35$ (25\% EtOAc-hexanes); ${ }^{1} \mathrm{H}$ NMR $\left(\mathrm{CDCl}_{3}, 400 \mathrm{MHz}\right): \delta 8.85-8.82(\mathrm{~m}, 2 \mathrm{H}), 8.02(\mathrm{t}, J=7.7 \mathrm{~Hz}$, $1 \mathrm{H}), 7.63-7.58(\mathrm{~m}, 2 \mathrm{H}), 5.72-5.69(\mathrm{~m}, 1 \mathrm{H}), 5.50-5.46(\mathrm{~m}, 1 \mathrm{H}), 1.80(\mathrm{~s}, 3 \mathrm{H}) ;{ }^{13} \mathrm{C} \mathrm{NMR}\left(\mathrm{CDCl}_{3}, 125 \mathrm{MHz}\right): \delta 146.3(\mathrm{br}$, 3C), 143.5 (2C), 140.8, 125.2 (2C), 124.3 (3C), 20.9 (3C); FTIR (thin film): $\mathrm{cm}^{-1}$ 2951, 1620, 1391, 1337, 1191, 937, 770, 691 .

4-Methoxy-2-(prop-1-en-2-yl)benzoic acid (1g) was synthesized using a previously reported procedure. ${ }^{6}$

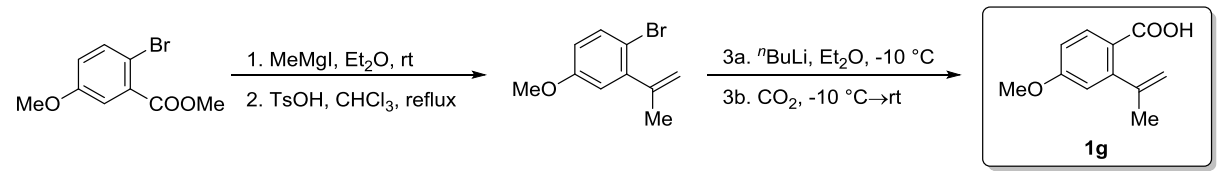

${ }^{1} \mathrm{H}$ NMR $\left(\mathrm{CDCl}_{3}, 400 \mathrm{MHz}\right): \delta 8.00(\mathrm{~d}, J=8.7 \mathrm{~Hz}, 1 \mathrm{H}), 6.85(\mathrm{dd}, J=8.7,2.6 \mathrm{~Hz}, 1 \mathrm{H}), 6.74(\mathrm{~d}, J=2.6 \mathrm{~Hz}, 1 \mathrm{H}), 5.12-5.10$ $(\mathrm{m}, 1 \mathrm{H}), 4.89-4.87(\mathrm{~m}, 1 \mathrm{H}), 3.87(\mathrm{~s}, 3 \mathrm{H}), 2.12-2.09(\mathrm{~m}, 3 \mathrm{H})$.

5-Chloro-2-(prop-1-en-2-yl)benzoic acid (1h).

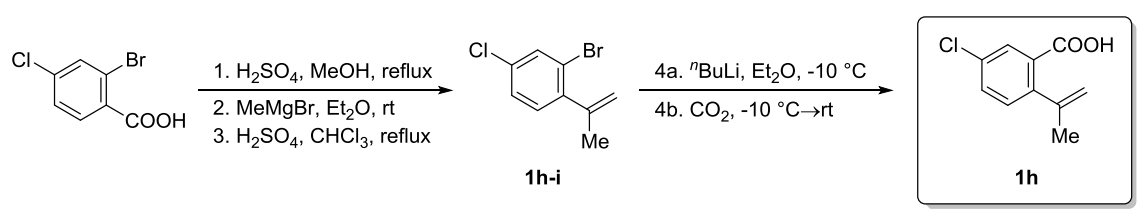

To a solution of 2-bromo-4-chlorobenzoic acid (5.89 g, $25.0 \mathrm{mmol}, 1.0$ equiv) in methanol (80 $\mathrm{mL})$ was added concentrated $\mathrm{H}_{2} \mathrm{SO}_{4}(2.8 \mathrm{~mL}, 2.0$ equiv). The resulting solution was refluxed for $26 \mathrm{~h}$. The reaction was cooled to room temperature and concentrated in vacuo to remove $\sim 50 \%$ of the $\mathrm{MeOH}$. The resulting residue was slowly quenched with a saturated aqueous solution of $\mathrm{NaHCO}_{3}(20 \mathrm{~mL})$. The aqueous layer was extracted with EtOAc $(50 \mathrm{~mL} \times 3)$. The combined organic layers were washed with sat. $\mathrm{NaHCO}_{3}\left(50 \mathrm{~mL}\right.$ x 3), brine $(50 \mathrm{~mL})$, dried with $\mathrm{Na}_{2} \mathrm{SO}_{4}$ and concentrated in vacuo to afford the ester as a clear oil $(6.09 \mathrm{~g})$, which was used without further purification.

The ester (5.74 g, $23.0 \mathrm{mmol}, 1.0$ equiv) was added to a reaction vessel and vacuum purged three times, backfilling with $\mathrm{N}_{2}$. $\mathrm{Et}_{2} \mathrm{O}(46 \mathrm{~mL})$ was added and the solution cooled to $0{ }^{\circ} \mathrm{C}$. To this solution was added $\operatorname{MeMgBr}\left(3 \mathrm{M} \mathrm{in} \mathrm{Et}_{2} \mathrm{O}, 23 \mathrm{~mL}\right.$, $69.0 \mathrm{mmol}$ ) dropwise over $30 \mathrm{~min}$. The solution was stirred for $16 \mathrm{~h}$ at room temperature. The solution was slowly quenched with a saturated aqueous solution of $\mathrm{NH}_{4} \mathrm{Cl}(25 \mathrm{~mL})$, followed by $\mathrm{HCl}(2 \mathrm{M}, 20 \mathrm{~mL})$. The aqueous layer was extracted with EtOAc (30 mL x 3). The combined organic layers were washed with brine (15 mL), dried with $\mathrm{Na}_{2} \mathrm{SO}_{4}$, and concentrated in vacuo to afford the crude alcohol as a tan oil $(5.28 \mathrm{~g})$, which was used without further purification.

To a solution of the above alcohol $\left(1.25 \mathrm{~g}, 5.0 \mathrm{mmol}, 1.0\right.$ equiv) in chloroform $(13 \mathrm{~mL})$ was added concentrated $\mathrm{H}_{2} \mathrm{SO}_{4}(84$ $\mu \mathrm{L}, 0.3$ equiv). The solution was refluxed for $4.5 \mathrm{~h}$. The reaction was quenched with a saturated aqueous solution of $\mathrm{NaHCO}_{3}(25 \mathrm{~mL})$. The aqueous layer was extracted with EtOAc $(15 \mathrm{~mL}$ x 3). The combined organic layers were washed with brine $(30 \mathrm{~mL})$, dried with $\mathrm{Na}_{2} \mathrm{SO}_{4}$, and concentrated in vacuo to afford the crude olefin. Purification by column chromatography (5\% EtOAc-hexanes) afforded olefin $\mathbf{1 h}-\mathbf{i}$ as a clear oil $\left(924.8 \mathrm{mg}, 4.0 \mathrm{mmol}, 72 \%\right.$ over three steps). $\mathbf{R}_{f}=$ 0.88 (25\% EtOAc-hexanes); ${ }^{1} \mathrm{H}$ NMR $\left(\mathrm{CDCl}_{3}, 400 \mathrm{MHz}\right): \delta 7.56(\mathrm{~d}, J=2.1 \mathrm{~Hz}, 1 \mathrm{H}), 7.24(\mathrm{dd}, J=8.2,2.1 \mathrm{~Hz}, 1 \mathrm{H}), 7.12$ $(\mathrm{d}, J=8.2 \mathrm{~Hz}, 1 \mathrm{H}), 5.25-5.22(\mathrm{~m}, 1 \mathrm{H}), 4.94-4.92(\mathrm{~m}, 1 \mathrm{H}), 2.11-2.04(\mathrm{~m}, 3 \mathrm{H})$. Further characterization not performed due to compound instability.

Olefin $\mathbf{1 h}$-i ( $882.1 \mathrm{mg}, 3.8 \mathrm{mmol}, 1.0$ equiv) was added to a reaction vessel and vacuum purged three times, backfilling with $\mathrm{N}_{2} . \mathrm{Et}_{2} \mathrm{O}(7.5 \mathrm{~mL})$ was added and the solution was cooled to $-10{ }^{\circ} \mathrm{C} . n$-BuLi $(2.5 \mathrm{M}$ in hexanes, $1.83 \mathrm{~mL}, 1.2 \mathrm{equiv})$ was added dropwise over $8 \mathrm{~min}$ and the resulting pale yellow solution was held at $-10{ }^{\circ} \mathrm{C}$ for 20 min. $\mathrm{CO}_{2}$ was bubbled through the solution for $15 \mathrm{~min}$. at $-10{ }^{\circ} \mathrm{C}$ and the resulting yellow solution was allowed to reach room temperature over 1 h. The reaction was quenched with a saturated aqueous solution of $\mathrm{NaHCO}_{3}(10 \mathrm{~mL})$. The organic layer was extracted with aqueous $\mathrm{NaOH}(2 \mathrm{M}, 15 \mathrm{~mL}$ x 3). The combined aqueous layers were acidified with concentrated $\mathrm{HCl}$ to $\mathrm{pH} 1$ extracted with EtOAc (30 mL x 3). The combined organic layers were washed with brine $(25 \mathrm{~mL})$, dried with $\mathrm{Na}_{2} \mathrm{SO}_{4}$, and concentrated in vacuo to yield the crude acid. Purification by column chromatography (10\% EtOAc-hexanes) afforded acid $\mathbf{1 h}$ as a white solid (499.9 mg, $2.54 \mathrm{mmol}, 49 \%) . \mathrm{R}_{f}=0.55\left(25 \%\right.$ EtOAc-hexanes); ${ }^{1} \mathrm{H} \mathrm{NMR}(\mathrm{CDCl}, 400 \mathrm{MHz}): \delta$ $7.93(\mathrm{~d}, J=2.3 \mathrm{~Hz}, 1 \mathrm{H}), 7.47(\mathrm{dd}, J=8.2,2.3 \mathrm{~Hz}, 1 \mathrm{H}), 7.21(\mathrm{~d}, J=8.2 \mathrm{~Hz}, 1 \mathrm{H}), 5.17-5.13(\mathrm{~m}, 1 \mathrm{H}), 4.90-4.87(\mathrm{~m}, 1 \mathrm{H})$, 2.12-2.07 (m, 3H); ${ }^{13} \mathrm{C}$ NMR $\left(\mathrm{CDCl}_{3}, 125 \mathrm{MHz}\right): \delta 172.2,145.3,144.6,132.9,132.6,131.2,130.6,129.3,114.6,24.1$; 
FTIR (thin film): $\mathrm{cm}^{-1} 2872$ (br), 1690, 1415, 1289, 1248, 1108, 896, 832, 744, 556; HRMS-ESI (m/z) Calcd for $\left(\mathrm{C}_{10} \mathrm{H}_{8} \mathrm{ClO}_{2}\right)\left([\mathrm{M}-\mathrm{H}]^{-}\right): 195.0218$; found: 195.0216 .

\section{4-Phenylpent-4-enoic acid (1i).}

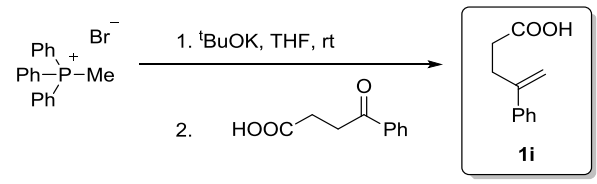

To a suspension of methyltriphenylphosphonium bromide $(39.1 \mathrm{~g}, 109.5 \mathrm{mmol})$ in THF (200 mL) was added sodium tertbutoxide $(21.0 \mathrm{~g}, 218.9 \mathrm{mmol})$. The mixture was stirred at room temperature for $0.5 \mathrm{~h} .3$-Benzoylpropionic acid (15.0 g, $84.2 \mathrm{mmol}$ ) was added and the reaction was stirred at room temperature overnight. Solvent was removed in vacuo and the residue diluted with dichloromethane $(100 \mathrm{~mL})$ and aqueous $\mathrm{NaOH}(1 \mathrm{M}, 100 \mathrm{~mL})$. The aqueous layer was separated, washed with dichloromethane $(50 \mathrm{~mL})$, and acidified to $\mathrm{pH} 1$ with concentrated $\mathrm{HCl}$. Olefin 1i was obtained in quantitative yield via Büchner filtration. $\mathrm{R}_{f}=0.32$ (10\% EtOAc-hexanes); ${ }^{1} \mathrm{H} \mathrm{NMR}\left(\mathrm{CDCl}_{3}, 500 \mathrm{MHz}\right): \delta 7.43(\mathrm{~d}, J=8.0$ $\mathrm{Hz}, 2 \mathrm{H}), 7.36(\mathrm{dd}, J=8.0,7.2 \mathrm{~Hz}, 2 \mathrm{H}), 7.30(\mathrm{t}, J=7.2 \mathrm{~Hz}, 1 \mathrm{H}), 5.35(\mathrm{~s}, 1 \mathrm{H}), 5.13(\mathrm{~s}, 1 \mathrm{H}), 2.87(\mathrm{t}, J=7.6 \mathrm{~Hz}, 2 \mathrm{H}), 2.58(\mathrm{t}$, $J=7.6 \mathrm{~Hz}, 2 \mathrm{H}) ;{ }^{13} \mathrm{C} \mathrm{NMR}\left(\mathrm{CDCl}_{3}, 125 \mathrm{MHz}\right): \delta 179.9,146.5,140.3,128.4,127.8,126.0,112.9,33.0$, 30.1; FTIR (thin film): $\mathrm{cm}^{-1} 2922$ (br), 1697, 1625, 1443, 1341, 1222, 899, 701; HRMS-ESI (m/z) Calcd for $\left(\mathrm{C}_{11} \mathrm{H}_{11} \mathrm{O}_{2}\right)\left([\mathrm{M}-\mathrm{H}]^{-}\right)$: 175.0765; found: 175.0762 .

2,2-Dimethyl-4-phenylpent-4-enoic acid (1j) was synthesized following the same procedure described for 11.

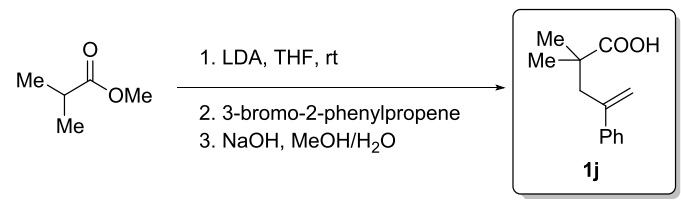

Purification by column chromatography ( $5 \%$ EtOAc-hexanes) gave the product $\mathbf{1 j}$ as a white solid in $44 \%$ yield. $\mathrm{R}_{f}=0.32$ (10\% EtOAc-hexanes); ${ }^{1} \mathrm{H}$ NMR $\left(\mathrm{CDCl}_{3}, 400 \mathrm{MHz}\right): \delta 7.36-7.32(\mathrm{~m}, 2 \mathrm{H}), 7.29-7.18(\mathrm{~m}, 3 \mathrm{H}), 5.26(\mathrm{~d}, J=1.6 \mathrm{~Hz}, 1 \mathrm{H})$, $5.08(\mathrm{~d}, J=1.6 \mathrm{~Hz}, 1 \mathrm{H}), 2.80(\mathrm{~s}, 2 \mathrm{H}), 1.09(\mathrm{~s}, 3 \mathrm{H}) ;{ }^{13} \mathrm{C} \mathrm{NMR}\left(\mathrm{CDCl}_{3}, 100 \mathrm{MHz}\right): \delta 184.4,145.7,142.2,128.0(2 \mathrm{C}), 127.3$, 126.6 (2C), 117.1, 45.1, 42.7, 25.0 (2C); FTIR (thin film): $\mathrm{cm}^{-1}$ 2974, 1696, 1473, 1293, 1161, 903, 774, 713, 578; HRMSESI (m/z) Calcd for $\left(\mathrm{C}_{13} \mathrm{H}_{16} \mathrm{O}_{2}\right)\left([\mathrm{M}-\mathrm{H}]^{-}\right): 203.1078$; found: 203.1076.

\section{4-Methylpent-4-enoic acid (1k).}

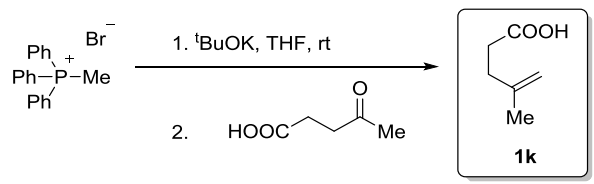

To a suspension of methyltriphenylphophonium bromide (5.75 g, 1.6 equiv) in THF (23 mL) was added a suspension of potassium tert-butoxide (2.65 g, 2.4 equiv) in THF $(12 \mathrm{~mL})$. The resulting yellow solution was stirred at room temperature for $1.5 \mathrm{~h}$. Levulinic acid (1.16 g, $10.0 \mathrm{mmol}, 1.0$ equiv) was added and the solution was refluxed overnight. The reaction was quenched with acetic acid $(0.6 \mathrm{~mL})$ and filtered through a plug of Celite. The resulting mixture was concentrated in vacuo and was diluted with EtOAc $(25 \mathrm{~mL})$. The organic layer was extracted with a saturated aqueous solution of $\mathrm{NaHCO}_{3}(25 \mathrm{~mL}$ x 3). The combined aqueous layers were acidified to $\mathrm{pH} 1$ with concentrated $\mathrm{HCl}$ and extracted with EtOAc $(50 \mathrm{~mL})$. The organic layer was washed with water $(50 \mathrm{~mL})$, brine $(50 \mathrm{~mL})$, dried with $\mathrm{Na}_{2} \mathrm{SO}_{4}$, and concentrated in vacuo to afford the crude olefin. Purification by column chromatography (25\% EtOAc-hexanes) afforded olefin $\mathbf{1 k}$ as a clear oil (667.2 mg, $5.8 \mathrm{mmol}, 58 \%) . \mathrm{R}_{f}=0.52$ (25\% EtOAc-hexanes); ${ }^{1} \mathrm{H} \mathrm{NMR}\left(\mathrm{CDCl}_{3}, 400 \mathrm{MHz}\right): \delta 4.78-4.75(\mathrm{~m}, 1 \mathrm{H})$, $4.72-4.70(\mathrm{~m}, 1 \mathrm{H}), 2.51(\mathrm{dd}, J=8.8,6.8 \mathrm{~Hz}, 2 \mathrm{H}), 2.37-2.31(\mathrm{~m}, 2 \mathrm{H}), 1.77-1.74(\mathrm{~m}, 3 \mathrm{H}) ;{ }^{13} \mathrm{C} \mathrm{NMR}\left(\mathrm{CDCl}_{3}, 125 \mathrm{MHz}\right): \delta$ 180.0, 143.7, 110.5, 32.4, 32.2, 22.5; FTIR (thin film): $\mathrm{cm}^{-1} 2915$ (br), 1707, 1651, 1443, 1290, 1290, 890; HRMS-ESI $(\mathrm{m} / \mathrm{z})$ Calcd for $\left(\mathrm{C}_{6} \mathrm{H}_{9} \mathrm{O}_{2}\right)\left([\mathrm{M}-\mathrm{H}]^{-}\right): 113.0608$; found: 113.0606 .

\section{2,2,4-Trimethylpent-4-enoic acid (11).}




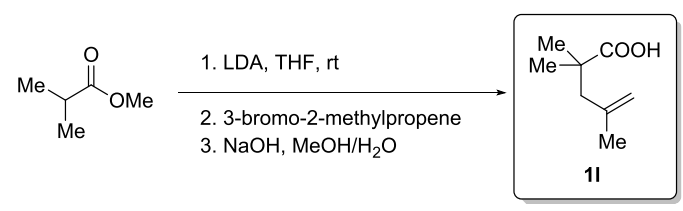

To a solution of LDA (18.8 mL, $2 \mathrm{M}$ in THF, 1.25 equiv) at room temperature was added methyl iso-butyrate (3.5 mL, 30 mmol, 1 equiv). The resulting mixture was stirred at room temperature for $0.5 \mathrm{~h}$. Then, 3-bromo-2-methylpropene (5 g, $37.5 \mathrm{mmol}, 1.25$ equiv) was added at room temperature. The resulting mixture was stirred at $60{ }^{\circ} \mathrm{C}$ overnight. The reaction was quenched with $\mathrm{HCl}(2 \mathrm{M}, 10 \mathrm{~mL})$. The organic layer was separated and the aqueous layer was extracted by EtOAc. The combined organic extracts were concentrated in vacuo. The residue was diluted in aqueous $\mathrm{NaOH}(6 \mathrm{~g}, 150 \mathrm{mmol}, 5$ equiv), $\mathrm{MeOH}(20 \mathrm{~mL})$, and $\mathrm{H}_{2} \mathrm{O}(5 \mathrm{~mL})$. The resulting mixture was stirred at $60{ }^{\circ} \mathrm{C}$ for $5 \mathrm{~h}$. The solvent was removed in vacuo. The residue was dissolved in $\mathrm{H}_{2} \mathrm{O}(20 \mathrm{~mL})$ and washed with EtOAc $(20 \mathrm{~mL})$. The aqueous layer was acidified to $\mathrm{pH} 1$ with concentrated $\mathrm{HCl}$ and extracted with EtOAc $(50 \mathrm{~mL}$ x 2). The combined organic layers were washed with water $(50 \mathrm{~mL})$, brine $(50 \mathrm{~mL})$, dried with $\mathrm{Na}_{2} \mathrm{SO}_{4}$, and concentrated in vacuo to afford the crude olefin. Purification by column chromatography (5\% EtOAc-hexanes) afforded olefin 11 as a yellow oil $(2.26 \mathrm{~g}, 15.9 \mathrm{mmol}, 53 \%) . \mathrm{R}_{f}=0.42(10 \%$ EtOAc-hexanes); ${ }^{1} \mathrm{H}$ NMR $\left(\mathrm{CDCl}_{3}, 400 \mathrm{MHz}\right): \delta 4.83-4.80(\mathrm{~m}, 1 \mathrm{H}), 4.70-4.67(\mathrm{~m}, 1 \mathrm{H}), 2.33(\mathrm{~s}, 2 \mathrm{H}), 1.71-1.68(\mathrm{~m}, 3 \mathrm{H})$, $1.20(\mathrm{~s}, 6 \mathrm{H}) ;{ }^{13} \mathrm{C} \mathrm{NMR}\left(\mathrm{CDCl}_{3}, 100 \mathrm{MHz}\right): \delta 185.1,142.2,114.4,48.2,42.0,25.3(2 \mathrm{C}), 23.5 ;$ FTIR (thin film): $\mathrm{cm}^{-1} 2971$, 1696, 1473, 1221, 892, 547; HRMS-ESI (m/z) Calcd for $\left(\mathrm{C}_{8} \mathrm{H}_{13} \mathrm{O}_{2}\right)\left([\mathrm{M}-\mathrm{H}]{ }^{-}\right): 141.0921$; found: 141.0919.

4-Methyl-2,2-diphenylpent-4-enoic acid (1m).
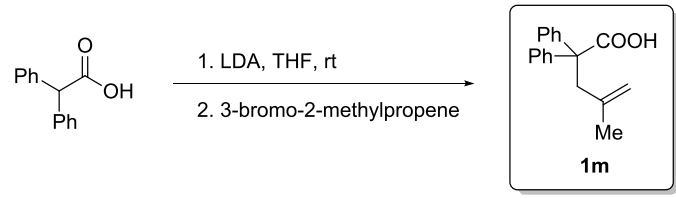

To a solution of LDA (37.5 mL, $2 \mathrm{M}$ in THF, 2.5 equiv) at room temperature was added a solution of 2,2-diphenylacetic acid $(3.2 \mathrm{~g}, 15 \mathrm{mmol})$ in dry THF $(10 \mathrm{~mL})$. The resulting mixture was stirred at room temperature for $0.5 \mathrm{~h}$. Then, $3-$ bromo-2-methylpropene ( $5 \mathrm{~g}, 37.5 \mathrm{mmol}, 1.25$ equiv) was added at room temperature. The resulting mixture was stirred at room temperature overnight. The reaction was quenched with aqueous $\mathrm{HCl}(2 \mathrm{M}, 20 \mathrm{~mL})$. The organic layer was separated and the aqueous layer was extracted by EtOAc. The combined organic layers were washed with water $(50 \mathrm{~mL})$, brine $(50$ $\mathrm{mL}$ ), dried with $\mathrm{Na}_{2} \mathrm{SO}_{4}$, and concentrated in vacuo to afford the crude olefin. Purification by column chromatography (10\% EtOAc-hexanes) afforded olefin $1 \mathrm{~m}$ as a white solid $(2.63 \mathrm{~g}, 9.9 \mathrm{mmol}, 66 \%) . \mathrm{R}_{f}=0.28(10 \% \mathrm{EtOAc}-$ hexanes $) ;{ }^{1} \mathrm{H}$ NMR (CDCl $3,400 \mathrm{MHz}): \delta$ 7.41-7.34 (m, 4H), 7.34-7.23 (m, 6H), $4.74(\mathrm{~s}, 1 \mathrm{H}), 4.59$ (s, 1H), 3.22 (s, $2 \mathrm{H}), 1.36(\mathrm{~s}, 3 \mathrm{H})$; ${ }^{13} \mathrm{C} \mathrm{NMR}\left(\mathrm{CDCl}_{3}, 100 \mathrm{MHz}\right): \delta 180.2,142.6$ (2C), 141.8, 129.0 (4C), 127.8 (4C), 126.9 (2C), 115.5, 60.2, 45.6, 24.3; FTIR (thin film): $\mathrm{cm}^{-1} 2941,1691,1443,1270,1215,939,695 ;$ HRMS-ESI $(\mathrm{m} / \mathrm{z})$ Calcd for $\left(\mathrm{C}_{17} \mathrm{H}_{17}\right)\left(\left[\mathrm{M}_{-} \mathrm{H}-\mathrm{CO}\right]^{-}\right)$: 221.1336; found: 221.1331.

4-Methyl-2-phenylpent-4-enoic acid (1n) was synthesized follow the same procedure described for $1 \mathbf{m}$.

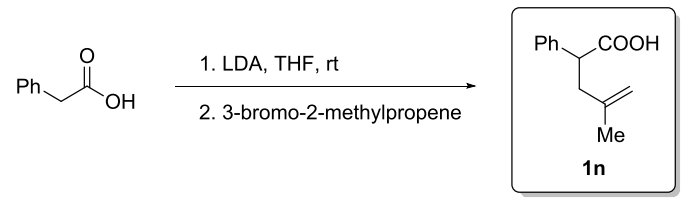

Purification by column chromatography (5\% EtOAc-hexanes) gave the product 1n as a yellow solid in quantitative yield. $\mathrm{R}_{f}=0.28$ (10\% EtOAc-hexanes); ${ }^{1} \mathrm{H}$ NMR $\left(\mathrm{CDCl}_{3}, 400 \mathrm{MHz}\right): \delta 7.40-7.28(\mathrm{~m}, 5 \mathrm{H}), 4.80(\mathrm{~s}, 1 \mathrm{H}), 4.76(\mathrm{~s}, 1 \mathrm{H}), 3.85(\mathrm{dd}, J$ $=8.7,6.7 \mathrm{~Hz}, 1 \mathrm{H}), 2.87(\mathrm{dd}, J=14.7,8.7 \mathrm{~Hz}, 1 \mathrm{H}), 2.49(\mathrm{dd}, J=14.7,6.7 \mathrm{~Hz}, 1 \mathrm{H}), 1.75(\mathrm{~s}, 3 \mathrm{H}) ;{ }^{13} \mathrm{C} \mathrm{NMR}\left(\mathrm{CDCl}{ }_{3}, 125\right.$ MHz): $\delta 180.1,142.1,138.0,128.6$ (2C), 128.0 (2C), 127.5, 112.4, 49.9, 40.7, 22.5; FTIR (thin film): $\mathrm{cm}^{-1} 2909,1692$, 1417, 1288, 1200, 947, 886, 699; HRMS-ESI (m/z) Calcd for $\left(\mathrm{C}_{12} \mathrm{H}_{13} \mathrm{O}_{2}\right)\left([\mathrm{M}-\mathrm{H}]^{-}\right)$: 189.0921; found: 189.0921.

\section{(E)-2-(1-Phenylprop-1-en-1-yl)benzoic acid (10).}




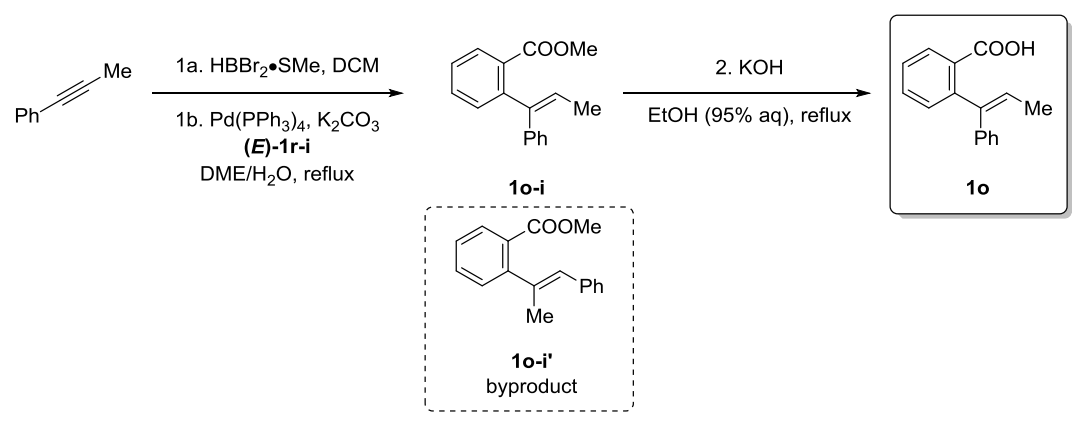

Following a reported procedure, ${ }^{7} 1$-phenyl-1-propyne $(1.25 \mathrm{~mL}, 10.0 \mathrm{mmol}, 1.0$ equiv) was added to a reaction vessel and cooled to $0{ }^{\circ} \mathrm{C}$. $\mathrm{HBBr}_{2} \cdot \mathrm{SMe}\left(1.0 \mathrm{M}\right.$ in DCM, $12.0 \mathrm{~mL}, 1.2$ equiv) was added and the reaction was stirred at $0{ }^{\circ} \mathrm{C}$ for $5 \mathrm{~min}$. The solution was allowed to reach room temperature and stirred for $12 \mathrm{~h}$. The reaction was then refluxed for $2 \mathrm{~h}$. The reaction was removed from heat, slowly quenched with $\mathrm{Et}_{2} \mathrm{O}: \mathrm{H}_{2} \mathrm{O}(2.5: 1,50 \mathrm{~mL})$, and allowed to stir at room temperature for $2 \mathrm{~h}$. The mixture was diluted with $\mathrm{Et}_{2} \mathrm{O}(40 \mathrm{~mL})$, extracted with water $(15 \mathrm{~mL}$ x 3), washed with brine $(15 \mathrm{~mL})$, dried with $\mathrm{Na}_{2} \mathrm{SO}_{4}$, and concentrated in vacuo to afford the crude boronic acid (1.35 g), which was used without further purification.

A solution of ester $(\boldsymbol{E})-\mathbf{1 r}-\mathbf{i}$ (see synthesis of $(\boldsymbol{E})-\mathbf{1 r}) \quad(581.0 \mathrm{mg}, \quad 2.7 \mathrm{mmol}, 1.0$ equiv) and tetrakis (triphenylphosphine)palladium $(31.2 \mathrm{mg}, 0.01$ equiv) in DME $(21.6 \mathrm{~mL})$ was stirred at room temperature for $20 \mathrm{~min}$. At this time, $\mathrm{K}_{2} \mathrm{CO}_{3}$ (373.0 mg, 1.0 equiv), the crude boronic acid above (1.30 g, 3.0 equiv), and DI $\mathrm{H}_{2} \mathrm{O}(6.5 \mathrm{~mL})$ were added and the reaction was refluxed for $3 \mathrm{~h}$. The reaction was cooled to room temperature and quenched with a saturated aqueous solution of $\mathrm{NH}_{4} \mathrm{Cl}(25 \mathrm{~mL})$. The aqueous layer was extracted with $\mathrm{Et}_{2} \mathrm{O}(40 \mathrm{~mL}$ x 3). The combined organic layers were washed with brine $(50 \mathrm{~mL})$, dried with $\mathrm{Na}_{2} \mathrm{SO}_{4}$, and concentrated in vacuo to afford the crude product as a 1.4:1 mixture of regioisomers 1o-i and 1o-i'. Purification by column chromatography (100\% hexanes $\rightarrow 5 \%$ EtOAc-hexanes) afforded olefin 1o-i as an isomerically pure white solid $\left(365.7 \mathrm{mg}, 1.4 \mathrm{mmol}, 45 \%\right.$ over two steps). $\mathrm{R}_{f}=0.82(25 \% \mathrm{EtOAc}-$ hexanes); ${ }^{1} \mathrm{H}$ NMR $\left(\mathrm{CDCl}_{3}, 400 \mathrm{MHz}\right): \delta 7.62(\mathrm{dd}, J=7.6,1.4 \mathrm{~Hz}, 1 \mathrm{H}), 7.41(\mathrm{td}, J=7.4,1.4 \mathrm{~Hz}, 1 \mathrm{H}), 7.32-7.25(\mathrm{~m}, 4 \mathrm{H})$, $7.23-7.14(\mathrm{~m}, 3 \mathrm{H}), 5.84(\mathrm{q}, J=7.1 \mathrm{~Hz}, 1 \mathrm{H}), 3.55(\mathrm{~s}, 3 \mathrm{H}), 1.88(\mathrm{~d}, J=7.1 \mathrm{~Hz}, 3 \mathrm{H}) ;{ }^{13} \mathrm{C} \mathrm{NMR}\left(\mathrm{CDCl}_{3}, 125 \mathrm{MHz}\right): \delta 168.9$, 144.2, 141.7, 139.3, 131.4, 130.9, 130.7, 129.8 (2C), 129.1, 127.5 (2C), 126.7, 126.6, 125.7, 51.6, 15.5; FTIR (thin film): $\mathrm{cm}^{-1}$ 2947, 1721, 1597, 1494, 1441, 1431, 1287, 1250, 1123, 1079, 758, 700; HRMS-ESI (m/z) Calcd for $\left(\mathrm{C}_{17} \mathrm{H}_{17} \mathrm{O}_{2}\right)$ $\left([\mathrm{M}+\mathrm{H}]^{+}\right): 253.1223$; found: 253.1224 .

Following a slight modification from a reported procedure, ${ }^{8}$ olefin 1o-i (302.8 mg, $1.2 \mathrm{mmol}, 1.0$ equiv) was added to a solution of $\mathrm{KOH}$ (268.6 mg, 4.0 equiv) in $95 \%$ aqueous EtOH $(24 \mathrm{~mL})$. The reaction was refluxed for $20.5 \mathrm{~h}$. The reaction was cooled to room temperature and the $\mathrm{EtOH}$ removed in vacuo. The reaction was quenched with a saturated aqueous solution of $\mathrm{NH}_{4} \mathrm{Cl}(10 \mathrm{~mL})$, followed by $\mathrm{HCl}(2 \mathrm{M}, 5 \mathrm{~mL})$. The aqueous layer was extracted with EtOAc (70 mL x 3$)$. The combined organic layers were washed with brine $(50 \mathrm{~mL})$, dried with $\mathrm{Na}_{2} \mathrm{SO}_{4}$, and concentrated in vacuo to afford the crude olefin. Purification by column chromatography (100\% hexanes $\rightarrow 30 \%$ EtOAc-hexanes) afforded olefin 1o as a white solid (263.1 mg, $1.1 \mathrm{mmol}, 92 \%) . \mathrm{R}_{f}=0.63$ (25\% EtOAc-hexanes); ${ }^{1} \mathrm{H} \mathrm{NMR}\left(\mathrm{CDCl}_{3}, 500 \mathrm{MHz}\right): \delta 7.87(\mathrm{~d}, J=7.9$ $\mathrm{Hz}, 1 \mathrm{H}), 7.53(\mathrm{t}, J=7.5 \mathrm{~Hz}, 1 \mathrm{H}), 7.41-7.36(\mathrm{~m}, 2 \mathrm{H}), 7.35-7.29(\mathrm{~m}, 2 \mathrm{H}), 7.28-7.22(\mathrm{~m}, 3 \mathrm{H}), 5.89(\mathrm{q}, J=7.1 \mathrm{~Hz}, 1 \mathrm{H}), 1.95$ $(\mathrm{d}, J=7.1 \mathrm{~Hz}, 3 \mathrm{H}) ;{ }^{13} \mathrm{C}$ NMR $\left(\mathrm{CDCl}_{3}, 125 \mathrm{MHz}\right): \delta 173.9,145.6,141.7,139.4,131.8,131.3,130.1,130.0(2 \mathrm{C}), 129.9$, 127.5 (2C), 126.8, 126.6, 125.9, 15.6; FTIR (thin film): $\mathrm{cm}^{-1} 2912$ (br), 1687, 1596, 1570, 1493, 1405, 1263, 907, 757, 729, 699; HRMS-ESI (m/z) Calcd for $\left(\mathrm{C}_{16} \mathrm{H}_{13} \mathrm{O}_{2}\right)\left([\mathrm{M}-\mathrm{H}]^{-}\right): 237.0921$; found: 237.0918.

\section{2-(2-Methylallyl)benzoic acid (1p).}

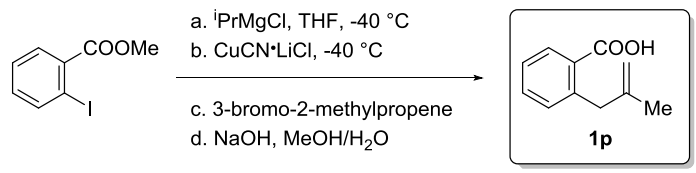

To a solution of methyl 2-iodobenzoate $\left(1.5 \mathrm{~mL}, 10 \mathrm{mmol}, 1.0\right.$ equiv) in THF $(20 \mathrm{~mL})$ at $-40{ }^{\circ} \mathrm{C}$ was added ${ }^{\mathrm{i}} \mathrm{PrMgCl}(1.4$ $\mathrm{M}$ in THF, $11 \mathrm{~mL}, 1.5$ equiv). The mixture was stirred at $-40{ }^{\circ} \mathrm{C}$ for $1 \mathrm{~h}$. A freshly prepared solution of CuCN (896 mg, 10 mmol) and $\mathrm{LiCl}(848 \mathrm{mg}, 20 \mathrm{mmol})$ in THF $(20 \mathrm{~mL})$ was added and stirred for $30 \mathrm{~min}$ at $-40{ }^{\circ} \mathrm{C}$, followed by dropwise addition of 3-bromo-2-methylpropene (3.0 mL, 3.0 equiv). The mixture was allowed to warm to room temperature. After stirring for $2 \mathrm{~h}$, the mixture was filtered through a pale of Celite. A saturated aqueous solution of $\mathrm{NH}_{4} \mathrm{Cl}(50 \mathrm{~mL})$ was added to the filtrate and the aqueous layer was extracted with EtOAc. The combined organic layers were concentrated in 
vacuo. To the resulting residue was added $\mathrm{NaOH}\left(1 \mathrm{~g}, 25 \mathrm{mmol}, 2.5\right.$ equiv), $\mathrm{MeOH}(4 \mathrm{~mL})$ and $\mathrm{H}_{2} \mathrm{O}(1 \mathrm{~mL})$. The resulting mixture was stirred at $60{ }^{\circ} \mathrm{C}$ for $5 \mathrm{~h}$. The solvent was removed in vacuo and the residue was dissolved in $\mathrm{H}_{2} \mathrm{O}(20 \mathrm{~mL})$. The aqueous solution was washed with EtOAc $(20 \mathrm{~mL})$, acidified to $\mathrm{pH} 1$ with concentrated $\mathrm{HCl}$, and extracted with EtOAc $(50 \mathrm{~mL} \times 2)$. The combined organic layers were washed with water $(50 \mathrm{~mL})$, brine $(50 \mathrm{~mL}), \mathrm{dried}_{\mathrm{with}} \mathrm{Na}_{2} \mathrm{SO}_{4}$, and concentrated in vacuo to afford the crude olefin. Purification by column chromatography (5\% EtOAc-hexanes) afforded olefin $1 p$ as a white solid $(1.26 \mathrm{~g}, 7.2 \mathrm{mmol}, 72 \%) . \mathrm{R}_{f}=0.32(10 \% \mathrm{EtOAc}-$ hexanes $) ;{ }^{1} \mathrm{H}$ NMR $\left(\mathrm{CDCl}_{3}, 400\right.$ $\mathrm{MHz}): \delta 8.08(\mathrm{~d}, J=7.8 \mathrm{~Hz}, 1 \mathrm{H}), 7.51(\mathrm{t}, J=7.5 \mathrm{~Hz}, 1 \mathrm{H}), 7.37-7.31(\mathrm{~m}, 2 \mathrm{H}), 4.84(\mathrm{~s}, 1 \mathrm{H}), 4.51(\mathrm{~s}, 1 \mathrm{H}), 3.82(\mathrm{~s}, 2 \mathrm{H}), 1.80$ $(\mathrm{s}, 3 \mathrm{H}) ;{ }^{13} \mathrm{C} \mathrm{NMR}\left(\mathrm{CDCl}_{3}, 100 \mathrm{MHz}\right): \delta$ 173.7, 145.4, 142.2, 132.7, 131.6, 131.5, 128.9, 126.3, 111.6, 41.8, 22.9; FTIR (thin film): $\mathrm{cm}^{-1}$ 2968, 1679, 1412, 1311, 1276, 923, 737; HRMS-ESI (m/z) Calcd for $\left(\mathrm{C}_{11} \mathrm{H}_{11} \mathrm{O}_{2}\right)\left([\mathrm{M}-\mathrm{H}]^{-}\right): 175.0765$; found: 175.0762 .

\section{2-(3-Methylbut-3-en-1-yl)benzoic acid (1q).}

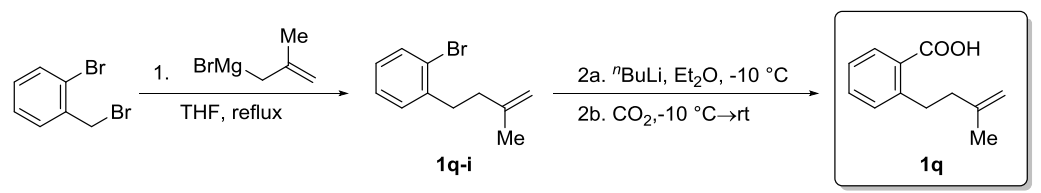

Compound 1q-i was synthesized from 2-bromobenzyl bromide by a previously published procedure. ${ }^{\mathbf{9}} \mathrm{R}_{f}=0.91(25 \%$ EtOAc-hexanes); ${ }^{1} \mathrm{H}$ NMR $\left(\mathrm{CDCl}_{3}, 400 \mathrm{MHz}\right): \delta 7.53(\mathrm{~d}, J=8.0 \mathrm{~Hz}, 1 \mathrm{H}), 7.25-7.21(\mathrm{~m}, 2 \mathrm{H}), 7.09-7.03(\mathrm{~m}, 1 \mathrm{H}), 4.77(\mathrm{~s}$, $1 \mathrm{H}), 4.74(\mathrm{~s}, 1 \mathrm{H}), 2.91-2.84(\mathrm{~m}, 2 \mathrm{H}), 2.35-2.27(\mathrm{~m}, 2 \mathrm{H}), 1.81(\mathrm{~s}, 3 \mathrm{H}) ;{ }^{13} \mathrm{C} \mathrm{NMR}\left(\mathrm{CDCl}_{3}, 125 \mathrm{MHz}\right): \delta 144.9,141.3$, 132.7, 130.2, 127.5, 127.3, 124.3, 110.4, 37.9, 34.7; FTIR (thin film): $\mathrm{cm}^{-1} 2934,1649,1470,1439,1374,1022,886,744$, $658,534$.

Aryl bromide 1q-i (900.5 mg, $4.0 \mathrm{mmol}, 1.0$ equiv) was added to a reaction vessel and vacuum purged three times, backfilling with $\mathrm{N}_{2} . \mathrm{Et}_{2} \mathrm{O}(8 \mathrm{~mL})$ was added and the solution was lowered to $-10{ }^{\circ} \mathrm{C} . n-\mathrm{BuLi}(2.5 \mathrm{M}$ in hexanes, $1.9 \mathrm{~mL}$, 1.2 equiv) was added dropwise and the resulting clear yellow solution was held at $-10{ }^{\circ} \mathrm{C}$ for 20 min. $\mathrm{CO}_{2}$ was bubbled through the solution (instantaneous, bright color changes) for $15 \mathrm{~min}$. at $-10{ }^{\circ} \mathrm{C}$. The resulting clear yellow solution was allowed to reach room temperature over $1 \mathrm{~h}$. The solution was quenched with a saturated aqueous solution of $\mathrm{NaHCO}_{3}(15$ $\mathrm{mL})$. The organic layer was extracted with aqueous $\mathrm{NaOH}(1 \mathrm{M}, 10 \mathrm{~mL}$ x 2). The combined aqueous layers were acidified with $1.0 \mathrm{M} \mathrm{HCl}$ to $\mathrm{pH} 1$ and extracted with EtOAc $\left(15 \mathrm{~mL}\right.$ x 3). The combined organic layers were dried with $\mathrm{Na}_{2} \mathrm{SO}_{4}$ and concentrated in vacuo to yield the crude olefin. Purified by column chromatography (10\% EtOAc-hexanes) afforded olefin 1q as a white solid (416.0 mg, $2.19 \mathrm{mmol}, 55 \%) . \mathrm{R}_{f}=0.28\left(10 \%\right.$ EtOAc-hexanes); ${ }^{1} \mathrm{H} \mathrm{NMR}\left(\mathrm{CDCl}{ }_{3}, 400 \mathrm{MHz}\right): \delta$ $8.06(\mathrm{dd}, J=8.1,1.4 \mathrm{~Hz}, 1 \mathrm{H}), 7.49(\mathrm{td} J=7.6,1.4 \mathrm{~Hz}, 1 \mathrm{H}), 7.33-7.28(\mathrm{~m}, 2 \mathrm{H}), 4.75(\mathrm{~d}, J=0.8 \mathrm{~Hz}, 1 \mathrm{H}), 4.73(\mathrm{~d}, J=0.8$ $\mathrm{Hz}, 1 \mathrm{H}), 3.17(\mathrm{t}, J=8.3 \mathrm{~Hz}, 2 \mathrm{H}), 2.33(\mathrm{t}, J=8.3 \mathrm{~Hz}, 2 \mathrm{H}) ; 1.81(\mathrm{~s}, 3 \mathrm{H}) ;{ }^{13} \mathrm{C} \mathrm{NMR}\left(\mathrm{CDCl}_{3}, 125 \mathrm{MHz}\right): \delta 173.4,145.6$, 145.3, 133.0, 131.7, 131.3, 128.0, 126.0, 110.3, 39.9, 33.4, 22.4; FTIR (thin film): $\mathrm{cm}^{-1} 2932,1688,1602,1575,1454$, 1405, 1301, 1269, 887, 772, 749; HRMS-ESI (m/z) Calcd for $\left(\mathrm{C}_{12} \mathrm{H}_{13} \mathrm{O}_{2}\right)\left([\mathrm{M}-\mathrm{H}]^{-}\right): 189.0921$; found: 189.0921.

(E)-2'-Carboxystyrene ((E)-1r).
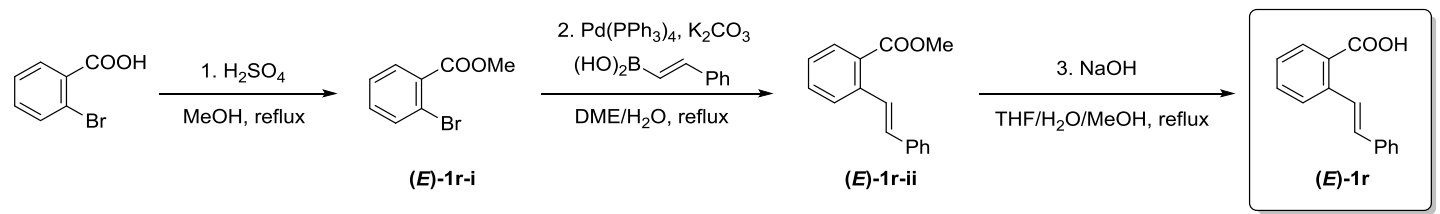

To a solution of 2-bromobenzoic acid (3.02 g, $15.0 \mathrm{mmol}, 1.0$ equiv) in methanol $(45 \mathrm{~mL})$ was added concentrated $\mathrm{H}_{2} \mathrm{SO}_{4}$ ( $1.7 \mathrm{~mL}, 2.0$ equiv). The resulting solution was refluxed for $11 \mathrm{~h}$. The solution was concentrated in vacuo to remove $\sim 75 \%$ of the $\mathrm{MeOH}$. The resulting residue was slowly quenched with a saturated aqueous solution of $\mathrm{NaHCO}_{3}(20 \mathrm{~mL})$. The aqueous layer was extracted with EtOAc $(60 \mathrm{~mL}$ x 3). The combined organic layers were washed brine $(100 \mathrm{~mL})$, dried with $\mathrm{Na}_{2} \mathrm{SO}_{4}$, and concentrated in vacuo to afford the crude ester. Purification by column chromatography (25\% EtOAchexanes) afforded ester $(\boldsymbol{E})-\mathbf{1 r}-\mathbf{i}$ as a clear oil (3.08 g, $14.3 \mathrm{mmol}, 95 \%) . \mathrm{R}_{f}=0.69\left(25 \%\right.$ EtOAc-hexanes); ${ }^{1} \mathrm{H}$ NMR $\left(\mathrm{CDCl}_{3}, 400 \mathrm{MHz}\right): \delta 7.79(\mathrm{dd}, J=7.5,2.0 \mathrm{~Hz}, 1 \mathrm{H}), 7.66(\mathrm{dd}, J=7.7,1.5 \mathrm{~Hz}, 1 \mathrm{H}), 7.39-7.30(\mathrm{~m}, 2 \mathrm{H}), 3.93(\mathrm{~s}, 3 \mathrm{H}) ;{ }^{13} \mathrm{C}$ NMR (CDCl $3,125 \mathrm{MHz}): \delta 166.2,134.0,132.3,131.8,131.0,126.9,121.3,52.1$; FTIR (thin film): $\mathrm{cm}^{-1} 2951,1732,1590$, 1433, 1293, 1253, 1111, 1029, 744; HRMS-ESI (m/z) Calcd for $\left(\mathrm{C}_{8} \mathrm{H}_{8} \mathrm{BrO}_{2}\right)\left([\mathrm{M}+\mathrm{H}]^{+}\right)$: 214.9702; found: 214.9702.

A solution of the purified ester $(\boldsymbol{E})-\mathbf{1 r}-\mathbf{i}(752.6 \mathrm{mg}, 3.5 \mathrm{mmol}, 1.0$ equiv) and tetrakis (triphenylphosphine)palladium (40.4 $\mathrm{mg}, 0.01$ equiv) in DME $(28 \mathrm{~mL})$ was stirred at room temperature for $20 \mathrm{~min}$. At this time, $\mathrm{K}_{2} \mathrm{CO}_{3}(483.7 \mathrm{mg}, 1.0 \mathrm{equiv})$, 
(E)-styrylboronic acid (621.5 g, 1.2 equiv), and $\mathrm{DI} \mathrm{H}_{2} \mathrm{O}(8.4 \mathrm{~mL})$ were added and the reaction was refluxed for $4 \mathrm{~h}$. The reaction was cooled to room temperature and quenched with a saturated aqueous solution of $\mathrm{NH}_{4} \mathrm{Cl}(20 \mathrm{~mL})$. The aqueous layer was extracted with $\mathrm{Et}_{2} \mathrm{O}(25 \mathrm{~mL}$ x 4). The combined organic layers were washed with brine $(50 \mathrm{~mL})$, dried with $\mathrm{Na}_{2} \mathrm{SO}_{4}$, and concentrated in vacuo to afford the crude olefin. Purification by column chromatography (100\% hexanes $\rightarrow$ $5 \%$ EtOAc-hexanes) afforded olefin $(\boldsymbol{E})-\mathbf{1 r}$-ii as a light-orange oil $(745.5 \mathrm{mg}, 3.1 \mathrm{mmol}, 90 \%)$. $\mathrm{R}_{f}=0.75(25 \% \mathrm{EtOAc}-$ hexanes); ${ }^{1} \mathrm{H}$ NMR $\left(\mathrm{CDCl}_{3}, 500 \mathrm{MHz}\right): \delta 7.99(\mathrm{~d}, J=16.2 \mathrm{~Hz}, 1 \mathrm{H}), 7.94(\mathrm{~d}, J=7.8 \mathrm{~Hz}, 1 \mathrm{H}), 7.73(\mathrm{~d}, J=7.8 \mathrm{~Hz}, 1 \mathrm{H}), 7.56$ $(\mathrm{d}, J=7.4 \mathrm{~Hz}, 2 \mathrm{H}), 7.52(\mathrm{t}, J=7.8 \mathrm{~Hz}, 1 \mathrm{H}), 7.37(\mathrm{t}, J=7.4 \mathrm{~Hz}, 2 \mathrm{H}), 7.33(\mathrm{t}, J=7.8 \mathrm{~Hz}, 1 \mathrm{H}), 7.28(\mathrm{t}, J=7.4 \mathrm{~Hz}, 1 \mathrm{H}), 7.02$ $(\mathrm{d}, J=16.2 \mathrm{~Hz}, 1 \mathrm{H}), 3.93(\mathrm{~s}, 3 \mathrm{H}) ;{ }^{13} \mathrm{C} \mathrm{NMR}\left(\mathrm{CDCl}_{3}, 125 \mathrm{MHz}\right): \delta 167.9,139.2,137.4,132.1,131.4,130.6,128.6(2 \mathrm{C})$, 128.5, 127.8, 127.4, 127.1, 127.0, 126.8 (2C), 52.1; FTIR (thin film): $\mathrm{cm}^{-1} 3027,1717,1271,1247,1130,1077,964,760$; HRMS-ESI $(\mathrm{m} / \mathrm{z})$ Calcd for $\left(\mathrm{C}_{16} \mathrm{H}_{15} \mathrm{O}_{2}\right)\left([\mathrm{M}+\mathrm{H}]^{+}\right)$: 239.1067; found: 239.1066 .

Following a modification from a reported procedure, ${ }^{5}$ olefin $(\boldsymbol{E})-\mathbf{1 r}$-ii $(595.7 \mathrm{mg}, 2.5 \mathrm{mmol}, 1.0$ equiv) was added to a solution of $\mathrm{NaOH}\left(1.20 \mathrm{~g}, 12.0\right.$ equiv) in THF: $\mathrm{H}_{2} \mathrm{O}: \mathrm{MeOH}(4: 1: 1,15 \mathrm{~mL})$. The reaction was refluxed for $5 \mathrm{~h}$. The reaction was cooled to room temperature and the MeOH/THF removed in vacuo. The resulting residue was quenched with a saturated aqueous solution of $\mathrm{NH}_{4} \mathrm{Cl}(10 \mathrm{~mL})$. The aqueous layer was extracted with EtOAc $(40 \mathrm{~mL}$ x 3$)$. The combined organic layers were washed with brine $(60 \mathrm{~mL})$, dried with $\mathrm{Na}_{2} \mathrm{SO}_{4}$, and concentrated in vacuo to afford the crude olefin. Purification by column chromatography (100\% hexanes $\rightarrow 15 \%$ EtOAc-hexanes) afforded olefin $(\boldsymbol{E})$-1r as a white solid (481.7 mg, $2.1 \mathrm{mmol}, 86 \%)$. $\mathrm{R}_{f}=0.55$ (25\% EtOAc-hexanes); ${ }^{1} \mathrm{H}$ NMR $\left(\mathrm{CDCl}_{3}, 400 \mathrm{MHz}\right): \delta 8.12-8.09(\mathrm{~m}, 1 \mathrm{H}), 8.08(\mathrm{~d}$, $J=16.2 \mathrm{~Hz}, 1 \mathrm{H}), 7.76(\mathrm{~d}, J=7.5 \mathrm{~Hz}, 1 \mathrm{H}), 7.61-7.55(\mathrm{~m}, 3 \mathrm{H}), 7.41-7.34(\mathrm{~m}, 3 \mathrm{H}), 7.29(\mathrm{t}, J=7.5 \mathrm{~Hz}, 1 \mathrm{H}), 7.04(\mathrm{~d}, J=$ $16.2 \mathrm{~Hz}, 1 \mathrm{H}) ;{ }^{13} \mathrm{C} \mathrm{NMR}\left(\mathrm{CDCl}_{3}, 125 \mathrm{MHz}\right): \delta 173.2,140.2,137.3,133.1,131.8,131.7,128.7(2 \mathrm{C}), 127.9,127.5,127.3$, 127.3, 127.2, 126.9 (2C); FTIR (thin film): $\mathrm{cm}^{-1}$ 3061, 3022 (br), 1683, 1302, 1276, 1250, 962, 759; HRMS-ESI (m/z) Calcd for $\left(\mathrm{C}_{15} \mathrm{H}_{11} \mathrm{O}_{2}\right)\left([\mathrm{M}-\mathrm{H}]^{-}\right): 223.0765$; found: 223.0764 .

\section{(Z)-2'-Carboxystyrene ((Z)-1r).}

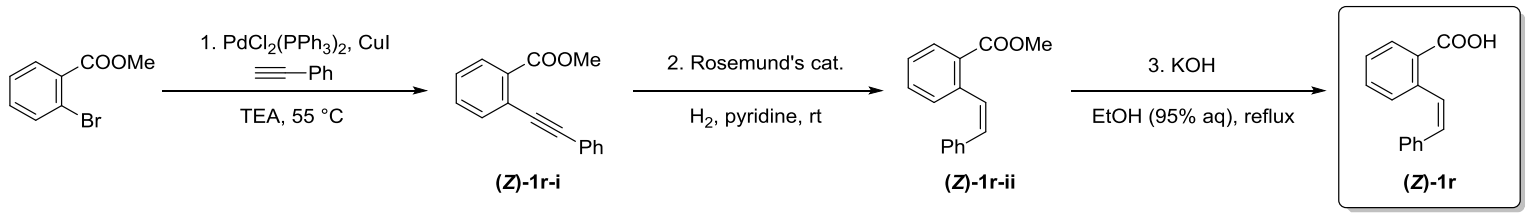

Starting from methyl 2-bromobenzoate (see synthesis of $(\boldsymbol{E})-\mathbf{1 r}),(\boldsymbol{Z})-\mathbf{1 r}-\mathbf{i}$ was synthesized by a previously reported method. ${ }^{8} \mathrm{R}_{f}=0.67$ (25\% EtOAc-hexanes); ${ }^{1} \mathrm{H} \mathrm{NMR}\left(\mathrm{CDCl}_{3}, 500 \mathrm{MHz}\right): \delta 7.89(\mathrm{~d}, J=7.7 \mathrm{~Hz}, 1 \mathrm{H}), 7.56(\mathrm{~d}, J=7.7,1 \mathrm{H})$, $7.52-7.48(\mathrm{~m}, 2 \mathrm{H}), 7.39(\mathrm{td}, J=7.7,1.3 \mathrm{~Hz}, 1 \mathrm{H}), 7.30-7.23(\mathrm{~m}, 4 \mathrm{H}), 3.87(\mathrm{~s}, 3 \mathrm{H}) ;{ }^{13} \mathrm{C} \mathrm{NMR}\left(\mathrm{CDCl}{ }_{3}, 125 \mathrm{MHz}\right): \delta 166.8$, 133.9, 131.7, 131.6 (3C), 130.4, 128.4, 128.3 (2C), 127.8, 123.6, 123.2, 94.3, 88.2, 52.1; FTIR (thin film): $\mathrm{cm}^{-1} 3060$, 1728, 1598, 1494, 1292, 1251, 1127, 1078, 755, 691; HRMS-ESI (m/z) Calcd for $\left(\mathrm{C}_{16} \mathrm{H}_{13} \mathrm{O}_{2}\right)\left([\mathrm{M}+\mathrm{H}]^{+}\right)$: 237.0910; found: 237.0910 .

Following a slight modification from a previously reported procedure, ${ }^{10}$ a solution of alkyne $(\boldsymbol{Z})-\mathbf{1 r}-\mathbf{i}(637.9 \mathrm{mg}, 2.7 \mathrm{mmol}$, 1.0 equiv) in pyridine $(30 \mathrm{~mL})$ was vacuum purged three times, backfilling with $\mathrm{N}_{2}$. Rosemund's catalyst $(5 \%$ Pd on $\mathrm{BaSO}_{4}, 364.0 \mathrm{mg}$ ) was added and the solution was vacuumed purged once more, backfilling with $\mathrm{H}_{2}$. The solution was allowed to stir at room temperature for $4 \mathrm{~h}$ until reaction completion (moitored by TLC). The reaction was vacuum purged and backfilled with $\mathrm{N}_{2}$, upon which the reaction was filtered through Celite, rinsing with EtOAc $(200 \mathrm{~mL})$. The organic solution was concentrated in vacuo to give an orange oil, which was dissolved in $50 \mathrm{~mL}$ EtOAc. The organic solution was washed with $\mathrm{HCl}\left(2 \mathrm{M}, 30 \mathrm{~mL}\right.$ x 2), water $(50 \mathrm{~mL})$, and brine $(50 \mathrm{~mL})$. The organic layer was then dried with $\mathrm{Na}_{2} \mathrm{SO}_{4}$ and concentrated in vacuo to afford the crude olefin. Purification by column chromatography (100\% hexanes $\rightarrow 5 \%$ EtOAchexanes) afforded olefin (Z)-1r-ii as a pale oil $(527.2 \mathrm{mg}, 2.2 \mathrm{mmol}, 82 \%) . \mathrm{R}_{f}=0.56\left(10 \%\right.$ EtOAc-hexanes); ${ }^{1} \mathrm{H}$ NMR $\left(\mathrm{CDCl}_{3}, 400 \mathrm{MHz}\right): \delta 7.98-7.93(\mathrm{~m}, 1 \mathrm{H}), 7.28-7.22(\mathrm{~m}, 1 \mathrm{H}), 7.22(\mathrm{~d}, J=12.0 \mathrm{~Hz}, 1 \mathrm{H}), 7.12-7.05(\mathrm{~m}, 3 \mathrm{H}), 7.04-6.98(\mathrm{~m}$, $3 \mathrm{H}), 6.60(\mathrm{~d}, J=12.0 \mathrm{~Hz}, 1 \mathrm{H}), 3.84(\mathrm{~s}, 3 \mathrm{H}) ;{ }^{13} \mathrm{C} \mathrm{NMR}\left(\mathrm{CDCl}_{3}, 125 \mathrm{MHz}\right): \delta 167.3,139.5,136.6,131.9,131.0,130.5$, 130.3, 129.4, 129.1 (2C), 128.6, 127.9 (2C), 127.0, 126.8, 51.9; FTIR (thin film): $\mathrm{cm}^{-1} 3023,1720,1445,1293,1259$, 1130, 1077, 778; HRMS-ESI (m/z) Calcd for $\left(\mathrm{C}_{16} \mathrm{H}_{15} \mathrm{O}_{2}\right)\left([\mathrm{M}+\mathrm{H}]^{+}\right)$: 239.1067; found: 239.1068 .

Following a slight modification from a reported procedure, ${ }^{8}$ olefin $(\boldsymbol{Z})$-1r-ii $(476.6 \mathrm{mg}, 2.0 \mathrm{mmol}, 1.0$ equiv) was added to a solution of $\mathrm{KOH}(403.0 \mathrm{mg}, 7.2$ equiv) in $95 \%$ aqueous EtOH $(40 \mathrm{~mL})$. The reaction was refluxed for $1.5 \mathrm{~h}$. The reaction was cooled to room temperature and the EtOH removed in vacuo. The reaction was quenched with a saturated aqueous solution of $\mathrm{NH}_{4} \mathrm{Cl}(10 \mathrm{~mL})$, followed by $\mathrm{HCl}(2 \mathrm{M}, 10 \mathrm{~mL})$. The aqueous layer was extracted with EtOAc $(25 \mathrm{~mL} \times 3)$. The combined organic layers were washed with brine $(50 \mathrm{~mL})$, dried with $\mathrm{Na}_{2} \mathrm{SO}_{4}$, and concentrated in vacuo to afford the crude olefin. Purification by column chromatography (100\% hexanes $\rightarrow$ 15\% EtOAc-hexanes) afforded olefin (Z)-1r as a 
white solid (430.9 mg, $1.9 \mathrm{mmol}, 96 \%) . \mathrm{R}_{f}=0.13$ (10\% EtOAc-hexanes); ${ }^{1} \mathrm{H} \mathrm{NMR}\left(\mathrm{CDCl}_{3}, 500 \mathrm{MHz}\right): \delta 8.30-8.25(\mathrm{~m}$, $1 \mathrm{H}), 7.48-7.44(\mathrm{~m}, 2 \mathrm{H}), 7.40-7.36(\mathrm{~m}, 1 \mathrm{H}), 7.30-7.23(\mathrm{~m}, 4 \mathrm{H}), 7.23-7.19(\mathrm{~m}, 2 \mathrm{H}), 6.81(\mathrm{~d}, J=12.2 \mathrm{~Hz}, 1 \mathrm{H}) ;{ }^{13} \mathrm{C} \mathrm{NMR}$ $\left(\mathrm{CDCl}_{3}, 125 \mathrm{MHz}\right): \delta 172.3,140.6,136.8,132.8,131.5,131.4,130.4,129.9,129.3$ (2C), 128.2, 128.1 (2C), 127.2, 127.0 ; FTIR (thin film): $\mathrm{cm}^{-1} 3025$ (br), 1687, 1445, 1303, 1269, 774, 696; HRMS-ESI (m/z) Calcd for $\left(\mathrm{C}_{15} \mathrm{H}_{11} \mathrm{O}_{2}\right)\left([\mathrm{M}-\mathrm{H}]^{-}\right)$: 223.0765; found: 223.0764 .

\section{(E)-2-(Prop-1-en-1-yl)benzoic acid ((E)-1s).}

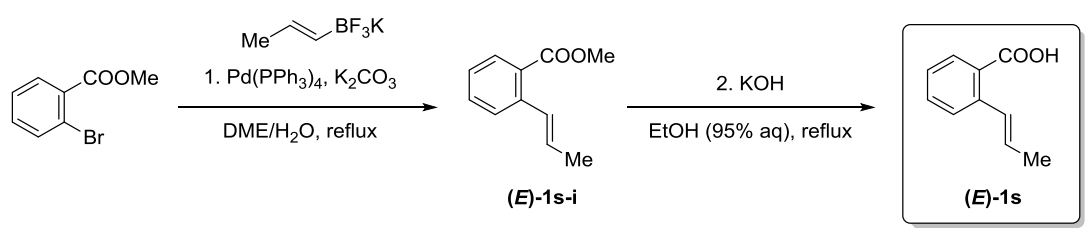

A solution of ester $(\boldsymbol{E})-\mathbf{1 r}-\mathbf{i}$ (see synthesis of $(\boldsymbol{E})-\mathbf{1 r}) \quad(645.1 \mathrm{mg}, \quad 3.0 \mathrm{mmol}, \quad 1.0$ equiv) and tetrakis (triphenylphosphine)palladium (34.7 mg, 0.01 equiv) in DME (24 mL) was stirred at room temperature for $18 \mathrm{~min}$. At this time, $\mathrm{K}_{2} \mathrm{CO}_{3}$ (414.6 mg, 1.0 equiv), potassium trans-1-propenyltrifluoroborate $(665.9 \mathrm{~g}, 1.5 \mathrm{equiv})$, and $\mathrm{DI} \mathrm{H} \mathrm{H}_{2} \mathrm{O}(7.2 \mathrm{~mL})$ were added and the reaction was heated at $90{ }^{\circ} \mathrm{C}$ for $4.5 \mathrm{~h}$. The reaction was cooled to room temperature and quenched with a saturated aqueous solution of $\mathrm{NH}_{4} \mathrm{Cl}(25 \mathrm{~mL})$. The aqueous layer was extracted with $\mathrm{Et}_{2} \mathrm{O}(40 \mathrm{~mL} \times 3)$. The combined organic layers were washed with brine $(50 \mathrm{~mL})$, dried with $\mathrm{Na}_{2} \mathrm{SO}_{4}$, and concentrated in vacuo to afford the crude olefin. Purification by column chromatography (100\% hexanes $\rightarrow$ 5\% EtOAc-hexanes) afforded $(\boldsymbol{E})$-1s-i as a clear oil (343.0 mg, $1.9 \mathrm{mmol}, 65 \%) . \mathrm{R}_{f}=0.83$ (25\% EtOAc-hexanes); ${ }^{1} \mathrm{H}$ NMR $\left(\mathrm{CDCl}_{3}, 400 \mathrm{MHz}\right): \delta 7.83$ (dd, $J=15.6,1.4$ $\mathrm{Hz}, 1 \mathrm{H}), 7.51(\mathrm{dd}, J=15.6,1.1 \mathrm{~Hz}, 1 \mathrm{H}), 7.42(\mathrm{td}, J=15.6,1.4 \mathrm{~Hz}, 1 \mathrm{H}), 7.24(\mathrm{td}, J=15.6,1.1 \mathrm{~Hz}, 1 \mathrm{H}), 7.17(\mathrm{dd}, J=15.6$, $1.7 \mathrm{~Hz}, 1 \mathrm{H}), 6.15(\mathrm{dq}, J=15.6,6.6 \mathrm{~Hz}, 1 \mathrm{H}), 3.88(\mathrm{~s}, 3 \mathrm{H}), 1.92(\mathrm{dd}, J=6.6,1.7 \mathrm{~Hz}, 3 \mathrm{H}) ;{ }^{13} \mathrm{C} \mathrm{NMR}\left(\mathrm{CDCl} \mathrm{N}_{3}, 125 \mathrm{MHz}\right): \delta$ $167.3,139.2,131.4,129.8,129.3,127.9,127.5,126.6,125.9,51.3,18.3$; FTIR (thin film): $\mathrm{cm}^{-1} 2950,1716,1567,1432$, 1281, 1243, 1130, 1098, 1074, 962, 740, 704; HRMS-ESI $(\mathrm{m} / \mathrm{z})$ Calcd for $\left(\mathrm{C}_{11} \mathrm{H}_{13} \mathrm{O}_{2}\right)\left([\mathrm{M}+\mathrm{H}]^{+}\right)$: 177.0910; found: 177.0911.

Following a slight modification from a reported procedure, ${ }^{8}$ olefin $(\boldsymbol{E})-\mathbf{1 s}-\mathbf{i}(317.2 \mathrm{mg}, 1.8 \mathrm{mmol}, 1.0$ equiv) was added to a solution of $\mathrm{KOH}(364.7 \mathrm{mg}, 3.6$ equiv) in $95 \%$ aqueous EtOH $(36 \mathrm{~mL})$. The reaction was refluxed for $2 \mathrm{~h}$. The reaction was cooled to room temperature and the $\mathrm{EtOH}$ removed in vacuo. The reaction was quenched with a saturated aqueous solution of $\mathrm{NH}_{4} \mathrm{Cl}(10 \mathrm{~mL})$, followed by $\mathrm{HCl}(2 \mathrm{M}, 10 \mathrm{~mL})$. The aqueous layer was extracted with EtOAc (25 mL x 3$)$. The combined organic layers were washed with brine $(25 \mathrm{~mL})$, dried with $\mathrm{Na}_{2} \mathrm{SO}_{4}$, and concentrated in vacuo to afford the crude olefin. Purification by column chromatography (100\% hexanes $\rightarrow 20 \%$ EtOAc-hexanes) afforded olefin $(\boldsymbol{E})$-1s as a white solid (265.9 mg, $1.6 \mathrm{mmol}, 91 \%) . \mathrm{R}_{f}=0.63\left(25 \%\right.$ EtOAc-hexanes); ${ }^{1} \mathrm{H} \mathrm{NMR}\left(\mathrm{CDCl}_{3}, 500 \mathrm{MHz}\right): \delta 8.10(\mathrm{dd}, J=$ 7.7, $1.0 \mathrm{~Hz}, 1 \mathrm{H}), 7.59(\mathrm{~d}, J=7.7 \mathrm{~Hz}, 1 \mathrm{H}), 7.51(\mathrm{td}, J=7.7,1.0 \mathrm{~Hz}, 1 \mathrm{H}), 7.39(\mathrm{dd}, J=15.6,1.6 \mathrm{~Hz}, 1 \mathrm{H}), 7.32(\mathrm{td}, J=7.7$, $1.0 \mathrm{~Hz}, 1 \mathrm{H}), 6.22(\mathrm{dq}, J=15.6,6.7 \mathrm{~Hz}, 1 \mathrm{H}), 2.00(\mathrm{dd}, J=6.7,1.6 \mathrm{~Hz}, 3 \mathrm{H}) ;{ }^{13} \mathrm{C} \mathrm{NMR}\left(\mathrm{CDCl}_{3}, 125 \mathrm{MHz}\right): \delta 173.7,140.5$, 132.8, 131.2, 129.7, 128.8, 127.3, 126.5, 126.4, 18.7; FTIR (thin film): $\mathrm{cm}^{-1} 2911$ (br), 1678, 1566, 1480, 1404, 1295, 1252, 961, 791, 737, 662; HRMS-ESI (m/z) Calcd for $\left(\mathrm{C}_{10} \mathrm{H}_{9} \mathrm{O}_{2}\right)\left([\mathrm{M}-\mathrm{H}]^{-}\right)$: 163.0754; found: 163.0754.

\section{(Z)-2-(Prop-1-en-1-yl)benzoic acid ((Z)-1s).}

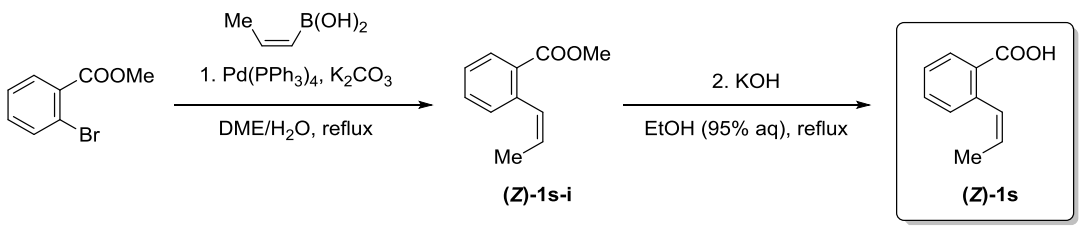

A solution of ester $(\boldsymbol{E})-\mathbf{- 1 r}-\mathbf{i}$ (see synthesis of $(\boldsymbol{E})-1 \mathbf{r}) \quad(860.0 \mathrm{mg}, 4.0 \mathrm{mmol}, 1.0$ equiv) and tetrakis (triphenylphosphine)palladium $(46.2 \mathrm{mg}, 0.01$ equiv) in DME (32 mL) was stirred at room temperature for $20 \mathrm{~min}$. At this time, $\mathrm{K}_{2} \mathrm{CO}_{3}\left(553.0 \mathrm{mg}, 1.0\right.$ equiv), cis-1-propen-1-ylboronic acid (515.0 g, 1.5 equiv), and DI $\mathrm{H}_{2} \mathrm{O}(9.6 \mathrm{~mL})$ were added and the reaction was heated at $70{ }^{\circ} \mathrm{C}$ for $4 \mathrm{~h}$. The reaction was cooled to room temperature and quenched with a saturated aqueous solution of $\mathrm{NH}_{4} \mathrm{Cl}(25 \mathrm{~mL})$. The aqueous layer was extracted with $\mathrm{Et}_{2} \mathrm{O}(40 \mathrm{~mL} \times 3)$. The combined organic layers were washed with brine $(50 \mathrm{~mL})$, dried with $\mathrm{Na}_{2} \mathrm{SO}_{4}$, and concentrated in vacuo to afford the crude olefin. Purification by column chromatography (100\% hexanes $\rightarrow 5 \%$ EtOAc-hexanes) afforded (Z)-1s-i as a clear oil (533.5 mg, $3.0 \mathrm{mmol}, 76 \%) . \mathrm{R}_{f}=0.83\left(25 \%\right.$ EtOAc-hexanes); ${ }^{1} \mathrm{H}$ NMR $\left(\mathrm{CDCl}_{3}, 400 \mathrm{MHz}\right): \delta 7.96-7.92(\mathrm{~m}, 1 \mathrm{H}), 7.47(\mathrm{td}, J=7.5$, $1.4 \mathrm{~Hz}, 1 \mathrm{H}), 7.33-7.27(\mathrm{~m}, 2 \mathrm{H}), 6.89(\mathrm{dd}, J=11.6,1.7 \mathrm{~Hz}, 1 \mathrm{H}), 5.84(\mathrm{dq}, J=11.6,7.0 \mathrm{~Hz}, 1 \mathrm{H}), 3.87(\mathrm{~s}, 3 \mathrm{H}), 1.73(\mathrm{dd}, J=$ 
7.0, 1.7 Hz, 3H); ${ }^{13} \mathrm{C} \mathrm{NMR}\left(\mathrm{CDCl}_{3}, 125 \mathrm{MHz}\right): \delta 167.5,138.6,131.3,130.7,130.2,129.5,129.1,126.4,126.0,51.7,14.1$; FTIR (thin film): $\mathrm{cm}^{-1}$ 2950, 1719, 1568, 1432, 1257, 1129, 1076, 760, 704; HRMS-ESI (m/z) Calcd for $\left(\mathrm{C}_{11} \mathrm{H}_{13} \mathrm{O}_{2}\right)$ $\left([\mathrm{M}+\mathrm{H}]^{+}\right): 177.0910$; found: 177.1910 .

Following a slight modification from a reported procedure, ${ }^{8}$ olefin $(\boldsymbol{Z})-\mathbf{1 s}-\mathbf{i}(475.8 \mathrm{mg}, 2.7 \mathrm{mmol}, 1.0$ equiv) was added to a solution of $\mathrm{KOH}(544.3 \mathrm{mg}, 3.6$ equiv) in $95 \%$ aqueous EtOH $(54 \mathrm{~mL})$. The reaction was refluxed for $2 \mathrm{~h}$. The reaction was cooled to room temperature and the EtOH removed in vacuo. The reaction was quenched with a saturated aqueous solution of $\mathrm{NH}_{4} \mathrm{Cl}(10 \mathrm{~mL})$, followed by $\mathrm{HCl}(2 \mathrm{M}, 5 \mathrm{~mL})$. The aqueous layer was extracted with EtOAc (40 mL x 3$)$. The combined organic layers were washed with brine $(50 \mathrm{~mL})$, dried with $\mathrm{Na}_{2} \mathrm{SO}_{4}$, and concentrated in vacuo to afford the crude olefin. Purification by column chromatography (100\% hexanes $\rightarrow 20 \%$ EtOAc-hexanes) afforded $(\boldsymbol{Z})$-1s as a white solid (428.3 mg, $2.6 \mathrm{mmol}, 98 \%) . \mathrm{R}_{f}=0.63$ (25\% EtOAc-hexanes); ${ }^{1} \mathrm{H} \mathrm{NMR}\left(\mathrm{CDCl}_{3}, 400 \mathrm{MHz}\right): \delta 8.10$ (dd, $J=8.1,1.2$ $\mathrm{Hz}, 1 \mathrm{H}), 7.54(\mathrm{td}, J=7.5,1.0 \mathrm{~Hz}, 1 \mathrm{H}), 7.38-7.33(\mathrm{~m}, 2 \mathrm{H}), 6.96(\mathrm{dd}, J=11.6,1.8 \mathrm{~Hz}, 1 \mathrm{H}), 5.87(\mathrm{dq}, J=11.6,7.1 \mathrm{~Hz}, 1 \mathrm{H})$, $1.76(\mathrm{dd}, J=7.1,1.8 \mathrm{~Hz}, 3 \mathrm{H}) ;{ }^{13} \mathrm{C} \mathrm{NMR}\left(\mathrm{CDCl}_{3}, 125 \mathrm{MHz}\right): \delta 173.2,139.6,132.4,131.4,131.1,129.7,128.0,126.7$, 126.4, 14.3; FTIR (thin film): $\mathrm{cm}^{-1} 2901$ (br), 1674, 1596, 1569, 1485, 1408, 1309, 1278, 1080, 911, 755, 703; HRMS-ESI $(\mathrm{m} / \mathrm{z})$ Calcd for $\left(\mathrm{C}_{10} \mathrm{H}_{9} \mathrm{O}_{2}\right)\left([\mathrm{M}-\mathrm{H}]^{-}\right)$: 161.0608 ; found: 161.0608 .

2-(2-Methylprop-1-en-1-yl)benzoic acid (1t).

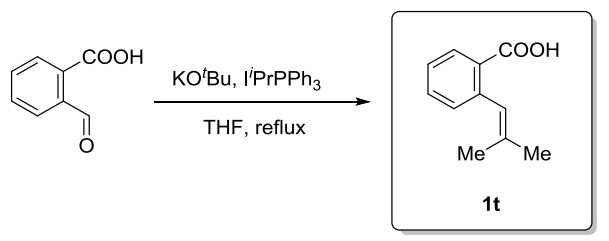

A solution of isopropyltriphenylphosphinium iodide $(6.9 \mathrm{~g}, 2.0$ equiv) and potassium tert-butoxide (2.7 g, 3.0 equiv) in THF $(30 \mathrm{~mL})$ was stirred at room temperature for $1.5 \mathrm{~h}$. 2-Carboxybenzaldehyde $(1.20 \mathrm{~g}, 8.0 \mathrm{mmol}, 1.0$ equiv $)$ was added and the solution was refluxed overnight. The reaction was cooled to room temperature and quenched with a saturated aqueous solution of $\mathrm{NH}_{4} \mathrm{Cl}(30 \mathrm{~mL})$. The solution was diluted with EtOAc $(20 \mathrm{~mL})$ and the organic layer extracted with aqueous $\mathrm{NaOH}(2 \mathrm{M}, 50 \mathrm{~mL} \times 6)$. The combined aqueous layers were acidified with concentrated $\mathrm{HCl}$ to $\mathrm{pH} 1$ and extracted with EtOAc $\left(75 \mathrm{~mL}\right.$ x 6). The combined organic layers were dried with $\mathrm{Na}_{2} \mathrm{SO}_{4}$ and concentrated in vacuo to yield the crude olefin. Purification by column chromatography (100\% hexanes $\rightarrow 10 \%$ EtOAc-hexanes) afforded olefin $\mathbf{1 t}$ as a white solid $(1.30 \mathrm{~g}, 7.4 \mathrm{mmol}, 92 \%) . \mathrm{R}_{f}=0.87$ (25\% EtOAc-hexanes); ${ }^{1} \mathrm{H} \mathrm{NMR}\left(\mathrm{CDCl}_{3}, 400 \mathrm{MHz}\right): \delta 8.05(\mathrm{~d}, J=7.9$ $\mathrm{Hz}, 1 \mathrm{H}), 7.50(\mathrm{t}, J=7.5 \mathrm{~Hz}, 1 \mathrm{H}), 7.33-7.26(\mathrm{~m}, 2 \mathrm{H}), 6.72(\mathrm{~s}, 1 \mathrm{H}), 1.95(\mathrm{~s}, 3 \mathrm{H}), 1.73(\mathrm{~s}, 3 \mathrm{H}) ;{ }^{13} \mathrm{C} \mathrm{NMR}\left(\mathrm{CDCl}_{3}, 125\right.$ MHz): $\delta 173.5,140.8,135.0,132.3,131.5,131.2,128.1,126.1,124.8,26.3,19.3$; FTIR (thin film): $\mathrm{cm}^{-1} 2909$ (br), 1682 , 1598, 1568, 1403, 1300, 1262, 741; HRMS-ESI (m/z) Calcd for $\left(\mathrm{C}_{11} \mathrm{H}_{11} \mathrm{O}_{2}\right)\left([\mathrm{M}-\mathrm{H}]^{-}\right): 175.0765$; found: 175.0763.

\section{2-(2-Cyclopropylvinyl)benzoic acid (1u).}

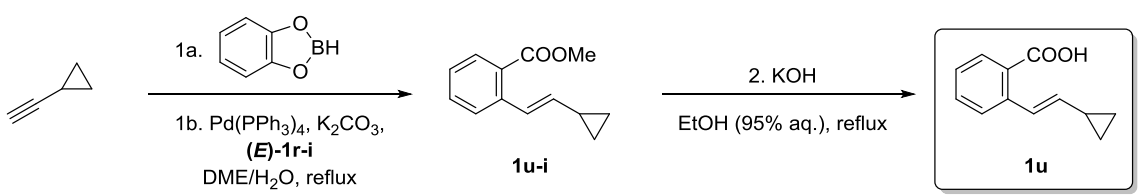

Following a reported procedure, ${ }^{11}$ cyclopropylacetylene $(1.0 \mathrm{~mL}, 12.0 \mathrm{mmol}, 1.0$ equiv) was added to a reaction vessel and vacuum purged three times, backfilling with nitrogen. Catecholborane (1.3 mL, 1.0 equiv) was added and the reaction was heated to $70{ }^{\circ} \mathrm{C}$ and stirred for $3 \mathrm{~h}$. The solution was cooled to room temperature to afford the catecholboronate ester ( 2.22 g, 11.9 mmol, quant.), which was used without further purification.

A solution of ester $(\boldsymbol{E})-\mathbf{1 r}-\mathbf{i}$ (see synthesis of $(\boldsymbol{E})-\mathbf{1 r}) \quad\left(\begin{array}{lllllll}1.29 & \mathrm{~g}, & 6.0 & \mathrm{mmol}, & 1.0 & \text { equiv }) & \text { and tetrakis }\end{array}\right.$ (triphenylphosphine)palladium (138.6 mg, 0.02 equiv) in DME (48 mL) was stirred at room temperature for $20 \mathrm{~min}$. At this time, $\mathrm{K}_{2} \mathrm{CO}_{3}\left(829.0 \mathrm{mg}, 1.0\right.$ equiv), the crude boronate ester above (2.12 g, 1.9 equiv), and $\mathrm{DI} \mathrm{H}_{2} \mathrm{O}(14.4 \mathrm{~mL})$ were added and the reaction was refluxed for $14 \mathrm{~h}$. The reaction was cooled to room temperature and quenched with a saturated aqueous solution of $\mathrm{NH}_{4} \mathrm{Cl}(35 \mathrm{~mL})$. The aqueous layer was extracted with $\mathrm{Et}_{2} \mathrm{O}(50 \mathrm{~mL} \times 3)$. The combined organic layers were washed with brine $(70 \mathrm{~mL})$, dried with $\mathrm{Na}_{2} \mathrm{SO}_{4}$, and concentrated in vacuo to afford the crude olefin. Purification by column chromatography (100\% hexanes $\rightarrow 20 \%$ EtOAc-hexanes) afforded olefin $\mathbf{1 u}$-i as a clear oil (1.16 g, $5.8 \mathrm{mmol}, 96 \%$ over two steps). $\mathrm{R}_{f}=0.80$ (25\% EtOAc-hexanes); ${ }^{1} \mathrm{H}$ NMR $\left(\mathrm{CDCl}_{3}, 400 \mathrm{MHz}\right): \delta 7.83(\mathrm{dd}, J=7.8,1.4$ $\mathrm{Hz}, 1 \mathrm{H}), 7.49(\mathrm{dd}, J=7.8,1.4 \mathrm{~Hz}, 1 \mathrm{H}), 7.40(\mathrm{td}, J=7.8,1.4 \mathrm{~Hz}, 1 \mathrm{H}), 7.27(\mathrm{~d}, J=15.6,1 \mathrm{H}), 7.22(\mathrm{td}, J=7.8,1.4 \mathrm{~Hz}, 1 \mathrm{H})$, 
$5.65(\mathrm{dd}, J=15.6,9.0 \mathrm{~Hz}, 1 \mathrm{H}), 3.90(\mathrm{~s}, 3 \mathrm{H}), 1.68-1.59(\mathrm{~m}, 1 \mathrm{H}), 0.84(\mathrm{ddd}, J=8.0,6.5,4.5 \mathrm{~Hz}, 2 \mathrm{H}), 0.53(\mathrm{dt}, J=6.5,4.5$ $\mathrm{Hz}, 2 \mathrm{H}) ;{ }^{13} \mathrm{C} \mathrm{NMR}\left(\mathrm{CDCl}_{3}, 125 \mathrm{MHz}\right): \delta 167.9,139.3,137.8,131.7,130.2,127.5,126.5,126.0,125.6,51.8,14.8,7.4$ (2C); FTIR (thin film): $\mathrm{cm}^{-1} 3003,1717,1641,1433,1244,1126,1075,953,747$; HRMS-ESI (m/z) Calcd for $\left(\mathrm{C}_{13} \mathrm{H}_{15} \mathrm{O}_{2}\right)$ $\left([\mathrm{M}+\mathrm{H}]^{+}\right): 203.1067$; found: 203.1067.

Following a slight modification from a reported procedure, ${ }^{8}$ olefin $\mathbf{1 u - i}(809.0 \mathrm{mg}, 4.0 \mathrm{mmol}, 1.0$ equiv) was added to a solution of $\mathrm{KOH}(808.0 \mathrm{mg}, 3.6$ equiv) in $95 \%$ aqueous EtOH $(24 \mathrm{~mL})$. The reaction was refluxed for $2 \mathrm{~h}$. The reaction was cooled to room temperature and the $\mathrm{EtOH}$ removed in vacuo. The reaction was quenched with a saturated aqueous solution of $\mathrm{NH}_{4} \mathrm{Cl}(20 \mathrm{~mL})$, followed by $\mathrm{HCl}(2 \mathrm{M}, 10 \mathrm{~mL})$. The aqueous layer was extracted with EtOAc $(70 \mathrm{~mL} \times 3)$. The combined organic layers were washed with brine $(50 \mathrm{~mL})$, dried with $\mathrm{Na}_{2} \mathrm{SO}_{4}$, and concentrated in vacuo to afford the crude olefin. Purification by column chromatography (100\% hexanes $\rightarrow 25 \%$ EtOAc-hexanes) afforded olefin 1u as a white solid (720.5 mg, $3.8 \mathrm{mmol}, 96 \%) . \mathrm{R}_{f}=0.67$ (25\% EtOAc-hexanes); ${ }^{1} \mathrm{H} \mathrm{NMR}\left(\mathrm{CDCl}_{3}, 400 \mathrm{MHz}\right): \delta 8.03(\mathrm{dd}, J=$ $7.9,0.7 \mathrm{~Hz}, 1 \mathrm{H}), 7.53(\mathrm{~d}, J=7.4 \mathrm{~Hz}, 1 \mathrm{H}), 7.48(\mathrm{td}, J=7.4,0.7 \mathrm{~Hz}, 1 \mathrm{H}), 7.41(\mathrm{~d}, J=15.6 \mathrm{~Hz}, 1 \mathrm{H}), 7.30-7.25(\mathrm{~m}, 1 \mathrm{H})$, $5.68(\mathrm{dd}, J=15.6,9.1 \mathrm{~Hz}, 1 \mathrm{H}), 1.73-1.63(\mathrm{~m}, 1 \mathrm{H}), 0.87(\mathrm{ddd}, J=7.9,6.6,4.5 \mathrm{~Hz}, 2 \mathrm{H}), 0.56(\mathrm{dt}, J=6.6,4.5 \mathrm{~Hz}, 2 \mathrm{H}) ;{ }^{13} \mathrm{C}$ NMR (CDCl $3,125 \mathrm{MHz}): \delta 173.7,140.4,138.4,131.4,126.9,126.3,125.9,15.0$, 7.6; FTIR (thin film): $\mathrm{cm}^{-1} 3004$ (br), 1679, 1566, 1403, 1251, 951, 745, 660; HRMS-ESI (m/z) Calcd for $\left(\mathrm{C}_{12} \mathrm{H}_{11} \mathrm{O}_{2}\right)\left([\mathrm{M}+\mathrm{H}]^{+}\right)$: 189.0910; found: 189.0909 .

\section{2-(1-Cyclopropylvinyl)benzoic acid (1v).}

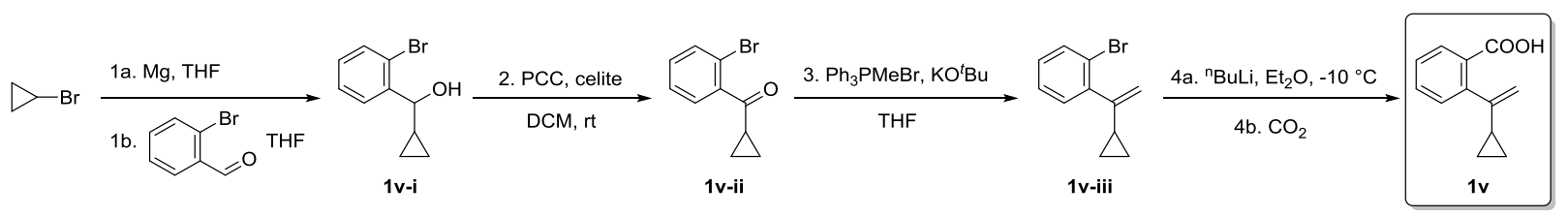

Magnesium turnings (1.03 g, 1.7 equiv) and an iodine spike were added to a reaction vessel and vacuum purged three times, backfilling with $\mathrm{N}_{2}$. THF $(25 \mathrm{~mL})$ was added, followed by dropwise addition of cyclopropyl bromide $(2.0 \mathrm{~mL}, 25$ mmol, 1.0 equiv). The solution was stirred at room temperature for $8 \mathrm{~h}$ at room temperature and Schlenk filtered.

To a vessel vacuum purged three times (backfilling with $\mathrm{N}_{2}$ ) was added THF $(30 \mathrm{~mL})$ and 2-bromobenzaldehyde (1.75 $\mathrm{mL}, 15.0 \mathrm{mmol}, 1.0$ equiv). The solution was cooled to $0{ }^{\circ} \mathrm{C}$. After $10 \mathrm{~min}$, the cyclopropylmagnesium bromide solution was slowly added. The solution was stirred at room temperature for $13 \mathrm{~h}$. The reaction was quenched with a saturated aqueous solution of $\mathrm{NH}_{4} \mathrm{Cl}(20 \mathrm{~mL})$. The aqueous layer was extracted with EtOAc $(30 \mathrm{~mL} \times 3)$. The combined organic layers were washed with brine $(20 \mathrm{~mL})$, dried with $\mathrm{Na}_{2} \mathrm{SO}_{4}$, and concentrated in vacuo to yield the crude alcohol. Purification by column chromatography (5\% EtOAc-hexanes) afforded alcohol 1v-i as a clear oil (2.78 g, $12.2 \mathrm{mmol}$, $49 \%) . \mathrm{R}_{f}=0.62\left(25 \%\right.$ EtOAc-hexanes); ${ }^{1} \mathrm{H} \mathrm{NMR}\left(\mathrm{CDCl}_{3}, 400 \mathrm{MHz}\right): \delta 7.61(\mathrm{dd}, J=7.8,1.5 \mathrm{~Hz}, 1 \mathrm{H}), 7.53(\mathrm{dd}, J=7.8$, $1.0 \mathrm{~Hz}, 1 \mathrm{H}), 7.35(\mathrm{td}, J=7.8,1.0 \mathrm{~Hz}, 1 \mathrm{H}), 7.14(\mathrm{td}, J=7.8,1.5 \mathrm{~Hz}, 1 \mathrm{H}), 4.64(\mathrm{dd}, J=7.6,3.4 \mathrm{~Hz}, 1 \mathrm{H}), 2.15-2.09(\mathrm{~m}$, $1 \mathrm{H}), 1.34-1.24(\mathrm{~m}, 1 \mathrm{H}), 0.67-0.58(\mathrm{~m}, 1 \mathrm{H}), 0.58-0.42(\mathrm{~m}, 3 \mathrm{H}) ;{ }^{13} \mathrm{C} \mathrm{NMR}\left(\mathrm{CDCl}_{3}, 125 \mathrm{MHz}\right): \delta 142.6,132.6,128.9$, 128.1, 127.6, 122.5, 75.4, 17.7, 3.3, 2.0; FTIR (thin film): $\mathrm{cm}^{-1} 3353$ (br), 3007, 1468, 1438, 1014, 772, 750; HRMS-ESI $(\mathrm{m} / \mathrm{z})$ Calcd for $\left(\mathrm{C}_{10} \mathrm{H}_{11} \mathrm{BrO}\right)\left(\left[\mathrm{M}+\mathrm{H}-\mathrm{H}_{2} \mathrm{O}\right]^{+}\right): 208.9960$; found: 208.9960 .

Following a slight modification of a reported procedure, ${ }^{12}$ an anhydrous solution of alcohol 1v-i $(2.27 \mathrm{~g}, 10.0 \mathrm{mmol}, 1.0$ equiv) in DCM $(11.5 \mathrm{~mL})$ was added dropwise over $20 \mathrm{~min}$ to an anhydrous suspension of pyridinium chlorochromate $(4.31 \mathrm{~g}, 2.0$ equiv) and Celite $(4.31 \mathrm{~g})$ in DCM $(61.5 \mathrm{~mL})$. The reaction was stirred for $12 \mathrm{~h}$ at room temperature. The reaction was quenched with DCM $(35 \mathrm{~mL})$ and filtered through a pad of silica. Purified by column chromatography $(25 \%$ EtOAc-hexanes) afforded ketone $\mathbf{1 v}$-ii as an pale oil $(2.13 \mathrm{~g}, 9.47 \mathrm{mmol}, 95 \%) . \mathrm{R}_{f}=0.78(25 \%$ EtOAc-hexanes $) ;{ }^{1} \mathrm{H}$ NMR $\left(\mathrm{CDCl}_{3}, 400 \mathrm{MHz}\right): \delta 7.62(\mathrm{dd}, J=7.6,1.1 \mathrm{~Hz}, 1 \mathrm{H}), 7.46(\mathrm{dd}, J=7.6,1.8 \mathrm{~Hz}, 1 \mathrm{H}), 7.38(\mathrm{td}, J=7.6,1.1 \mathrm{~Hz}, 1 \mathrm{H})$, $7.29(\mathrm{td}, J=7.6,1.8 \mathrm{~Hz}, 1 \mathrm{H}), 2.45(\mathrm{tt}, J=7.8,4.6 \mathrm{~Hz}, 1 \mathrm{H}), 1.36-1.30(\mathrm{~m}, 2 \mathrm{H}), 1.15-1.09(\mathrm{~m}, 2 \mathrm{H}) ;{ }^{13} \mathrm{C} \mathrm{NMR}\left(\mathrm{CDCl}_{3}, 125\right.$ MHz): $\delta 204.3,142.3,133.5,131.5,128.9,127.3,119.1,21.9,13.4$; FTIR (thin film): $\mathrm{cm}^{-1} 2945,1681,1587,1379,1215$, 987, 769, 741; HRMS-ESI (m/z) Calcd for $\left(\mathrm{C}_{10} \mathrm{H}_{10} \mathrm{BrO}\right)\left([\mathrm{M}+\mathrm{H}]^{+}\right)$: 224.9910; found: 224.9909 .

To a suspension of methyltriphenylphosphonium bromide (5.25 g, 1.6 equiv) in THF (21.1 mL) was added a suspension of $\mathrm{KO} t$-Bu (2.68 g, 2.6 equiv) in THF (10.6 mL). The solution was stirred at room temperature for $2.5 \mathrm{~h}$. Ketone 1v-ii (2.07 $\mathrm{g}, 9.2 \mathrm{mmol}, 1.0$ equiv) was slowly added to the ylide solution and refluxed for $15 \mathrm{~h}$. The reaction was quenched with a saturated aqueous solution of $\mathrm{NH}_{4} \mathrm{Cl}(15 \mathrm{~mL})$. The organic layer was extracted with EtOAc $(15 \mathrm{~mL} \times 3)$. The combined organic layers were washed with brine $(30 \mathrm{~mL})$, dried with $\mathrm{Na}_{2} \mathrm{SO}_{4}$, and concentrated in vacuo to yield the crude olefin. Purification by column chromatography (10\% EtOAc-hexanes) afforded olefin 1v-iii as a yellow oil (1.77 g, $8.0 \mathrm{mmol}$, $86 \%) . \mathrm{R}_{f}=0.86\left(25 \%\right.$ EtOAc-hexanes); ${ }^{1} \mathrm{H} \mathrm{NMR}\left(\mathrm{CDCl}_{3}, 400 \mathrm{MHz}\right): \delta 7.68(\mathrm{~d}, J=8.3 \mathrm{~Hz}, 1 \mathrm{H}), 7.38(\mathrm{td}, J=7.6,1.2 \mathrm{~Hz}$, 1H), 7.27-7.21 (m, 2H), $5.30(\mathrm{~s}, 1 \mathrm{H}), 4.99(\mathrm{~d}, J=1.4 \mathrm{~Hz}, 1 \mathrm{H}), 1.87-1.77(\mathrm{~m}, 1 \mathrm{H}), 0.90-0.80(\mathrm{~m}, 2 \mathrm{H}), 0.63-0.56(\mathrm{~m}, 2 \mathrm{H})$; 
${ }^{13} \mathrm{C} \mathrm{NMR}\left(\mathrm{CDCl}_{3}, 125 \mathrm{MHz}\right): \delta 150.8,142.7,132.5,130.5,128.4,126.8,122.6,112.4,16.9,6.7$ (2C); FTIR (thin film): $\mathrm{cm}^{-1} 3083,3008,1628,1468,1426,1023,759,735$.

Olefin 1v-iii (1.56 g, $7.0 \mathrm{mmol}, 1.0$ equiv) was added to a reaction vessel and vacuum purged three times, backfilling with $\mathrm{N}_{2} . \mathrm{Et}_{2} \mathrm{O}(14 \mathrm{~mL})$ was added and the solution was lowered to $-10{ }^{\circ} \mathrm{C} . n$-BuLi $(3.4 \mathrm{~mL}, 2.5 \mathrm{M}$ in hexanes, 1.2 equiv) was added dropwise over $6 \mathrm{~min}$ and the resulting yellow solution was held at $-10{ }^{\circ} \mathrm{C}$ for $20 \mathrm{~min} . \mathrm{CO}_{2}$ was bubbled through the solution for $15 \mathrm{~min}$ at $-10{ }^{\circ} \mathrm{C}$. The resulting yellow solution was allowed to reach room temperature over $1.5 \mathrm{~h}$. The solution was quenched with a saturated aqueous solution of $\mathrm{NaHCO}_{3}(20 \mathrm{~mL})$ and diluted with EtOAc (10 mL). The organic layer was extracted with aqueous $\mathrm{NaOH}(2 \mathrm{M}, 15 \mathrm{~mL}$ x 4). The combined aqueous layers were acidified with concentrated $\mathrm{HCl}$ to $\mathrm{pH} 1$ and extracted with EtOAc $(40 \mathrm{~mL}$ x 3). The combined organic layers were washed with brine $(50 \mathrm{~mL})$, dried with $\mathrm{Na}_{2} \mathrm{SO}_{4}$, and concentrated in vacuo to yield the crude olefin. Purification by column chromatography (10\% EtOAc-hexanes) afforded olefin $1 \mathrm{v}$ as a white solid $(1.03 \mathrm{~g}, 5.5 \mathrm{mmol}, 78 \%) . \mathrm{R}_{f}=0.60\left(25 \%\right.$ EtOAc-hexanes); ${ }^{1} \mathrm{H}$ NMR $\left(\mathrm{CDCl}_{3}, 400 \mathrm{MHz}\right): \delta 7.96(\mathrm{dd}, J=7.5,1.4 \mathrm{~Hz}, 1 \mathrm{H}), 7.49(\mathrm{td}, J=7.5,1.4 \mathrm{~Hz}, 1 \mathrm{H}), 7.37(\mathrm{td}, J=7.5,1.3 \mathrm{~Hz}, 1 \mathrm{H})$, $7.22(\mathrm{dd}, J=7.5,1.3 \mathrm{~Hz}, 1 \mathrm{H}), 5.08-5.04(\mathrm{~m}, 1 \mathrm{H}), 4.90-4.86(\mathrm{~m}, 1 \mathrm{H}), 1.76-1.68(\mathrm{~m}, 1 \mathrm{H}), 0.76-0.71(\mathrm{~m}, 2 \mathrm{H}), 0.52-0.47$ $(\mathrm{m}, 2 \mathrm{H}) ;{ }^{13} \mathrm{C} \mathrm{NMR}\left(\mathrm{CDCl}_{3}, 125 \mathrm{MHz}\right): \delta 173.5,151.7,144.3,132.1,130.4,130.4,128.7,127.0,110.4,17.4,7.1 ;$ FTIR (thin film): $\mathrm{cm}^{-1} 3008$ (br), 1688, 1406, 1298, 1266, 893, 765; HRMS-ESI (m/z) Calcd for $\left(\mathrm{C}_{12} \mathrm{H}_{11} \mathrm{O}_{2}\right)\left([\mathrm{M}-\mathrm{H}]^{-}\right): 187.0765$; found: 187.0764 .

\section{2-(2-Methylprop-1-en-1-yl)benzoic acid (1w).}

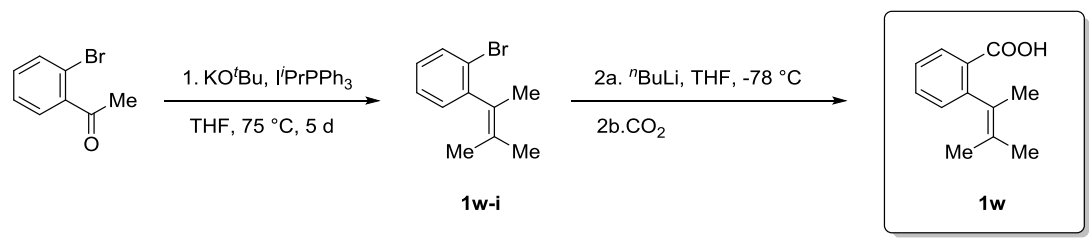

Compound 1w-i was synthesized by a previously published procedure and matched previously reported spectra. ${ }^{13}{ }^{1} \mathrm{H}$ NMR $\left(\mathrm{CDCl}_{3}, 400 \mathrm{MHz}\right): \delta 7.55(\mathrm{~d}, J=8.4 \mathrm{~Hz}, 1 \mathrm{H}), 7.28-7.23(\mathrm{~m}, 1 \mathrm{H}), 7.10-7.04(\mathrm{~m}, 2 \mathrm{H}), 1.89(\mathrm{~s}, 3 \mathrm{H}), 1.81(\mathrm{~s}, 3 \mathrm{H}), 1.43(\mathrm{~s}$, $3 \mathrm{H})$.

Aryl bromide $\mathbf{1 x}-\mathbf{i}$ ( $303.1 \mathrm{mg}, 1.35 \mathrm{mmol}, 1.0$ equiv) was added to a reaction vessel and vacuum purged three times, backfilling with $\mathrm{N}_{2}$. THF $(3.3 \mathrm{~mL})$ was added and the solution was lowered to $-78{ }^{\circ} \mathrm{C}$. After $15 \mathrm{minutes}, n$-BuLi $(2.5 \mathrm{M}$ in hexanes, $0.70 \mathrm{~mL}, 1.3$ equiv) was added dropwise over $10 \mathrm{~min}$ and the resulting clear solution held at $-78{ }^{\circ} \mathrm{C}$ for $30 \mathrm{~min}$. $\mathrm{CO}_{2}$ was bubbled through the solution for $7 \mathrm{~min}$ at $-78{ }^{\circ} \mathrm{C}$. The resulting clear solution was allowed to reach room temperature over 50 minutes, while maintain $\mathrm{CO}_{2}$ bubbling. The solution was quenched with a saturated aqueous solution of $\mathrm{NH}_{4} \mathrm{Cl}(10 \mathrm{~mL})$. The organic layer was extracted with $\mathrm{NaOH}(2 \mathrm{M}, 20 \mathrm{~mL}$ x 4). The combined aqueous layers were acidified with concentrated $\mathrm{HCl}$ to $\mathrm{pH} 1$ and extracted with EtOAc $(25 \mathrm{~mL}$ x 5). The combined organic layers were dried with $\mathrm{Na}_{2} \mathrm{SO}_{4}$ and concentrated in vacuo to yield the crude olefin. Purification by column chromatography (15\% EtOAchexanes) afforded olefin $\mathbf{1 w}$ as a white solid $(229.4 \mathrm{mg}, 1.21 \mathrm{mmol}, 89 \%) . \mathrm{R}_{f}=0.61\left(25 \%\right.$ EtOAc-hexanes); ${ }^{1} \mathrm{H} \mathrm{NMR}$ $\left(\mathrm{CDCl}_{3}, 400 \mathrm{MHz}\right): \delta 8.02(\mathrm{dd}, J=7.7,1.4 \mathrm{~Hz}, 1 \mathrm{H}), 7.50(\mathrm{td}, J=7.7,1.4 \mathrm{~Hz}, 1 \mathrm{H}), 7.32(\mathrm{td}, J=7.7,1.3 \mathrm{~Hz}, 1 \mathrm{H}), 7.12(\mathrm{dd}$, $J=7.7,1.3 \mathrm{~Hz}, 1 \mathrm{H}), 1.96(\mathrm{~s}, 3 \mathrm{H}), 1.82(\mathrm{~s}, 3 \mathrm{H}), 1.43(\mathrm{~s}, 3 \mathrm{H}) ;{ }^{13} \mathrm{C} \mathrm{NMR}\left(\mathrm{CDCl}_{3}, 125 \mathrm{MHz}\right): \delta 173.1,147.4,132.7,130.9$, 130.3, 129.5, 128.4, 126.6, 126.2, 21.8, 20.7, 20.0; FTIR (thin film): $\mathrm{cm}^{-1}$ 2911, 2905 (br), 1693, 1598, 1568, 1484, 1406, 1291, 1260, 927 (br), 761, 712, 650; HRMS-ESI (m/z) Calcd for $\left(\mathrm{C}_{12} \mathrm{H}_{14} \mathrm{O}_{2}\right)\left([\mathrm{M}-\mathrm{H}]^{-}\right)$: 189.0921; found: 189.0921 .

\subsection{Amino lactonization Standard Protocols and Characterization Data of Synthesized Compounds}

\section{Typical Condition A (Amino lactonization Standard Conditions):}

To a $4 \mathrm{~mL}$ vial with Teflon-coated micro stir bar was added carboxylated olefin (0.4 mmol, 1.0 equiv), Oacylhydroxylamine ( $0.8 \mathrm{mmol}, 2.0$ equiv), copper (II) trifluoromethanesulfonate $(7.2 \mathrm{mg}, 0.04 \mathrm{mmol}, 0.01$ equiv), and bathocuproine $(7.2 \mathrm{mg}, 0.02 \mathrm{mmol}, 0.01$ equiv), followed by addition of anhydrous 1,2 -dichloroethane $(2.0 \mathrm{~mL})$. The resulting solution was stirred at $80{ }^{\circ} \mathrm{C}$ and monitored by TLC to consumption of O-acylhydroxylamine. The resulting reaction mixture was filtered through a plug of activated, neutral (Brockman grade I, 58-60^) $\mathrm{Al}_{2} \mathrm{O}_{3}$ and condensed by rotary evaporation to yield the crude product. NMR yields were determined by ${ }^{1} \mathrm{H}$ NMR spectroscopy with dibromomethane as a quantitative internal standard. 


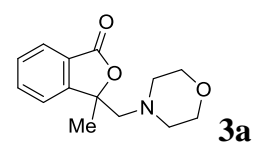

3-Methyl-3-(morpholinomethyl)isobenzofuran-1(3H)-one (3a). Synthesized using condition A. Isolated by Flash Column Chromatography (addition of $0.2 \mathrm{~mL}$ TFA to crude reaction mixture, 25\% EtOAc-hexanes, then $25 \%$ EtOAchexanes with $5 \%$ TEA) as a clear oil $(74.4 \mathrm{mg}, 75 \%) . \mathrm{R}_{f}=0.49(50 \%$ EtOAc-hexanes $) ;{ }^{1} \mathrm{H} \mathrm{NMR}\left(\mathrm{CDCl}_{3}, 400 \mathrm{MHz}\right): \delta$ $7.84(\mathrm{~d}, J=7.5 \mathrm{~Hz}, 1 \mathrm{H}), 7.61(\mathrm{t}, J=7.5 \mathrm{~Hz}, 1 \mathrm{H}), 7.48(\mathrm{t}, J=7.5 \mathrm{~Hz}, 1 \mathrm{H}), 7.38(\mathrm{~d}, J=7.5 \mathrm{~Hz}, 1 \mathrm{H}), 3.50(\mathrm{ddd}, J=11.2$, $6.2,2.9 \mathrm{~Hz}, 2 \mathrm{H}), 3.42(\mathrm{ddd}, J=11.2,6.2,2.9 \mathrm{~Hz}, 2 \mathrm{H}), 2.80(\mathrm{~d}, J=14.3 \mathrm{~Hz}, 1 \mathrm{H}), 2.63(\mathrm{~d}, J=14.3 \mathrm{~Hz}, 1 \mathrm{H}), 2.45(\mathrm{ddd}, J=$ 11.2, 6.2, $2.9 \mathrm{~Hz}, 2 \mathrm{H}), 2.36(\mathrm{ddd}, J=11.2,6.2,2.9 \mathrm{~Hz}, 2 \mathrm{H}), 1.62(\mathrm{~s}, 3 \mathrm{H}) ;{ }^{13} \mathrm{C} \mathrm{NMR}\left(\mathrm{CDCl}_{3}, 125 \mathrm{MHz}\right): \delta 169.7,152.4$, $133.5,128.8,126.7,125.1,121.0,87.5,66.9$ (2C), 65.5, 54.6 (2C), 22.6; FTIR (thin film): $\mathrm{cm}^{-1} 2850,1751,1285,1112$, 1032, 764, 694; HRMS-ESI (m/z) Calcd for $\left(\mathrm{C}_{14} \mathrm{H}_{18} \mathrm{NO}_{3}\right)\left([\mathrm{M}+\mathrm{H}]^{+}\right): 248.1281$; found: 248.1280 .

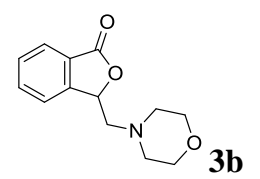

3-(Morpholinomethyl)isobenzofuran-1(3H)-one (3b). Synthesized using condition A, without BCP. Isolated by Flash Column Chromatography (addition of $0.2 \mathrm{~mL}$ TFA to crude reaction mixture, 25\% EtOAc-hexanes, then $25 \%$ EtOAchexanes with 5\% TEA) as clear oil $(39.4 \mathrm{mg}, 42 \%) . \mathrm{R}_{f}=0.10\left(50 \%\right.$ EtOAc-hexanes); ${ }^{1} \mathrm{H} \mathrm{NMR}\left(\mathrm{CDCl}_{3}, 400 \mathrm{MHz}\right): \delta 7.89$ $(\mathrm{d}, J=7.5 \mathrm{~Hz}, 1 \mathrm{H}), 7.67(\mathrm{t}, J=8.0 \mathrm{~Hz}, 1 \mathrm{H}), 7.57-7.51(\mathrm{~m}, 2 \mathrm{H}), 5.57(\mathrm{dd}, J=7.1,4.5 \mathrm{~Hz}, 1 \mathrm{H}), 3.77-3.66(\mathrm{~m}, 4 \mathrm{H}), 2.88$ $(\mathrm{dd}, J=13.7,4.5 \mathrm{~Hz}, 1 \mathrm{H}), 2.77-2.66(\mathrm{~m}, 3 \mathrm{H}), 2.61-2.52(\mathrm{~m}, 2 \mathrm{H}) ;{ }^{13} \mathrm{C} \mathrm{NMR}\left(\mathrm{CDCl}_{3}, 125 \mathrm{MHz}\right): \delta 170.2,148.5,133.9$, $129.3,126.0,125.6,122.3,79.3,66.8$ (2C), 62.3, 54.1 (2C); FTIR (thin film): $\mathrm{cm}^{-1} 2852,1754,1285,1115,1069,1009$, 866, 746, 696; HRMS-ESI (m/z) Calcd for $\left(\mathrm{C}_{13} \mathrm{H}_{16} \mathrm{NO}_{3}\right)\left([\mathrm{M}+\mathrm{H}]^{+}\right): 234.1125$; found: 234.1126.

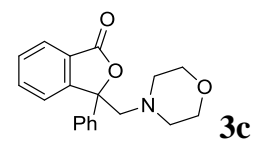

3-(Morpholinomethyl)-3-phenylisobenzofuran-1(3H)-one (3c). Synthesized using condition A. Isolated by Flash Column Chromatography (25\% EtOAc-hexanes) as a light red solid $(101.2 \mathrm{mg}, 82 \%) . \mathrm{R}_{f}=0.59$ (50\% EtOAc-hexanes); ${ }^{1} \mathrm{H}$ NMR $\left(\mathrm{CDCl}_{3}, 400 \mathrm{MHz}\right): \delta 7.91(\mathrm{~d}, J=7.6 \mathrm{~Hz}, 1 \mathrm{H}), 7.66-7.49(\mathrm{~m}, 5 \mathrm{H}), 7.41-7.29(\mathrm{~m}, 3 \mathrm{H}), 3.48-3.40(\mathrm{~m}, 2 \mathrm{H}), 3.38-$ $3.29(\mathrm{~m}, 2 \mathrm{H}), 3.21(\mathrm{~d}, J=14.2 \mathrm{~Hz}, 1 \mathrm{H}), 3.14(\mathrm{~d}, J=14.2 \mathrm{~Hz}, 1 \mathrm{H}), 2.60-2.50(\mathrm{~m}, 2 \mathrm{H}), 2.27-2.17(\mathrm{~m}, 2 \mathrm{H}) ;{ }^{13} \mathrm{C} \mathrm{NMR}$ $\left(\mathrm{CDCl}_{3}, 125 \mathrm{MHz}\right): \delta 169.8,151.0,138.5,133.5,129.1,128.6(2 \mathrm{C}), 128.4,127.2,125.3$ (2C), 125.3, 122.4, 89.7, 67.1 (2C), 65.8, 54.7 (2C); FTIR (thin film): $\mathrm{cm}^{-1}$ 2851, 1762, 1465, 1287, 1116, 1007, 701; HRMS-ESI (m/z) Calcd for $\left(\mathrm{C}_{19} \mathrm{H}_{20} \mathrm{NO}_{3}\right)\left([\mathrm{M}+\mathrm{H}]^{+}\right): 310.1438$; found: 310.1438 .

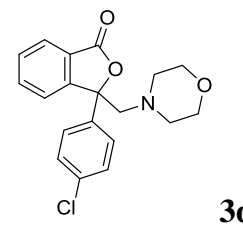

3-(4-Chlorophenyl)-3-(morpholinomethyl)isobenzofuran-1(3H)-one (3d). Synthesized using condition A. Isolated by Flash Column Chromatography (2\%EtOAc-hexanes $\rightarrow 30 \%$ EtOAc-hexanes) as a white solid $(122.0 \mathrm{mg}, 89 \%)$. $\mathrm{R}_{f}=0.61$ (50\% EtOAc-hexanes); ${ }^{1} \mathrm{H} \mathrm{NMR}\left(\mathrm{CDCl}_{3}, 400 \mathrm{MHz}\right): \delta 7.88(\mathrm{~d}, J=7.6 \mathrm{~Hz}, 1 \mathrm{H}), 7.65-7.59(\mathrm{~m}, 1 \mathrm{H}), 7.57-7.44(\mathrm{~m}, 4 \mathrm{H})$, $7.31(\mathrm{~d}, J=8.4 \mathrm{~Hz}, 2 \mathrm{H}), 3.47-3.37(\mathrm{~m}, 2 \mathrm{H}), 3.37-3.26(\mathrm{~m}, 2 \mathrm{H}), 3.15(\mathrm{~d}, J=14.2 \mathrm{~Hz}, 1 \mathrm{H}), 3.06(\mathrm{~d}, J=14.2 \mathrm{~Hz}, 1 \mathrm{H})$, 2.57-2.42 (m, 2H), 2.31-2.15 (m, 2H); ${ }^{13} \mathrm{C} \mathrm{NMR}\left(\mathrm{CDCl}_{3}, 125 \mathrm{MHz}\right): \delta 169.3,150.4,136.9,134.2,133.6,129.2,128.6$ (2C), 126.7 (3C), 125.3, 122.2, 88.9, 66.8 (2C), 65.4, 54.5 (2C); FTIR (thin film): $\mathrm{cm}^{-1}$ 2852, 1758, 1597, 1493, 1286, 1091, 1005, 758; HRMS-ESI (m/z) Calcd for $\left(\mathrm{C}_{19} \mathrm{H}_{19} \mathrm{ClNO}_{3}\right)\left([\mathrm{M}+\mathrm{H}]^{+}\right): 344.1048$; found: 344.1048 .

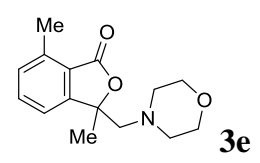

3,7-Dimethyl-3-(morpholinomethyl)isobenzofuran-1(3H)-one (3e). Synthesized using condition A. Isolated by Flash Column Chromatography (addition of $0.2 \mathrm{~mL}$ TFA to crude reaction mixture, $50 \%$ EtOAc-hexanes, then $25 \%$ EtOAchexanes with $5 \%$ TEA) as a clear oil $(80.1 \mathrm{mg}, 77 \%) . \mathrm{R}_{f}=0.54\left(50 \%\right.$ EtOAc-hexanes); ${ }^{1} \mathrm{H} \mathrm{NMR}\left(\mathrm{CDCl}_{3}, 400 \mathrm{MHz}\right): \delta$ 
$7.44(\mathrm{t}, J=7.5 \mathrm{~Hz}, 1 \mathrm{H}), 7.20(\mathrm{~d}, J=7.5 \mathrm{~Hz}, 1 \mathrm{H}), 7.16(\mathrm{~d}, J=7.5 \mathrm{~Hz}, 1 \mathrm{H}), 3.51(\mathrm{ddd}, J=11.1,6.0,3.2 \mathrm{~Hz}, 2 \mathrm{H}), 3.44(\mathrm{ddd}$, $J=11.1,6.0,3.2 \mathrm{~Hz}, 2 \mathrm{H}), 2.75(\mathrm{~d}, J=14.1 \mathrm{~Hz}, 1 \mathrm{H}), 2.65(\mathrm{~s}, 3 \mathrm{H}), 2.58(\mathrm{~d}, J=14.1 \mathrm{~Hz}, 1 \mathrm{H}), 2.47-2.35(\mathrm{~m}, 4 \mathrm{H}), 1.58(\mathrm{~s}$, $3 \mathrm{H}) ;{ }^{13} \mathrm{C} \mathrm{NMR}\left(\mathrm{CDCl}_{3}, 100 \mathrm{MHz}\right): \delta 169.9,153.1,139.2,133.2,130.4,124.1,118.3,86.3,67.0(2 \mathrm{C}), 65.8,54.7(2 \mathrm{C})$, 22.7, 17.2; FTIR (thin film): $\mathrm{cm}^{-1} 2851,1747,1601,1228,1116,1033,698 ;$ HRMS-ESI (m/z) Calcd for $\left(\mathrm{C}_{15} \mathrm{H}_{20} \mathrm{NO}_{3}\right)$ $\left([\mathrm{M}+\mathrm{H}]^{+}\right): 262.1438$; found: 262.1437 .

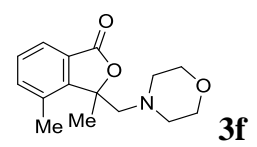

3,4-Dimethyl-3-(morpholinomethyl)isobenzofuran-1(3H)-one (3f). Synthesized using condition A. Isolated by Flash Column Chromatography (addition of $0.2 \mathrm{~mL}$ TFA to crude reaction mixture, $50 \%$ EtOAc-hexanes, then $25 \%$ EtOAchexanes with $5 \%$ TEA) as a clear oil $(78.0 \mathrm{mg}, 75 \%) . \mathrm{R}_{f}=0.46\left(50 \%\right.$ EtOAc-hexanes); ${ }^{1} \mathrm{H} \mathrm{NMR}\left(\mathrm{CDCl}_{3}, 400 \mathrm{MHz}\right): \delta$ $7.69(\mathrm{~d}, J=6.5 \mathrm{~Hz}, 1 \mathrm{H}), 7.40-7.33(\mathrm{~m}, 2 \mathrm{H}), 3.42(\mathrm{ddd}, J=11.1,6.3,2.8 \mathrm{~Hz}, 2 \mathrm{H}), 3.34(\mathrm{ddd}, J=11.1,6.3,2.8 \mathrm{~Hz}, 2 \mathrm{H})$, $2.90(\mathrm{~d}, J=14.4 \mathrm{~Hz}, 1 \mathrm{H}), 2.77(\mathrm{~d}, J=14.4 \mathrm{~Hz}, 1 \mathrm{H}), 2.50-2.39(\mathrm{~m}, 5 \mathrm{H}), 2.30-2.20(\mathrm{~m}, 2 \mathrm{H}), 1.66(\mathrm{~s}, 3 \mathrm{H}) ;{ }^{13} \mathrm{C} \mathrm{NMR}$ $\left(\mathrm{CDCl}_{3}, 125 \mathrm{MHz}\right): \delta 169.9,149.3,135.4,131.5,129.1,127.7,122.8,88.3,66.9$ (2C), 63.3, 54.5 (2C), 21.2, 18.2; FTIR (thin film): $\mathrm{cm}^{-1}$ 2852, 1754, 1454, 1284, 1116, 763; HRMS-ESI (m/z) Calcd for $\left(\mathrm{C}_{15} \mathrm{H}_{20} \mathrm{NO}_{3}\right)\left([\mathrm{M}+\mathrm{H}]^{+}\right): 262.1438 ;$ found: 262.1439 .

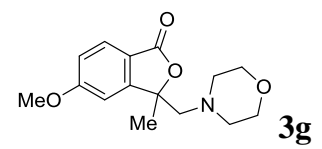

5-Methoxy-3-methyl-3-(morpholinomethyl)isobenzofuran-1(3H)-one (3g). Synthesized using condition A. Isolated by Flash Column Chromatography (addition of $0.2 \mathrm{~mL}$ TFA to crude reaction mixture, 50\% EtOAc-hexanes, then $25 \%$ EtOAc-hexanes with 5\% TEA) as a white solid $(81.3 \mathrm{mg}, 73 \%) . \mathrm{R}_{f}=0.10(25 \%$ EtOAc-hexanes $) ;{ }^{1} \mathrm{H} \mathrm{NMR}\left(\mathrm{CDCl}_{3}, 400\right.$ $\mathrm{MHz}): \delta 7.62(\mathrm{~d}, J=8.5 \mathrm{~Hz}, 1 \mathrm{H}), 6.86(\mathrm{dd}, J=8.5,2.2 \mathrm{~Hz}, 1 \mathrm{H}), 6.69(\mathrm{~d}, J=2.2 \mathrm{~Hz}, 1 \mathrm{H}), 3.75(\mathrm{~s}, 3 \mathrm{H}), 3.47-3.32(\mathrm{~m}, 4 \mathrm{H})$, $2.64(\mathrm{~d}, J=14.2 \mathrm{~Hz}, 1 \mathrm{H}), 2.49(\mathrm{~d}, J=14.2 \mathrm{~Hz}, 1 \mathrm{H}), 2.33(\mathrm{t}, J=4.6 \mathrm{~Hz}, 4 \mathrm{H}), 1.48(\mathrm{~s}, 3 \mathrm{H}) ;{ }^{13} \mathrm{C} \mathrm{NMR}\left(\mathrm{CDCl}_{3}, 125 \mathrm{MHz}\right): \delta$ 169.5, 164.3, 155.4, 126.9, 119.0, 115.8, 105.5, 86.8, 67.0 (2C), 65.7, 55.7, 54.8 (2C), 22.9; FTIR (thin film): $\mathrm{cm}^{-1} 2850$, 1745, 1606, 1490, 1296, 1250, 1114, 1035, 924, 863, 696; HRMS-ESI (m/z) Calcd for $\left(\mathrm{C}_{15} \mathrm{H}_{20} \mathrm{NO}_{4}\right)\left([\mathrm{M}+\mathrm{H}]^{+}\right): 278.1387$; found: 278.1389 .<smiles>CC1(CN2CCOCC2)OC(=O)c2cc(Cl)ccc21</smiles>

6-Chloro-3-methyl-3-(morpholinomethyl)isobenzofuran-1(3H)-one (3h). Synthesized using condition A. Isolated by Flash Column Chromatography (addition of $0.2 \mathrm{~mL}$ TFA to crude reaction mixture, 50\% EtOAc-hexanes, then $25 \%$ EtOAc-hexanes with 5\% TEA) as white solid $(81.6 \mathrm{mg}, 72 \%) . \mathrm{R}_{f}=0.46(50 \% \mathrm{EtOAc}-$ hexanes $) ;{ }^{1} \mathrm{H} \mathrm{NMR}\left(\mathrm{CDCl}_{3}, 400\right.$ $\mathrm{MHz}): \delta 7.82(\mathrm{~s}, 1 \mathrm{H}), 7.58(\mathrm{dd}, J=8.1,1.8 \mathrm{~Hz}, 1 \mathrm{H}), 7.34(\mathrm{~d}, J=8.1 \mathrm{~Hz}, 1 \mathrm{H}), 3.51(\mathrm{ddd}, J=11.1,6.2,2.9 \mathrm{~Hz}, 2 \mathrm{H}), 3.44$ (ddd, $J=11.1,6.2,2.9 \mathrm{~Hz}, 2 \mathrm{H}), 2.79$ (d, $J=14.3 \mathrm{~Hz}, 1 \mathrm{H}), 2.65$ (d, $J=14.3 \mathrm{~Hz}, 1 \mathrm{H}), 2.47$ (ddd, $J=11.2,6.2,2.9 \mathrm{~Hz}, 2 \mathrm{H}$ ), 2.35 (ddd, $J=11.2,6.2,2.9 \mathrm{~Hz}, 2 \mathrm{H}), 1.62(\mathrm{~s}, 3 \mathrm{H}) ;{ }^{13} \mathrm{C} \mathrm{NMR}\left(\mathrm{CDCl}_{3}, 100 \mathrm{MHz}\right): \delta 168.2,150.7,135.2,133.8,128.8$, 125.2, 122.4, 87.6, 67.0 (2C), 65.5, 54.8 (2C), 22.6; FTIR (thin film): $\mathrm{cm}^{-1}$ 2807, 1758, 1423, 1225, 1115, 1067, 837, 647; HRMS-ESI (m/z) Calcd for $\left(\mathrm{C}_{14} \mathrm{H}_{17} \mathrm{ClNO}_{3}\right)\left([\mathrm{M}+\mathrm{H}]^{+}\right)$: 282.0893 ; found: 282.0895 .

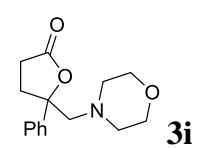

5-(Morpholinomethyl)-5-phenyldihydrofuran-2(3H)-one (3i). Synthesized using condition A, without BCP. Isolated by Flash Column Chromatography (addition of $0.2 \mathrm{~mL}$ TFA to crude reaction mixture, 50\% EtOAc-hexanes, then $25 \%$ EtOAc-hexanes with 5\% TEA) as a light red oil $(47.2 \mathrm{mg}, 45 \%) . \mathrm{R}_{f}=0.17(25 \%$ EtOAc-hexanes $) ;{ }^{1} \mathrm{H} \mathrm{NMR}\left(\mathrm{CDCl}_{3}, 400\right.$ $\mathrm{MHz}): \delta 7.43-7.27(\mathrm{~m}, 5 \mathrm{H}), 3.64(\mathrm{t}, J=4.6 \mathrm{~Hz}, 4 \mathrm{H}), 2.88-2.60(\mathrm{~m}, 6 \mathrm{H}), 2.57-2.45(\mathrm{~m}, 3 \mathrm{H}), 2.38(\mathrm{ddd}, J=11.9,10.0,5.9$ $\mathrm{Hz}, 1 \mathrm{H}) ;{ }^{13} \mathrm{C} \mathrm{NMR}\left(\mathrm{CDCl}_{3}, 125 \mathrm{MHz}\right): \delta 176.6,142.4,128.4(2 \mathrm{C}), 127.8,124.6(2 \mathrm{C}), 89.8,68.1,67.3(2 \mathrm{C}), 55.4(2 \mathrm{C})$, 31.9, 29.4; FTIR (thin film): $\mathrm{cm}^{-1} 2851,1773,1453,1159,1117,1010,703$; HRMS-ESI (m/z) $\mathrm{Calcd}$ for $\left(\mathrm{C}_{15} \mathrm{H}_{20} \mathrm{NO}_{3}\right)$ $\left([\mathrm{M}+\mathrm{H}]^{+}\right): 262.1438$; found: 262.1438 . 


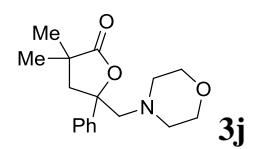

3,3-Dimethyl-5-(morpholinomethyl)-5-phenyldihydrofuran-2(3H)-one (3j). Synthesized using condition A, without BCP. Isolated by Flash Column Chromatography (addition of $0.2 \mathrm{~mL}$ TFA to crude reaction mixture, 50\% EtOAchexanes, then $25 \%$ EtOAc-hexanes with $5 \%$ TEA) as a yellow oil $(60.3 \mathrm{mg}, 53 \%)$. $\mathrm{R}_{f}=0.37(33 \%$ EtOAc-hexanes with $2 \%$ TEA); ${ }^{1} \mathrm{H}$ NMR $\left(\mathrm{CDCl}_{3}, 400 \mathrm{MHz}\right): \delta 7.30-7.24(\mathrm{~m}, 4 \mathrm{H}), 7.23-7.16(\mathrm{~m}, 1 \mathrm{H}), 3.60-3.47(\mathrm{~m}, 4 \mathrm{H}), 2.68-2.55(\mathrm{~m}, 4 \mathrm{H})$, 2.46-2.37 (m, 3H), $2.24\left(\mathrm{~d}, J=12.6 \mathrm{~Hz}, 1.29(\mathrm{~s}, 3 \mathrm{H}), 0.90(\mathrm{~s}, 3 \mathrm{H}) ;{ }^{13} \mathrm{C} \mathrm{NMR}\left(\mathrm{CDCl}_{3}, 100 \mathrm{MHz}\right): \delta 181.6,143.4,128.4\right.$ (2C), 127.5, 124.6 (2C), 86.5, 68.0, 67.2 (2C), 55.2 (2C), 45.5, 40.4, 26.7, 25.8; FTIR (thin film): $\mathrm{cm}^{-1} 2967,2851,2807$, 1761, 1448, 1241, 1115, 1043, 701; HRMS-ESI (m/z) Calcd for $\left(\mathrm{C}_{17} \mathrm{H}_{24} \mathrm{NO}_{3}\right)\left([\mathrm{M}+\mathrm{H}]^{+}\right): 290.1751$; found: 290.1751.

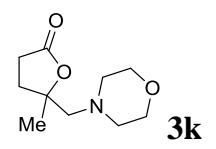

5-Methyl-5-(morpholinomethyl)dihydrofuran-2(3H)-one (3k). Synthesized using condition A, without BCP. Isolated by Flash Column Chromatography (addition of $0.2 \mathrm{~mL}$ TFA to crude reaction mixture, $50 \%$ EtOAc-hexanes, then $25 \%$

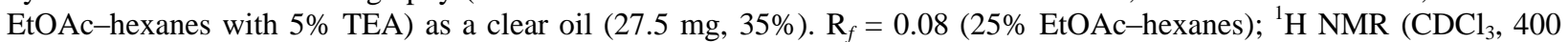
$\mathrm{MHz}): \delta 3.65(\mathrm{t}, J=4.6 \mathrm{~Hz}, 4 \mathrm{H}), 2.73(\mathrm{ddd}, J=17.9,10.5,6.7 \mathrm{~Hz}, 1 \mathrm{H}), 2.65-2.49(\mathrm{~m}, 6 \mathrm{H}), 2.39(\mathrm{~d}, J=14.3 \mathrm{~Hz}, 1 \mathrm{H})$, $2.23(\mathrm{ddd}, J=12.8,10.3,6.7 \mathrm{~Hz}, 1 \mathrm{H}), 1.93(\mathrm{ddd}, J=12.7,10.3,6.7 \mathrm{~Hz}, 1 \mathrm{H}), 1.36(\mathrm{~s}, 3 \mathrm{H}) ;{ }^{13} \mathrm{C} \mathrm{NMR}\left(\mathrm{CDCl}_{3}, 125 \mathrm{MHz}\right): \delta$ 177.0, 87.0, 67.2 (2C), 66.7, 55.4 (2C), 31.5, 29.6, 24.7; FTIR (thin film): $\mathrm{cm}^{-1}$ 2851, 1769, 1163, 1118, 943, 864; HRMSESI $(\mathrm{m} / \mathrm{z})$ Calcd for $\left(\mathrm{C}_{10} \mathrm{H}_{18} \mathrm{NO}_{3}\right)\left([\mathrm{M}+\mathrm{H}]^{+}\right): 200.1281$; found: 200.1279 .

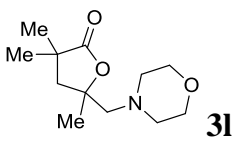

3,3,5-Trimethyl-5-(morpholinomethyl)dihydrofuran-2(3H)-one (3I). Synthesized using condition A, without BCP. Isolated by Flash Column Chromatography (addition of $0.2 \mathrm{~mL}$ TFA to crude reaction mixture, 50\% EtOAc-hexanes, then $25 \%$ EtOAc-hexanes with 5\% TEA) as a colorless oil $(27.2 \mathrm{mg}, 30 \%) . \mathrm{R}_{f}=0.27\left(33 \%\right.$ EtOAc-hexanes with $2 \%$ TEA); ${ }^{1} \mathrm{H}$ NMR $\left(\mathrm{CDCl}_{3}, 400 \mathrm{MHz}\right): \delta 3.70-3.59(\mathrm{~m}, 4 \mathrm{H}), 2.67-2.58(\mathrm{~m}, 2 \mathrm{H}), 2.57-2.48(\mathrm{~m}, 3 \mathrm{H}), 2.26(\mathrm{~d}, J=14.2 \mathrm{~Hz}, 1 \mathrm{H}), 2.23(\mathrm{~d}$, $J=12.8 \mathrm{~Hz}, 1 \mathrm{H}), 1.82(\mathrm{~d}, J=12.8 \mathrm{~Hz}, 1 \mathrm{H}), 1.38(\mathrm{~s}, 3 \mathrm{H}), 1.35(\mathrm{~s}, 3 \mathrm{H}), 1.32(\mathrm{~s}, 3 \mathrm{H}) ;{ }^{13} \mathrm{C} \mathrm{NMR}\left(\mathrm{CDCl}_{3}, 100 \mathrm{MHz}\right): \delta 182.1$, 83.4, 67.1 (2C), 67.0, 55.3 (2C), 45.4, 40.8, 28.3, 26.7, 26.3; FTIR (thin film): $\mathrm{cm}^{-1}$ 2967, 2851, 2807, 1755, 1237, 1115, 942, 862; HRMS-ESI (m/z) Calcd for $\left(\mathrm{C}_{12} \mathrm{H}_{22} \mathrm{NO}_{3}\right)\left([\mathrm{M}+\mathrm{H}]^{+}\right): 228.1594$; found: 228.1594 .

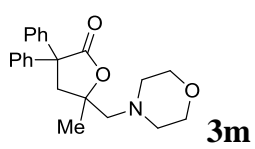

5-Methyl-5-(morpholinomethyl)-3-phenyldihydrofuran-2(3H)-one (3m). Synthesized using condition A. Isolated by Flash Column Chromatography (addition of $0.2 \mathrm{~mL}$ TFA to crude reaction mixture, 50\% EtOAc-hexanes, then $25 \%$ EtOAc-hexanes with 5\% TEA) as a white solid (76.8 mg, 55\%). $\mathrm{R}_{f}=0.27\left(33 \%\right.$ EtOAc-hexanes with $2 \%$ TEA); ${ }^{1} \mathrm{H} \mathrm{NMR}$ $\left(\mathrm{CDCl}_{3}, 400 \mathrm{MHz}\right): \delta 7.48-7.22(\mathrm{~m}, 10 \mathrm{H}), 3.72-3.59(\mathrm{~m}, 4 \mathrm{H}), 3.33(\mathrm{~d}, J=13.3 \mathrm{~Hz}, 1 \mathrm{H}), 2.86(\mathrm{~d}, J=13.3 \mathrm{~Hz}, 1 \mathrm{H}), 2.70-$ $2.58(\mathrm{~m}, 3 \mathrm{H}), 2.58-2.50(\mathrm{~m}, 2 \mathrm{H}), 2.35(\mathrm{~d}, J=14.4 \mathrm{~Hz}, 1 \mathrm{H}), 1.21(\mathrm{~s}, 3 \mathrm{H}) ;{ }^{13} \mathrm{C} \mathrm{NMR}\left(\mathrm{CDCl}_{3}, 125 \mathrm{MHz}\right): \delta 177.1,143.7$, 141.9, 128.7 (2C), 128.3 (2C), 127.8 (2C), 127.6 (2C), 127.3, 127.0, 84.2, 67.1 (2C), 66.4, 58.7, 55.2 (2C), 46.0, 25.0; FTIR (thin film): $\mathrm{cm}^{-1}$ 2968, 2846, 2809, 1751, 1446, 1318, 1140, 890, 697; HRMS-ESI (m/z) Calcd for $\left(\mathrm{C}_{22} \mathrm{H}_{26} \mathrm{NO}_{3}\right)$ $\left([\mathrm{M}+\mathrm{H}]^{+}\right): 325.1907$; found: 325.1908 .<smiles>C[C@]1(CN2CCO[C@H]([AlH2])C2)C[C@H](c2ccccc2)C(=O)O1</smiles>

5-Methyl-5-(morpholinomethyl)-3-phenyldihydrofuran-2(3H)-one (3n). Synthesized using condition A, without BCP. Two diastereomers of $\mathbf{3 n}$ were observed in a ratio of $1.2: 1$ by ${ }^{1} \mathrm{H}$ NMR of the crude reaction mixture. Isolated by Flash Column Chromatography (addition of $0.2 \mathrm{~mL}$ TFA to crude reaction mixture, 50\% EtOAc-hexanes, then 50\% EtOAchexanes with $2 \%$ TEA) as a yellow oil $\left(48.3 \mathrm{mg}, 44 \%\right.$, an inseparable 1.3:1 mixture of two diastereomers). $\mathrm{R}_{f}=0.27(33 \%$ EtOAc-hexanes with $2 \%$ TEA); ${ }^{1} \mathrm{H} \mathrm{NMR}\left(\mathrm{CDCl}_{3}, 400 \mathrm{MHz}\right.$, signals including both diastereomers): $\delta 7.38-7.22(\mathrm{~m}, 10 \mathrm{H})$, $4.31(\mathrm{t}, J=10.3 \mathrm{~Hz}, 1 \mathrm{H}), 3.96(\mathrm{t}, J=10.6 \mathrm{~Hz}, 1 \mathrm{H}), 3.67(\mathrm{~b}, \mathrm{t}, J=4.4 \mathrm{~Hz}, 8 \mathrm{H}), 2.74-2.51(\mathrm{~m}, 11 \mathrm{H}), 2.50-2.29(\mathrm{~m}, 4 \mathrm{H})$, 
$2.14(\mathrm{dd}, J=12.8,10.5 \mathrm{~Hz}, 1 \mathrm{H}), 1.42(\mathrm{~b}, \mathrm{~s}, 6 \mathrm{H}) ;{ }^{13} \mathrm{C} \mathrm{NMR}\left(\mathrm{CDCl}_{3}, 125 \mathrm{MHz}\right.$, signals including both diastereomers): $\delta$ 176.9, 176.4, 138.1, 137.3, 128.7 (2C), 128.7 (2C), 128.1 (2C), 127.8 (2C), 127.4, 127.3, 84.7, 84.5, 67.3 (2C), 67.0 (2C), 66.7, 66.0, 55.5 (2C), 55.1 (2C), 47.9, 46.2, 41.7, 39.9, 25.8, 24.0; FTIR (thin film): $\mathrm{cm}^{-1} 2968,2855,2804,1762,1453$, 1113, 910, 801, 633; HRMS-ESI (m/z) Calcd for $\left(\mathrm{C}_{16} \mathrm{H}_{22} \mathrm{NO}_{3}\right)\left([\mathrm{M}+\mathrm{H}]^{+}\right): 276.1594$; found: 276.1596.

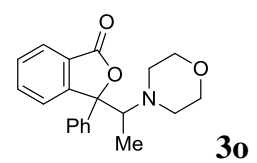

3-(1-Morpholinoethyl)-3-phenylisobenzofuran-1(3H)-one (3o). Synthesized using condition A. Two diastereomers of 3o were observed in a ratio of $1.4: 1$ by ${ }^{1} \mathrm{H}$ NMR of the crude reaction mixture. Isolated by Flash Column Chromatography (100\% hexanes $\rightarrow 30 \%$ EtOAc-hexanes) as a light clear oil $(129.0 \mathrm{mg}$, 99\%, an inseparable 1.4:1 mixture of two diastereomers). $\mathrm{R}_{f}=0.3,0.19$ (25\% EtOAc-hexanes); ${ }^{1} \mathrm{H} \mathrm{NMR}\left(\mathrm{CDCl}_{3}, 400 \mathrm{MHz}\right.$, signals including both diastereomers): $\delta 7.90-7.81(\mathrm{~m}, 2 \mathrm{H}), 7.77(\mathrm{~d}, J=7.8 \mathrm{~Hz}, 1 \mathrm{H}), 7.69-7.54(\mathrm{~m}, 6 \mathrm{H}), 7.53-7.41(\mathrm{~m}, 2 \mathrm{H}), 7.40-7.30(\mathrm{~m}, 4 \mathrm{H}), 7.30-7.23(\mathrm{~m}$, $3 \mathrm{H}), 3.54-3.12(\mathrm{~m}, 10 \mathrm{H}), 2.72-2.61(\mathrm{~m}, 2 \mathrm{H}), 2.51-2.30(\mathrm{~m}, 4 \mathrm{H}), 2.26-2.13(\mathrm{~m}, 2 \mathrm{H}), 1.05(\mathrm{~d}, J=7.0 \mathrm{~Hz}, 3 \mathrm{H}), 0.92(\mathrm{~d}, J=$ $5.8 \mathrm{~Hz}, 3 \mathrm{H}) ;{ }^{13} \mathrm{C} \mathrm{NMR}\left(\mathrm{CDCl}_{3}, 125 \mathrm{MHz}\right.$, signals including both diastereomers): $\delta 170.2,169.9,152.8,150.6,139.3$, 138.6 133.6, 133.5, 129.2128 .7 (2C), 128.5, 128.1, 127.8, 127.8 126.5, 125.7 (2C), 125.6 (2C), 124.9, 124.4 (2C), 123.0, 121.5, 93.7, 92.8, 77.2, 67.2 (2C), 67.1 (2C), 66.3 (2C), 66.2 (2C), 51.4, 50.4, 9.5, 7.9; FTIR (thin film): $\mathrm{cm}^{-1} 2852,1754$, 1448, 1287, 1115, 751, 701; HRMS-ESI (m/z) Calcd for $\left(\mathrm{C}_{20} \mathrm{H}_{22} \mathrm{NO}_{3}\right)\left([\mathrm{M}+\mathrm{H}]^{+}\right): 324.1594$; found: 324.1594.

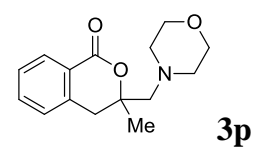

3-Methyl-3-(morpholinomethyl)isochroman-1-one (3p). Synthesized using condition A. Isolated by Flash Column Chromatography (addition of $0.2 \mathrm{~mL}$ TFA to crude reaction mixture, 50\% EtOAc-hexanes, then 50\% EtOAc-hexanes with $2 \%$ TEA) as a yellow solid $(92.1 \mathrm{mg}, 89 \%) . \mathrm{R}_{f}=0.27\left(33 \%\right.$ EtOAc-hexanes); ${ }^{1} \mathrm{H} \mathrm{NMR}\left(\mathrm{CDCl}_{3}, 400 \mathrm{MHz}\right): \delta 7.93$ $(\mathrm{d}, J=7.5 \mathrm{~Hz}, 1 \mathrm{H}), 7.38(\mathrm{td}, J=7.5,1.3 \mathrm{~Hz}, 1 \mathrm{H}), 7.22(\mathrm{t}, J=7.5 \mathrm{~Hz}, 1 \mathrm{H}), 7.08(\mathrm{~d}, J=7.5 \mathrm{~Hz}, 1 \mathrm{H}), 3.46(\mathrm{ddd}, J=11.2$, $6.2,3.2 \mathrm{~Hz}, 2 \mathrm{H}), 3.40$ (ddd, $J=11.2,6.2,3.2 \mathrm{~Hz}, 2 \mathrm{H}), 3.27(\mathrm{~d}, J=16.5 \mathrm{~Hz}, 1 \mathrm{H}), 2.71(\mathrm{~d}, J=16.5 \mathrm{~Hz}, 1 \mathrm{H}), 2.49-2.42(\mathrm{~m}$, $2 \mathrm{H}), 2.48(\mathrm{~d}, J=14.3 \mathrm{~Hz}, 1 \mathrm{H}), 2.40-2.33(\mathrm{~m}, 2 \mathrm{H}), 2.35(\mathrm{~d}, J=14.3 \mathrm{~Hz}, 1 \mathrm{H}), 1.23(\mathrm{~s}, 3 \mathrm{H}) ;{ }^{13} \mathrm{C} \mathrm{NMR}\left(\mathrm{CDCl}_{3}, 100 \mathrm{MHz}\right): \delta$ $164.6,137.9,133.6,129.5,127.7,127.2,124.9,83.3,66.9$ (2C), 66.1, 55.0 (2C), 35.7, 24.4; FTIR (thin film): $\mathrm{cm}^{-1} 2851$, 1708, 1289, 1109, 1065, 741; HRMS-ESI (m/z) Calcd for $\left(\mathrm{C}_{15} \mathrm{H}_{20} \mathrm{NO}_{3}\right)\left([\mathrm{M}+\mathrm{H}]^{+}\right): 262.1438$; found: 262.1438.

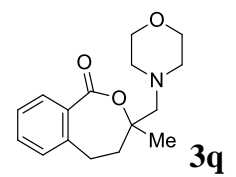

3-Methyl-3-(morpholinomethyl)-4,5-dihydrobenzo[c]oxepin-1(3H)-one (3q). Synthesized using condition A. Isolated by Flash Column Chromatography (addition of $0.2 \mathrm{~mL}$ TFA to crude reaction mixture, 50\% EtOAc-hexanes, then $25 \%$ EtOAc-hexanes with 5\% TEA) as a clear oil $(29.4 \mathrm{mg}, 27 \%) . \mathrm{R}_{f}=0.19(50 \%$ EtOAc-hexanes $) ;{ }^{1} \mathrm{H} \mathrm{NMR}\left(\mathrm{CDCl}_{3}, 400\right.$ $\mathrm{MHz}): \delta 7.82(\mathrm{~d}, J=7.5 \mathrm{~Hz}, 1 \mathrm{H}), 7.43(\mathrm{t}, J=7.5 \mathrm{~Hz}, 1 \mathrm{H}), 7.32(\mathrm{t}, J=7.5 \mathrm{~Hz}, 1 \mathrm{H}), 7.18(\mathrm{~d}, J=7.5 \mathrm{~Hz}, 1 \mathrm{H}), 3.71-3.54(\mathrm{~m}$, $4 \mathrm{H}), 3.00-2.83(\mathrm{~m}, 2 \mathrm{H}), 2.67-2.55(\mathrm{~m}, 2 \mathrm{H}), 2.55-2.45(\mathrm{~m}, 2 \mathrm{H}), 2.39-2.25(\mathrm{~m}, 3 \mathrm{H}), 1.93(\mathrm{ddd}, J=14.0,8.7,5.4 \mathrm{~Hz}, 1 \mathrm{H})$; ${ }^{13} \mathrm{C} \mathrm{NMR}\left(\mathrm{CDCl}_{3}, 125 \mathrm{MHz}\right): \delta 170.0,140.3,132.8,132.5,130.9,128.4,127.0,84.0,68.3,67.0(2 \mathrm{C}), 54.9(2 \mathrm{C}), 38.8$, 30.4, 27.5; FTIR (thin film): $\mathrm{cm}^{-1}$ 2853, 1706, 1603, 1454, 1290, 1248, 1117, 1085, 772; HRMS-ESI (m/z) Calcd for $\left(\mathrm{C}_{16} \mathrm{H}_{22} \mathrm{NO}_{3}\right)\left([\mathrm{M}+\mathrm{H}]^{+}\right): 276.1594$; found: 276.1594 .

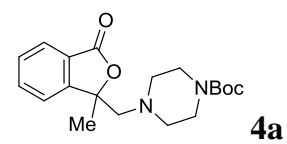

Tert-butyl 4-((1-methyl-3-oxo-1,3-dihydroisobenzofuran-1-yl)methyl)piperazine-1-carboxylate (4a). Synthesized using condition A. Isolated by Flash Column Chromatography (20\% EtOAc-hexanes with 2\% TEA) as a white solid (98.0 mg, 71\%). $\mathrm{R}_{f}=0.27$ (33\% EtOAc-hexanes with $2 \%$ TEA); ${ }^{1} \mathrm{H}$ NMR $\left(\mathrm{CDCl}_{3}, 400 \mathrm{MHz}\right): \delta 7.83(\mathrm{~d}, J=7.6 \mathrm{~Hz}, 1 \mathrm{H}), 7.60$ $(\mathrm{t}, J=7.6 \mathrm{~Hz}, 1 \mathrm{H}), 7.47(\mathrm{t}, J=7.6 \mathrm{~Hz}, 1 \mathrm{H}), 7.38(\mathrm{~d}, J=7.6 \mathrm{~Hz}, 1 \mathrm{H}), 3.30-3.00(\mathrm{~m}, 4 \mathrm{H}), 2.82(\mathrm{~d}, J=14.4 \mathrm{~Hz}, 1 \mathrm{H}), 2.65$ $(\mathrm{d}, J=14.4 \mathrm{~Hz}, 1 \mathrm{H}), 2.45-2.35(\mathrm{~m}, 2 \mathrm{H}), 2.35-2.28(\mathrm{~m}, 2 \mathrm{H}), 1.60(\mathrm{~s}, 3 \mathrm{H}), 1.38(\mathrm{~s}, 9 \mathrm{H}) ;{ }^{13} \mathrm{C} \mathrm{NMR}\left(\mathrm{CDCl}_{3}, 125 \mathrm{MHz}, 60\right.$ $\left.{ }^{\circ} \mathrm{C}\right): \delta 169.4,154.6,152.5,133.4,128.8,127.1,125.1,121.0,87.4,79.3,65.2,54.1$ (2C), 43.8 (2C), 28.3, (3C), 22.6; FTIR (thin film): $\mathrm{cm}^{-1}$ 2980, 2927, 2822, 1748, 1686, 1425, 1245, 1167, 1120, 1037, 761, 710; HRMS-ESI (m/z) Calcd for $\left(\mathrm{C}_{19} \mathrm{H}_{27} \mathrm{~N}_{2} \mathrm{O}_{4}\right)\left([\mathrm{M}+\mathrm{H}]^{+}\right): 347.1965$; found: 347.1965 . 


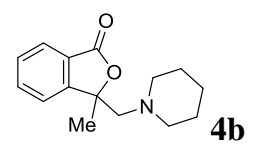

3-Methyl-3-(piperidin-1-ylmethyl)isobenzofuran-1(3H)-one (4b). Synthesized using condition A. Isolated by Flash Column Chromatography (25\% EtOAc-hexanes) as a clear oil $(49.6 \mathrm{mg}, 51 \%) . \mathrm{R}_{f}=0.12\left(50 \%\right.$ EtOAc-hexanes); ${ }^{1} \mathrm{H}$ NMR $\left(\mathrm{CDCl}_{3}, 400 \mathrm{MHz}\right): \delta 7.83(\mathrm{~d}, J=7.5 \mathrm{~Hz}, 1 \mathrm{H}), 7.60(\mathrm{t}, J=7.5 \mathrm{~Hz}, 1 \mathrm{H}), 7.46(\mathrm{t}, J=7.5 \mathrm{~Hz}, 1 \mathrm{H}), 7.42(\mathrm{~d}, J=7.5 \mathrm{~Hz}$, $1 \mathrm{H}), 2.75(\mathrm{~d}, J=14.1 \mathrm{~Hz}, 1 \mathrm{H}), 2.62(\mathrm{~d}, J=14.1 \mathrm{~Hz}, 1 \mathrm{H}), 2.41-2.33(\mathrm{~m}, 4 \mathrm{H}), 1.61(\mathrm{~s}, 3 \mathrm{H}), 1.44-1.26(\mathrm{~m}, 6 \mathrm{H}) ;{ }^{13} \mathrm{C} \mathrm{NMR}$ $\left(\mathrm{CDCl}_{3}, 125 \mathrm{MHz}\right): \delta 169.9,152.9,133.4 .128 .7,126.7,125.2,125.1,121.3,87.7,65.8,55.9(2 \mathrm{C}), 26.0(2 \mathrm{C}), 23.7,22.7$; FTIR (thin film): $\mathrm{cm}^{-1} 2930,1752,1285,1034,763,694$; HRMS-ESI (m/z) Calcd for $\left(\mathrm{C}_{15} \mathrm{H}_{20} \mathrm{NO}_{2}\right)\left([\mathrm{M}+\mathrm{H}]^{+}\right): 246.1489$; found: 246.1490 .

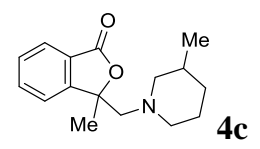

3-Methyl-3-((3-methylpiperidin-1-yl)methyl)isobenzofuran-1(3H)-one (4c). Synthesized using condition A. Two diastereomers of $\mathbf{4 c}$ were observed in a ratio of $1: 1$ by ${ }^{1} \mathrm{H}$ NMR of the crude reaction mixture. Isolated by Flash Column Chromatography (100\% hexanes $\rightarrow 20 \%$ EtOAc-hexanes) as a clear oil $(59.2 \mathrm{mg}, 57 \%$, an inseparable 1:1 mixture of two diastereomers). $\mathrm{R}_{f}=0.20$ (50\% EtOAc-hexanes); ${ }^{1} \mathrm{H} \mathrm{NMR}\left(\mathrm{CDCl}_{3}, 400 \mathrm{MHz}\right.$, signals including both diastereomers): $\delta$ $7.82(\mathrm{~d}, J=7.6 \mathrm{HZ}, 2 \mathrm{H}), 7.60(\mathrm{t}, J=7.6 \mathrm{~Hz}, 2 \mathrm{H}), 7.50-7.40(\mathrm{~m}, 4 \mathrm{H}), 2.86-2.76(\mathrm{~m}, 2 \mathrm{H}), 2.75-2.50(\mathrm{~m}, 6 \mathrm{H}), 2.16-2.04$ $(\mathrm{m}, 2 \mathrm{H}), 1.88-1.73(\mathrm{~m}, 2 \mathrm{H}), 1.63(\mathrm{~s}, 3 \mathrm{H}), 1.63(\mathrm{~s}, 3 \mathrm{H}), 1.72-1.32(\mathrm{~m}, 14 \mathrm{H}), 1.32-1.17(\mathrm{~m}, 2 \mathrm{H}), 0.71(\mathrm{~d}, J=6.5 \mathrm{~Hz}, 3 \mathrm{H})$, $0.69(\mathrm{~d}, J=6.5 \mathrm{~Hz}, 3 \mathrm{H}) ;{ }^{13} \mathrm{C} \mathrm{NMR}\left(\mathrm{CDCl}_{3}, 100 \mathrm{MHz}\right.$, signals including both diastereomers): $\delta 169.9$ (2C), $152.8,152.7$, 133.5 (2C), 128.8 (2C), 126.7, 126.6, 125.2 (2C), 121.4, 121.3, 87.6, 87.6, 65.5, 65.4, 63.4, 62.7, 55.6, 54.6, 32.1, 32.0, 31.0, 30.8, 25.3, 25.1, 22.9, 22.8, 19.3, 19.1; FTIR (thin film): $\mathrm{cm}^{-1}$ 2926, 1754, 1614, 1466, 1286, 1110, 1032, 763, 694; HRMS-ESI (m/z) Calcd for $\left(\mathrm{C}_{16} \mathrm{H}_{22} \mathrm{NO}_{2}\right)\left([\mathrm{M}+\mathrm{H}]^{+}\right): 260.1645$; found: 260.1646.

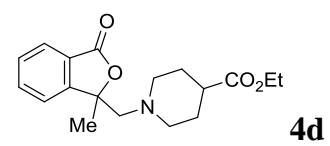

Ethyl 1-((1-methyl-3-oxo-1,3-dihydroisobenzofuran-1-yl)methyl)piperidine-4-carboxylate (4d). Synthesized using condition A. Isolated by Flash Column Chromatography (25\% EtOAc-hexanes) as a clear oil $(74.9 \mathrm{mg}, 59 \%) . \mathrm{R}_{f}=0.45$ (50\% EtOAc-hexanes); ${ }^{1} \mathrm{H}$ NMR $\left(\mathrm{CDCl}_{3}, 400 \mathrm{MHz}\right): \delta 7.85(\mathrm{~d}, J=7.5 \mathrm{~Hz}, 1 \mathrm{H}), 7.62(\mathrm{t}, J=7.5 \mathrm{~Hz}, 1 \mathrm{H}), 7.48(\mathrm{t}, J=7.5$ $\mathrm{Hz}, 1 \mathrm{H}), 7.42(\mathrm{~d}, J=7.5 \mathrm{~Hz}, 1 \mathrm{H}), 4.08(\mathrm{q}, J=7.1 \mathrm{~Hz}, 2 \mathrm{H}), 2.79-2.72(\mathrm{~m}, 1 \mathrm{H}), 2.76(\mathrm{~d}, J=14.2 \mathrm{~Hz}, 1 \mathrm{H}), 2.67-2.60(\mathrm{~m}$, $1 \mathrm{H}), 2.66(\mathrm{~d}, J=14.2 \mathrm{~Hz}, 1 \mathrm{H}), 2.24-2.08(\mathrm{~m}, 3 \mathrm{H}), 1.78-1.56(\mathrm{~m}, 3 \mathrm{H}), 1.62(\mathrm{~s}, 3 \mathrm{H}), 1.47-1.36(\mathrm{~m}, 1 \mathrm{H}), 1.21(\mathrm{t}, J=7.1 \mathrm{~Hz}$, $3 \mathrm{H}) ;{ }^{13} \mathrm{C} \mathrm{NMR}\left(\mathrm{CDCl}_{3}, 125 \mathrm{MHz}\right): \delta 174.9,169.8,152.6,133.4,128.7,126.7,125.1,121.1,87.6,65.2,60.1,54.5,53.9$, 40.4, 28.4, 28.3, 22.6, 14.0; FTIR (thin film): $\mathrm{cm}^{-1}$ 2929, 1756, 1724, 1285, 1032, 762, 693; HRMS-ESI (m/z) Calcd for $\left(\mathrm{C}_{18} \mathrm{H}_{24} \mathrm{NO}_{4}\right)\left([\mathrm{M}+\mathrm{H}]^{+}\right): 318.1700$; found: 318.1702 .

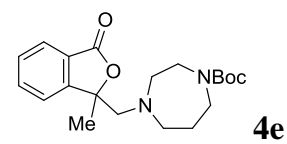

Tert-butyl 4-((1-methyl-3-oxo-1,3-dihydroisobenzofuran-1-yl)methyl)-1,4-diazepane-1-carboxylate (4e). Synthesized using condition $\mathrm{A}$, without $\mathrm{BCP}$ and with $20 \% \mathrm{Cu}(\mathrm{OTf})_{2}$. Isolated as a mixture of conformers by Flash Column Chromatography (25\% EtOAc-hexanes) as a clear oil $(63.3 \mathrm{mg}, 44 \%) . \mathrm{R}_{f}=0.39\left(50 \%\right.$ EtOAc-hexanes); ${ }^{1} \mathrm{H}$ NMR $\left(\mathrm{CDCl}_{3}, 400 \mathrm{MHz}\right): \delta 7.86(\mathrm{~d}, J=7.5 \mathrm{~Hz}, 1 \mathrm{H}), 7.63(\mathrm{t}, J=7.5 \mathrm{~Hz}, 1 \mathrm{H}), 7.49(\mathrm{t}, J=7.5 \mathrm{~Hz}, 1 \mathrm{H}), 7.37(\mathrm{~d}, J=7.5 \mathrm{~Hz}, 1 \mathrm{H})$, $3.33-2.87(\mathrm{~m}, 6 \mathrm{H}), 2.71-2.44(\mathrm{~m}, 4 \mathrm{H}), 1.64-1.26(5 \mathrm{H}), 1.40(\mathrm{~s}, 9 \mathrm{H}) ;{ }^{13} \mathrm{C} \mathrm{NMR}\left(\mathrm{CDCl}_{3}, 125 \mathrm{MHz}\right): \delta 169.7,155.2,152.6$, 133.4, 128.8, 127.5, 125.2, 121.1, 88.0, 79.1, 65.2, 57.9, 56.7, 47.8, 47.3, 45.9, 45.2, 28.4 (3C), 27.7, 22.4; FTIR (thin film): $\mathrm{cm}^{-1} 2974,1758,1684,1412,1365,1166,1035,765,695 ;$ HRMS-ESI (m/z) Calcd for $\left(\mathrm{C}_{20} \mathrm{H}_{29} \mathrm{~N}_{2} \mathrm{O}_{4}\right)\left([\mathrm{M}+\mathrm{H}]^{+}\right)$: 361.2123; found: 361.2123 .

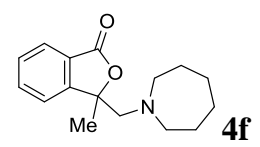

3-(Azepan-1-ylmethyl)-3-methylisobenzofuran-1(3H)-one (4f). Synthesized using condition A, without BCP and with $20 \% \mathrm{Cu}(\mathrm{OTf})_{2}$. Isolated pure following $\mathrm{Al}_{2} \mathrm{O}_{3}$ filtration as a tan oil $(35.2 \mathrm{mg}, 34 \%)$. $\mathrm{R}_{f}=0.19(50 \%$ EtOAc-hexanes $) ;{ }^{1} \mathrm{H}$ 
NMR $\left(\mathrm{CDCl}_{3}, 400 \mathrm{MHz}\right): \delta 7.86(\mathrm{~d}, J=7.5 \mathrm{~Hz}, 1 \mathrm{H}), 7.62(\mathrm{t}, J=7.5 \mathrm{~Hz}, 1 \mathrm{H}), 7.48(\mathrm{t}, J=7.5 \mathrm{~Hz}, 1 \mathrm{H}), 7.39(\mathrm{~d}, J=7.5 \mathrm{~Hz}$, $1 \mathrm{H}), 3.04(\mathrm{~d}, J=14.6 \mathrm{~Hz}, 1 \mathrm{H}), 2.94(\mathrm{~d}, J=14.6 \mathrm{~Hz}, 1 \mathrm{H}), 2.66-2.58(\mathrm{~m}, 2 \mathrm{H}), 2.56-2.48(\mathrm{~m}, 2 \mathrm{H}), 1.60$ (s, 3H), $1.47-1.29$ $(\mathrm{m}, 6 \mathrm{H}), 1.27-1.15(\mathrm{~m}, 3 \mathrm{H}) ;{ }^{13} \mathrm{C} \mathrm{NMR}\left(\mathrm{CDCl}_{3}, 125 \mathrm{MHz}\right): \delta ; 170.1,152.9,133.3,128.6,127.4,125.1,121.2,88.6,66.2$, 57.5 (2C), 28.8 (2C), 26.8 (2C), 22.5; FTIR (thin film): $\mathrm{cm}^{-1} 2924,1756,1286,1121,1035,765,694$; HRMS-ESI (m/z) Calcd for $\left(\mathrm{C}_{16} \mathrm{H}_{22} \mathrm{NO}_{2}\right)\left([\mathrm{M}+\mathrm{H}]^{+}\right): 260.1445$; found: 260.1447 .

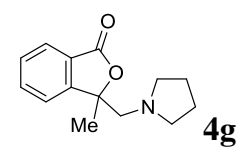

3-Methyl-3-(pyrrolidin-1-ylmethyl)isobenzofuran-1(3H)-one (4g). Synthesized using condition A, without BCP and with $20 \% \mathrm{Cu}(\mathrm{OTf})_{2}$. Isolated pure following $\mathrm{Al}_{2} \mathrm{O}_{3}$ filtration as a tan oil $(29.2 \mathrm{mg}, 32 \%)$. $\mathrm{R}_{f}=0.12(50 \%$ EtOAc-hexanes $)$; ${ }^{1} \mathrm{H}$ NMR $\left(\mathrm{CDCl}_{3}, 400 \mathrm{MHz}\right): \delta 7.85(\mathrm{~d}, J=7.5 \mathrm{~Hz}, 1 \mathrm{H}), 7.63(\mathrm{t}, J=7.5 \mathrm{~Hz}, 1 \mathrm{H}), 7.49(\mathrm{t}, J=7.5 \mathrm{~Hz}, 1 \mathrm{H}), 7.41(\mathrm{~d}, J=7.5$ $\mathrm{Hz}, 1 \mathrm{H}), 3.09(\mathrm{~d}, J=13.8 \mathrm{~Hz}, 1 \mathrm{H}), 2.70(\mathrm{~d}, J=13.8 \mathrm{~Hz}, 1 \mathrm{H}), 2.54-2.50(\mathrm{~m}, 4 \mathrm{H}), 1.68-1.62(\mathrm{~m}, 4 \mathrm{H}) 1.65(\mathrm{~s}, 3 \mathrm{H}) ;{ }^{13} \mathrm{C} \mathrm{NMR}$ $\left(\mathrm{CDCl}_{3}, 125 \mathrm{MHz}\right): \delta 170.0,153.3,133.7,128.8,126.4,125.4,121.3,87.9,63.9,55.5$ (2C), 23.9 (2C), 23.3; FTIR (thin film): $\mathrm{cm}^{-1}$ 2968, 1755, 1111, 1034, 765, 696; HRMS-ESI $(\mathrm{m} / \mathrm{z})$ Calcd for $\left(\mathrm{C}_{14} \mathrm{H}_{18} \mathrm{NO}_{2}\right)\left([\mathrm{M}+\mathrm{H}]^{+}\right): 232.1332$; found: 232.1331 .

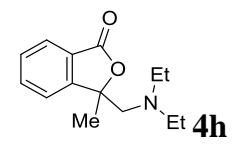

3-((Diethylamino)methyl)-3-methylisobenzofuran-1(3H)-one (4h). Synthesized using condition A, without BCP and with $20 \% \mathrm{Cu}(\mathrm{OTf})_{2}$. Isolated pure following $\mathrm{Al}_{2} \mathrm{O}_{3}$ filtration as a tan oil $(43.1 \mathrm{mg}, 46 \%) . \mathrm{R}_{f}=0.09$ (50\% EtOAc-hexanes); ${ }^{1} \mathrm{H}$ NMR $\left(\mathrm{CDCl}_{3}, 400 \mathrm{MHz}\right): \delta 7.83(\mathrm{~d}, J=7.5 \mathrm{~Hz}, 1 \mathrm{H}), 7.60(\mathrm{t}, J=7.5 \mathrm{~Hz}, 1 \mathrm{H}), 7.46(\mathrm{t}, J=7.5 \mathrm{~Hz}, 1 \mathrm{H}), 7.39(\mathrm{~d}, J=7.5$ $\mathrm{Hz}, 1 \mathrm{H}), 2.87(\mathrm{~d}, J=14.5 \mathrm{~Hz}, 1 \mathrm{H}), 2.77(\mathrm{~d}, J=14.5 \mathrm{~Hz}, 1 \mathrm{H}), 2.43(\mathrm{q}, J=7.1 \mathrm{~Hz}, 2 \mathrm{H}), 2.42(\mathrm{q}, J=7.1 \mathrm{~Hz}, 2 \mathrm{H}), 1.60(\mathrm{~s}$, $3 \mathrm{H}), 0.79(\mathrm{t}, J=7.1 \mathrm{~Hz}, 6 \mathrm{H}) ;{ }^{13} \mathrm{C}$ NMR $\left(\mathrm{CDCl}_{3}, 125 \mathrm{MHz}\right): \delta 170.1,152.9,133.3,128.7,127.1,125.2,121.4,88.1,61.8$, 48.2 (2C), 22.9, 11.8 (2C); FTIR (thin film): $\mathrm{cm}^{-1}$ 2970, 1759, 1287, 1032, 763, 695; HRMS-ESI (m/z) Calcd for $\left(\mathrm{C}_{14} \mathrm{H}_{20} \mathrm{NO}_{2}\right)\left([\mathrm{M}+\mathrm{H}]^{+}\right): 234.1489$; found: 234.1488 .

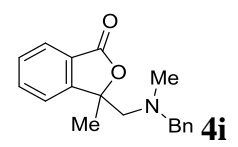

3-((Benzyl(methyl)amino)methyl)-3-methylisobenzofuran-1(3H)-one (4i). Synthesized using condition A, without BCP and with $20 \% \mathrm{Cu}(\mathrm{OTf})_{2}$. Isolated by Flash Column Chromatography (10\% EtOAc-hexanes) as a clear oil (50.6 mg, $\left.45 \%\right)$. $\mathrm{R}_{f}=0.69\left(50 \%\right.$ EtOAc-hexanes); ${ }^{1} \mathrm{H} \mathrm{NMR}\left(\mathrm{CDCl}_{3}, 400 \mathrm{MHz}\right): \delta 7.89(\mathrm{~d}, J=7.5 \mathrm{~Hz}, 1 \mathrm{H}), 7.58(\mathrm{t}, J=7.5 \mathrm{~Hz}, 1 \mathrm{H}), 7.49(\mathrm{t}$, $J=7.5 \mathrm{~Hz}, 1 \mathrm{H}), 7.26-7.18(\mathrm{~m}, 4 \mathrm{H}), 7.08-7.02(\mathrm{~m}, 2 \mathrm{H}), 3.52(\mathrm{~d}, J=13.2 \mathrm{~Hz}, 1 \mathrm{H}), 3.46(\mathrm{~d}, J=13.2 \mathrm{~Hz}, 1 \mathrm{H}), 2.97(\mathrm{~d}, J=$ $14.3 \mathrm{~Hz}, 1 \mathrm{H}), 2.79(\mathrm{~d}, J=14.3 \mathrm{~Hz}, 1 \mathrm{H}), 2.20(\mathrm{~s}, 3 \mathrm{H}), 1.62(\mathrm{~s}, 3 \mathrm{H}) ;{ }^{13} \mathrm{C} \mathrm{NMR}\left(\mathrm{CDCl}_{3}, 125 \mathrm{MHz}\right): \delta 169.9,152.9,138.9$, 133.7, 128.8, 128.7 (2C), 128.1 (2C), 126.7, 125.3, 121.2, 88.3, 64.4, 63.7, 44.0, 23.2; FTIR (thin film): $\mathrm{cm}^{-1} 2791,1754$, 1286, 1286, 1112, 1032, 695; HRMS-ESI (m/z) Calcd for $\left(\mathrm{C}_{18} \mathrm{H}_{20} \mathrm{NO}_{2}\right)\left([\mathrm{M}+\mathrm{H}]^{+}\right)$: 282.1489; found: 282.1488 .

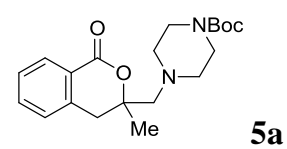

Tert-butyl 4-((3-methyl-1-oxoisochroman-3-yl)methyl)piperazine-1-carboxylate (5a). Synthesized using condition A. Isolated by Flash Column Chromatography (10\% EtOAc-hexanes $\rightarrow$ 25\% EtOAc-hexanes) as a clear oil (101.0 mg, $70 \%) . \mathrm{R}_{f}=0.50\left(50 \%\right.$ EtOAc-hexanes); ${ }^{1} \mathrm{H} \mathrm{NMR}\left(\mathrm{CDCl}_{3}, 500 \mathrm{MHz}\right): \delta 8.02(\mathrm{~d}, J=7.6 \mathrm{~Hz}, 1 \mathrm{H}), 7.48(\mathrm{t}, J=7.6 \mathrm{~Hz}, 1 \mathrm{H})$, $7.31(\mathrm{t}, J=7.6 \mathrm{~Hz}, 1 \mathrm{H}), 7.18(\mathrm{~d}, J=7.6 \mathrm{~Hz}, 1 \mathrm{H}), 3.35(\mathrm{~d}, J=16.5 \mathrm{~Hz}, 1 \mathrm{H}), 3.32-3.15(\mathrm{~m}, 4 \mathrm{H}), 2.82(\mathrm{~d}, J=16.5 \mathrm{~Hz}, 1 \mathrm{H})$, $2.59(\mathrm{~d}, J=14.4 \mathrm{~Hz}, 1 \mathrm{H}), 2.54-2.37(\mathrm{~m}, 5 \mathrm{H}), 1.38(\mathrm{~s}, 9 \mathrm{H}), 1.33(\mathrm{~s}, 3 \mathrm{H}) ;{ }^{13} \mathrm{C} \mathrm{NMR}\left(\mathrm{CDCl}_{3}, 125 \mathrm{MHz}, 60{ }^{\circ} \mathrm{C}\right): \delta 164.6$, 154.7, 137.9, 133.6, 129.7, 127.7, 127.3, 125.2, 83.2, 79.5, 65.9, 54.6 (2C), 43.7 (2C), 36.0, 28.4 (3C), 24.6; FTIR (thin film): $\mathrm{cm}^{-1} 2975,1711,1688,1421,1291,1244,1165,1115,1008,729 ;$ HRMS-ESI $(\mathrm{m} / \mathrm{z})$ Calcd for $\left(\mathrm{C}_{20} \mathrm{H}_{29} \mathrm{~N}_{2} \mathrm{O}_{4}\right)$ $\left([\mathrm{M}+\mathrm{H}]^{+}\right): 361.2122$; found: 361.2122 .

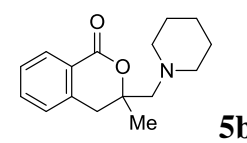


3-Methyl-3-(piperidin-1-ylmethyl)isochroman-1-one (5b). Synthesized using condition A. Isolated by Flash Column Chromatography (25\% EtOAc-hexanes) as a clear oil $(48.5 \mathrm{mg}, 47 \%) . \mathrm{R}_{f}=0.07\left(50 \%\right.$ EtOAc-hexanes); ${ }^{1} \mathrm{H} \mathrm{NMR}\left(\mathrm{CDCl} \mathrm{N}_{3}\right.$, $400 \mathrm{MHz}): \delta 8.06(\mathrm{~d}, J=7.6 \mathrm{~Hz}, 1 \mathrm{H}), 7.50(\mathrm{t}, J=7.6 \mathrm{~Hz}, 1 \mathrm{H}), 7.34(\mathrm{t}, J=7.6 \mathrm{~Hz}, 1 \mathrm{H}), 7.20(\mathrm{~d}, J=7.6 \mathrm{~Hz}, 1 \mathrm{H}), 3.37(\mathrm{~d}, J$ $=16.4 \mathrm{~Hz}, 1 \mathrm{H}), 2.86(\mathrm{~d}, J=16.4 \mathrm{~Hz}, 1 \mathrm{H}), 2.57(\mathrm{~d}, J=14.2 \mathrm{~Hz}, 1 \mathrm{H}), 2.54-2.36(\mathrm{~m}, 5 \mathrm{H}), 1.53-1.27(\mathrm{~m}, 6 \mathrm{H}), 1.36(\mathrm{~s}, 3 \mathrm{H})$; ${ }^{13} \mathrm{C} \mathrm{NMR}\left(\mathrm{CDCl}_{3}, 125 \mathrm{MHz}\right): \delta 164.9,138.2,133.6,129.6,127.8,127.1,125.1,83.5,66.0,56.3(2 \mathrm{C}), 35.7,26.1(2 \mathrm{C})$, 24.6, 23.8; FTIR (thin film): $\mathrm{cm}^{-1} 2932,1710,1607,1459,1289,1109,742 ;$ HRMS-ESI (m/z) Calcd for $\left(\mathrm{C}_{16} \mathrm{H}_{22} \mathrm{NO}_{2}\right)$ $\left([\mathrm{M}+\mathrm{H}]^{+}\right): 260.1645$; found: 260.1645 .

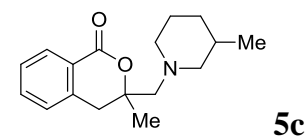

3-Methyl-3-((3-methylpiperidin-1-yl)methyl)isochroman-1-one (5c). Synthesized using condition A. Two diastereomers of $\mathbf{5 c}$ were observed in a ratio of $1: 1$ by ${ }^{1} \mathrm{H}$ NMR of the crude reaction mixture. Isolated by Flash Column Chromatography (100\% hexanes $\rightarrow 30 \%$ EtOAc-hexanes) as a clear oil (55.0 $\mathrm{mg}, 50 \%$, an inseparable 1:1 mixture of two diastereomers). $\mathrm{R}_{f}=0.26$ (50\% EtOAc-hexanes); ${ }^{1} \mathrm{H} \mathrm{NMR}\left(\mathrm{CDCl}_{3}, 400 \mathrm{MHz}\right.$, signals including both diastereomers): $\delta$ $8.05(\mathrm{~d}, J=7.6 \mathrm{~Hz}, 2 \mathrm{H}), 7.49(\mathrm{t}, J=7.6 \mathrm{~Hz}, 2 \mathrm{H}), 7.49(\mathrm{t}, J=7.6 \mathrm{~Hz}, 2 \mathrm{H}), 7.20(\mathrm{~d}, J=7.6 \mathrm{~Hz}, 2 \mathrm{H}), 3.38(\mathrm{~d}, J=9.2 \mathrm{~Hz}$, $1 \mathrm{H}), 3.34(\mathrm{~d}, J=9.1 \mathrm{~Hz}, 1 \mathrm{H}), 2.85(\mathrm{~d}, J=16.4 \mathrm{~Hz}, 2 \mathrm{H}), 2.79-2.60(\mathrm{~m}, 4 \mathrm{H}), 2.56(\mathrm{dd}, J=14.2,5.8 \mathrm{~Hz}, 2 \mathrm{H}), 2.43(\mathrm{dd}, J=$ $14.2,8.6 \mathrm{~Hz}, 2 \mathrm{H}), 2.17-1.98(\mathrm{~m}, 2 \mathrm{H}), 1.84(\mathrm{t}, J=10.5 \mathrm{~Hz}, 1 \mathrm{H}), 1.77(\mathrm{t}, J=10.5 \mathrm{~Hz}, 1 \mathrm{H}), 1.62-1.19(\mathrm{~m}, 10 \mathrm{H}), 1.36(\mathrm{~s}$, $6 \mathrm{H}), 0.78(\mathrm{~s}, 3 \mathrm{H}), 0.76(\mathrm{~s}, 3 \mathrm{H}) ;{ }^{13} \mathrm{C} \mathrm{NMR}\left(\mathrm{CDCl}_{3}, 125 \mathrm{MHz}\right.$, signals including both diastereomers): $\delta 164.8(2 \mathrm{C}), 138.1$ (2C), 133.6, 129.6 (2C), 127.8 (2C), 127.1 (2C), 125.0 (2C), 83.4 (2C), 65.7 (2C), 63.8, 63.4, 55.8, 55.5, 35.8, 35.7, 32.2 (2C), 30.9 (2C), 25.3 (2C), 24.7, 24.6, 19.4, 19.4; FTIR (thin film): $\mathrm{cm}^{-1}$ 2927, 1712, 1607, 1459, 1292, 1113, 1066, 742; HRMS-ESI (m/z) Calcd for $\left(\mathrm{C}_{17} \mathrm{H}_{24} \mathrm{NO}_{2}\right)\left([\mathrm{M}+\mathrm{H}]^{+}\right)$: 274.1802; found: 274.1801 .

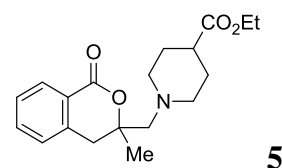

$5 d$

Ethyl 1-((3-methyl-1-oxoisochroman-3-yl)methyl)piperidine-4-carboxylate (5d). Synthesized using condition A. Isolated by Flash Column Chromatography (25\% EtOAc-hexanes) as a clear oil $(65.4 \mathrm{mg}, 49 \%)$. $\mathrm{R}_{f}=0.23(50 \%$ EtOAchexanes); ${ }^{1} \mathrm{H}$ NMR $\left(\mathrm{CDCl}_{3}, 400 \mathrm{MHz}\right): \delta 8.05(\mathrm{~d}, J=7.5 \mathrm{~Hz}, 1 \mathrm{H}), 7.50(\mathrm{td}, J=7.5,1.3 \mathrm{~Hz}, 1 \mathrm{H}), 7.34(\mathrm{t}, J=7.5 \mathrm{~Hz}, 1 \mathrm{H})$, $7.20(\mathrm{~d}, J=7.5 \mathrm{~Hz}, 1 \mathrm{H}), 4.09(\mathrm{q}, J=7.1 \mathrm{~Hz}, 2 \mathrm{H}), 3.36(\mathrm{~d}, J=16.4 \mathrm{~Hz}, 1 \mathrm{H}), 2.90-2.76(\mathrm{~m}, 2 \mathrm{H}), 2.84(\mathrm{~d}, J=16.4 \mathrm{~Hz}, 1 \mathrm{H})$, 2.90-2.76 (m, 2H), $2.84(\mathrm{~d}, J=16.4 \mathrm{~Hz}, 1 \mathrm{H}), 2.60(\mathrm{~d}, J=14.3 \mathrm{~Hz}, 1 \mathrm{H}), 2.47(\mathrm{~d}, J=14.3 \mathrm{~Hz}, 1 \mathrm{H}), 2.28(\mathrm{td}, J=11.2,1.9$ $\mathrm{Hz}, 1 \mathrm{H}), 2.24-2.13(\mathrm{~m}, 2 \mathrm{H}), 1.81-1.51(\mathrm{~m}, 4 \mathrm{H}), 1.35(\mathrm{~s}, 3 \mathrm{H}), 1.22(\mathrm{t}, J=7.1 \mathrm{~Hz}, 3 \mathrm{H}) ;{ }^{13} \mathrm{C} \mathrm{NMR}\left(\mathrm{CDCl}_{3}, 125 \mathrm{MHz}\right): \delta$ 175.1, 164.8, 138.1, 133.6, 129.7, 127.8, 127.2, 125.0, 83.5, 65.5, 60.2, 54.8, 54.4, 40.6, 35.7, 28.4, 28.3, 24.5, 14.1; FTIR (thin film): $\mathrm{cm}^{-1} 2931,1713,1606,1460,1289,1289,1112,743$; HRMS-ESI (m/z) Calcd for $\left(\mathrm{C}_{19} \mathrm{H}_{26} \mathrm{NO}_{4}\right)\left([\mathrm{M}+\mathrm{H}]^{+}\right)$: 332.1856; found: 332.1857 .

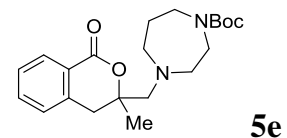

Tert-butyl 4-((3-methyl-1-oxoisochroman-3-yl)methyl)-1,4-diazepane-1-carboxylate (5e). Synthesized using condition $\mathrm{A}$, without $\mathrm{BCP}$ and with $20 \% \mathrm{Cu}(\mathrm{OTf})_{2}$. Isolated as a mixture of conformers by Flash Column Chromatography $(25 \%$ EtOAc-hexanes) as a clear oil $(53.1 \mathrm{mg}, 35 \%) . \mathrm{R}_{f}=0.27$ (50\% EtOAc-hexanes); ${ }^{1} \mathrm{H} \mathrm{NMR}\left(\mathrm{CDCl}_{3}, 400 \mathrm{MHz}\right): \delta 8.06(\mathrm{~d}, J$ $=7.5 \mathrm{~Hz}, 1 \mathrm{H}), 7.51(\mathrm{t}, J=7.5 \mathrm{~Hz}, 1 \mathrm{H}), 7.35(\mathrm{t}, J=7.5 \mathrm{~Hz}, 1 \mathrm{H}), 7.21(\mathrm{~d}, J=7.5 \mathrm{~Hz}, 1 \mathrm{H}), 3.45-3.25(\mathrm{~m}, 5 \mathrm{H}), 2.91-2.68$ $(\mathrm{m}, 6 \mathrm{H}), 2.63(\mathrm{~d}, J=14.6 \mathrm{~Hz}, 1 \mathrm{H}), 1.76-1.63(\mathrm{~m}, 2 \mathrm{H}), 1.41(\mathrm{~s}, 9 \mathrm{H}), 1.35(\mathrm{~s}, 3 \mathrm{H}) ;{ }^{13} \mathrm{C} \mathrm{NMR}\left(\mathrm{CDCl}_{3}, 125 \mathrm{MHz}\right): \delta 164.5$, $155.3,138.0,133.5,129.6,127.8,127.2,125.2,83.7,79.1,64.5,57.7,56.7,47.0,46.8,46.3,45.3,35.635 .6,28.4$ (3C), 27.7, 24.3; FTIR (thin film): $\mathrm{cm}^{-1} 2974,1714,1687,1607,1460,1414,1365,1291,1169,1116,744$; HRMS-ESI (m/z) Calcd for $\left(\mathrm{C}_{21} \mathrm{H}_{31} \mathrm{~N}_{2} \mathrm{O}_{4}\right)\left([\mathrm{M}+\mathrm{H}]^{+}\right)$: 375.2278; found: 375.2277 .

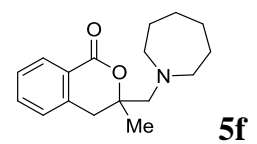

3-(Azepan-1-ylmethyl)-3-methylisochroman-1-one (5f). Synthesized using condition C. Isolated pure following $\mathrm{Al}_{2} \mathrm{O}_{3}$ filtration as a $\tan$ oil $(25.4 \mathrm{mg}, 23 \%) . \mathrm{R}_{f}=0.14\left(50 \%\right.$ EtOAc-hexanes); ${ }^{1} \mathrm{H} \mathrm{NMR}\left(\mathrm{CDCl}_{3}, 400 \mathrm{MHz}\right): \delta 8.07(\mathrm{~d}, J=7.5 \mathrm{~Hz}$, $1 \mathrm{H}), 7.51(\mathrm{t}, J=7.5 \mathrm{~Hz}, 1 \mathrm{H}), 7.35(\mathrm{t}, J=7.5 \mathrm{~Hz}, 1 \mathrm{H}), 7.22(\mathrm{~d}, J=7.5 \mathrm{~Hz}, 1 \mathrm{H}), 3.41(\mathrm{~d}, J=16.3 \mathrm{~Hz}, 1 \mathrm{H}), 2.84(\mathrm{~d}, J=16.3$ $\mathrm{Hz}, 1 \mathrm{H}), 2.81(\mathrm{~d}, J=14.5 \mathrm{~Hz}, 1 \mathrm{H}), 2.76-2.70(\mathrm{~m}, 4 \mathrm{H}), 2.64(\mathrm{~d}, J=14.5 \mathrm{~Hz}, 1 \mathrm{H}), 1.60-1.47(\mathrm{~m}, 8 \mathrm{H}), 1.37(\mathrm{~s}, 3 \mathrm{H}) ;{ }^{13} \mathrm{C}$ 
NMR $\left(\mathrm{CDCl}_{3}, 125 \mathrm{MHz}\right): \delta 165.0,138.4,133.6,129.7,127.9,127.2,125.1,84.3,65.1,57.6(2 \mathrm{C}), 35.4,28.6(2 \mathrm{C}), 27.1$ (2C), 24.4; FTIR (thin film): $\mathrm{cm}^{-1} 2923,1716,1607,1460,1292,1115,742 ;$ HRMS-ESI $(\mathrm{m} / \mathrm{z}) \mathrm{Calcd}_{\text {for }}\left(\mathrm{C}_{17} \mathrm{H}_{24} \mathrm{NO}_{2}\right)$ $\left([\mathrm{M}+\mathrm{H}]^{+}\right): 274.1802$; found: 274.1802 .

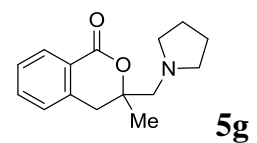

3-Methyl-3-(pyrrolidin-1-ylmethyl)isochroman-1-one (5g). Synthesized using condition A, without BCP and with 20\% $\mathrm{Cu}(\mathrm{OTf})_{2}$. Isolated pure following $\mathrm{Al}_{2} \mathrm{O}_{3}$ filtration as a tan oil $(25.4 \mathrm{mg}, 26 \%) . \mathrm{R}_{f}=0.09(50 \%$ EtOAc-hexanes $) ;{ }^{1} \mathrm{H} \mathrm{NMR}$ $\left(\mathrm{CDCl}_{3}, 400 \mathrm{MHz}\right): \delta 8.06(\mathrm{~d}, J=7.5 \mathrm{~Hz}, 1 \mathrm{H}), 7.51(\mathrm{t}, J=7.5 \mathrm{~Hz}, 1 \mathrm{H}), 7.34(\mathrm{t}, J=7.5 \mathrm{~Hz}, 1 \mathrm{H}), 7.21(\mathrm{~d}, J=7.5 \mathrm{~Hz}, 1 \mathrm{H})$, $3.43(\mathrm{~d}, J=16.4 \mathrm{~Hz}, 1 \mathrm{H}), 2.84(\mathrm{~d}, J=16.4 \mathrm{~Hz}, 1 \mathrm{H}), 2.80(\mathrm{~d}, J=13.8 \mathrm{~Hz}, 1 \mathrm{H}), 2.75(\mathrm{~d}, J=13.8 \mathrm{~Hz}, 1 \mathrm{H}), 2.72-2.59(\mathrm{~m}$, $4 \mathrm{H}), 1.77-1.66(\mathrm{~m}, 4 \mathrm{H}), 1.38(\mathrm{~s}, 3 \mathrm{H}) ;{ }^{13} \mathrm{C} \mathrm{NMR}\left(\mathrm{CDCl}_{3}, 125 \mathrm{MHz}\right): \delta 164.8,138.2,133.6,129.7,128.0,127.2,124.8$, 83.3, 64.0, 55.8 (2C), 35.9, 24.3, 23.8 (2C); FTIR (thin film): $\mathrm{cm}^{-1}$ 2968, 1714, 1607, 1459, 1290, 1116, 1067, 744; HRMS-ESI (m/z) Calcd for $\left(\mathrm{C}_{15} \mathrm{H}_{20} \mathrm{NO}_{2}\right)\left([\mathrm{M}+\mathrm{H}]^{+}\right)$: 246.1489 ; found: 246.1491 .<smiles>CCN(CC)CC1(C)Cc2ccccc2C(=O)O1</smiles>

3-((Diethylamino)methyl)-3-methylisochroman-1-one (5h). Synthesized using condition A, without BCP and with $20 \%$ $\mathrm{Cu}(\mathrm{OTf})_{2}$. Isolated by Flash Column Chromatography (25\% EtOAc-hexanes) as a clear oil $(39.0 \mathrm{mg}, 39 \%)$. $\mathrm{R}_{f}=0.13$ (50\% EtOAc-hexanes); ${ }^{1} \mathrm{H} \mathrm{NMR}\left(\mathrm{CDCl}_{3}, 400 \mathrm{MHz}\right): \delta 8.06(\mathrm{~d}, J=7.5 \mathrm{~Hz}, 1 \mathrm{H}), 7.50(\mathrm{t}, J=7.5 \mathrm{~Hz}, 1 \mathrm{H}), 7.33(\mathrm{t}, J=7.5$ $\mathrm{Hz}, 1 \mathrm{H}), 7.20$ (d, $J=7.5 \mathrm{~Hz}, 1 \mathrm{H}), 3.41(\mathrm{~d}, J=16.4 \mathrm{~Hz}, 1 \mathrm{H}), 2.79$ (d, $J=16.4 \mathrm{~Hz}, 1 \mathrm{H}), 2.69$ (d, $J=14.5 \mathrm{~Hz}, 1 \mathrm{H}), 2.65-$ $2.47(\mathrm{~m}, 5 \mathrm{H}), 1.35(\mathrm{~s}, 3 \mathrm{H}), 0.93(\mathrm{t}, J=7.1 \mathrm{~Hz}, 6 \mathrm{H}) ;{ }^{13} \mathrm{C} \mathrm{NMR}\left(\mathrm{CDCl}_{3}, 125 \mathrm{MHz}\right): \delta 165.0,138.4,133.6,129.7,127.9$, 127.1, 125.0, 83.9, 61.4, 48.2 (2C), 35.6, 24.5, 11.8 (2C); FTIR (thin film): $\mathrm{cm}^{-1}$ 2969, 1713, 1607, 1460, 1292, 1115, 1065, 743; HRMS-ESI (m/z) Calcd for $\left(\mathrm{C}_{15} \mathrm{H}_{22} \mathrm{NO}_{2}\right)\left([\mathrm{M}+\mathrm{H}]^{+}\right): 248.1645$; found: 248.1644 .

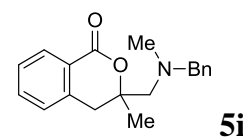

3-((Benzyl(methyl)amino)methyl)-3-methylisochroman-1-one (5i). Synthesized using condition A, without BCP and with $20 \% \mathrm{Cu}(\mathrm{OTf})_{2}$. Isolated by Flash Column Chromatography $(10 \%$ EtOAc-hexanes $)$ as a clear oil $(32.6 \mathrm{mg}, 28 \%) . \mathrm{R}_{f}=$ 0.35 (50\% EtOAc-hexanes); ${ }^{1} \mathrm{H}$ NMR $\left(\mathrm{CDCl}_{3}, 400 \mathrm{MHz}\right): \delta 8.05(\mathrm{~d}, J=7.6 \mathrm{~Hz}, 1 \mathrm{H}), 7.48(\mathrm{t}, J=7.6 \mathrm{~Hz}, 1 \mathrm{H}), 7.36-7.19$ $(\mathrm{m}, 6 \mathrm{H}), 7.16(\mathrm{~d}, J=7.6 \mathrm{~Hz}, 1 \mathrm{H}), 3.61(\mathrm{~d}, J=13.3 \mathrm{~Hz}, 1 \mathrm{H}), 3.54(\mathrm{~d}, J=13.3 \mathrm{~Hz}, 1 \mathrm{H}), 3.44(\mathrm{~d}, J=16.5 \mathrm{~Hz}, 1 \mathrm{H}), 2.70(\mathrm{~d}, J$ $=16.5 \mathrm{~Hz}, 1 \mathrm{H}), 2.69(\mathrm{~d}, J=14.2 \mathrm{~Hz}, 1 \mathrm{H}), 2.59(\mathrm{~d}, J=14.2 \mathrm{~Hz}, 1 \mathrm{H}), 2.29(\mathrm{~s}, 3 \mathrm{H}), 1.34(\mathrm{~s}, 3 \mathrm{H}) ;{ }^{13} \mathrm{C} \mathrm{NMR}\left(\mathrm{CDCl}{ }_{3}, 125\right.$ MHz): $\delta 164.8,139.1,138.3,133.7,129.8,128.8,128.2,128.0,127.2,127.0,124.8,83.9,64.5,64.0,44.4,35.6,24.3$; FTIR (thin film): $\mathrm{cm}^{-1} 2791,1712,1607,1494,1459,1291,1114,1030,742 ;$ HRMS-ESI (m/z) $\mathrm{Calcd}$ for $\left(\mathrm{C}_{19} \mathrm{H}_{22} \mathrm{NO}_{2}\right)$ $\left([\mathrm{M}+\mathrm{H}]^{+}\right): 296.1645$; found: 296.1647 .

\subsection{Mechanistic Study Protocols and Characterization Data of Synthesized Compounds}
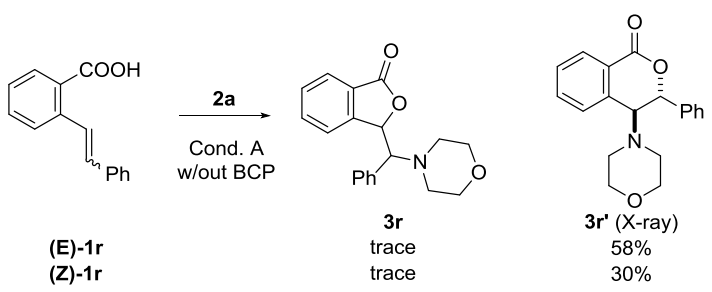

4-Morpholino-3-phenylisochroman-1-one (3r').

From $(\boldsymbol{E})$-1r. Synthesized using condition A, without BCP. Isolated by Flash Column Chromatography $(100 \%$ hexanes $\rightarrow$ $30 \%$ EtOAc-hexanes) as a clear oil (71.5 mg, 58\%). 
From (Z)-1r. Synthesized using condition A, without BCP. Isolated by Flash Column Chromatography (100\% hexanes $\rightarrow$ $30 \%$ EtOAc-hexanes) as a clear oil $(41.0 \mathrm{mg}, 33 \%)$.

$\mathrm{R}_{f}=0.07\left(25 \%\right.$ EtOAc-hexanes); ${ }^{1} \mathrm{H} \mathrm{NMR}\left(\mathrm{CDCl}_{3}, 400 \mathrm{MHz}\right): \delta 8.26(\mathrm{~d}, J=7.6 \mathrm{~Hz}, 1 \mathrm{H}), 7.70(\mathrm{t}, J=7.6 \mathrm{~Hz}, 1 \mathrm{H}), 7.56(\mathrm{t}$, $J=7.6 \mathrm{~Hz}, 1 \mathrm{H}), 7.52(\mathrm{~d}, J=7.6 \mathrm{~Hz}, 1 \mathrm{H}), 7.44-7.32(\mathrm{~m}, 5 \mathrm{H}), 5.95(\mathrm{~d}, J=4.1 \mathrm{~Hz}, 1 \mathrm{H}), 4.19(\mathrm{~d}, J=4.1 \mathrm{~Hz}, 1 \mathrm{H}), 3.79(\mathrm{ddd}$, $J=11.1,6.1,3.2 \mathrm{~Hz}, 2 \mathrm{H}), 3.73(\mathrm{ddd}, J=11.1,6.1,3.2 \mathrm{~Hz}, 2 \mathrm{H}), 2.74(\mathrm{ddd}, J=10.9,6.1,3.2 \mathrm{~Hz}, 2 \mathrm{H}), 2.61(\mathrm{ddd}, J=10.9$, 6.1, $3.2 \mathrm{~Hz}, 2 \mathrm{H}) ;{ }^{13} \mathrm{C} \mathrm{NMR}\left(\mathrm{CDCl}_{3}, 125 \mathrm{MHz}\right): \delta 164.1137 .9,136.3,133.9,129.7,128.7,128.6,128.6(2 \mathrm{C}), 128.3,126.2$ (2C), 125.6, 80.6, 67.1 (2C), 65.1, 49.6; FTIR (thin film): $\mathrm{cm}^{-1}$ 2851, 1717, 1602, 1452, 1280, 1255, 1113, 1007, 742, 697; HRMS-ESI (m/z) Calcd for $\left(\mathrm{C}_{19} \mathrm{H}_{20} \mathrm{NO}_{3}\right)\left([\mathrm{M}+\mathrm{H}]^{+}\right): 310.1438$; found: 310.1438 .
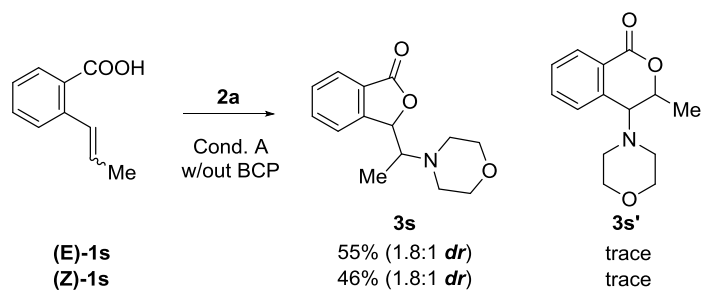

\section{3-(1-Morpholinoethyl)isobenzofuran-1(3H)-one (3s).}

From $(\boldsymbol{E})$-1s. Synthesized using condition A, without BCP. Two diastereomers of $3 \mathbf{s}$ were observed in the crude mixture in a ratio of $1.8: 1$ by ${ }^{1} \mathrm{H}$ NMR. Isolated by Flash Column Chromatography (addition of $0.2 \mathrm{~mL}$ TFA to crude reaction mixture, 50\% EtOAc-hexanes, then 50\% EtOAc-hexanes with 5\% TEA $\rightarrow$ 75\% EtOAc-hexanes with 5\% TEA) to yield 3s $(54.8 \mathrm{mg}, 55 \%)$.

From (Z)-1s. Synthesized using condition A, without BCP. Two diastereomers of $\mathbf{3 s}$ were observed in the crude mixture in a ratio of $1.8: 1$ by ${ }^{1} \mathrm{H}$ NMR. Isolated by Flash Column Chromatography (addition of $0.2 \mathrm{~mL}$ TFA to crude reaction mixture, 50\% EtOAc-hexanes, then 50\% EtOAc-hexanes with 5\% TEA $\rightarrow$ 75\% EtOAc-hexanes with 5\% TEA) to yield 3s $(45.9 \mathrm{mg}, 46 \%)$.

Major diastereomer. Isolated as an off-white solid. $\mathrm{R}_{f}=0.24\left(50 \%\right.$ EtOAc-hexanes); ${ }^{1} \mathrm{H} \mathrm{NMR}(\mathrm{CDCl} 3,400 \mathrm{MHz}): \delta$ $7.88(\mathrm{~d}, J=7.6 \mathrm{~Hz}, 1 \mathrm{H}), 7.64(\mathrm{t}, J=7.6 \mathrm{~Hz}, 1 \mathrm{H}), 7.58(\mathrm{~d}, J=7.6 \mathrm{~Hz}, 1 \mathrm{H}), 7.51(\mathrm{t}, J=7.6 \mathrm{~Hz}, 1 \mathrm{H}), 5.49(\mathrm{~d}, J=3.9 \mathrm{~Hz}$, $1 \mathrm{H}), 3.58(\mathrm{t}, J=4.6,4 \mathrm{H}), 3.05(\mathrm{qd}, J=6.9,3.9 \mathrm{~Hz}, 1 \mathrm{H}), 2.67-2.60(\mathrm{~m}, 2 \mathrm{H}), 2.59-2.51(\mathrm{~m}, 2 \mathrm{H}), 0.99(\mathrm{~d}, J=6.9 \mathrm{~Hz}, 3 \mathrm{H})$; ${ }^{13} \mathrm{C} \mathrm{NMR}\left(\mathrm{CDCl}_{3}, 125 \mathrm{MHz}\right): \delta 170.6,148.1,133.5,129.0,127.0,125.4,123.0,82.4,67.3$ (2C), 61.3, 51.2 (2C), 11.3; FTIR (thin film): $\mathrm{cm}^{-1} 2852,1760,1466,1285,1118,1057,753 ;$ HRMS-ESI $(\mathrm{m} / \mathrm{z})$ Calcd for $\left(\mathrm{C}_{14} \mathrm{H}_{18} \mathrm{NO}_{3}\right)\left([\mathrm{M}+\mathrm{H}]^{+}\right)$: 248.1281; found: 248.1282 .

Minor diastereomer. Isolated as an off-white solid. $\mathrm{R}_{f}=0.12(50 \%$ EtOAc-hexanes $) ;{ }^{1} \mathrm{H} \mathrm{NMR}\left(\mathrm{CDCl}{ }_{3}, 400 \mathrm{MHz}\right): \delta$ $7.89(\mathrm{~d}, J=7.6 \mathrm{~Hz}, 1 \mathrm{H}), 7.67(\mathrm{t}, J=7.6 \mathrm{~Hz}, 1 \mathrm{H}), 7.60(\mathrm{~d}, J=7.6 \mathrm{~Hz}, 1 \mathrm{H}), 7.53(\mathrm{t}, J=7.6 \mathrm{~Hz}, 1 \mathrm{H}), 5.56(\mathrm{~d}, J=4.5 \mathrm{~Hz}$, $1 \mathrm{H}), 3.76(\mathrm{t}, J=4.2 \mathrm{~Hz}, 4 \mathrm{H}), 2.99(\mathrm{qd}, J=6.8,4.5 \mathrm{~Hz}, 1 \mathrm{H}), 2.81-2.74(\mathrm{~m}, 2 \mathrm{H}), 2.69-2.61(\mathrm{~m}, 2 \mathrm{H}), 0.92(\mathrm{~d}, J=6.8 \mathrm{~Hz}$, $3 \mathrm{H}) ;{ }^{13} \mathrm{C} \mathrm{NMR}\left(\mathrm{CDCl}_{3}, 125 \mathrm{MHz}\right): \delta 170.6,148.7,133.9,129.2,126.0,125.5,122.6,82.1,67.2(2 \mathrm{C}), 63.1,49.6(2 \mathrm{C}), 7.9$; FTIR (thin film): $\mathrm{cm}^{-1}$ 2848, 1762, 1283, 1117, 1062; HRMS-ESI (m/z) Calcd for $\left(\mathrm{C}_{14} \mathrm{H}_{18} \mathrm{NO}_{3}\right)\left([\mathrm{M}+\mathrm{H}]^{+}\right): 248.1281$; found: 248.1282 .

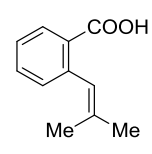

$1 t, R=M e$

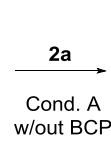

whout BCP

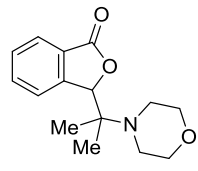

$3 \mathbf{t}(30 \%)$

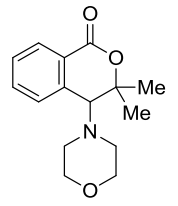

3t' $(16 \%)$

3-(2-Morpholinopropan-2-yl)isobenzofuran-1(3H)-one (3t). Synthesized using condition A, without BCP. Isolated by Flash Column Chromatography (addition of $0.2 \mathrm{~mL}$ TFA to crude reaction mixture, 50\% EtOAc-hexanes, then $25 \%$ EtOAc-hexanes with 5\% TEA) as a white solid $(31.1 \mathrm{mg}, 30 \%) . \mathrm{R}_{f}=0.15(25 \%$ EtOAc-hexanes $) ;{ }^{1} \mathrm{H} \mathrm{NMR}\left(\mathrm{CDCl}_{3}, 400\right.$ $\mathrm{MHz}): \delta 7.87(\mathrm{~d}, J=7.6 \mathrm{~Hz}, 1 \mathrm{H}), 7.86(\mathrm{~d}, J=7.6 \mathrm{~Hz}, 1 \mathrm{H}), 7.63(\mathrm{t}, J=7.6 \mathrm{~Hz}, 1 \mathrm{H}), 7.51(\mathrm{t}, J=7.6 \mathrm{~Hz}, 1 \mathrm{H}), 5.43(\mathrm{~s}, 1 \mathrm{H})$, $3.79(\mathrm{t}, J=4.6 \mathrm{~Hz}, 4 \mathrm{H}), 2.79-2.65(\mathrm{~m}, 4 \mathrm{H}), 1.21(\mathrm{~s}, 3 \mathrm{H}), 0.67(\mathrm{~s}, 3 \mathrm{H}) ;{ }^{13} \mathrm{C} \mathrm{NMR}\left(\mathrm{CDCl}_{3}, 125 \mathrm{MHz}\right): \delta 170.8,148.1$, 133.4, 129.0, 126.8, 125.3, 124.7, 83.1, 67.6 (2C), 59.7, 46.8 (2C), 19.8, 17.8; FTIR (thin film): $\mathrm{cm}^{-1} 2847,1760,1469$, 1120, 1069, 997, 735; HRMS-ESI (m/z) Calcd for $\left(\mathrm{C}_{15} \mathrm{H}_{20} \mathrm{NO}_{3}\right)\left([\mathrm{M}+\mathrm{H}]^{+}\right)$: 262.1438; found: 262.1440 .

3,3-Dimethyl-4-morpholinoisochroman-1-one (3t'). Synthesized using condition A, without BCP. Isolated by Flash Column Chromatography $(100 \%$ hexanes $\rightarrow 30 \%$ EtOAc-hexanes $)$ as an off-white solid $(16.7 \mathrm{mg}, 16 \%)$. $\mathrm{R}_{f}=0.54(50 \%$ EtOAc-hexanes); ${ }^{1} \mathrm{H} \mathrm{NMR}\left(\mathrm{CDCl}_{3}, 400 \mathrm{MHz}\right): \delta 8.17(\mathrm{~d}, J=7.6 \mathrm{~Hz}, 1 \mathrm{H}), 7.63(\mathrm{t}, J=7.6 \mathrm{~Hz}, 1 \mathrm{H}), 7.49(\mathrm{t}, J=7.6 \mathrm{~Hz}$, 
$1 \mathrm{H}), 7.23(\mathrm{~d}, J=7.6 \mathrm{~Hz}, 1 \mathrm{H}), 3.65(\mathrm{td}, J=5.5,3.4 \mathrm{~Hz}, 4 \mathrm{H}), 3.43(\mathrm{~s}, 1 \mathrm{H}), 2.71-2.64(\mathrm{~m}, 2 \mathrm{H}), 2.23-2.15(\mathrm{~m}, 2 \mathrm{H}), 1.66(\mathrm{~s}$, $3 \mathrm{H}), 1.31(\mathrm{~s}, 3 \mathrm{H}) ;{ }^{13} \mathrm{C} \mathrm{NMR}\left(\mathrm{CDCl}_{3}, 125 \mathrm{MHz}\right): \delta 164.6,134.5,133.4,129.8,129.4,128.7,125.3,83.9,67.0(2 \mathrm{C}), 66.9$, 49.7 (2C), 28.0, 25.1; FTIR (thin film): $\mathrm{cm}^{-1}$ 2851, 1712, 1603, 1456, 1289, 1113, 1087, 941, 706; HRMS-ESI (m/z) Calcd for $\left(\mathrm{C}_{15} \mathrm{H}_{20} \mathrm{NO}_{3}\right)\left([\mathrm{M}+\mathrm{H}]^{+}\right): 262.1438$; found: 262.1439 .

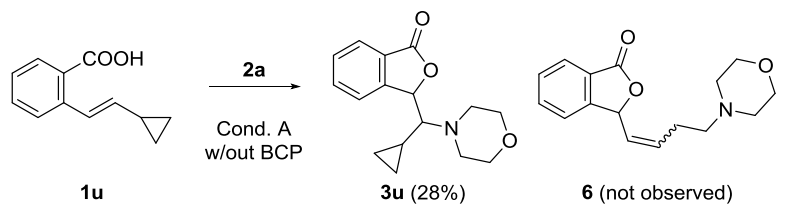

3-(Cyclopropyl(morpholino)methyl)isobenzofuran-1(3H)-one (3u). Synthesized using condition A, without BCP . Two diastereomers of $3 \mathbf{u}$ were observed in a ratio of $4.1: 1$ by ${ }^{1} \mathrm{H}$ NMR of the crude reaction mixture. Isolated by Flash Column Chromatography (addition of $0.2 \mathrm{~mL}$ TFA to crude reaction mixture, 50\% EtOAc-hexanes, then 50\% EtOAc-hexanes with $5 \%$ TEA) to yield $3 \mathbf{u}(30.6 \mathrm{mg}, 28 \%$ an inseparable 5:1 mixture of two diastereomers).

Major Diastereomer. Isolated as a white solid. $\mathrm{R}_{f}=0.33\left(50 \%\right.$ EtOAc-hexanes); ${ }^{1} \mathrm{H}$ NMR $\left(\mathrm{CDCl}_{3}, 400 \mathrm{MHz}\right): \delta 7.89(\mathrm{~d}$, $J=7.6 \mathrm{~Hz}, 1 \mathrm{H}), 7.66-7.58(\mathrm{~m}, 2 \mathrm{H}), 7.54-7.47(\mathrm{~m}, 1 \mathrm{H}), 5.60(\mathrm{~d}, J=2.9 \mathrm{~Hz}, 1 \mathrm{H}), 3.61-3.51(\mathrm{~m}, 4 \mathrm{H}), 2.80(\mathrm{dt}, J=11.4,4.5$ $\mathrm{Hz}, 2 \mathrm{H}), 2.63(\mathrm{dt}, J=11.4,4.5 \mathrm{~Hz}, 2 \mathrm{H}), 2.07(\mathrm{dd}, J=9.5,2.9 \mathrm{~Hz}, 1 \mathrm{H}), 0.61-0.50(\mathrm{~m}, 2 \mathrm{H}), 0.50-0.40$ (m, 1H), $0.37-0.25$ $(\mathrm{m}, 2 \mathrm{H}) ;{ }^{13} \mathrm{C}$ NMR $\left(\mathrm{CDCl}_{3}, 125 \mathrm{MHz}\right): \delta 170.8,148.6,133.4,128.8,127.2,125.2,122.7,82.5,71.8,67.5(2 \mathrm{C}), 52.2(2 \mathrm{C})$, 8.7, 4.4, 2.9; FTIR (thin film): $\mathrm{cm}^{-1} 2850,1759,1466,1287,1116,1068$; HRMS-ESI (m/z) Calcd for $\left(\mathrm{C}_{16} \mathrm{H}_{20} \mathrm{NO}_{3}\right)$ $\left([\mathrm{M}+\mathrm{H}]^{+}\right): 274.1438$; found: 274.1437 .

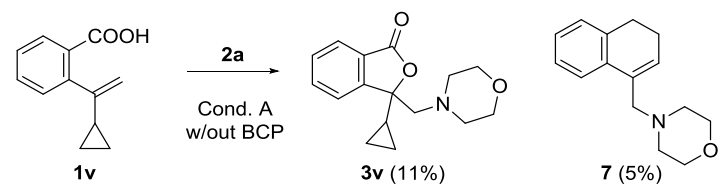

3-Cyclopropyl-3-(morpholinomethyl)isobenzofuran-1(3H)-one (3v). Synthesized using condition A, without BCP. Isolated by Flash Column Chromatography (100\% hexanes $\rightarrow 100 \%$ EtOAc) as a clear oil $(12.2 \mathrm{mg}, 11 \%)$. $\mathrm{R}_{f}=0.26$ (50\% EtOAc-hexanes); ${ }^{1} \mathrm{H}$ NMR $\left(\mathrm{CDCl}_{3}, 400 \mathrm{MHz}\right): \delta 7.86(\mathrm{dt}, J=7.5,0.9 \mathrm{~Hz}, 1 \mathrm{H}), 7.62(\mathrm{td}, J=7.5,0.9 \mathrm{~Hz}, 1 \mathrm{H}), 7.50$ $(\mathrm{td}, J=7.5,0.9 \mathrm{~Hz}, 1 \mathrm{H}), 7.46(\mathrm{dt}, J=7.5,0.9 \mathrm{~Hz}, 1 \mathrm{H}), 3.50(\mathrm{ddd}, J=11.1,6.3,2.9 \mathrm{~Hz}, 2 \mathrm{H}), 3.42(\mathrm{ddd}, J=11.1,6.3,2.9$ $\mathrm{Hz}, 2 \mathrm{H}), 2.92(\mathrm{~d}, J=14.2 \mathrm{~Hz}, 1 \mathrm{H}), 2.87(\mathrm{~d}, J=14.2 \mathrm{~Hz}, 1 \mathrm{H}), 2.51(\mathrm{ddd}, J=11.1,6.3,2.9 \mathrm{~Hz}, 2 \mathrm{H}), 2.35$ (ddd, $J=11.1$, 6.3, $2.9 \mathrm{~Hz}, 2 \mathrm{H}), 1.47(\mathrm{tt}, J=8.1,5.5 \mathrm{~Hz}, 1 \mathrm{H}), 0.61-0.50(\mathrm{~m}, 1 \mathrm{H}), 0.48-0.37(\mathrm{~m}, 2 \mathrm{H}), 0.37-0.26(\mathrm{~m}, 1 \mathrm{H}) ;{ }^{13} \mathrm{C} \mathrm{NMR}$ $\left(\mathrm{CDCl}_{3}, 125 \mathrm{MHz}\right): \delta$ 170.0, 151.2, 133.4, 128.9, 127.3, 125.3, 121.6, 88.5, 67.1 (2C), 64.9, 55.0 (2C), 16.2, 1.5, 0.6; FTIR (thin film): $\mathrm{cm}^{-1} 2850,1762,1287,1117,1008,695$; HRMS-ESI $(\mathrm{m} / \mathrm{z})$ Calcd for $\left(\mathrm{C}_{16} \mathrm{H}_{20} \mathrm{NO}_{3}\right)\left([\mathrm{M}+\mathrm{H}]^{+}\right): 274.1438$; found: 274.1437.

4-((3,4-Dihydronaphthalen-1-yl)methyl)morpholine (7). Synthesized using condition A, without BCP. Isolated by Flash Column Chromatography (100\% hexanes $\rightarrow 100 \%$ EtOAc) as a clear oil (4.8 mg, 5\%). $\mathrm{R}_{f}=0.08(50 \%$ EtOAc-hexanes); ${ }^{1} \mathrm{H}$ NMR $\left(\mathrm{CDCl}_{3}, 400 \mathrm{MHz}\right): \delta 7.57(\mathrm{~d}, J=7.6 \mathrm{~Hz}, 1 \mathrm{H}), 7.23-7.09(\mathrm{~m}, 3 \mathrm{H}), 6.00(\mathrm{t}, J=4.4 \mathrm{~Hz}, 1 \mathrm{H}), 3.69(\mathrm{t}, J=4.6 \mathrm{~Hz}$, $4 \mathrm{H}), 3.29(\mathrm{~s}, 2 \mathrm{H}), 2.76(\mathrm{t}, J=8.0 \mathrm{~Hz}, 2 \mathrm{H}), 2.34-2.26(\mathrm{~m}, 2 \mathrm{H}) ;{ }^{13} \mathrm{C} \mathrm{NMR}\left(\mathrm{CDCl}_{3}, 125 \mathrm{MHz}\right): \delta 136.3,134.5,128.8,127.3$, 126.8, 126.2, 123.8, 67.1 (2C), 62.1, 53.6 (2C), 28.1, 23.1; FTIR (thin film): $\mathrm{cm}^{-1}$ 2917, 1739, 1453, 1115, 1003, 866, 760, 735; HRMS-ESI (m/z) Calcd for $\left(\mathrm{C}_{15} \mathrm{H}_{20} \mathrm{NO}\right)\left([\mathrm{M}+\mathrm{H}]^{+}\right): 230.1539$; found: 230.1539 .

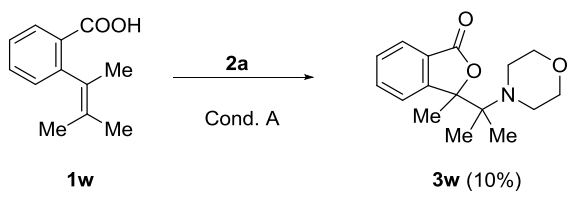

3-Methyl-3-(2-morpholinopropan-2-yl)isobenzofuran-1(3H)-one (3w). Synthesized using condition A. Isolated by Flash Column Chromatography (addition of $0.2 \mathrm{~mL}$ TFA to crude reaction mixture, 50\% EtOAc-hexanes, then 50\%

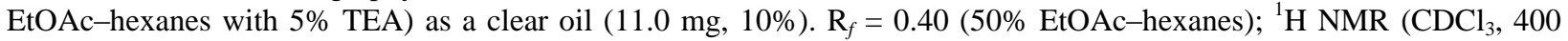
$\mathrm{MHz}): \delta 7.86(\mathrm{~d}, J=7.5 \mathrm{~Hz}, 1 \mathrm{H}), 7.62-7.53(\mathrm{~m}, 2 \mathrm{H}), 7.49(\mathrm{t}, J=7.5 \mathrm{~Hz}, 1 \mathrm{H}), 3.49(\mathrm{ddd}, J=10.9,6.5,2.8 \mathrm{~Hz}, 2 \mathrm{H}), 3.44-$ $3.35(\mathrm{~m}, 2 \mathrm{H}), 2.65-2.55(\mathrm{~m}, 2 \mathrm{H}), 2.48-2.35(\mathrm{~m}, 2 \mathrm{H}), 1.66(\mathrm{~s}, 3 \mathrm{H}), 1.23(\mathrm{~s}, 3 \mathrm{H}), 1.18(\mathrm{~s}, 3 \mathrm{H}) ;{ }^{13} \mathrm{C} \mathrm{NMR}\left(\mathrm{CDCl}_{3}, 125 \mathrm{MHz}\right)$ : $\delta 170.3,152.7,132.9,128.8,127.8,125.2,122.8,93.1,67.9(2 \mathrm{C}), 62.1,48.8(2 \mathrm{C}), 22.0,21.3,19.8$; FTIR (thin film): $\mathrm{cm}^{-1}$ 2851, 1757, 1464, 1273, 1120, 1034, 924, 763; HRMS-ESI (m/z) Calcd for $\left(\mathrm{C}_{16} \mathrm{H}_{22} \mathrm{NO}_{3}\right)\left([\mathrm{M}+\mathrm{H}]^{+}\right): 276.1594$; found: 276.1595 . 


\section{Additional Control Experiments}

Table S10. Effect of oxidative atmosphere upon reaction yield. ${ }^{a}$

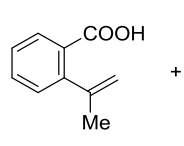

$1 \mathrm{a}$

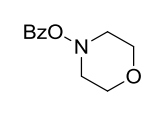

$2 a$

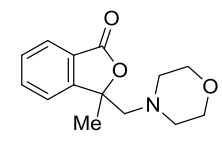

$3 \mathbf{a}$

\begin{tabular}{ccccc}
\hline Entry & Catalyst & Atmosphere & $\mathbf{3 a}^{\mathbf{b}}(\%)$ & Time (h) \\
\hline 1 & $\mathrm{Cu}(\mathrm{OTf})_{2}$ & $\mathrm{~N}_{2}$ & $87 \%$ & 0.25 \\
2 & {$[\mathrm{CuOTf}]_{2} \cdot$ tol } & $\mathrm{N}_{2}$ & $82 \%$ & 1.0 \\
3 & $\mathrm{Cu}(\mathrm{OTf})_{2}$ & $\mathrm{O}_{2}$ & $10 \%$ & 10.0 \\
4 & {$[\mathrm{CuOTf}]_{2} \cdot$ tol } & $\mathrm{O}_{2}$ & $14 \%$ & 43.5 \\
\hline
\end{tabular}

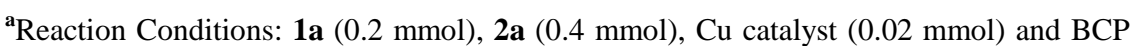
$(0.02 \mathrm{mmol})$ in DCE $(1.0 \mathrm{~mL})$ at $80{ }^{\circ} \mathrm{C}$. ${ }^{\mathbf{b}}$ Yield determined by ${ }^{1} \mathrm{H}$ NMR spectroscopy with dibromomethane as a quantitative internal standard.

Table S11. Probing possible cyclization independent of $\boldsymbol{O}$-benzoylhydroxylamine by means of Brønsted acid-catalyzed protonation from $\mathrm{Cu}(\mathrm{OTf})_{2}$ ligands. $^{a}$

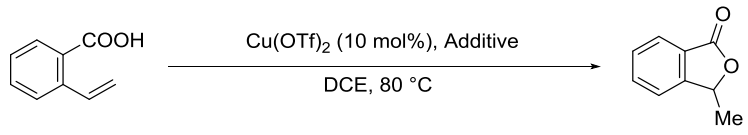

1c

11

\begin{tabular}{ccccc}
\hline Entry & $\mathrm{Cu}(\mathrm{OTf})_{2}(\mathrm{~mol} \%)$ & Additive (equiv) & $\mathbf{1 1}^{\mathbf{b}}(\%)$ & Time (h) \\
\hline 1 & $10 \%$ & $\sim$ & $2 \%$ & 2.0 \\
2 & $\sim$ & $\mathrm{MsOH}(0.2$ equiv) & $2 \%$ & 2.0 \\
3 & $10 \%$ & Morpholine (1.0 equiv) & $\mathrm{ND}$ & 2.0 \\
\hline
\end{tabular}

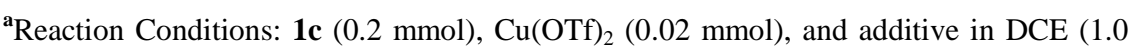
$\mathrm{mL}$ ) at $80{ }^{\circ} \mathrm{C}$. ${ }^{\mathbf{b}}$ Yield determined by ${ }^{1} \mathrm{H}$ NMR spectroscopy with dibromomethane as a quantitative internal standard. ${ }^{\mathrm{c}} \mathrm{ND}=$ not detected by GCMS.

Table S12. Radical-trapping attempts using TEMPO. ${ }^{a}$

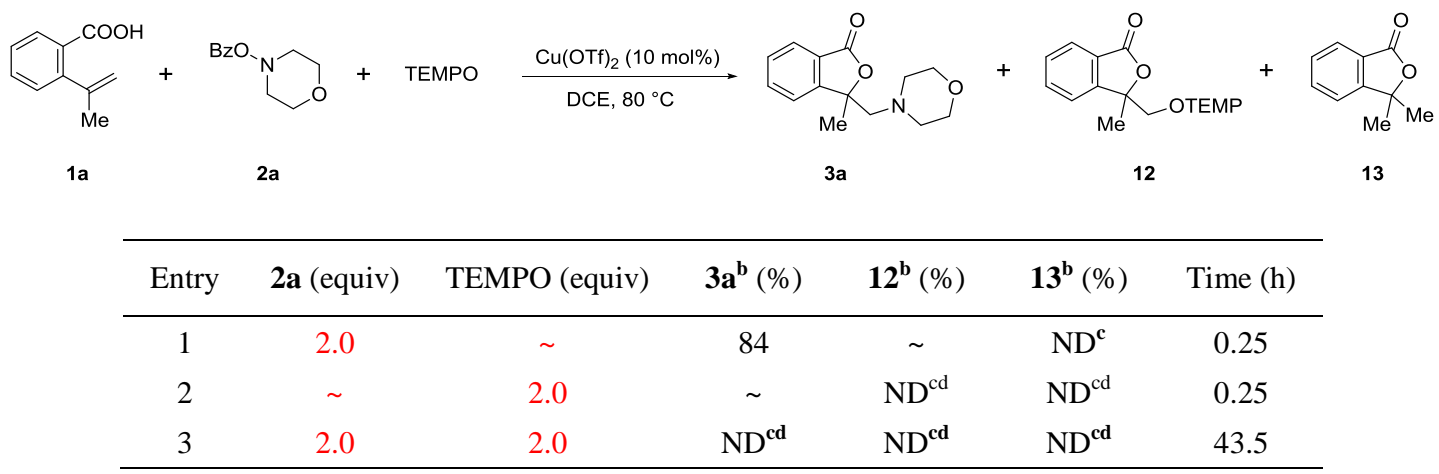

${ }^{\mathrm{a}}$ Reaction Conditions: 1a $(0.2 \mathrm{mmol}), \mathbf{2 a}$, TEMPO, and Cu catalyst $(0.02 \mathrm{mmol})$ in DCE $(1.0 \mathrm{~mL}), 80{ }^{\circ} \mathrm{C}, 2 \mathrm{~h} .{ }^{\mathrm{b}}$ Yield determined by ${ }^{1} \mathrm{H}$ NMR spectroscopy with dibromomethane as a quantitative internal standard. ${ }^{c} \mathrm{ND}=$ not detected by GCMS. ${ }^{d}$ Quantitative recovery of 1a. 


\subsection{Intermolecular Amino Oxygenation Standard Protocols and Characterization Data of Synthesized Compounds}

\section{Typical Condition B (Intermolecular Standard Conditions):}

To a 1 Dram vial with Teflon-coated micro stir bar was added carboxylic acid (1.2 mmol, 3 equiv), $O$ benzoylhydroxylamine ( $0.4 \mathrm{mmol}, 1$ equiv), and copper (II) acetate ( $0.08 \mathrm{mmol}, 0.2$ equiv), followed by addition of anhydrous 1,2-dichloroethane $(2.0 \mathrm{~mL})$ and olefin $\left(1.2 \mathrm{mmol}, 3\right.$ equiv). The resulting solution was stirred at $80{ }^{\circ} \mathrm{C}$ for $1 \mathrm{~h}$ until the consumption of $O$-benzoylhydroxylamine (monitored by TLC). The resulting reaction mixture was cooled to

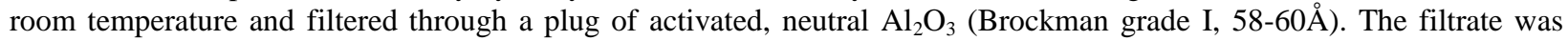
concentrated under reduced pressure, providing the crude reaction mixture. The crude reaction mixture was purified by silica column chromatography unless otherwise noted.

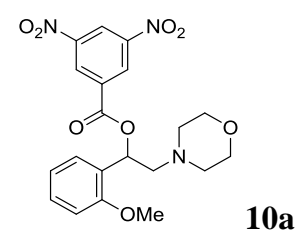

1-(2-Methoxyphenyl)-2-morpholinoethyl 3,5-dinitrobenzoate (10a). Synthesized using condition B. Purification by flash column chromatography $\left(100 \%\right.$ hexanes $\rightarrow 35 \%$ EtOAc-hexanes) gave 10a as a yellow oil $(169.7 .0 \mathrm{mg}, 98 \%)$. $\mathrm{R}_{f}=$ 0.53 (50\% EtOAc-hexanes); ${ }^{1} \mathrm{H} \mathrm{NMR}\left(\mathrm{CDCl}_{3}, 400 \mathrm{MHz}\right): \delta 9.21(\mathrm{t}, J=2.2 \mathrm{~Hz}, 1 \mathrm{H}), 9.19(\mathrm{~d}, J=2.2 \mathrm{~Hz}, 2 \mathrm{H}), 7.41(\mathrm{dd}, J$ $=7.5,1.6 \mathrm{~Hz}, 1 \mathrm{H}), 7.31(\mathrm{td}, J=7.5,1.6 \mathrm{~Hz}, 1 \mathrm{H}), 6.98(\mathrm{t}, J=7.5 \mathrm{~Hz}, 1 \mathrm{H}), 6.92(\mathrm{~d}, J=7.5 \mathrm{~Hz}, 1 \mathrm{H}), 6.71(\mathrm{dd}, J=9.6,2.8$ $\mathrm{Hz}, 1 \mathrm{H}), 3.90$ (s, 3H), 3.68-3.58 (m, 4H), $2.97(\mathrm{dd}, J=13.6,9.6 \mathrm{~Hz}, 1 \mathrm{H}), 2.78-2.67(\mathrm{~m}, 3 \mathrm{H}), 2.52-2.42(\mathrm{~m}, 2 \mathrm{H}) ;{ }^{13} \mathrm{C}$ NMR $\left(\mathrm{CDCl}_{3}, 125 \mathrm{MHz}\right): \delta$ 161.6, 156.1, 148.5 (2C), 134.3, 129.5, 129.3 (2C), 126.3, 126.0, 122.1, 120.6, 110.6, 70.1, 66.9 (2C), 62.9, 55.5, 53.6 (2C); FTIR (thin film): $\mathrm{cm}^{-1}$ 2958, 1730, 1542, 1494, 1456, 1343, 1272, 1166, 1115, 719; HRMS-ESI $(\mathrm{m} / \mathrm{z})$ Calcd for $\left(\mathrm{C}_{20} \mathrm{H}_{22} \mathrm{~N}_{3} \mathrm{O}_{8}\right)\left([\mathrm{M}+\mathrm{H}]^{+}\right)$: 432.1401 ; found: 432.1398 .

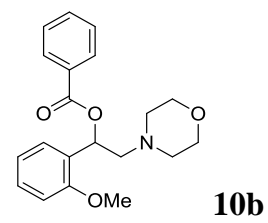

1-(2-Methoxyphenyl)-2-morpholinoethyl benzoate (10b). Synthesized using condition B. Purification by flash column chromatography $\left(100 \%\right.$ hexanes $\rightarrow 30 \%$ EtOAc-hexanes) gave $\mathbf{1 0 b}$ as a yellow oil $(80.6 \mathrm{mg}, 59 \%)$. $\mathrm{R}_{f}=0.57(50 \%$ EtOAc-hexanes); ${ }^{1} \mathrm{H} \mathrm{NMR}\left(\mathrm{CDCl}_{3}, 400 \mathrm{MHz}\right): \delta 8.15-8.10(\mathrm{~m}, 2 \mathrm{H}), 7.57(\mathrm{t}, J=7.4 \mathrm{~Hz}, 1 \mathrm{H}), 7.46(\mathrm{t}, J=7.4 \mathrm{~Hz}, 2 \mathrm{H})$, $7.41(\mathrm{dd}, J=7.6,1.6 \mathrm{~Hz}, 1 \mathrm{H}), 7.29-7.24(\mathrm{~m}, 1 \mathrm{H}), 6.94(\mathrm{t}, J=7.6 \mathrm{~Hz}, 1 \mathrm{H}), 6.89(\mathrm{~d}, J=7.6 \mathrm{~Hz}, 1 \mathrm{H}), 6.65(\mathrm{dd}, J=9.0,2.8$ $\mathrm{Hz}, 1 \mathrm{H}), 3.88(\mathrm{~s}, 3 \mathrm{H}), 3.67(\mathrm{t}, J=4.6 \mathrm{~Hz}, 4 \mathrm{H}), 2.93(\mathrm{dd}, J=13.6,9.0 \mathrm{~Hz}, 1 \mathrm{H}), 2.79-2.70(\mathrm{~m}, 3 \mathrm{H}), 2.61-2.48(\mathrm{~m}, 2 \mathrm{H}) ;{ }^{13} \mathrm{C}$ NMR $\left(\mathrm{CDCl}_{3}, 125 \mathrm{MHz}\right): \delta 165.5,155.9,132.8,130.5,129.6$ (2C), 128.8, 128.3 (2C), 127.7, 126.1, 120.5, 110.4, 68.3, 66.9 (2C), 63.0, 55.4, 53.6 (2C); FTIR (thin film): $\mathrm{cm}^{-1}$ 2957, 1716, 1493, 1451, 1268, 1239, 1109, 1026, 708; HRMS-ESI $(\mathrm{m} / \mathrm{z})$ Calcd for $\left(\mathrm{C}_{20} \mathrm{H}_{24} \mathrm{NO}_{4}\right)\left([\mathrm{M}+\mathrm{H}]^{+}\right): 342.1700$; found: 342.1700 .

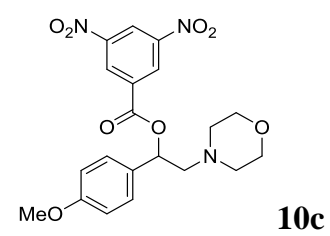

1-(4-Methoxyphenyl)-2-morpholinoethyl 3,5-dinitrobenzoate (10c). Synthesized using condition B. Purification by flash column chromatography $\left(100 \%\right.$ hexanes $\rightarrow 40 \%$ EtOAc-hexanes) gave 10c as a yellow oil $(148.4 \mathrm{mg}, 86 \%) . \mathrm{R}_{f}=$ 0.51 (50\% EtOAc-hexanes); ${ }^{1} \mathrm{H}$ NMR $\left(\mathrm{CDCl}_{3}, 400 \mathrm{MHz}\right): \delta 9.20-9.17(\mathrm{~m}, 1 \mathrm{H}), 9.16-9.11(\mathrm{~m}, 2 \mathrm{H}), 7.38(\mathrm{~d}, J=8.5 \mathrm{~Hz}$, $2 \mathrm{H}), 6.90(\mathrm{~d}, J=8.5 \mathrm{~Hz}, 2 \mathrm{H}), 6.25(\mathrm{dd}, J=9.4,3.6 \mathrm{~Hz}, 1 \mathrm{H}), 3.79(\mathrm{~s}, 3 \mathrm{H}), 3.67-3.56(\mathrm{~m}, 4 \mathrm{H}), 3.07(\mathrm{dd}, J=13.5,9.4 \mathrm{~Hz}$, 1H), 2.76-2.62 (m, 3H), 2.54-2.42 (m, 2H); ${ }^{13} \mathrm{C} \mathrm{NMR}\left(\mathrm{CDCl}_{3}, 125 \mathrm{MHz}\right): \delta 161.8,159.8,148.5$ (2C), $134.2,129.6,129.3$ (2C), 128.2 (2C), 122.2, 114.0 (2C), 74.9, 66.9 (2C), 63.6, 55.2, 53.8 (2C); FTIR (thin film): $\mathrm{cm}^{-1} 2813,1727,1542,1514$, 1455, 1343, 1270, 1248, 1164, 1114, 718; HRMS-ESI $(\mathrm{m} / \mathrm{z})$ Calcd for $\left(\mathrm{C}_{20} \mathrm{H}_{22} \mathrm{~N}_{3} \mathrm{O}_{8}\right)\left([\mathrm{M}+\mathrm{H}]^{+}\right)$: 531.2086; found: 531.2085. 


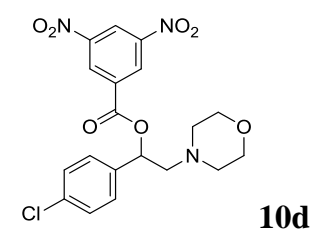

1-(4-Chlorophenyl)-2-morpholinoethyl 3,5-dinitrobenzoate (10d). Synthesized using condition B. Purification by flash column chromatography $\left(100 \%\right.$ hexanes $\rightarrow 35 \%$ EtOAc-hexanes) gave $\mathbf{1 0 d}$ as a yellow oil $(84.6 \mathrm{mg}, 49 \%) . \mathrm{R}_{f}=0.49$ (50\% EtOAc-hexanes); ${ }^{1} \mathrm{H} \mathrm{NMR}\left(\mathrm{CDCl}_{3}, 400 \mathrm{MHz}\right): \delta 9.23(\mathrm{t}, J=2.1 \mathrm{~Hz}, 1 \mathrm{H}), 9.15(\mathrm{~d}, J=2.1 \mathrm{~Hz}, 2 \mathrm{H}), 7.43-7.33(\mathrm{~m}$, $4 \mathrm{H}), 6.24(\mathrm{dd}, J=9.1,4.1 \mathrm{~Hz}, 1 \mathrm{H}), 3.63(\mathrm{t}, J=4.6 \mathrm{~Hz}, 4 \mathrm{H}), 3.03(\mathrm{dd}, J=13.6,9.1 \mathrm{~Hz}, 1 \mathrm{H}), 2.73(\mathrm{dd}, J=13.6,4.1 \mathrm{~Hz}$, 1H), 2.70-2.61 (m, 2H), 2.57-2.46 (m, 2H); ${ }^{13} \mathrm{C} \mathrm{NMR}\left(\mathrm{CDCl}_{3}, 125 \mathrm{MHz}\right): \delta 161.7,148.6(2 \mathrm{C}), 136.1,134.6,133.8,129.3$ (2C), 128.9 (2C), 128.1 (2C), 122.4, 74.4, 66.8 (2C), 63.5, 53.8 (2C); FTIR (thin film): $\mathrm{cm}^{-1}$ 3101, 2813, 1730, 1541, 1493, 1455, 1343, 1268, 1163, 1115, 1010, 717; HRMS-ESI (m/z) Calcd for $\left(\mathrm{C}_{19} \mathrm{H}_{19} \mathrm{ClN}_{3} \mathrm{O}_{7}\right)\left([\mathrm{M}+\mathrm{H}]^{+}\right)$: 436.0906; found: 436.0906.

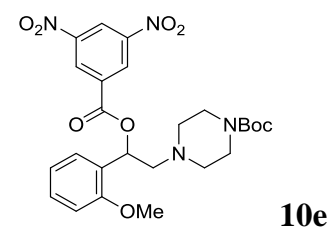

Tert-butyl 4-(2-((3,5-dinitrobenzoyl)oxy)-2-(2-methoxyphenyl)ethyl)piperazine-1-carboxylate (10e). Synthesized using condition B. Purification by flash column chromatography (100\% hexanes $\rightarrow 30 \%$ EtOAc-hexanes) gave 10e as a yellow oil (198.4 mg, 93\%). $\mathrm{R}_{f}=0.73\left(50 \%\right.$ EtOAc-hexanes); ${ }^{1} \mathrm{H} \mathrm{NMR}\left(\mathrm{CDCl}_{3}, 400 \mathrm{MHz}\right): \delta 9.20(\mathrm{t}, J=2.1 \mathrm{~Hz}, 1 \mathrm{H})$, $9.18(\mathrm{~d}, J=2.1 \mathrm{~Hz}, 2 \mathrm{H}), 7.39(\mathrm{dd}, J=7.6,1.6 \mathrm{~Hz}, 1 \mathrm{H}), 7.30(\mathrm{td}, J=7.6,1.6 \mathrm{~Hz}, 1 \mathrm{H}), 6.97(\mathrm{td}, J=7.6,0.8 \mathrm{~Hz}, 1 \mathrm{H}), 6.91$ $(\mathrm{d}, J=7.6 \mathrm{~Hz}, 1 \mathrm{H}), 6.70(\mathrm{dd}, J=9.5,2.8 \mathrm{~Hz}, 1 \mathrm{H}), 3.89(\mathrm{~s}, 3 \mathrm{H}), 3.34(\mathrm{t}, J=4.9 \mathrm{~Hz}, 4 \mathrm{H}), 2.98(\mathrm{dd}, J=13.7,9.5 \mathrm{~Hz}, 1 \mathrm{H})$, 2.75-2.63 (m, 3H), 2.46-2.37 (m, 2H); ${ }^{13} \mathrm{C} \mathrm{NMR}\left(\mathrm{CDCl}_{3}, 125 \mathrm{MHz}, 60{ }^{\circ} \mathrm{C}\right): \delta 161.6,156.4,154.6,148.7(2 \mathrm{C}), 134.6$, 129.5, 129.2 (2C), 126.5, 126.3, 122.0, 120.7, 110.9, 79.4, 70.7, 62.4, 55.6, 55.5, 53.1, 43.8 (2C), 28.3 (3C); FTIR (thin film): $\mathrm{cm}^{-1} 2976,1731,1684,1544,1365,1245,1164,1003,908,719 ;$ HRMS-ESI $(\mathrm{m} / \mathrm{z})$ Calcd for $\left(\mathrm{C}_{25} \mathrm{H}_{31} \mathrm{~N}_{4} \mathrm{O}_{9}\right)$ $\left([\mathrm{M}+\mathrm{H}]^{+}\right): 432.1401$; found: 432.1400 .

\subsection{References}

(1) Berman, A. M.; Johnson, J. S. J. Org. Chem. 2006, 71, 219-224.

(2) Le Quement, S. T.; Nielsen, T. E.; Meldal, M. J. Comb. Chem. 2007, 9, 1060-1072.

(3) Song, S.; Zhu, S.-F.; Yu, Y.-B.; Zhou, Q.-L. Angew. Chem. Int. Ed. 2013, 52, 1556-1559.

(4) Kerins, F.; O'Shea, D. F. J. Org. Chem. 2002, 67, 4968-4971.

(5) Dydio, P.; Reek, J. N. H. Angew. Chem. Int. Ed. 2013, 52, 3878-3882.

(6) Nicolai, S.; Erard, S.; González, D. F.; Waser, J. Org. Lett. 2010, 12, 384-387.

(7) Patil, A. S.; Mo, D.-L.; Wang, H.-Y.; Mueller, D. S.; Anderson, L. L. Angew. Chem. Int. Ed. 2012, 51, 7799-7803.

(8) Al-Amin, M.; Johnson, J. S.; Blum, S. A. Organometallics 2014, 33, 5448-5456.

(9) Watson, I. D. G.; Ritter, S.; Toste, F. D. J. Am. Chem. Soc. 2009, 131, 2056-2057.

(10) Bowden, K.; Ghadir, K. D. F. J. Chem. Soc., Perkin Trans. 2 1990, 1333-1338.

(11) Farney, E. P.; Yoon, T. P. Angew. Chem. Int. Ed. 2014, 53, 793-797.

(12) Zhang, L.; Herndon, J. W. Organometallics 2004, 23, 1231-1235.

(13) Inci, B.; Cheng, P.-N.; Beljanski, K.; Moore, J. S. ACS Macro Lett. 2013, 2, 935-938. 
1.8 Spectra

1a

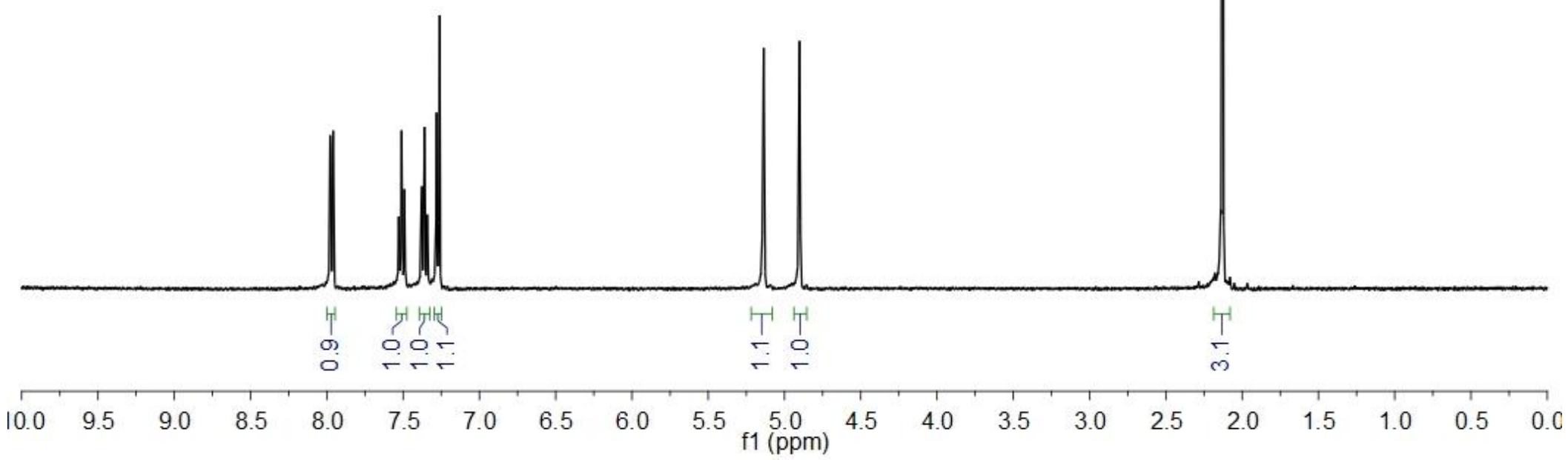



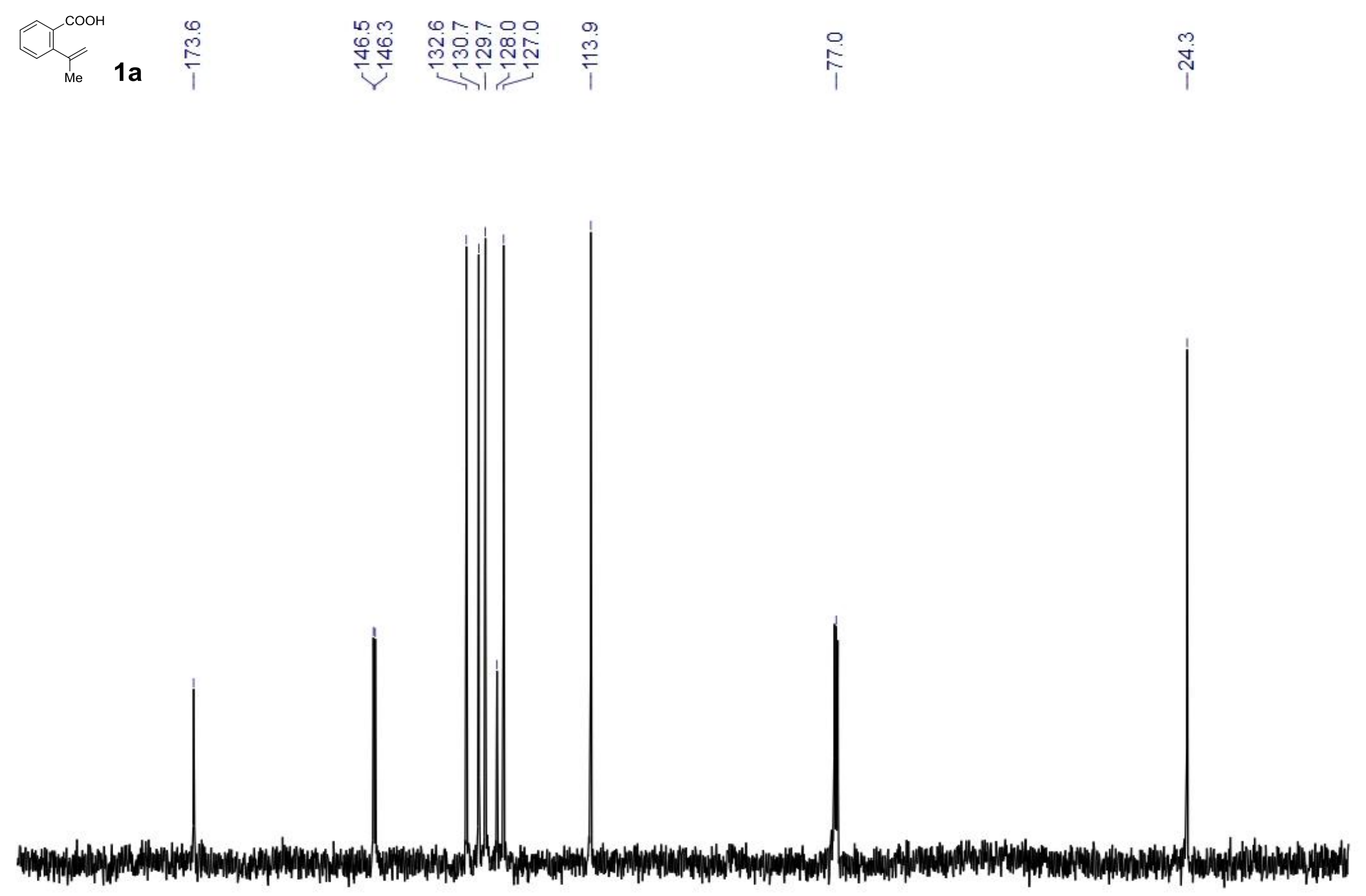


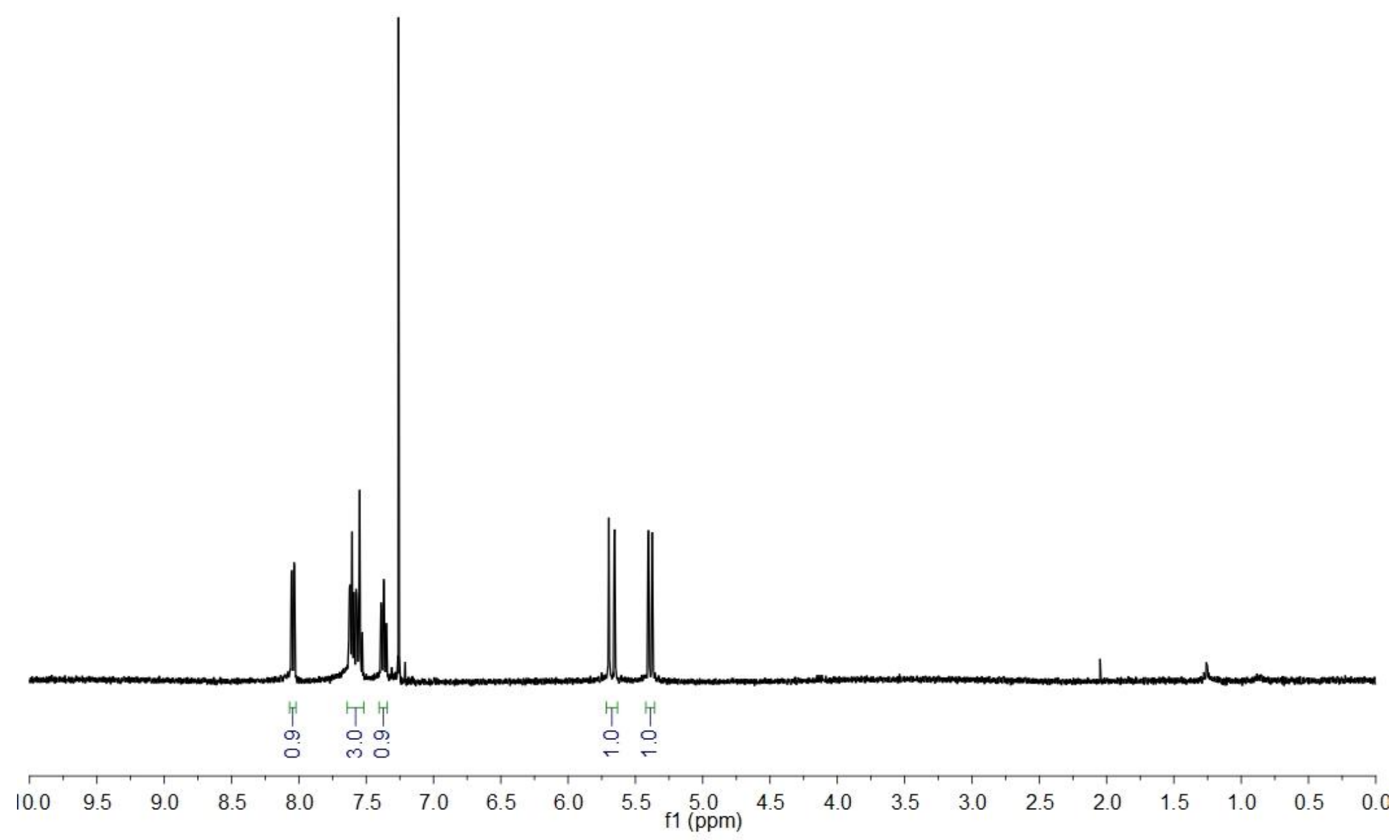




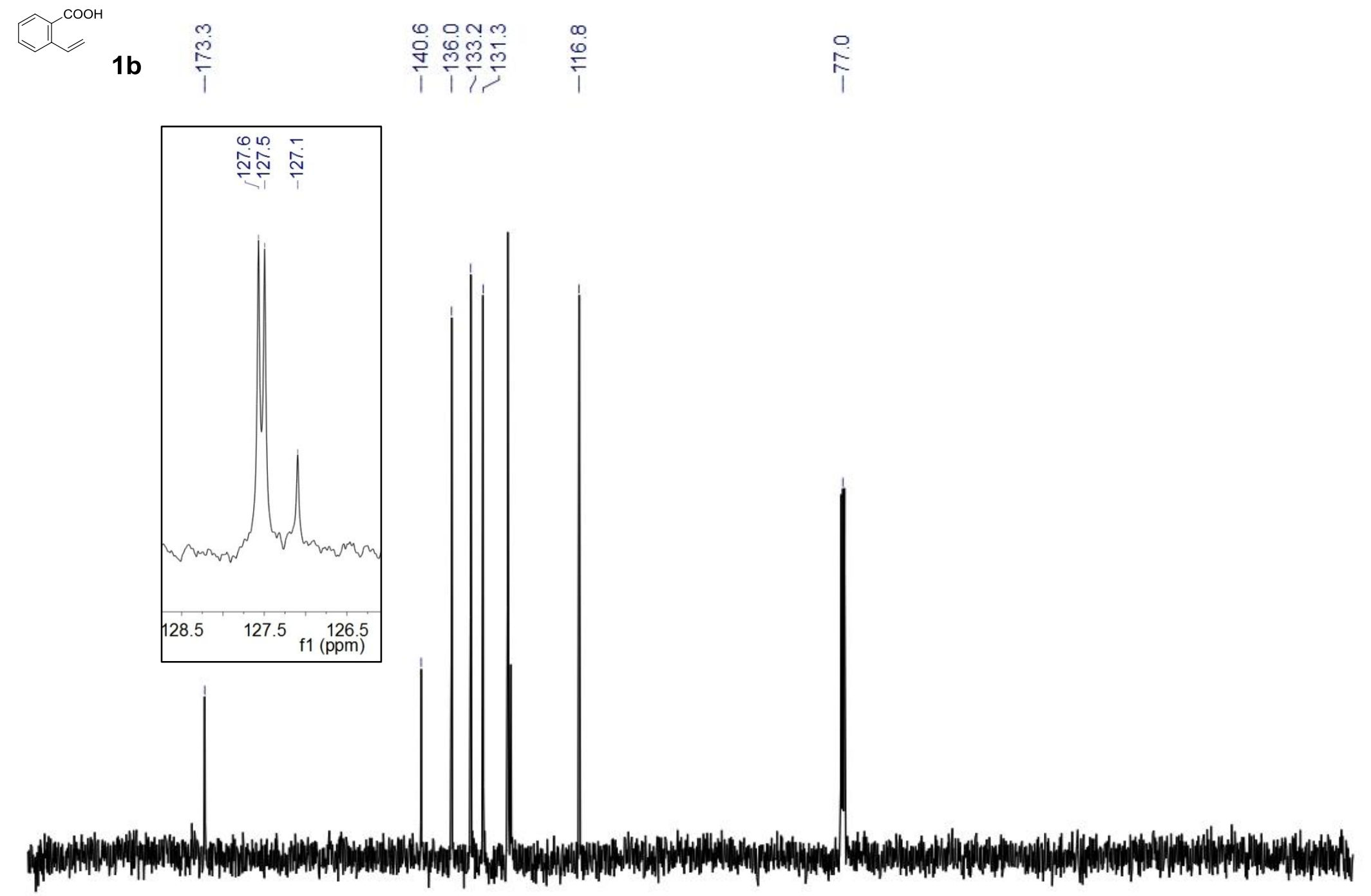

\begin{tabular}{lllllllllllllllllllllll}
\hline & 00 & 190 & 180 & 170 & 160 & 150 & 140 & 130 & 120 & 110 & 100 & 90 & 80 & 70 & 60 & 50 & 40 & 30 & 20 & 10 & 0
\end{tabular} 


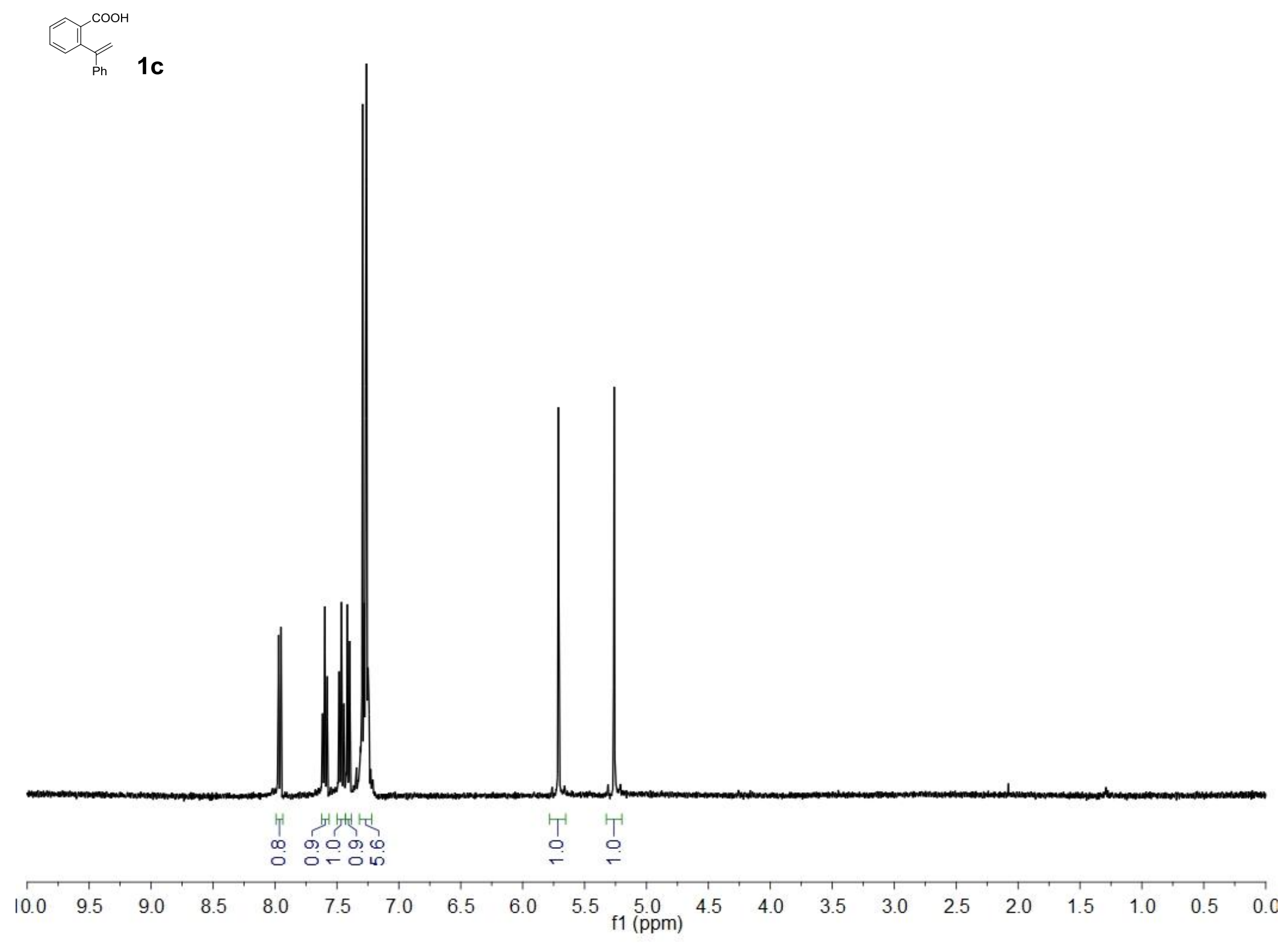



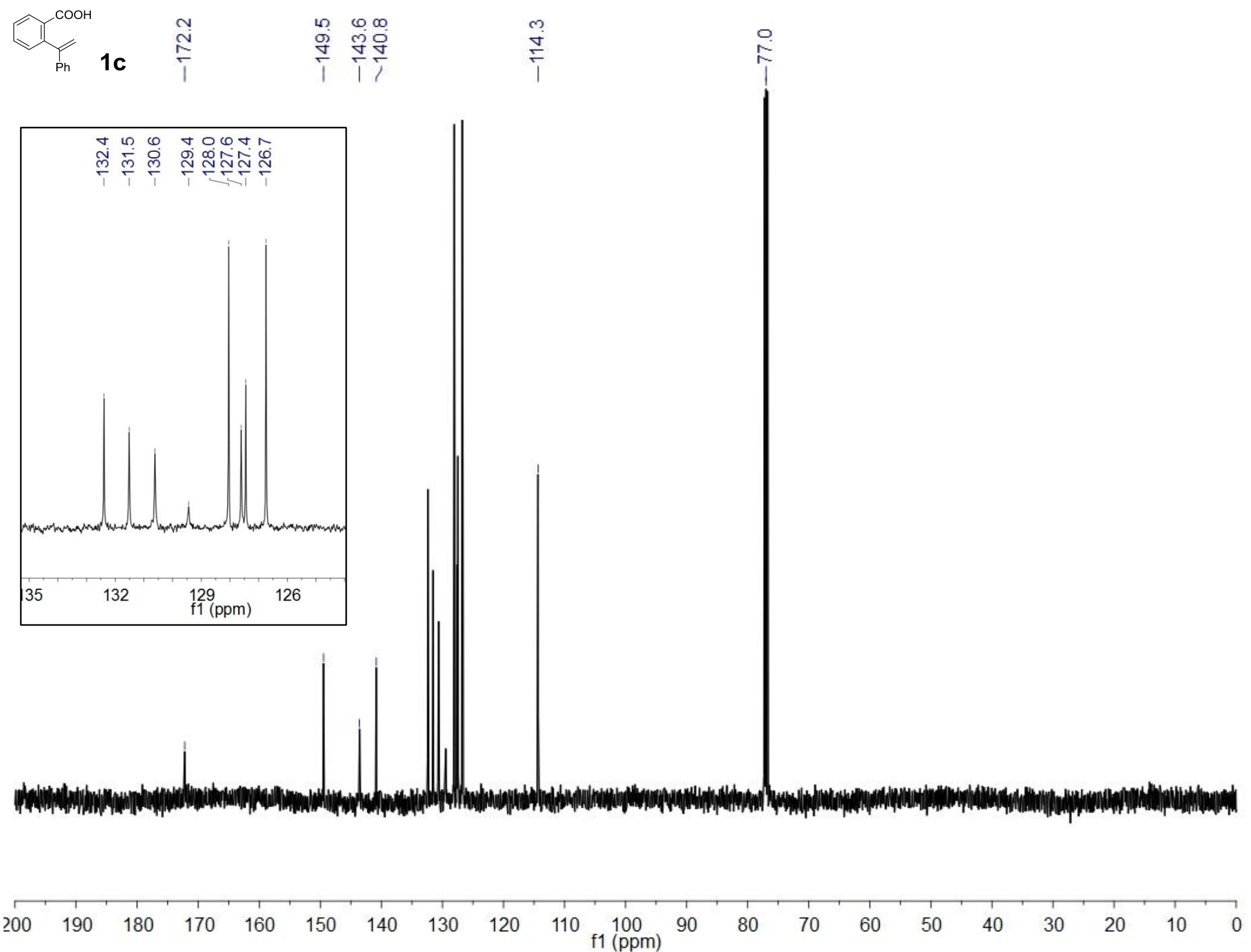
$1 d$

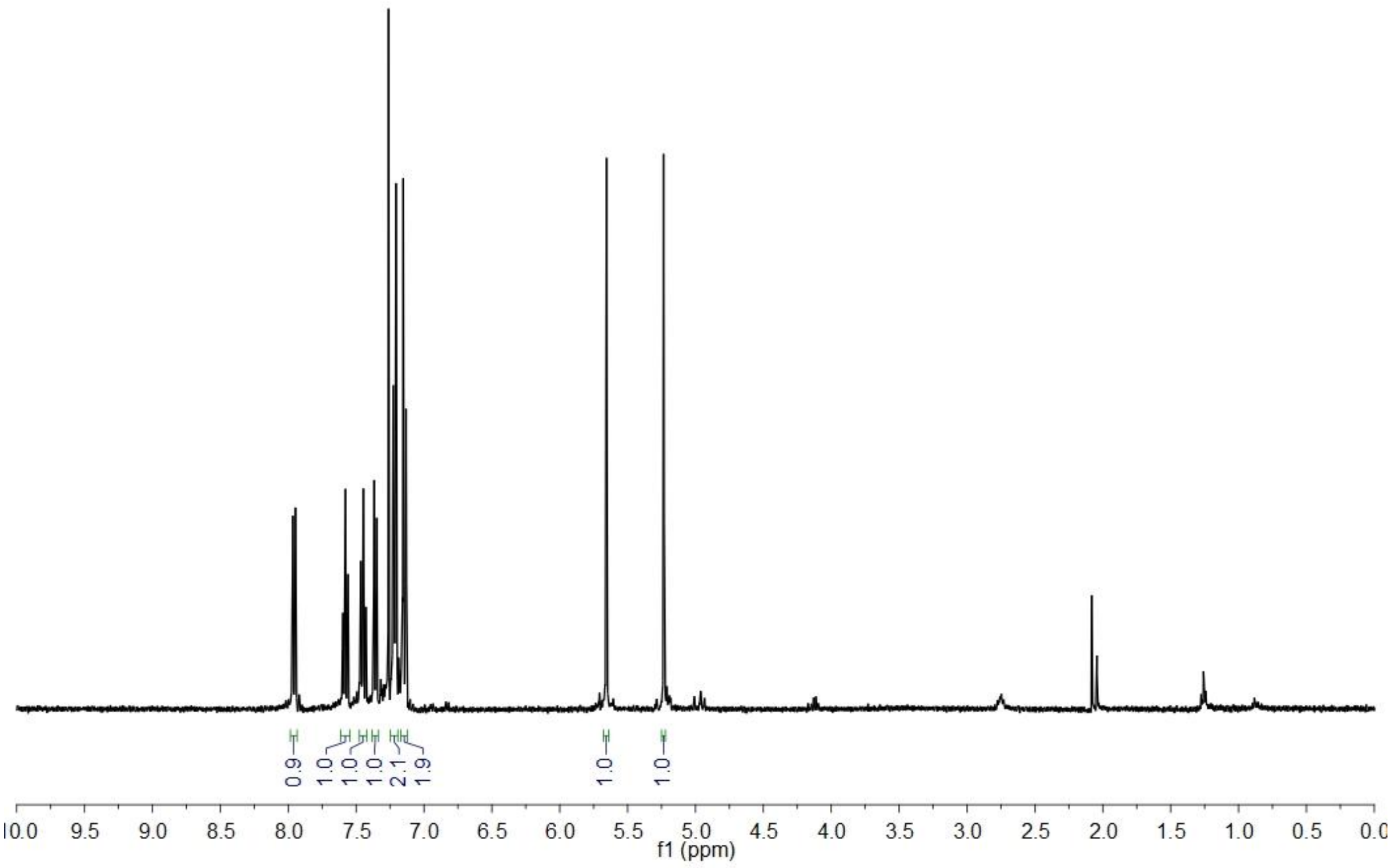




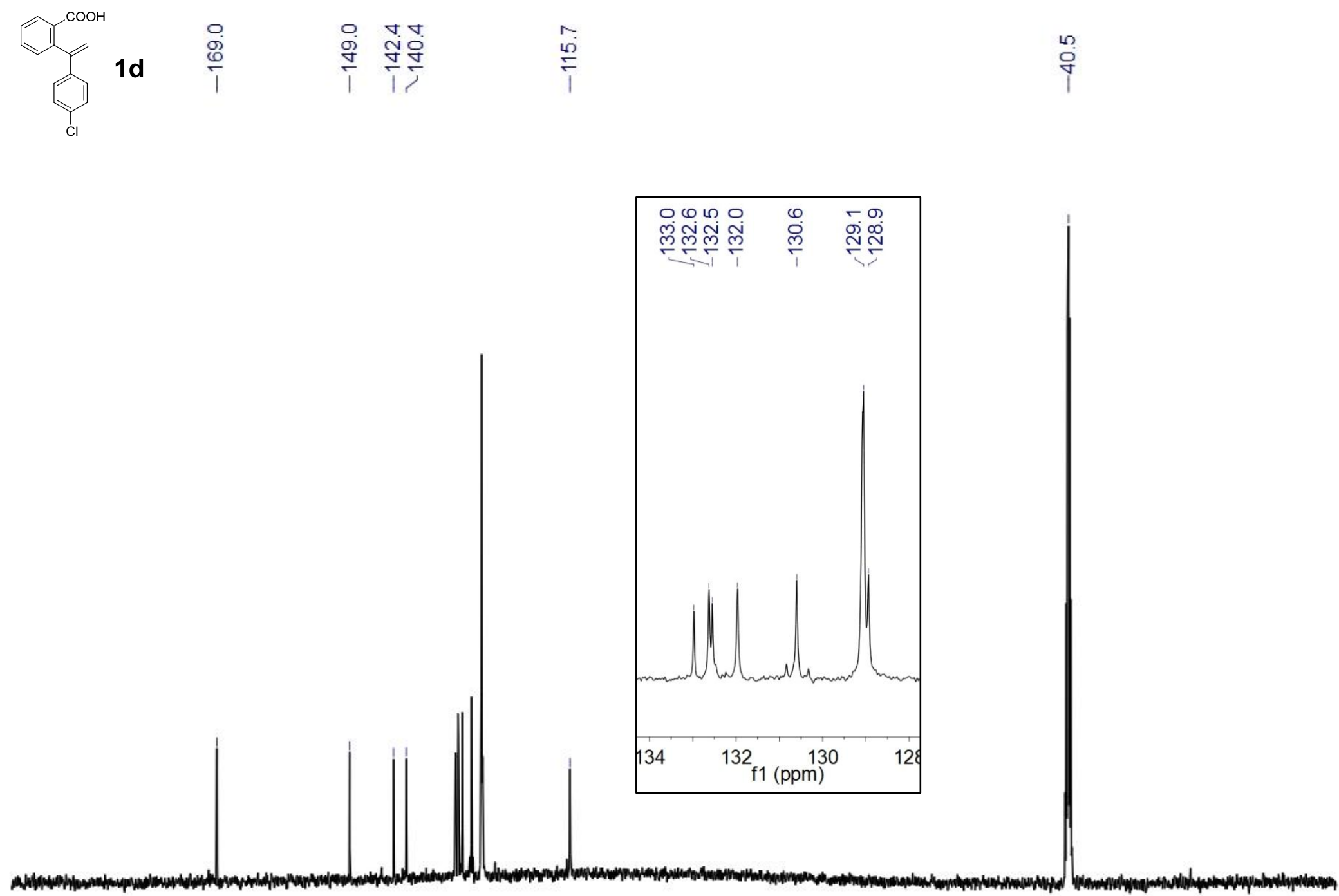

00

120

100
-10

80

$70 \quad 60$

50

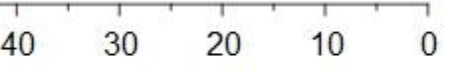




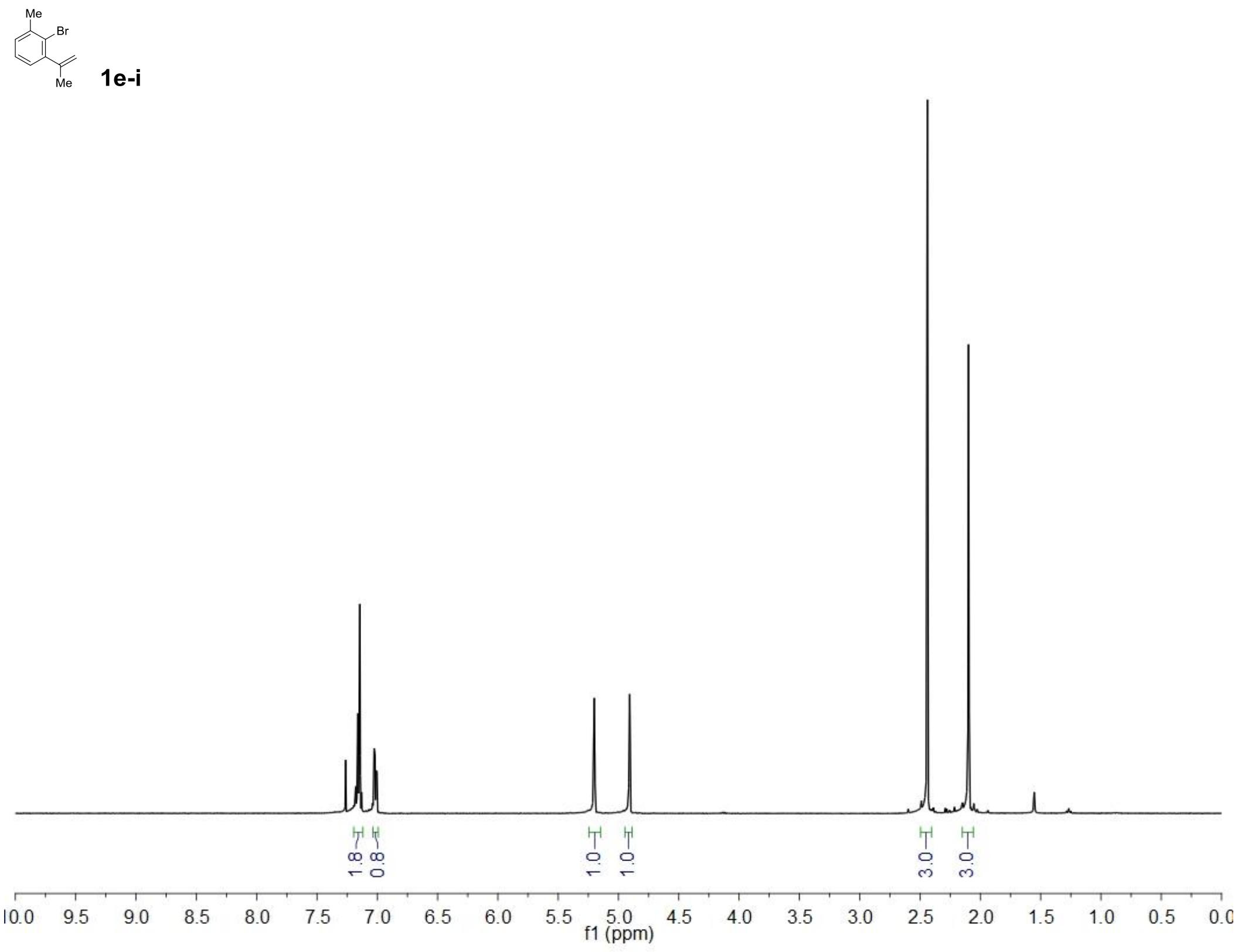


1e-i

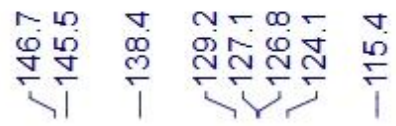

$\stackrel{\circ}{i}$

$\infty \omega$

N

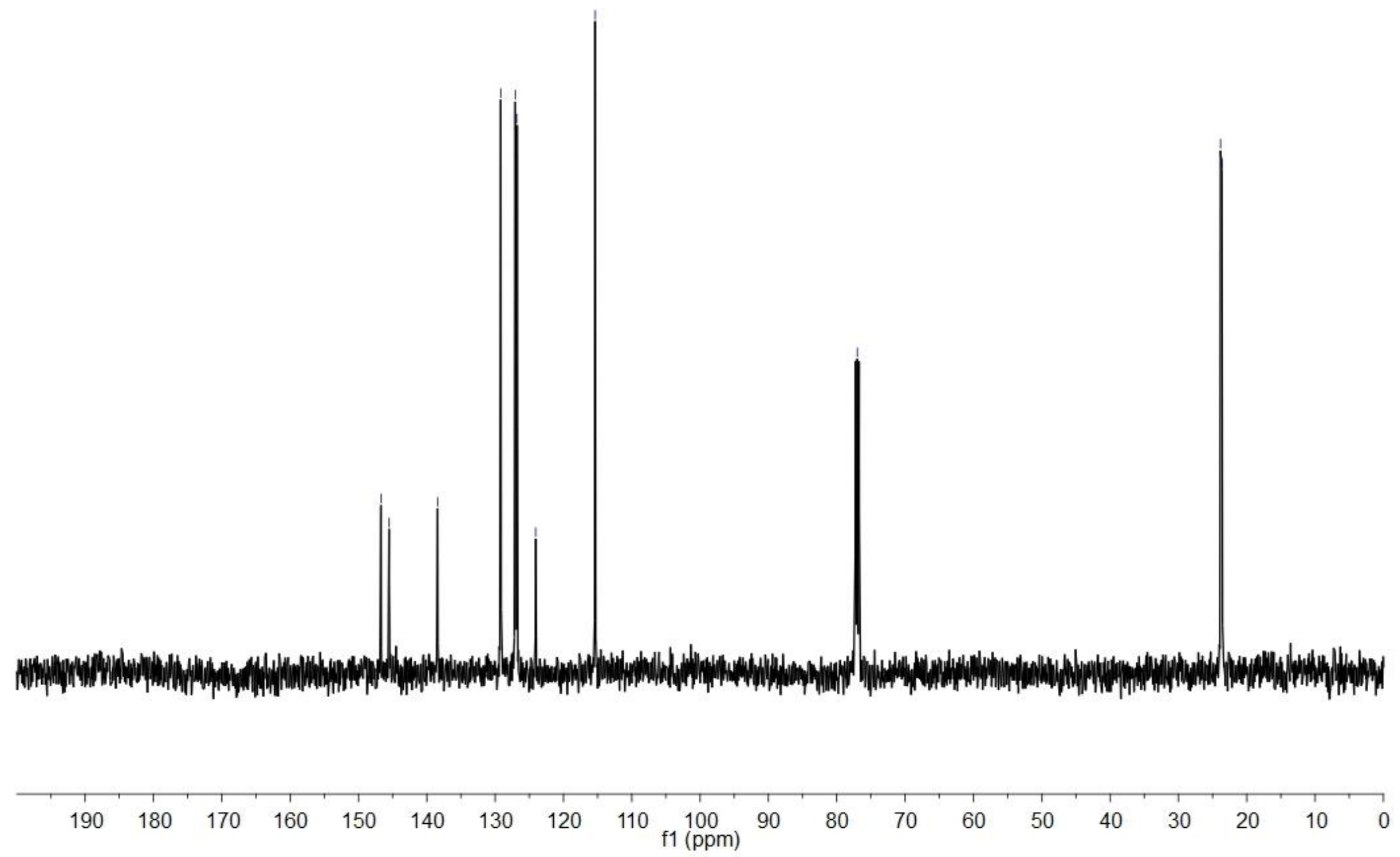




$$
\underbrace{\mathrm{Me}}_{\mathrm{Me}} 1 \mathrm{e}
$$

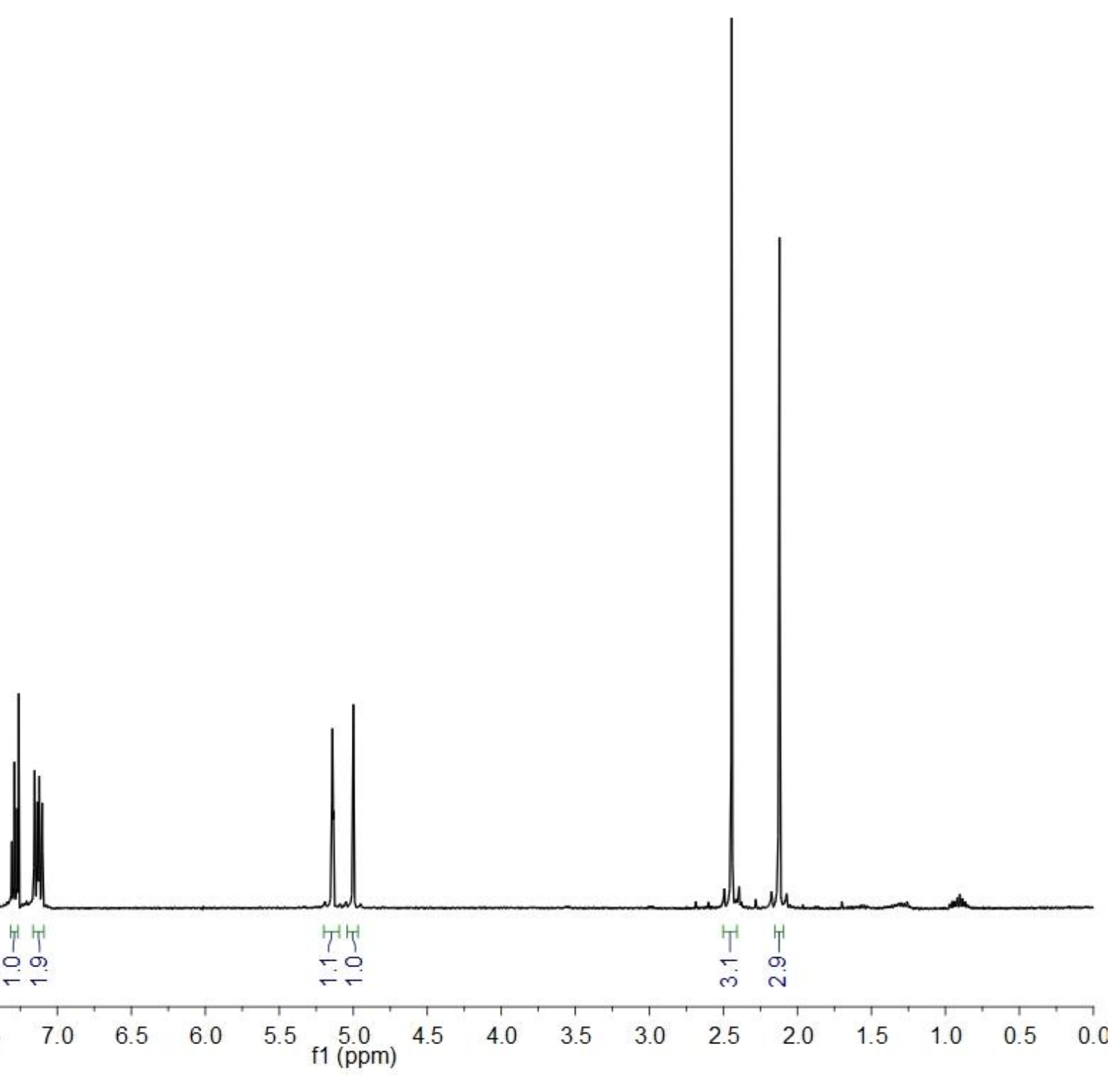




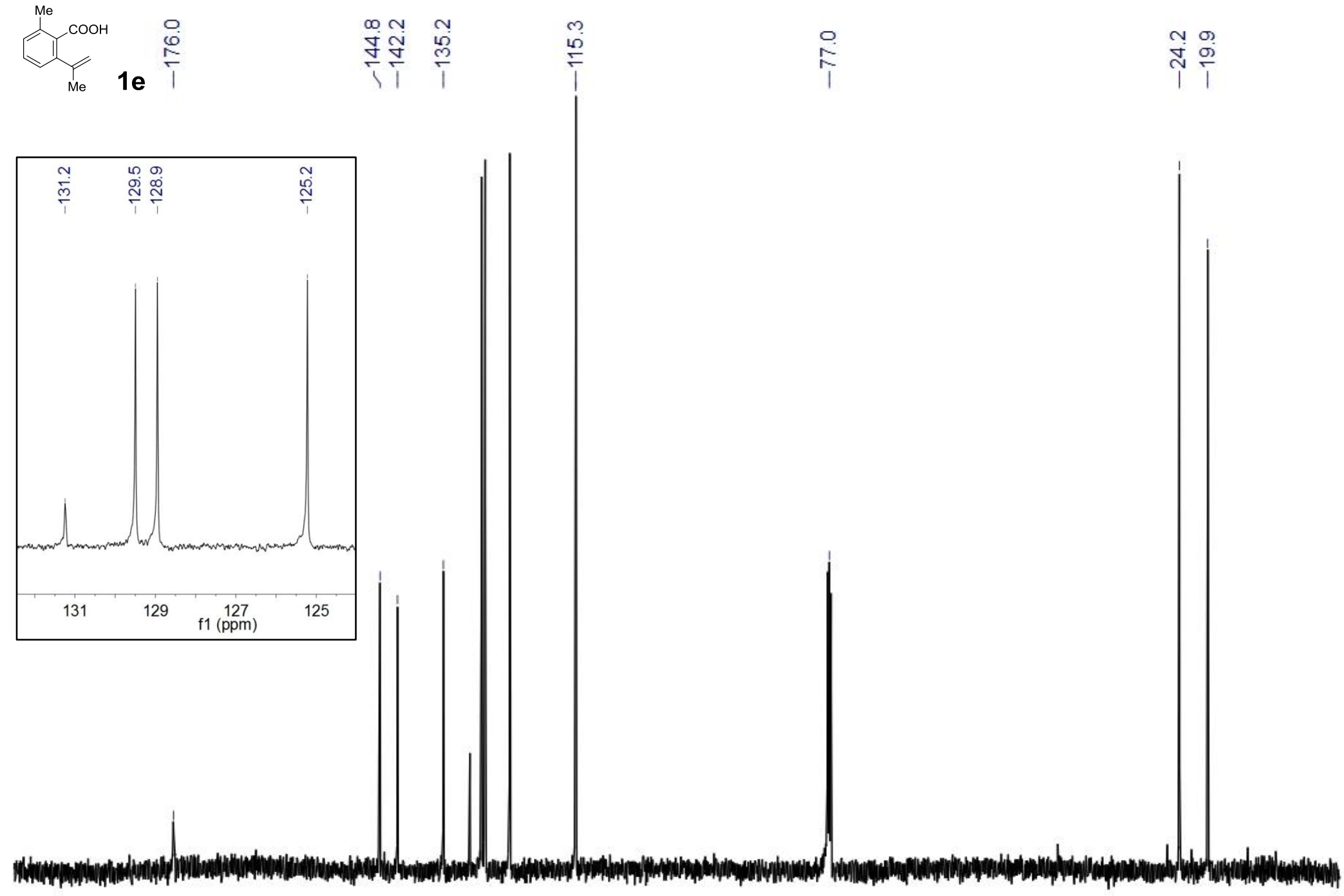

120

$110 \quad 100$

$80 \quad 70$

60

50

$40 \quad 30$

$20 \quad 10$ 


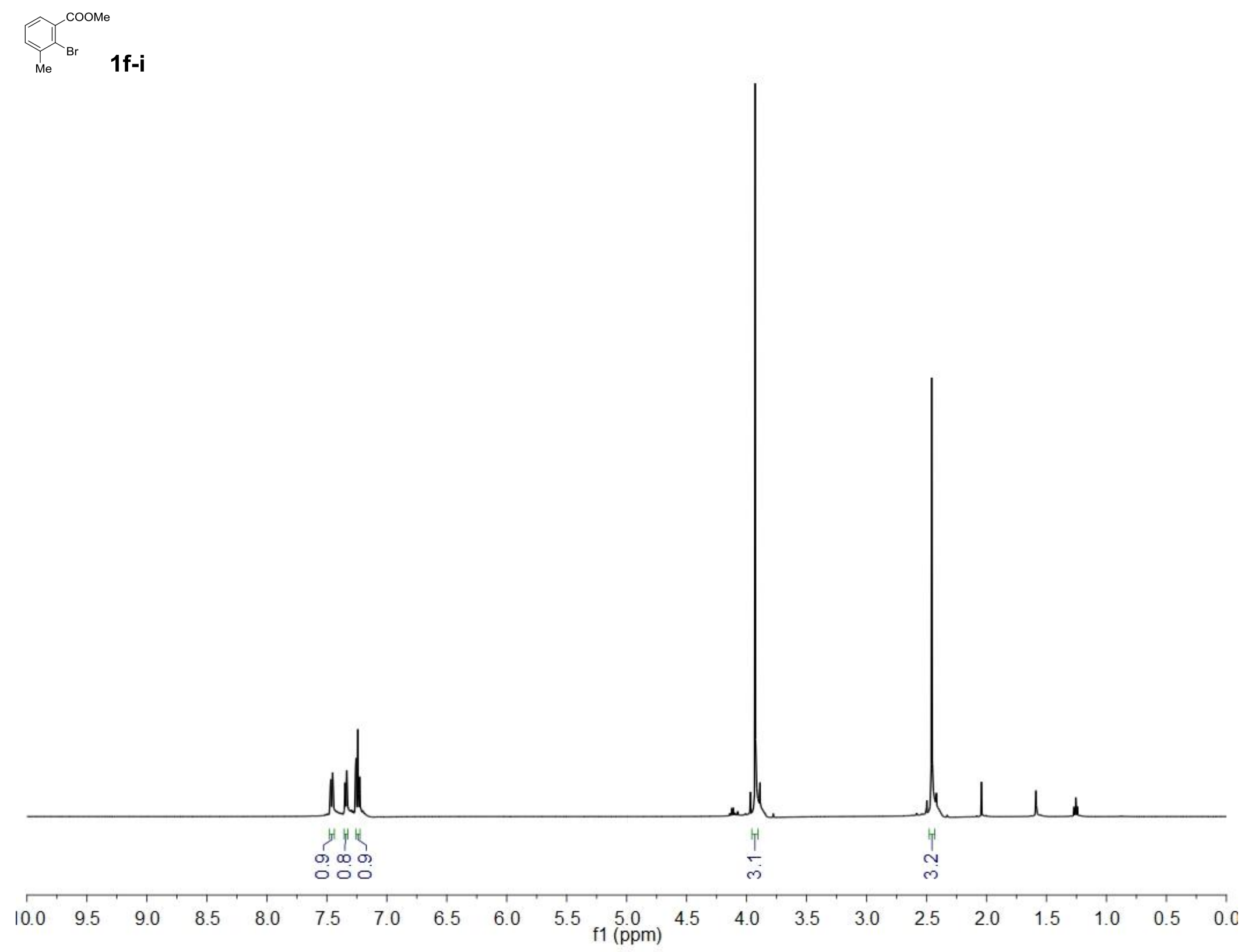




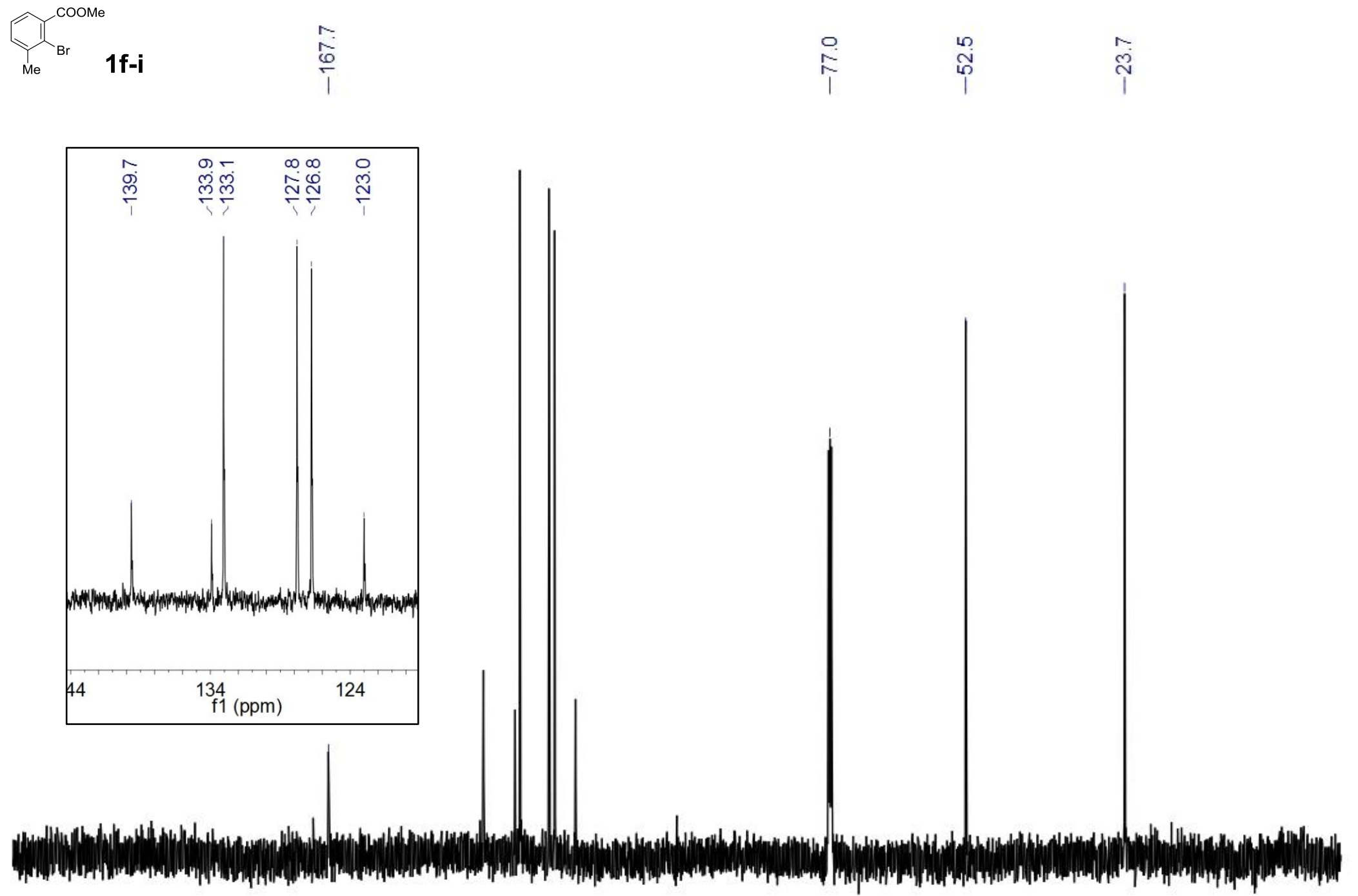

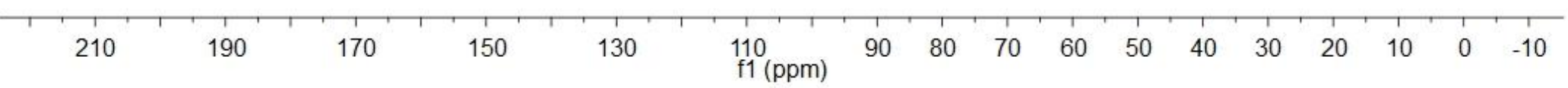




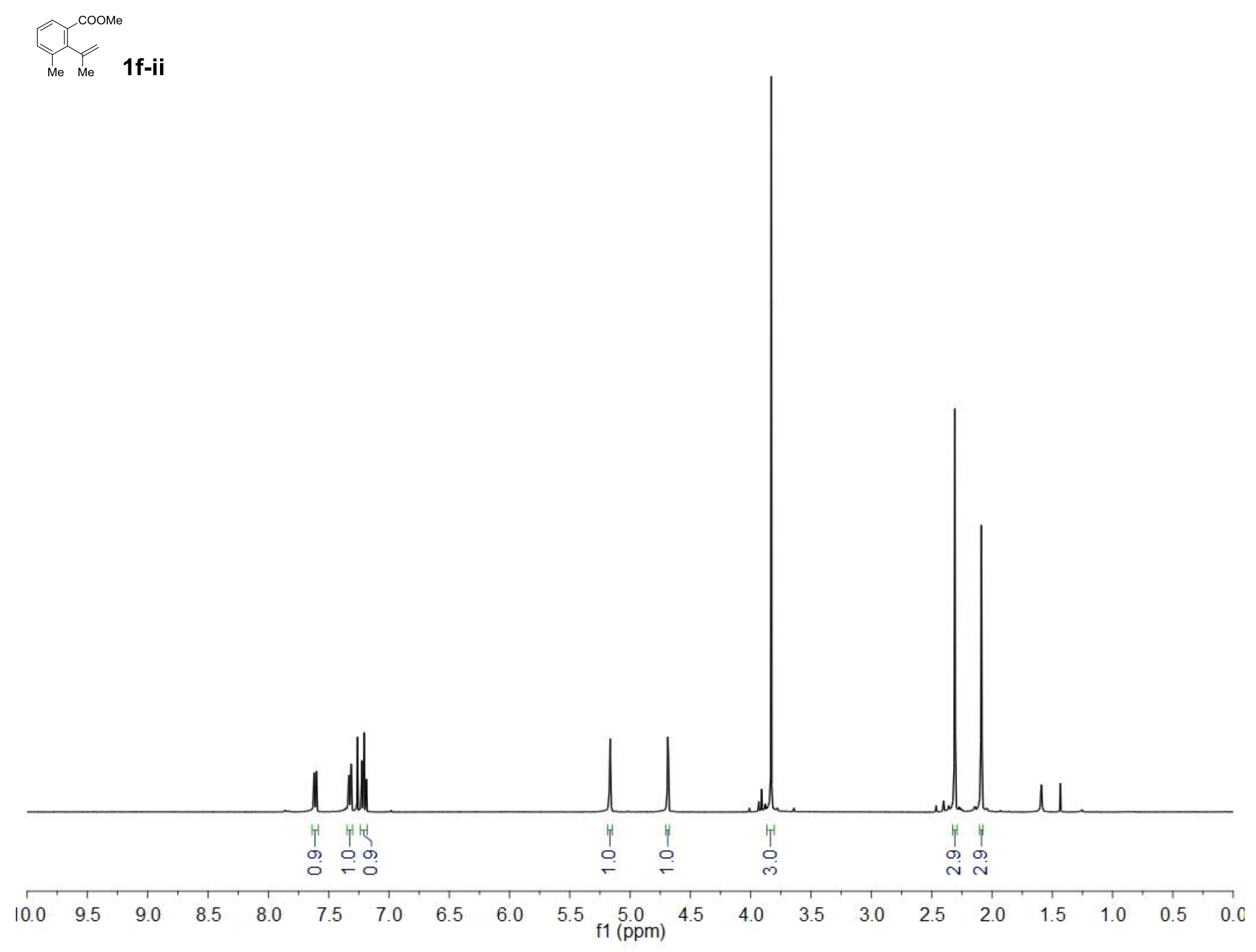


$\underbrace{1 f-i i}_{\text {Me }} \stackrel{i}{\frac{\infty}{\infty}}$

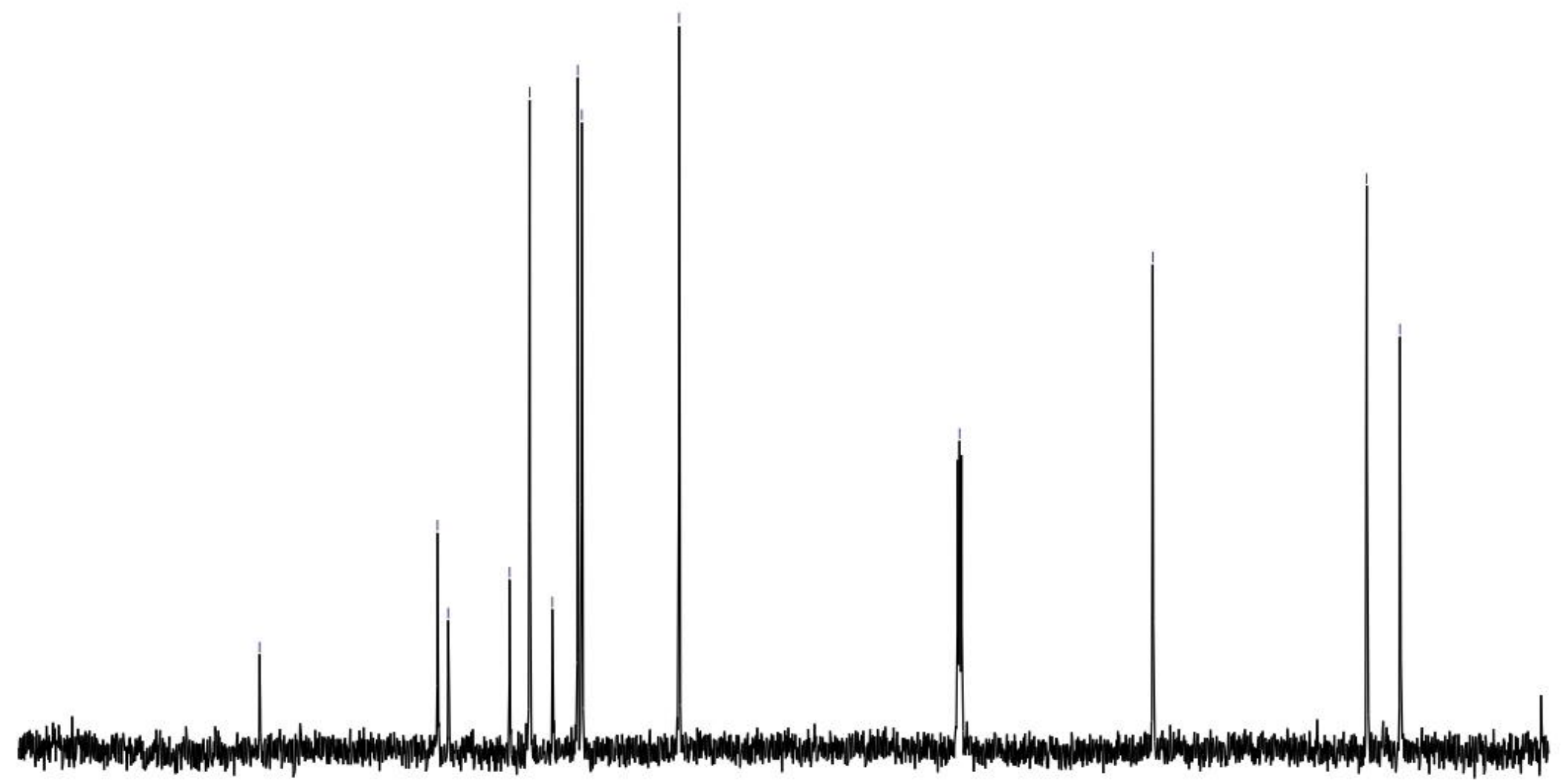




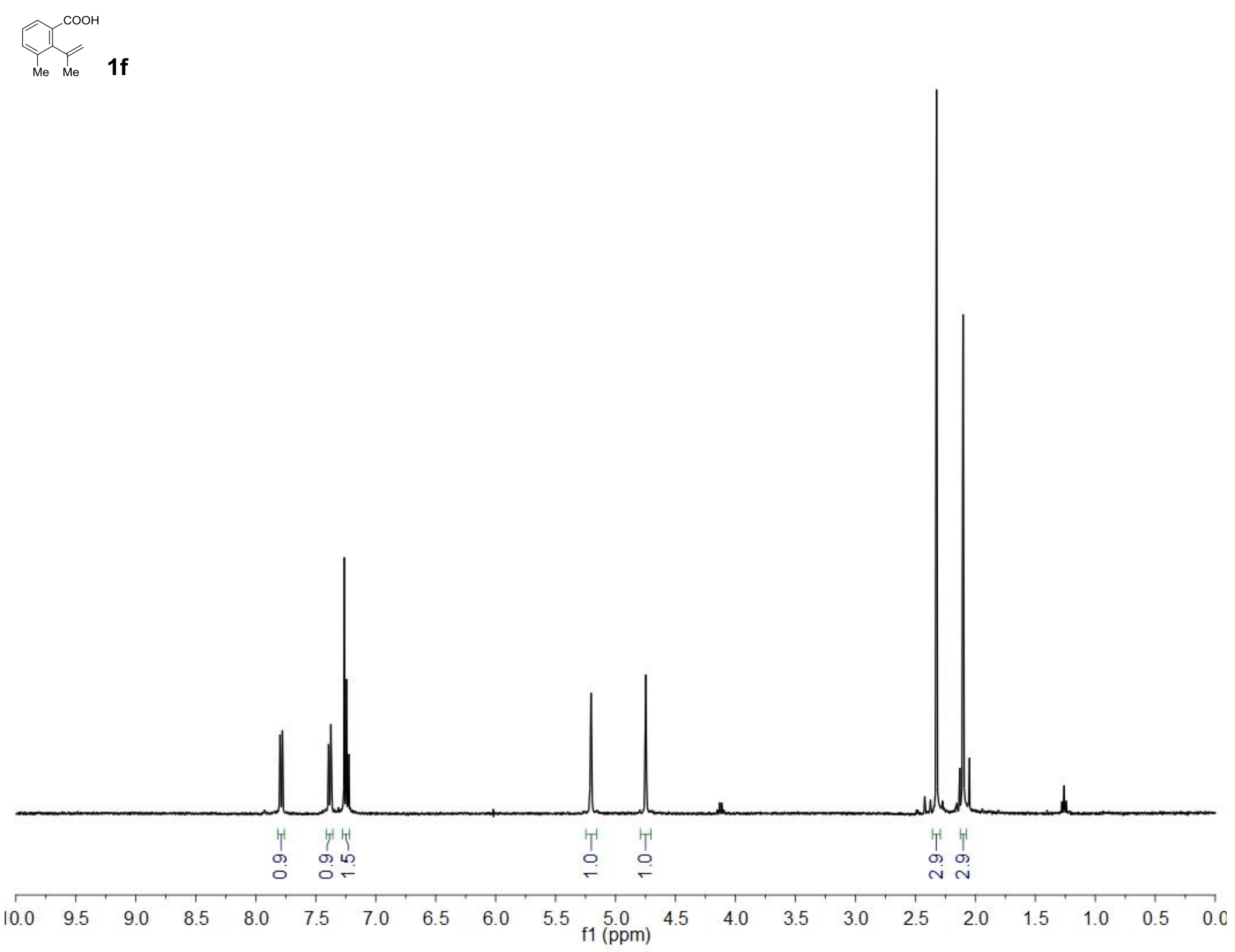




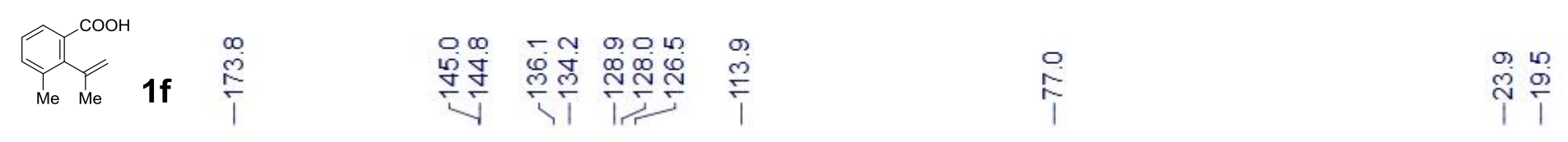

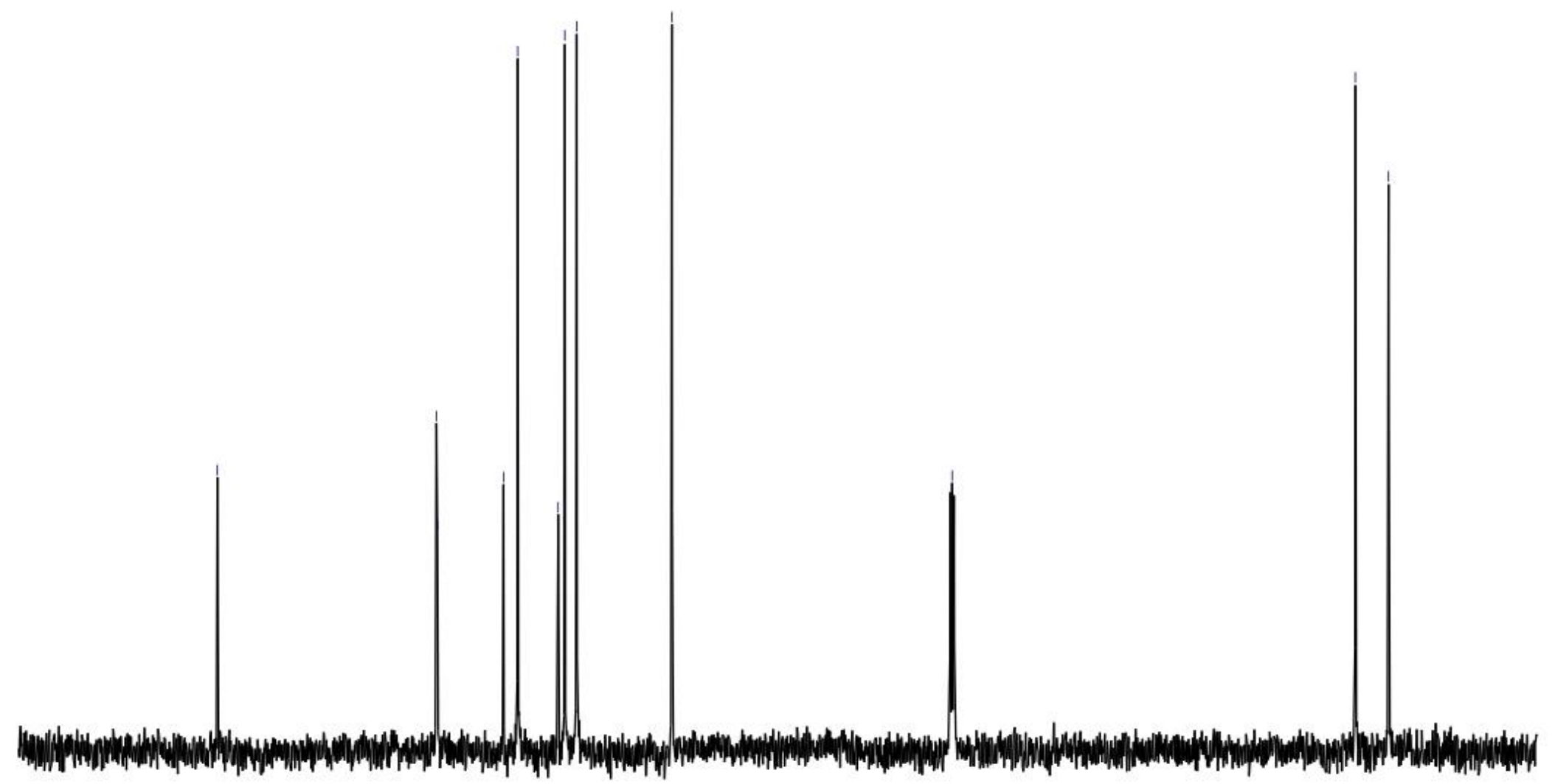




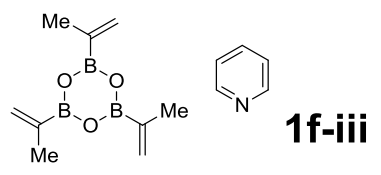

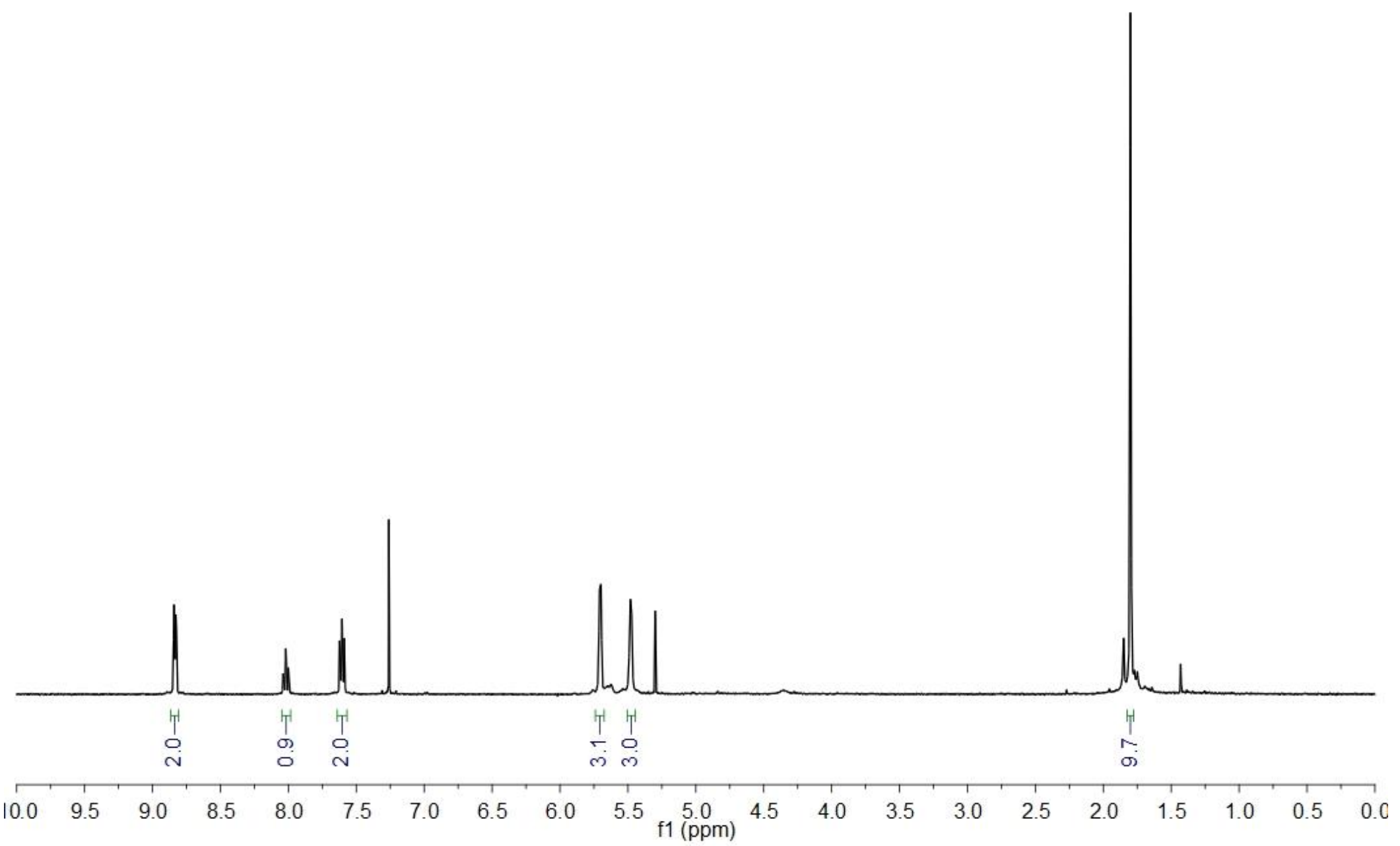




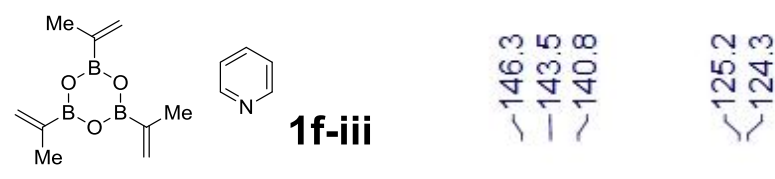

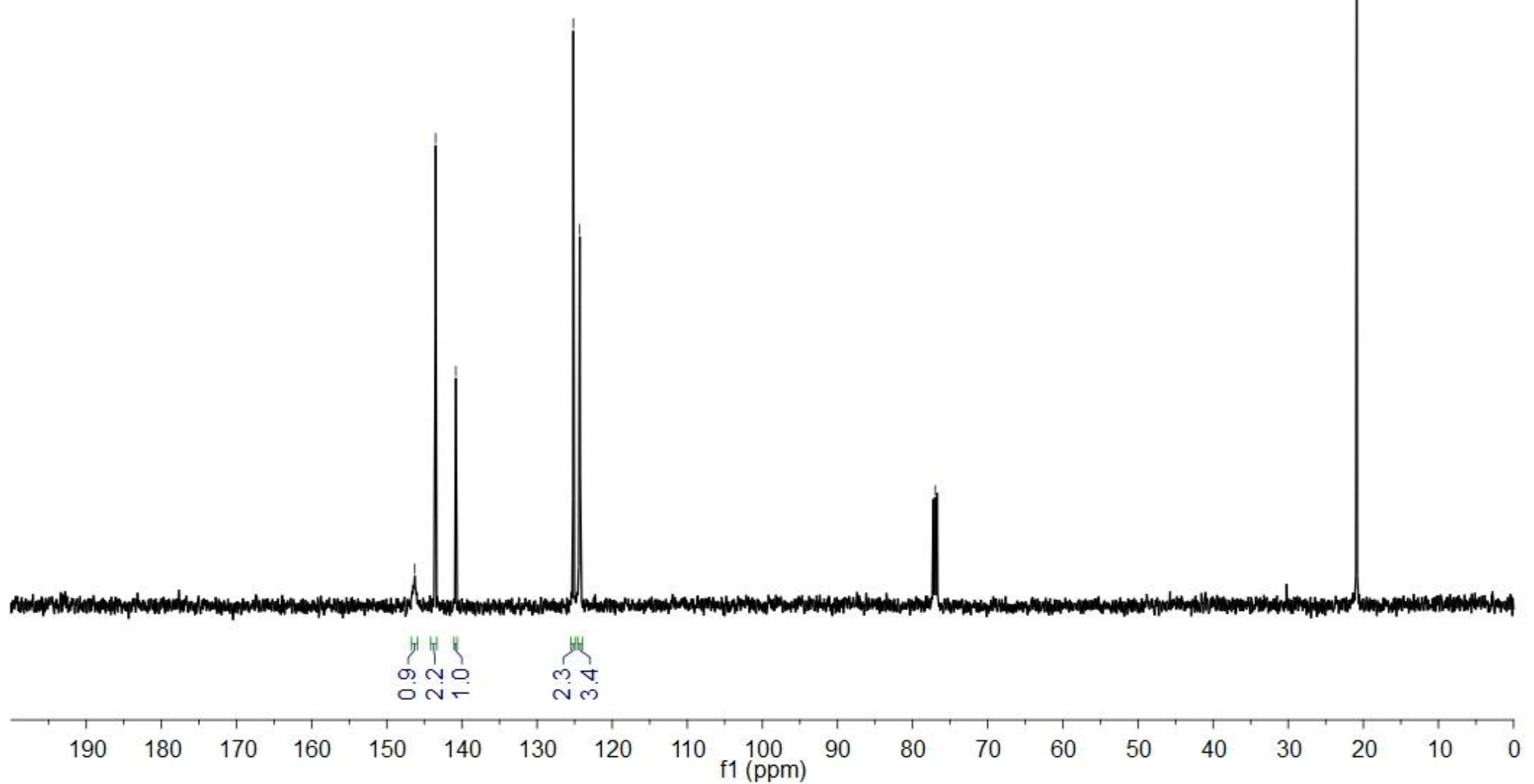


19

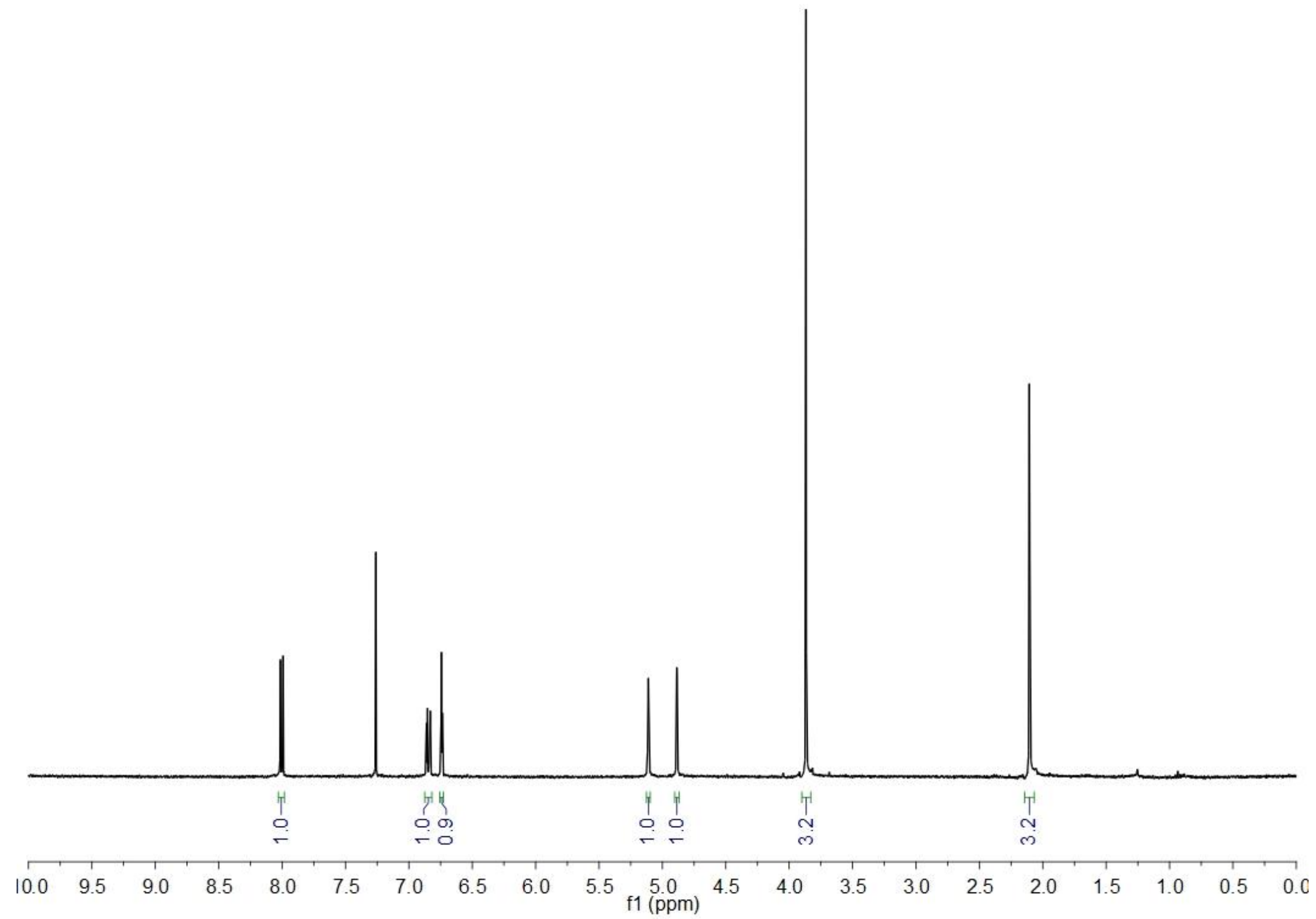




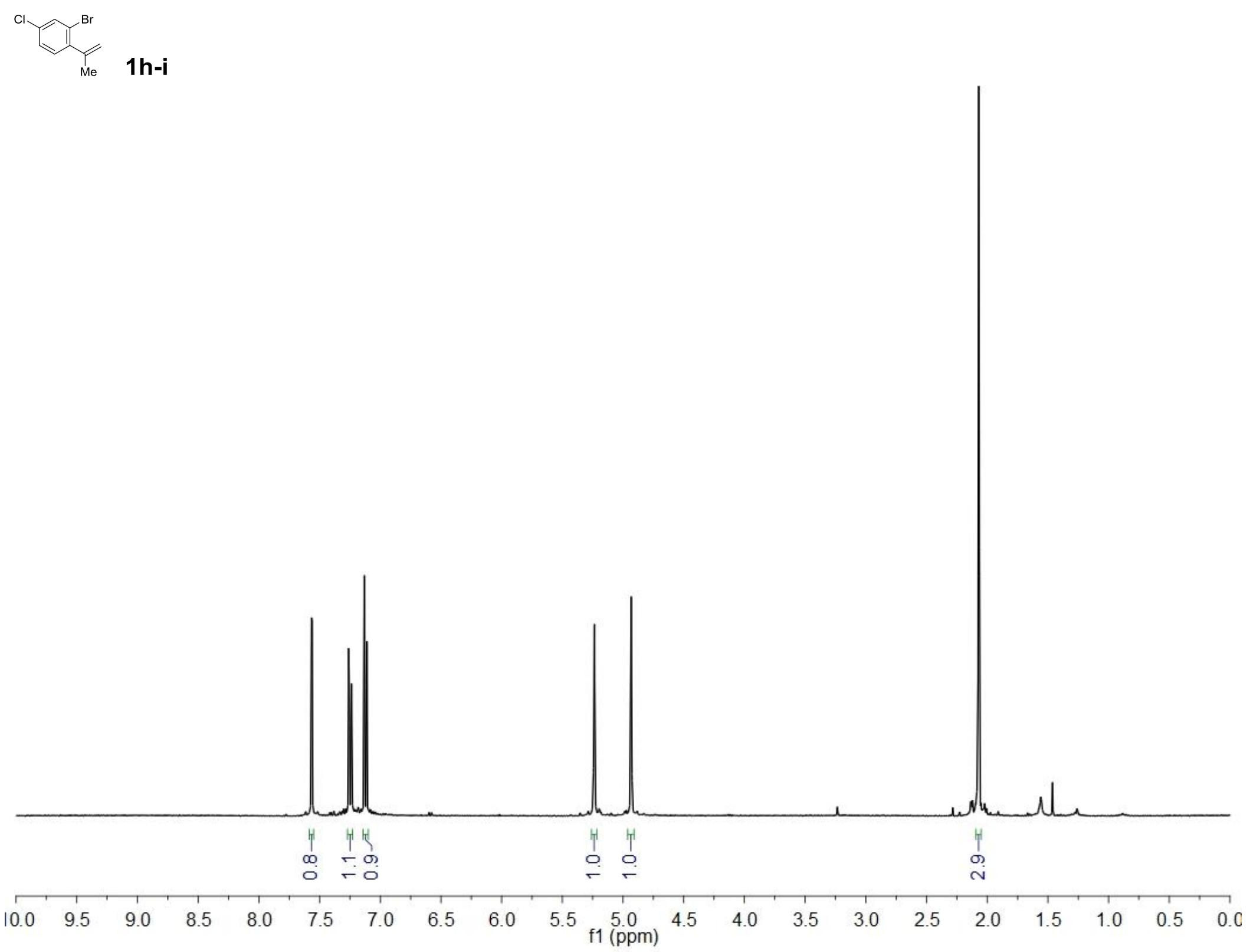




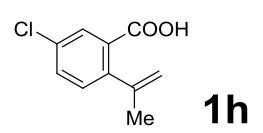

me $1 \mathrm{~h}$

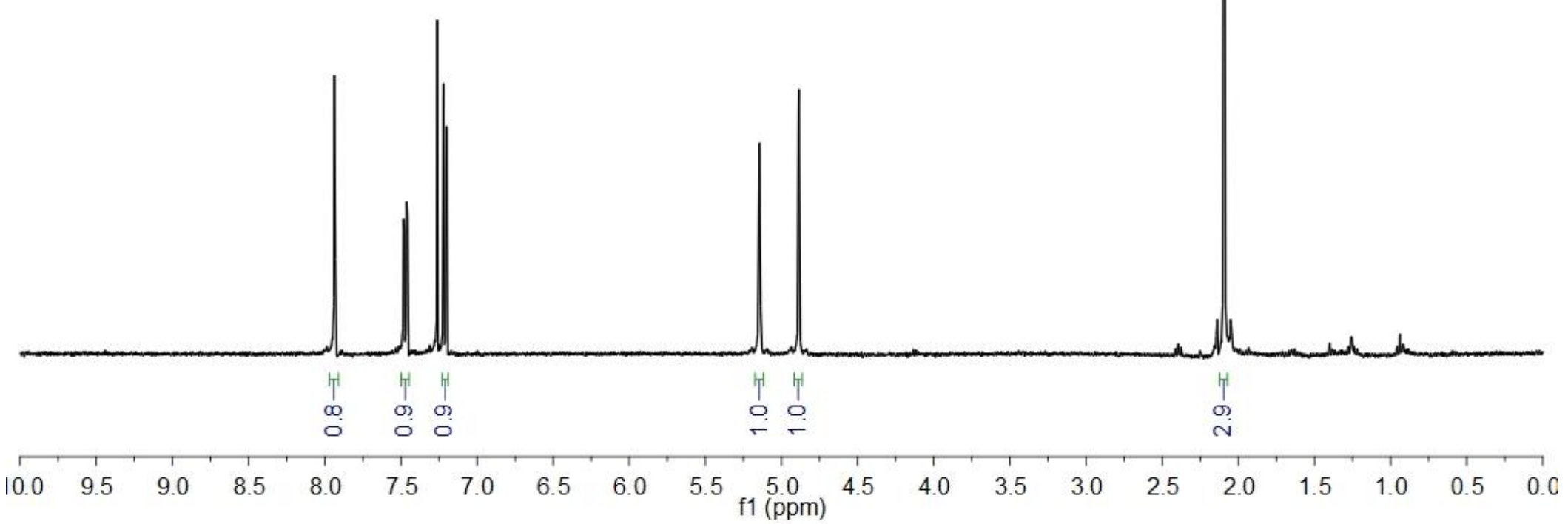




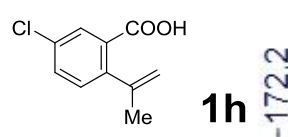

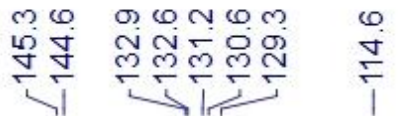

$\stackrel{\circ}{1}$

$\stackrel{\check{I}}{\text { I }}$

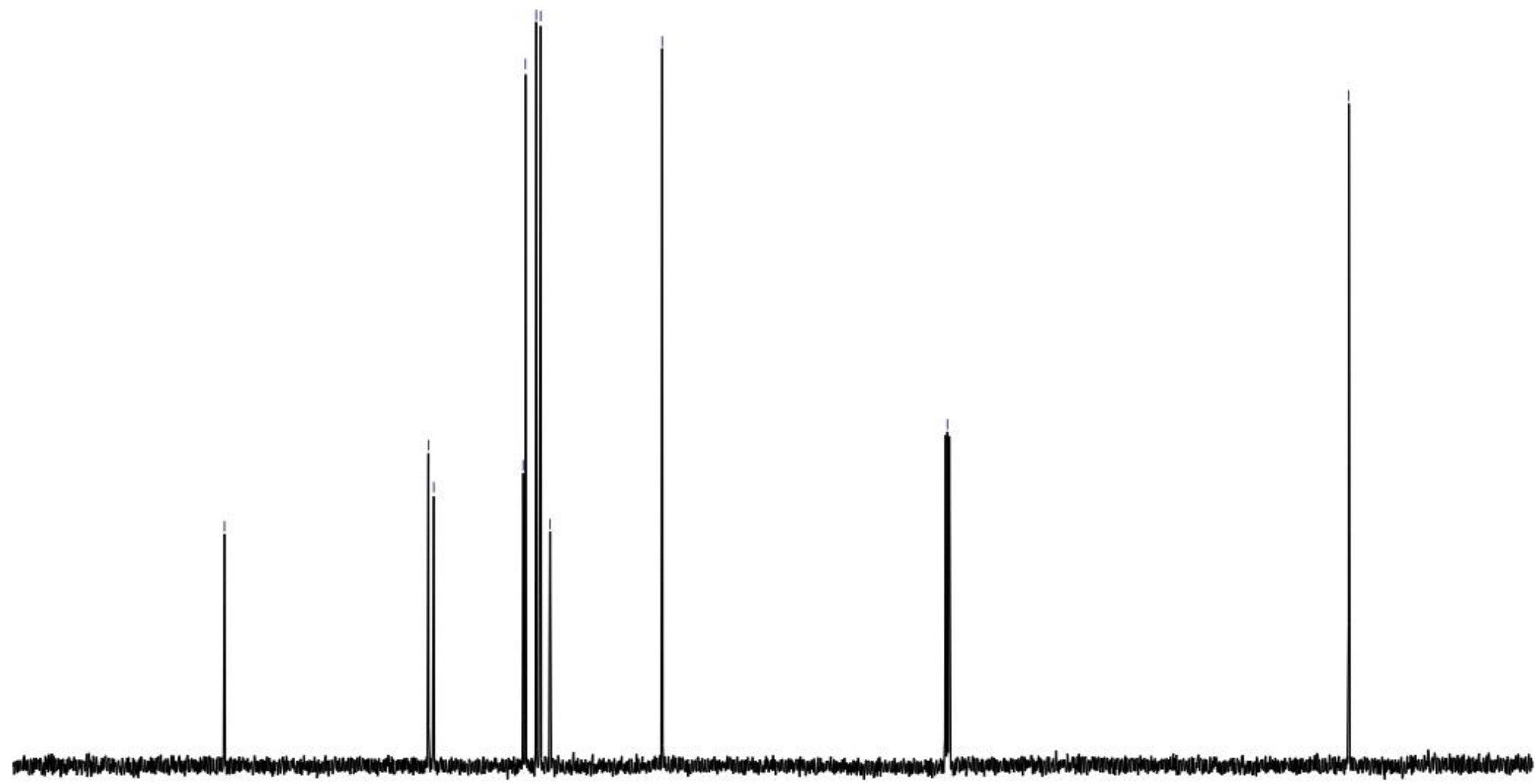

200

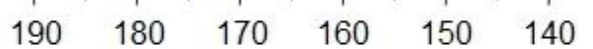

130

120

$\begin{array}{ll}110 & 100 \\ & \end{array}$

80

70

60

50

$40 \quad 30$

20

10 

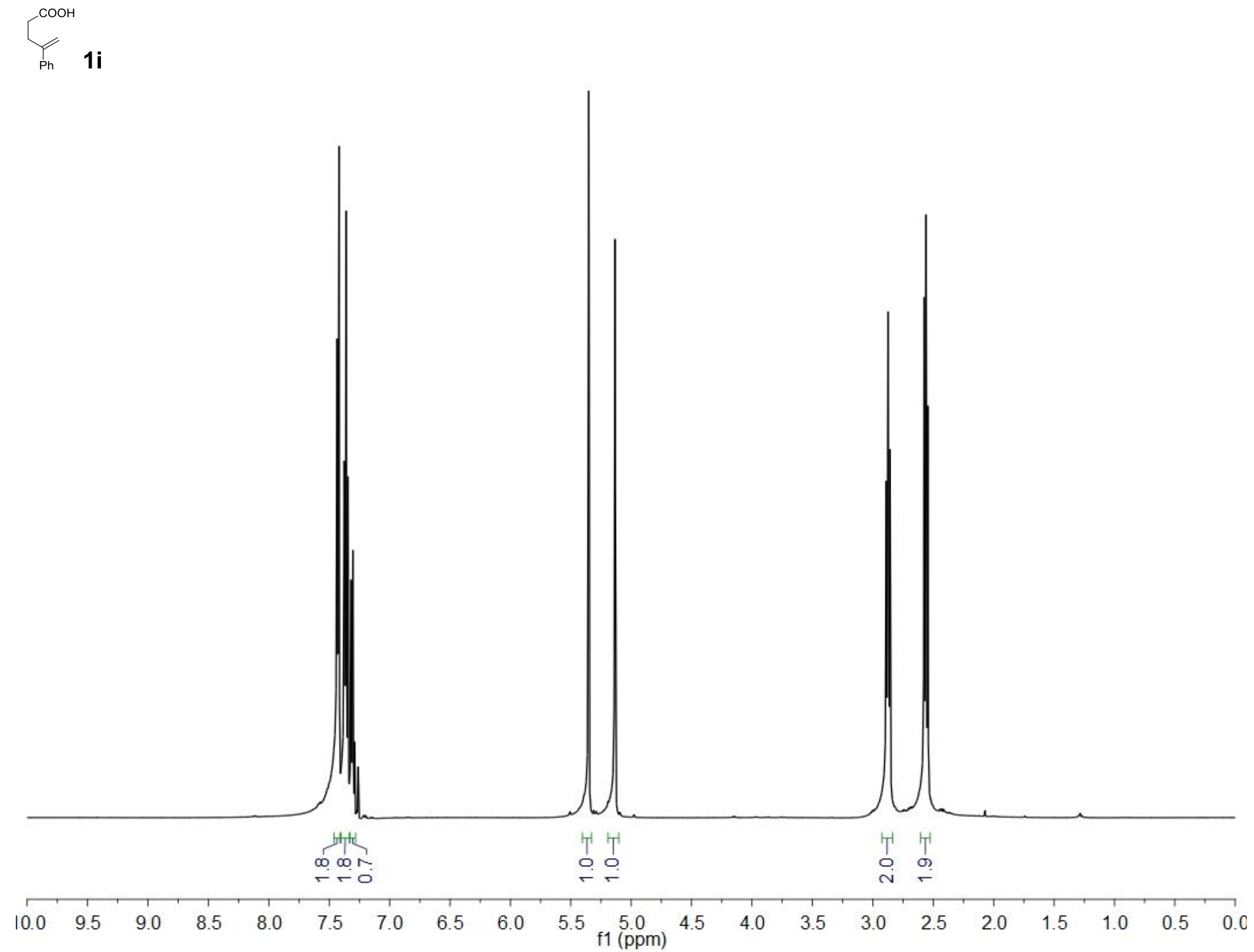

S56 


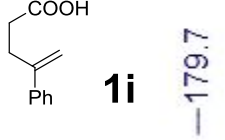
i
웅
윰ํํ

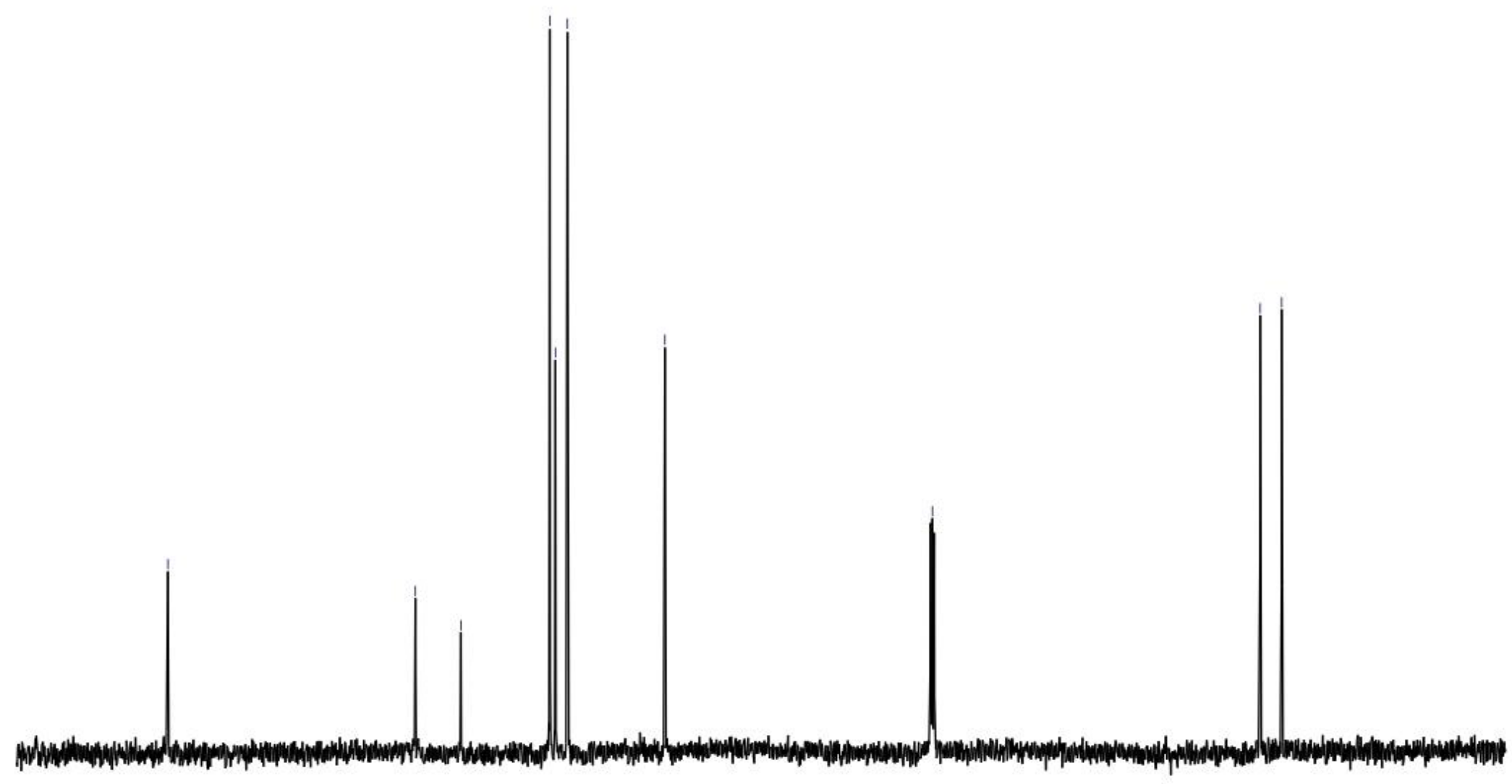

200

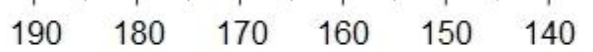

130

120

$110 \quad 100$

80

$70 \quad 60$

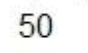

$40 \quad 30$

20 


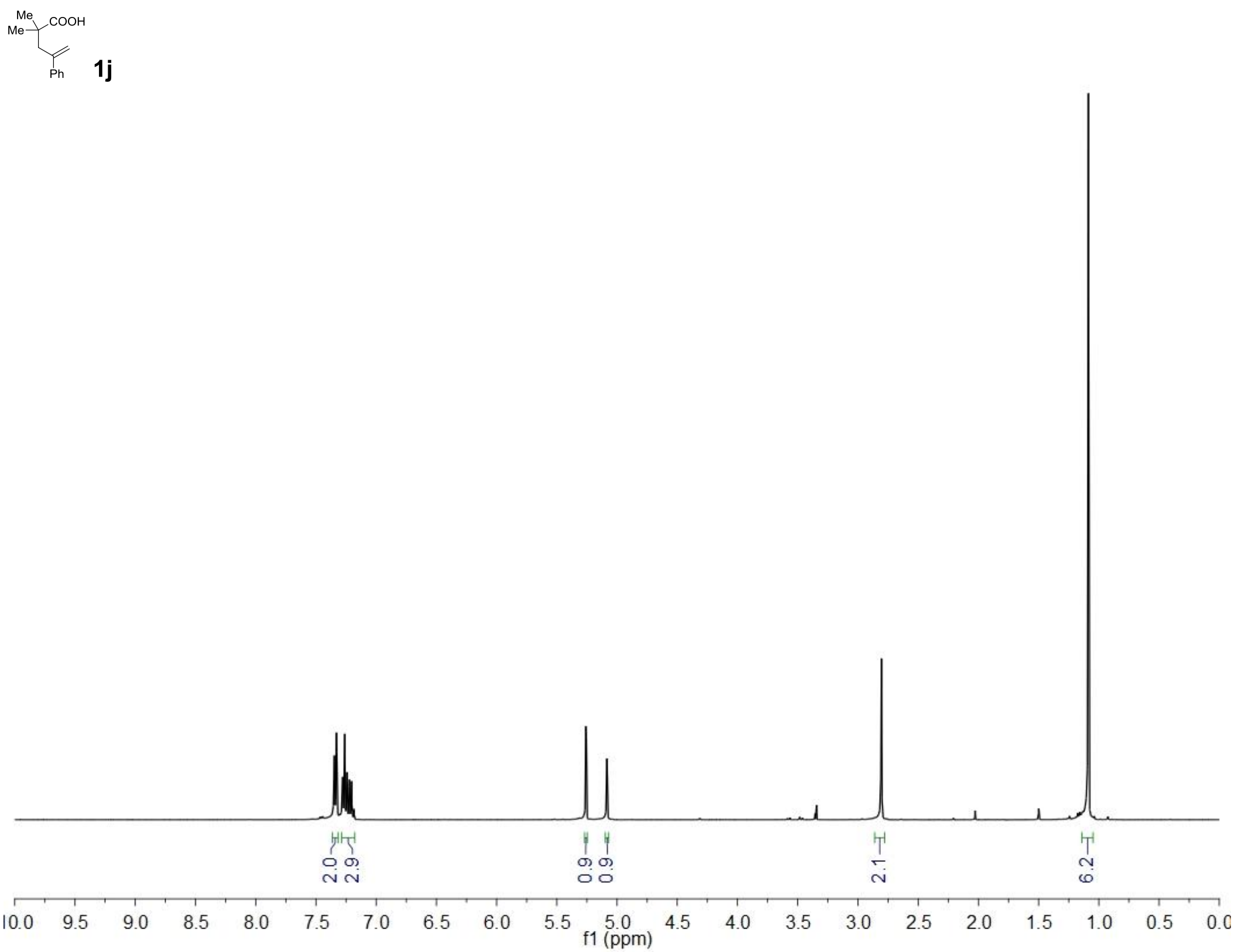



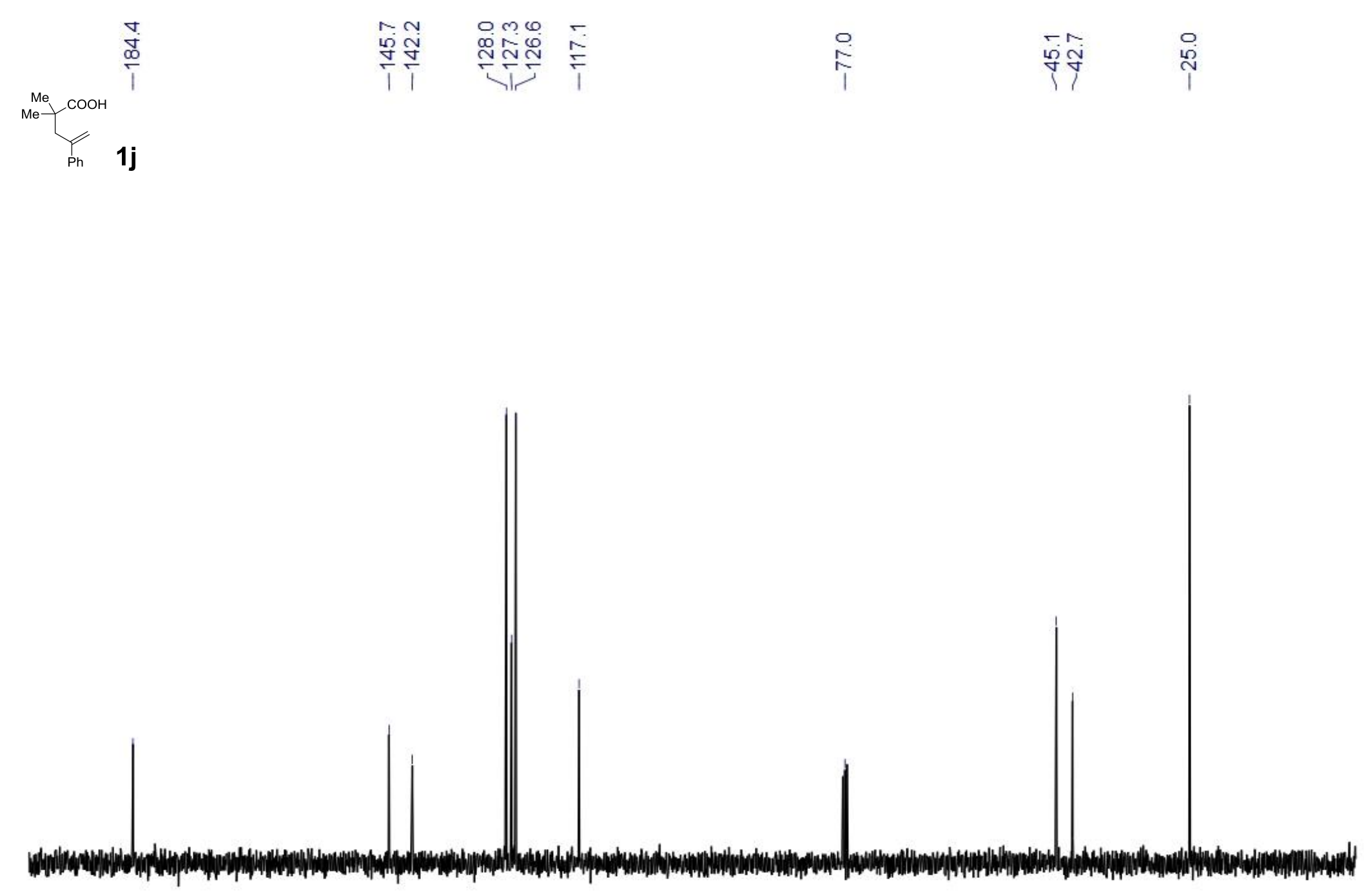

200

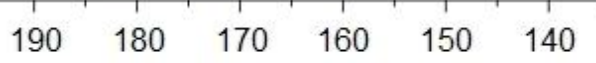

130

120

$110 \quad 100$

$90 \quad 80 \quad 70 \quad 60$

50

$40 \quad 30$

20

10 

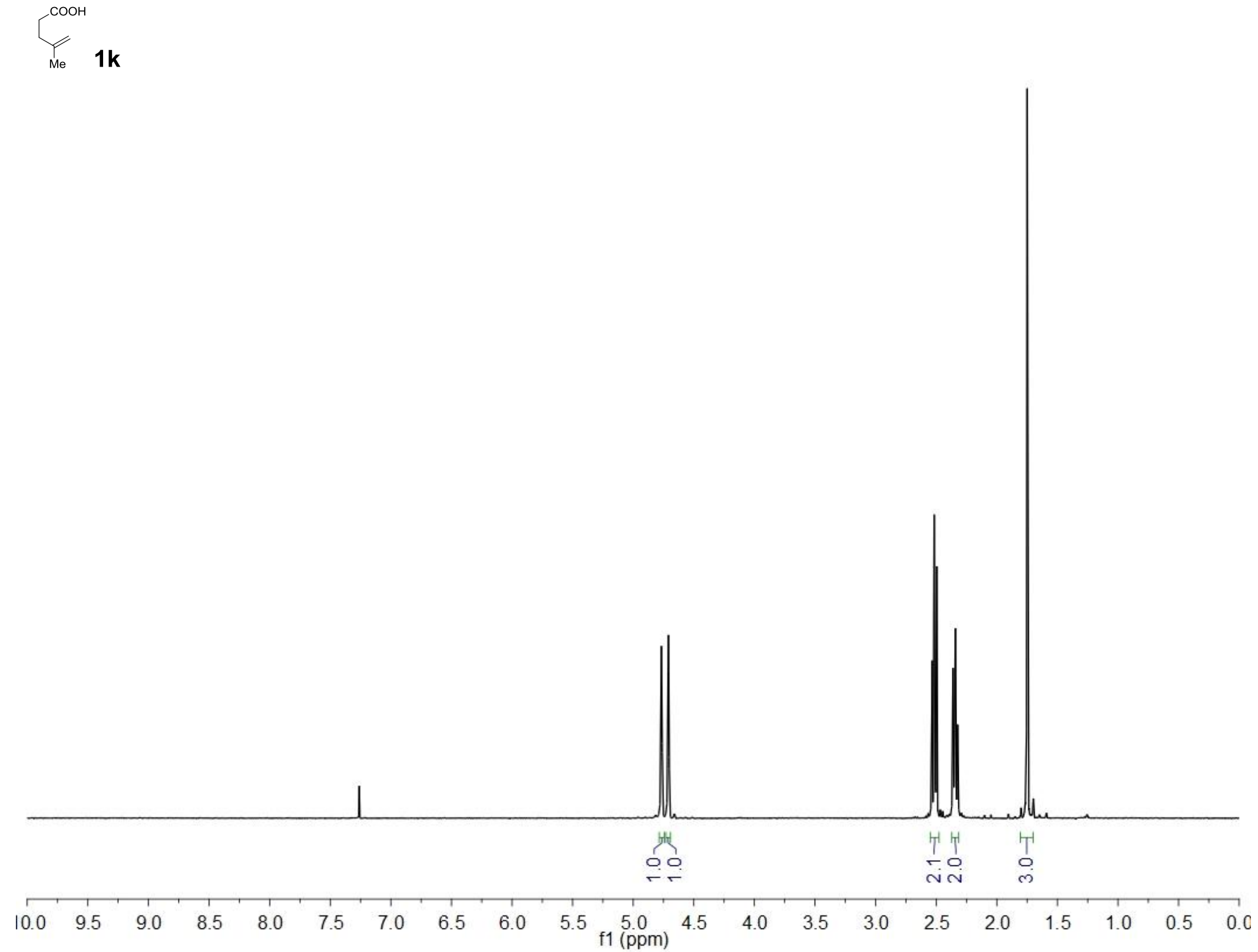

S60 


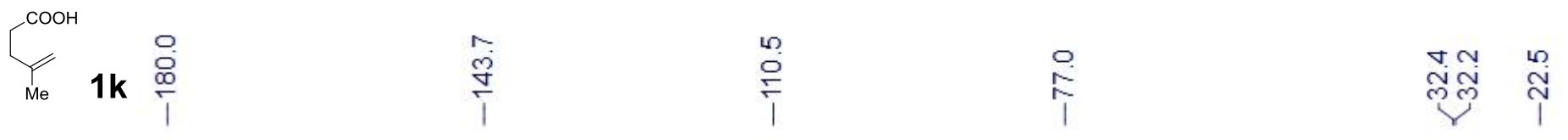

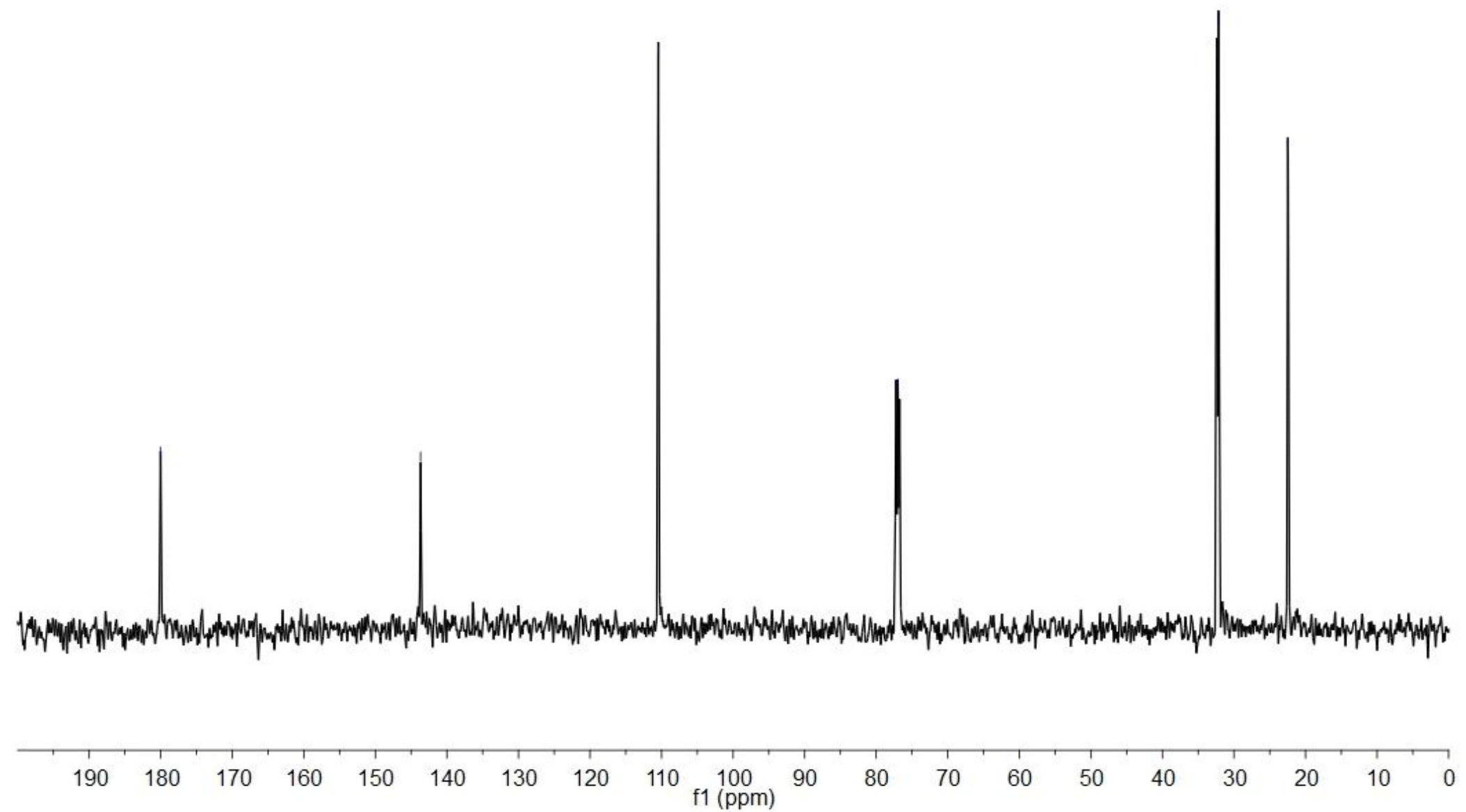




$$
\underbrace{\mathrm{Me}}_{\mathrm{Me}} \mathbf{1 I}
$$

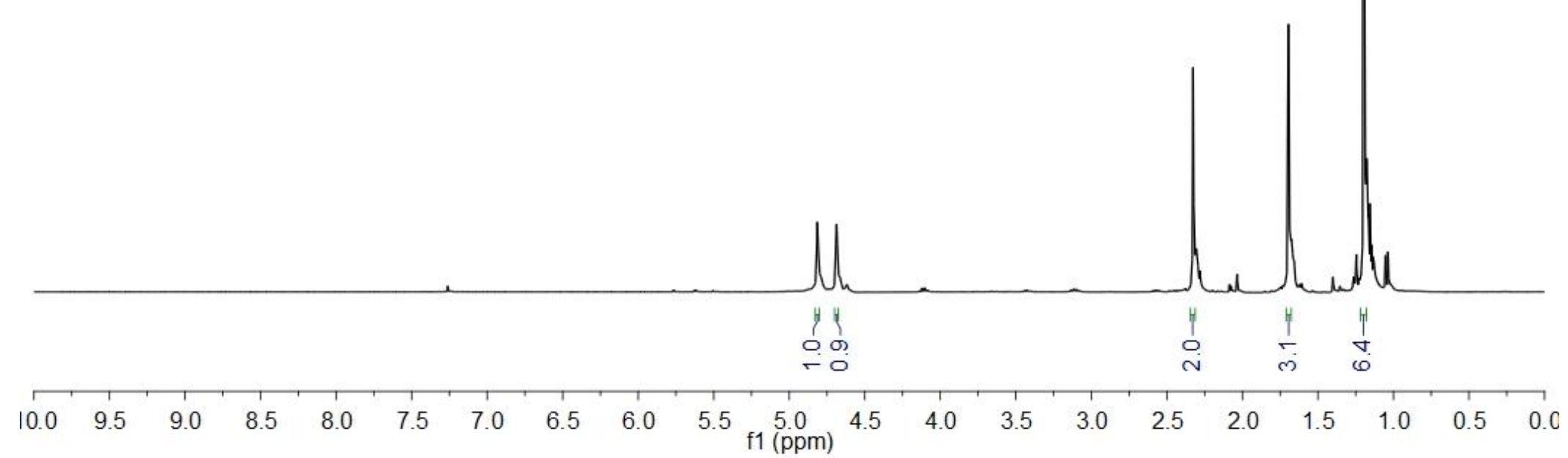




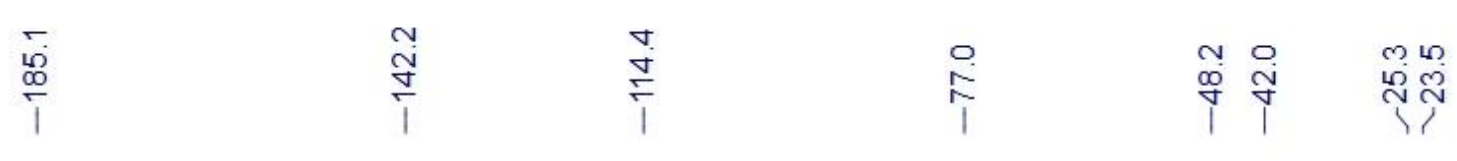

$\underbrace{\text { Me }}_{\text {Me }} \mathbf{1 1}$

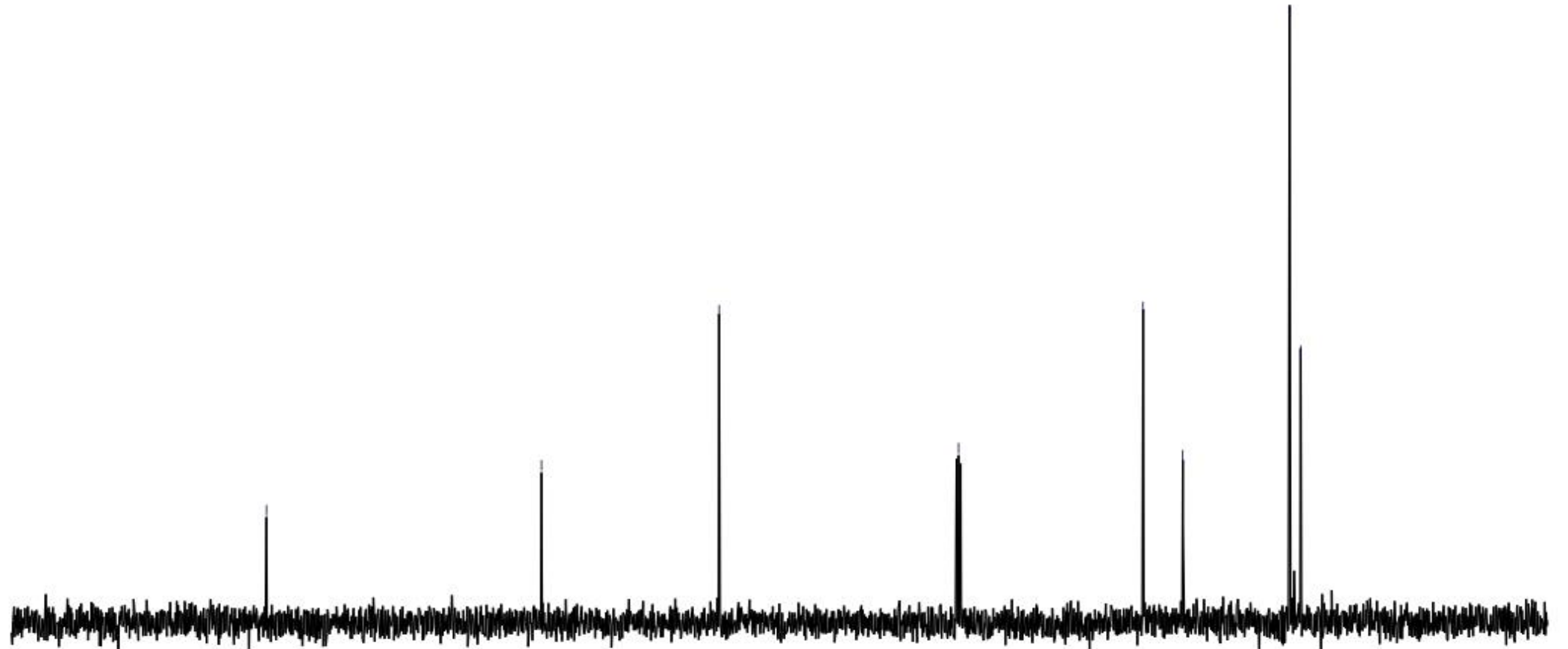

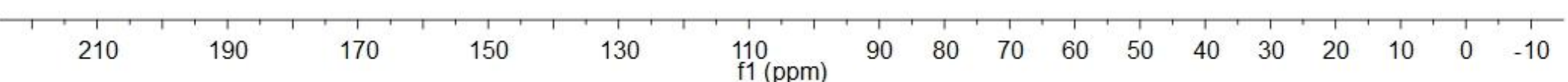




$$
\underbrace{\mathrm{Ph}}_{\mathrm{Me}} \mathbf{1 m}
$$

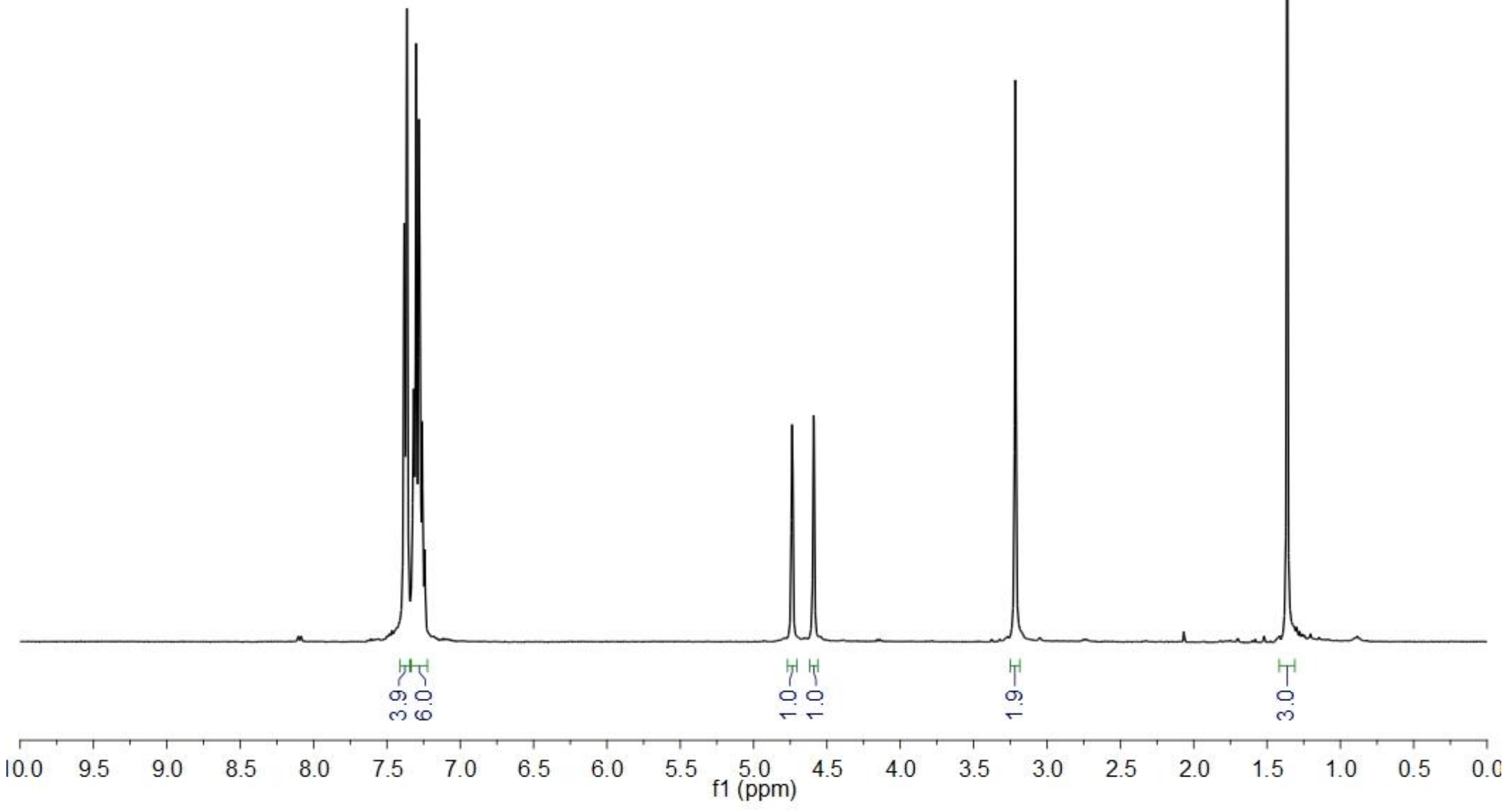



$\underbrace{}_{\text {Me }} \mathbf{1 m}$
\}
定
$\stackrel{1}{\stackrel{1}{+}}$

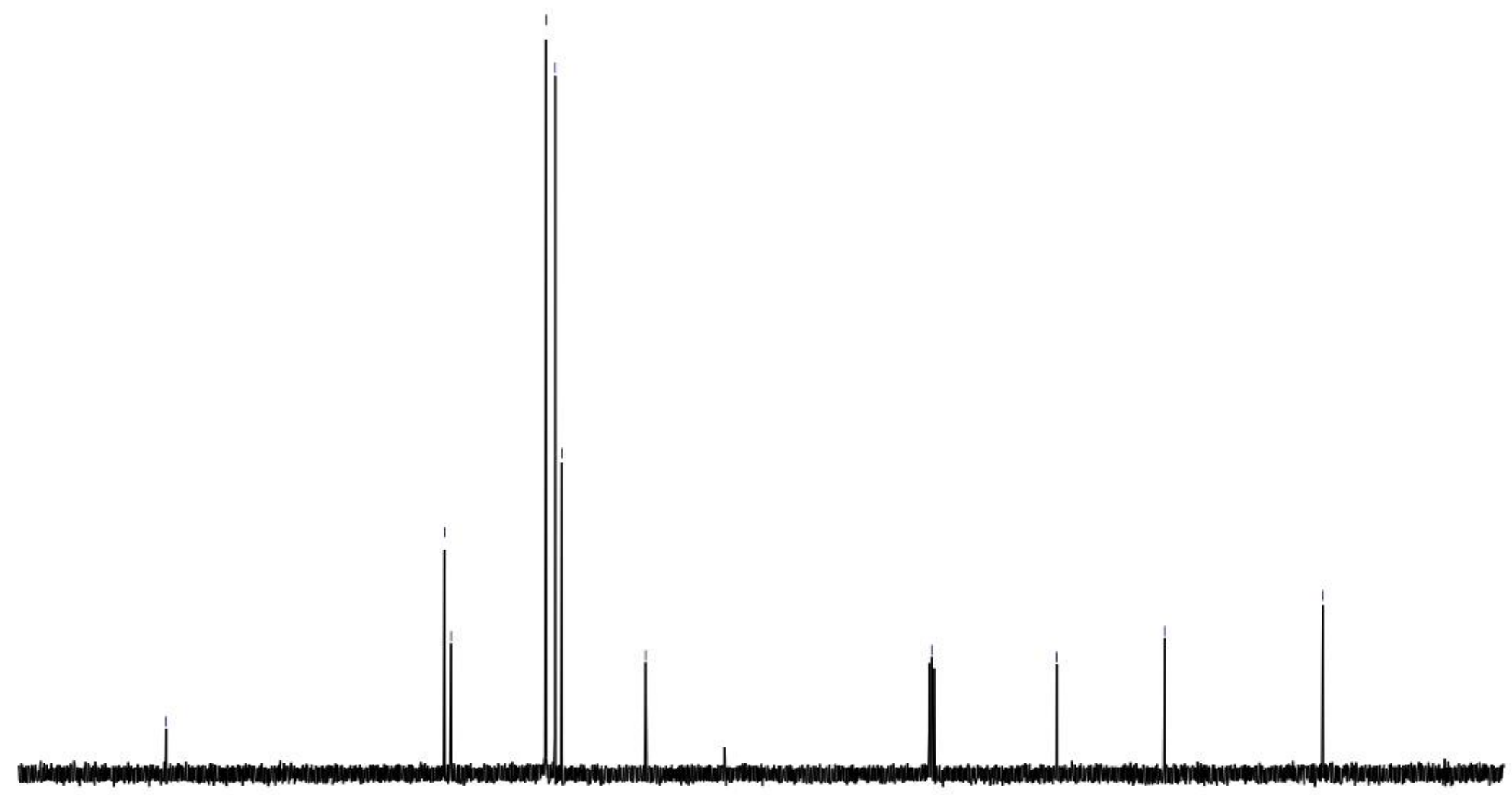




$$
\underbrace{\mathrm{Ph}}_{\mathrm{Me}} \mathbf{1 n}
$$

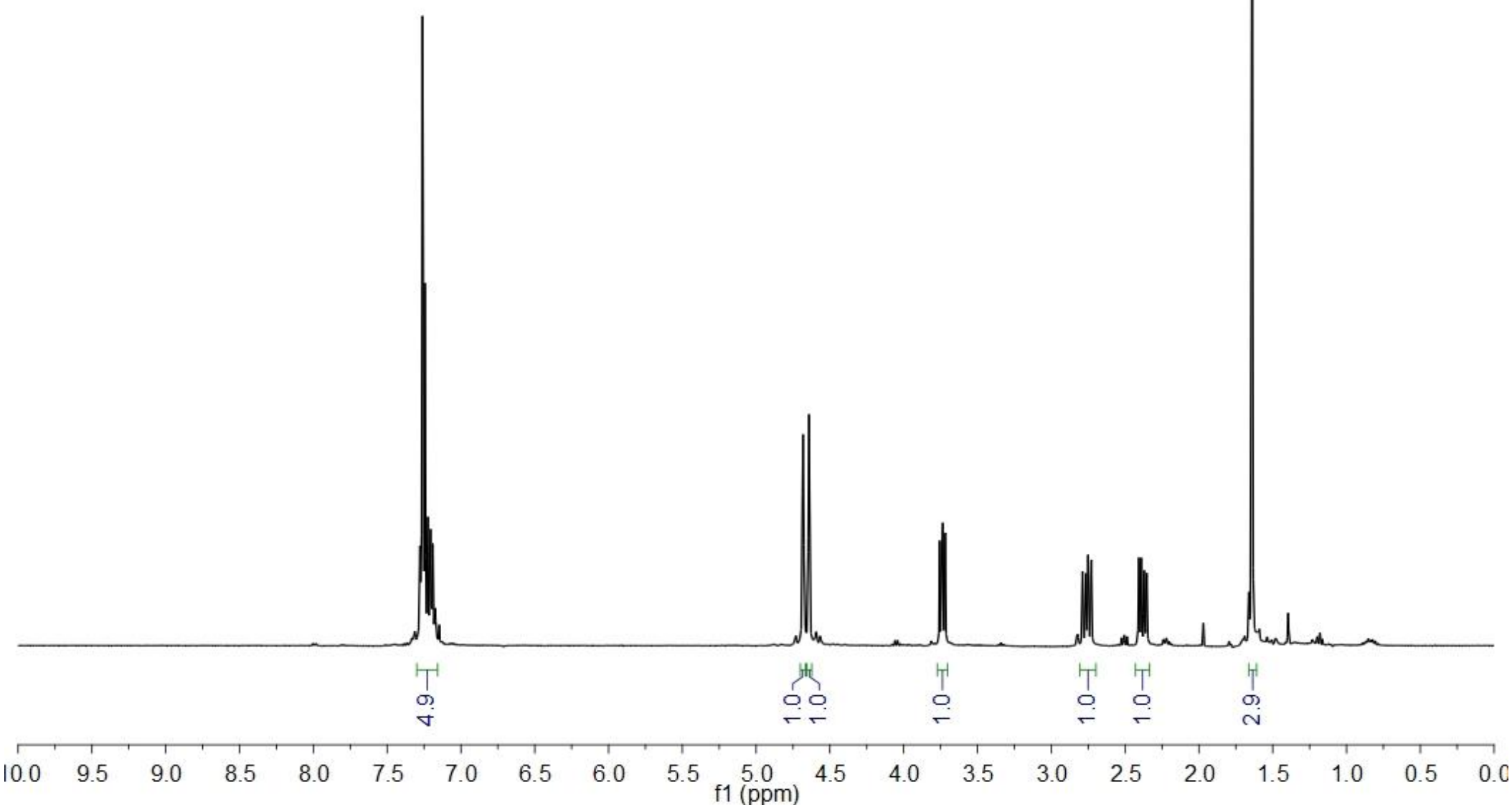




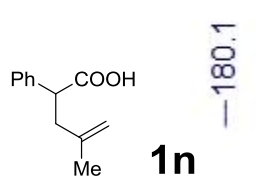

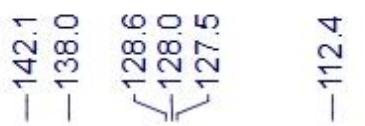

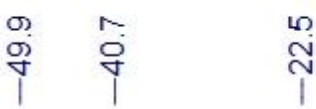

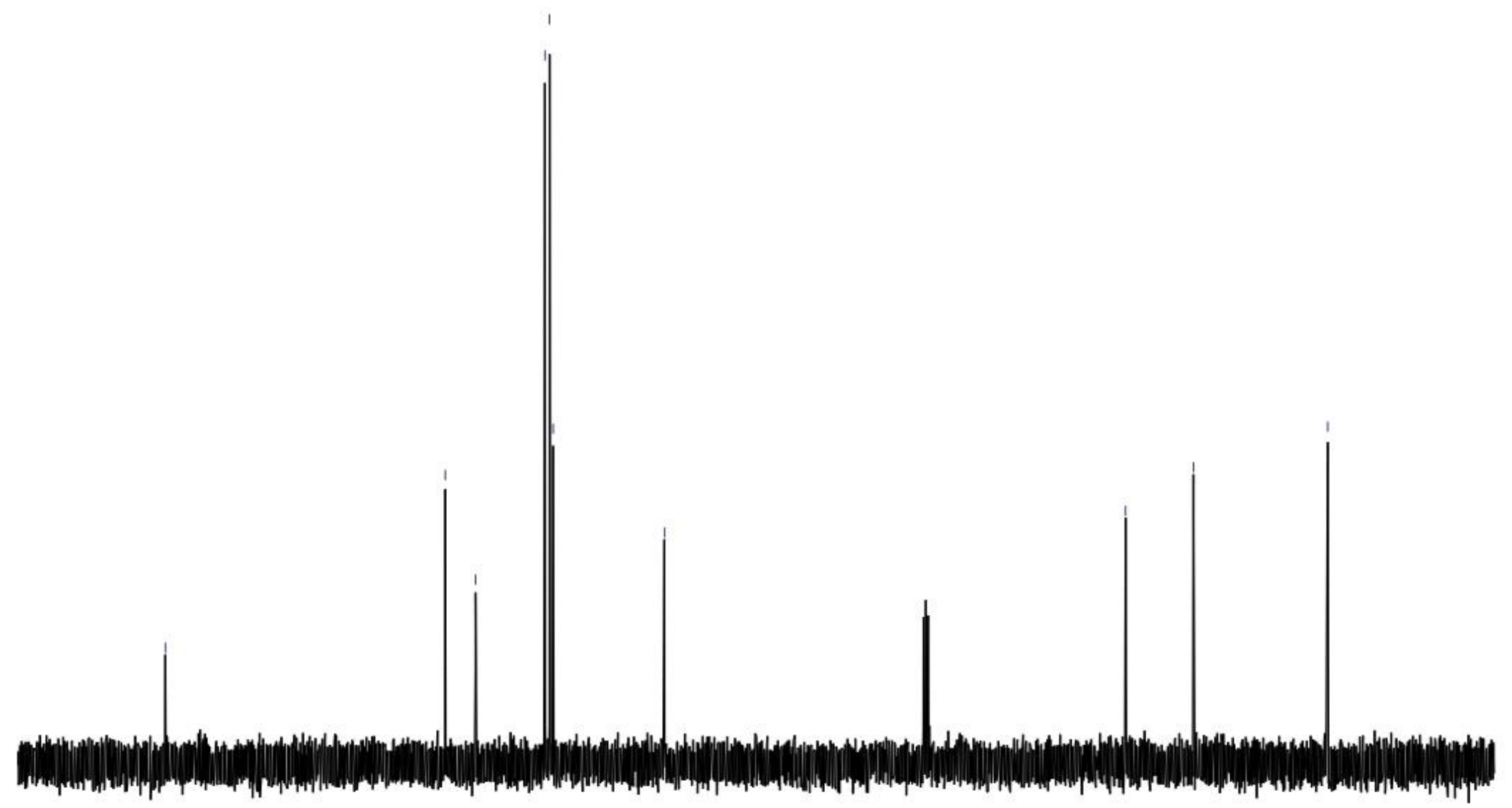




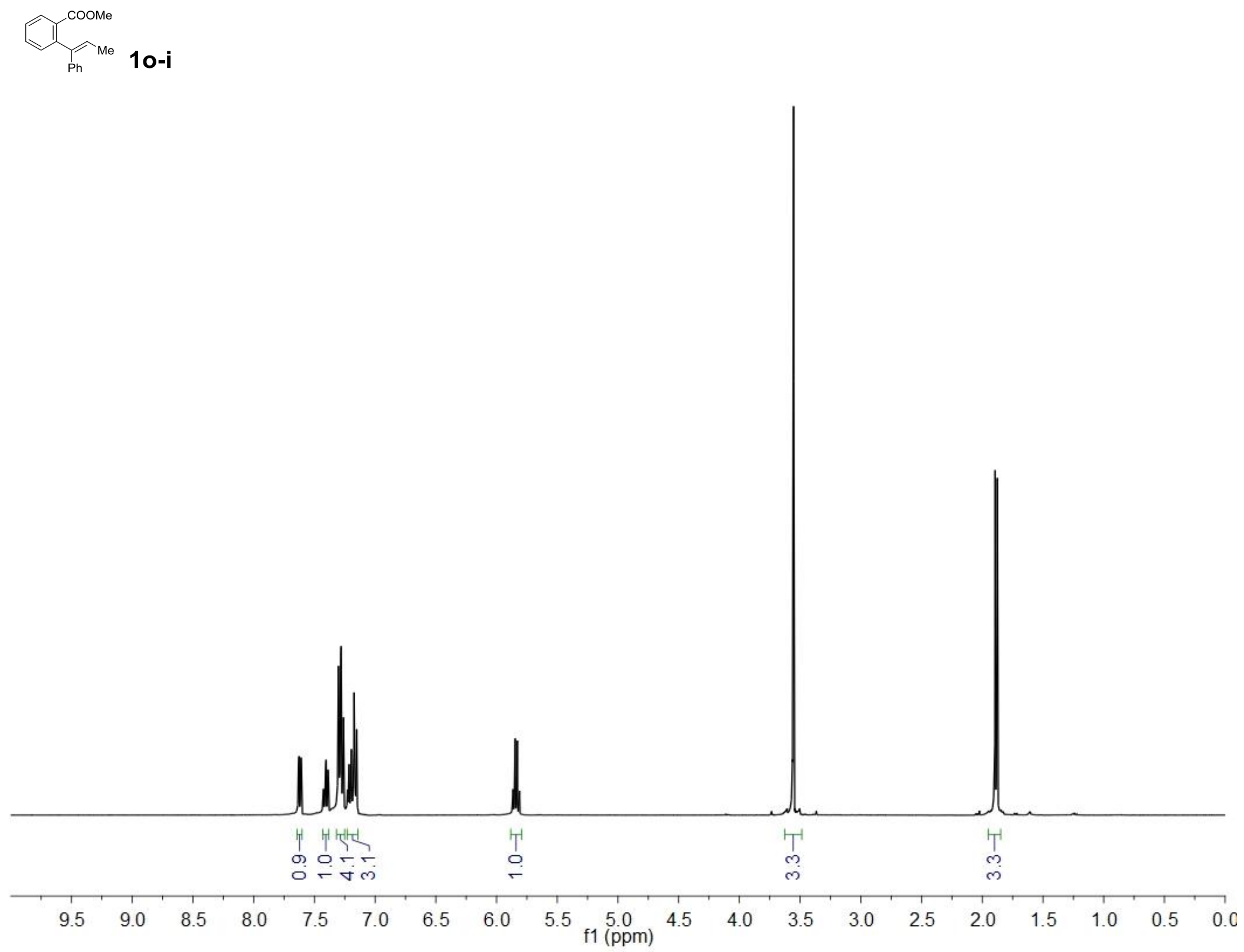



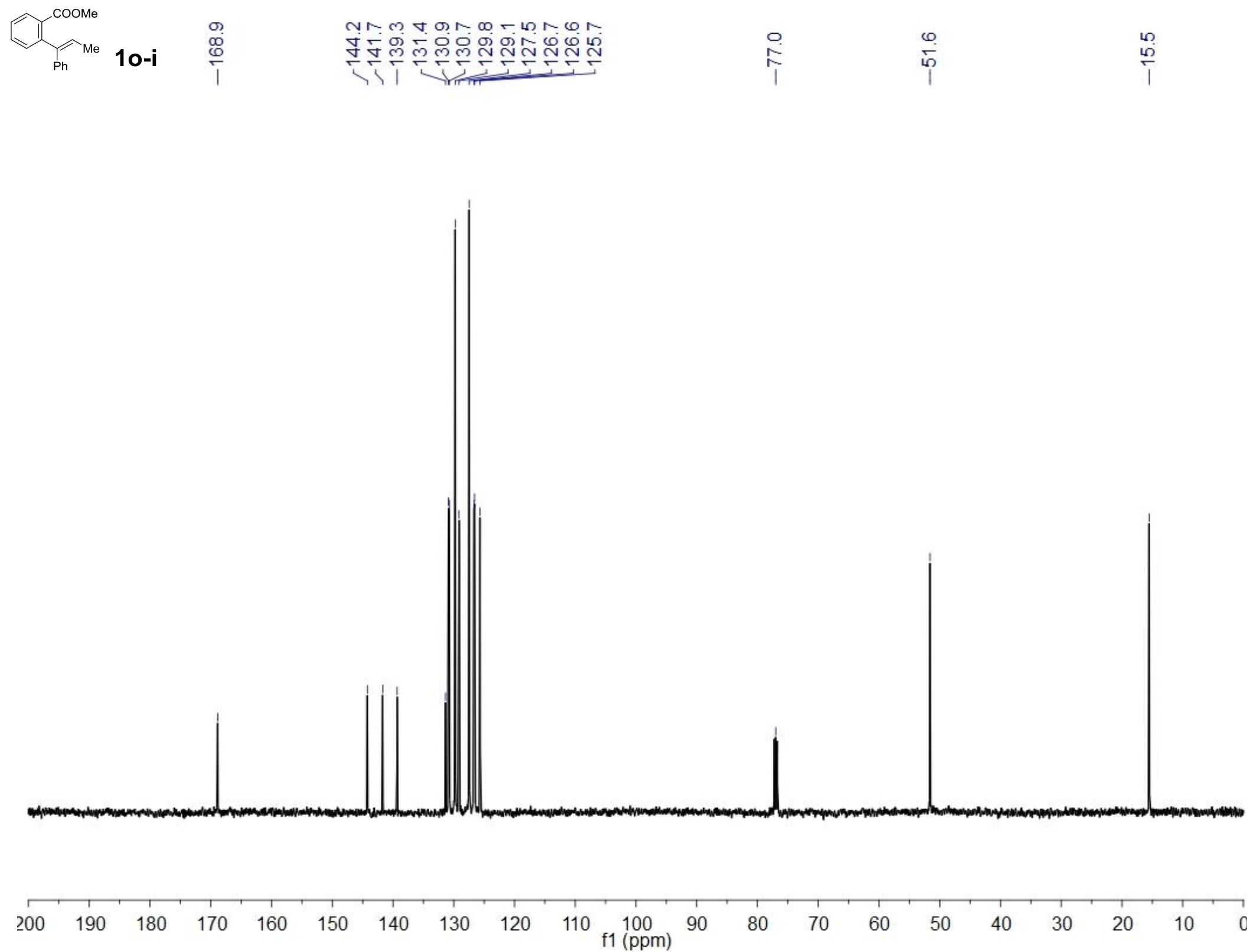

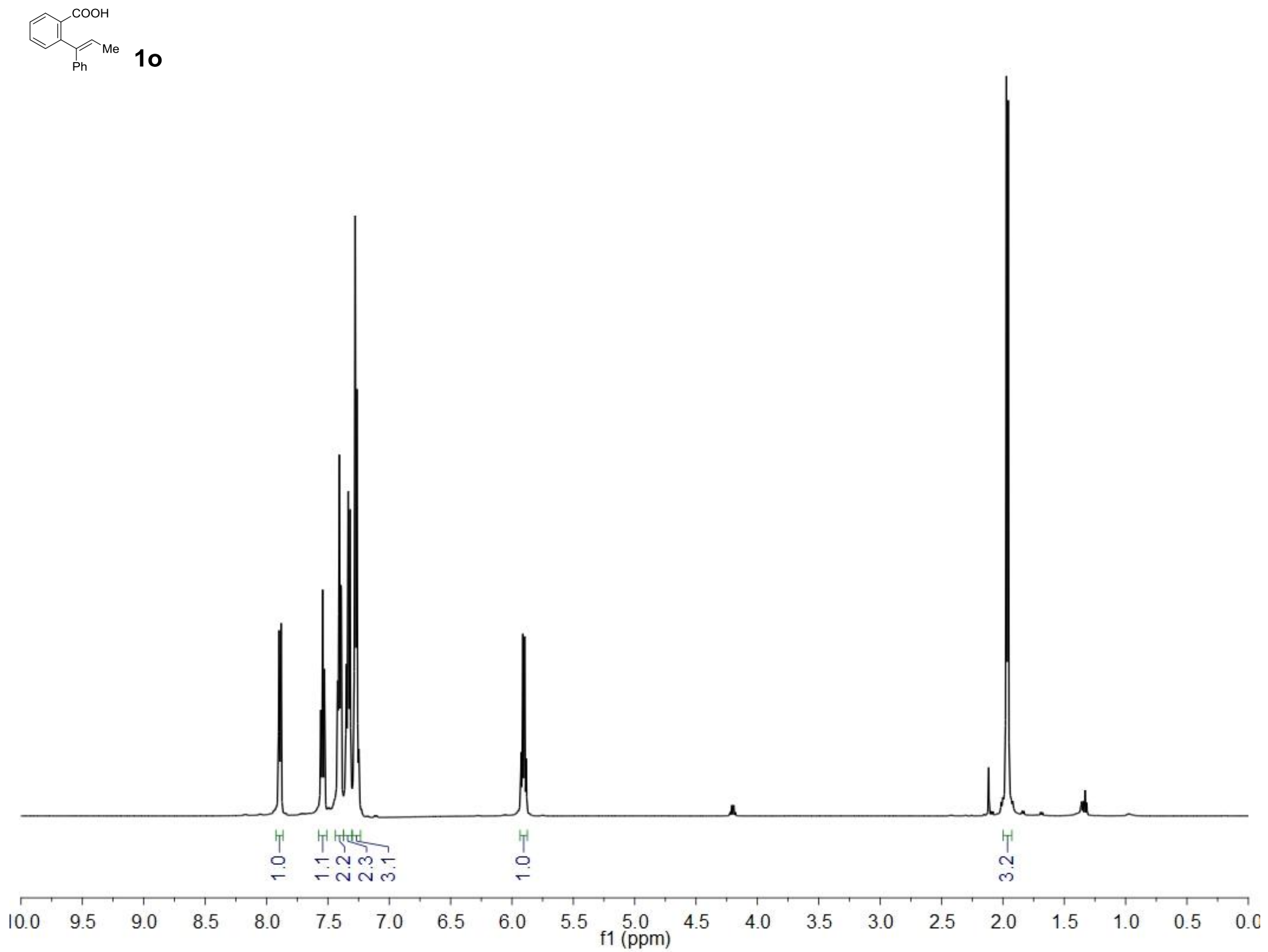

S70 


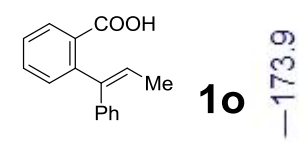

息离方

$\stackrel{\circ}{\gtrless}$

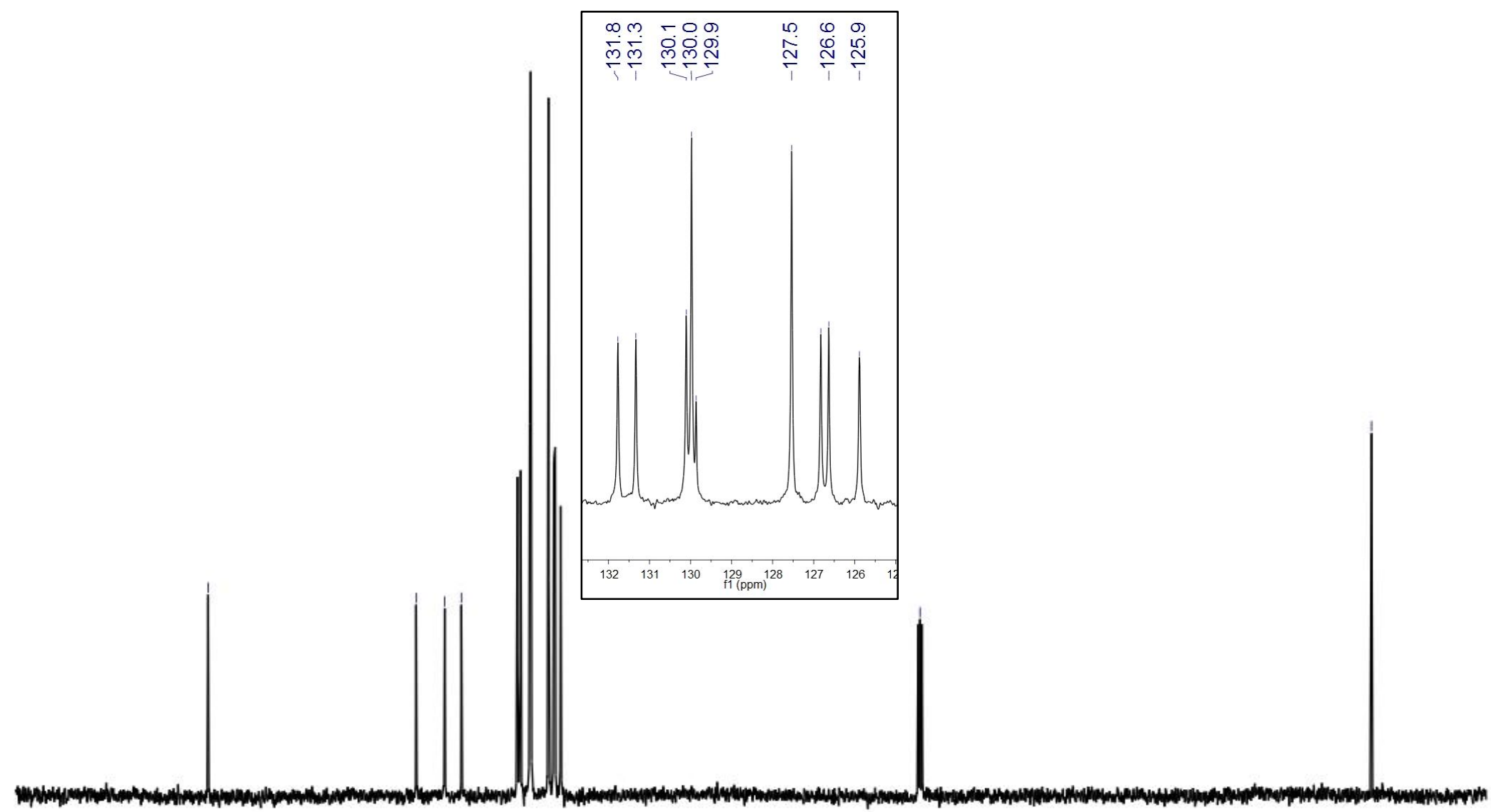

200 

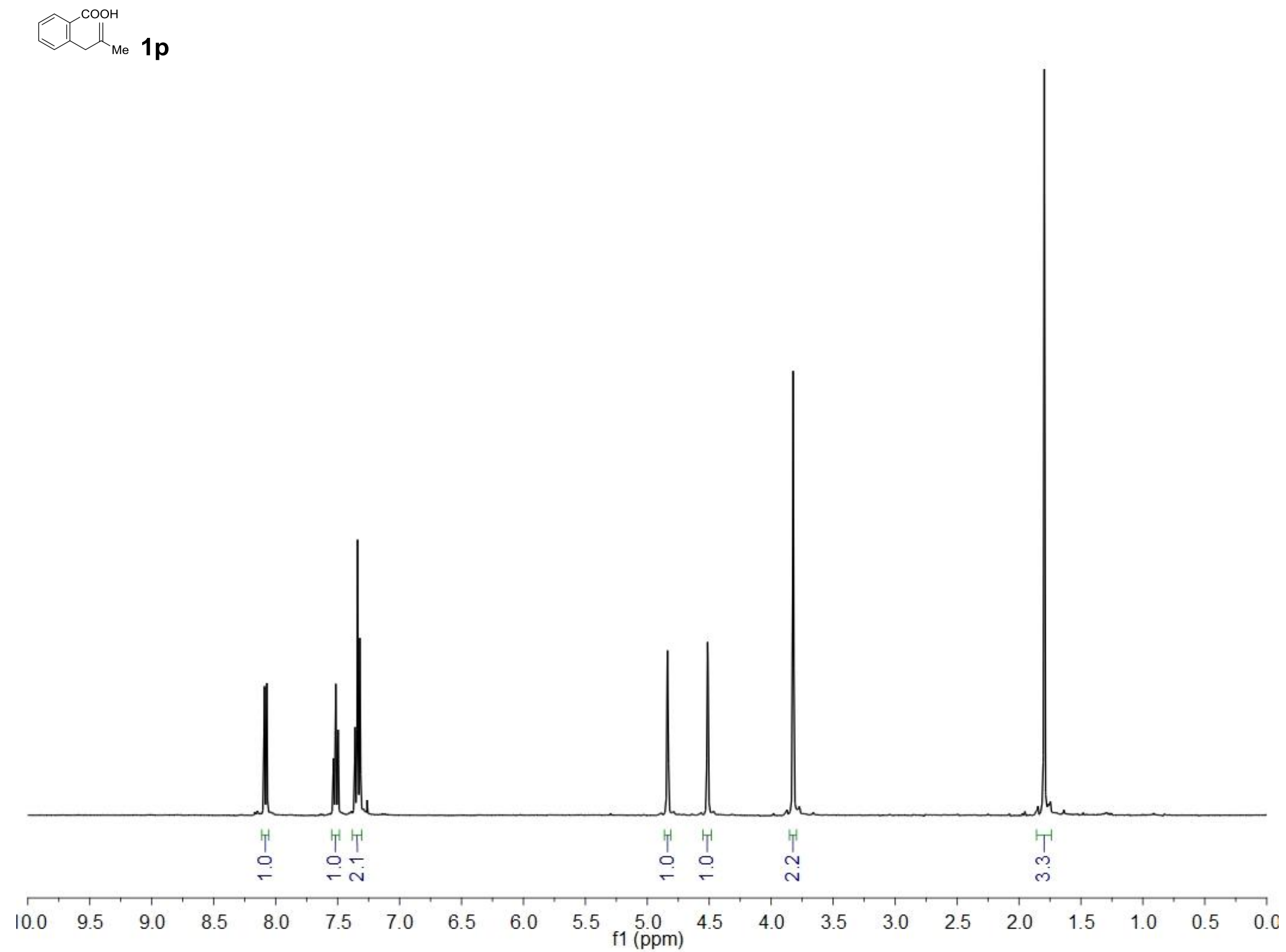

S72 


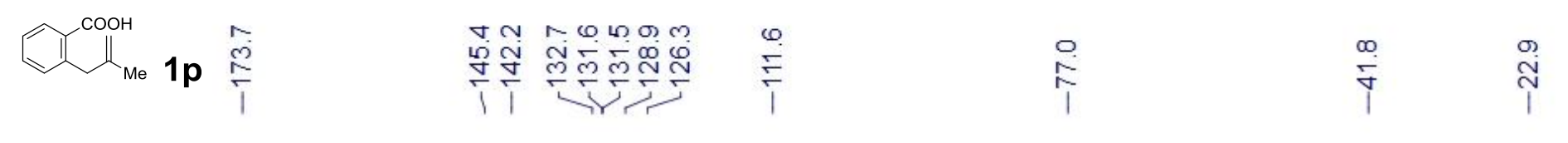

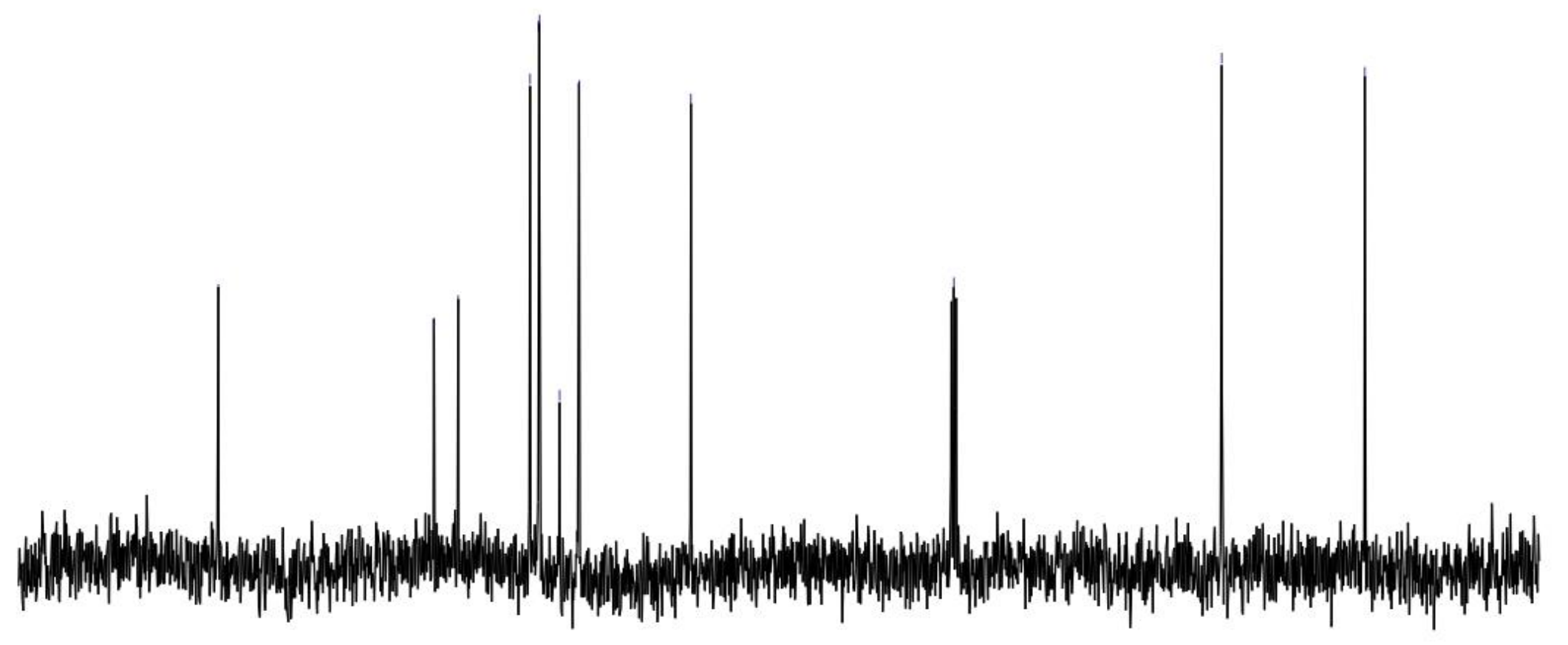


$\underbrace{B r}_{\text {Me }} \mathbf{1 q - i}$

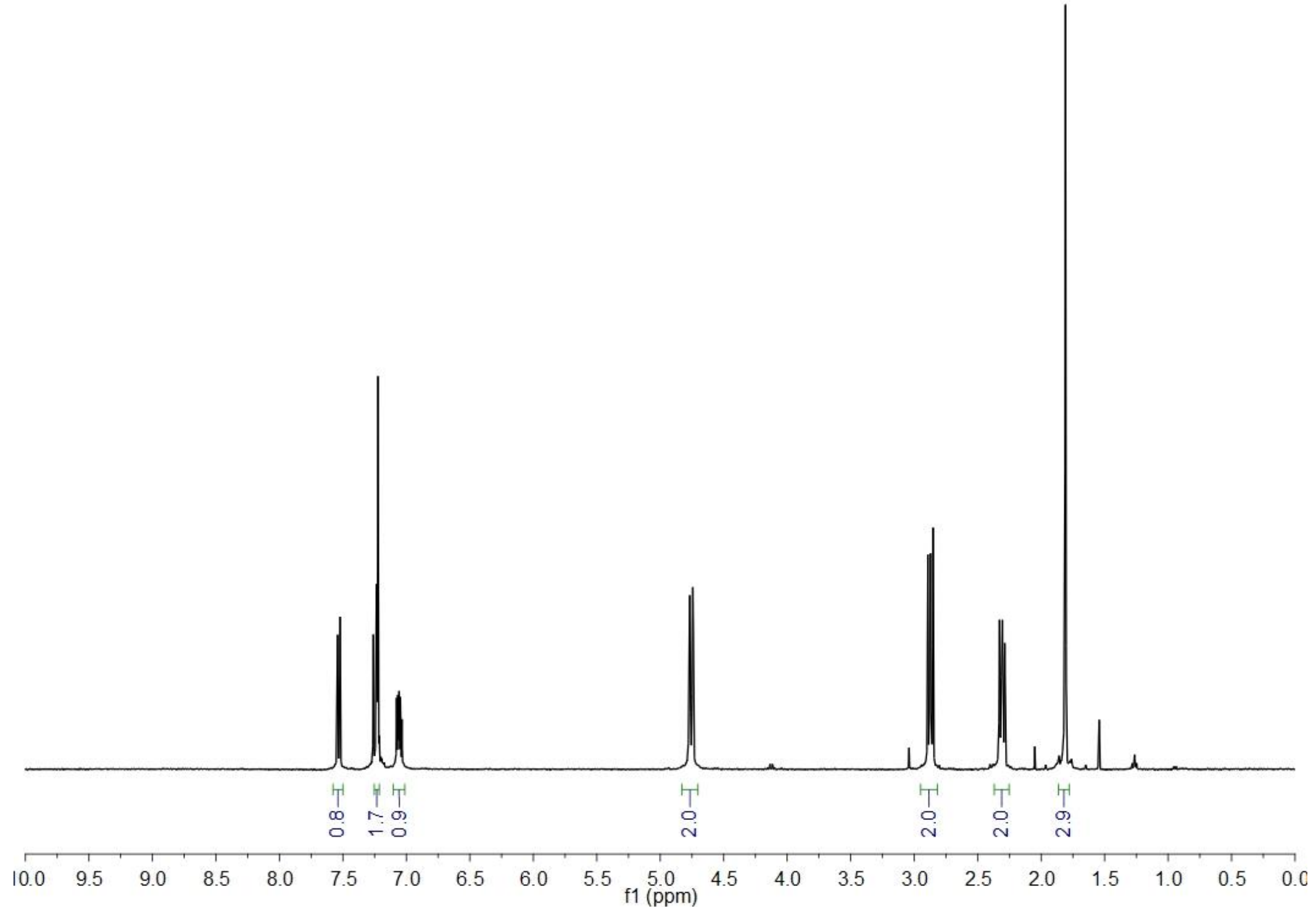




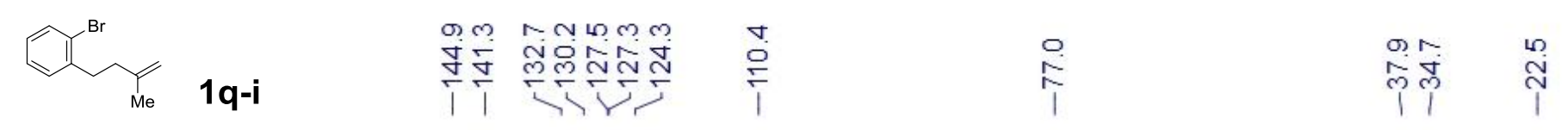

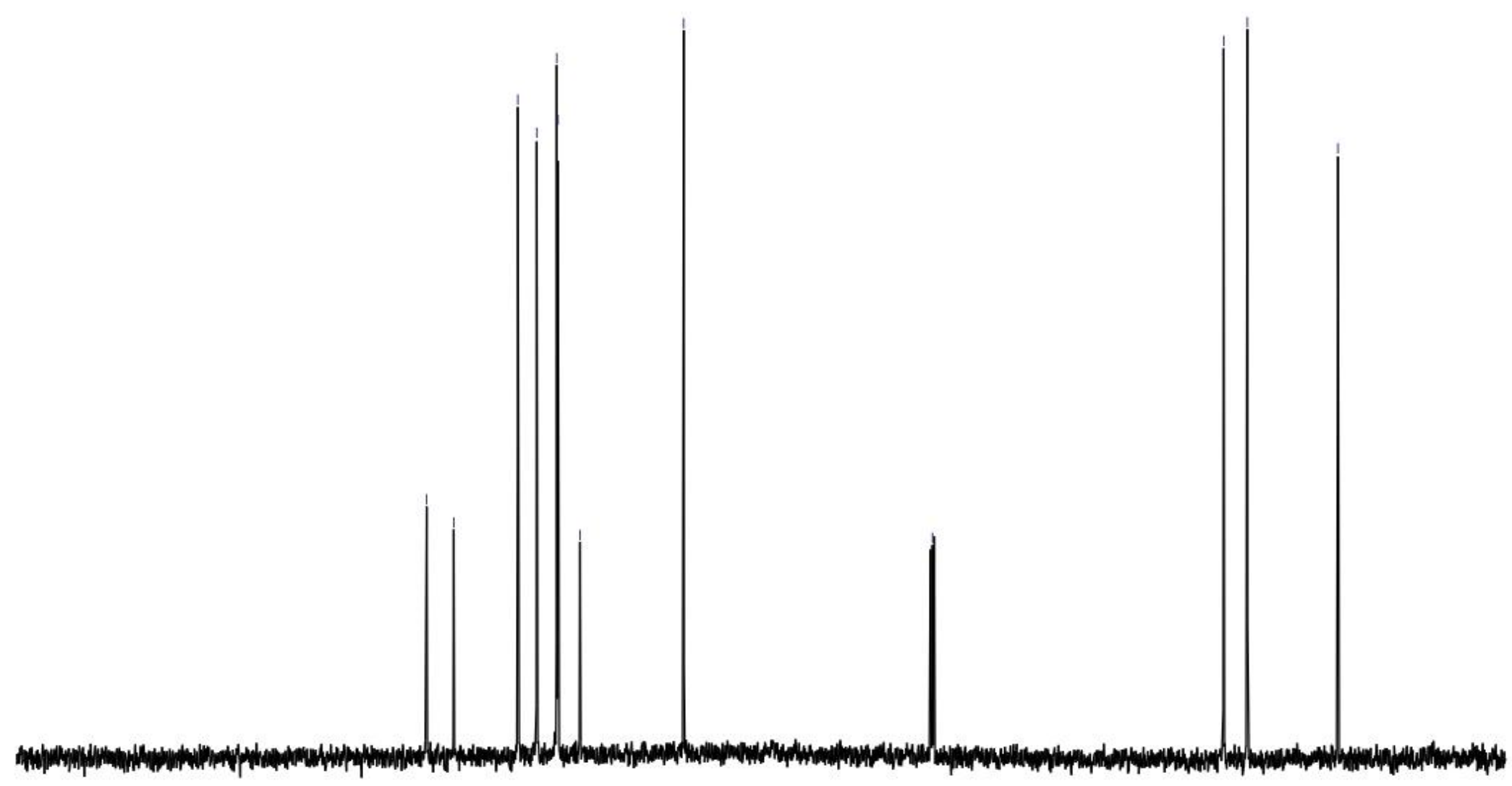



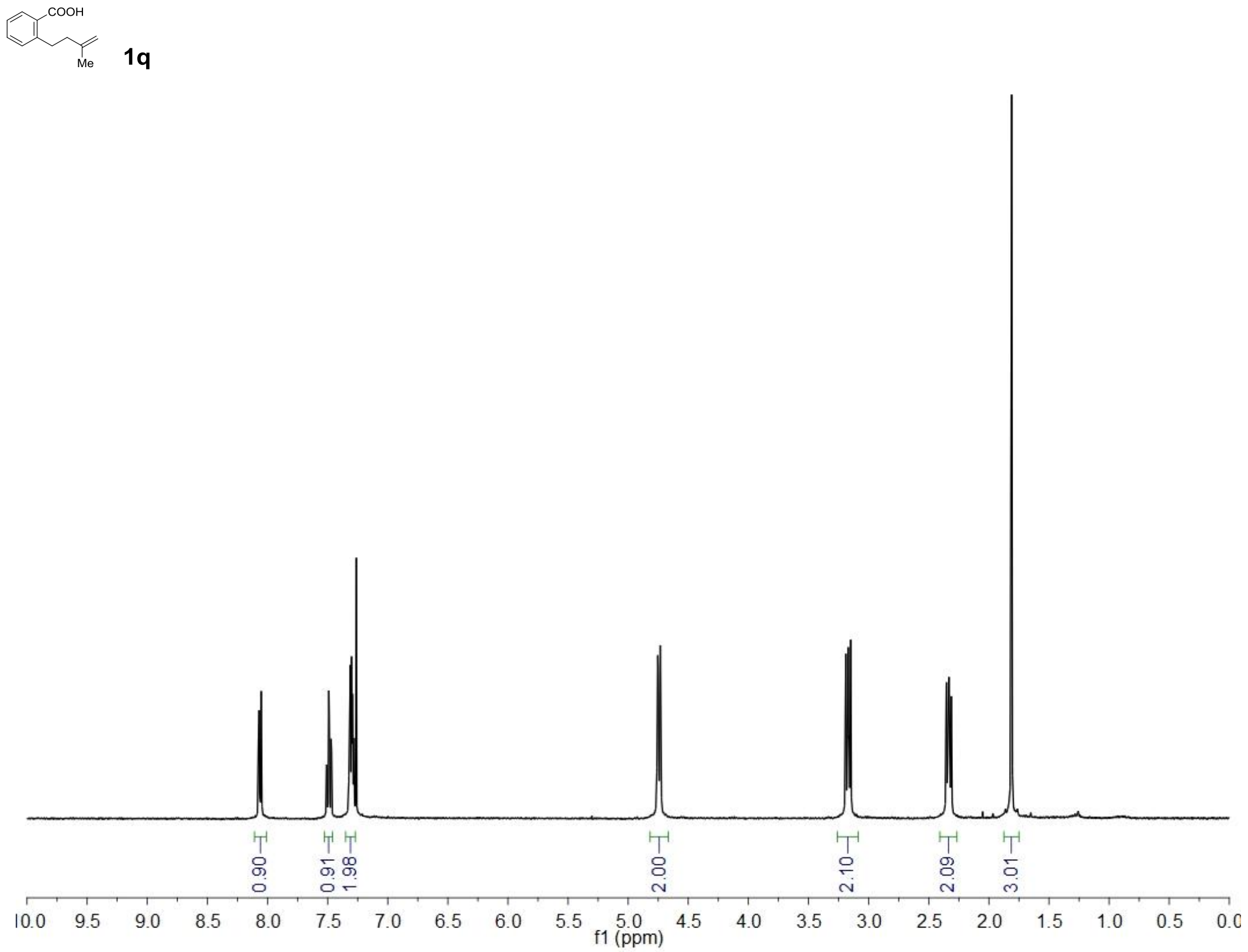

S76 

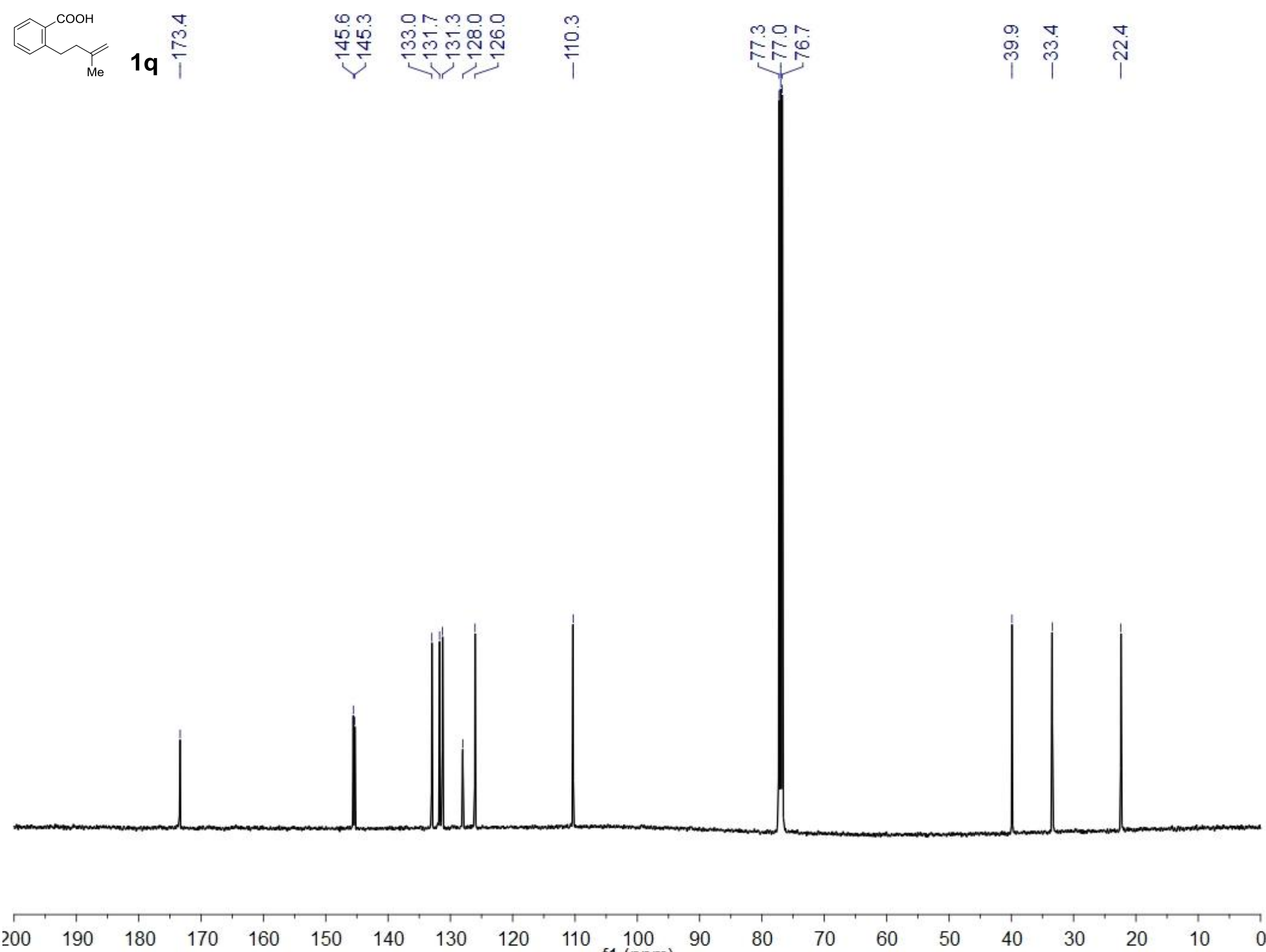

f1 $(p p m)$

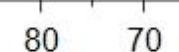

60

$50 \quad 40$

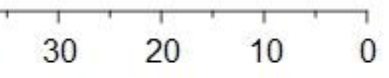




\section{$\overbrace{\mathrm{Br}}^{\mathrm{COOMe}}(E)-\mathbf{1 r}-\mathbf{i}$}

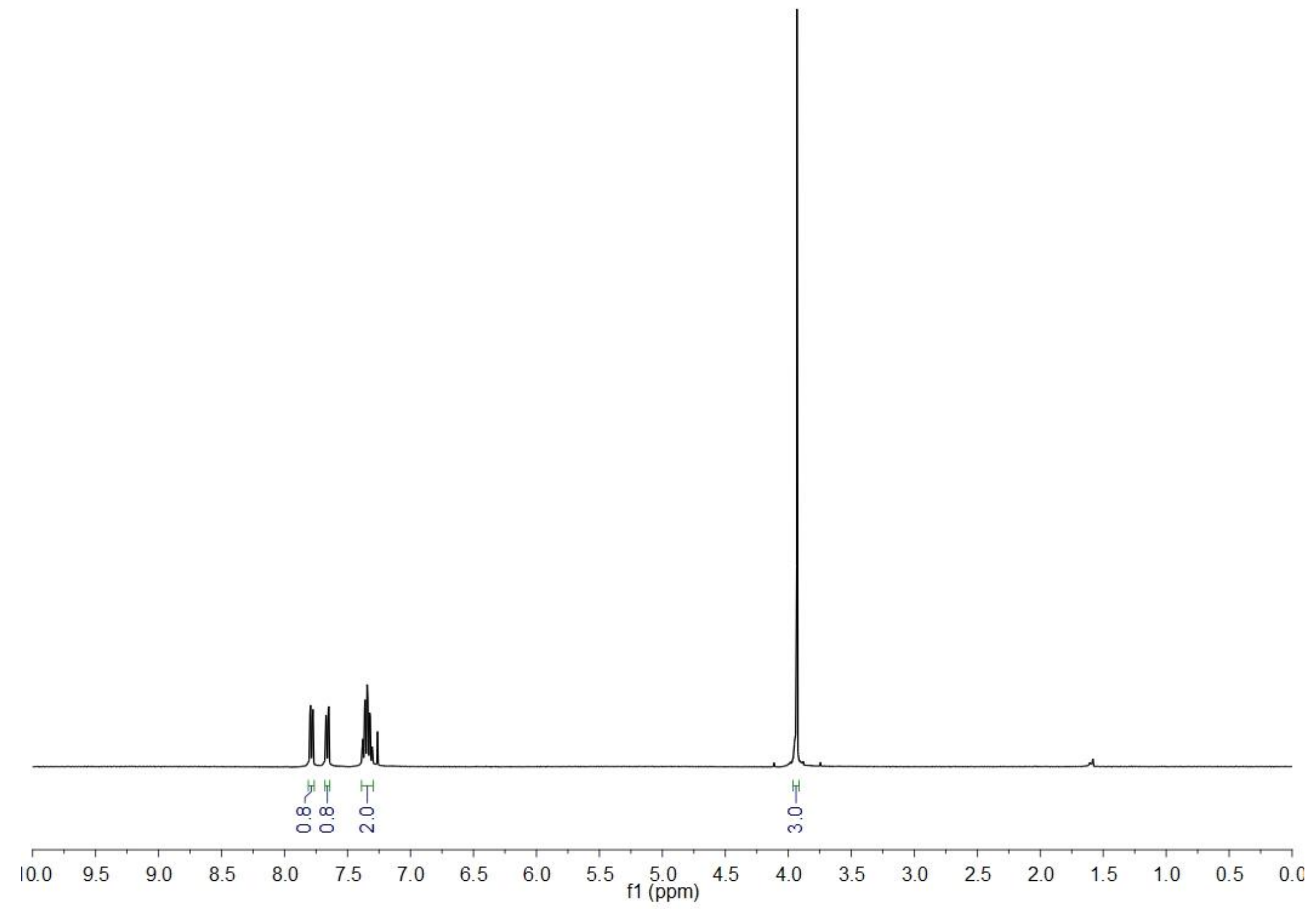




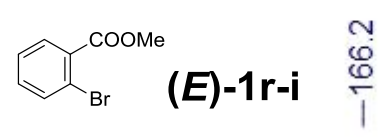

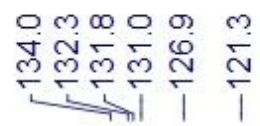
$\stackrel{0}{i}$
$\underset{\sim}{i}$

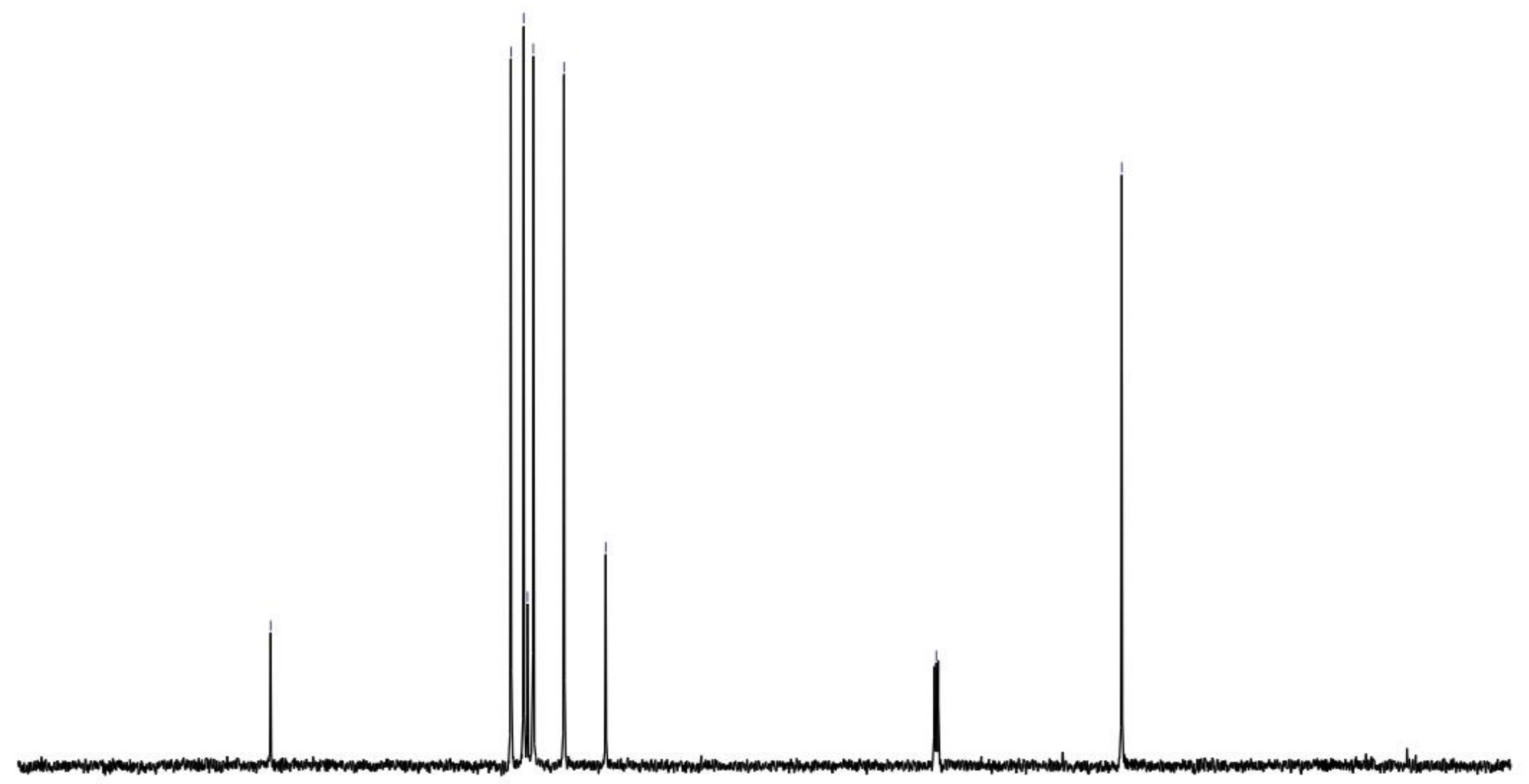

200

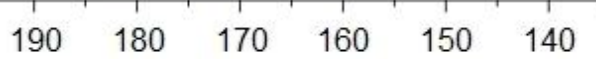




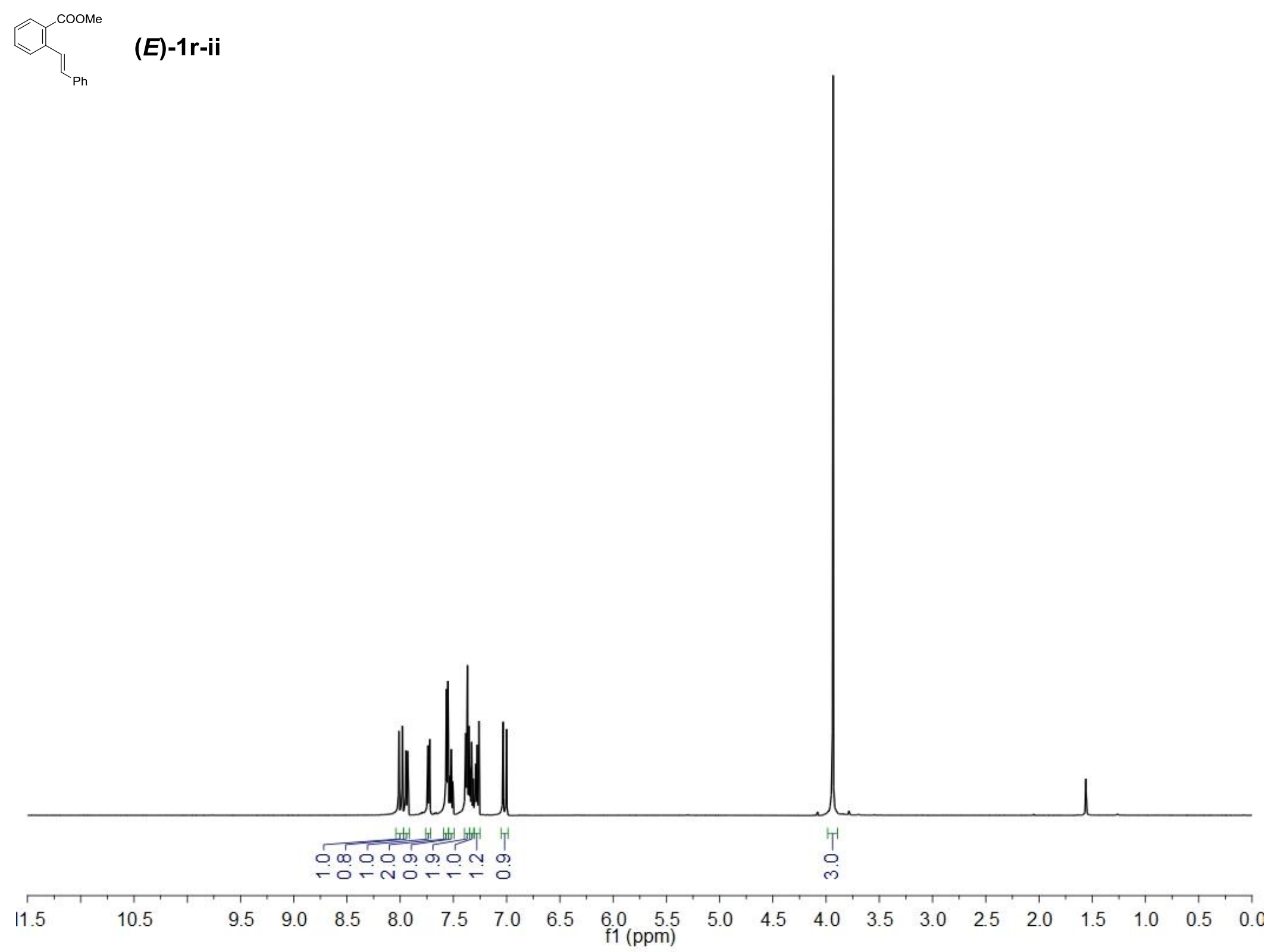




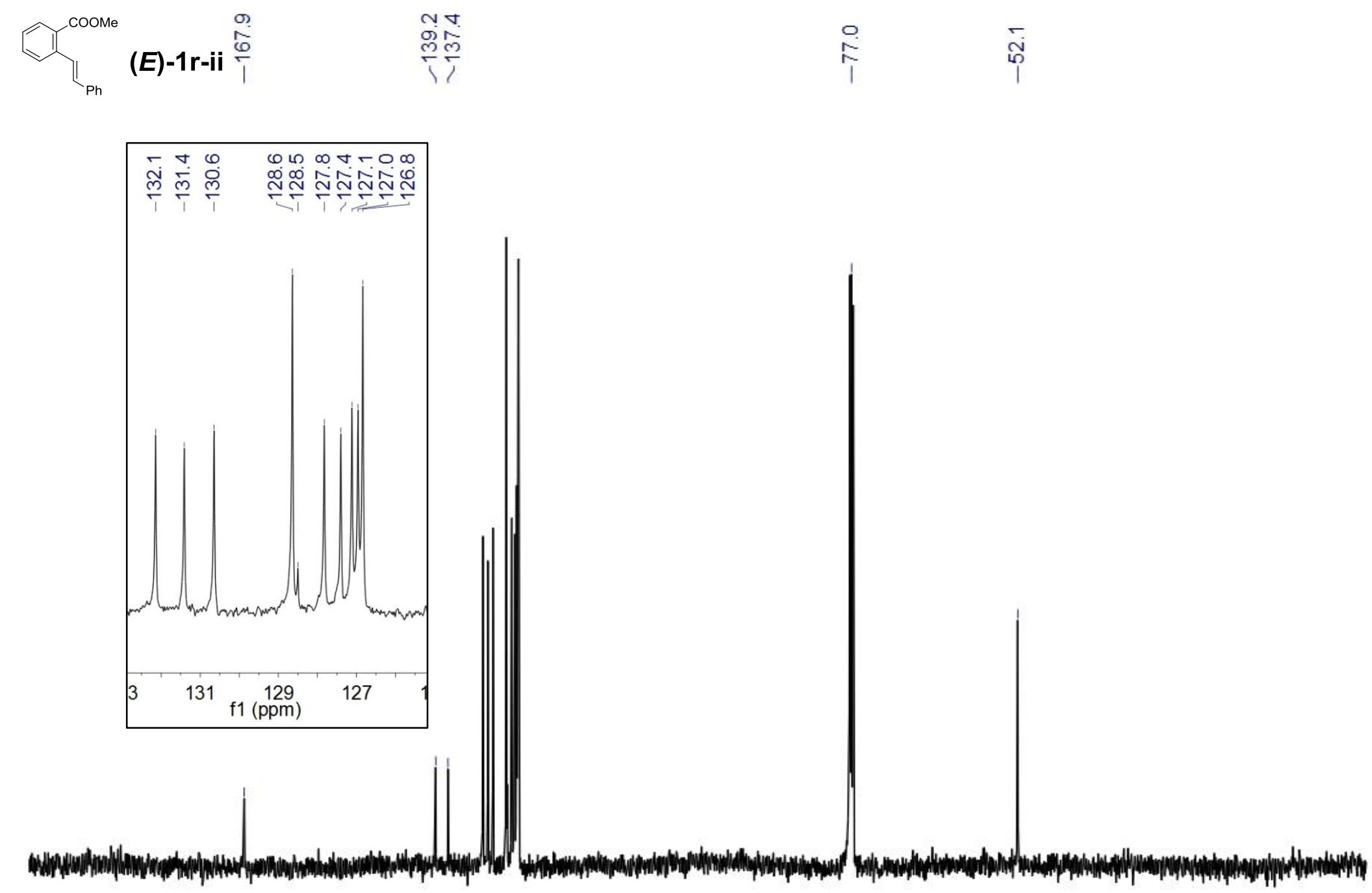

200

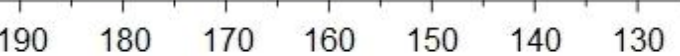

120

$110 \quad 100$

$80 \quad 70$

60

50

$40 \quad 30$

20

$10 \quad 0$ 


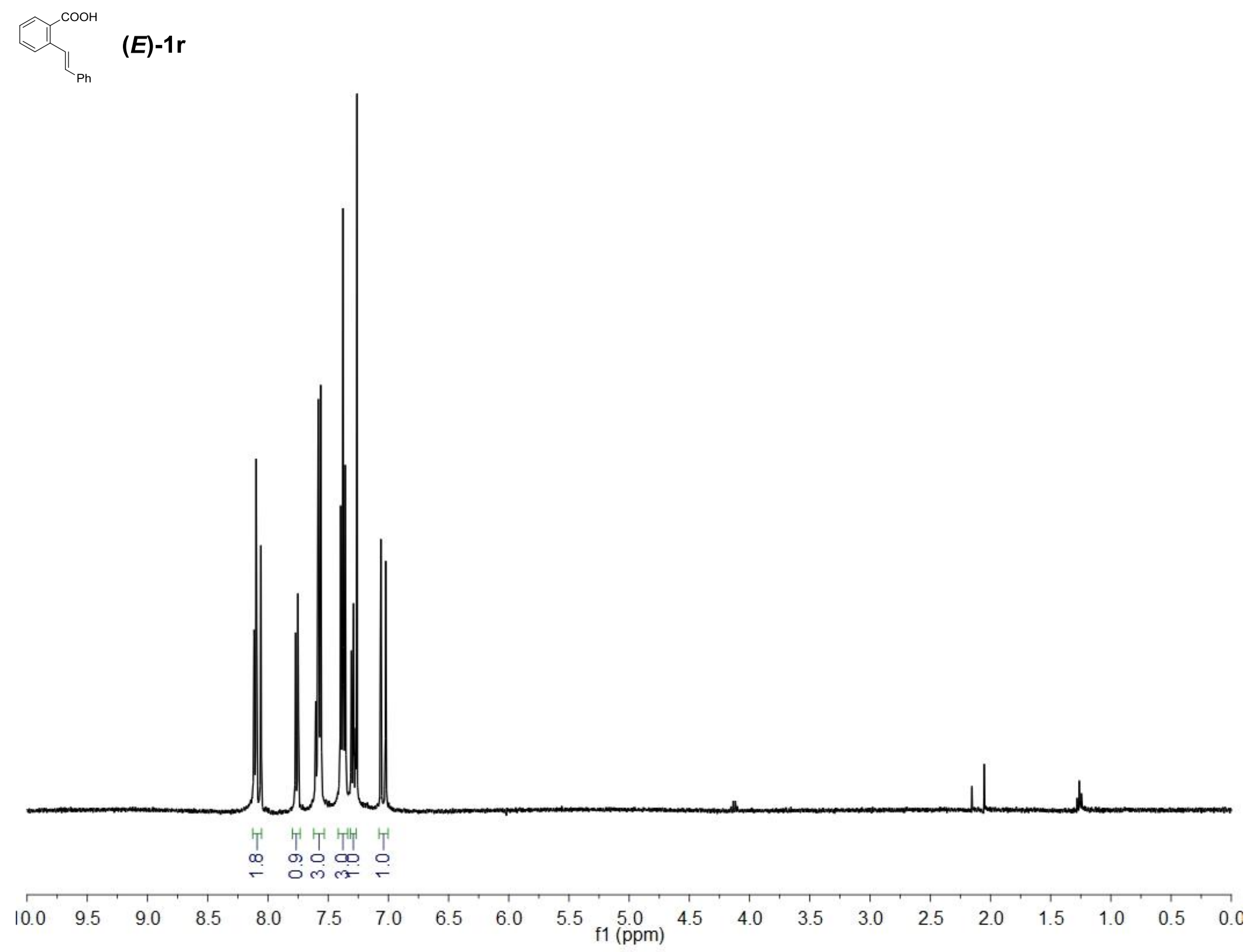




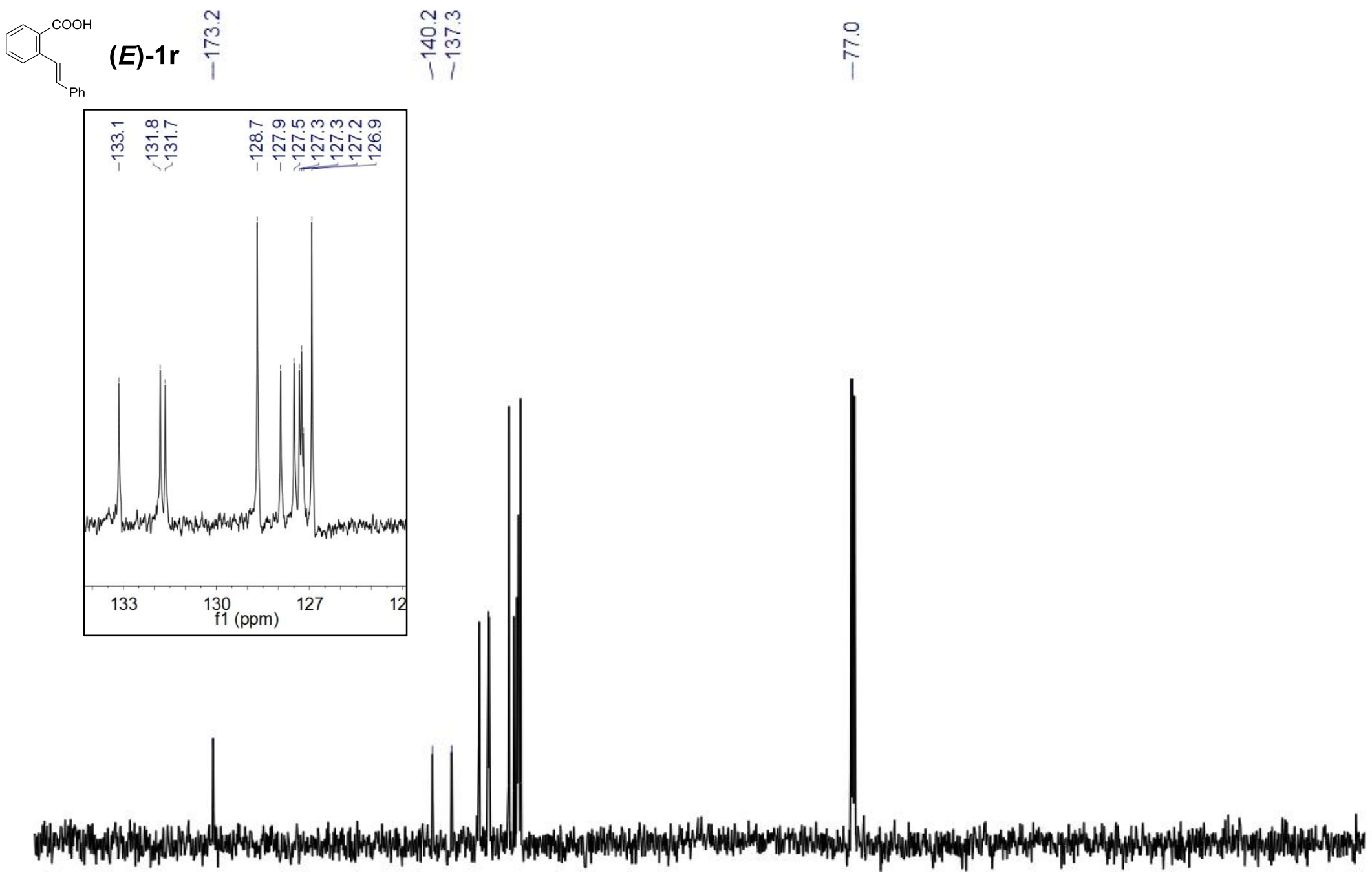




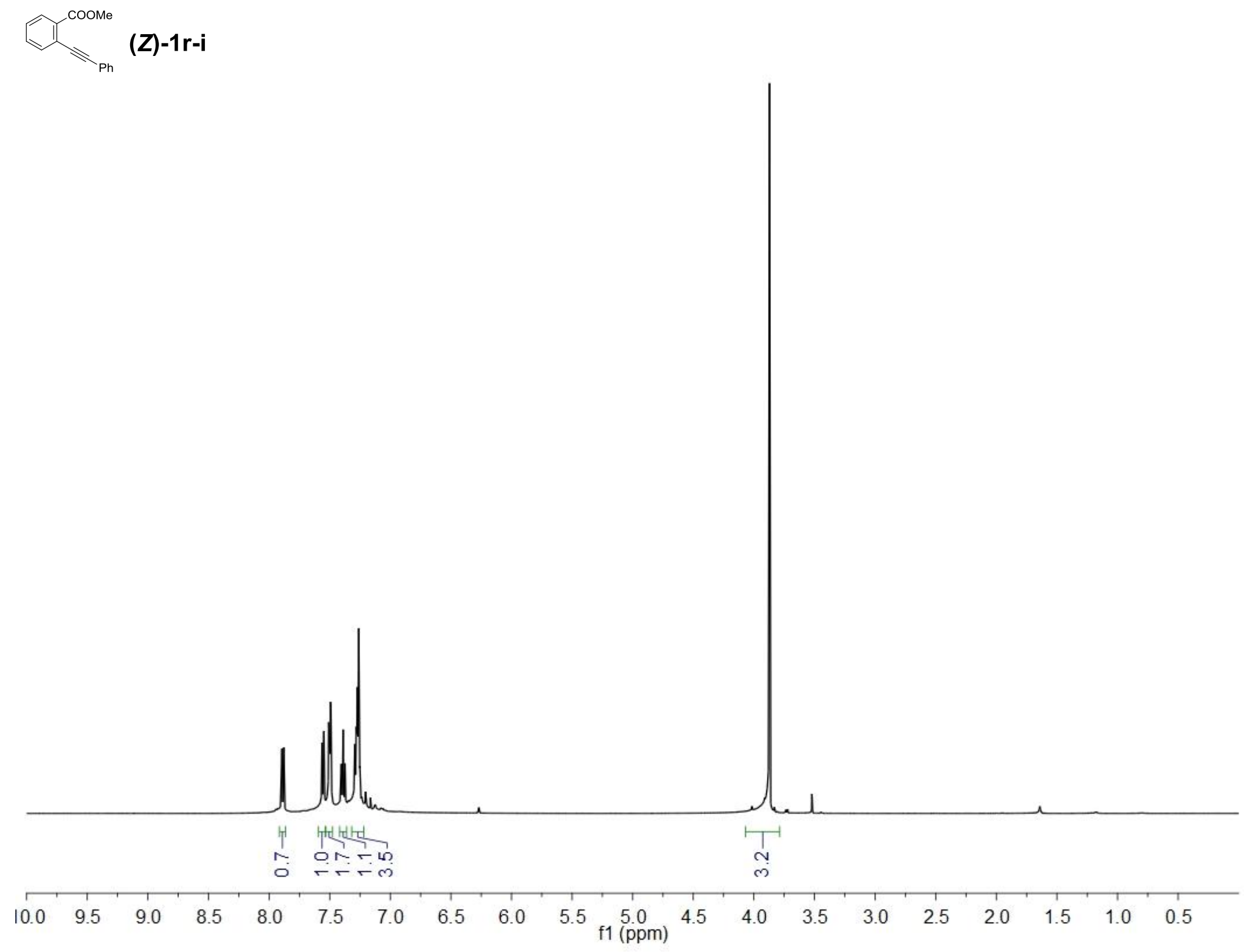




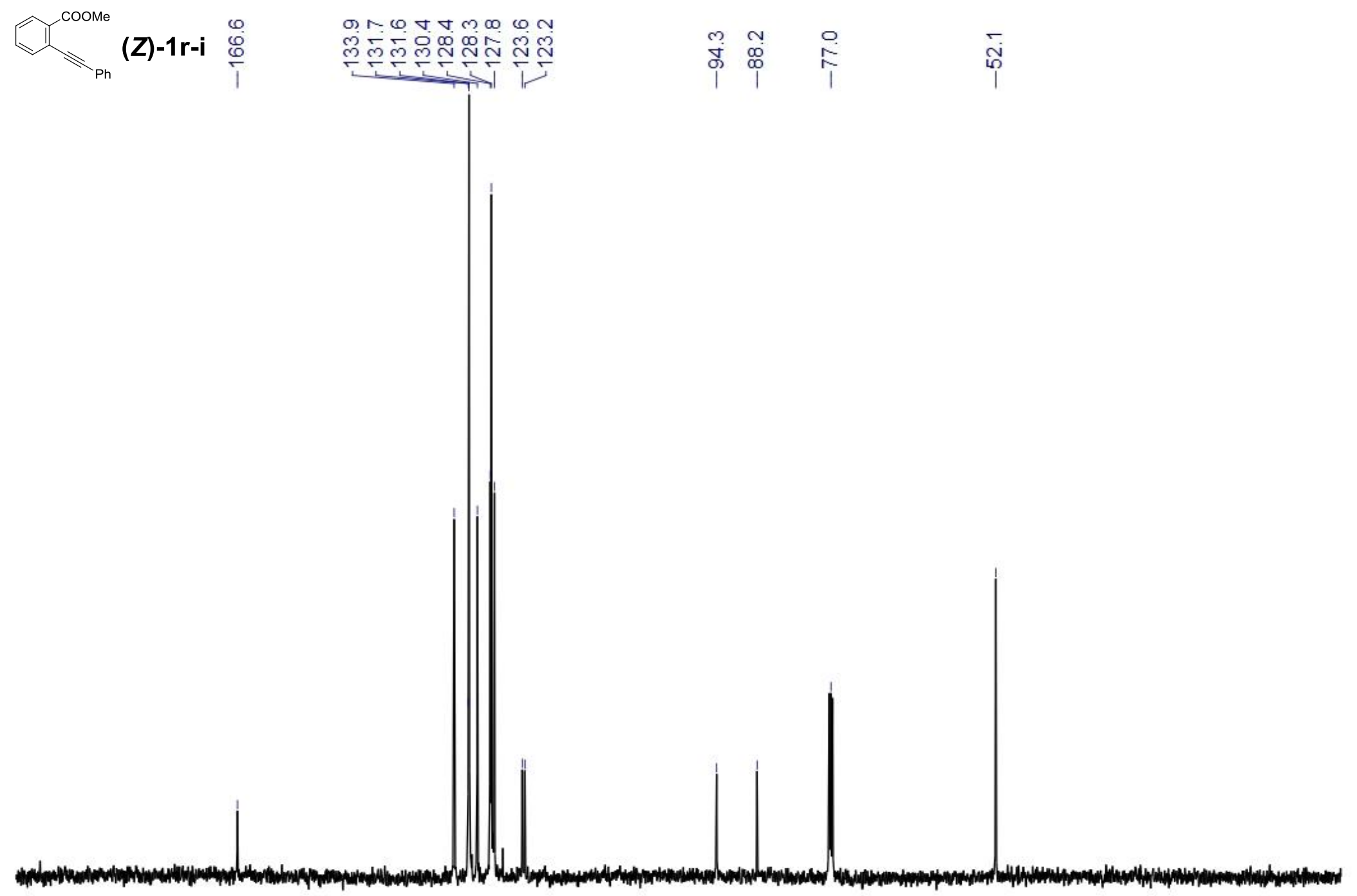

200

120

$110 \quad 100$

80

70

60

50

$40 \quad 30$

20

10 


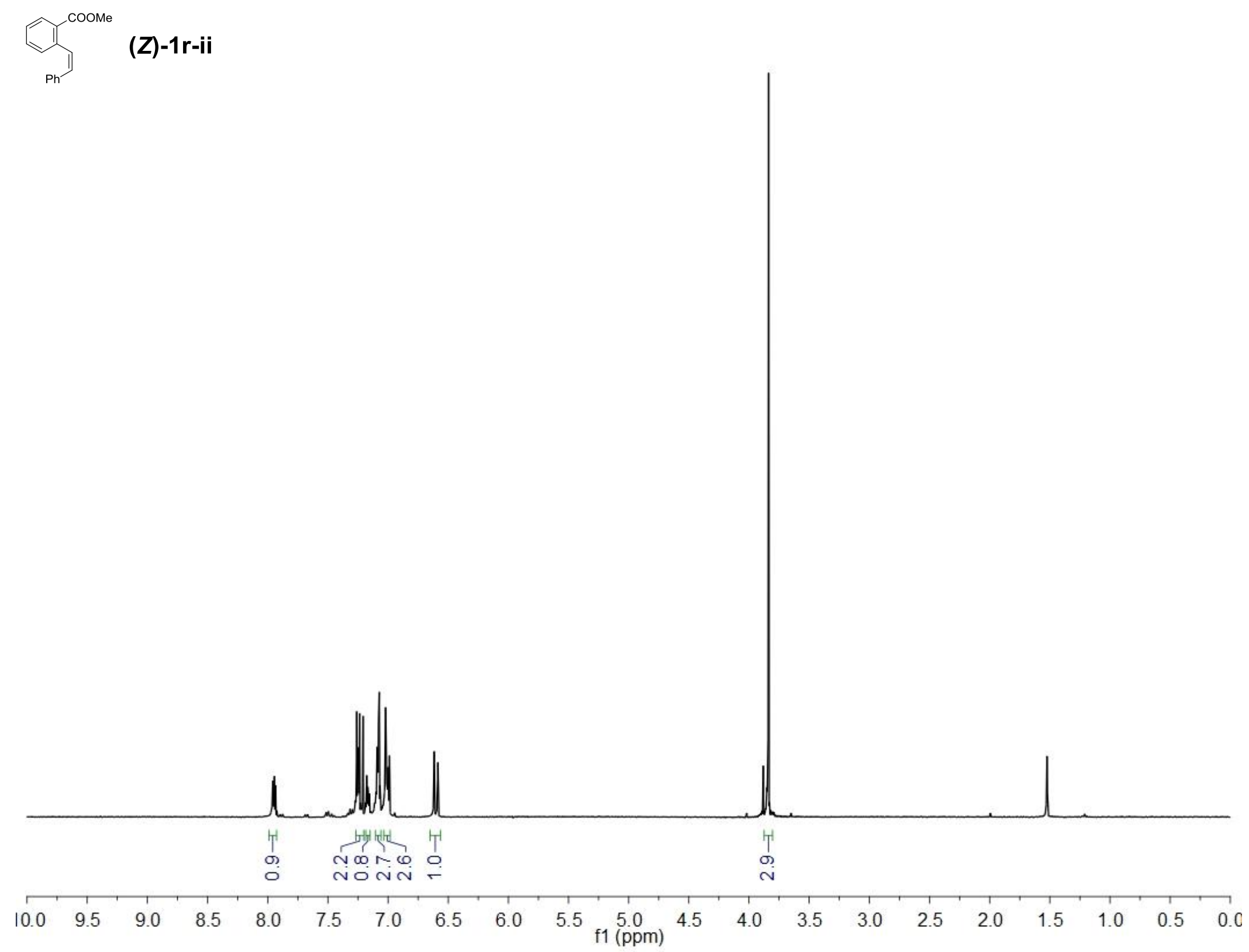




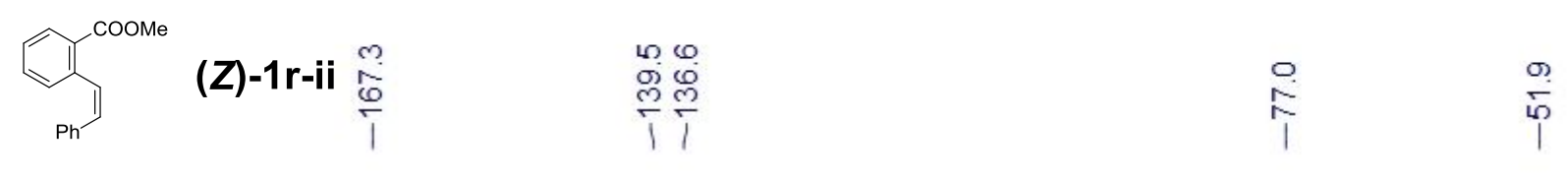

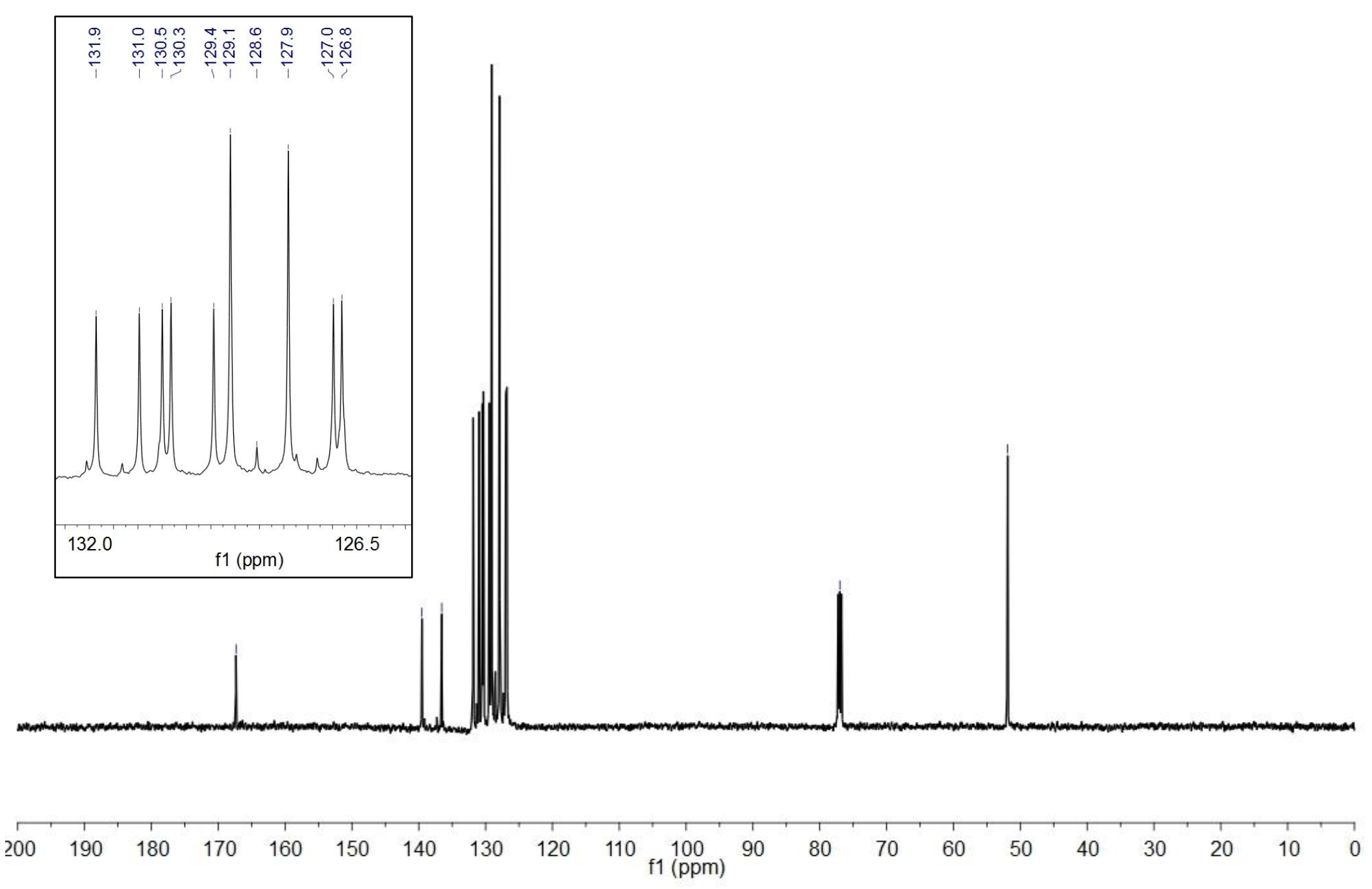




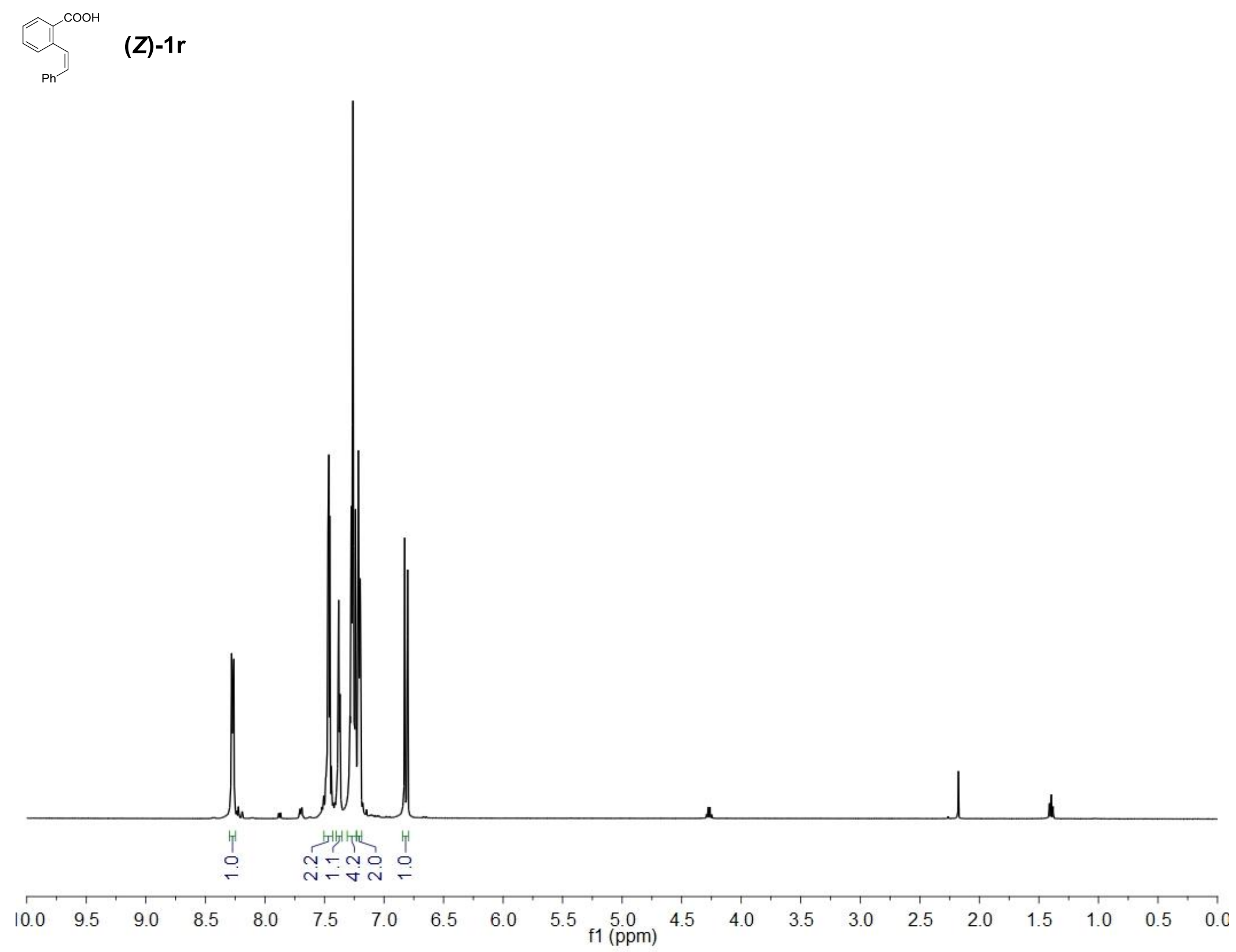




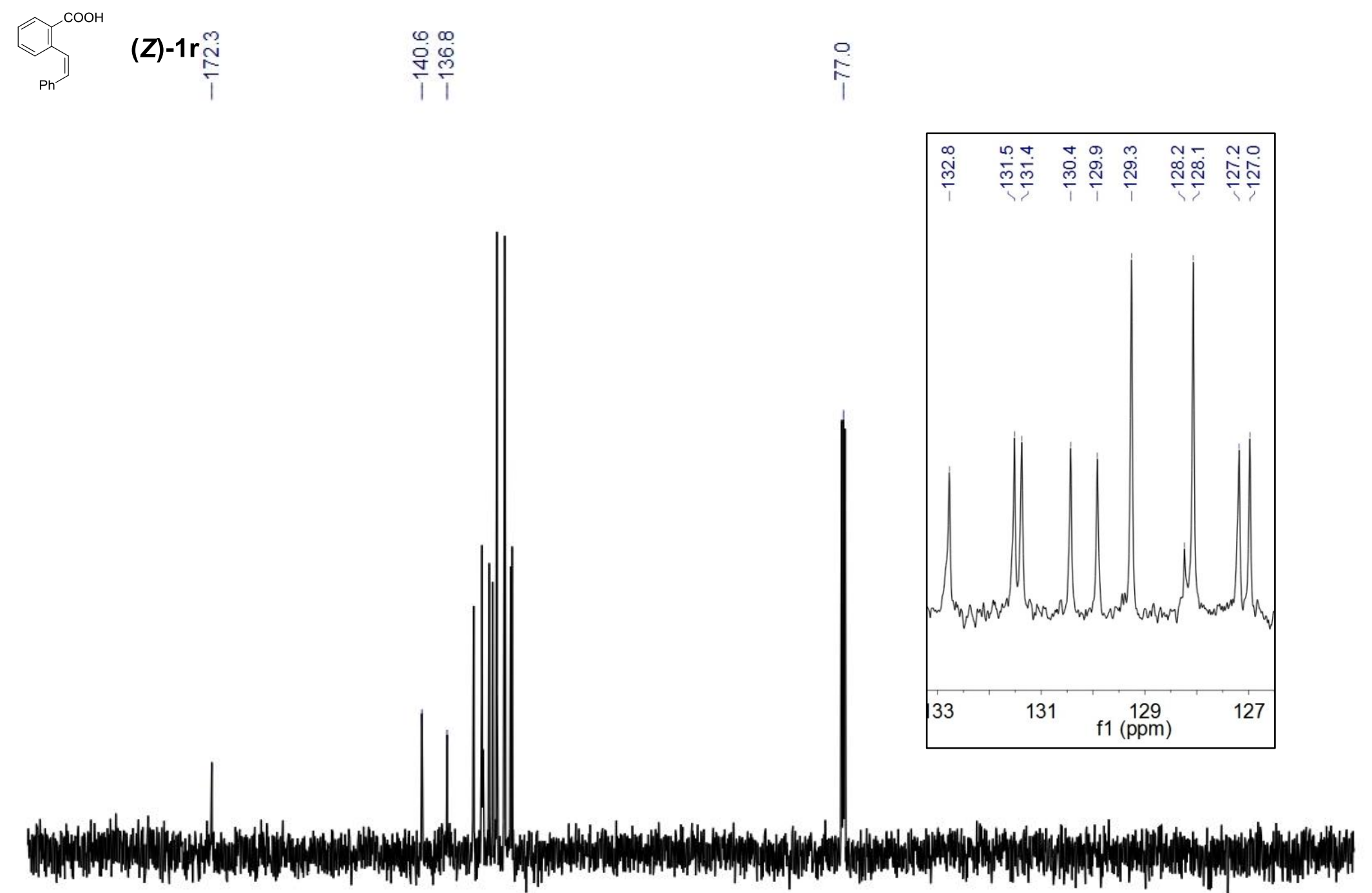




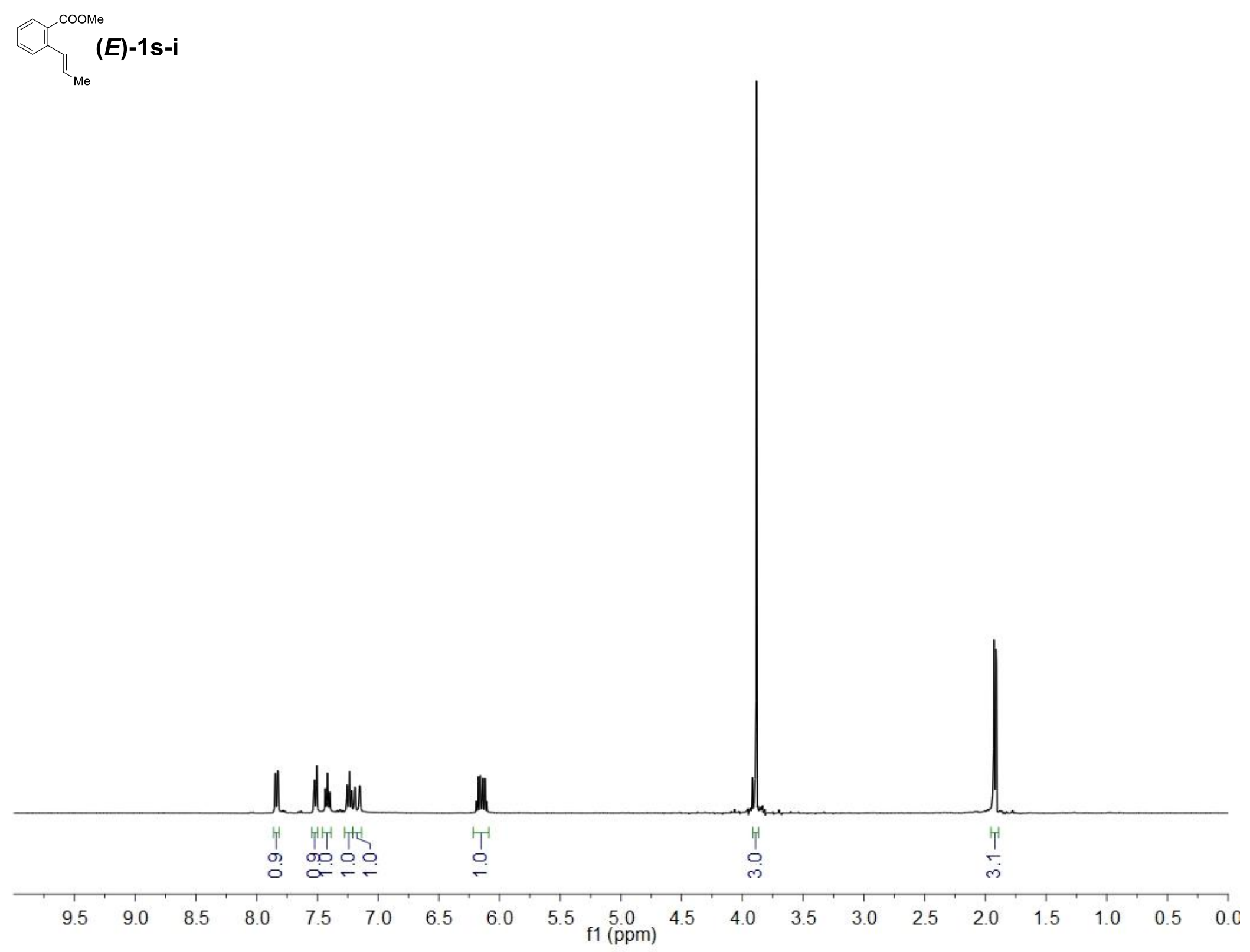




$$
\overbrace{\mathrm{Me}}^{\mathrm{coome}}(E)-1 \mathrm{~s}-\mathrm{i} \stackrel{\frac{m}{6}}{\frac{6}{1}}
$$

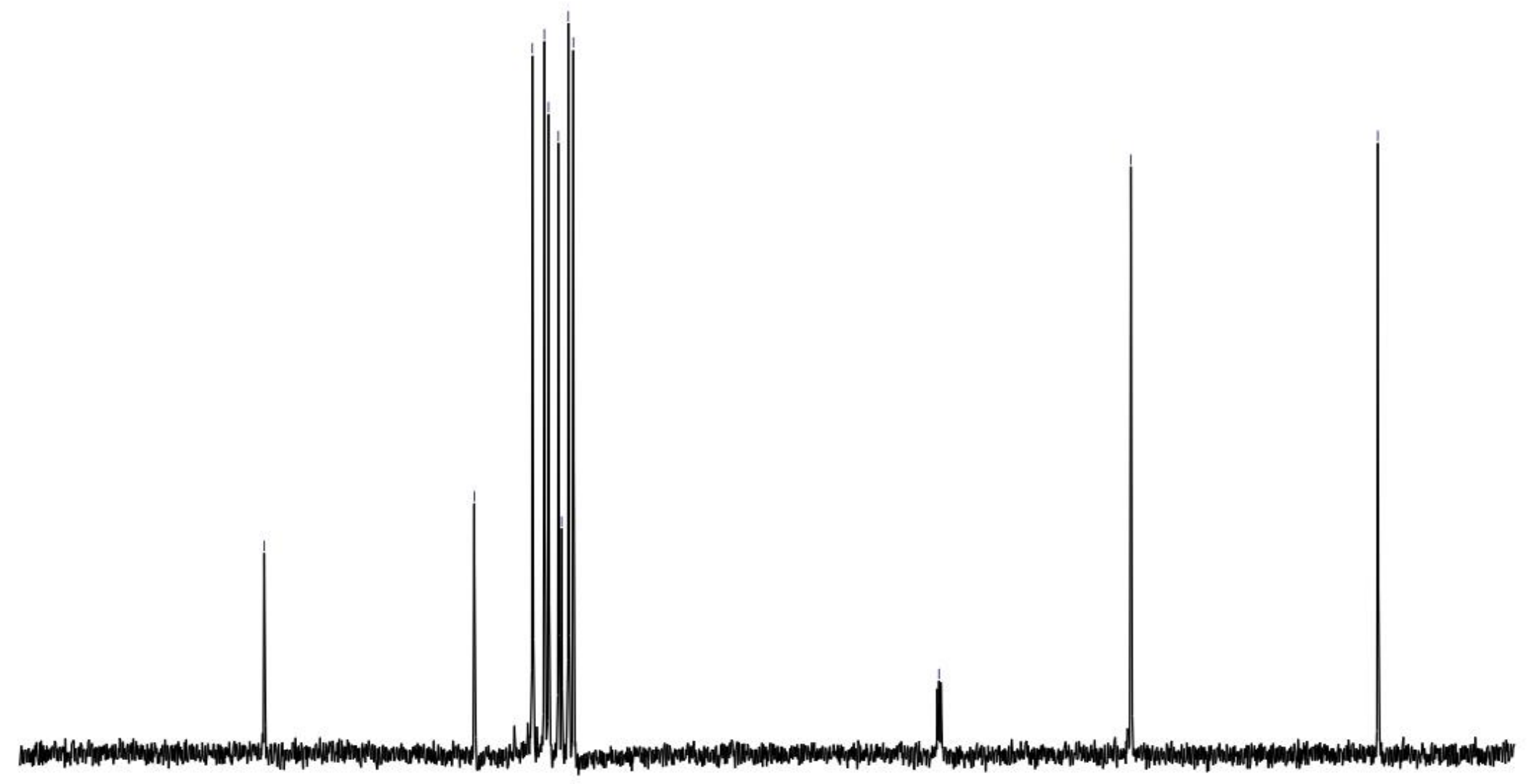



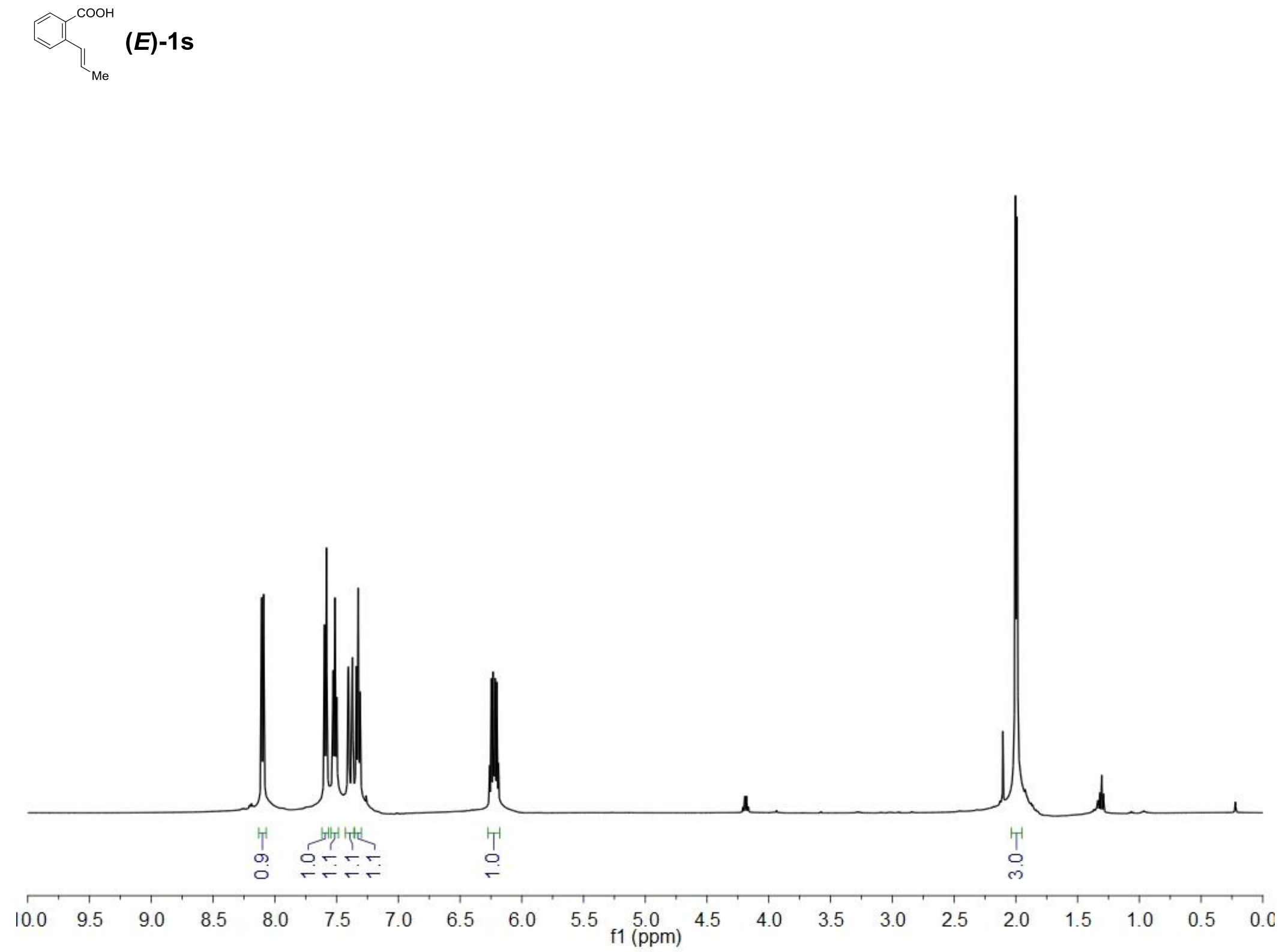

S92 


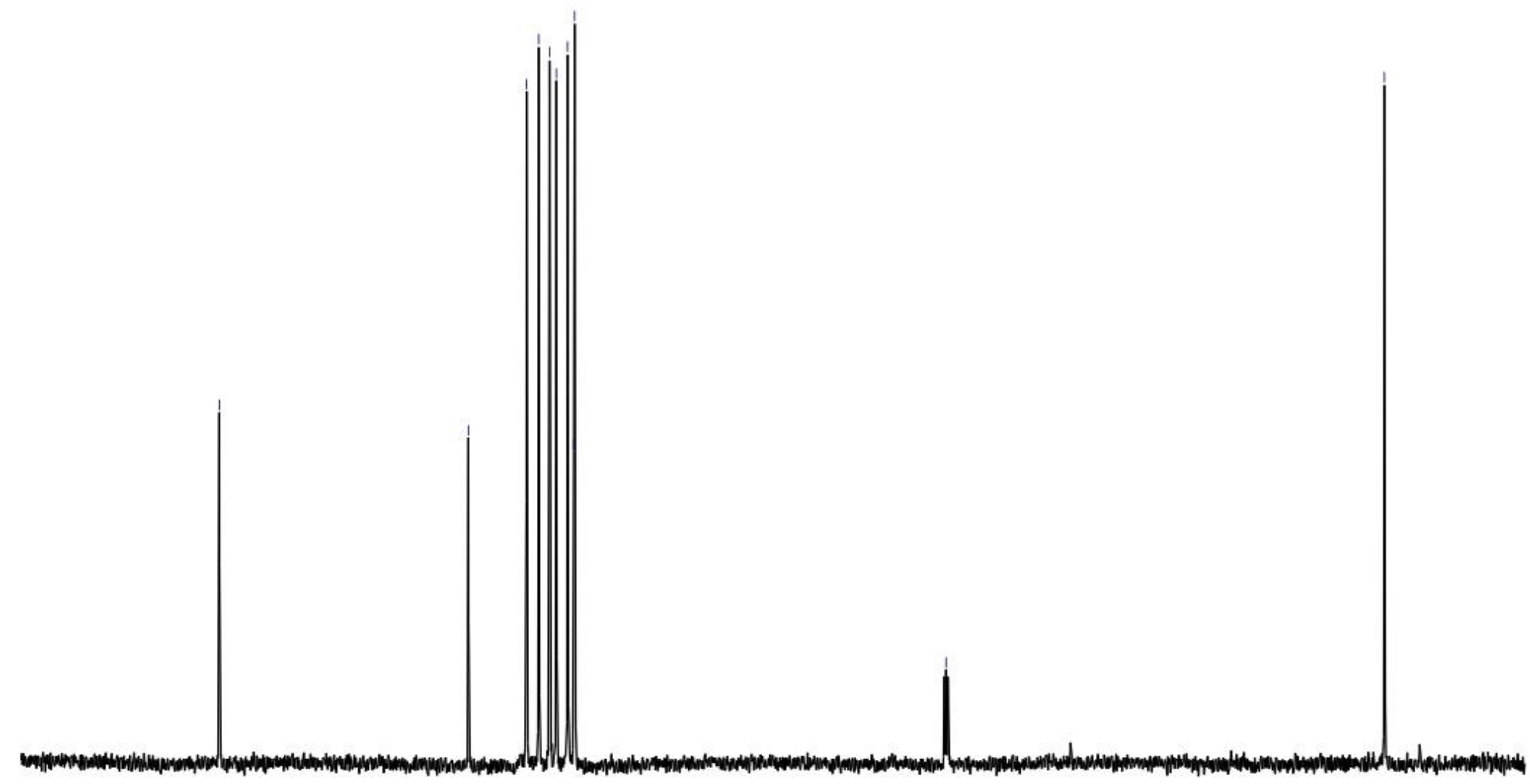




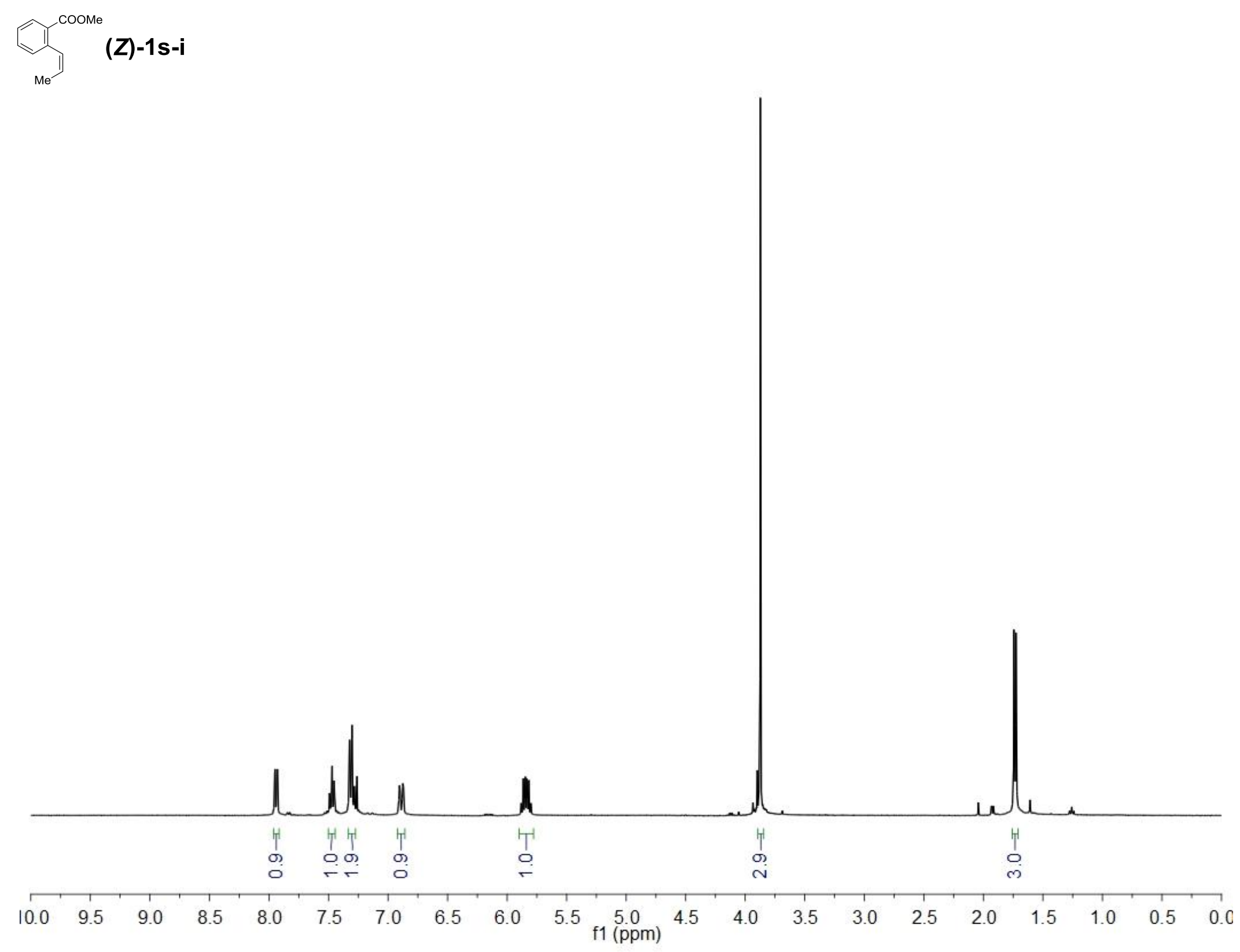




Me (Z)-1s-i

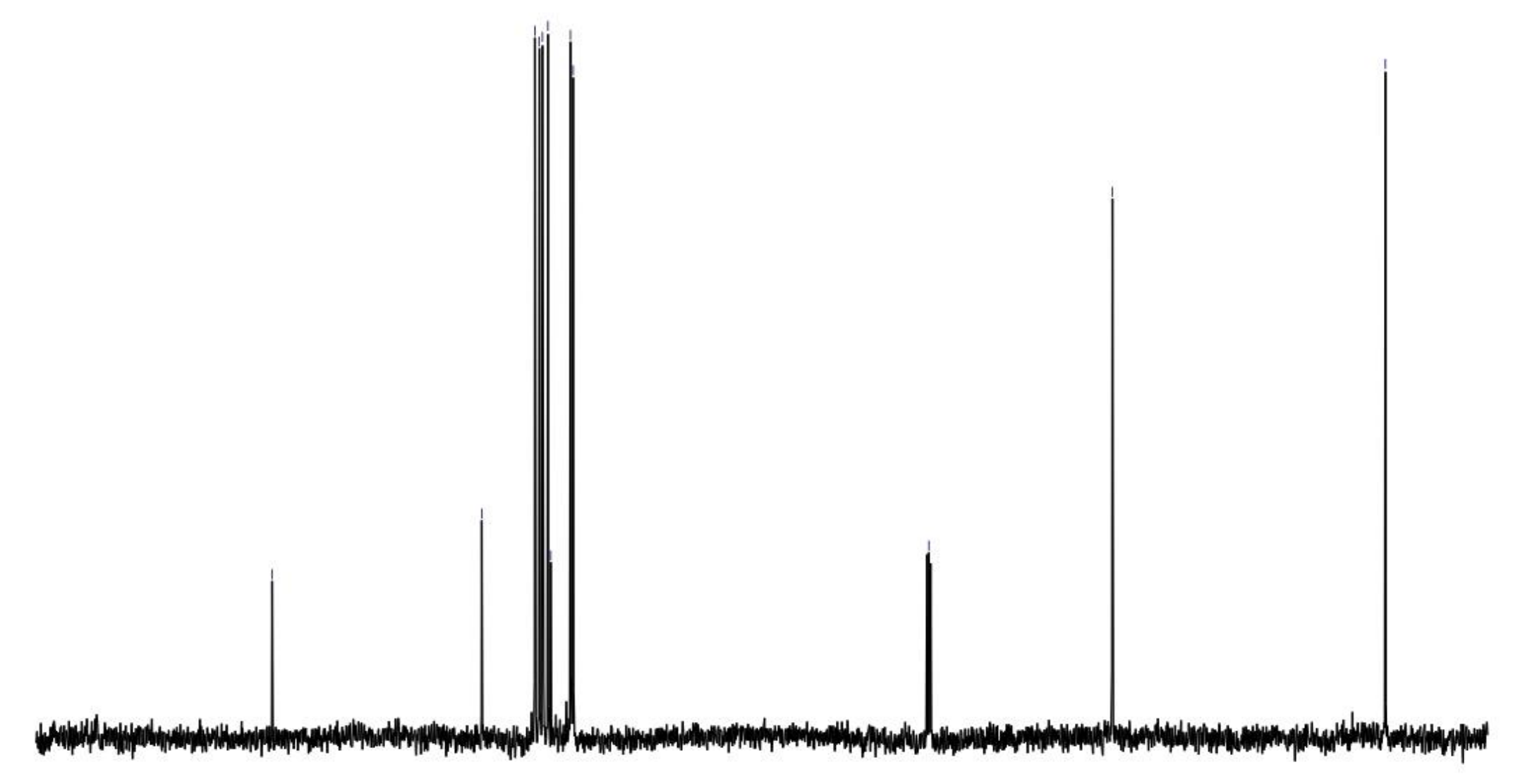

$\begin{array}{llllllllllllllllllllllll}200 & 190 & 180 & 170 & 160 & 150 & 140 & 130 & 120 & 110 & \begin{array}{l}100 \\ \mathrm{f} 1(\mathrm{ppm})\end{array} & 90 & 80 & 70 & 60 & 50 & 40 & 30 & 20 & 10 & 0\end{array}$ 


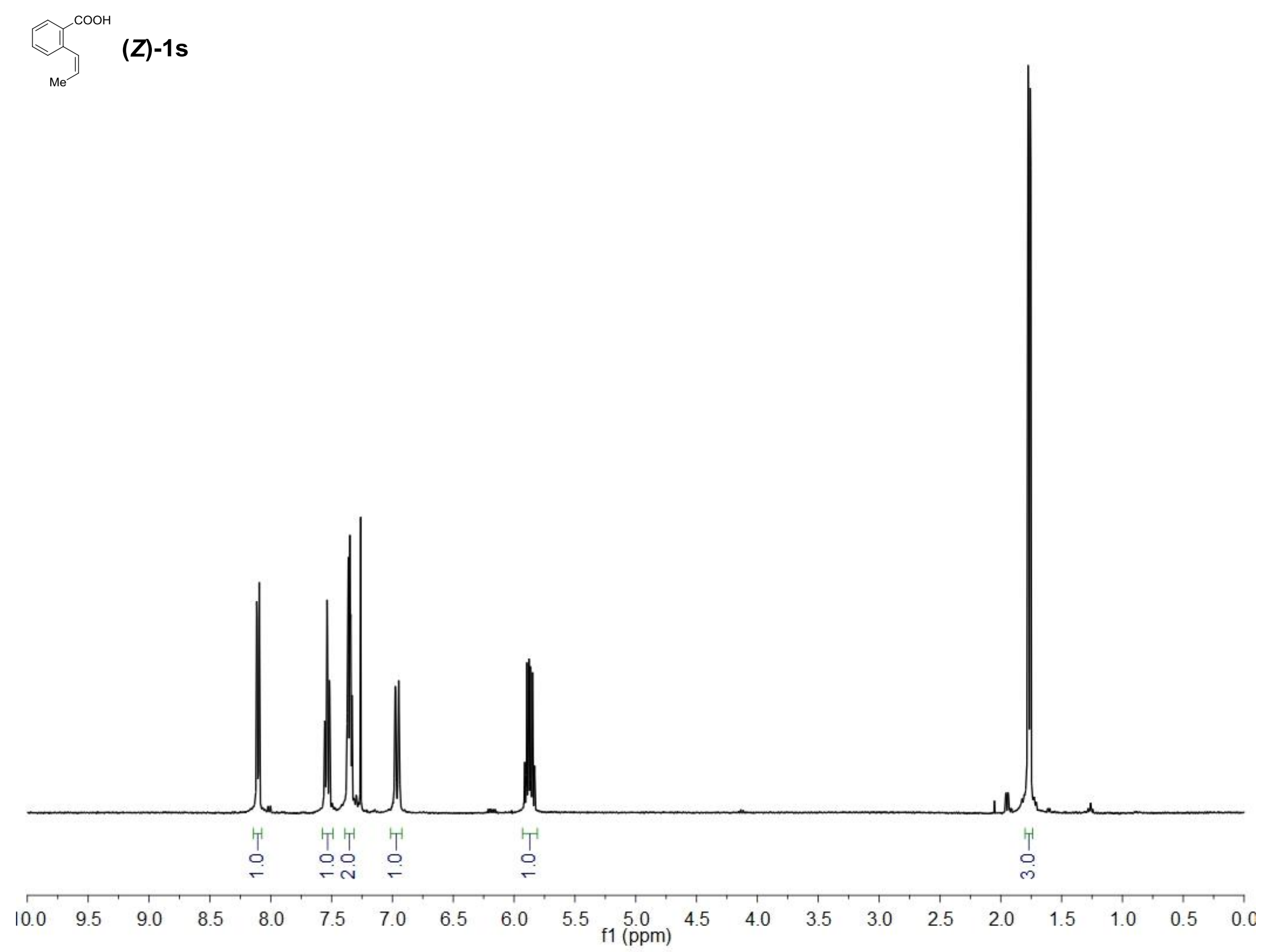




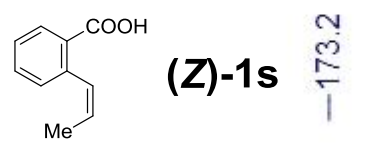

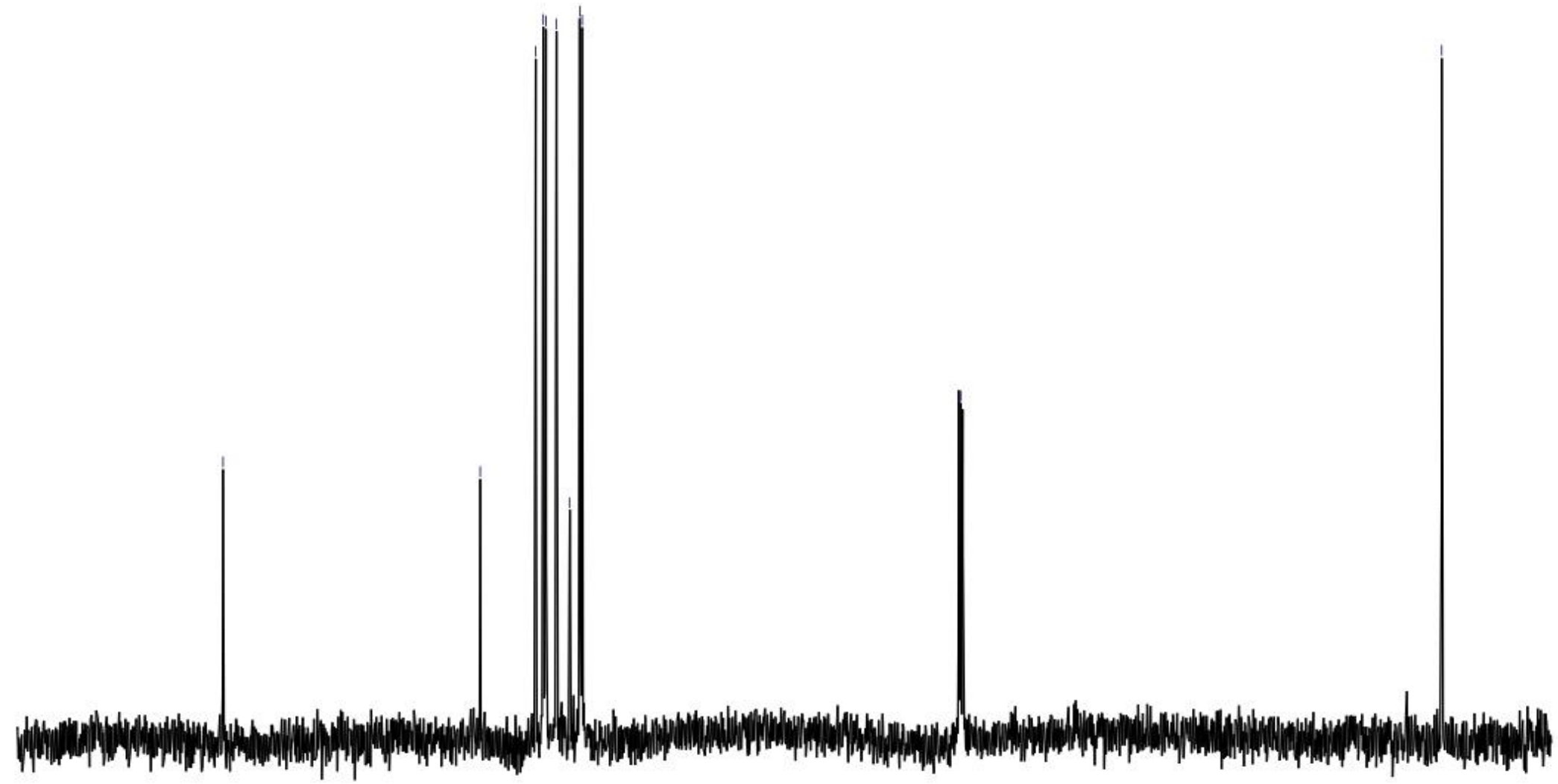




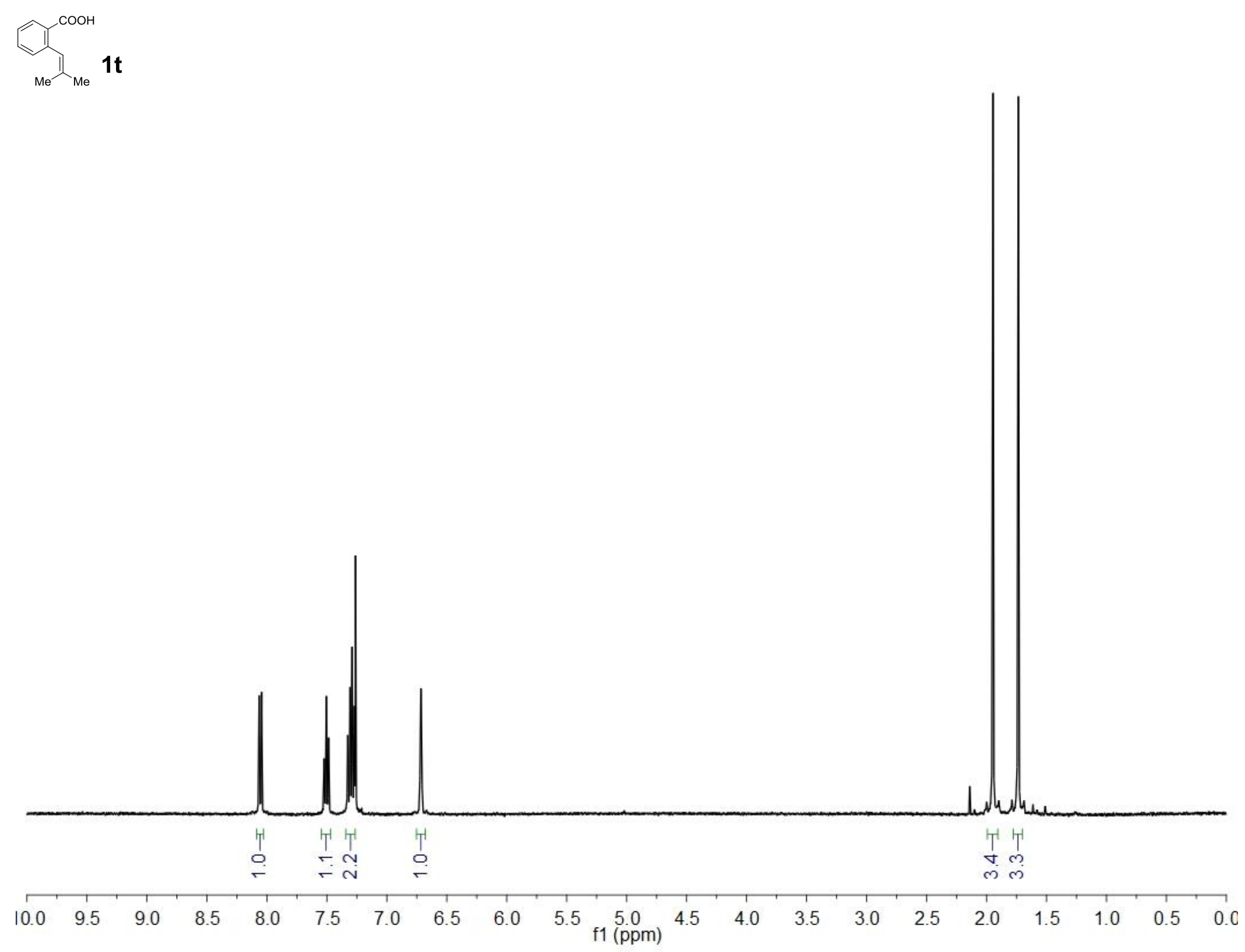


Me 11 i
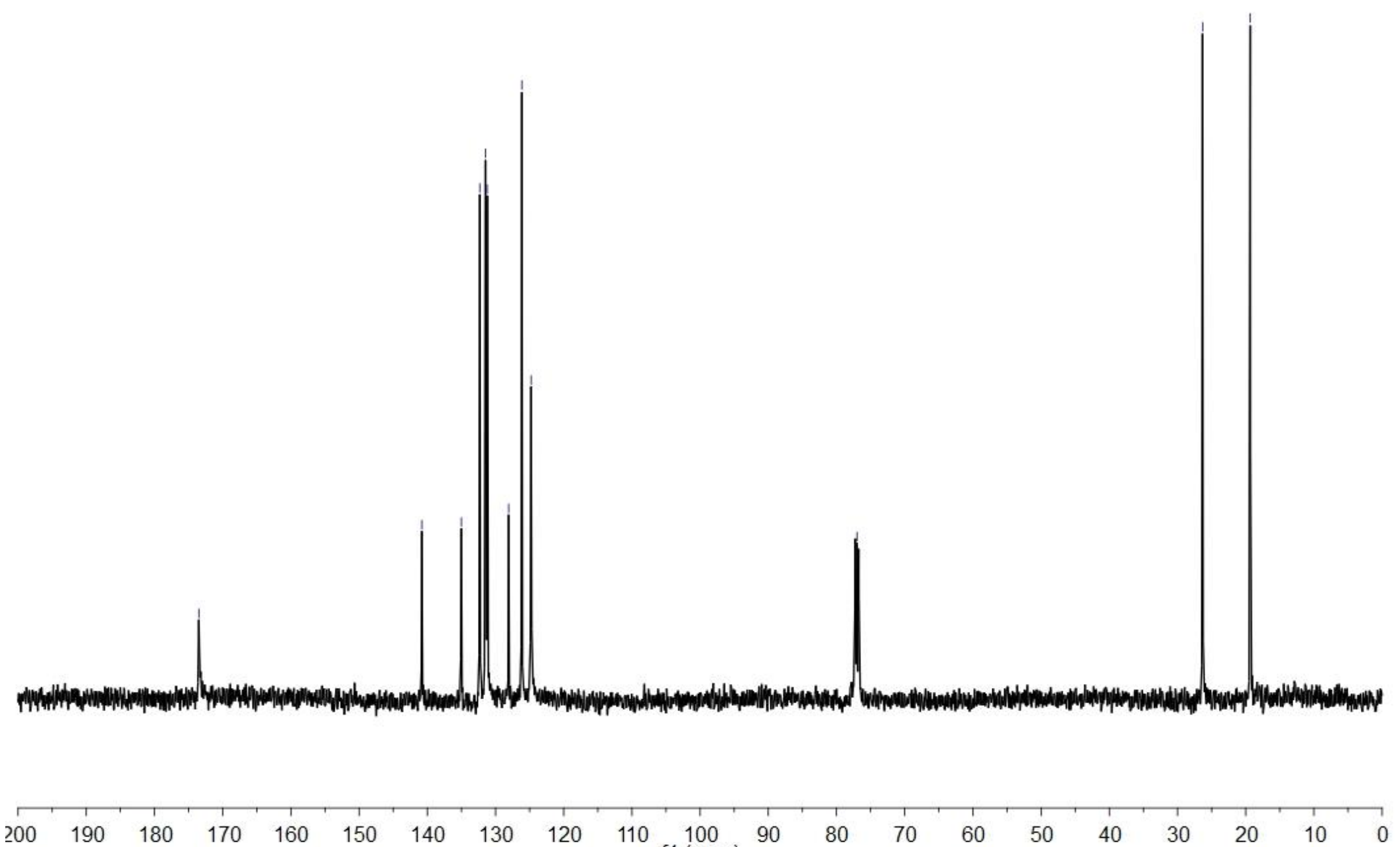

$110 \quad \begin{aligned} & 100 \\ & \end{aligned}$

80

60

50

$40 \quad 30$

20

10 


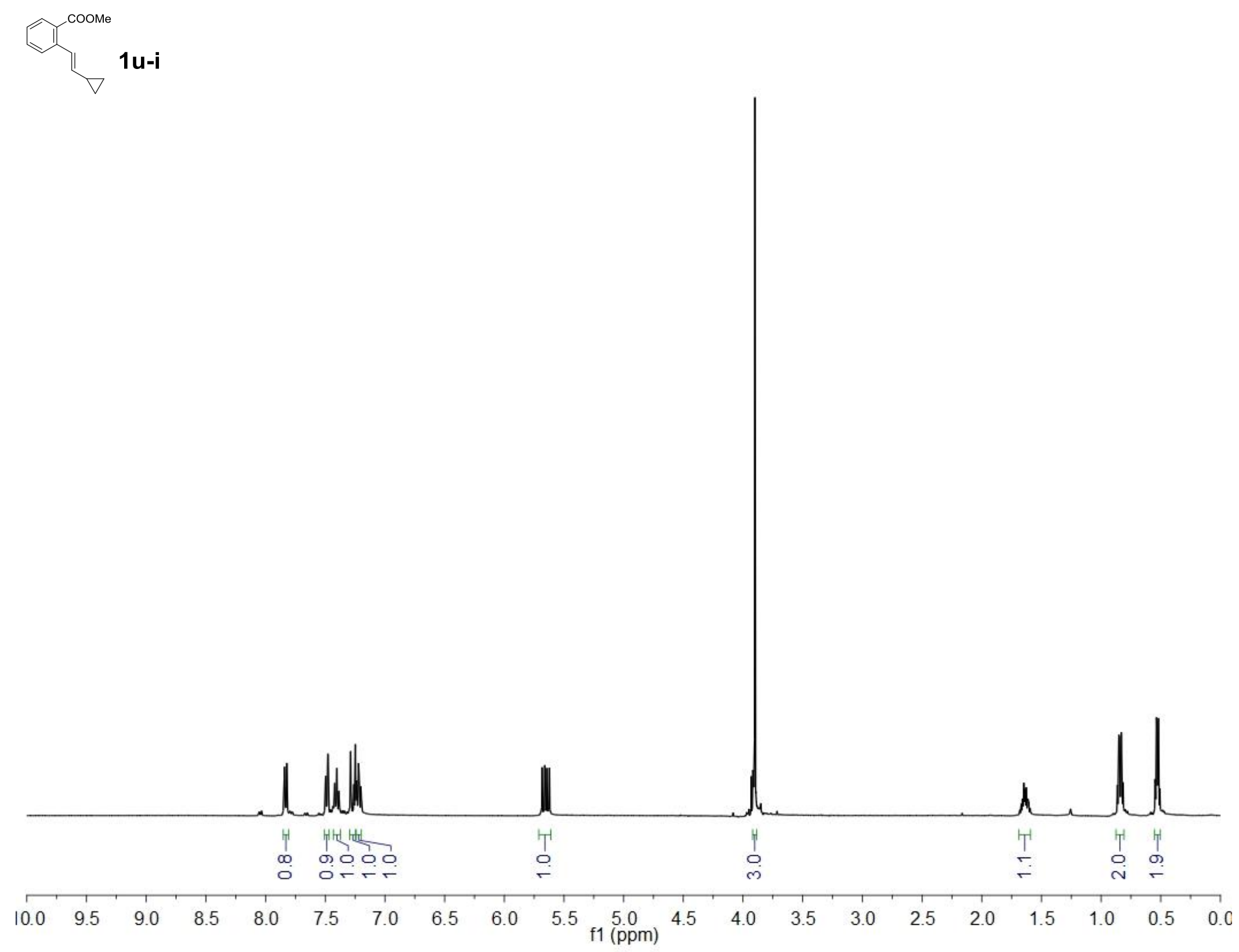



$\overbrace{\nabla}^{\text {coome }} \mathbf{u} i \stackrel{\infty}{\frac{\sigma}{i}}$
ल)
$\stackrel{0}{1}$
$\stackrel{\infty}{i \infty}$
$\stackrel{i}{i}$

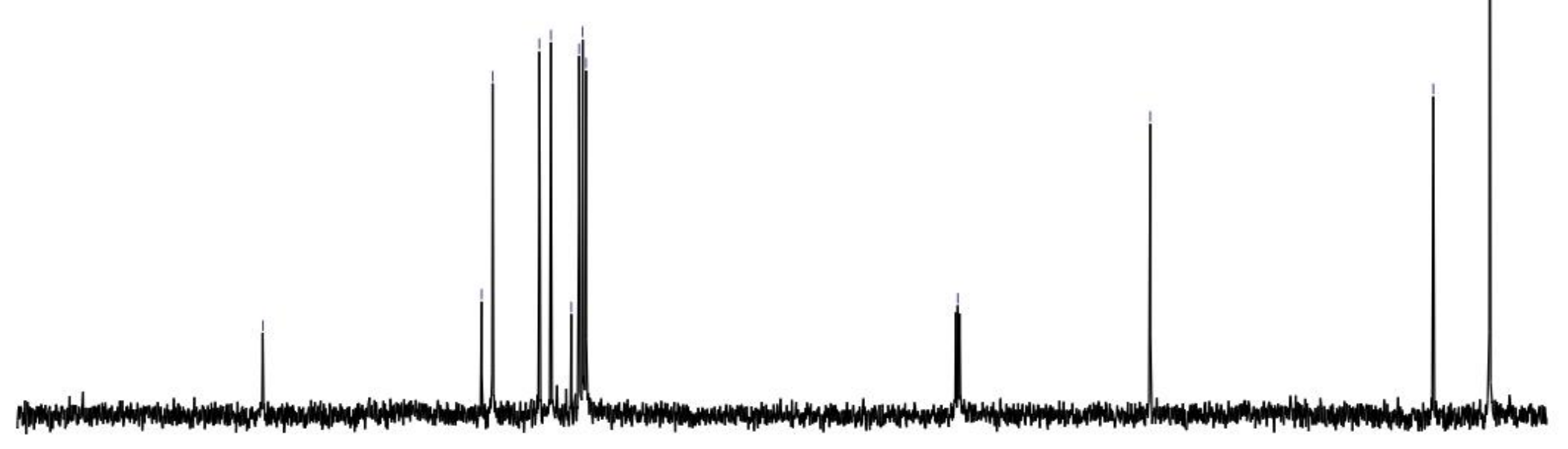

200

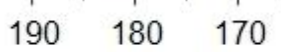

160

$150 \quad 140$

130

120

$110 \quad 100$

80

$70 \quad 60$

50

$40 \quad 30$

20

$10 \quad 0$ 


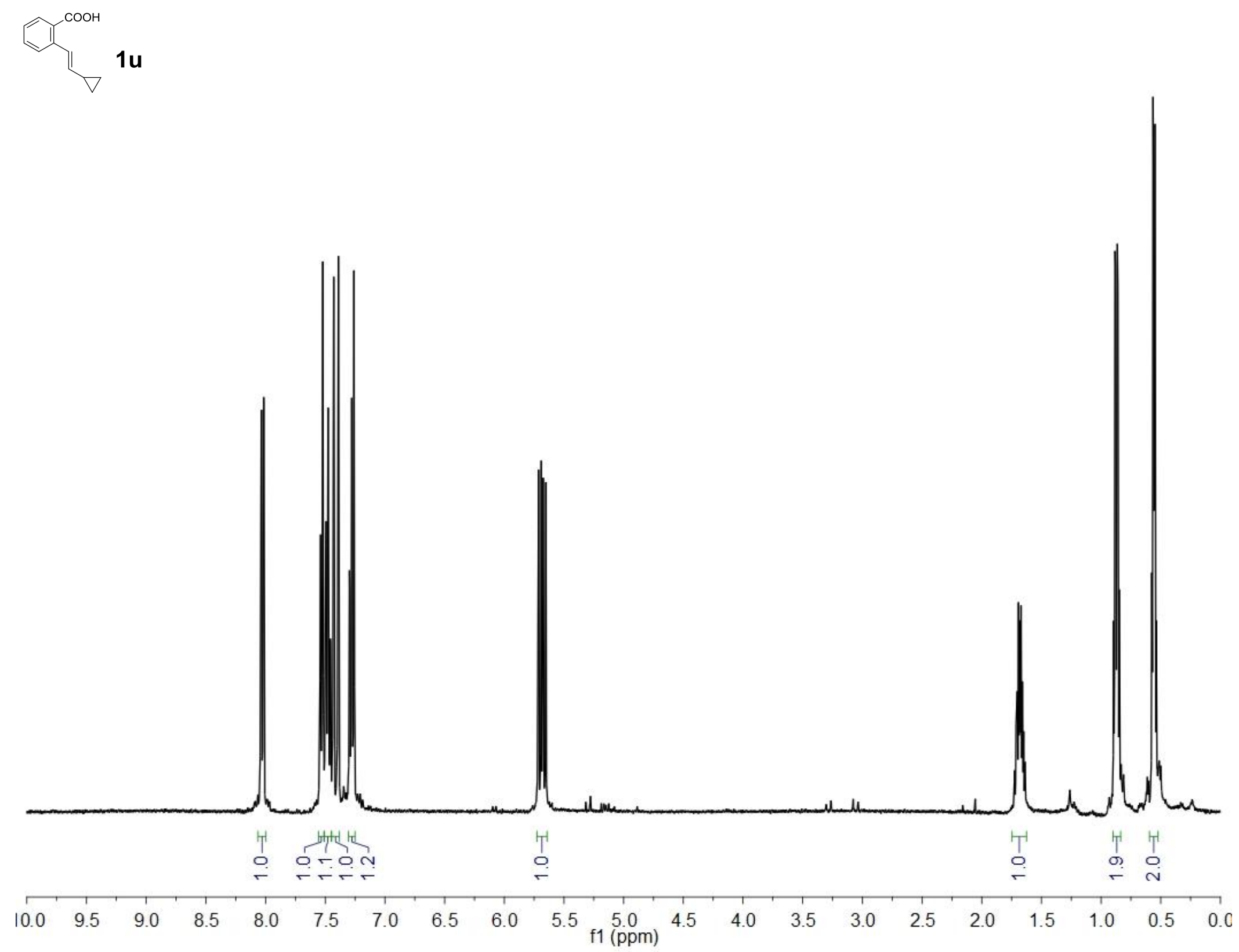



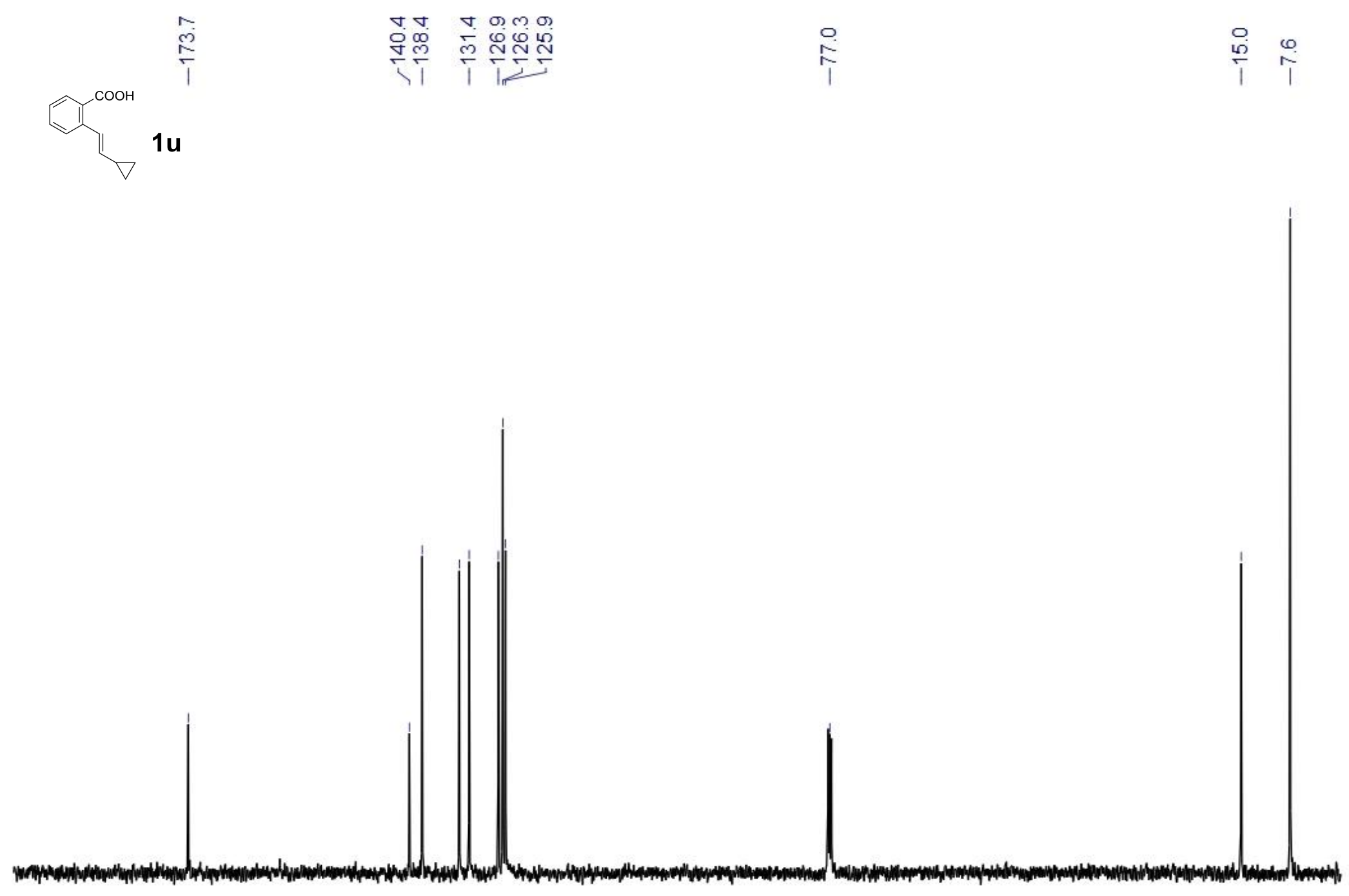

200

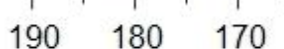




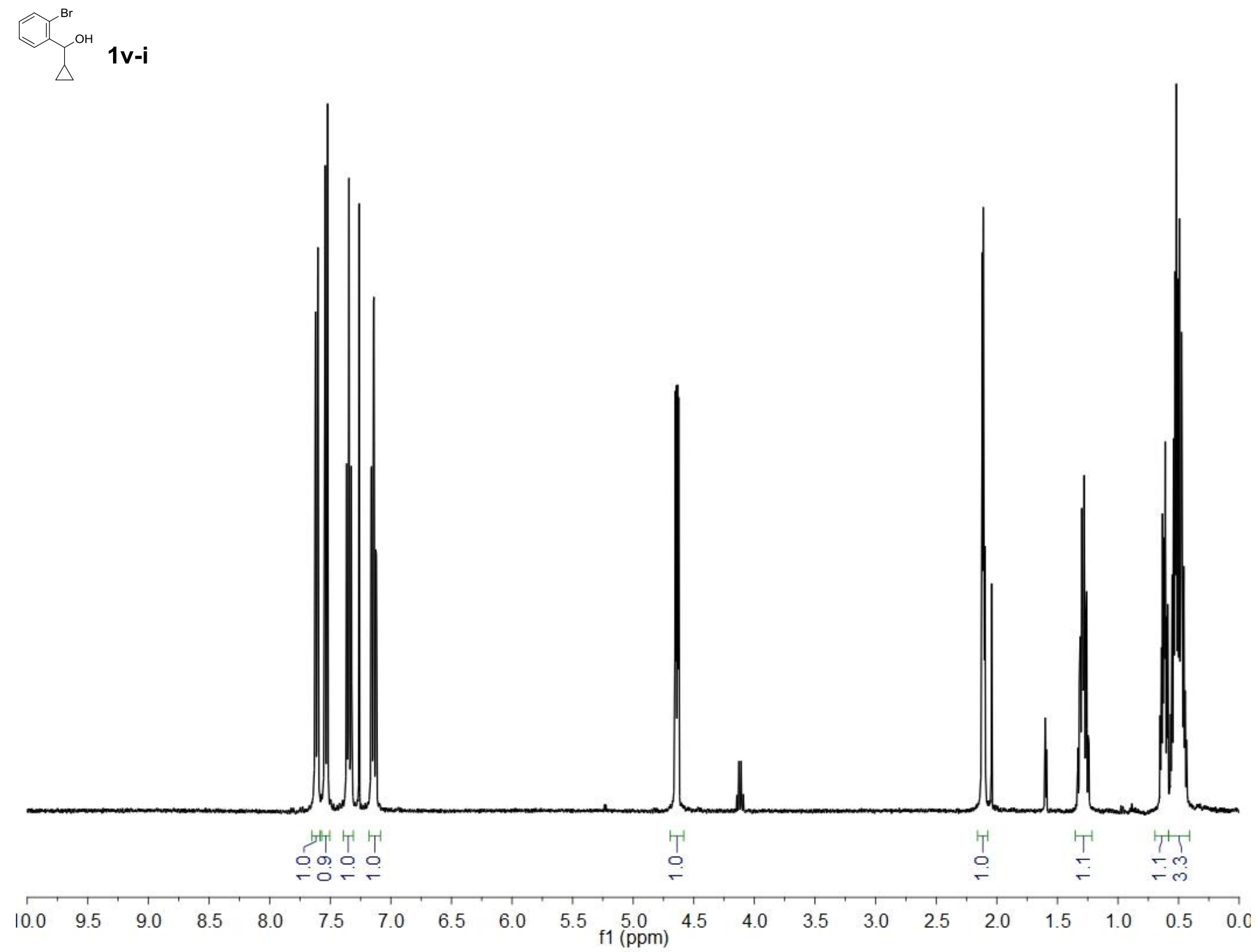




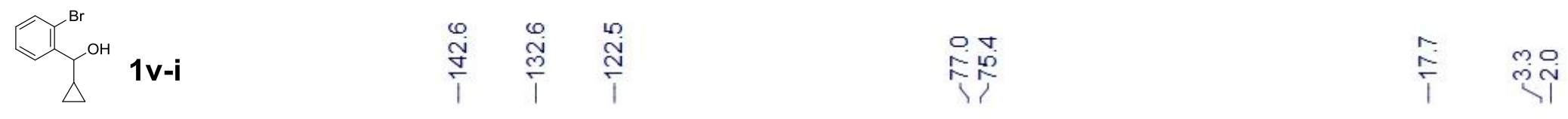

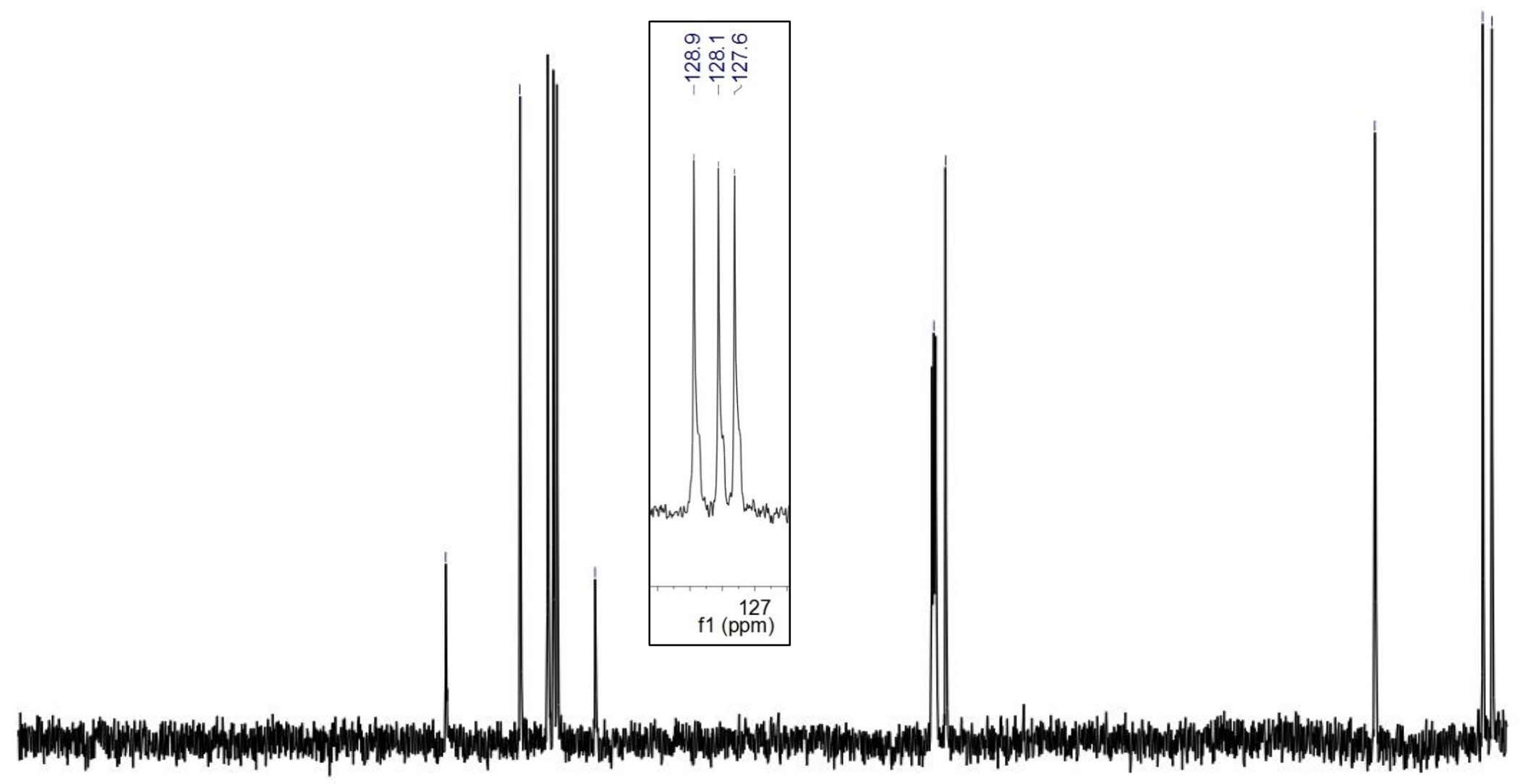




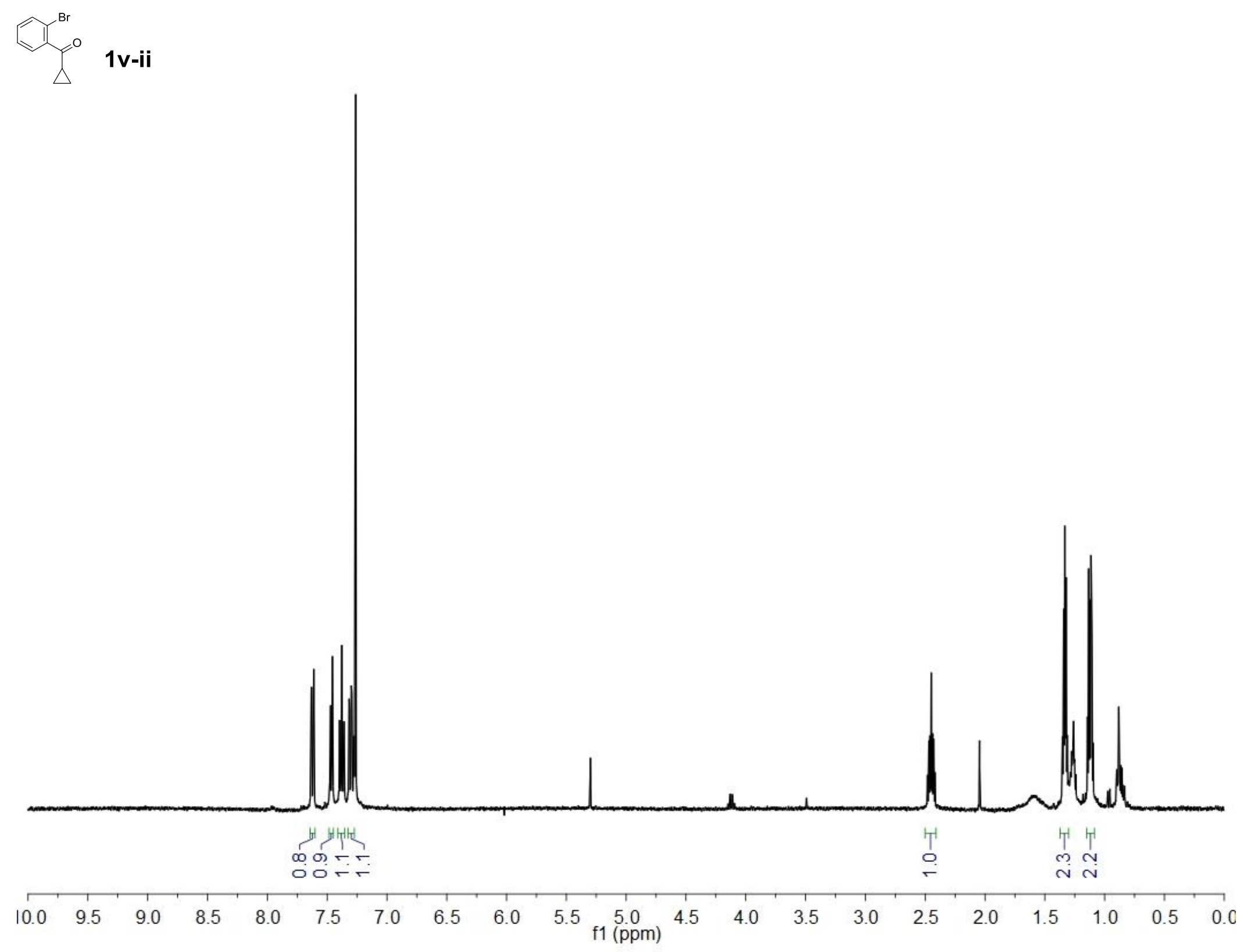


$\stackrel{m}{\stackrel{m}{*}}$

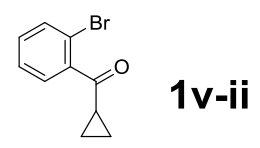

ঙำ

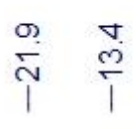
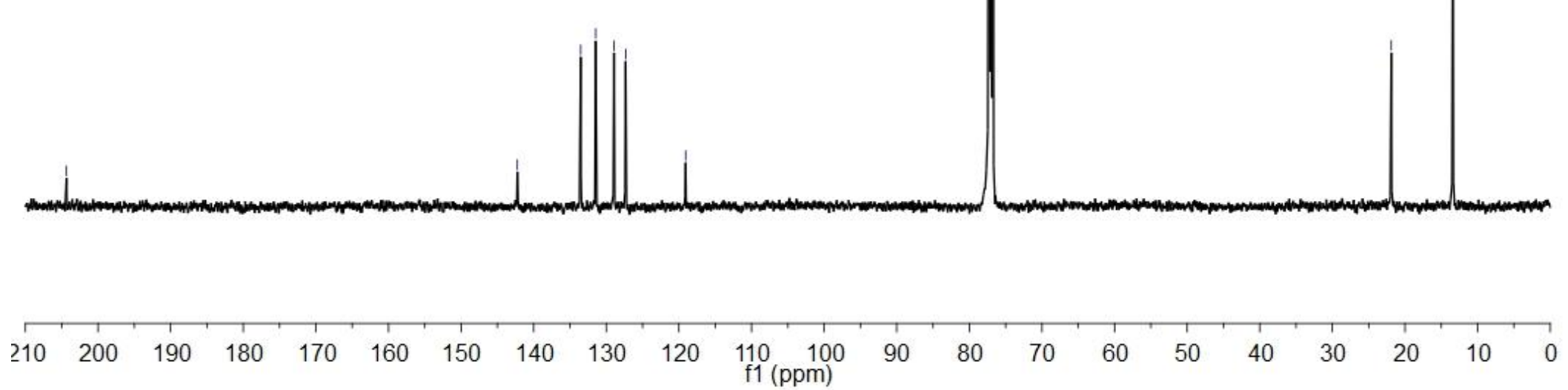

S107 


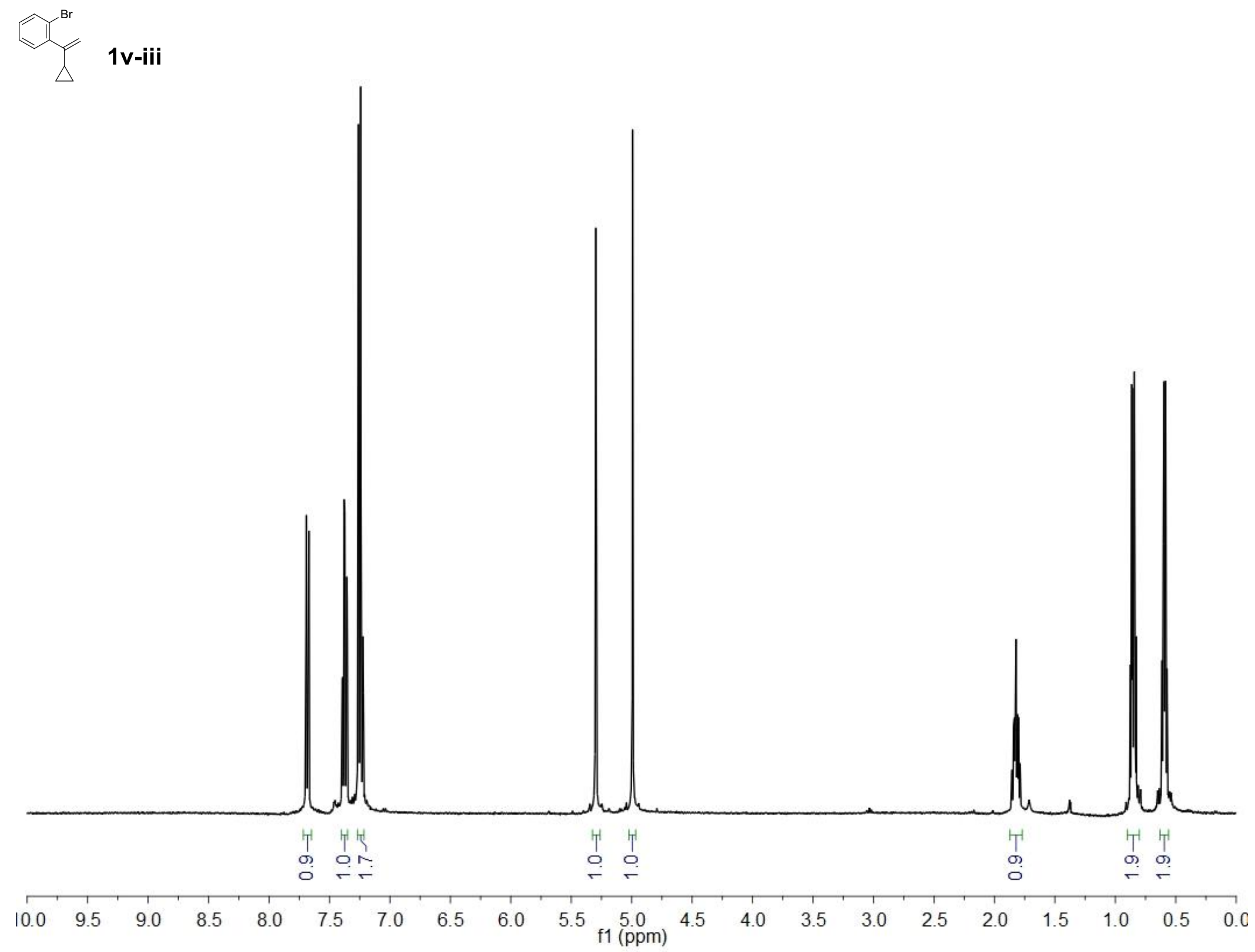


$\overbrace{1 \mathbf{v}-\mathrm{iii}}^{\mathrm{Br}}$

i

$\stackrel{0}{i}$

$\stackrel{0}{\infty} \underset{1}{0}$

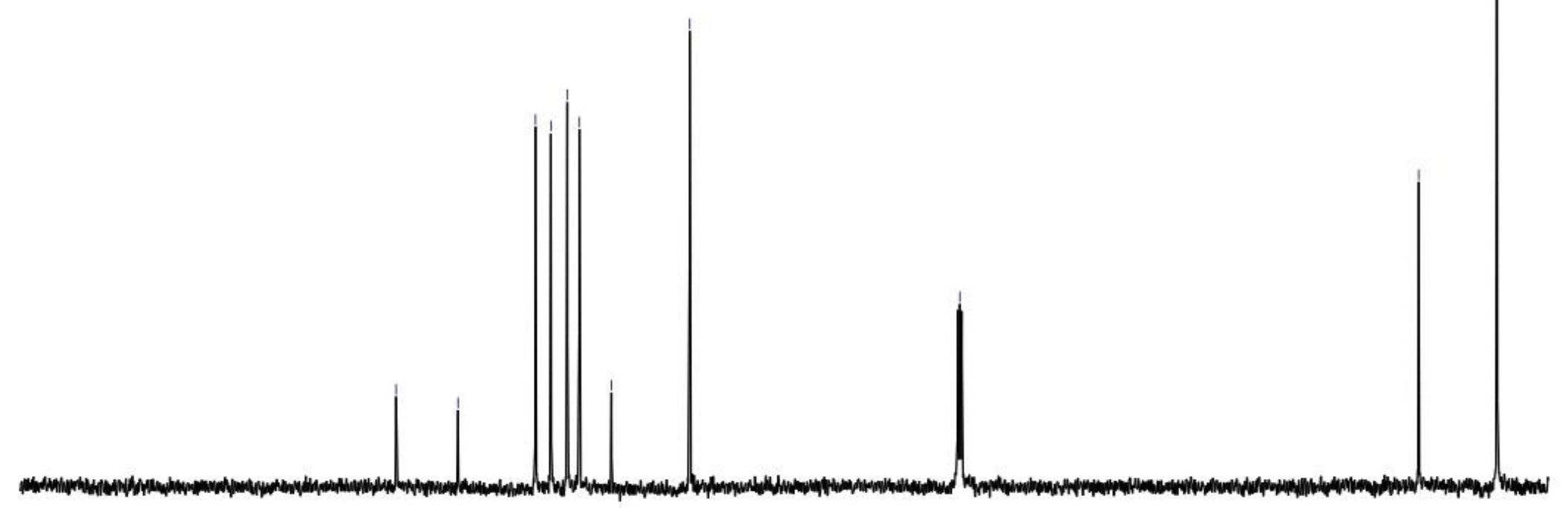

200

190

$180 \quad 170$ 


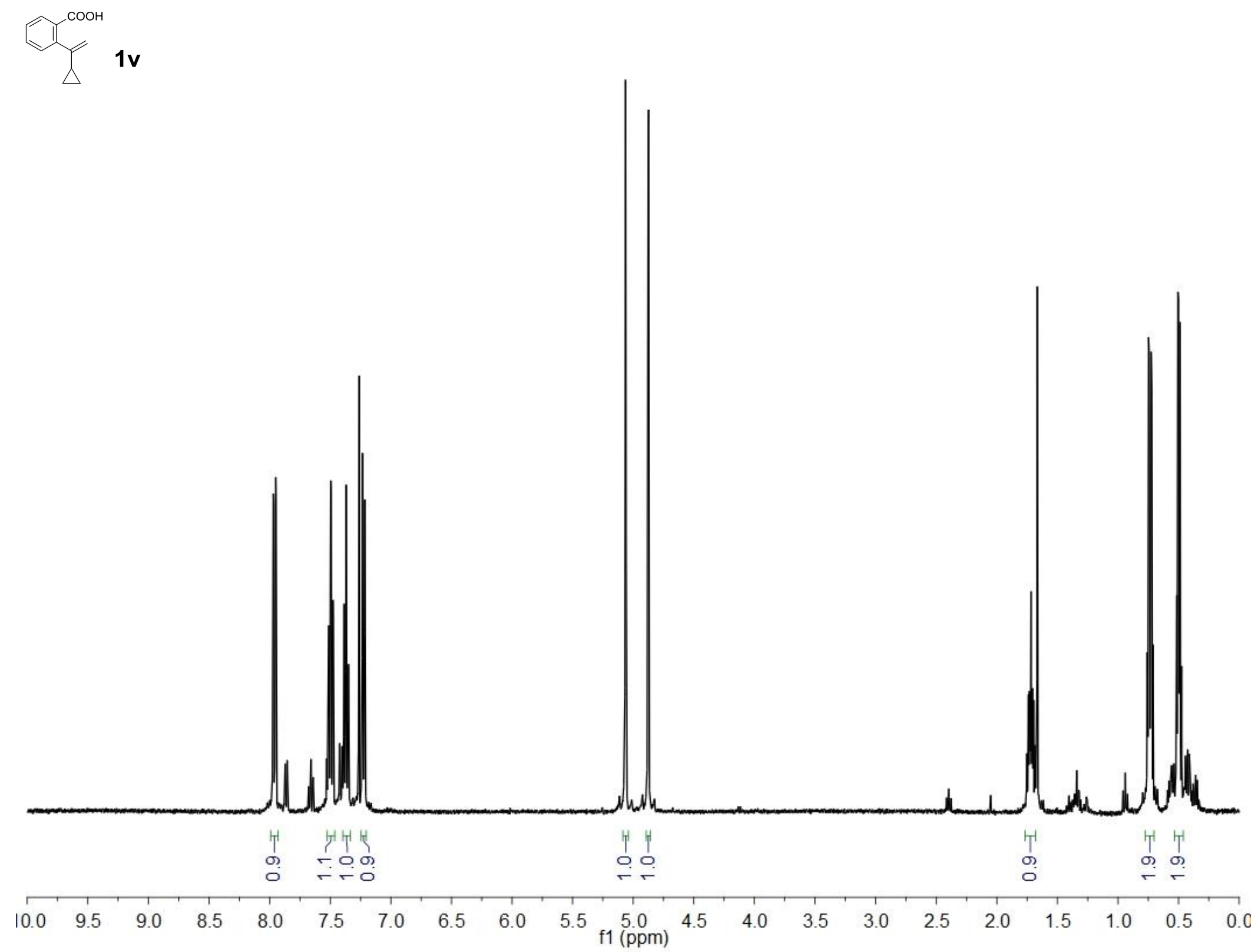




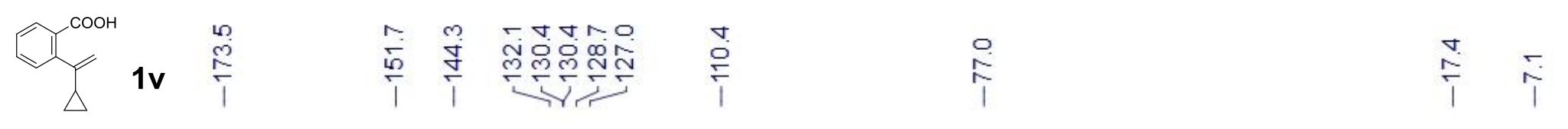

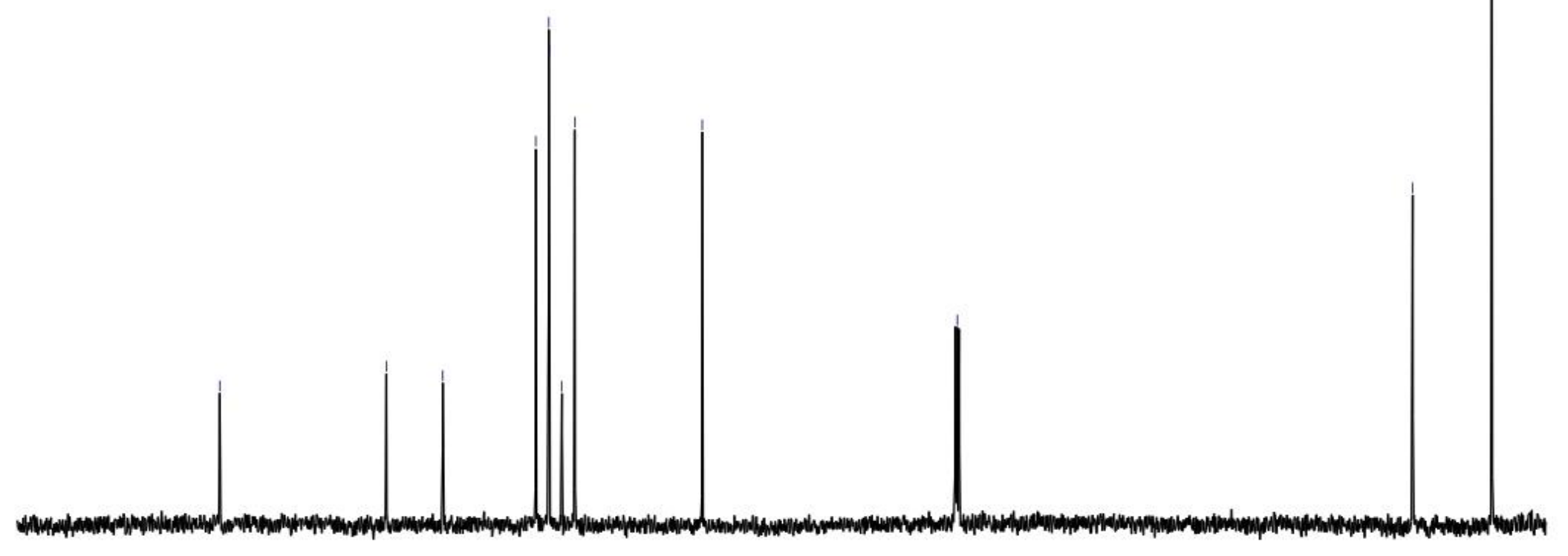

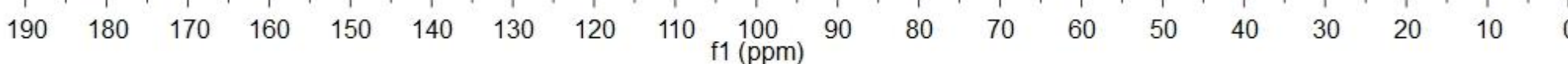




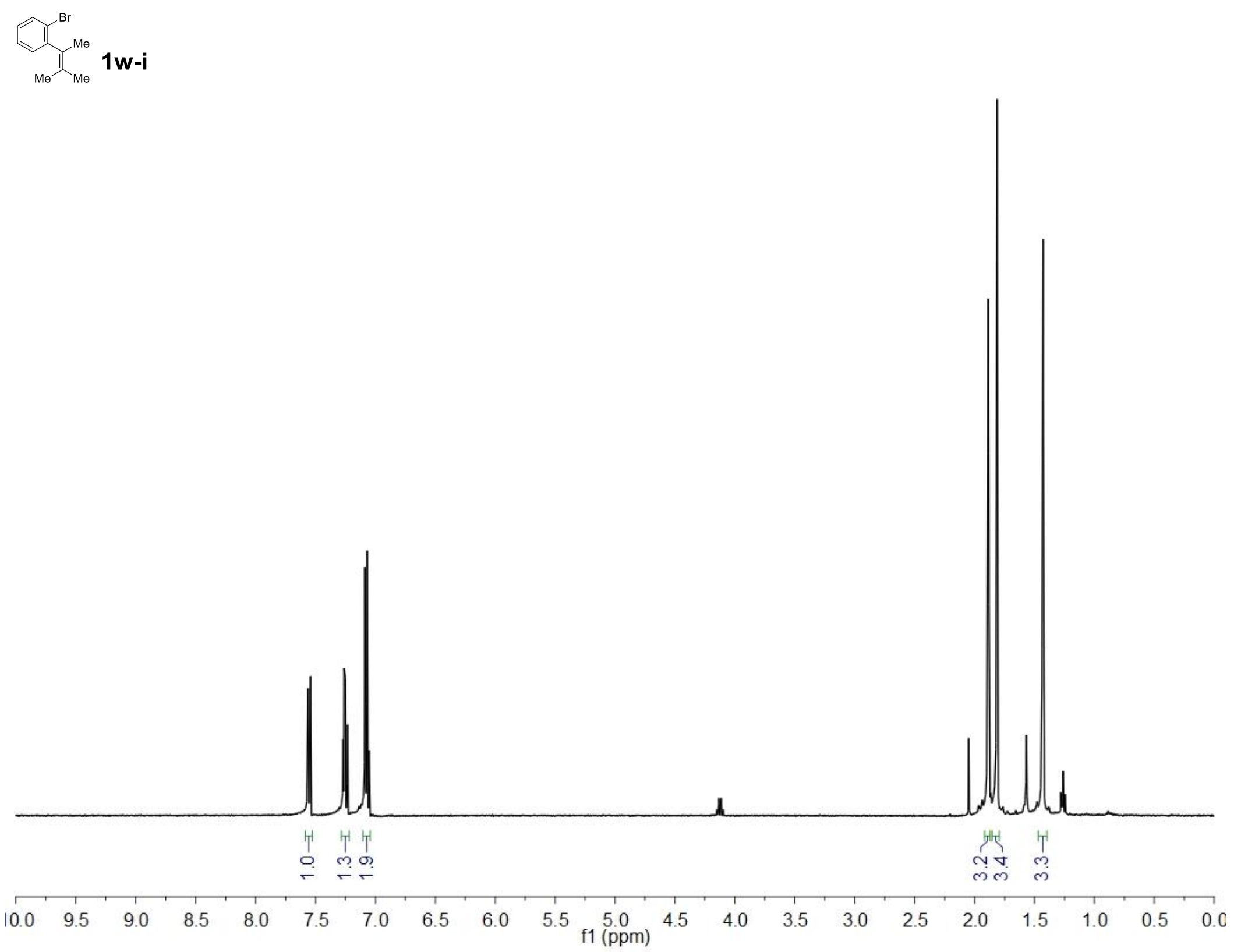




$$
\overbrace{\mathrm{Me}}^{\mathrm{Me}} \mathbf{1} \mathbf{w}
$$

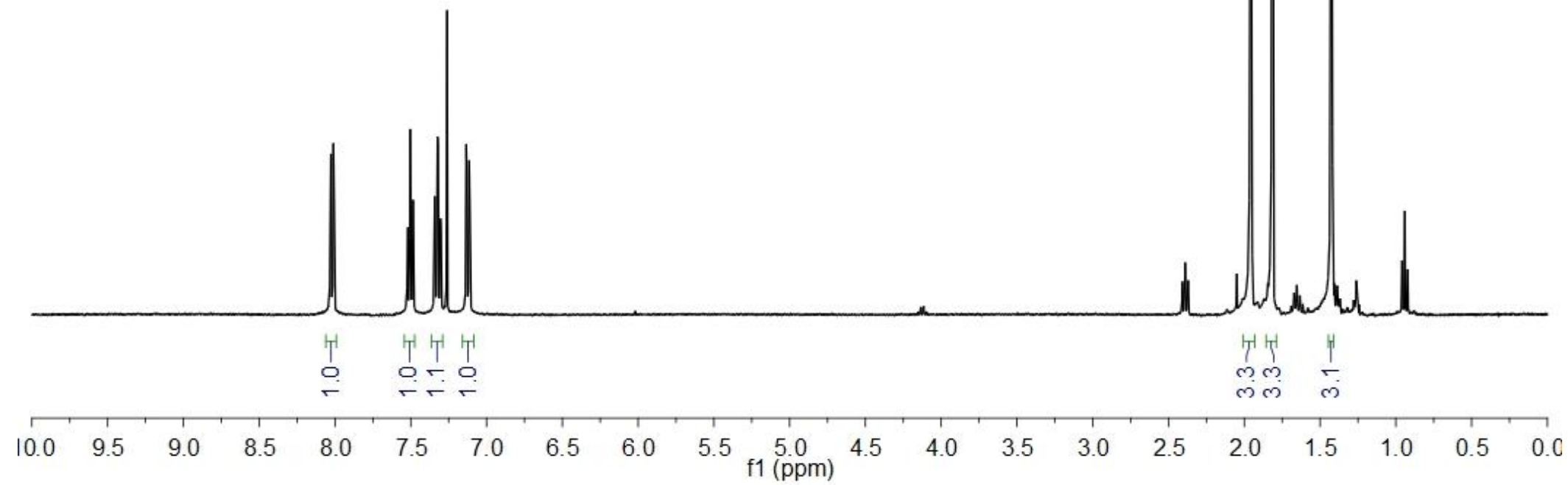


Me $1 \mathbf{i}$

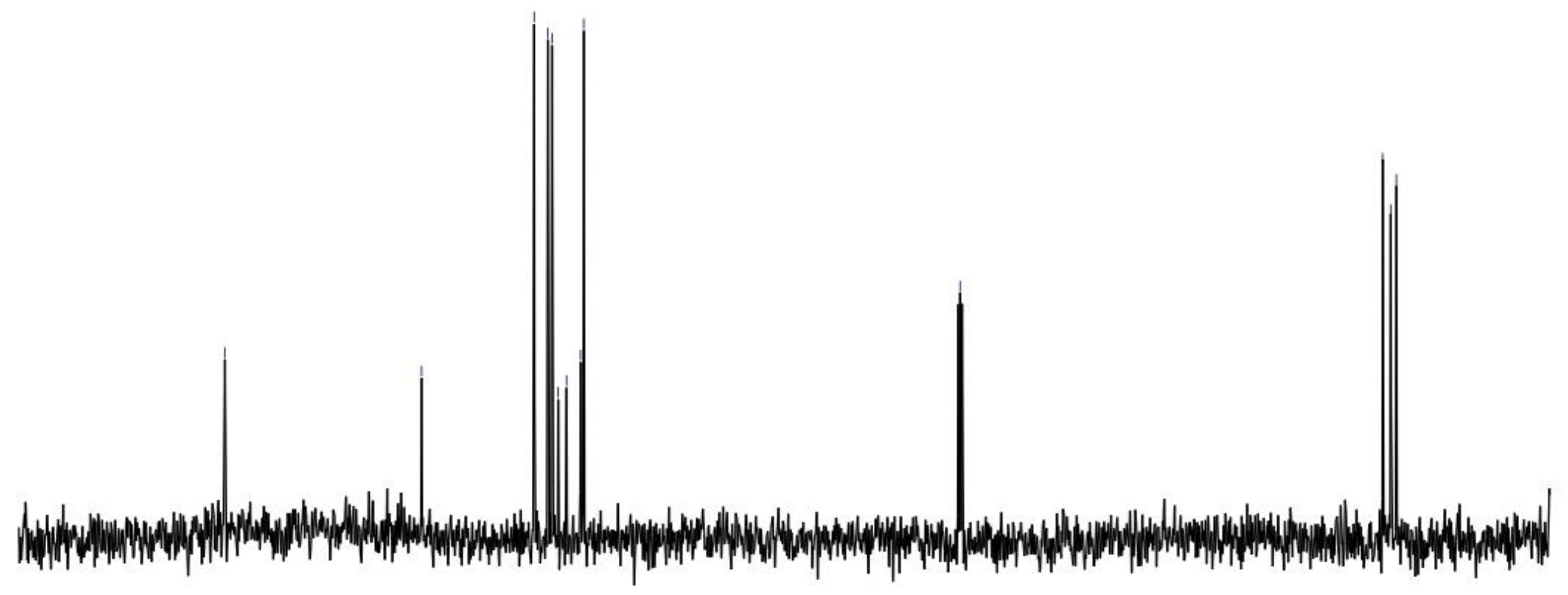



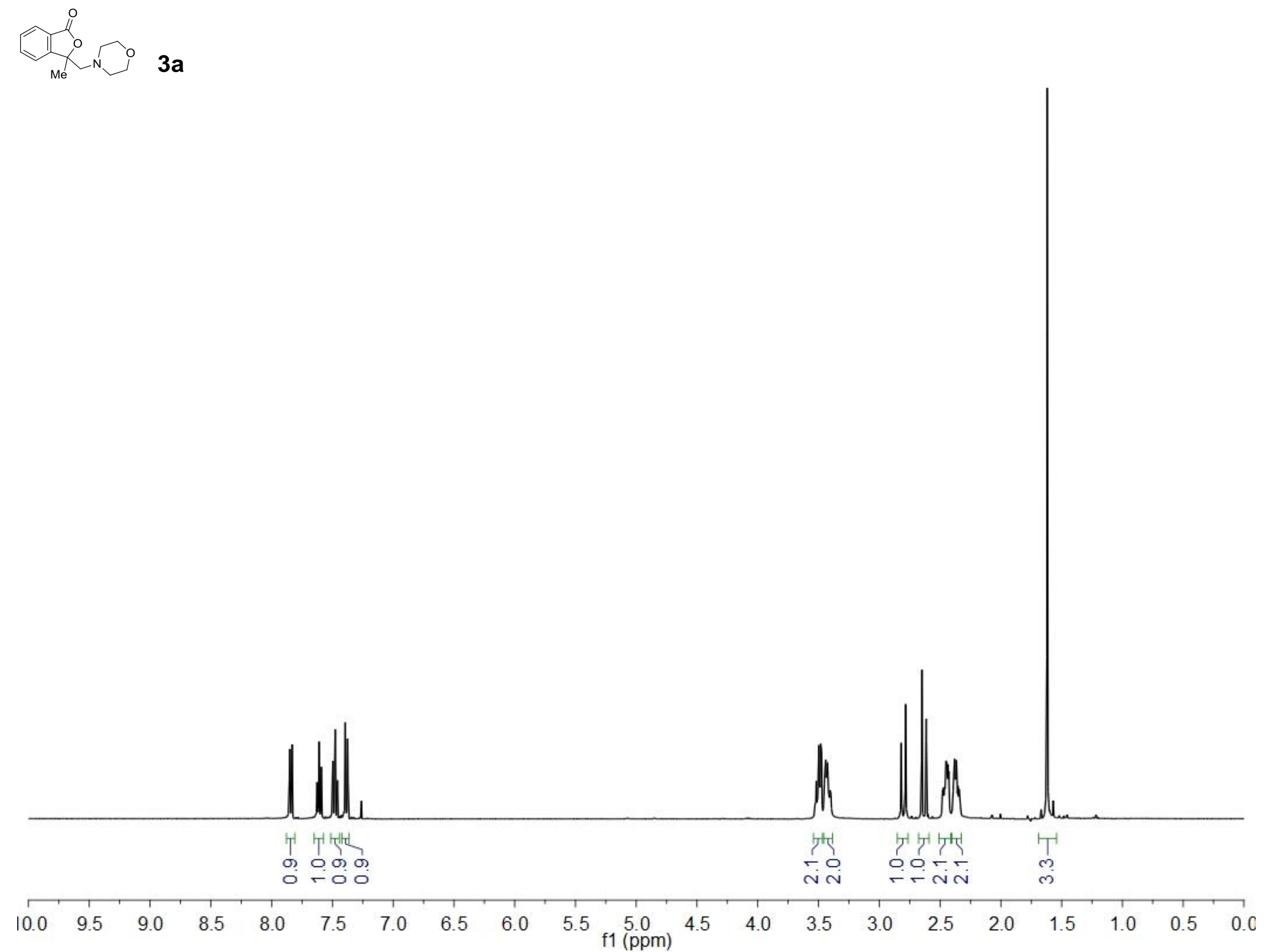

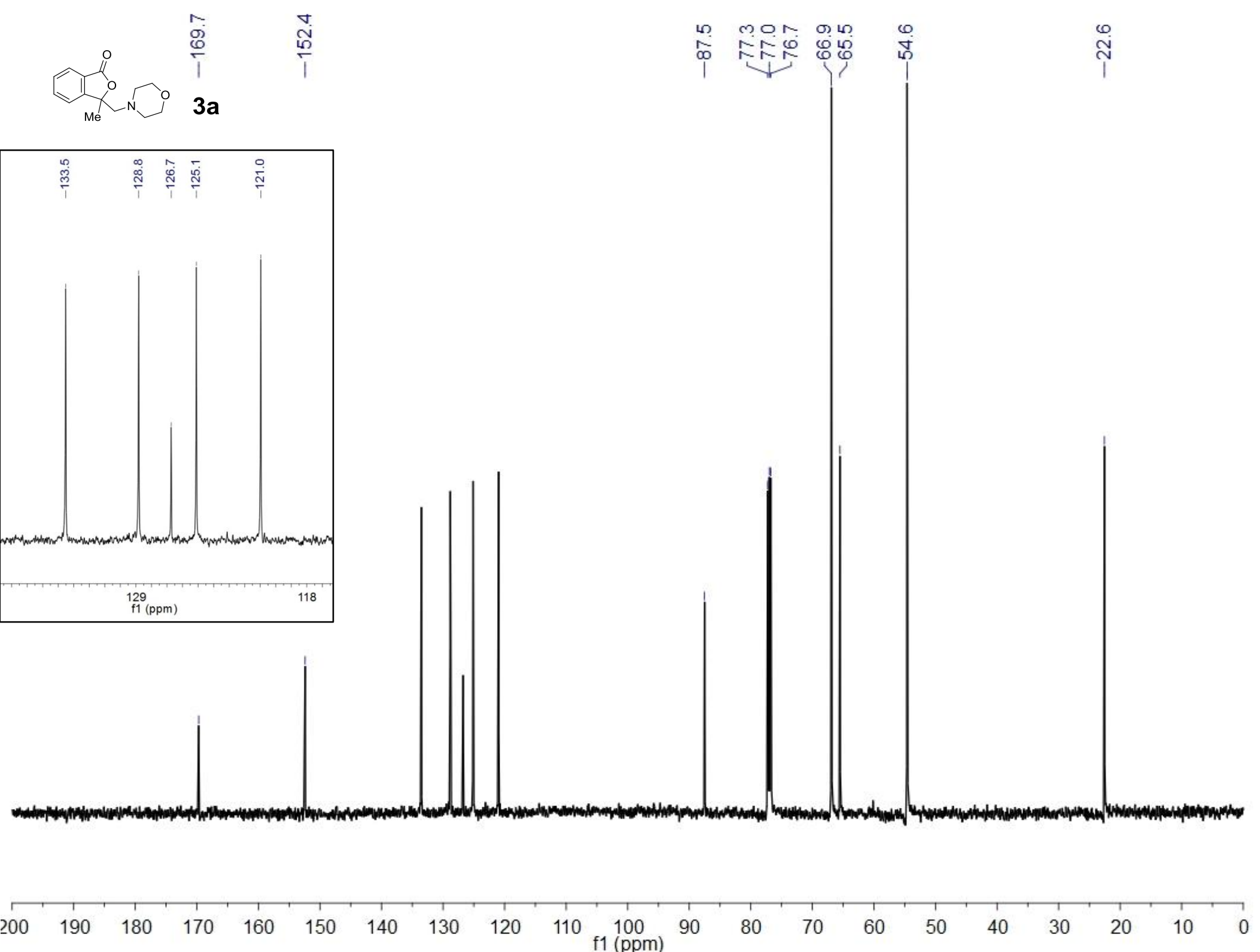


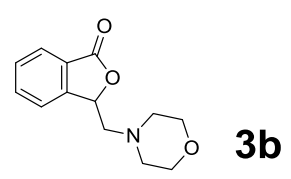

$3 b$

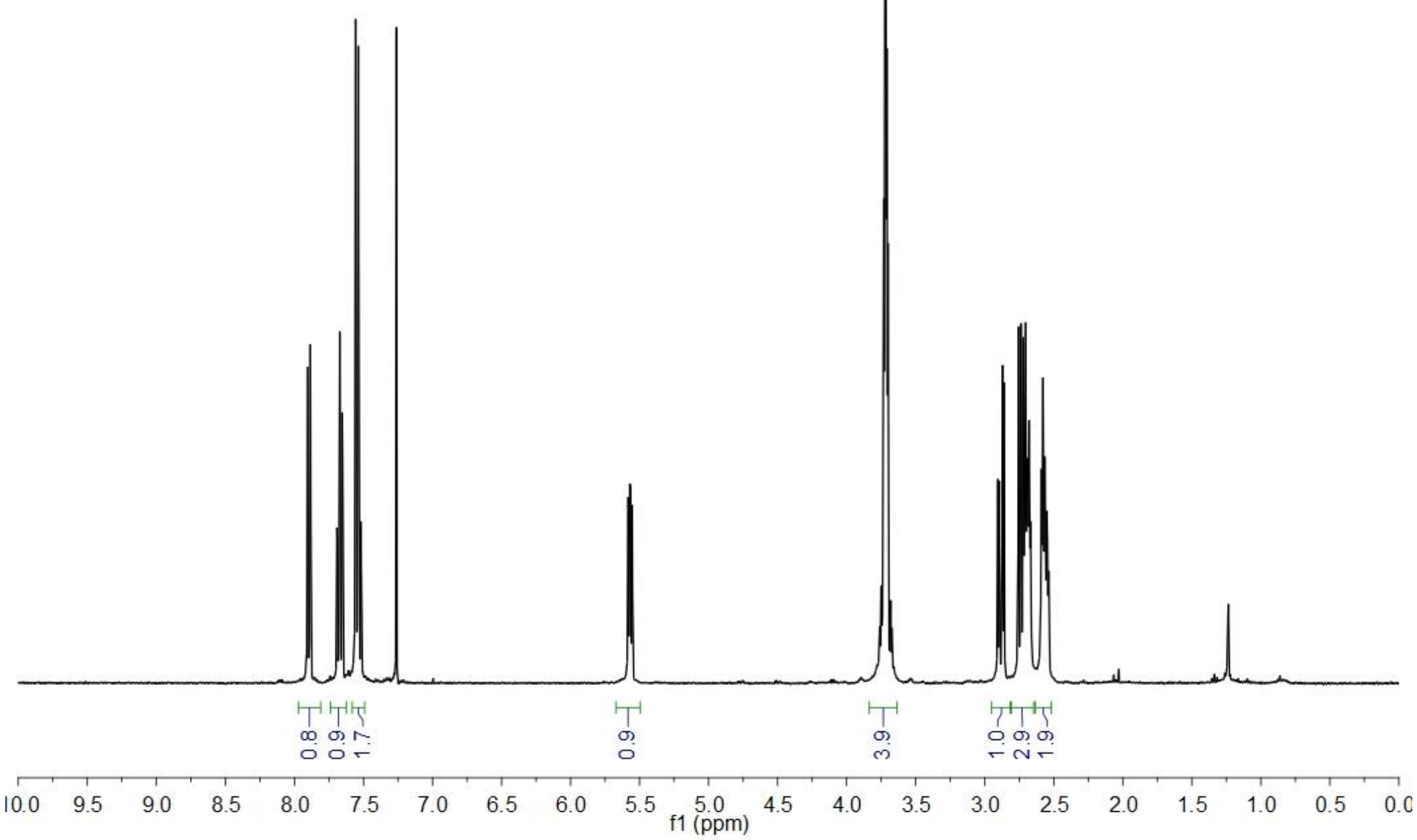



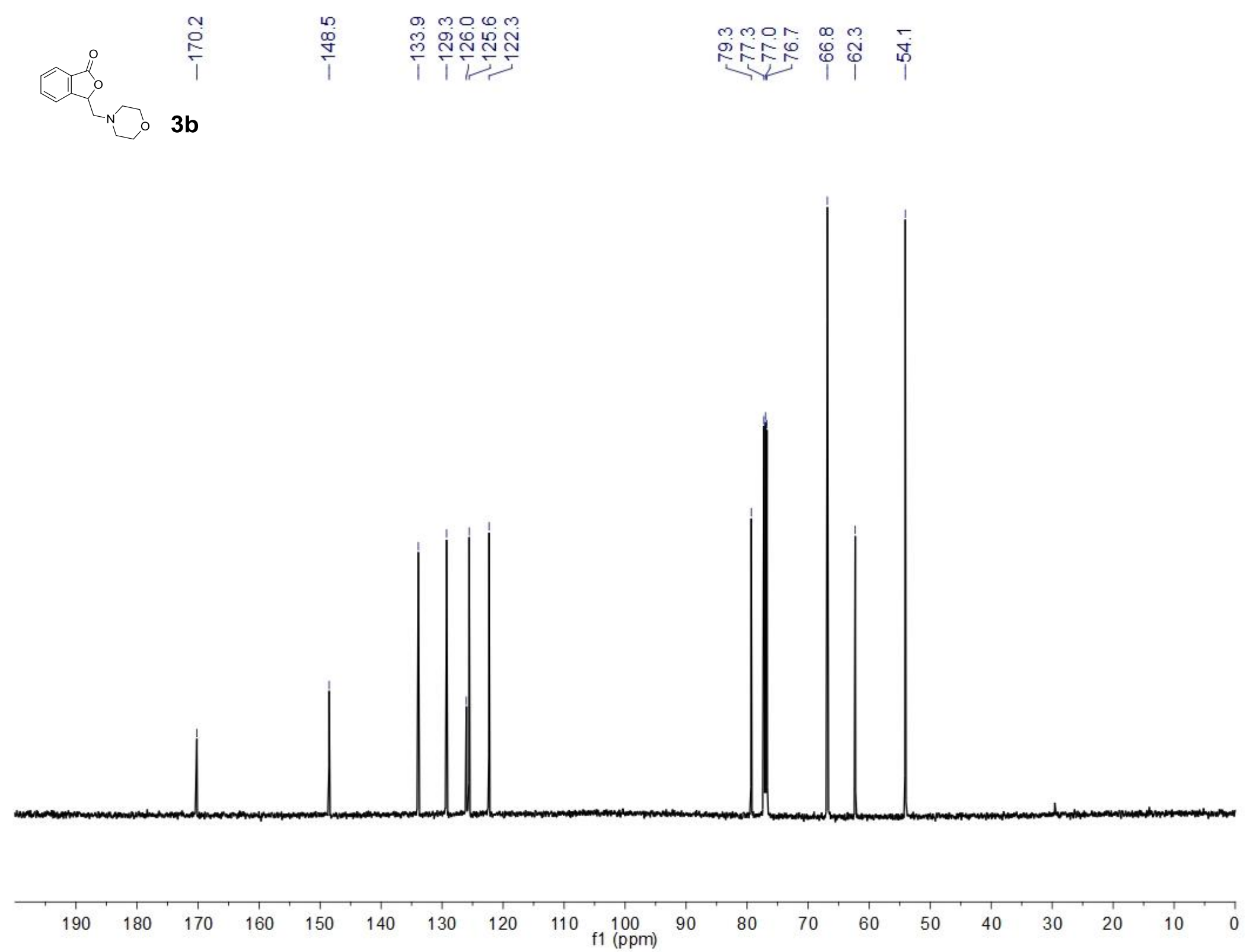

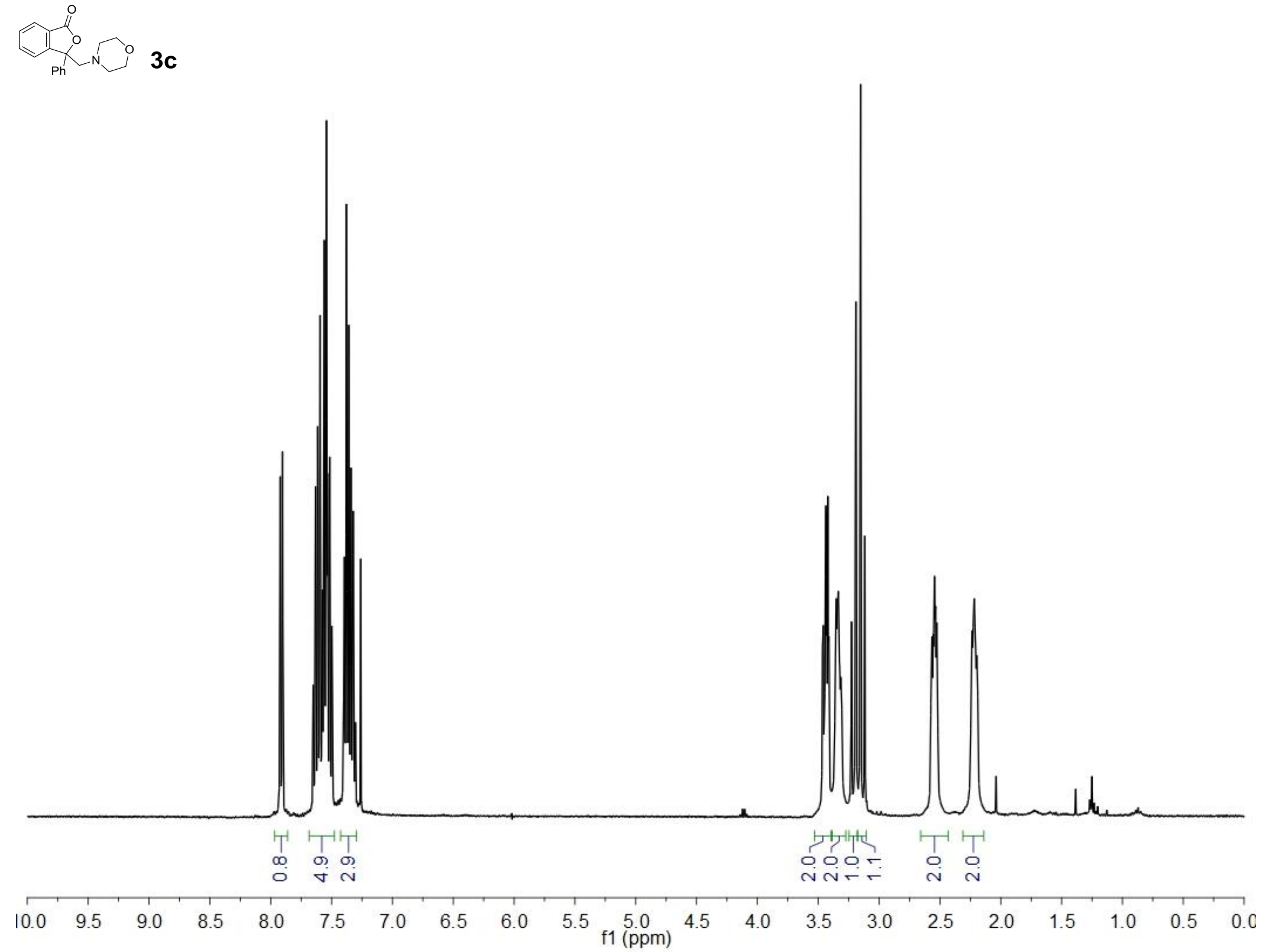

S119 


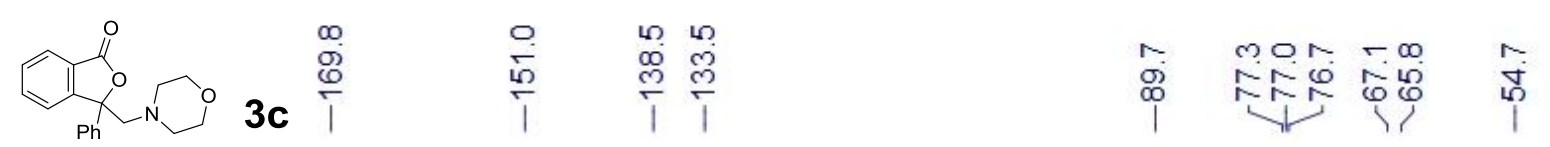
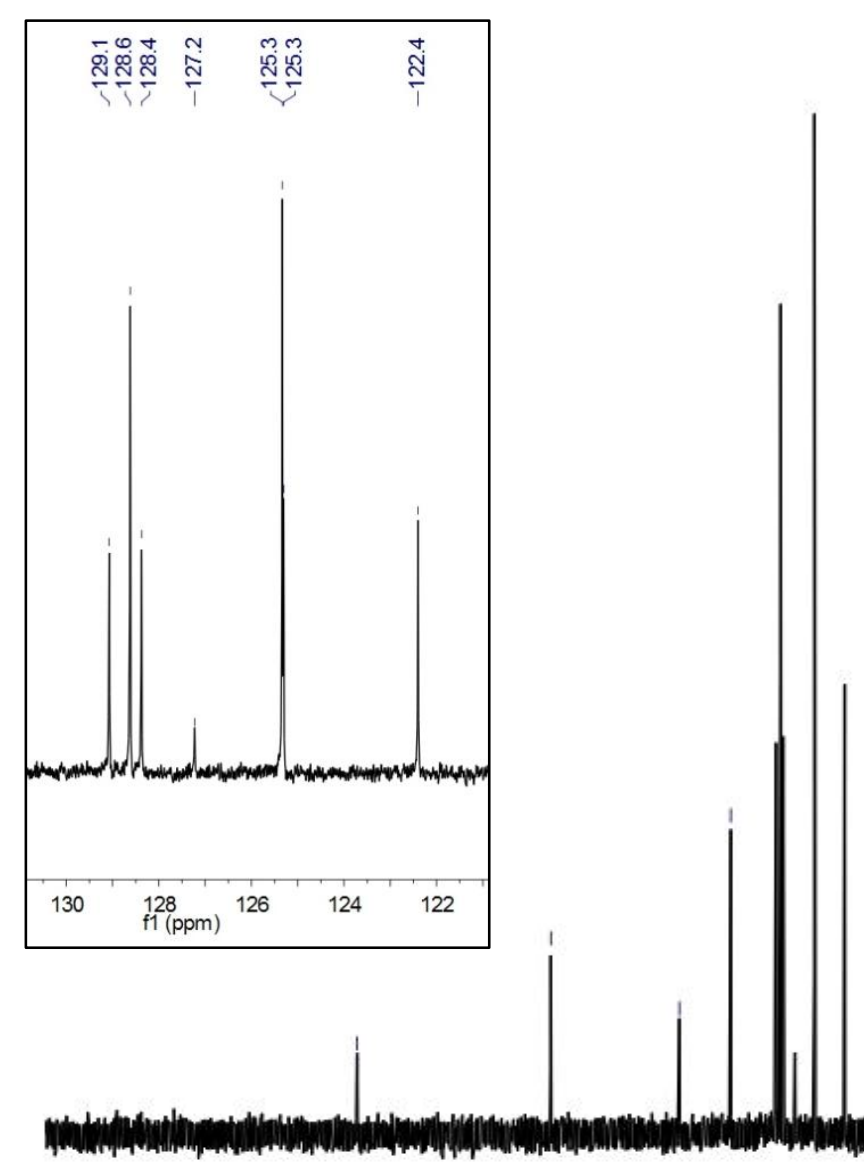

Nomining 


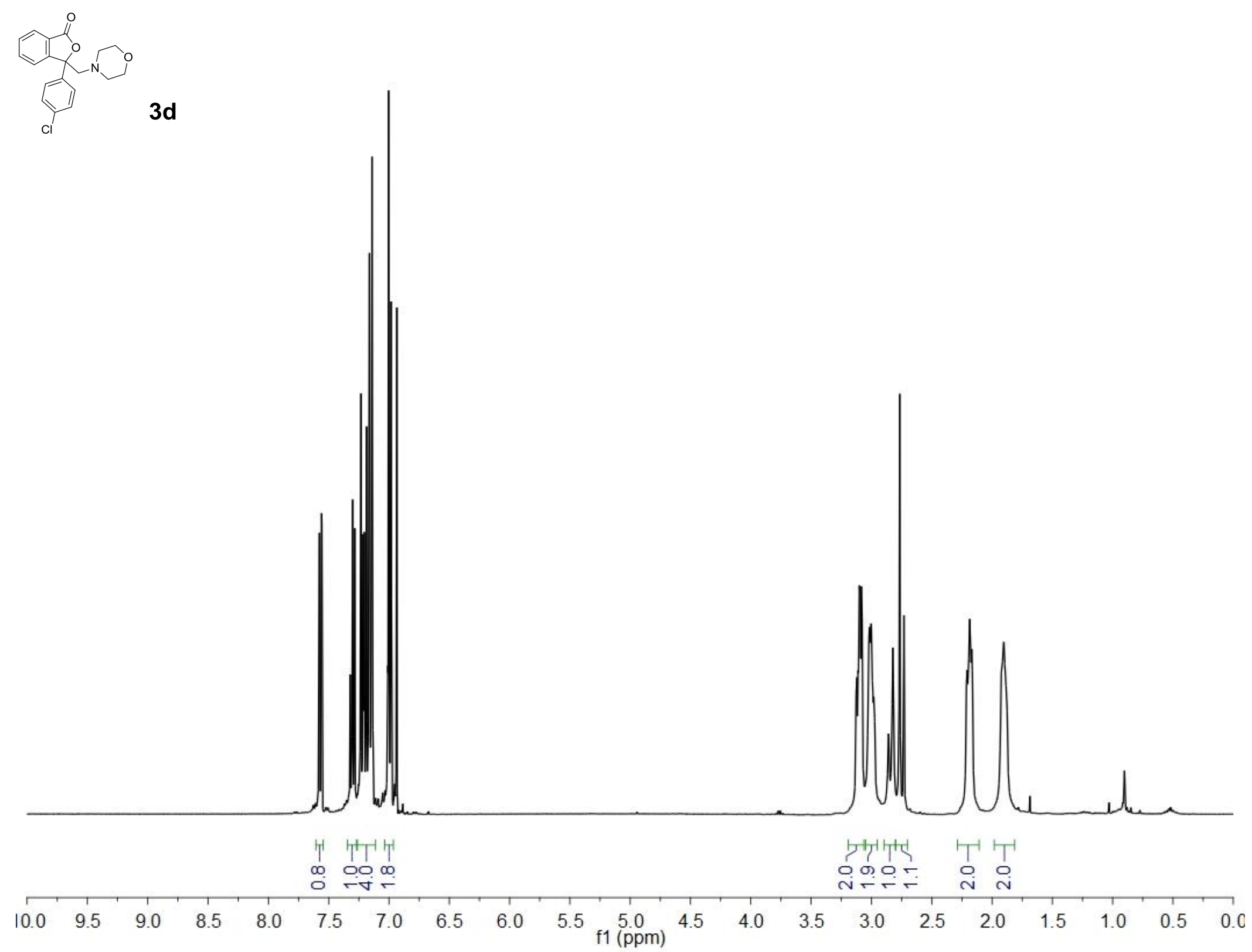



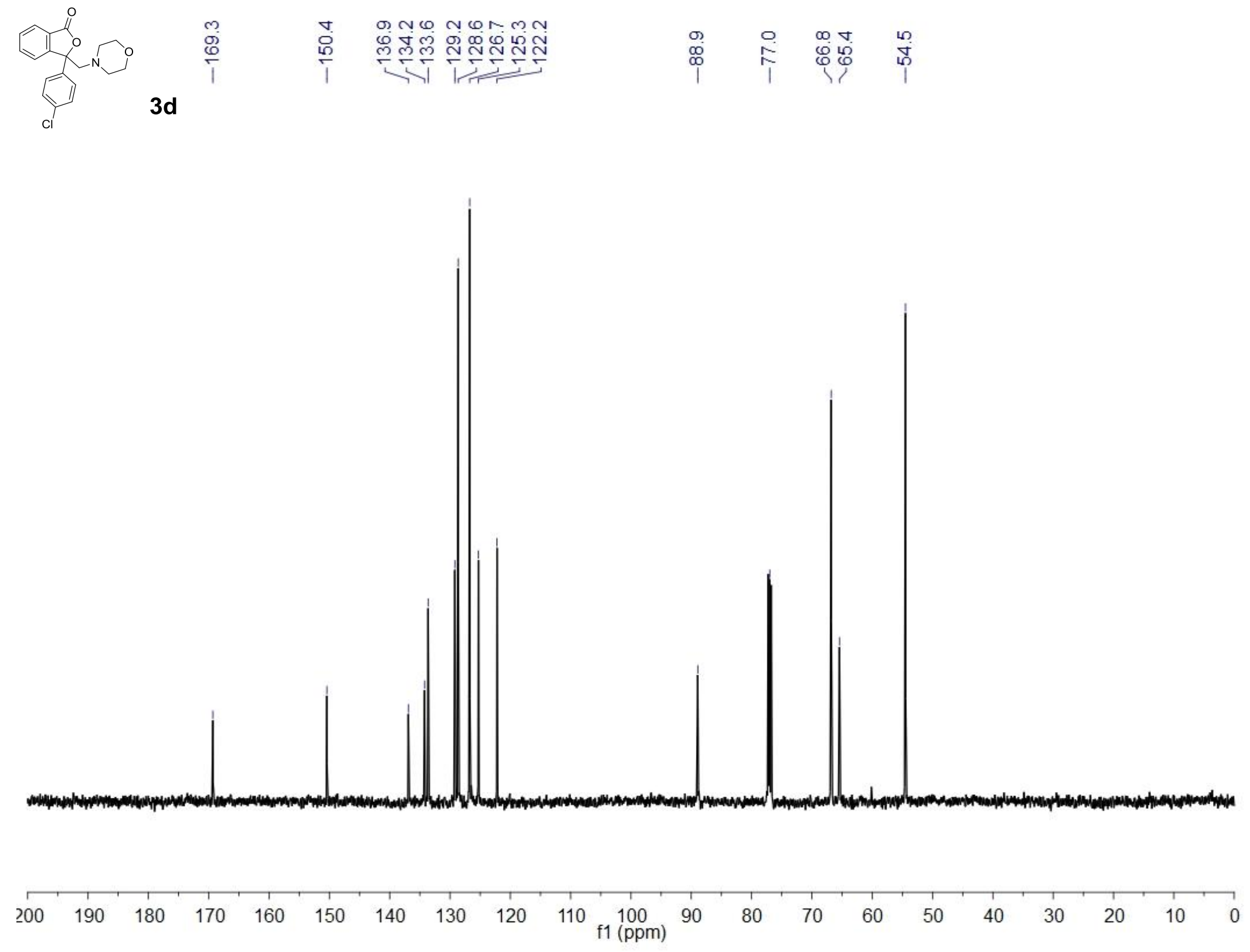


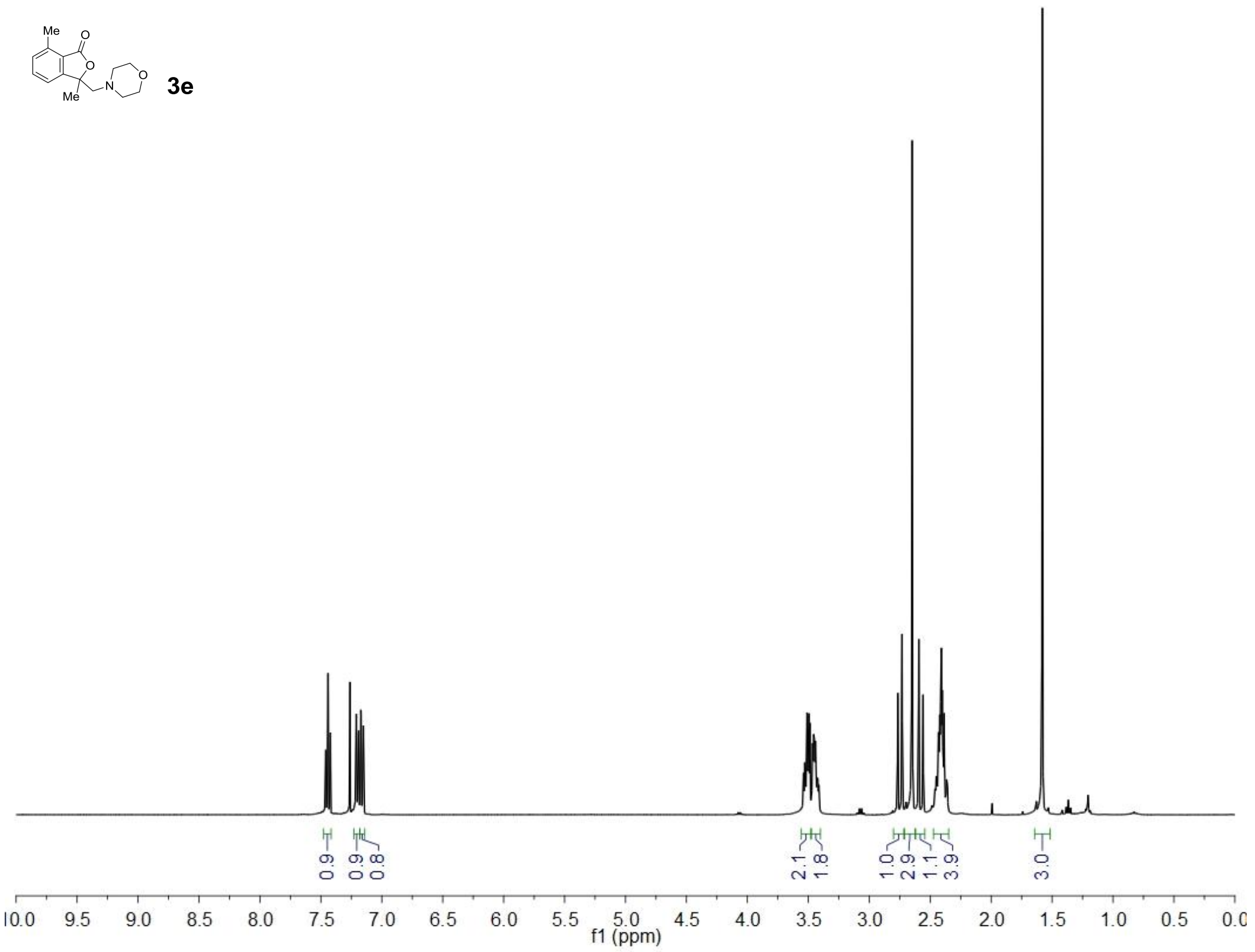



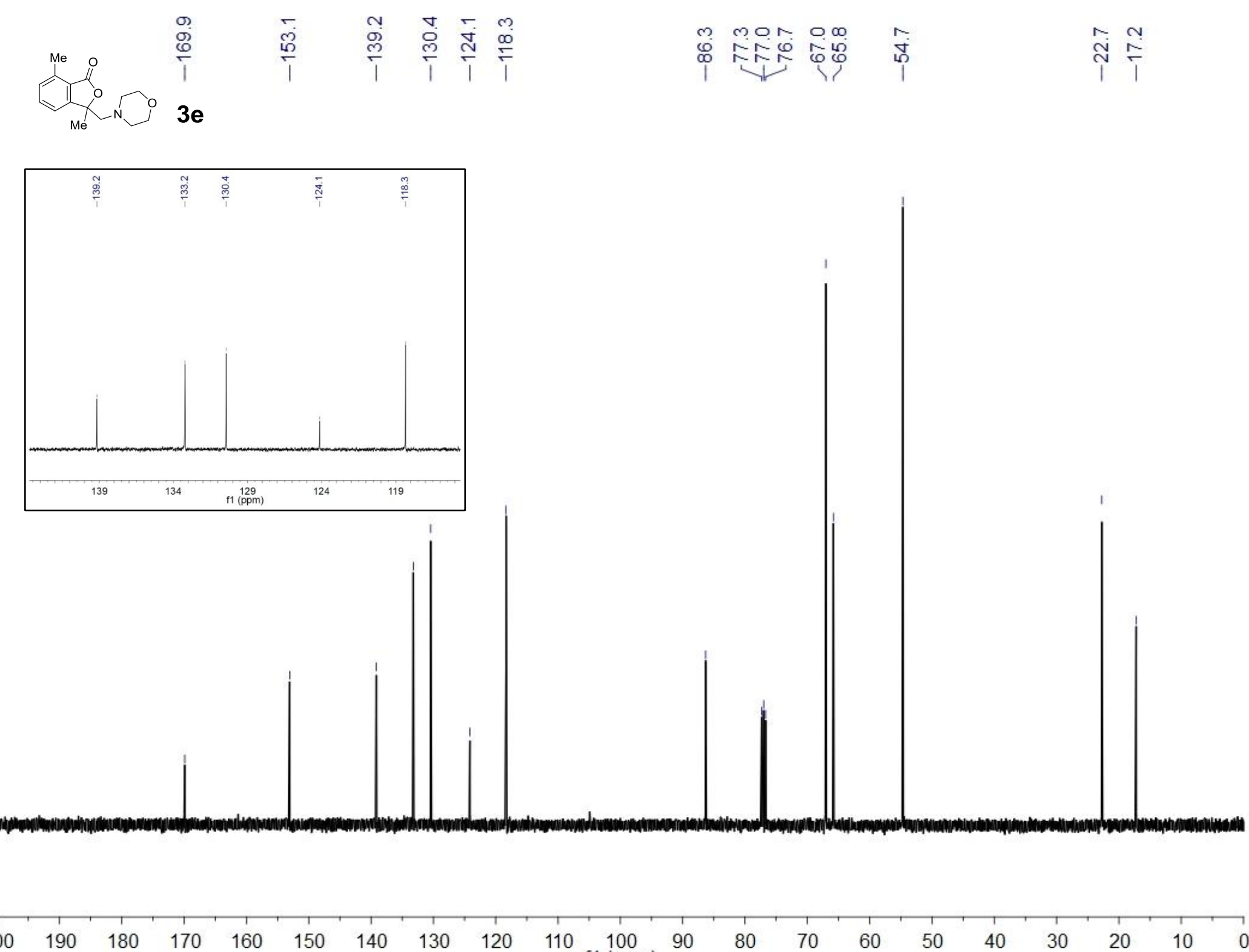

120

$10 \quad 100$

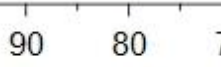

$70 \quad 60$

50

1 30

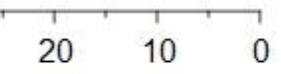




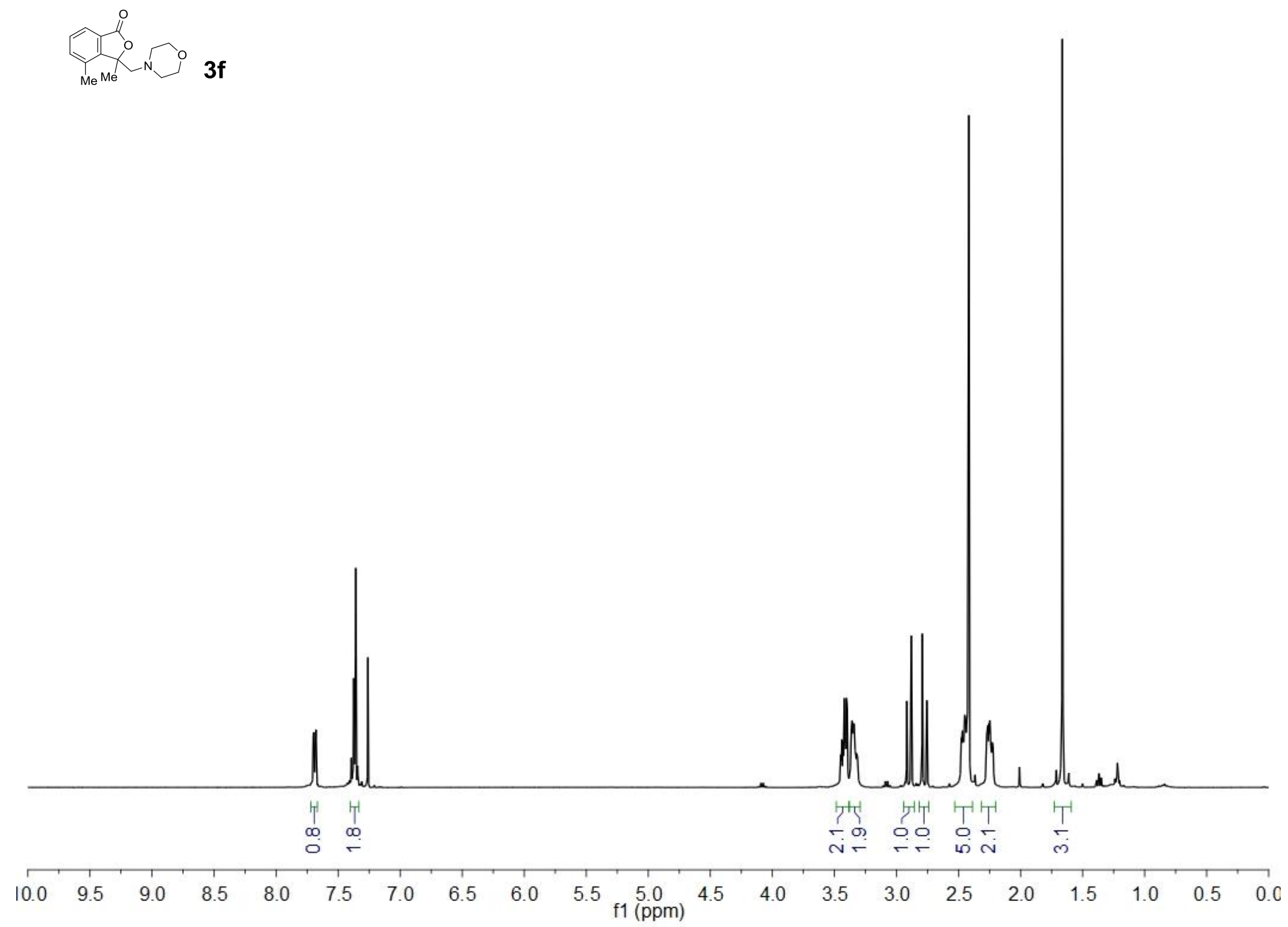




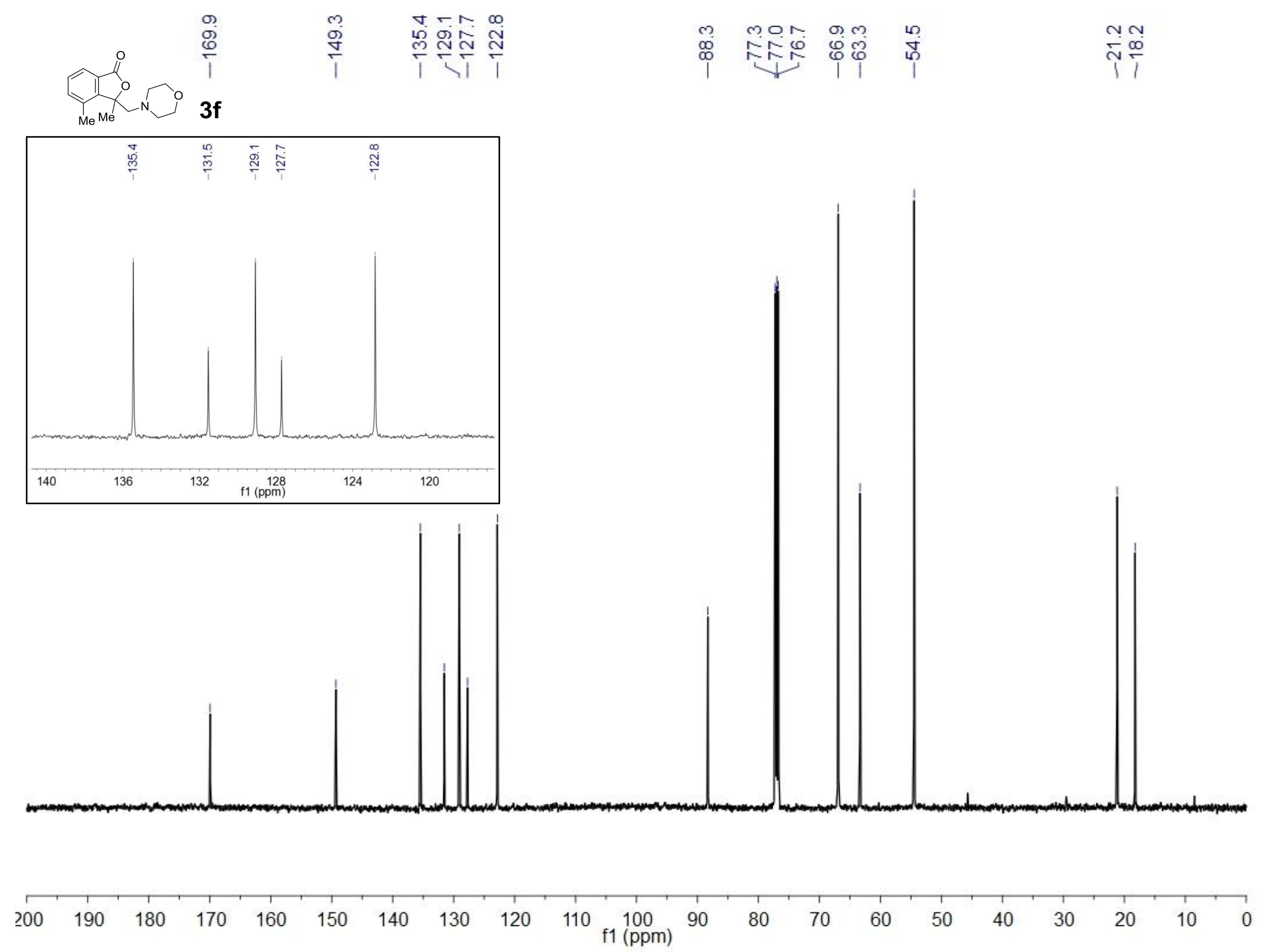




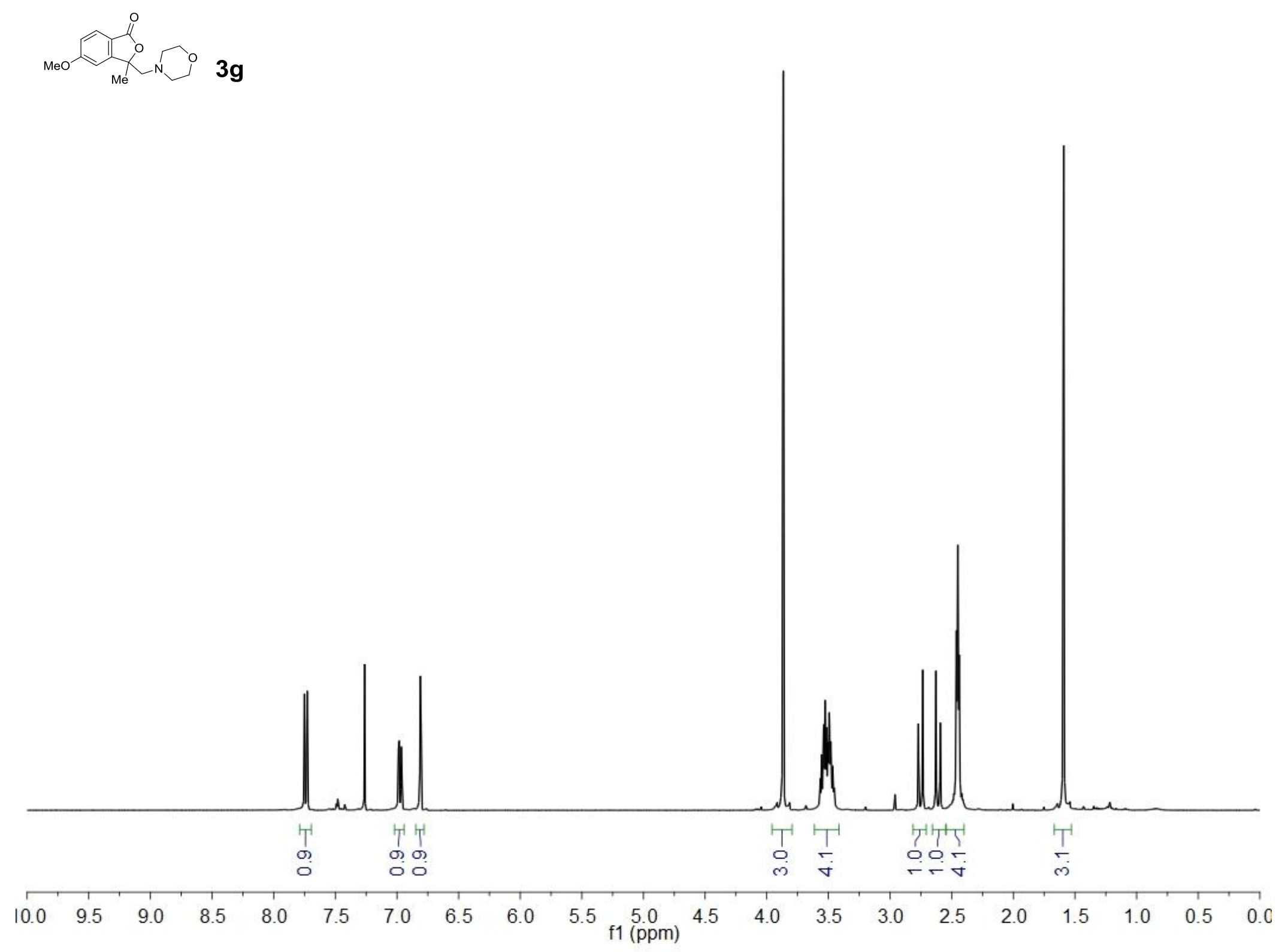




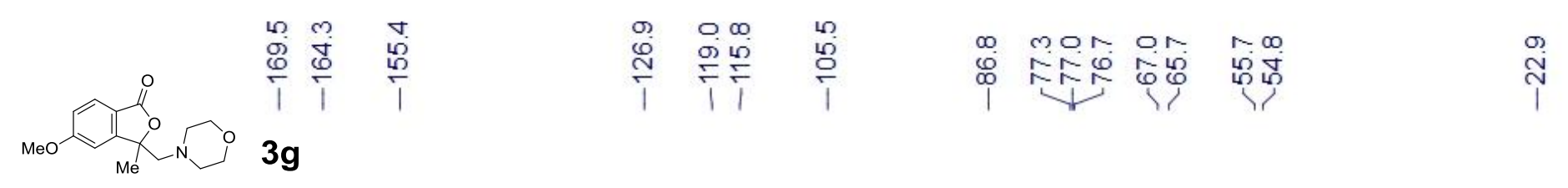
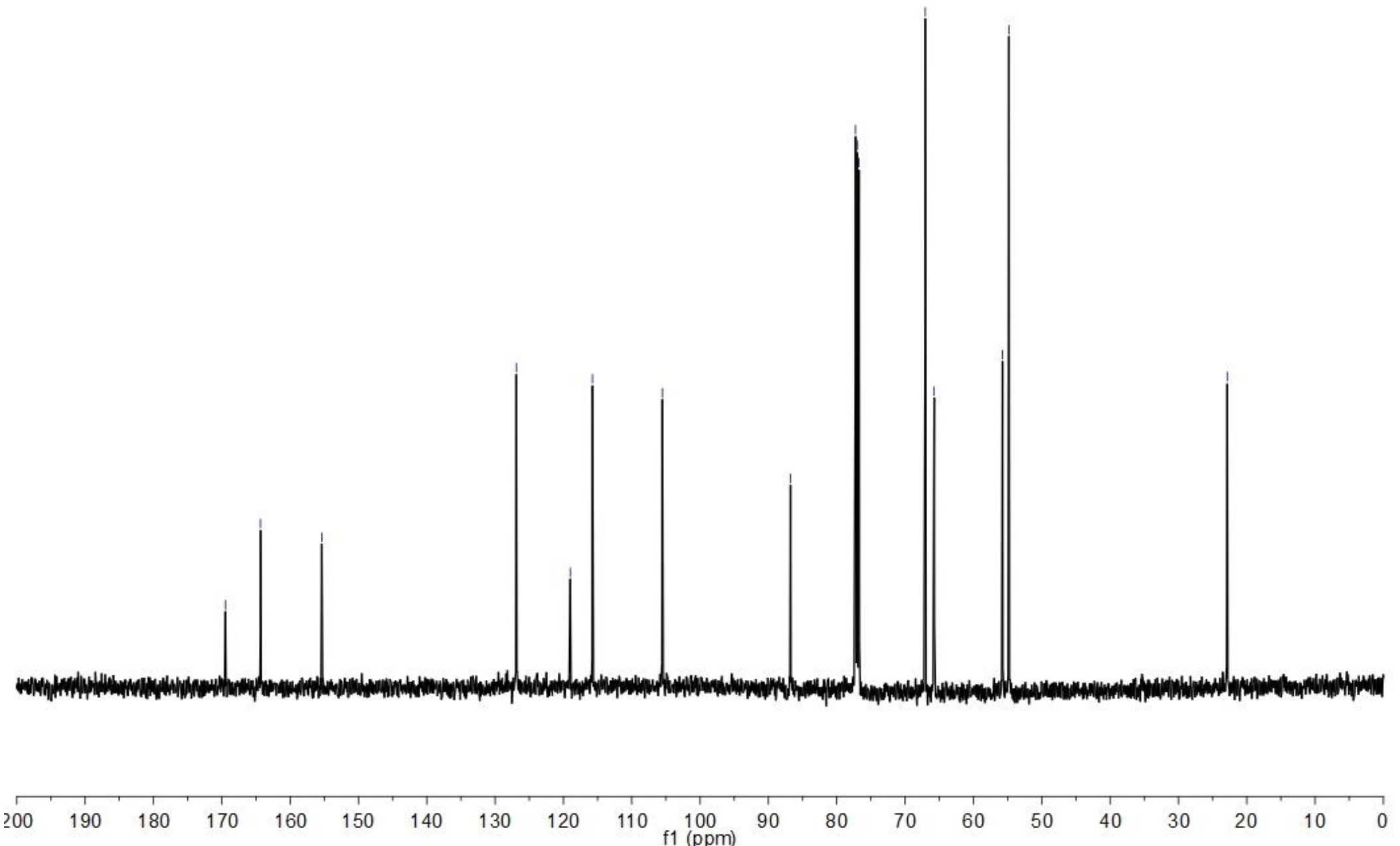

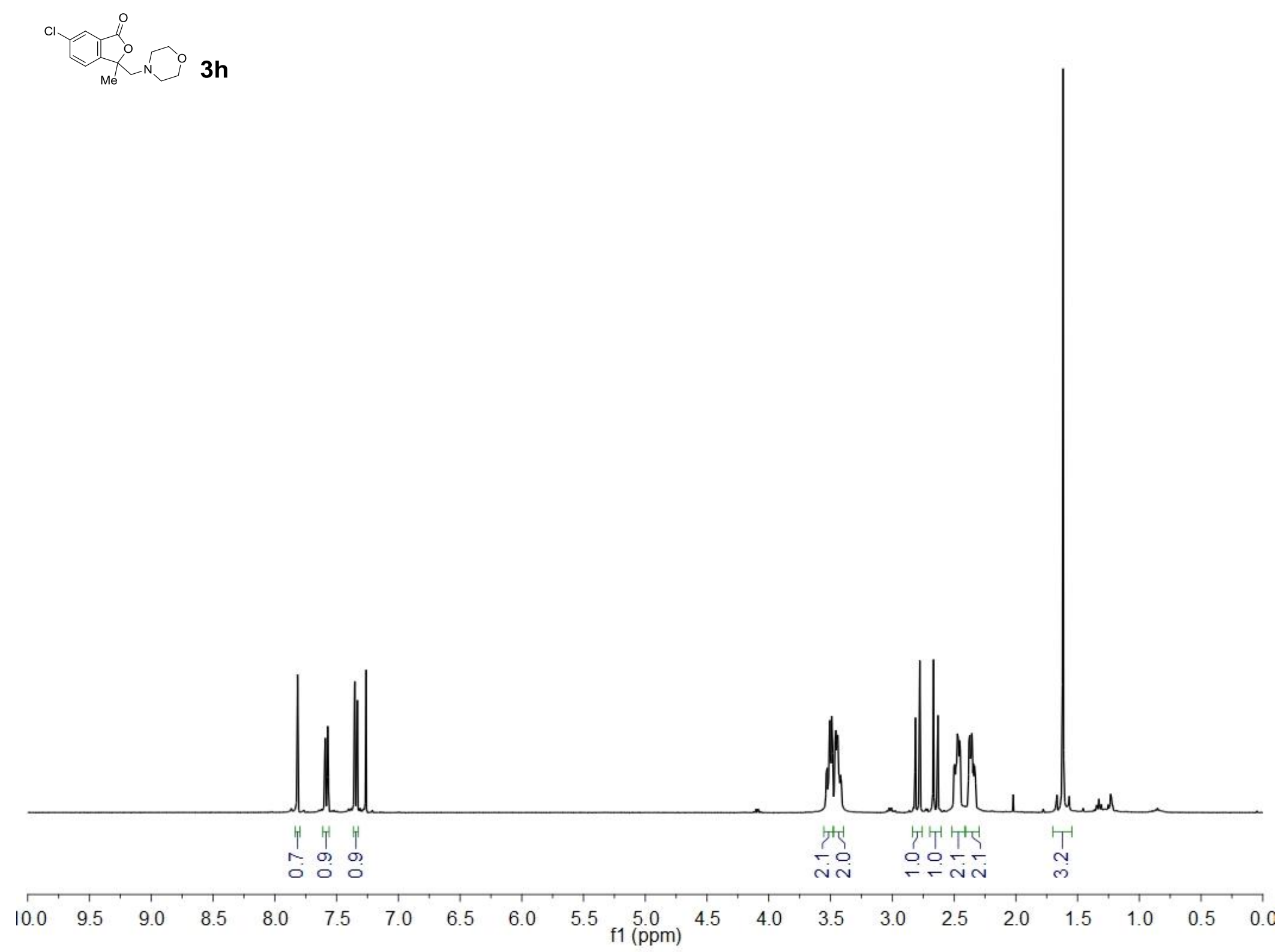


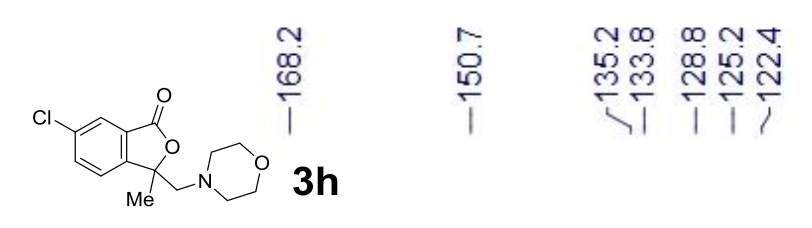

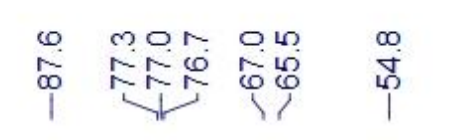

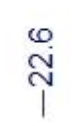

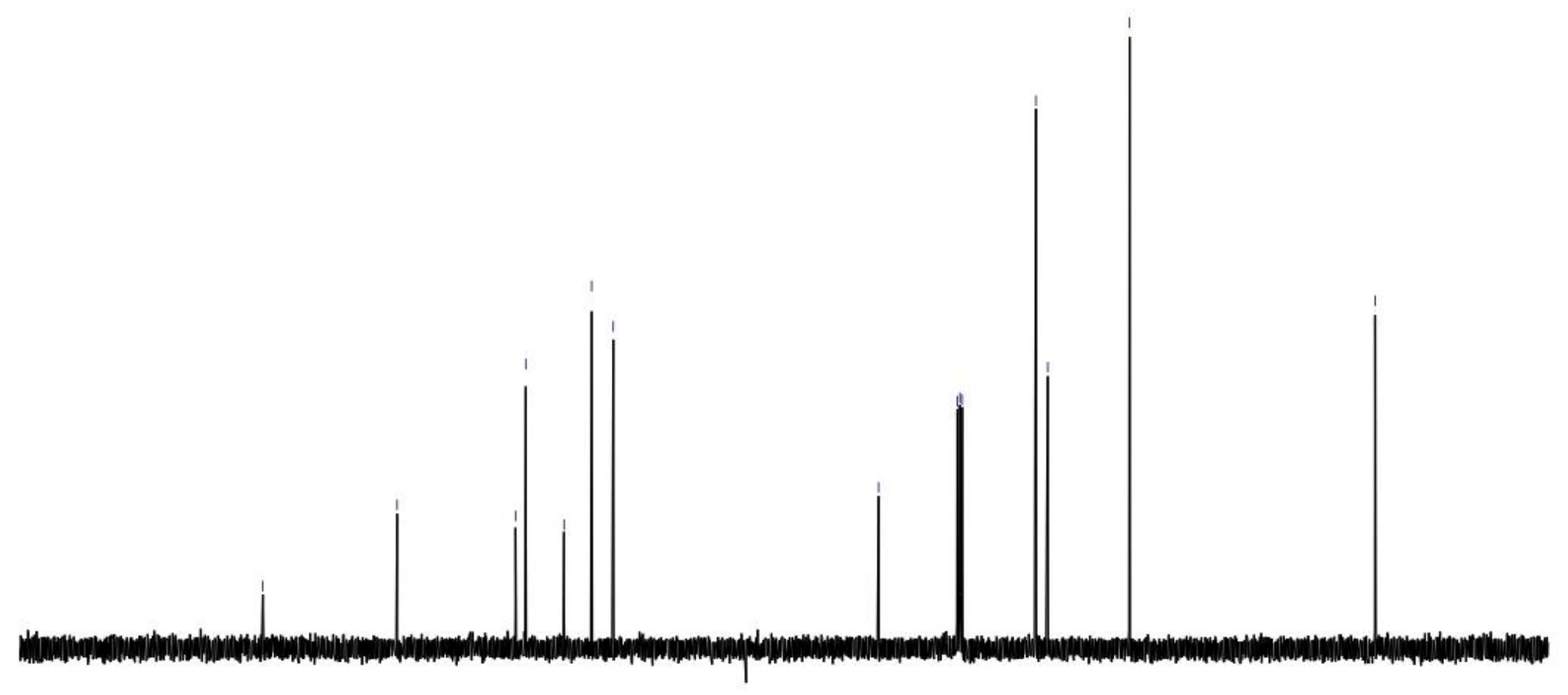

200

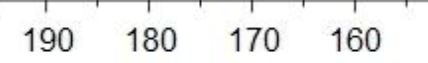

$150 \quad 140$

130

120

$110 \quad 100$

\begin{tabular}{ll|l|l}
1 & 1 & 1 \\
90 & 80 & 70 & 60
\end{tabular}

50

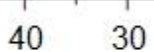


$\sum_{\text {Ph }} x_{n}{ }_{3 i}$

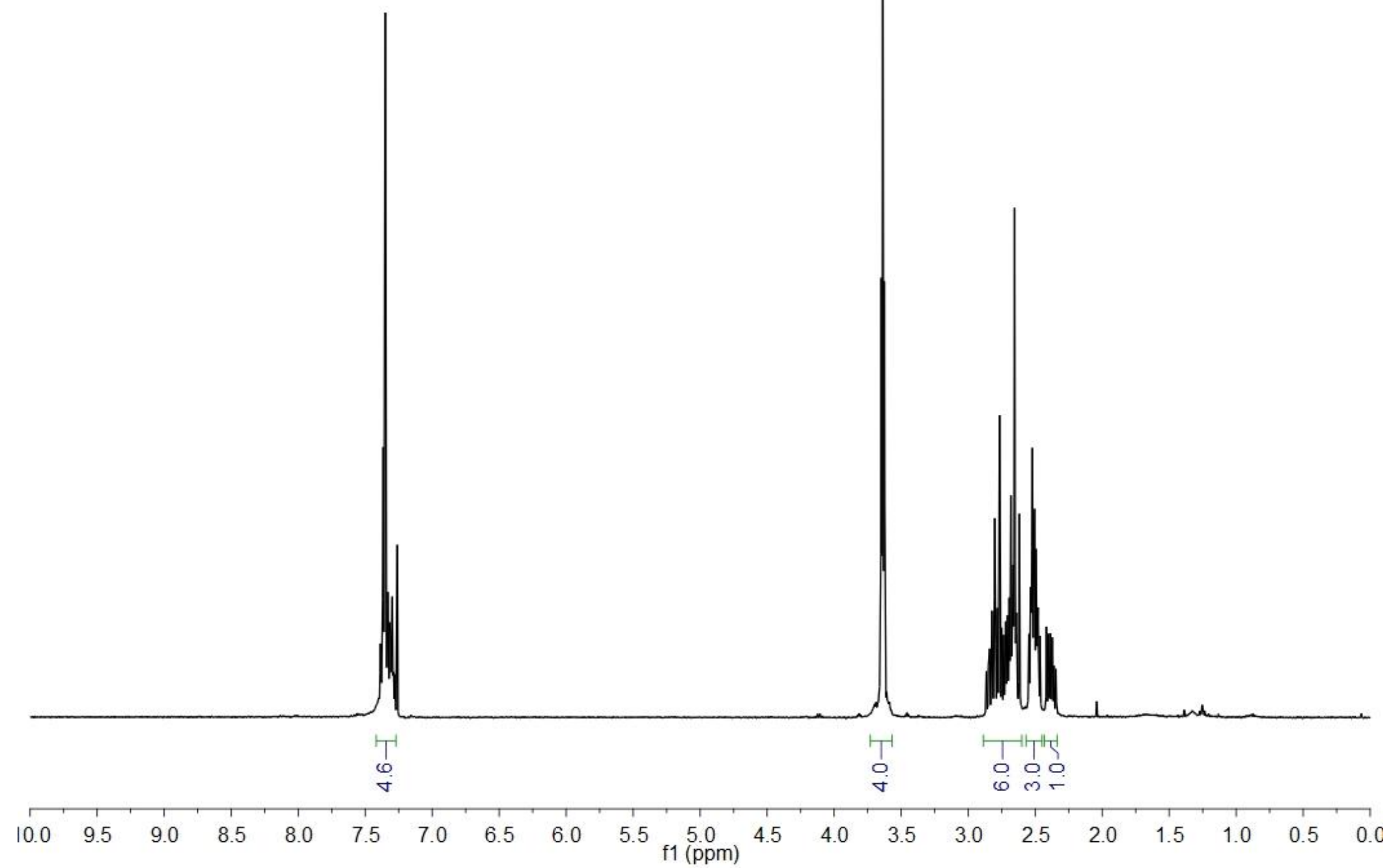




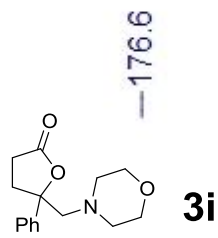

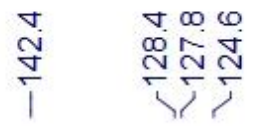

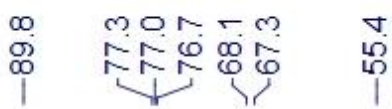
क्ष

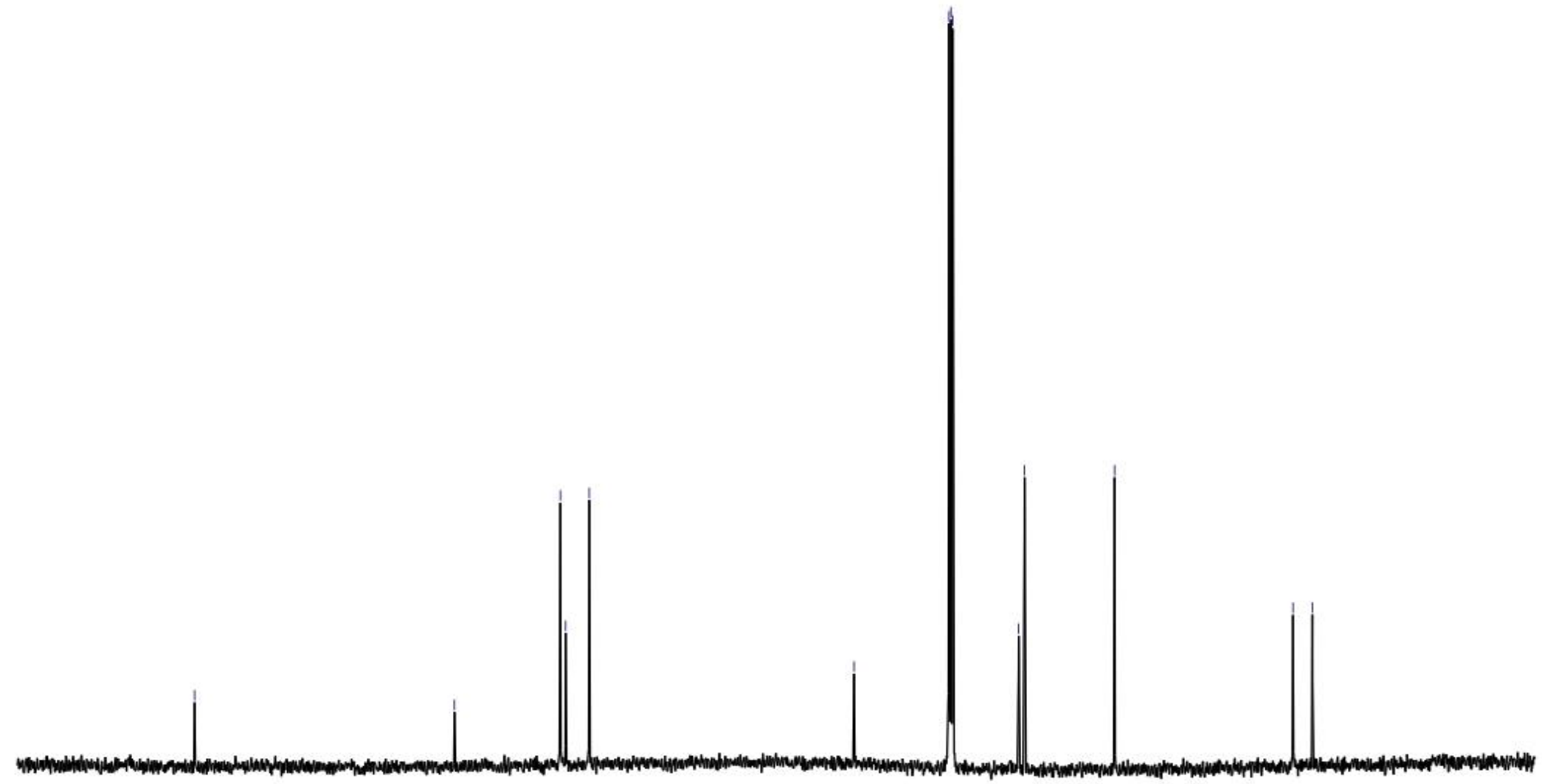


$\underbrace{\text { Me }}_{\text {Ph }}$

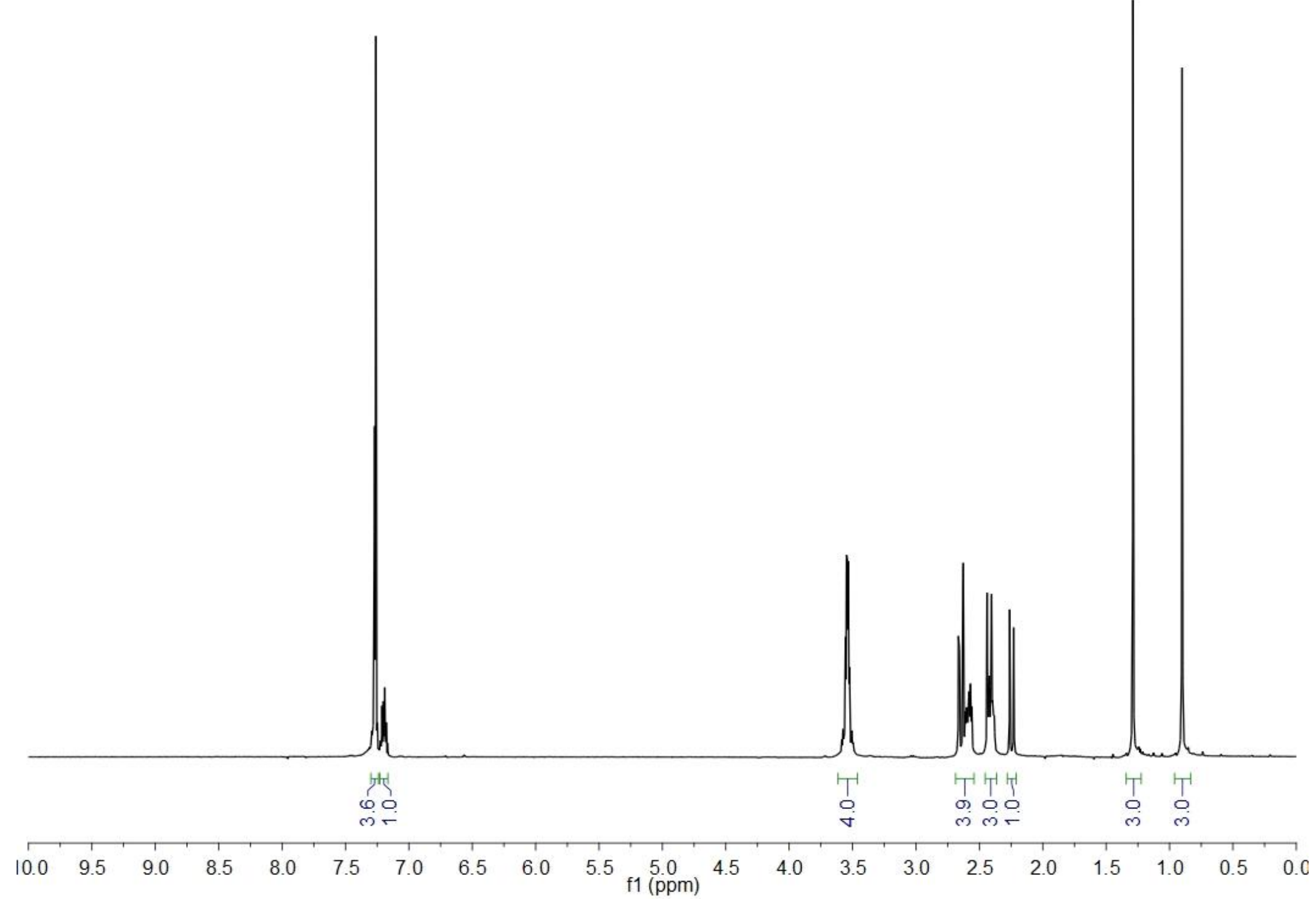




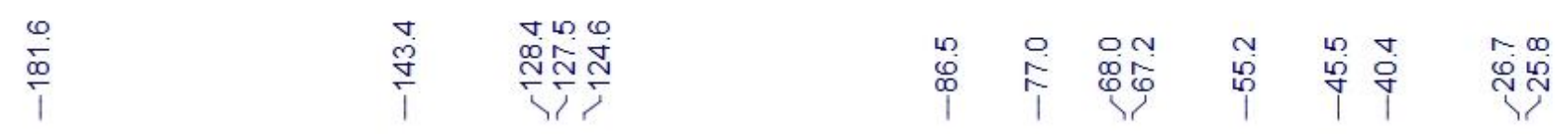

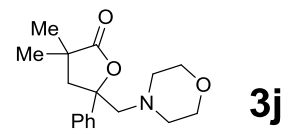

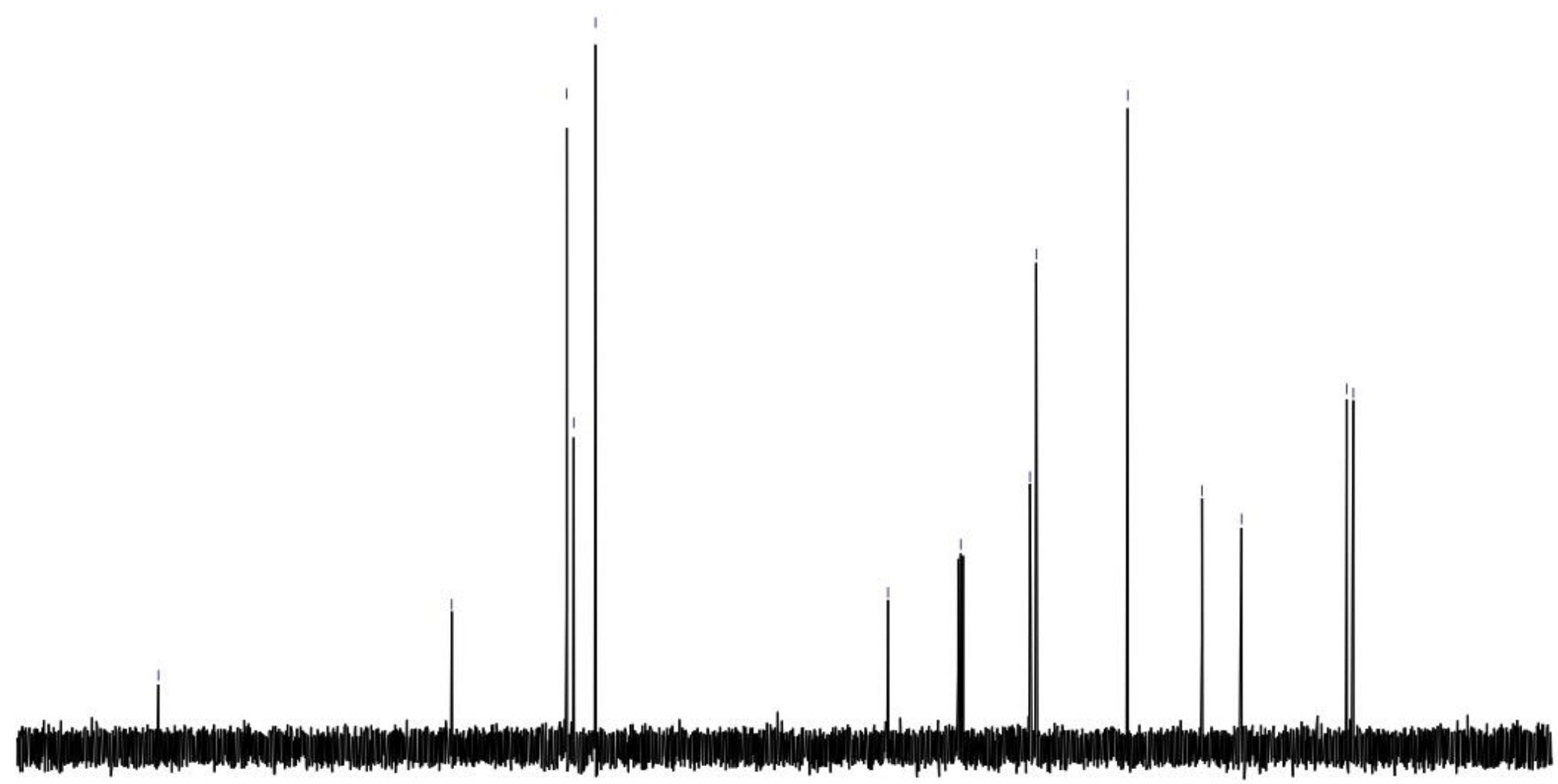




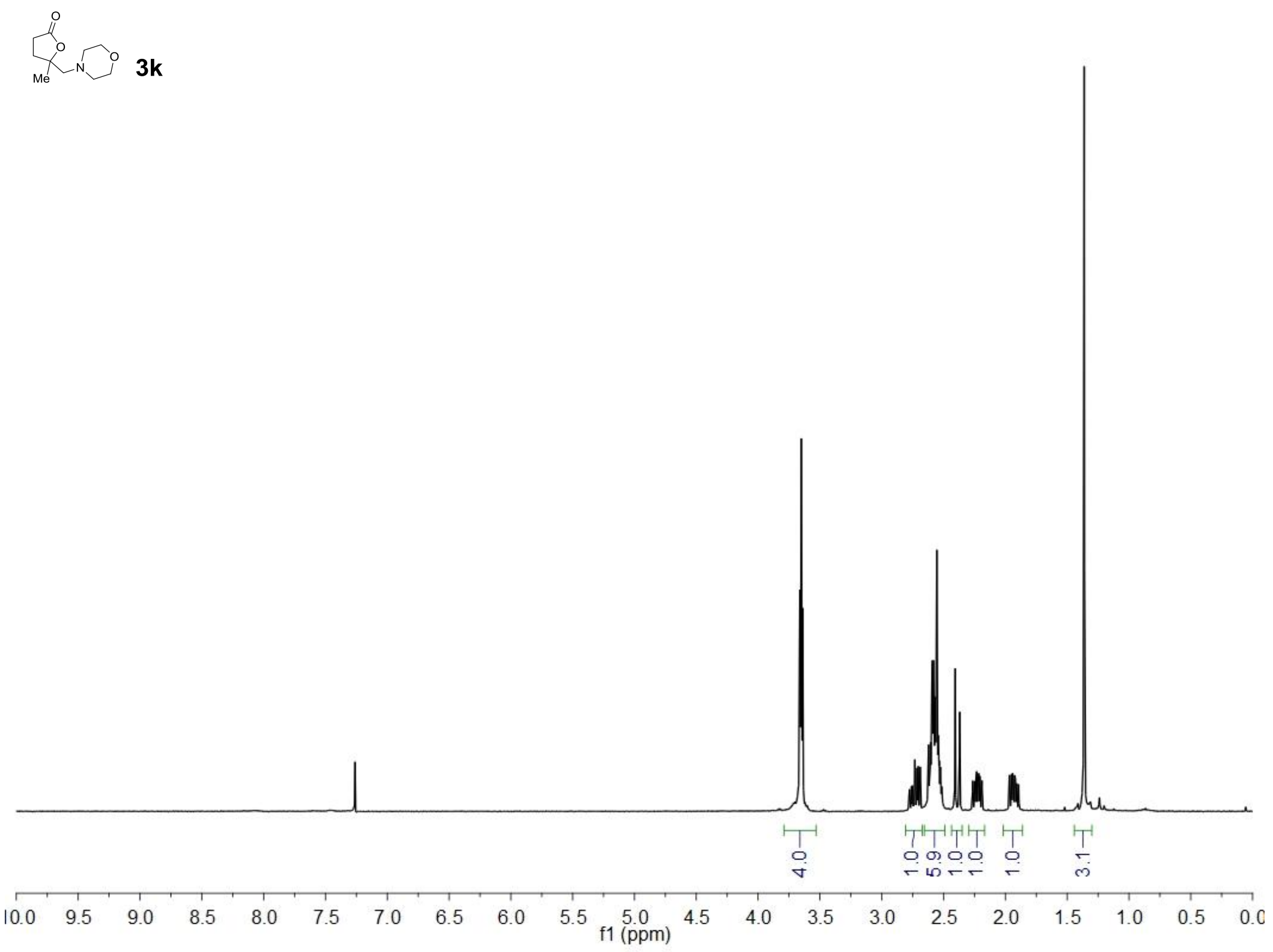




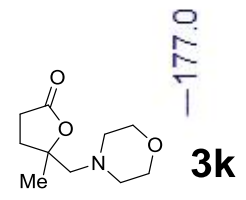
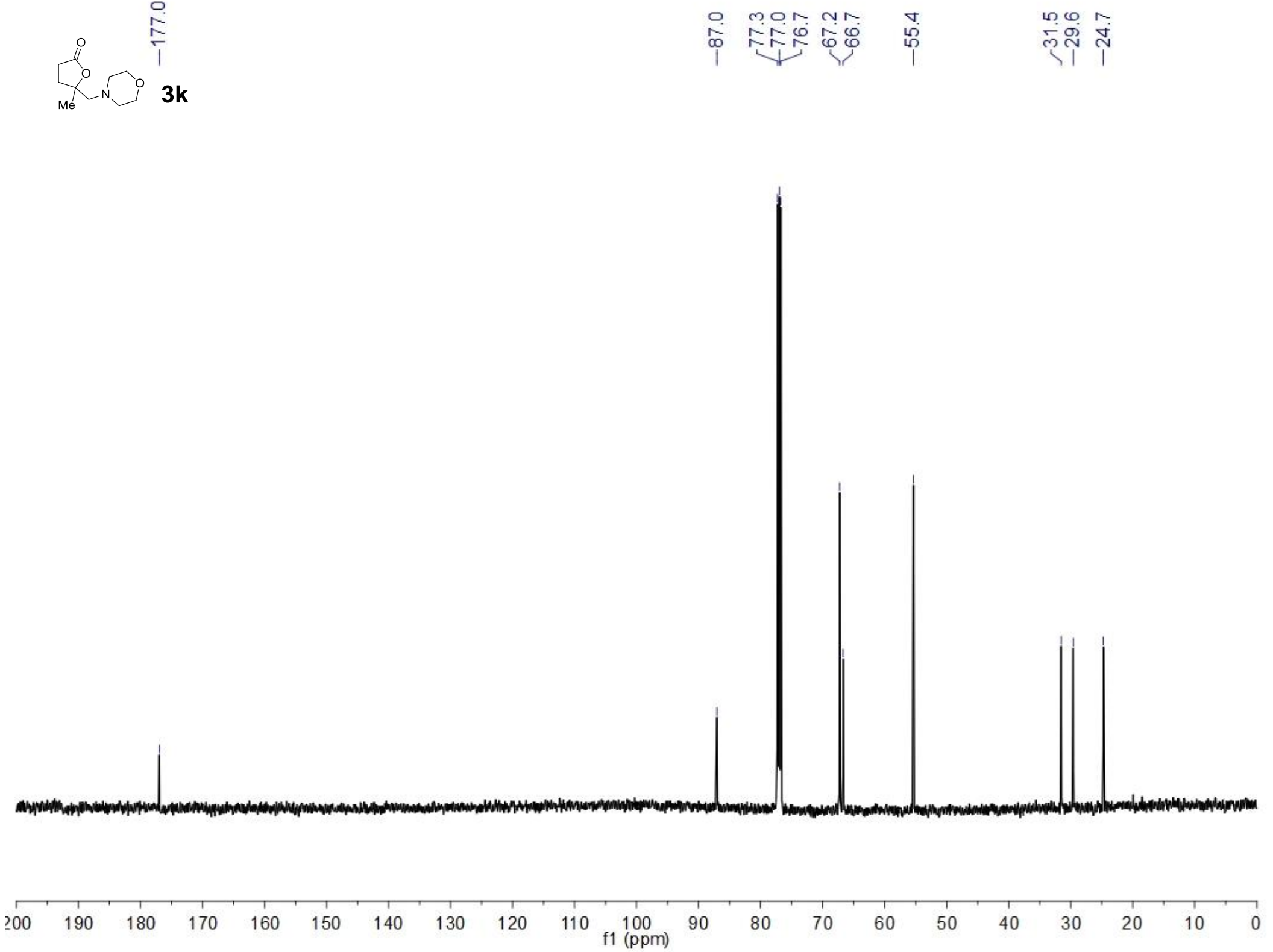


$$
\text { 3I }
$$

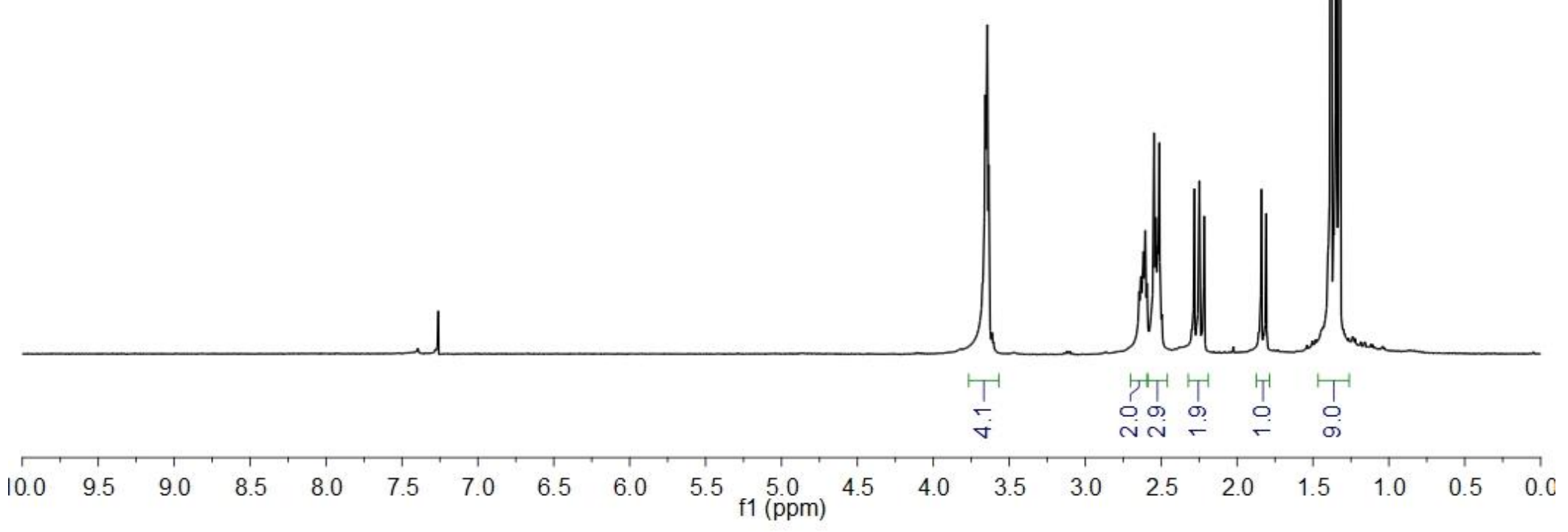




$$
\begin{aligned}
& \underset{\substack{\infty \\
i}}{\stackrel{\infty}{i}} \\
& \text { me } \underbrace{}_{\text {me }}
\end{aligned}
$$

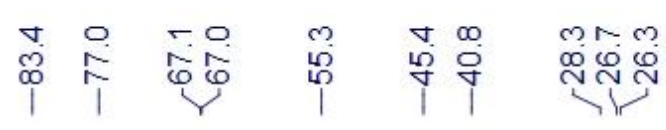

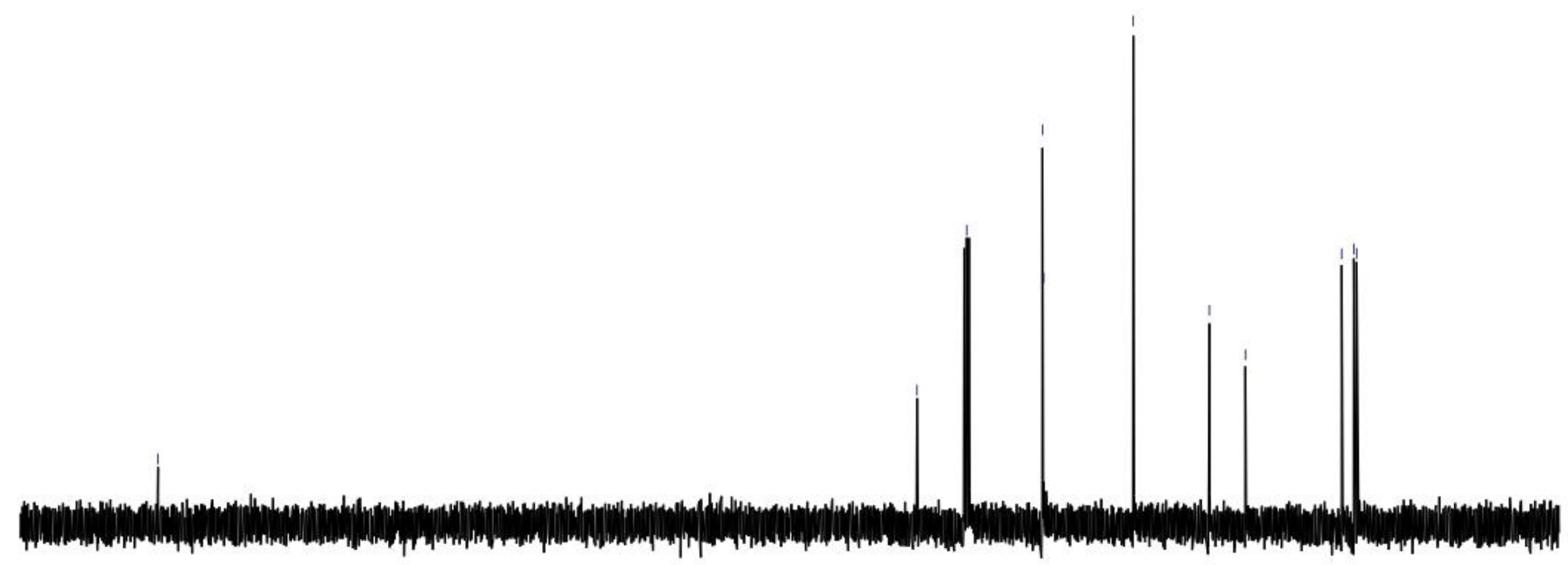




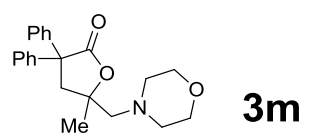

$$
3 m
$$

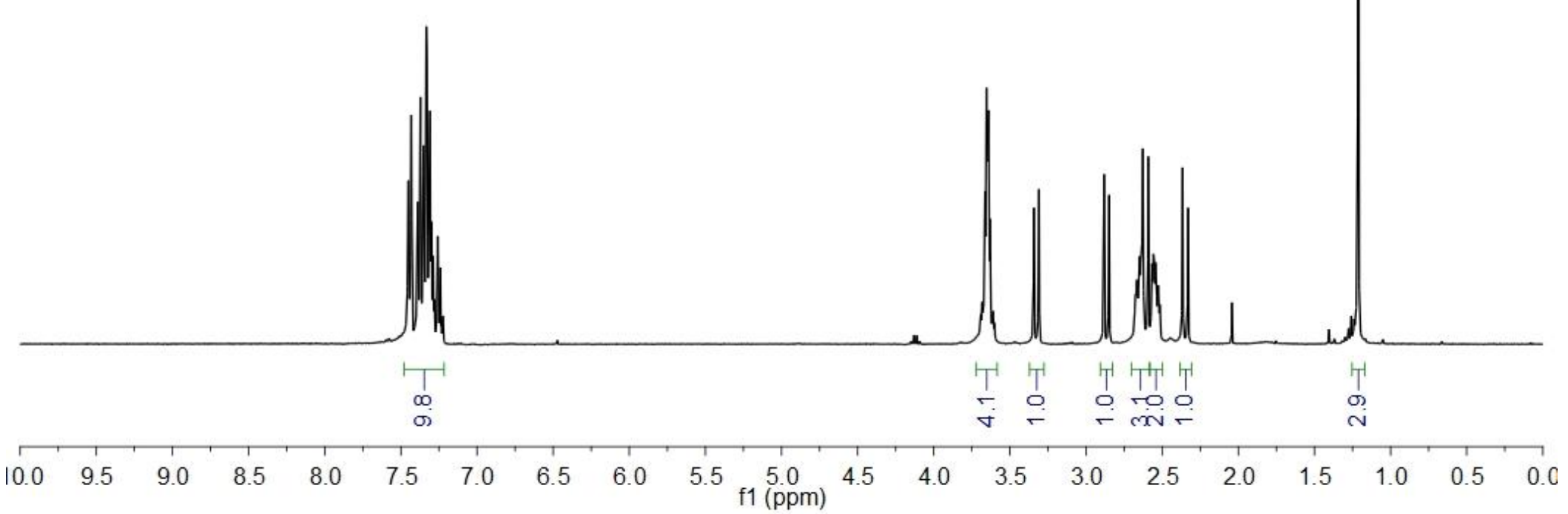



me
定

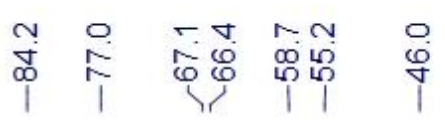
윰

.

200

$\begin{array}{lll}190 & 180 & 170\end{array}$

160

150

140

120

$110 \quad \begin{aligned} & 100 \\ & \text { f1 }(\mathrm{ppm})\end{aligned}$

80

70

60

50

$40 \quad 30$

20

10 

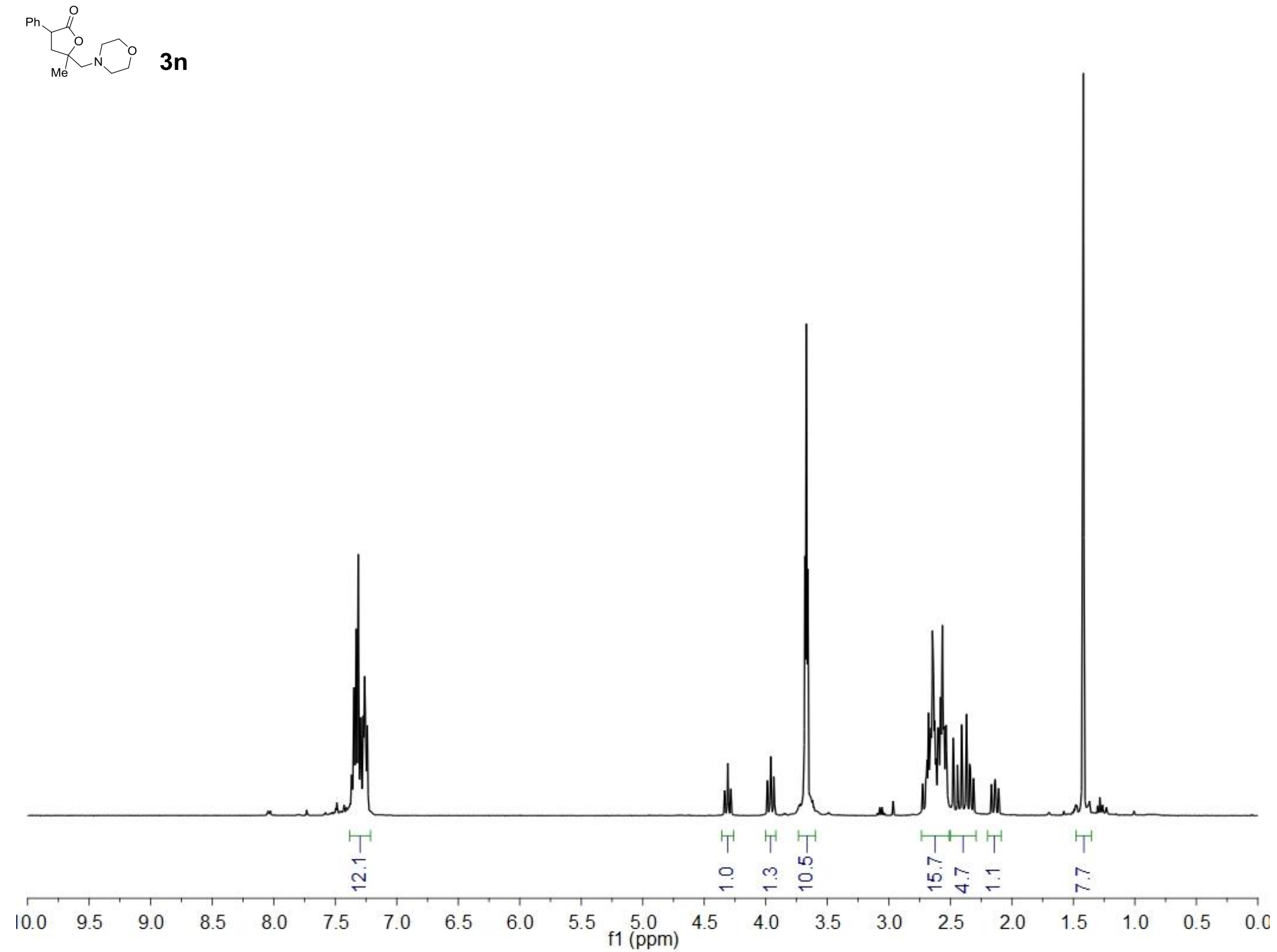

S141 

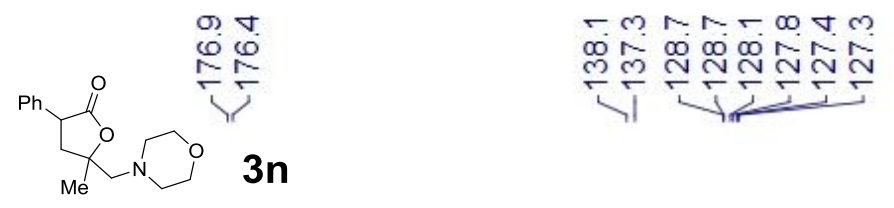

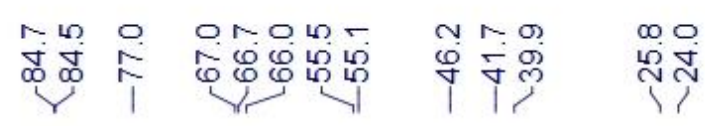

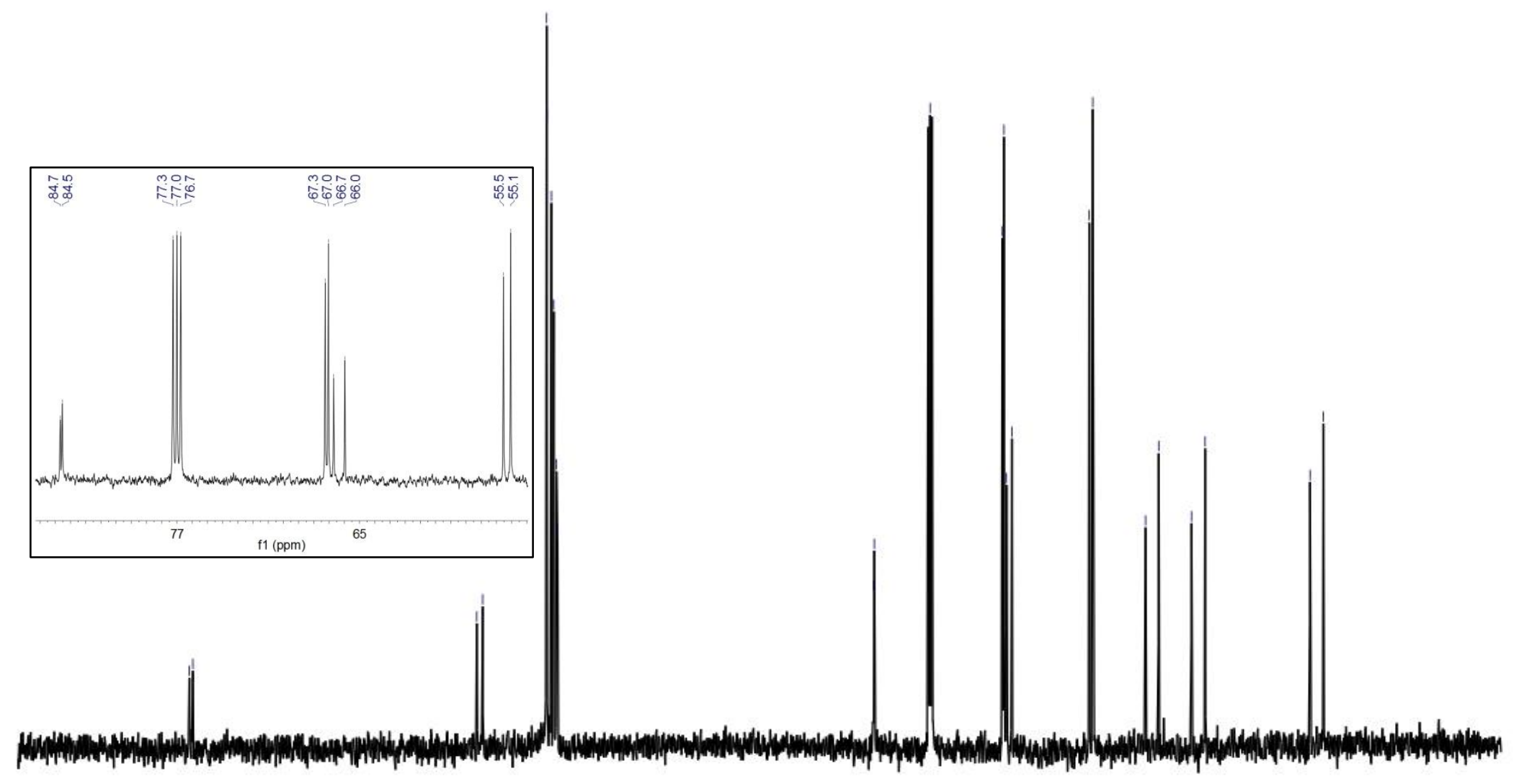




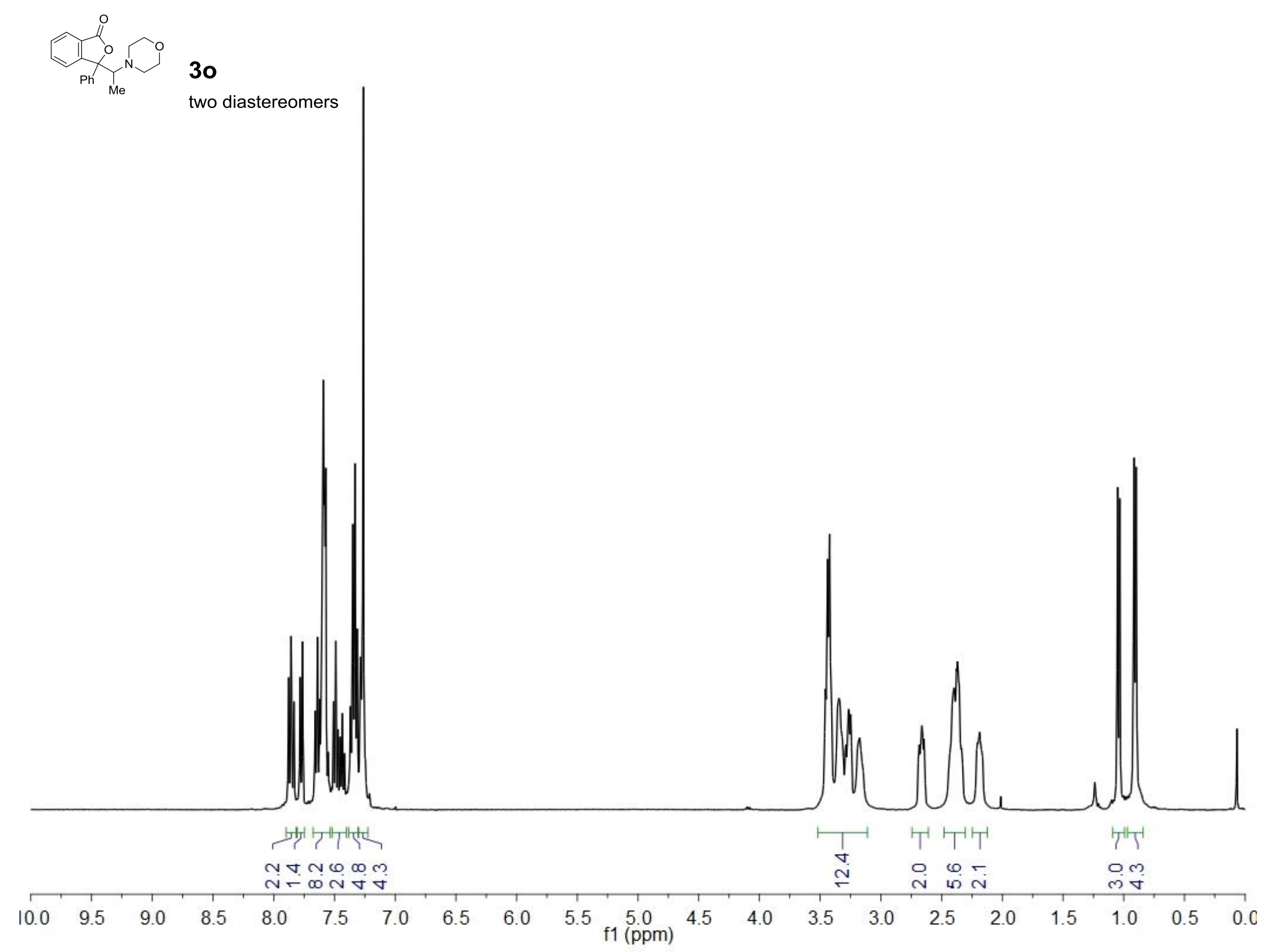



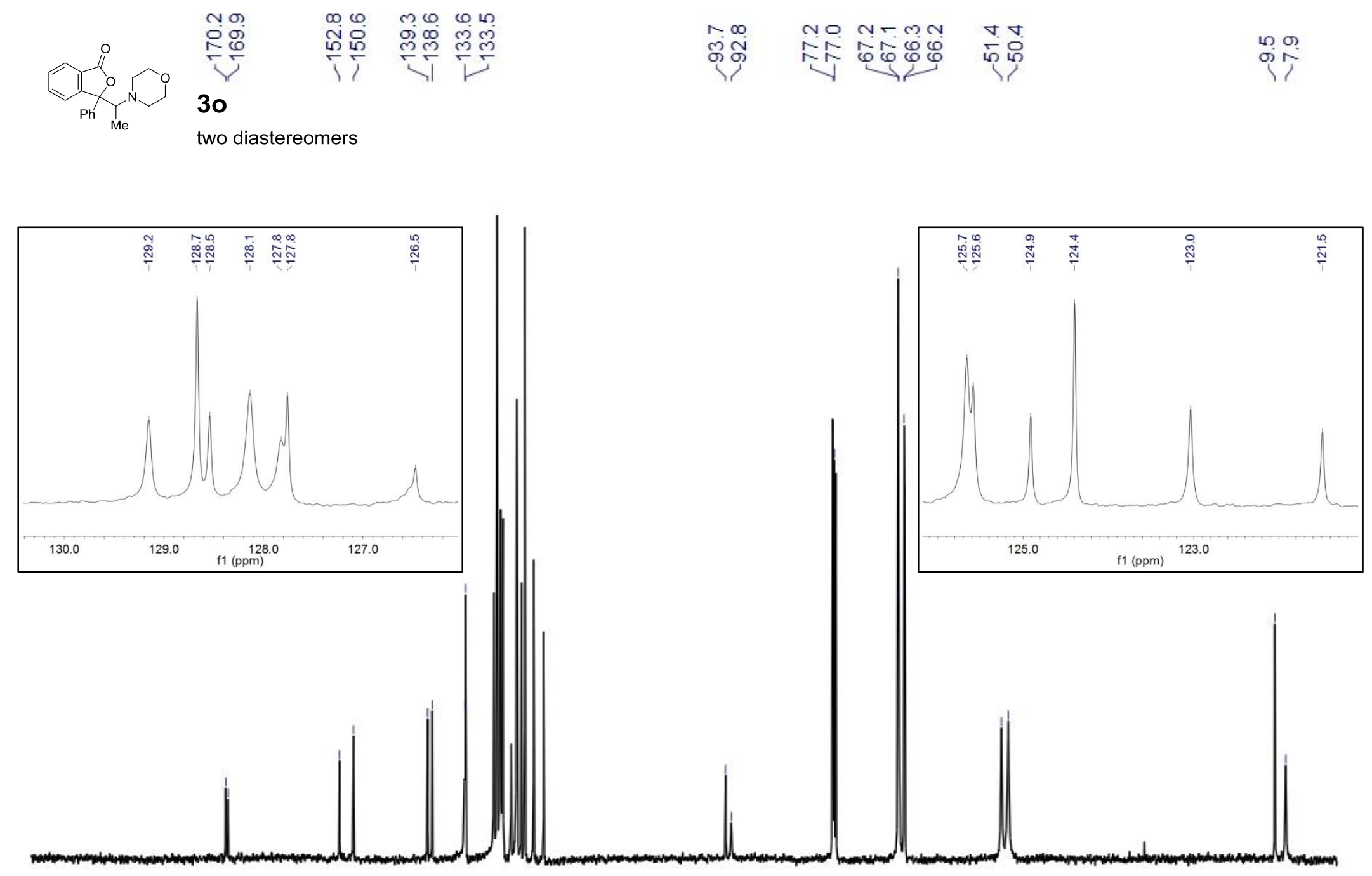

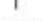

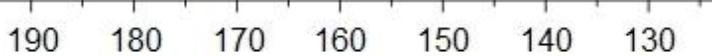

120

$110 \quad 100$

80

70

60

$50 \quad 40$

$\begin{array}{lllll}1 & 30 & 20 & 10 & 0\end{array}$ 


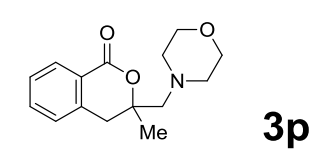

$3 p$

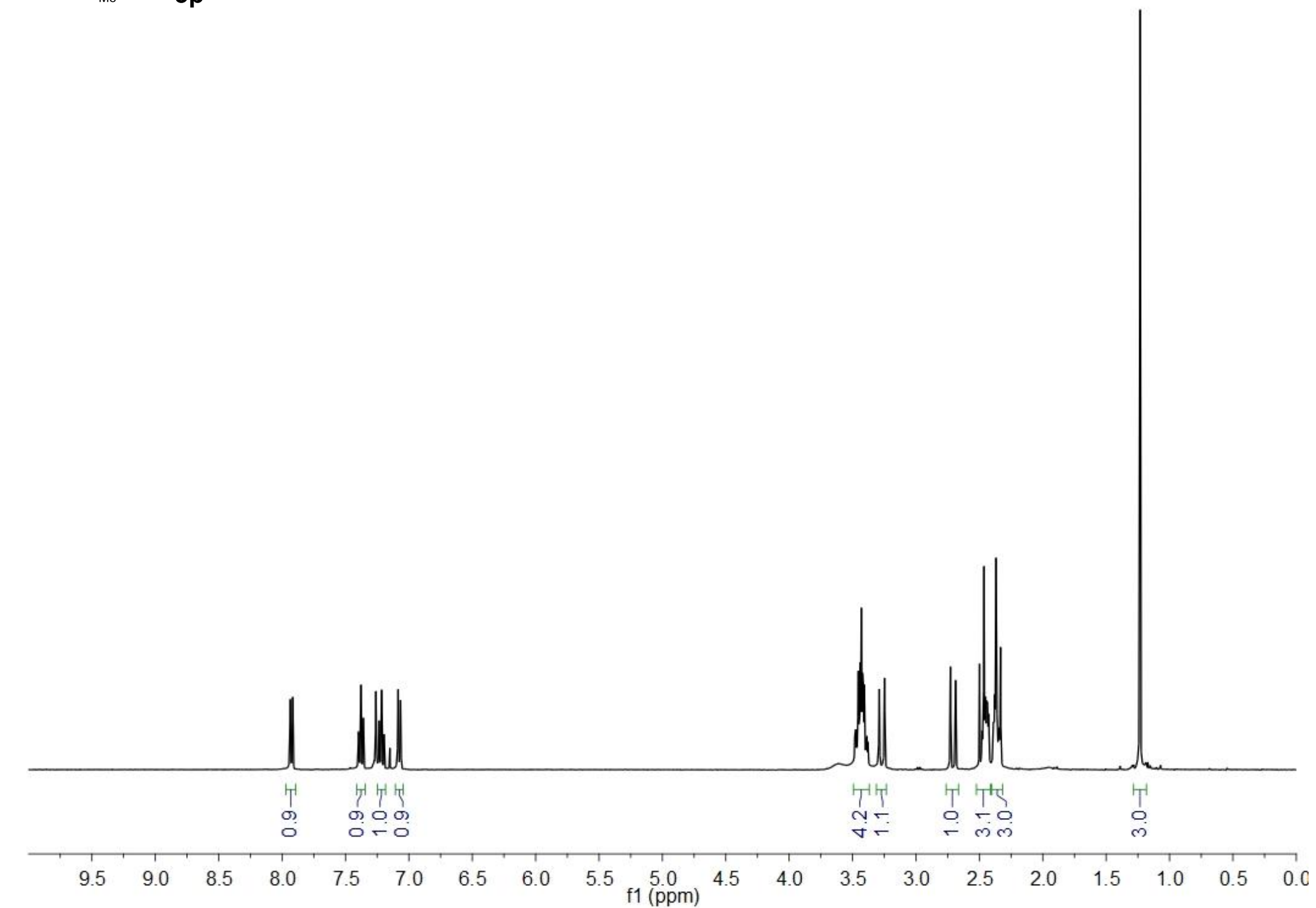




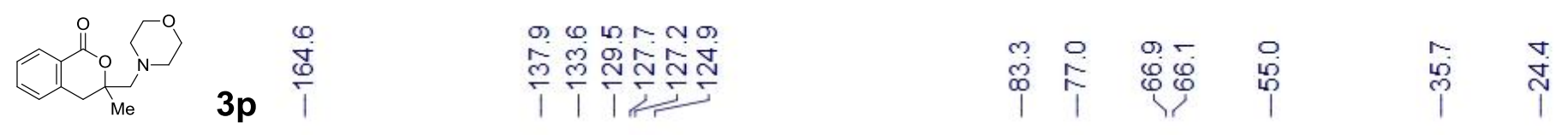

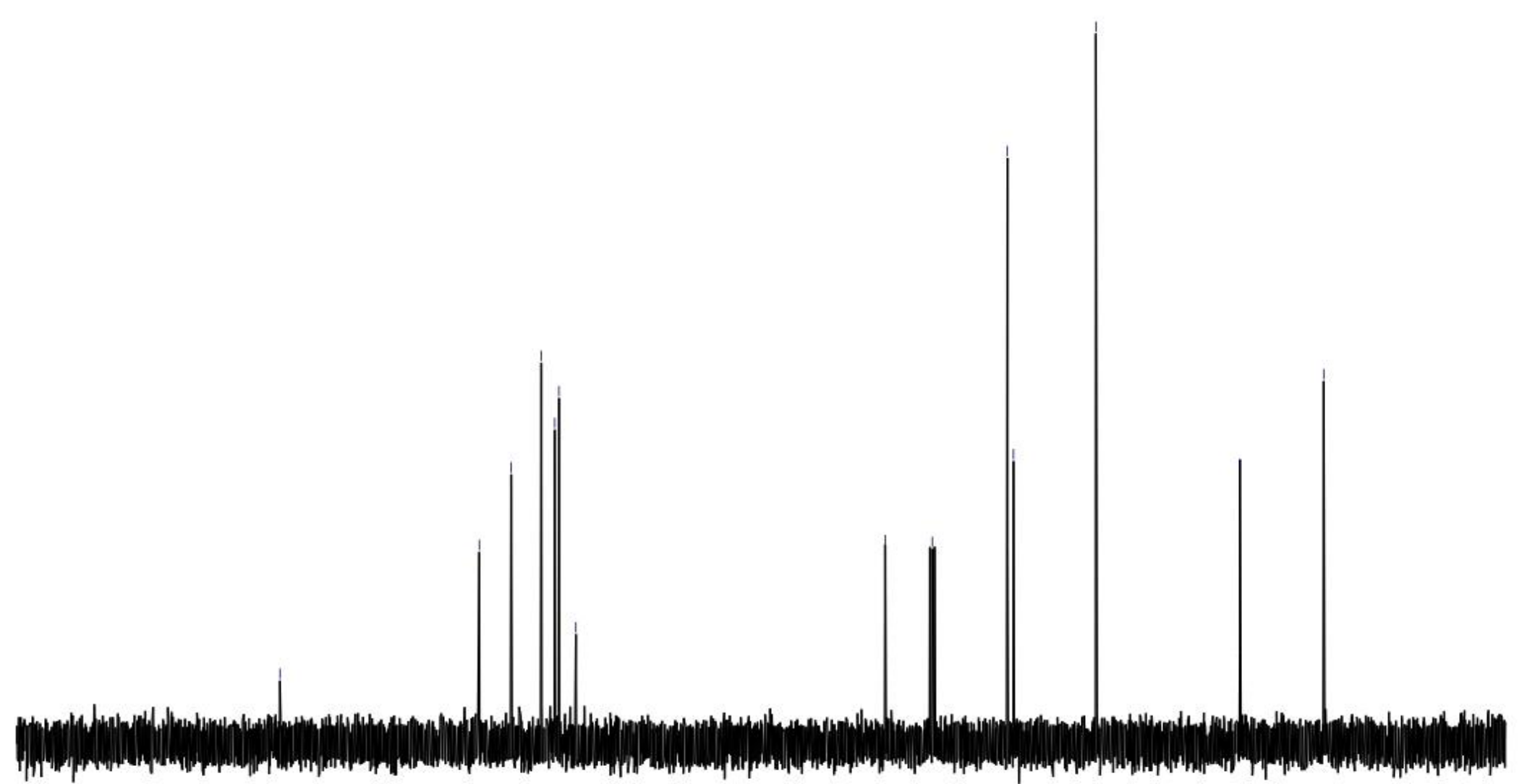




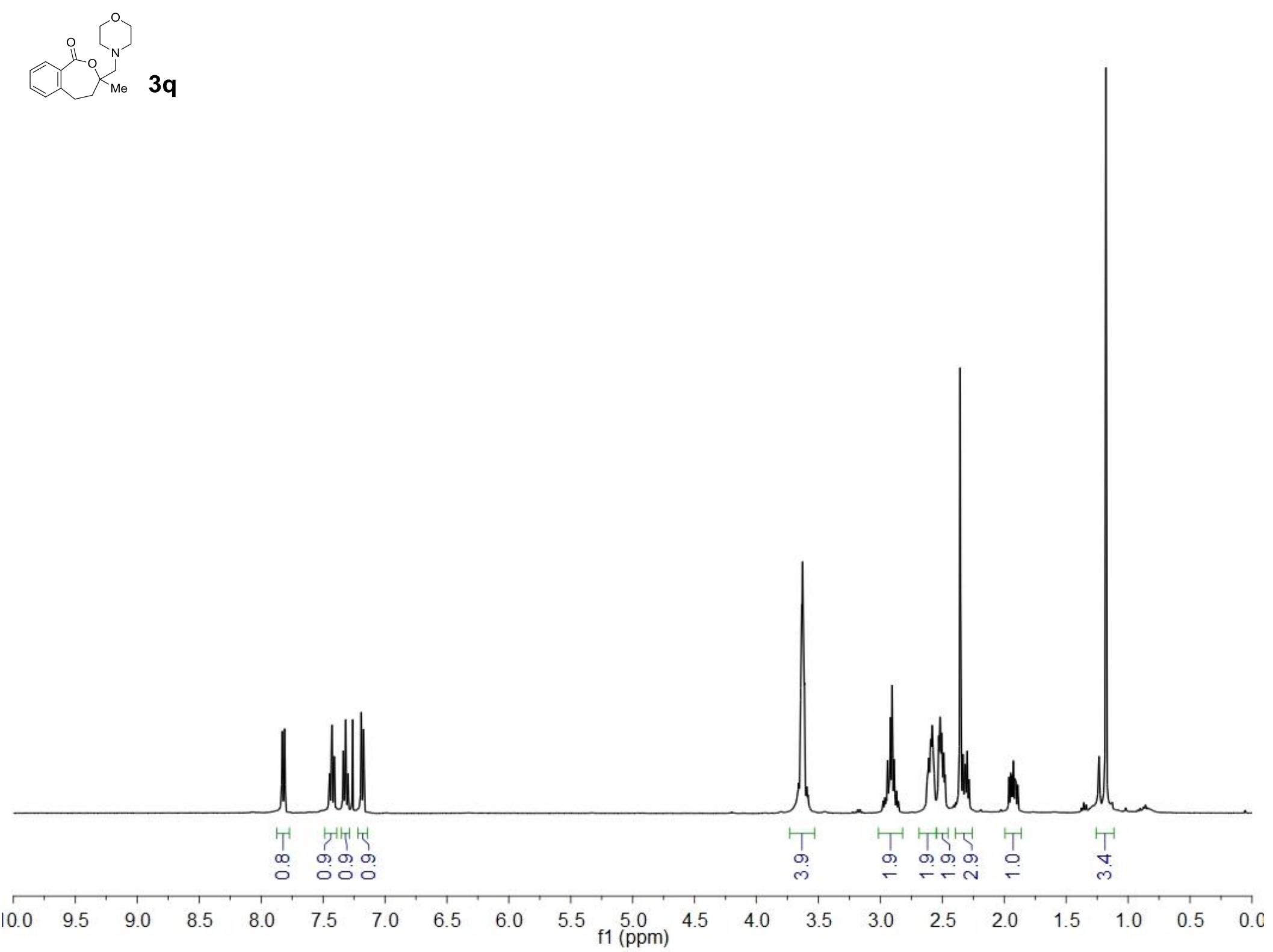



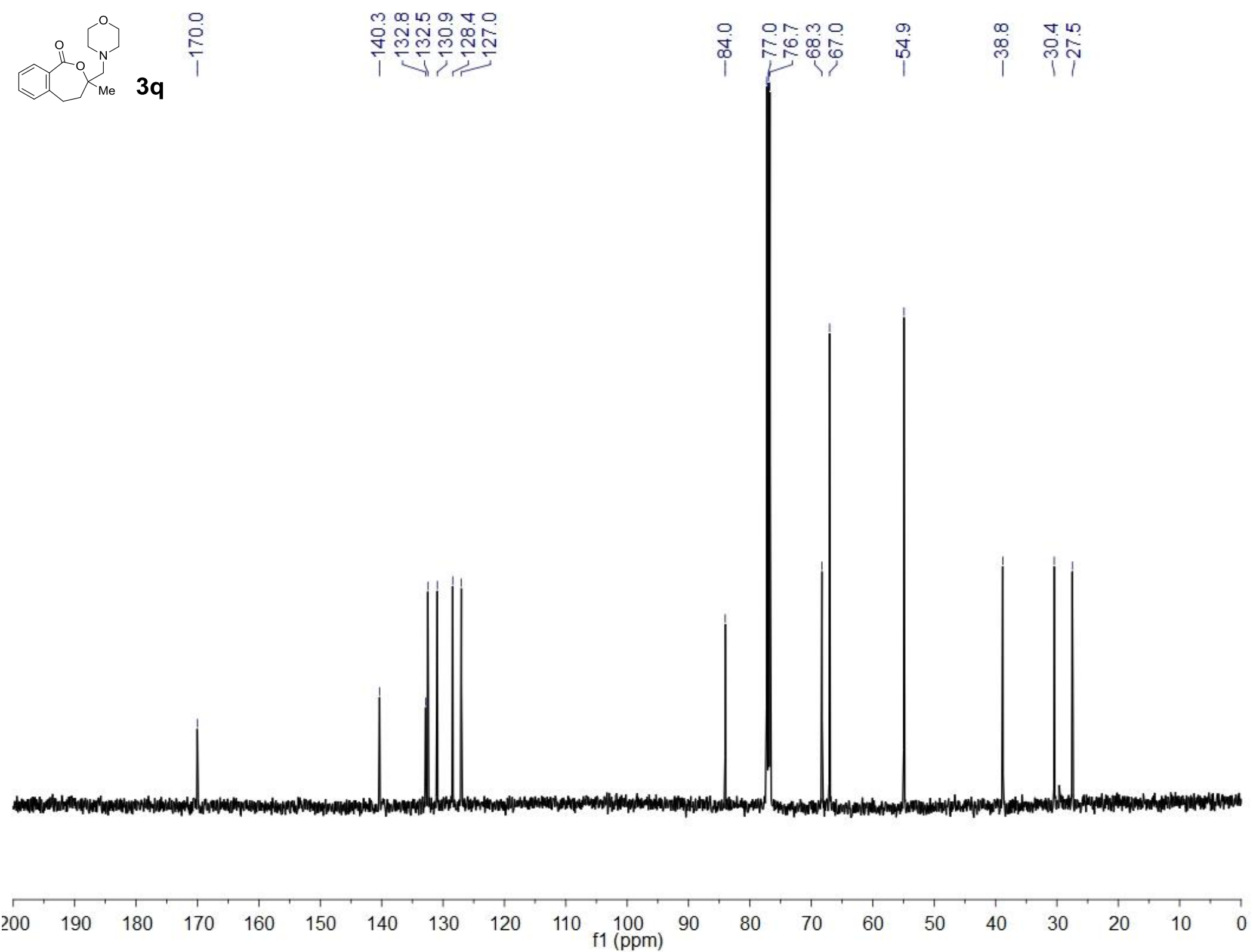

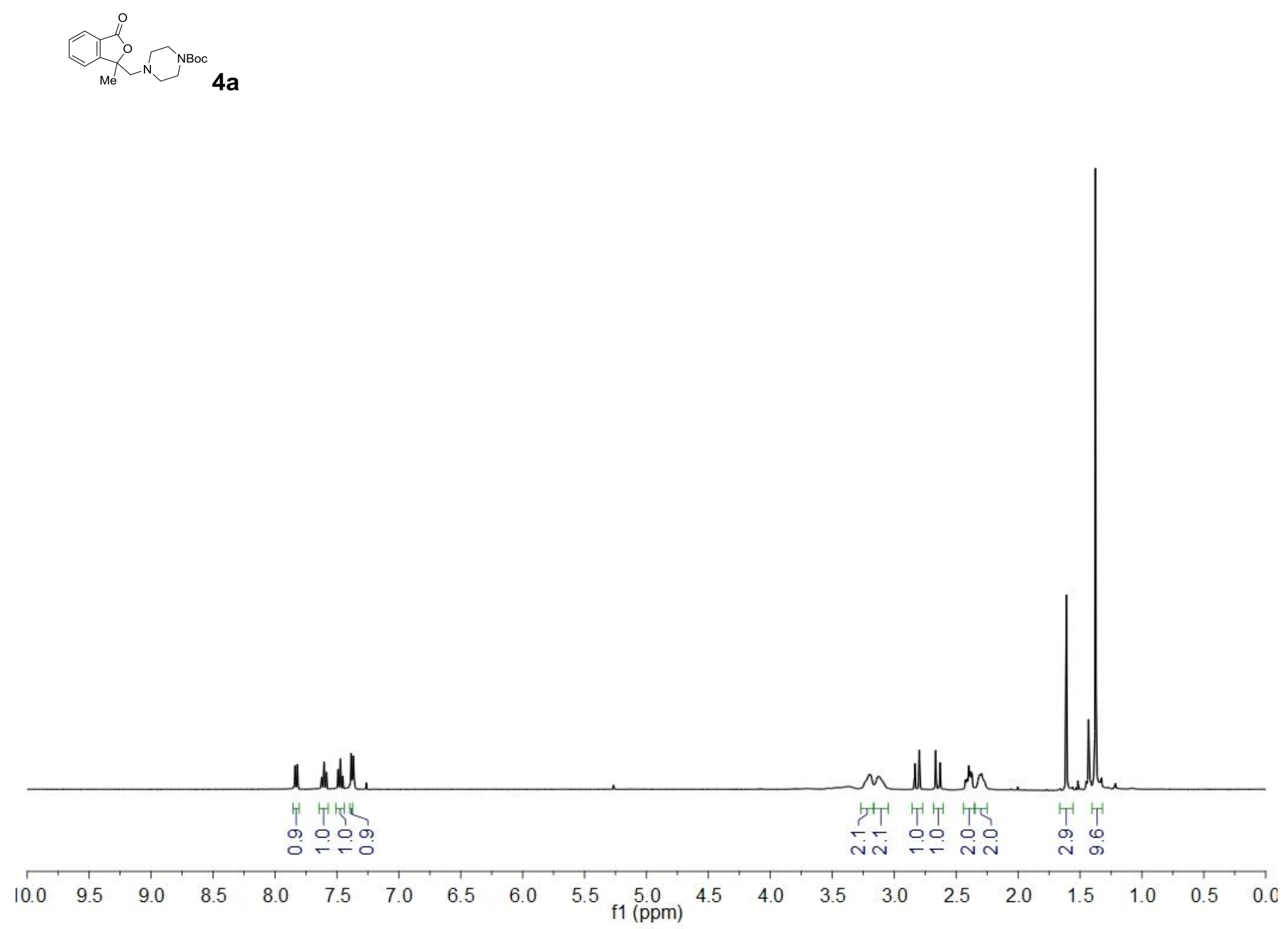


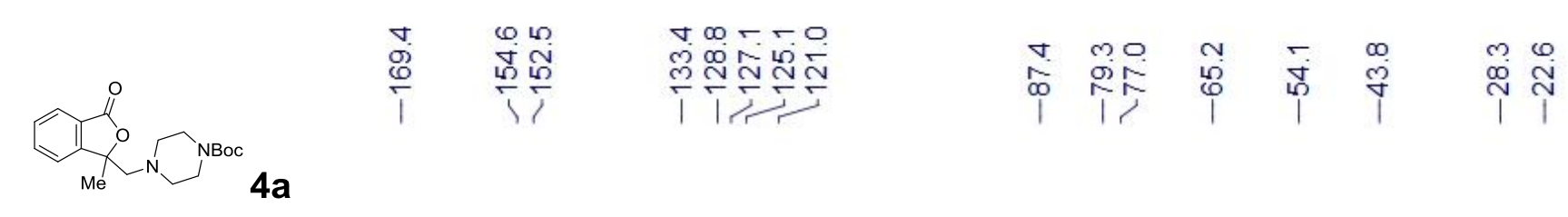
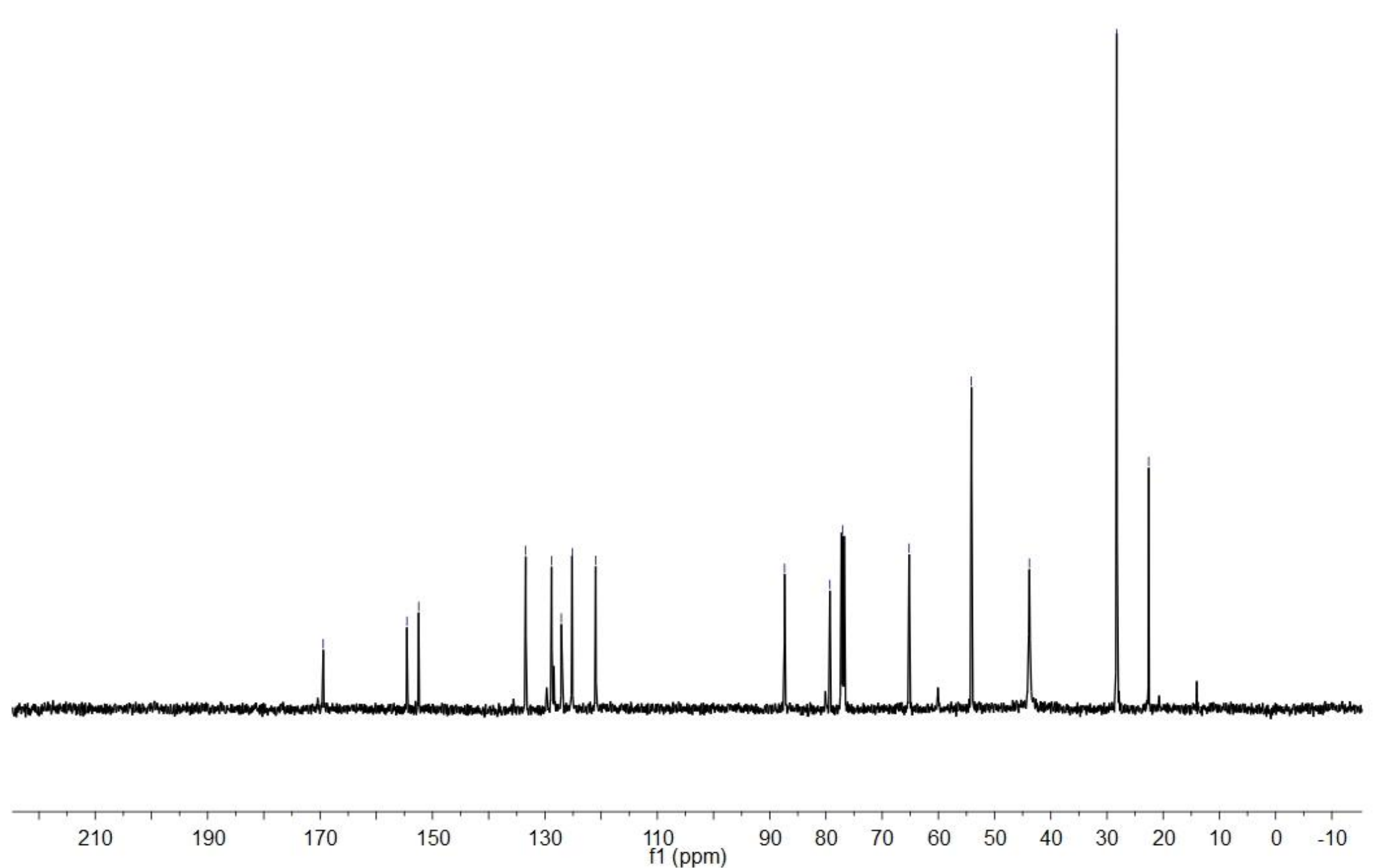

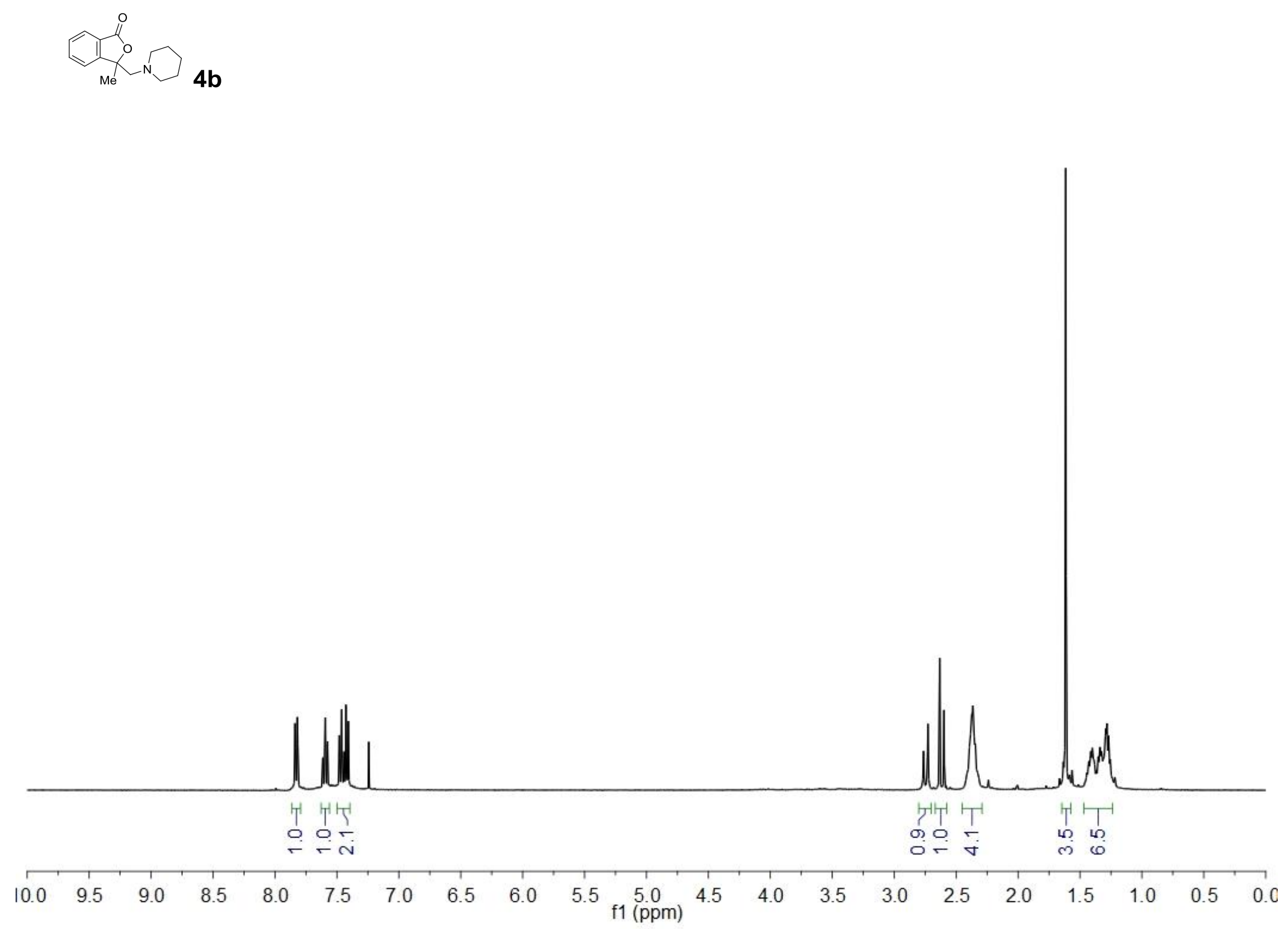

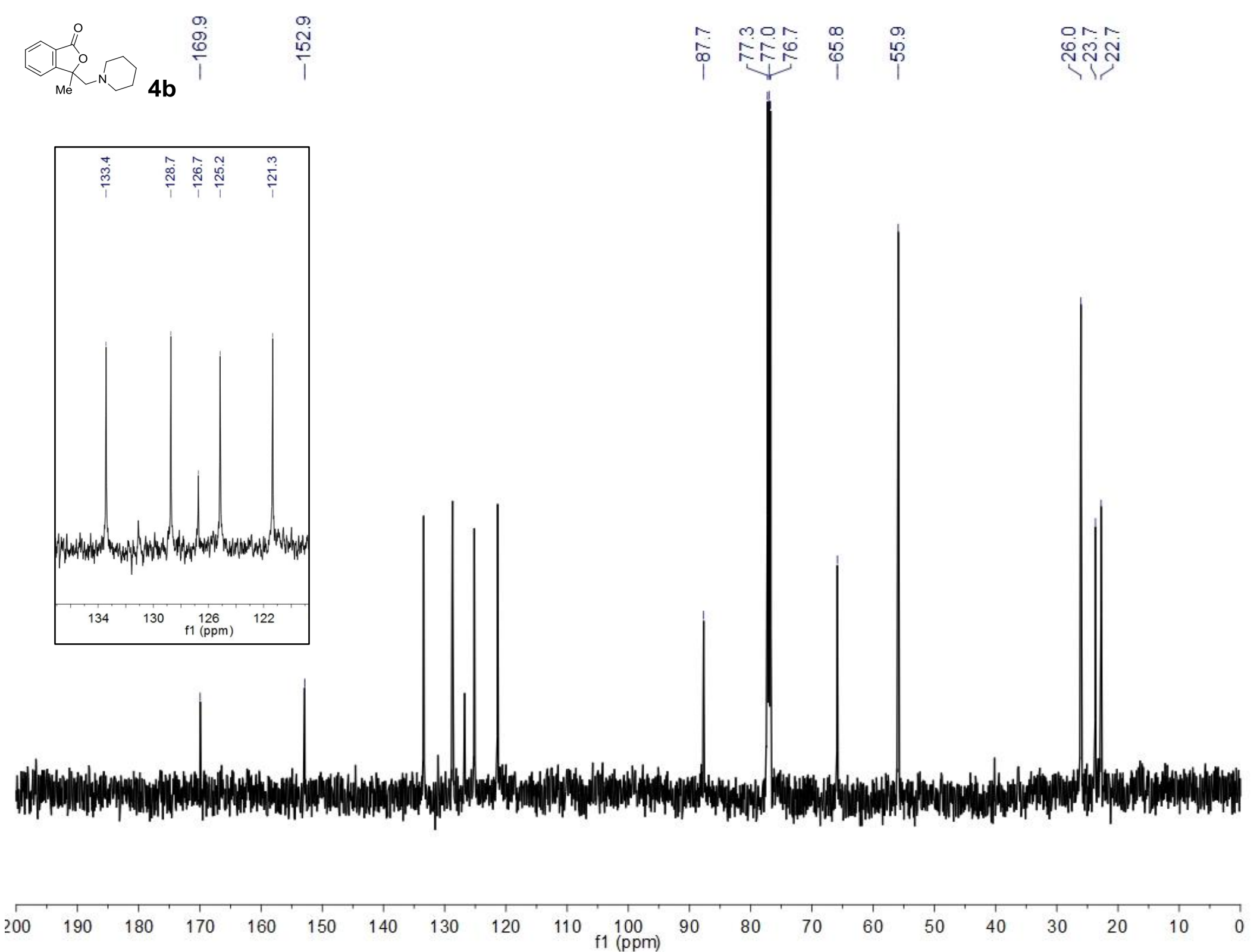
4c

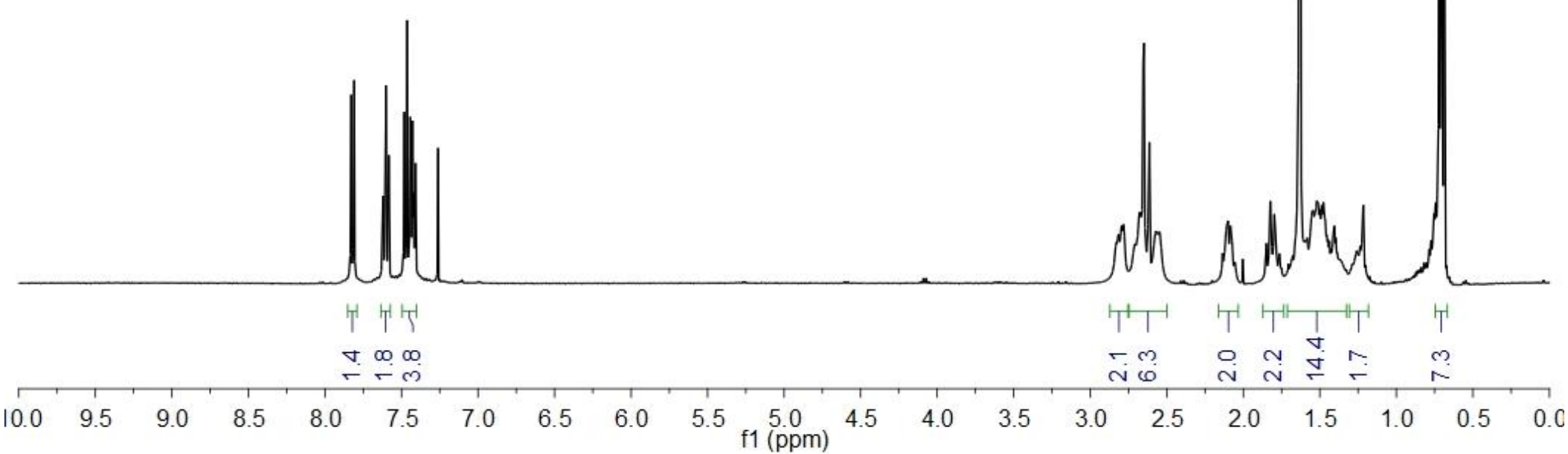




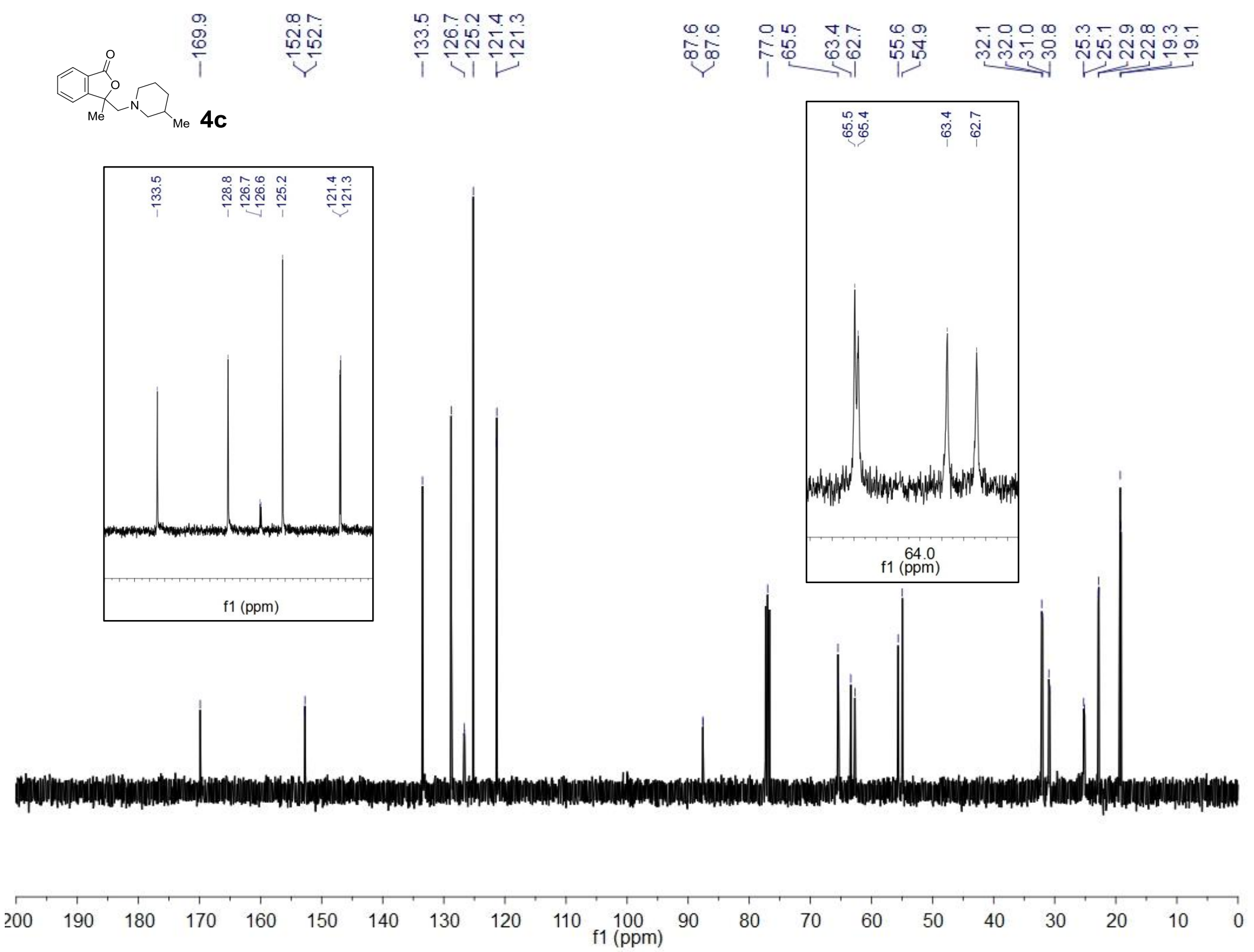



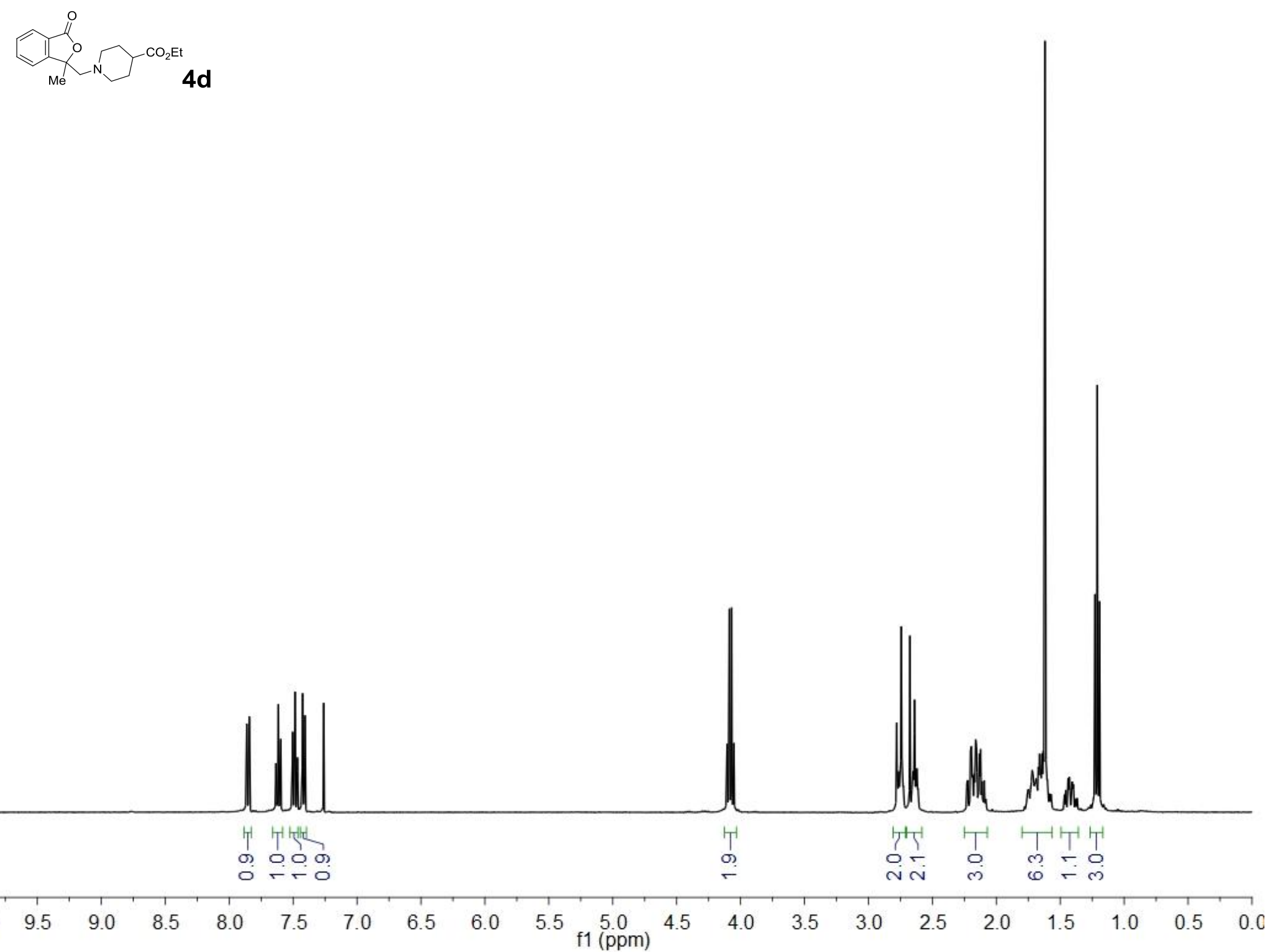


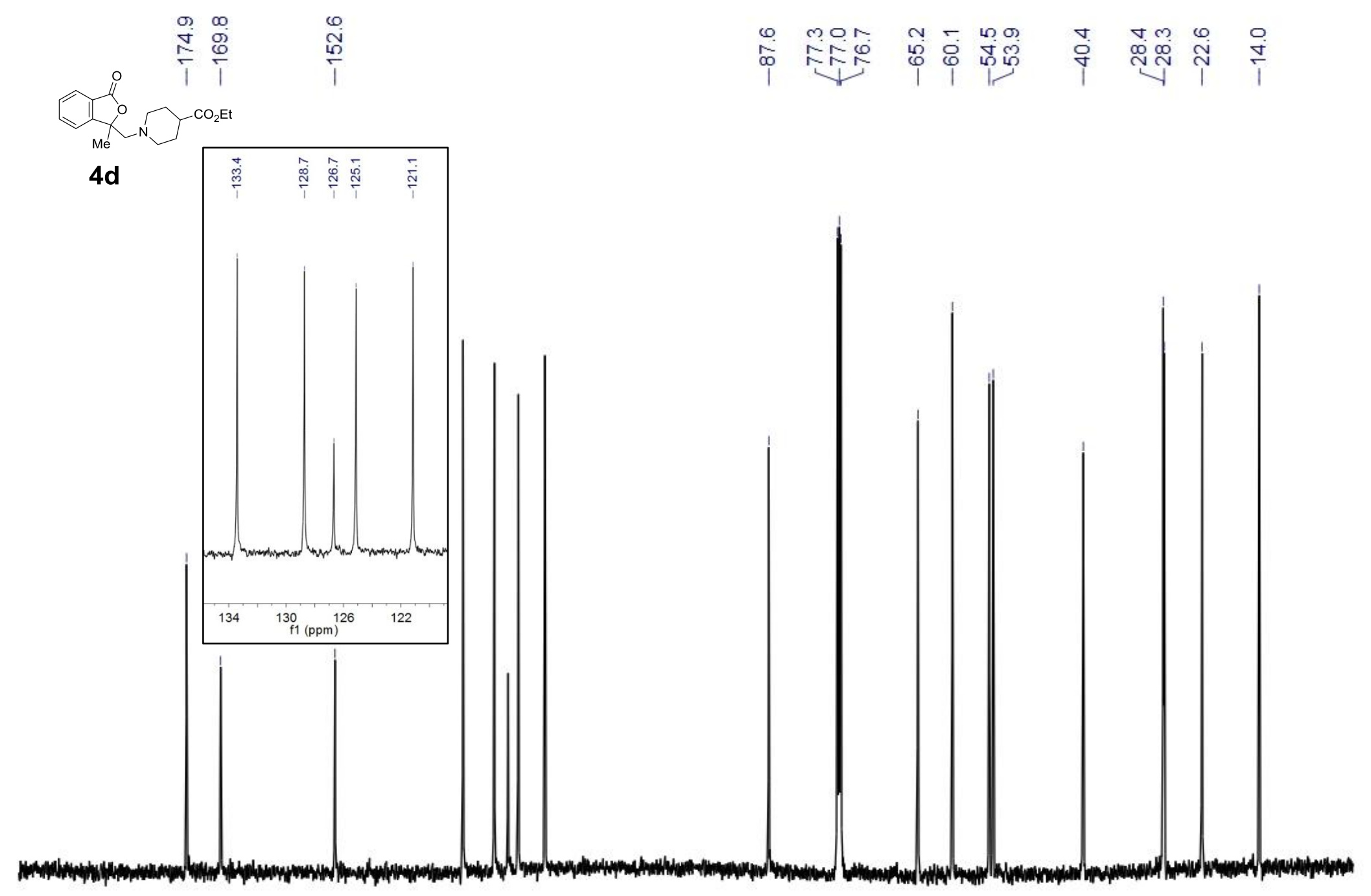



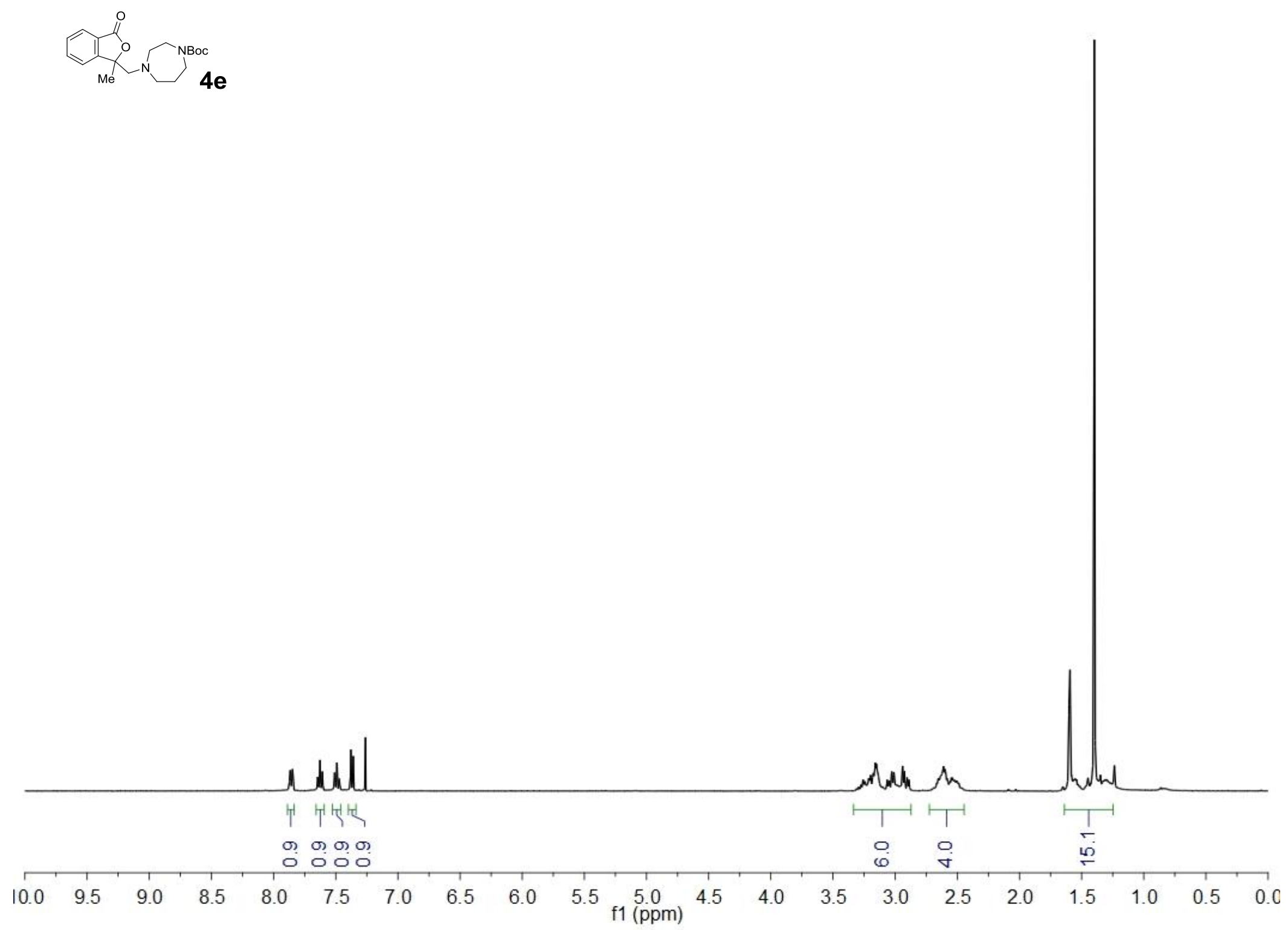
Pe

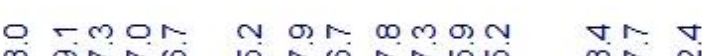

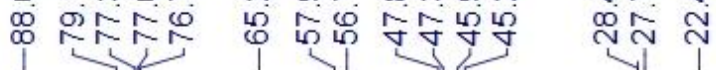

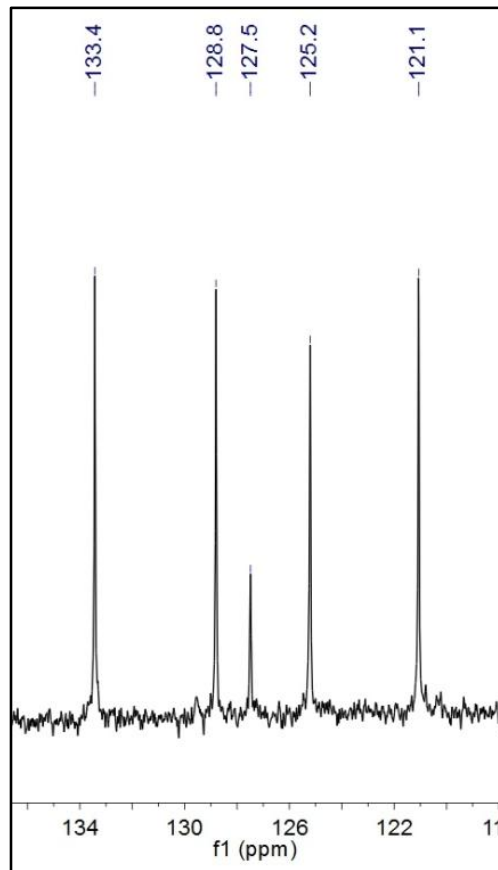

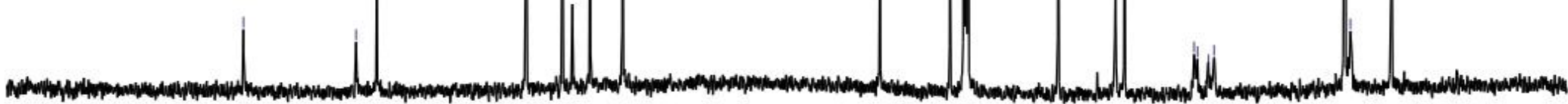

200

190

$180 \quad 170$

160

$150 \quad 140$

130

120

$110 \quad 100$

80

70

60

50

40

30

20

10 

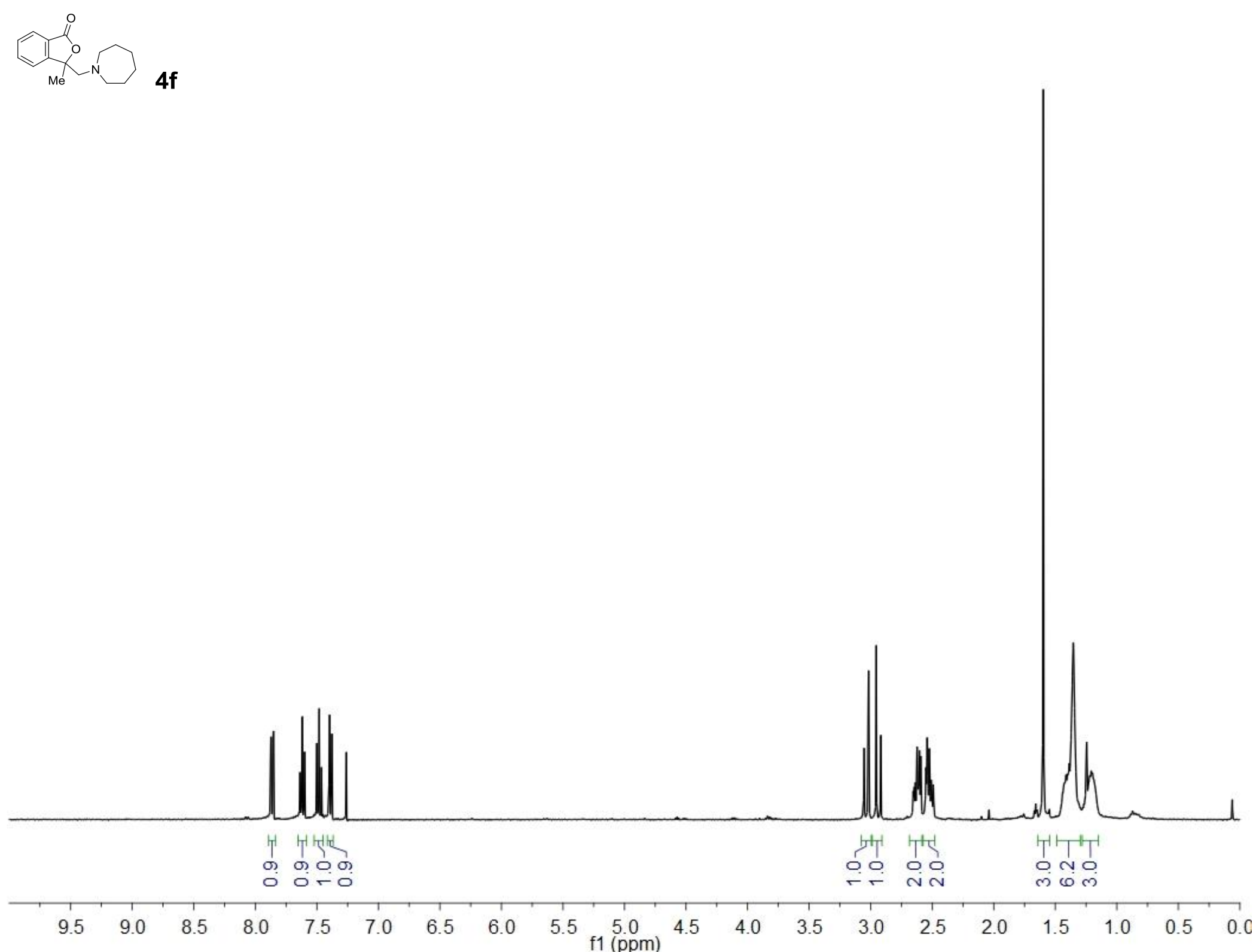
$\operatorname{men}_{4}^{2}$

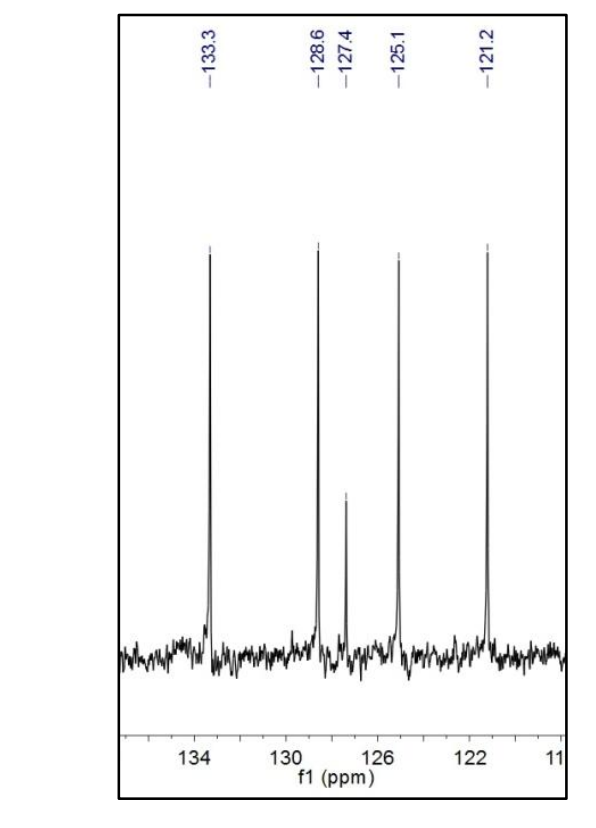

क

$\infty \infty$ in

N N

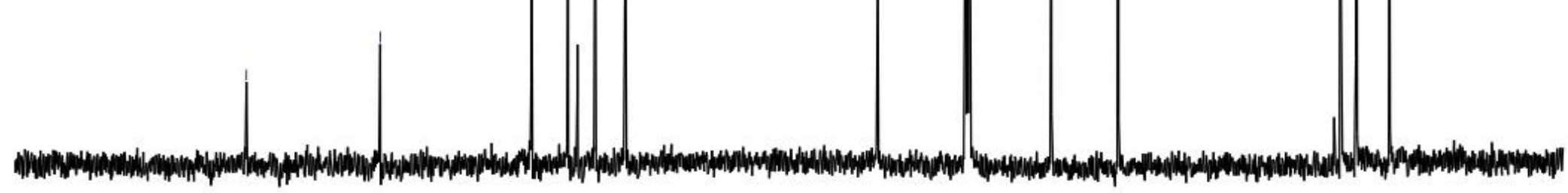

200

$\begin{array}{llllll}190 & 180 & 170 & 160 & 150 & 140\end{array}$

130

120

$110 \quad 100$

80

70

60

50

$40 \quad 30$

$2010 \quad 0$ 


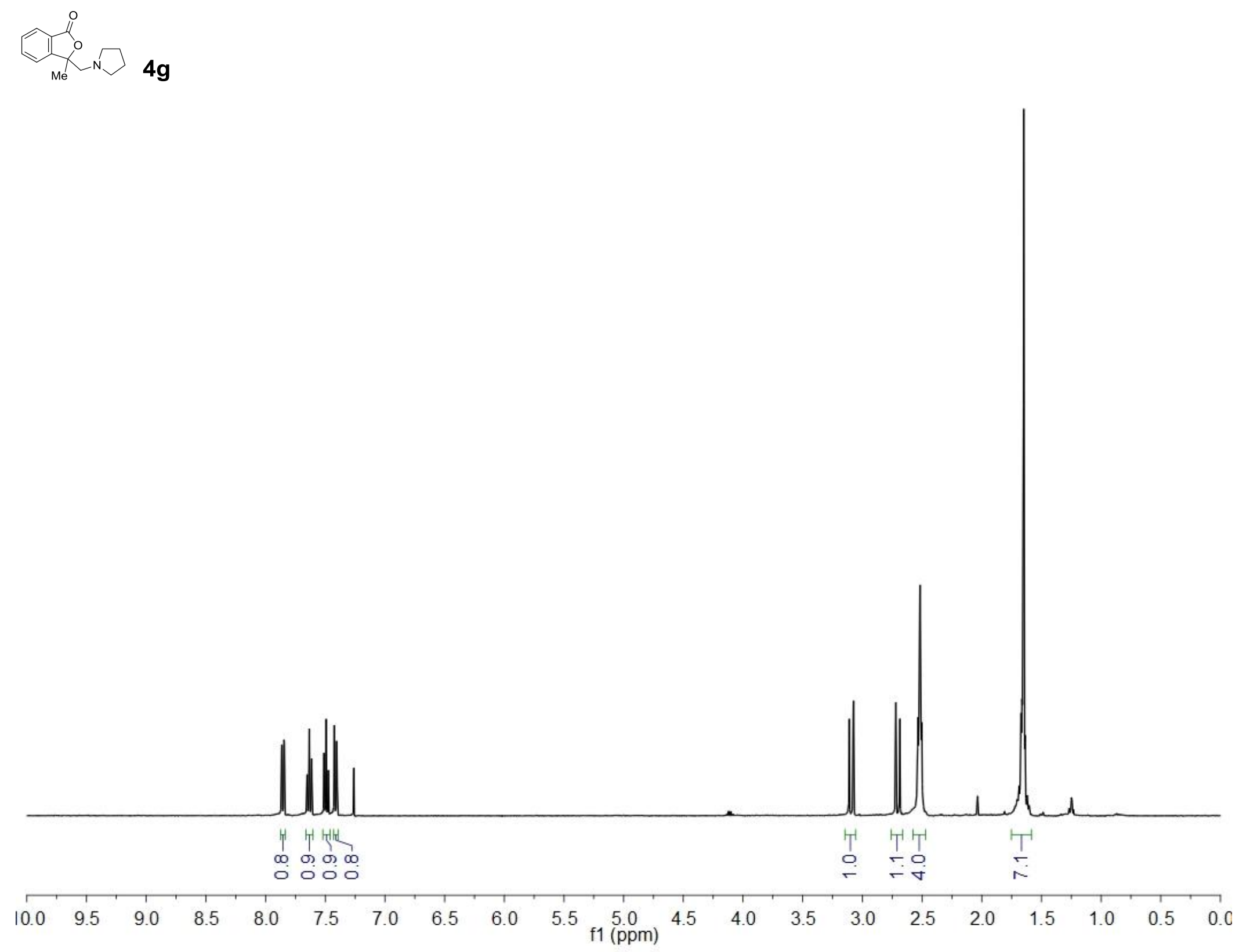




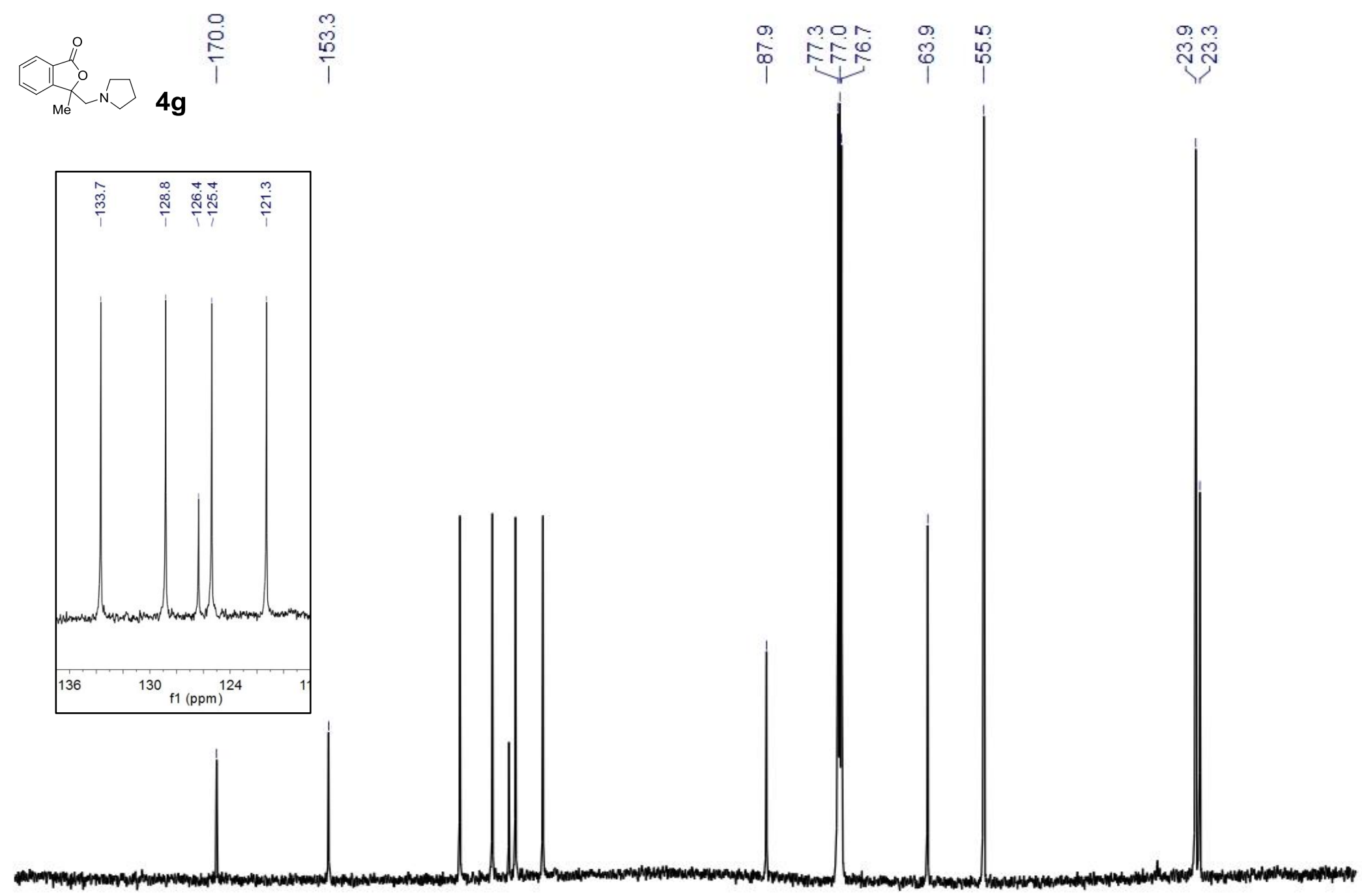

200

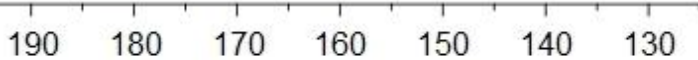

120

$110 \quad 100$

80

70

60

50

$40 \quad 30$

20

$10 \quad 0$ 

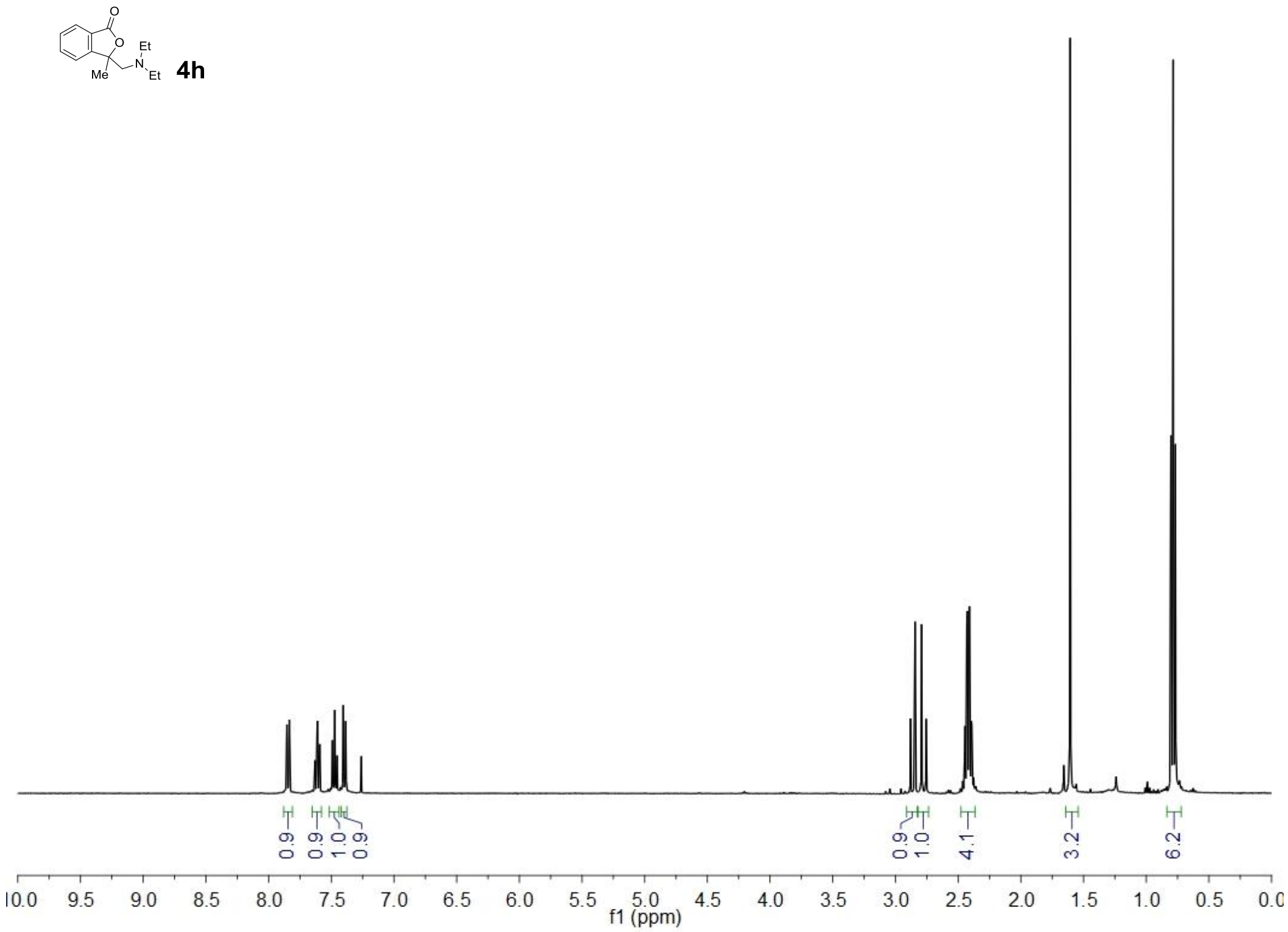


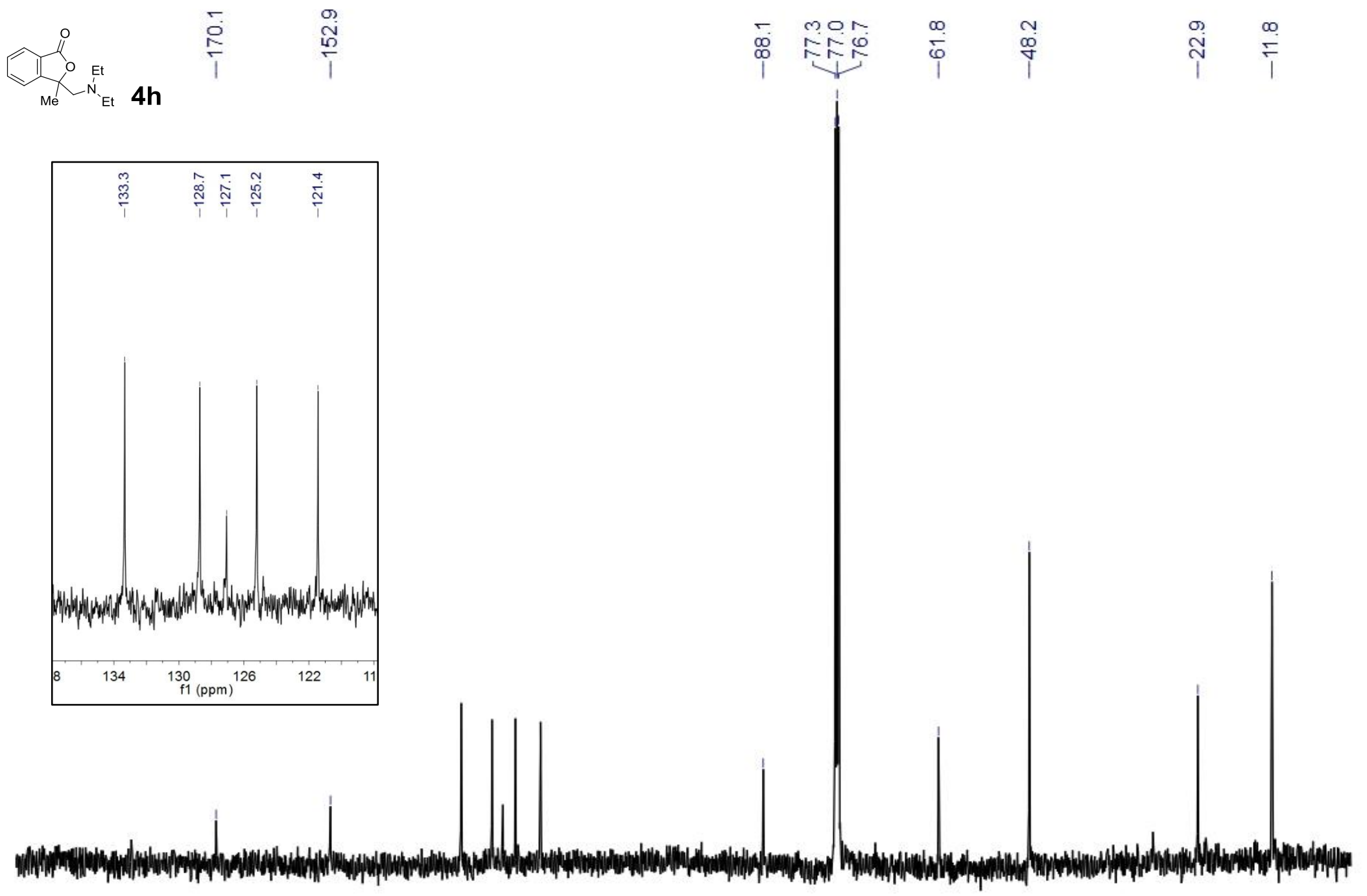




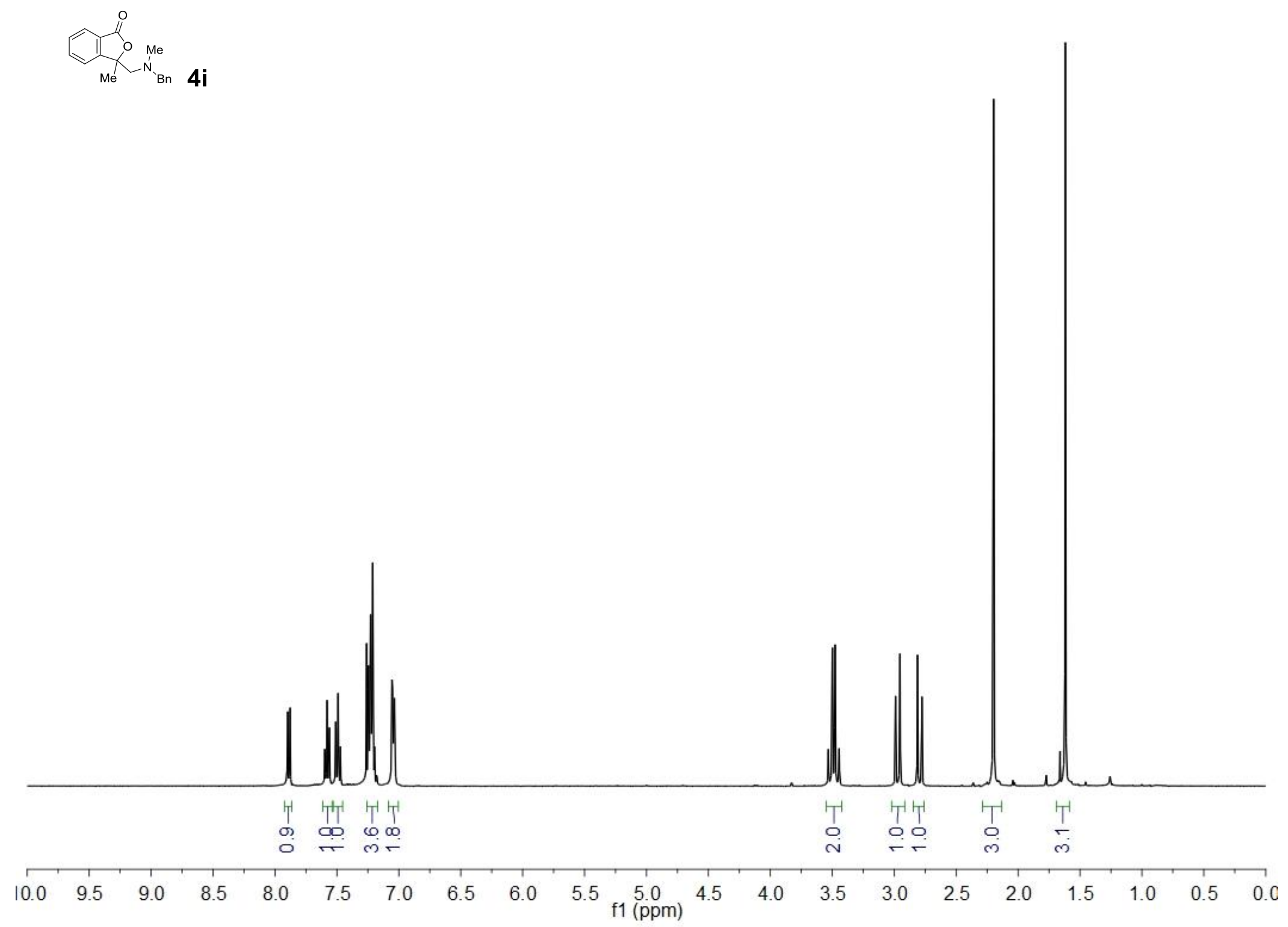




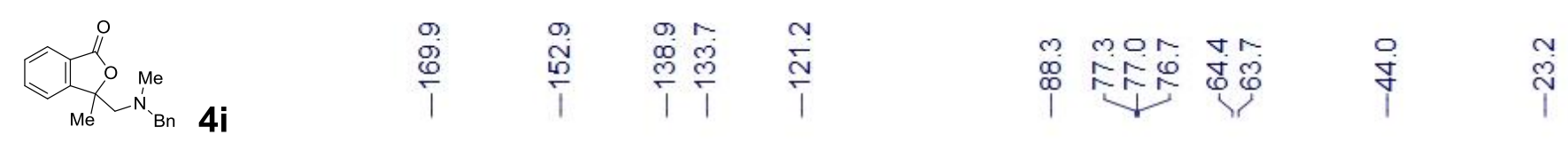
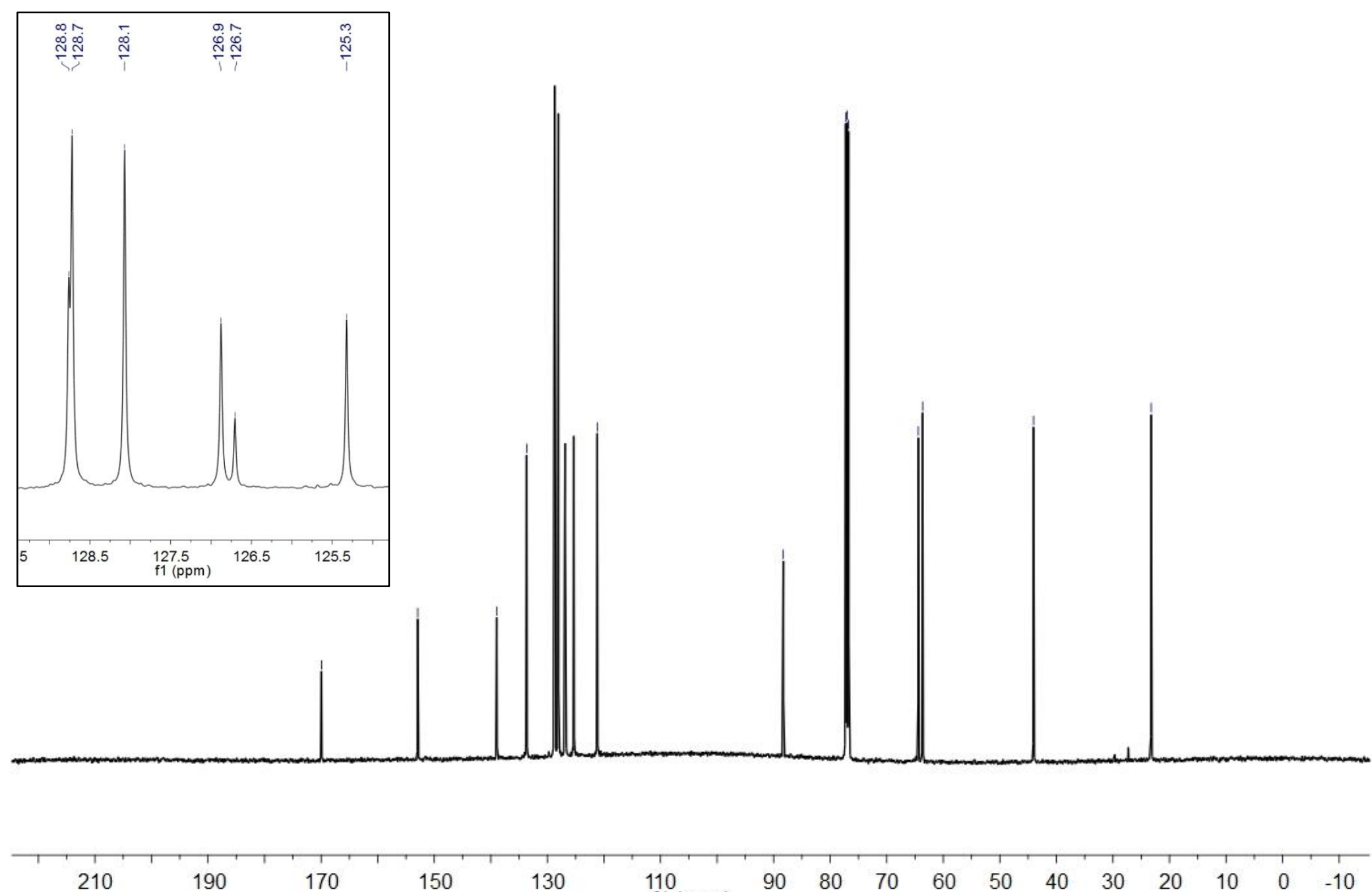

130 

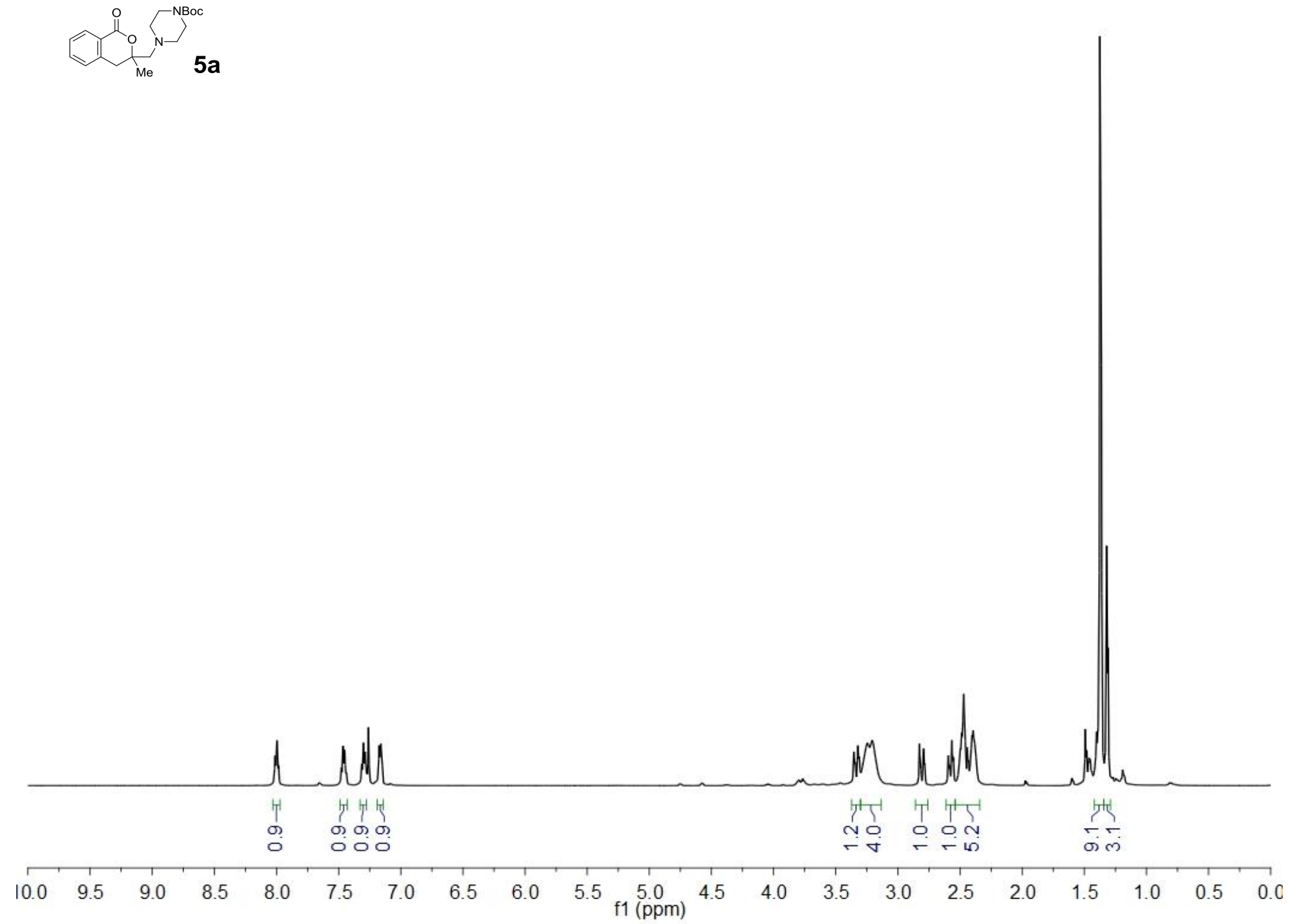

$\alpha^{2} x^{5}$

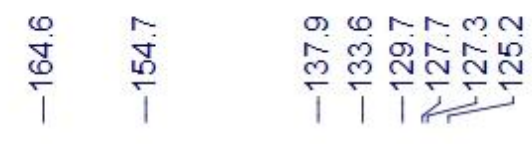

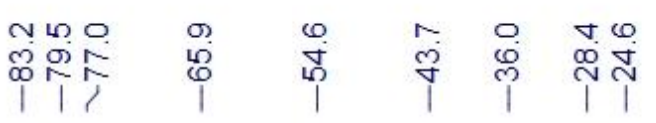$$
\text { . }
$$

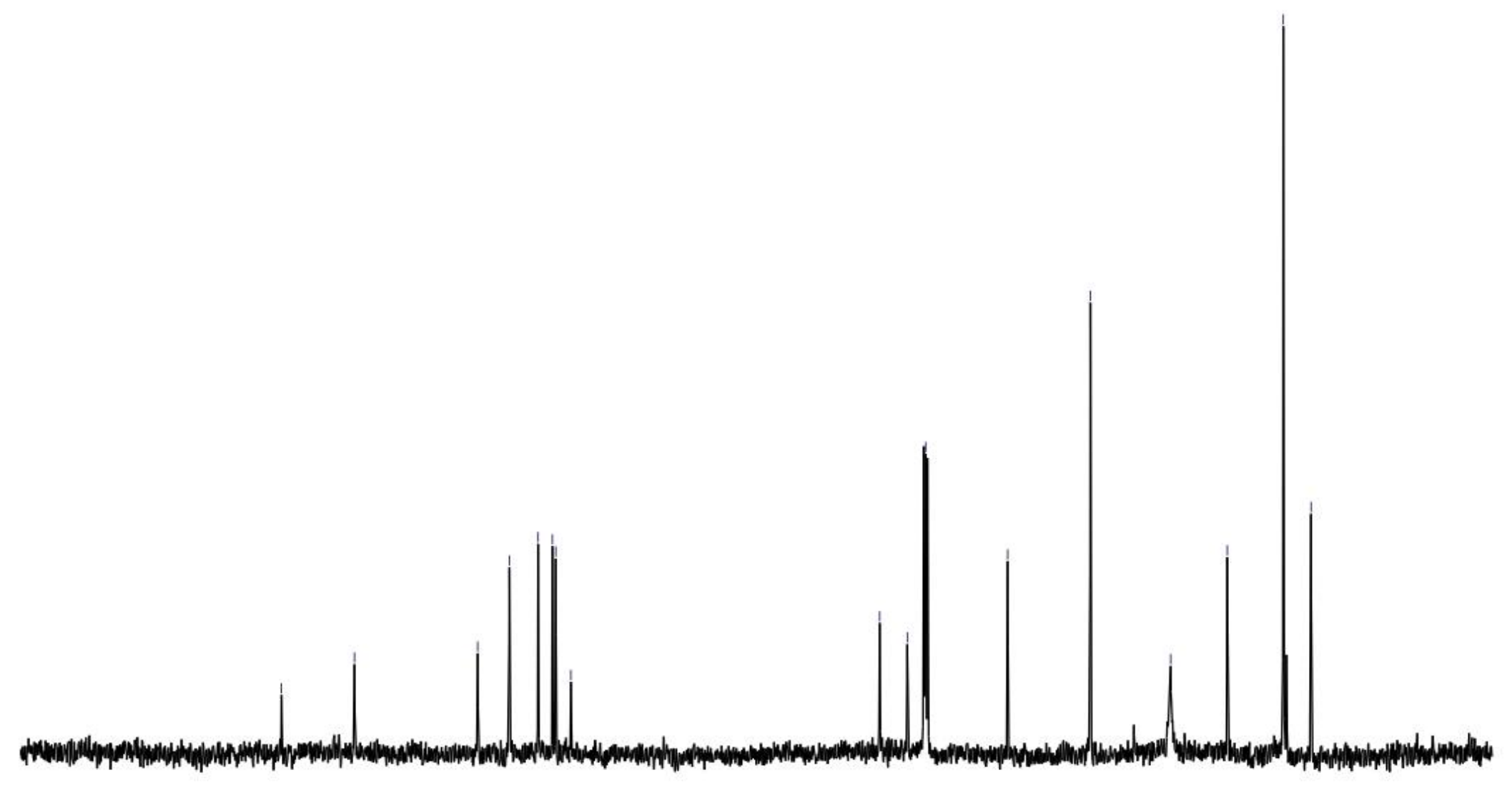

200

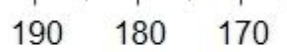

160

$150 \quad 140$

130

120

$110 \quad 100$

8070

60

50

$40 \quad 30$

20

10 

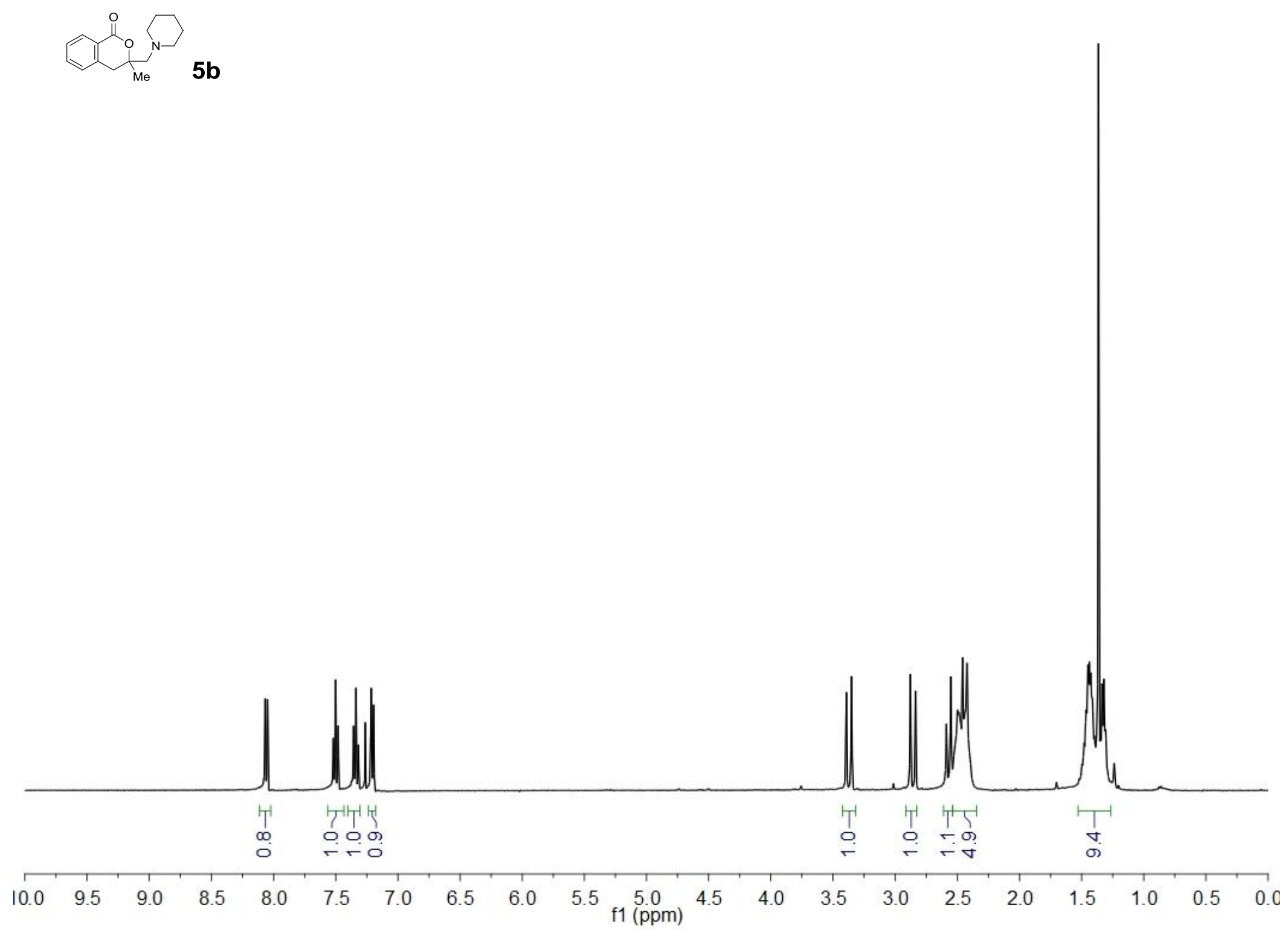


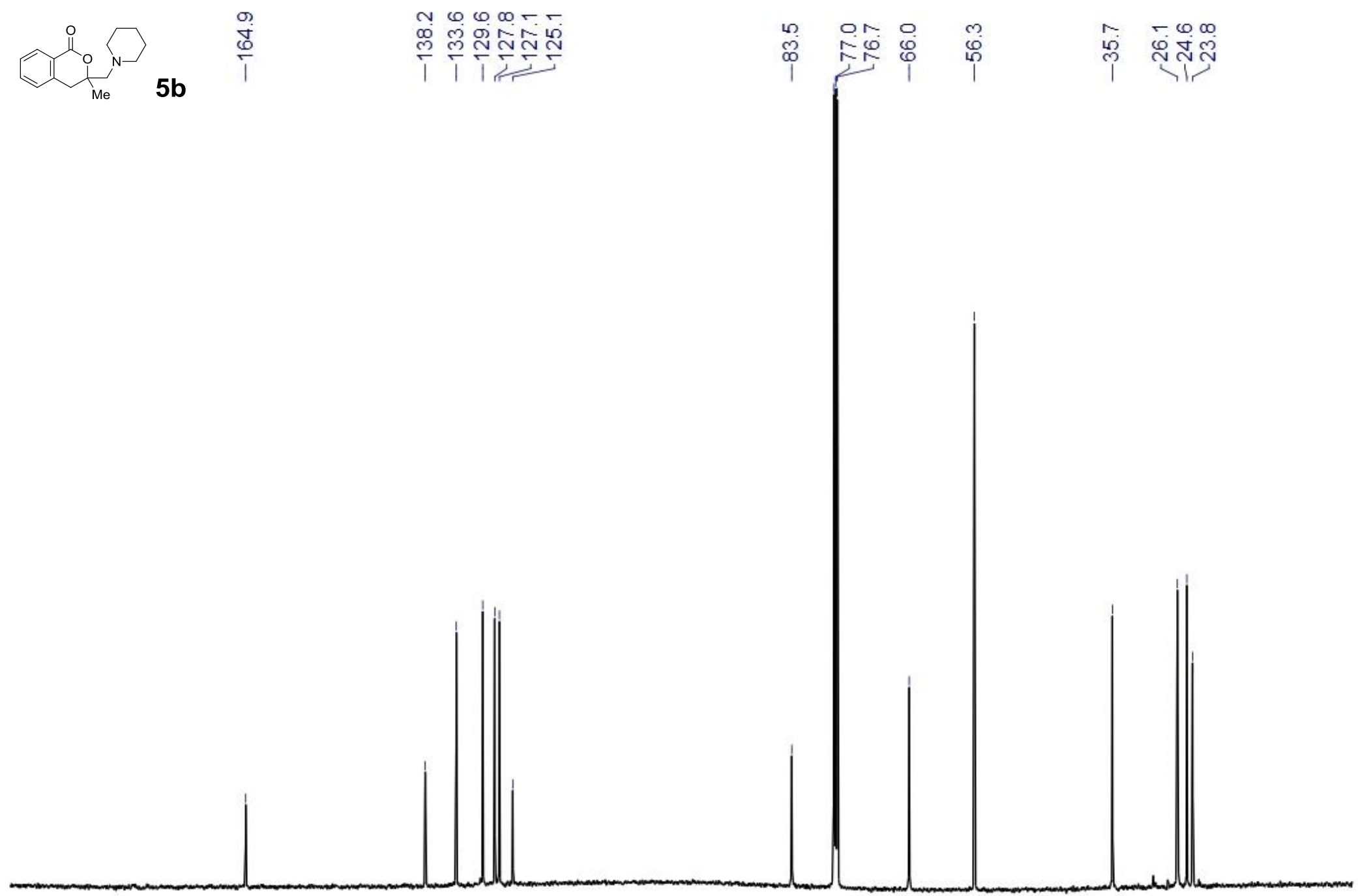



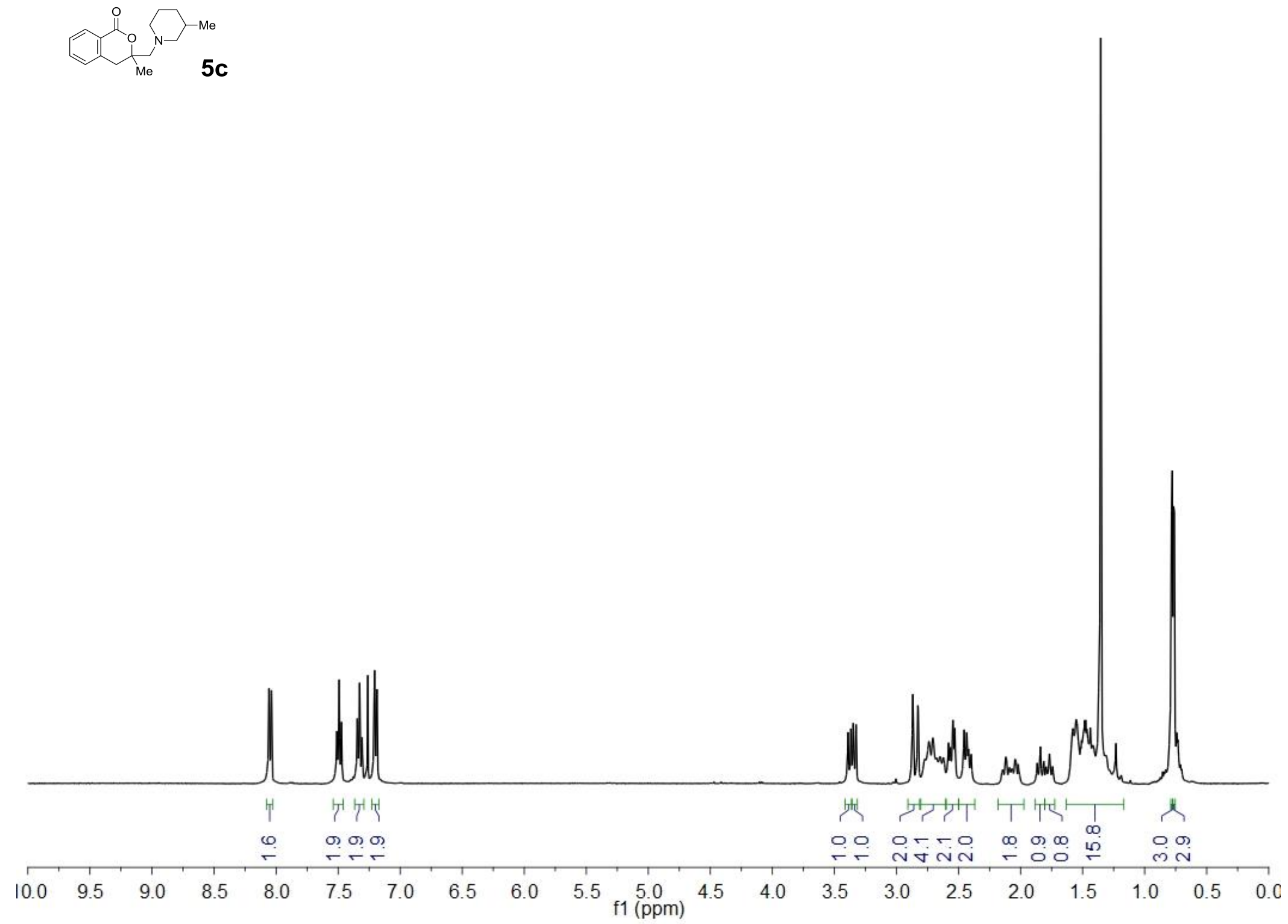

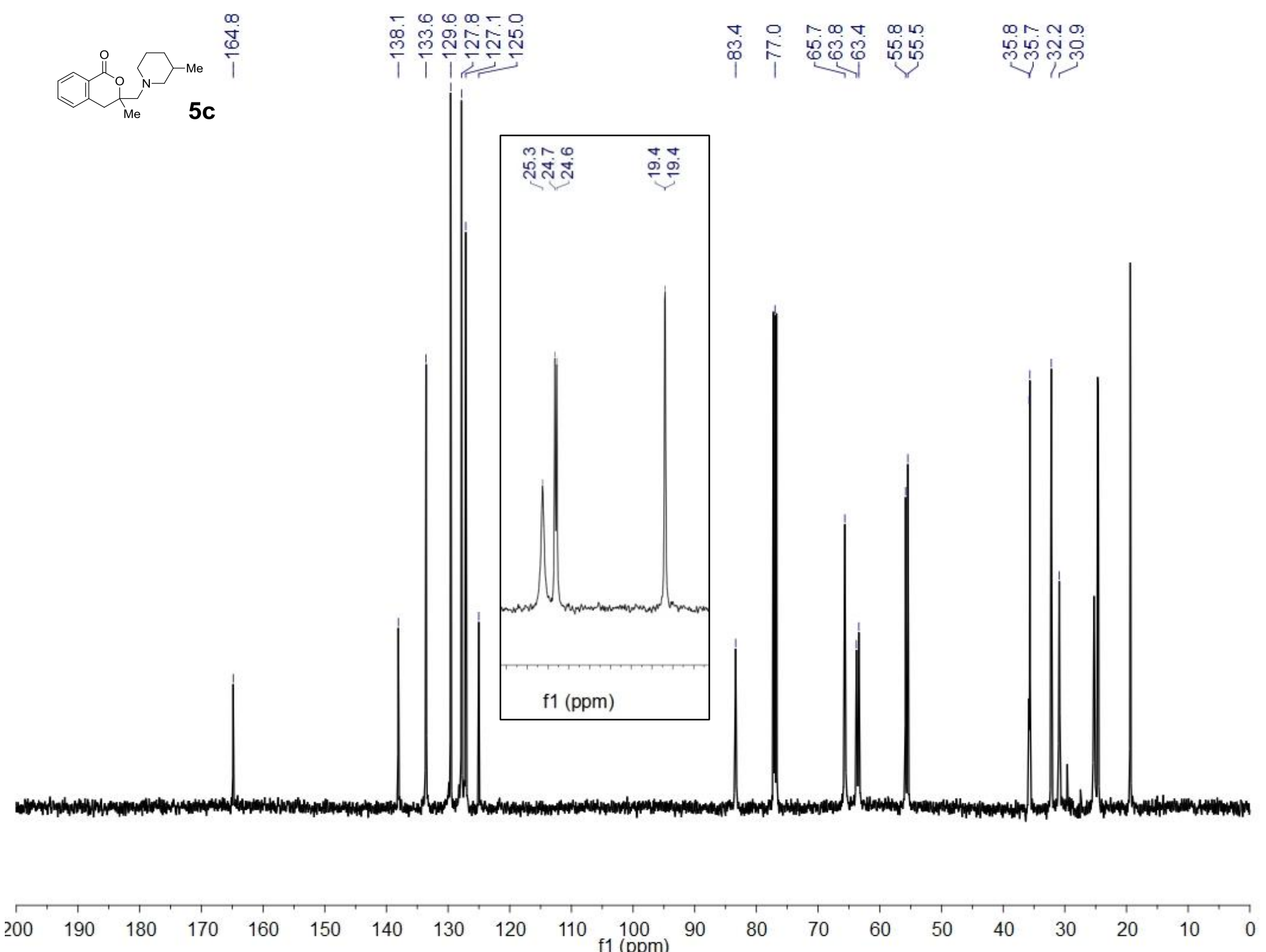

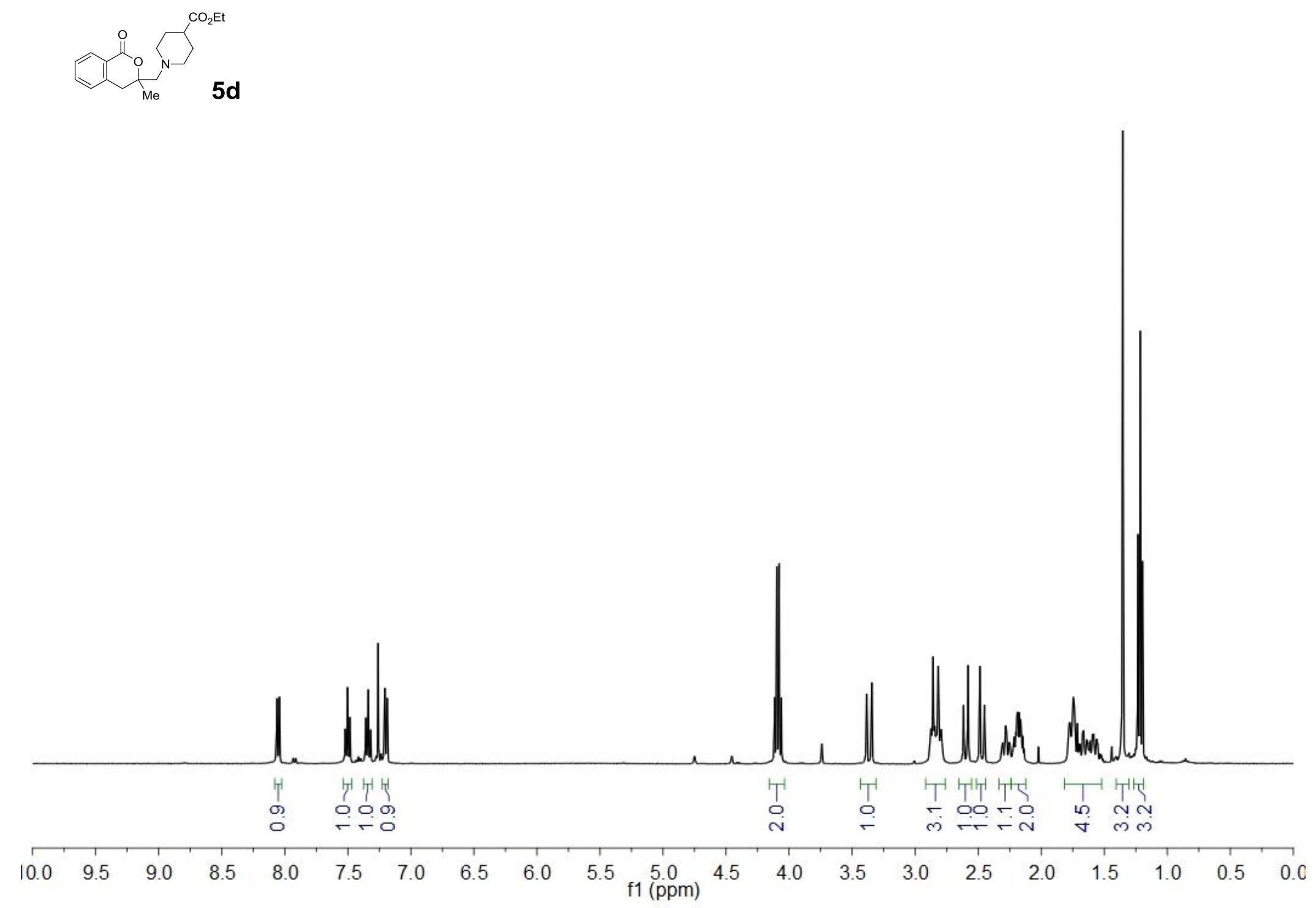


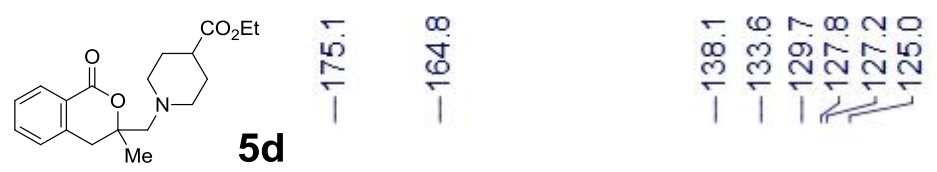

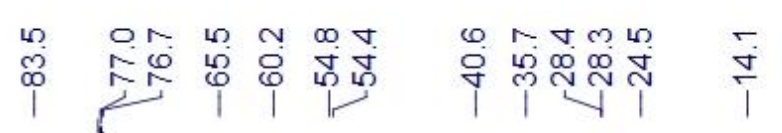

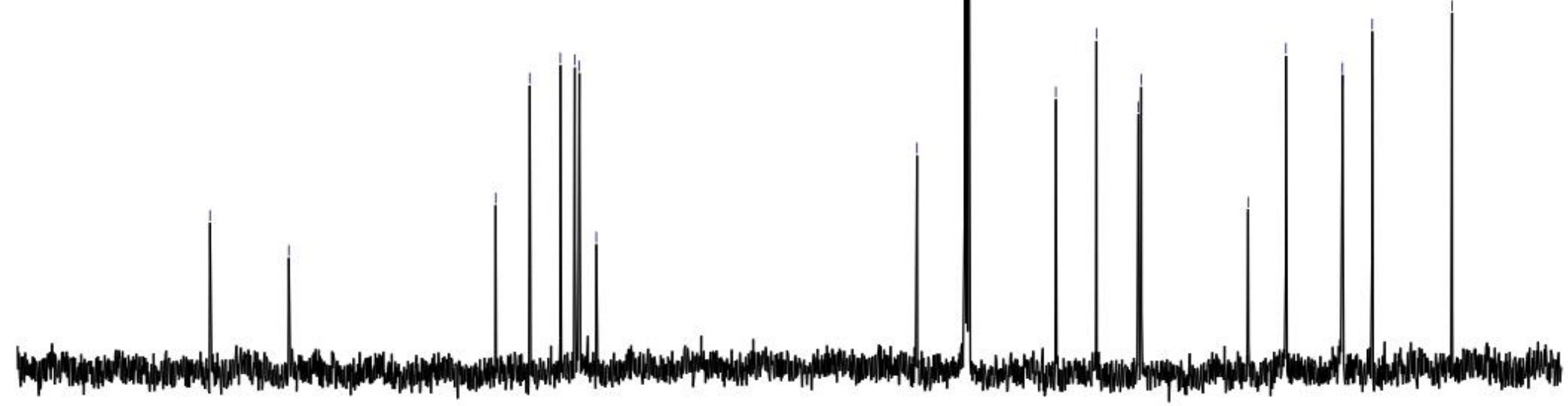



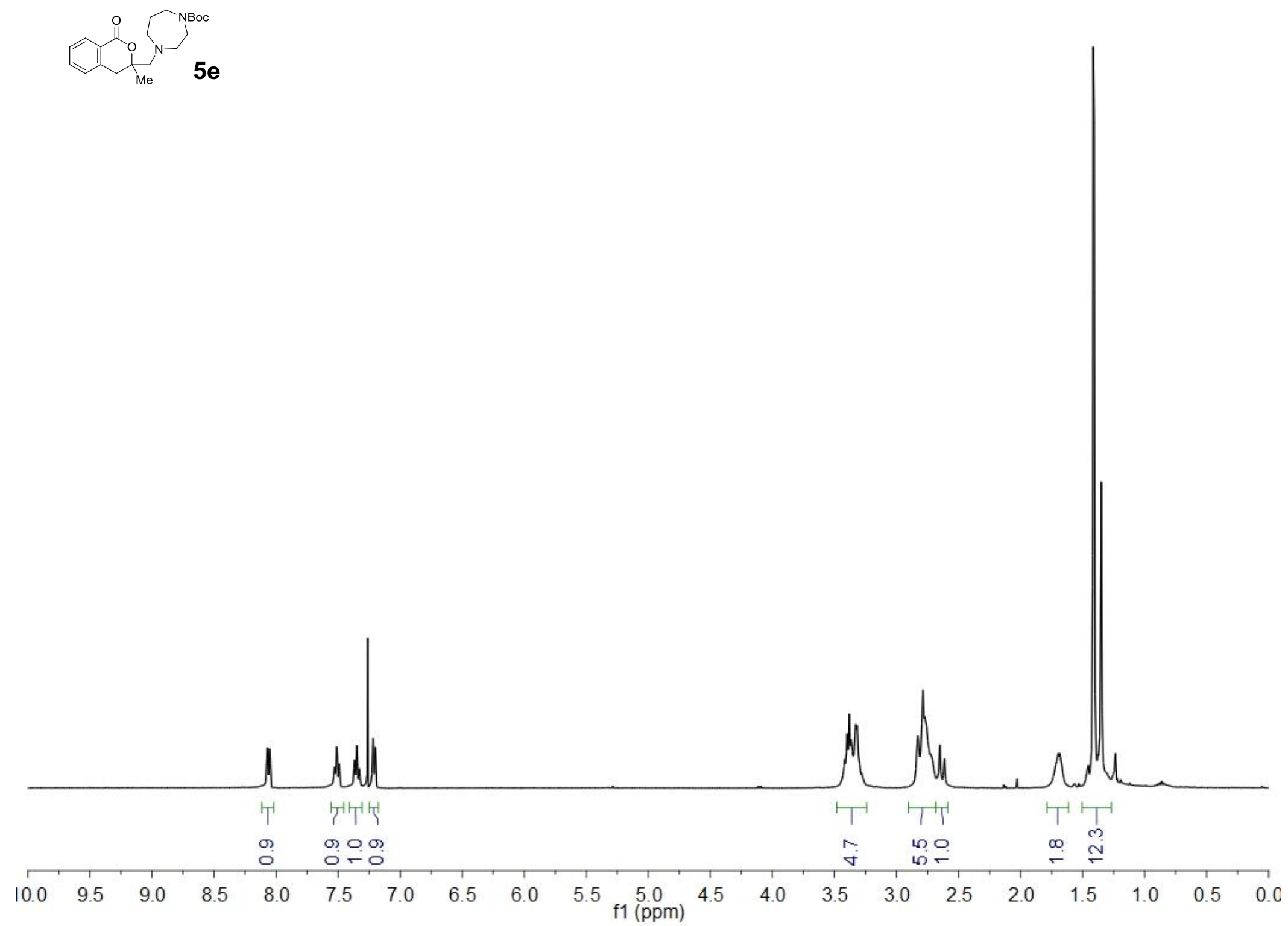

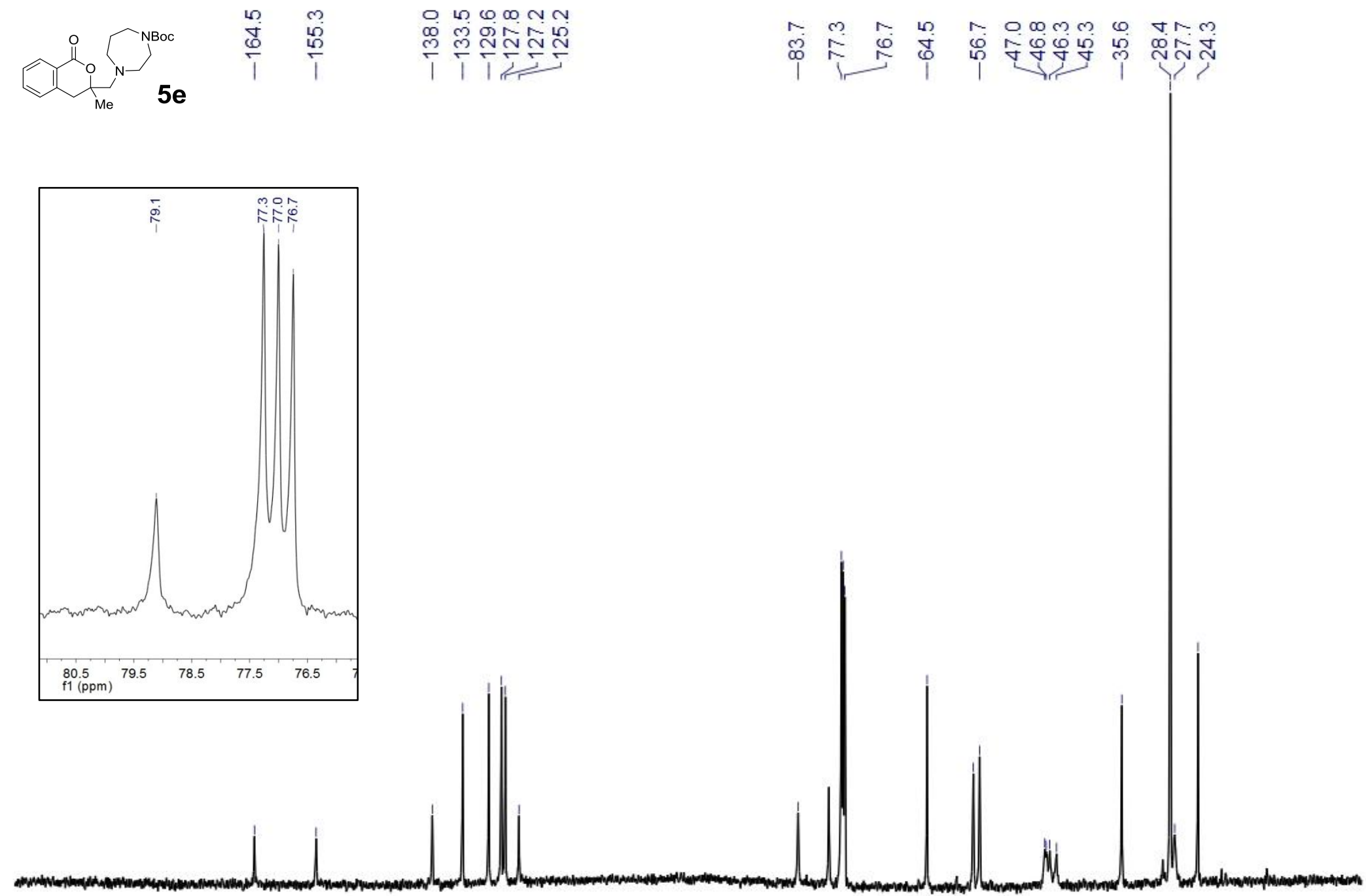

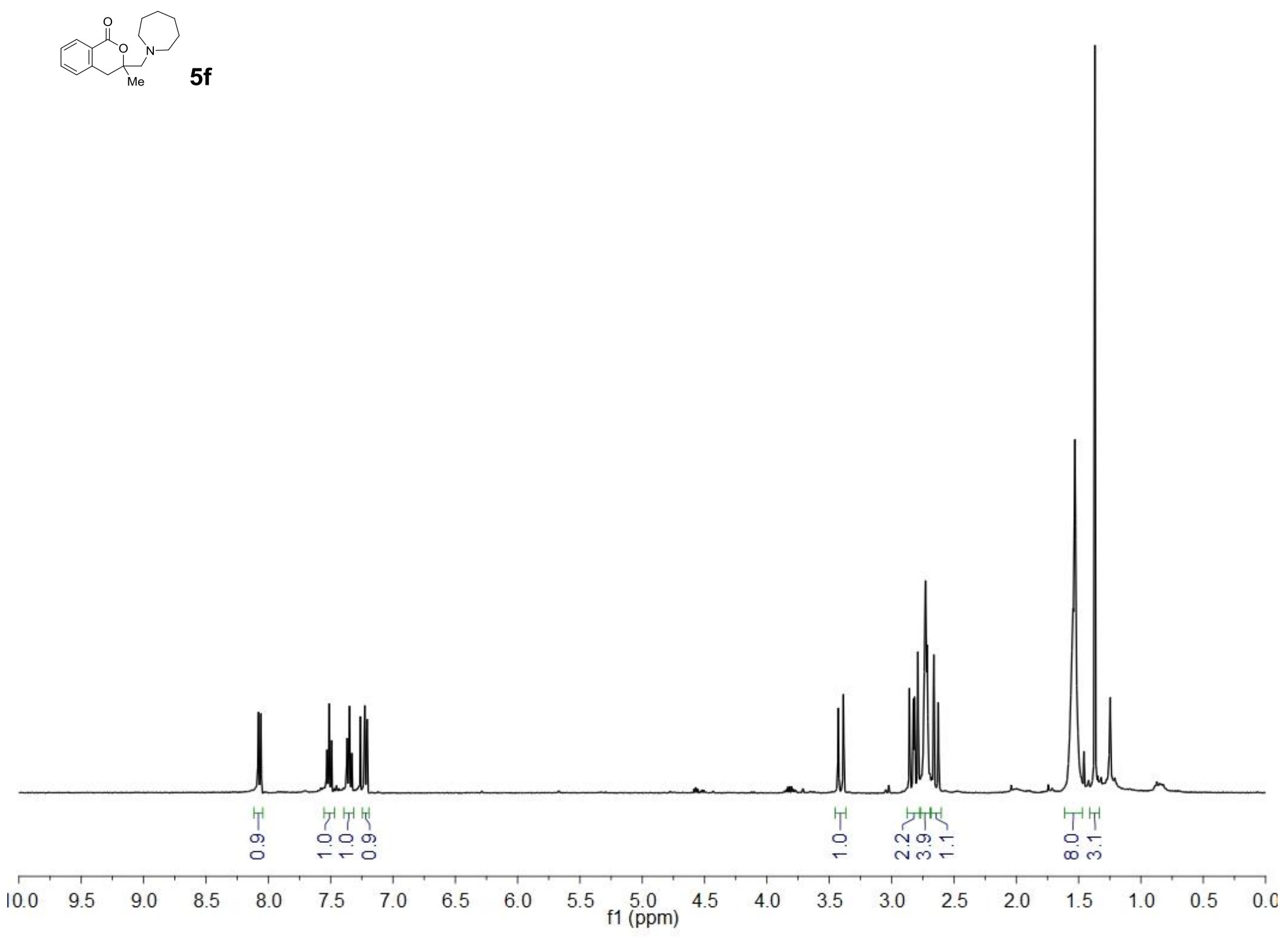

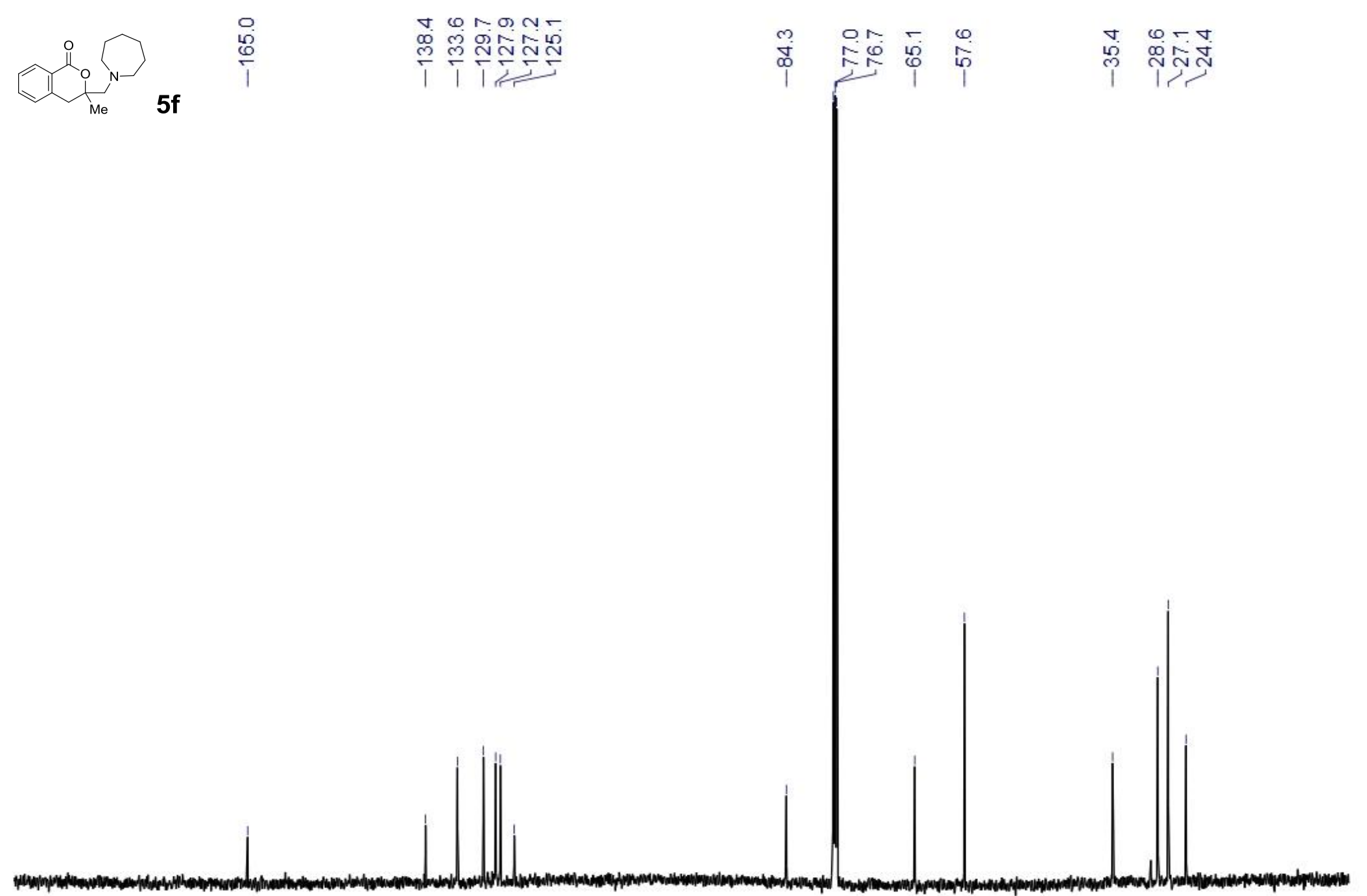


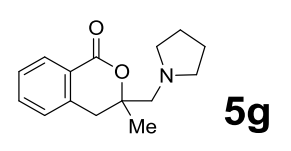

\section{$5 g$}

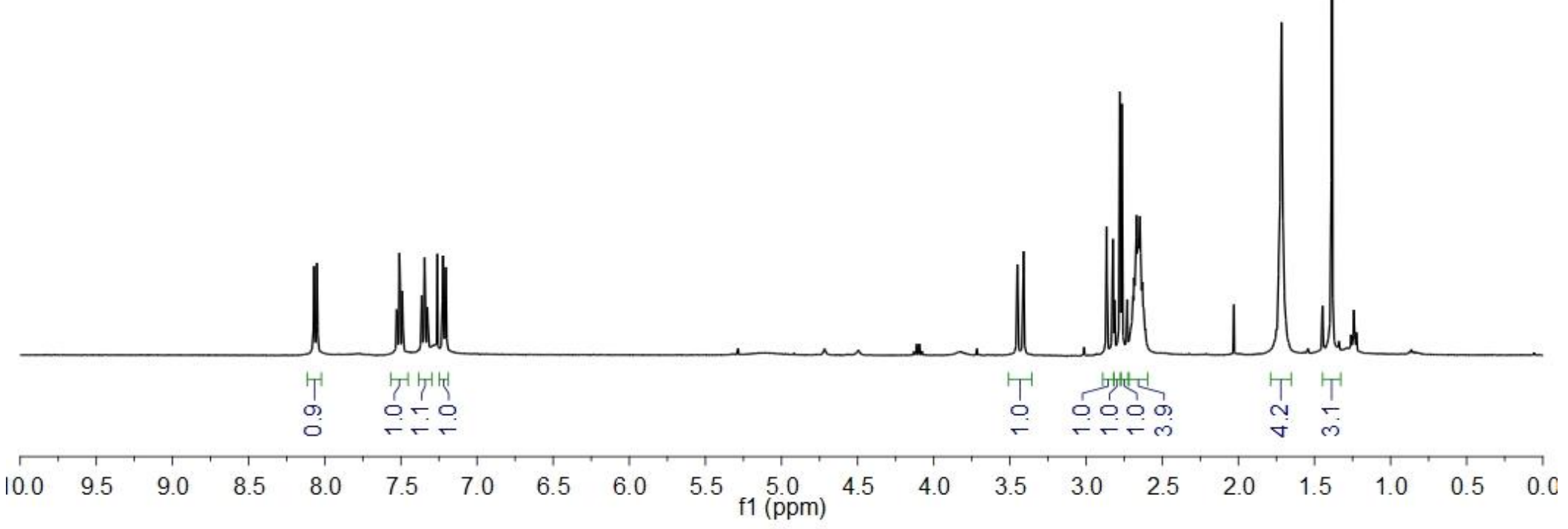




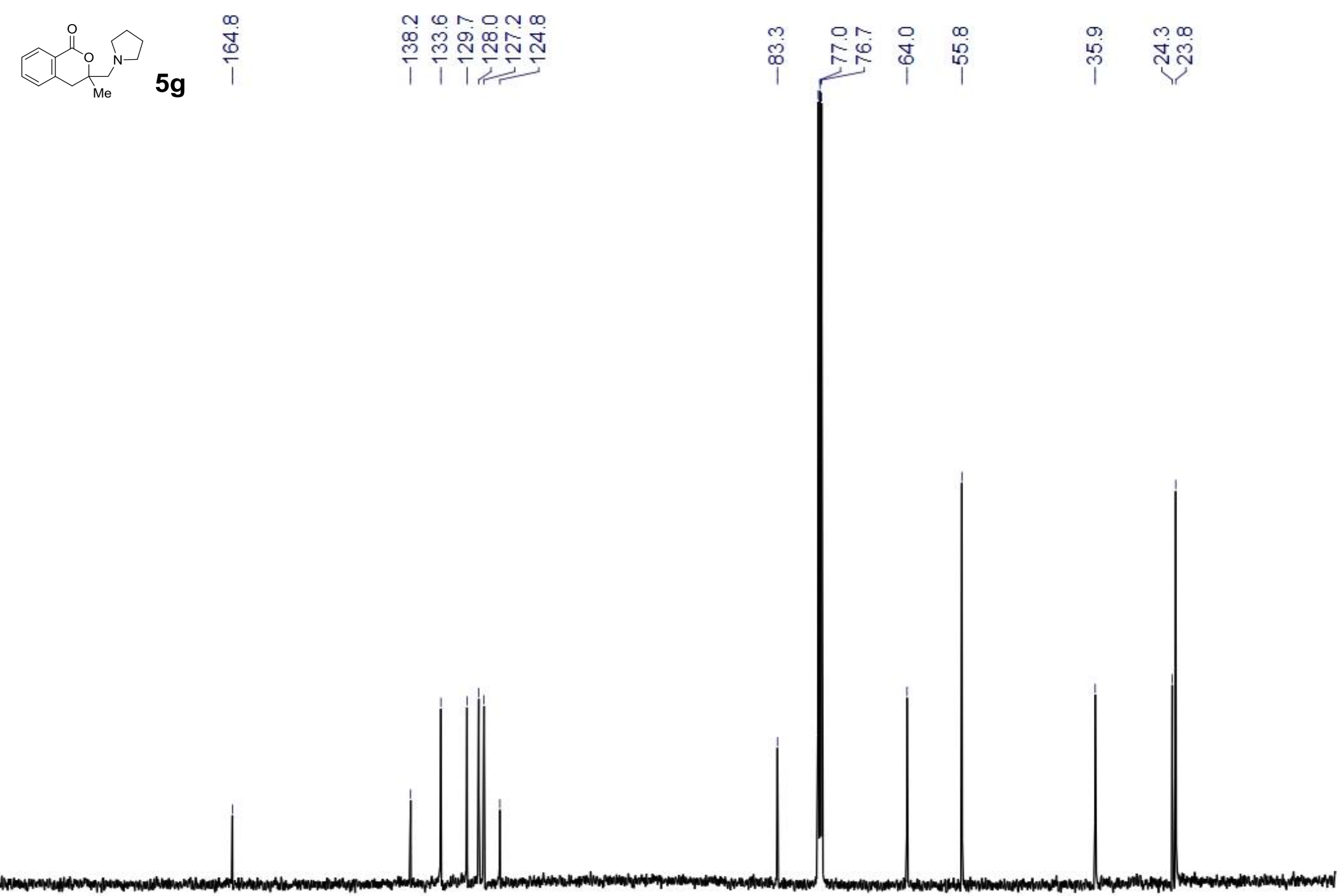

200

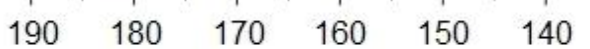

130

120

$110 \quad 100$

80

70

60

50

$40 \quad 30$

20

10 

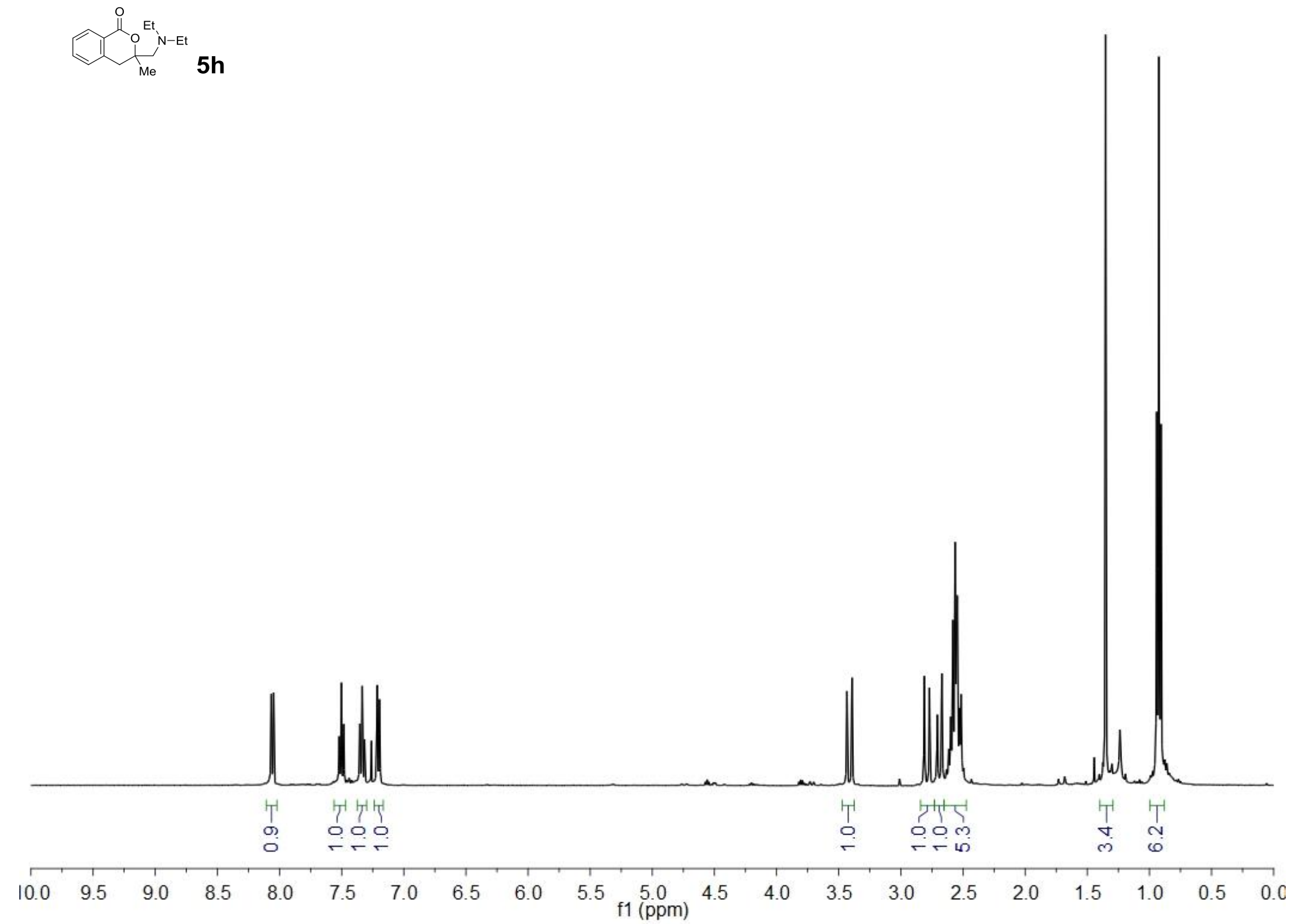


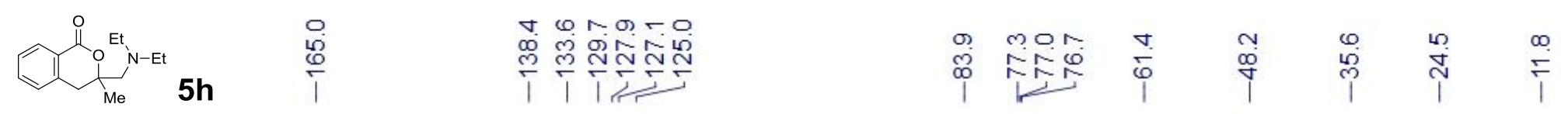

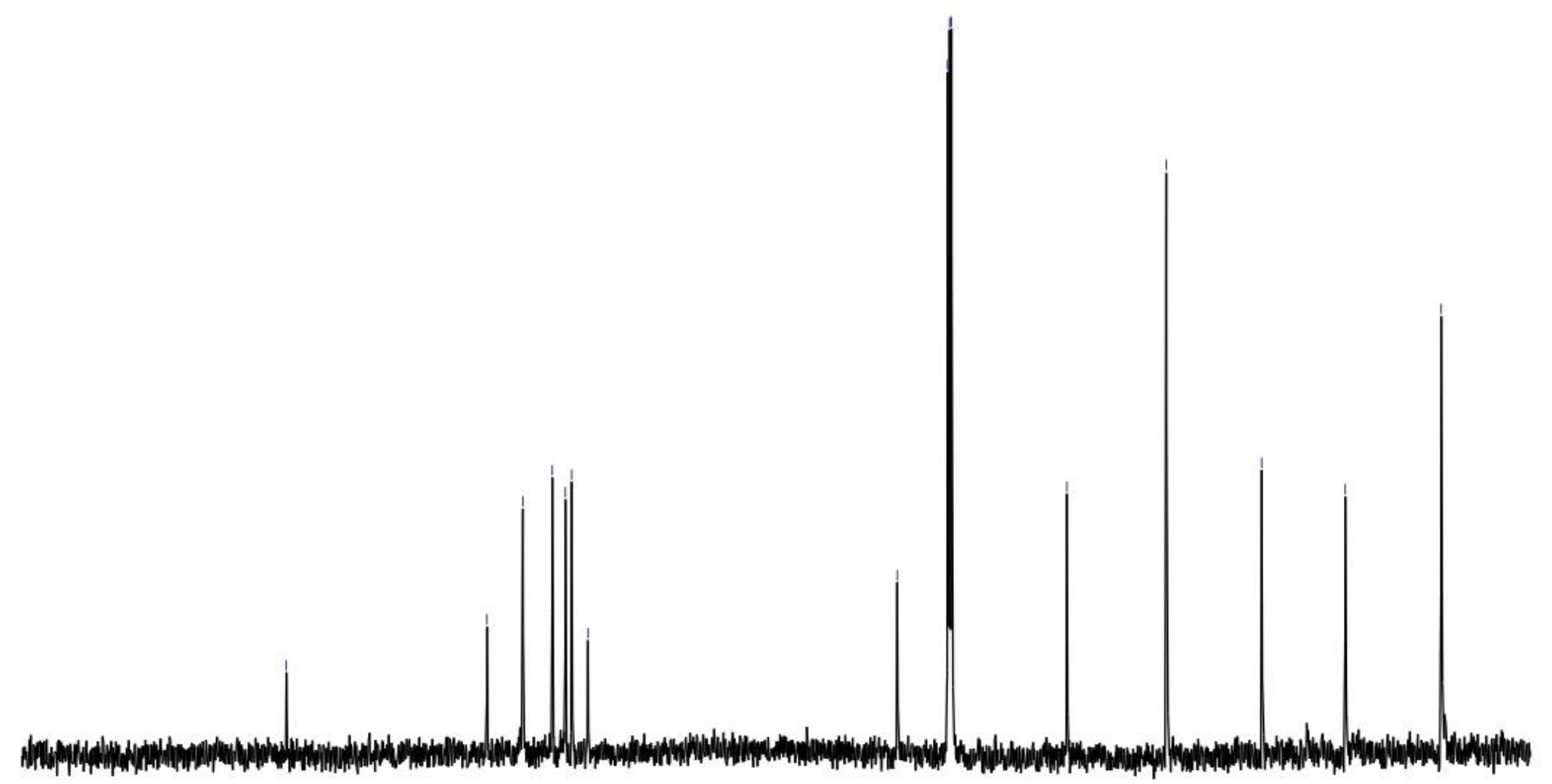




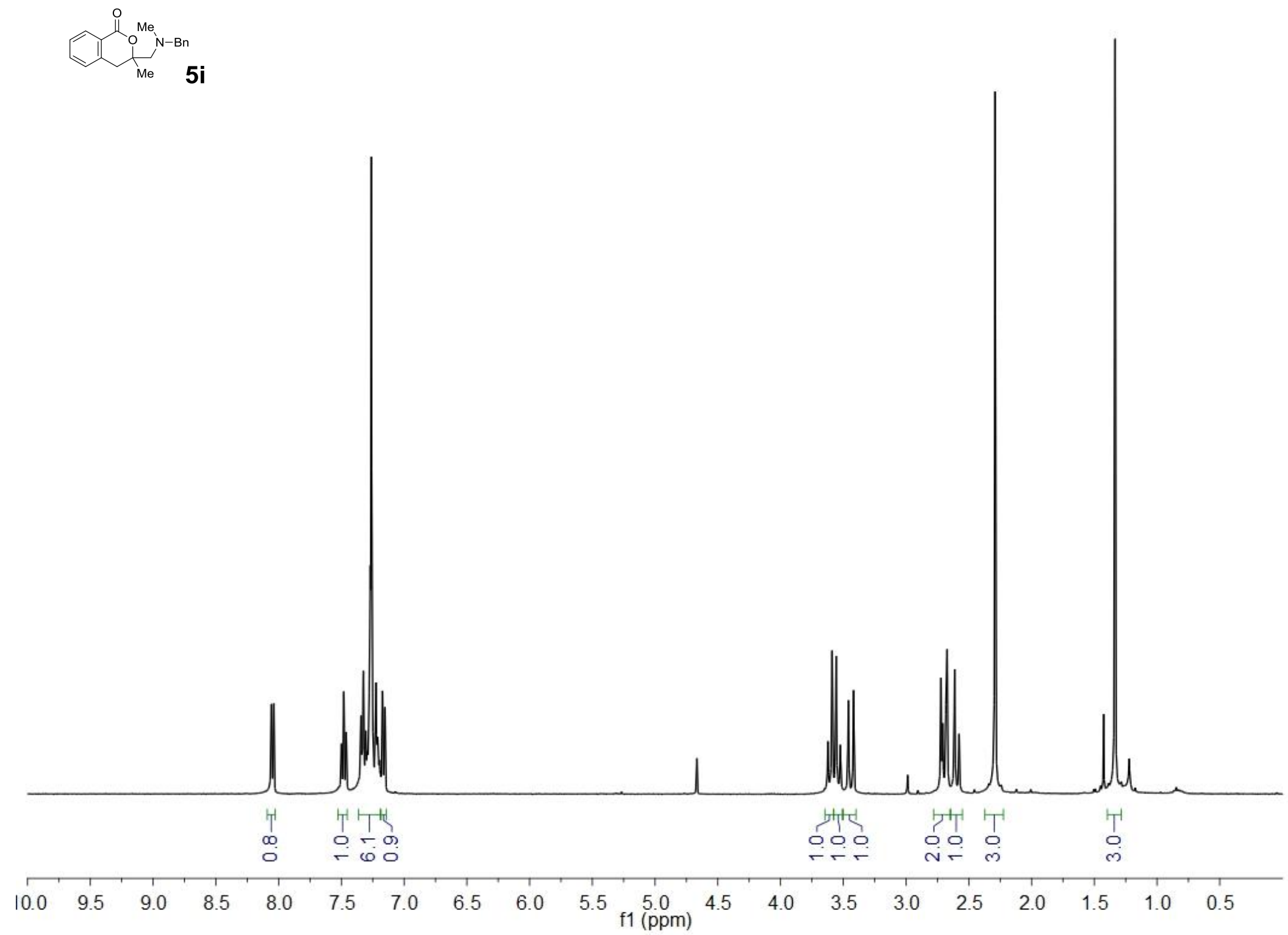




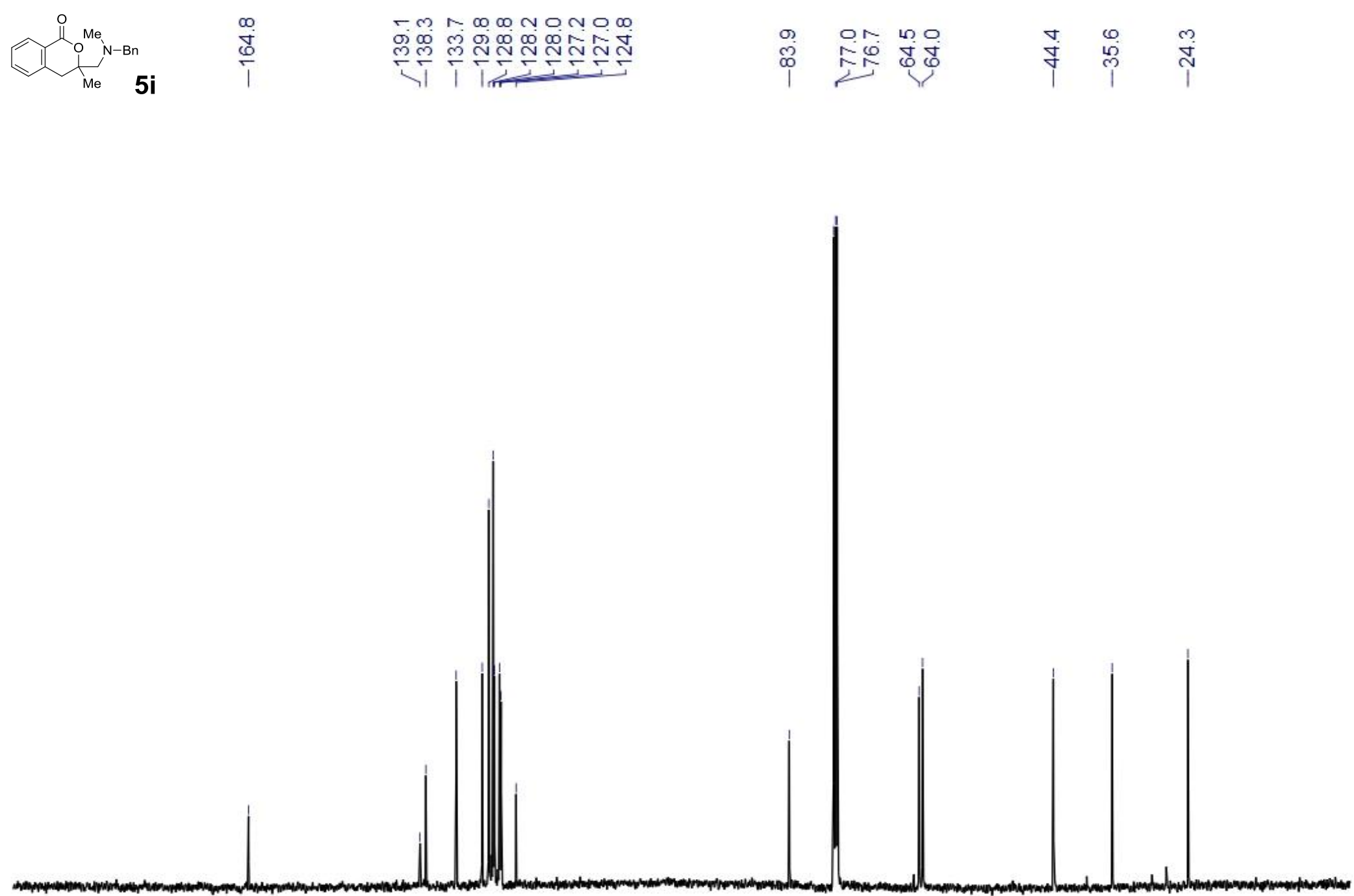

00

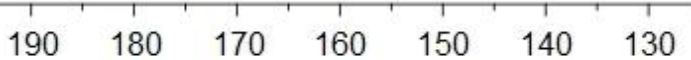

120

$\begin{array}{ll}110 & 100 \\ & \end{array}$

$90 \quad 8017$

60

50

$40 \quad 30$ 


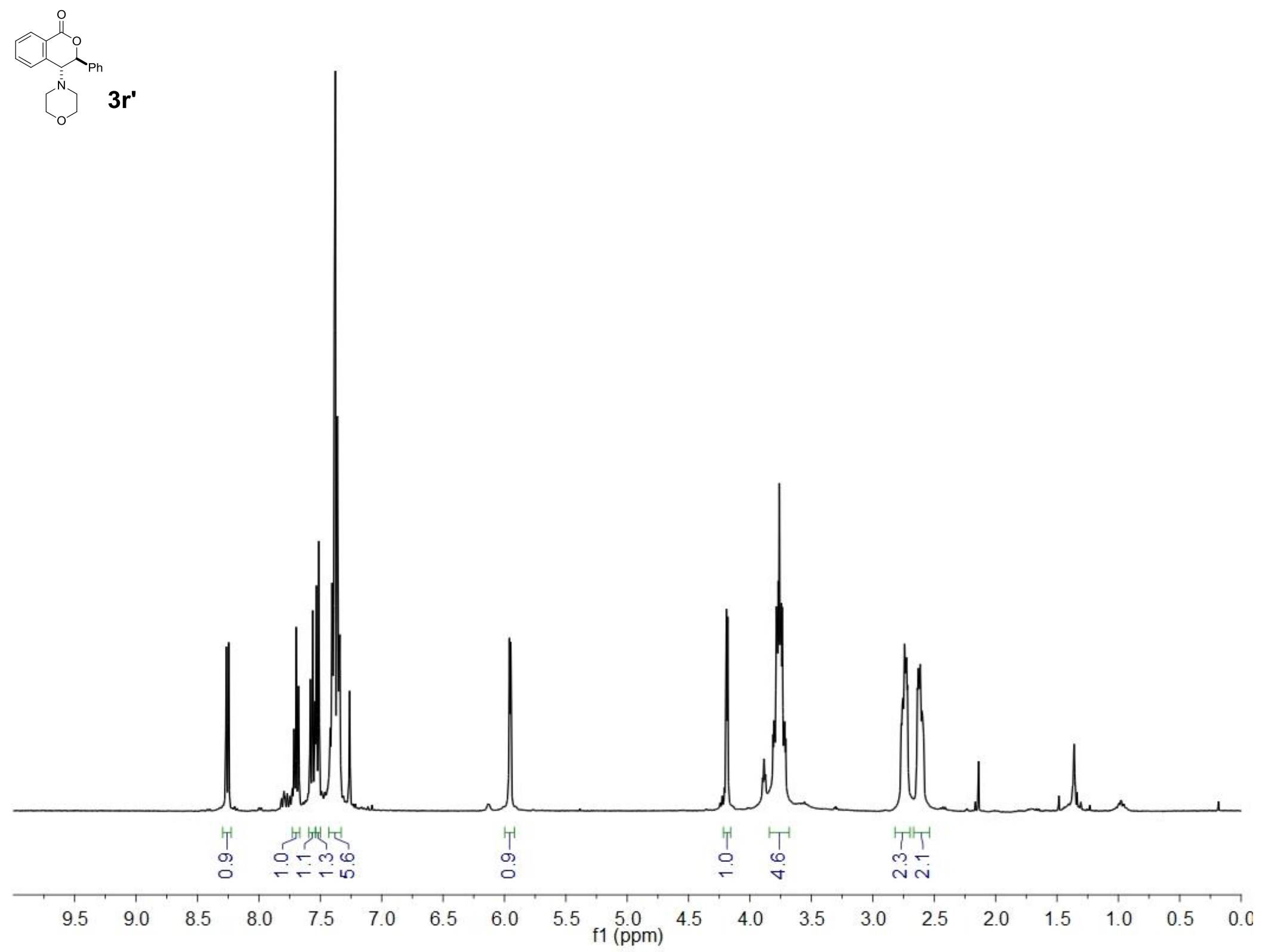




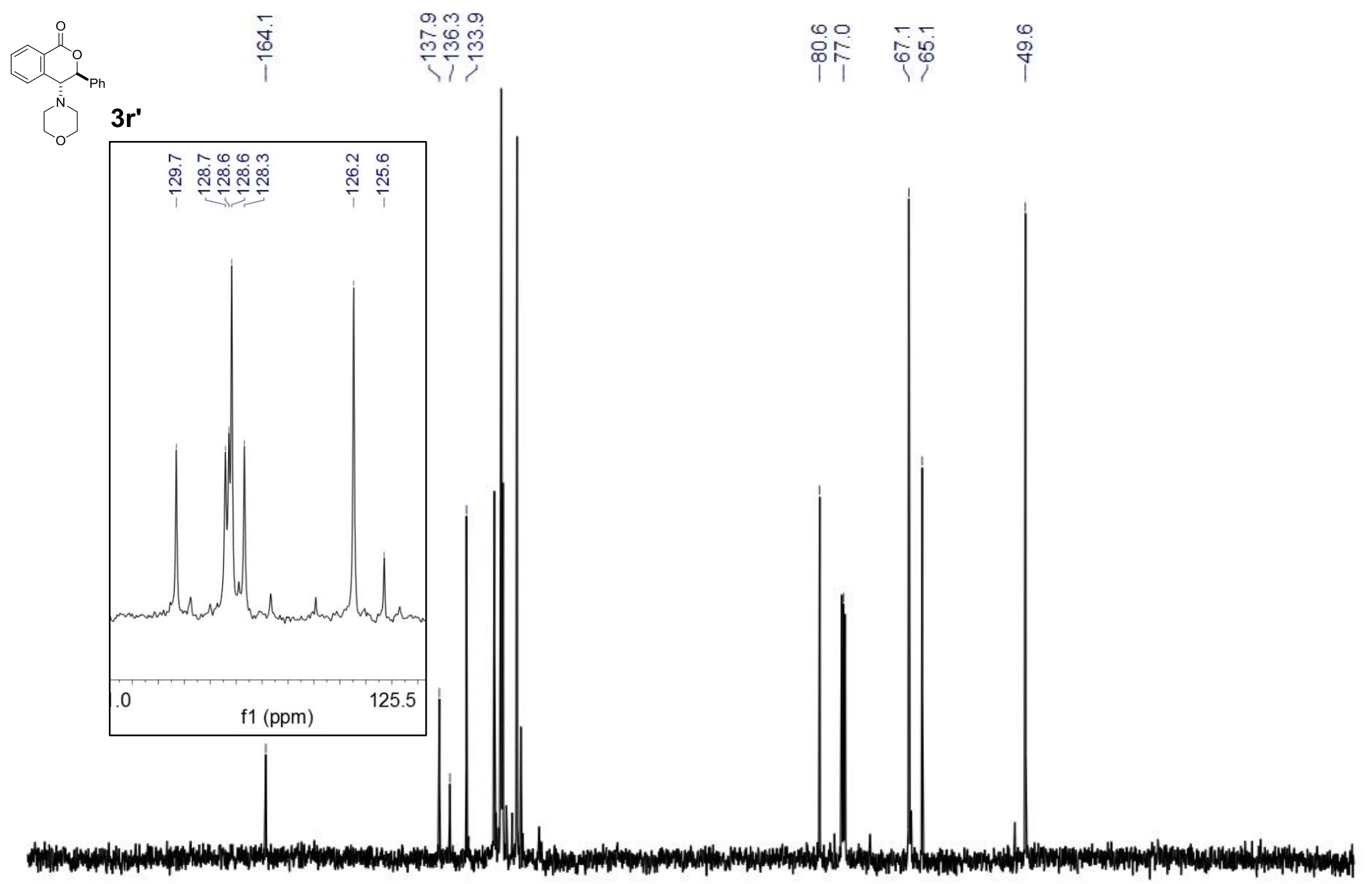

00

$110 \quad 100$

80

$70 \quad 60$

$50 \quad 40$

30

$20 \quad 10$ 


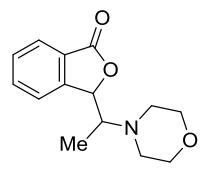

$3 s$

major diastereomer

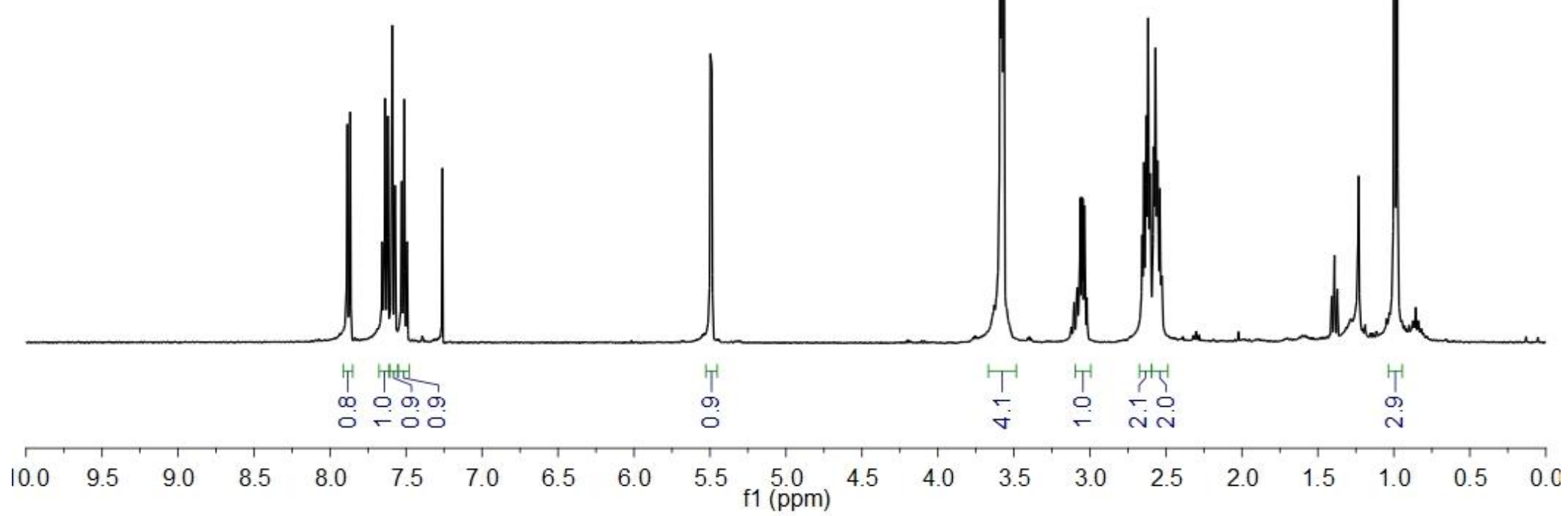

S187 


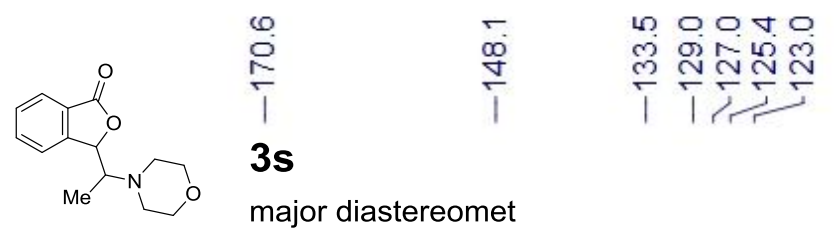

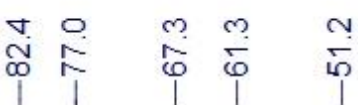

$\stackrel{m}{\leftarrow}$

major diastereomet

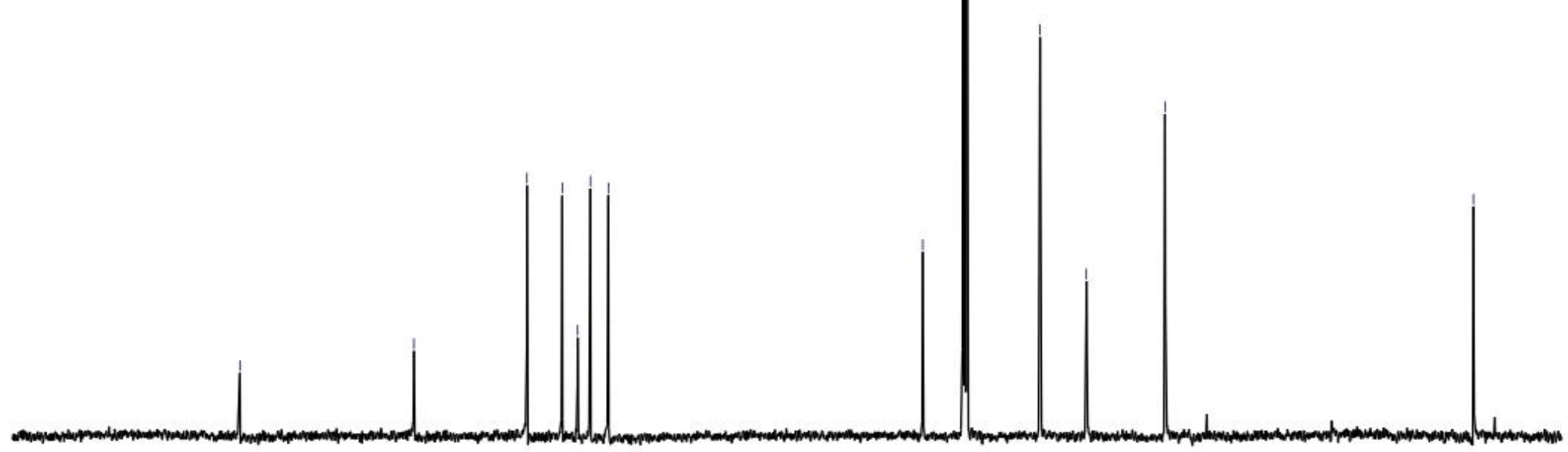


minor diastereomer

w/ trace regioisomer

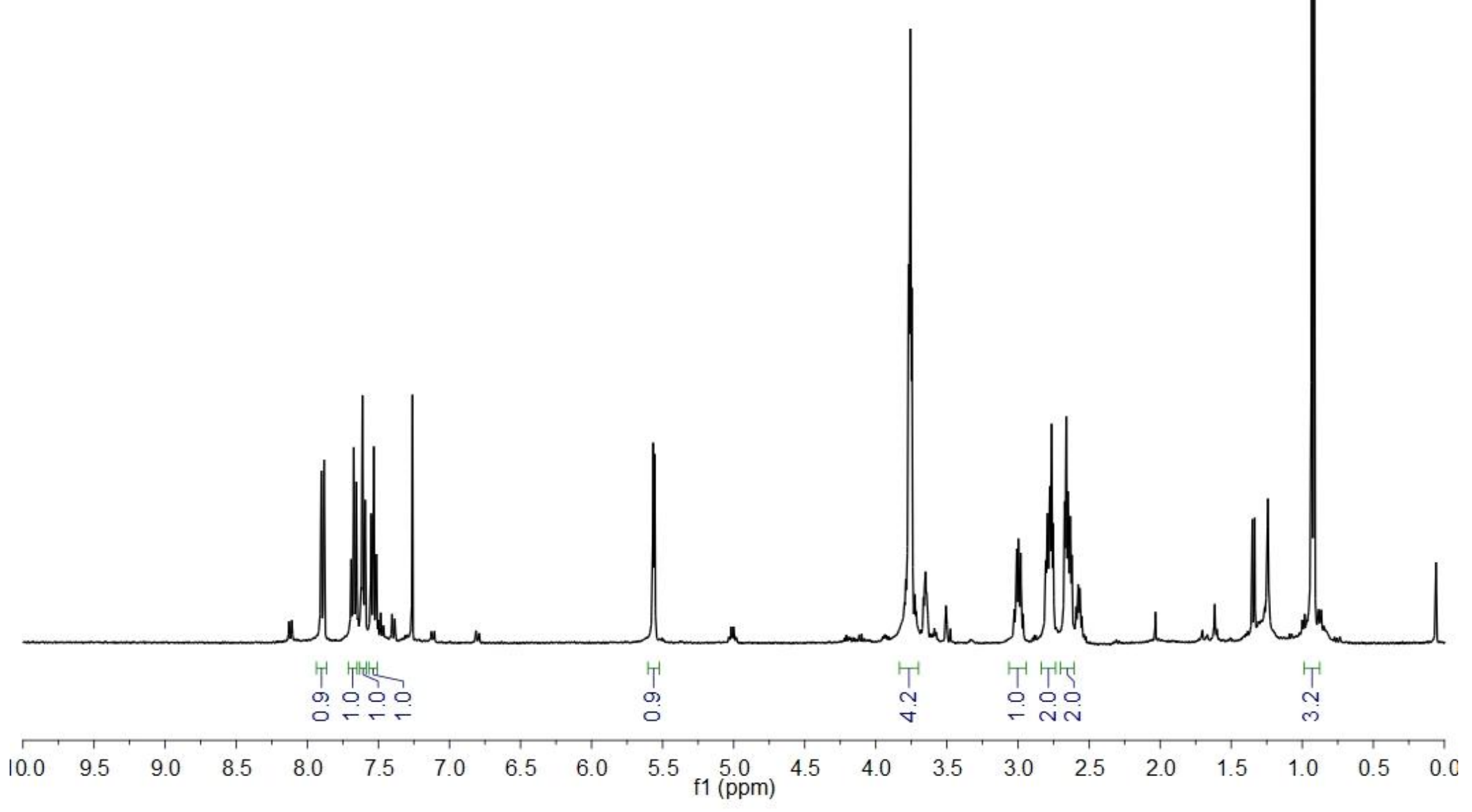



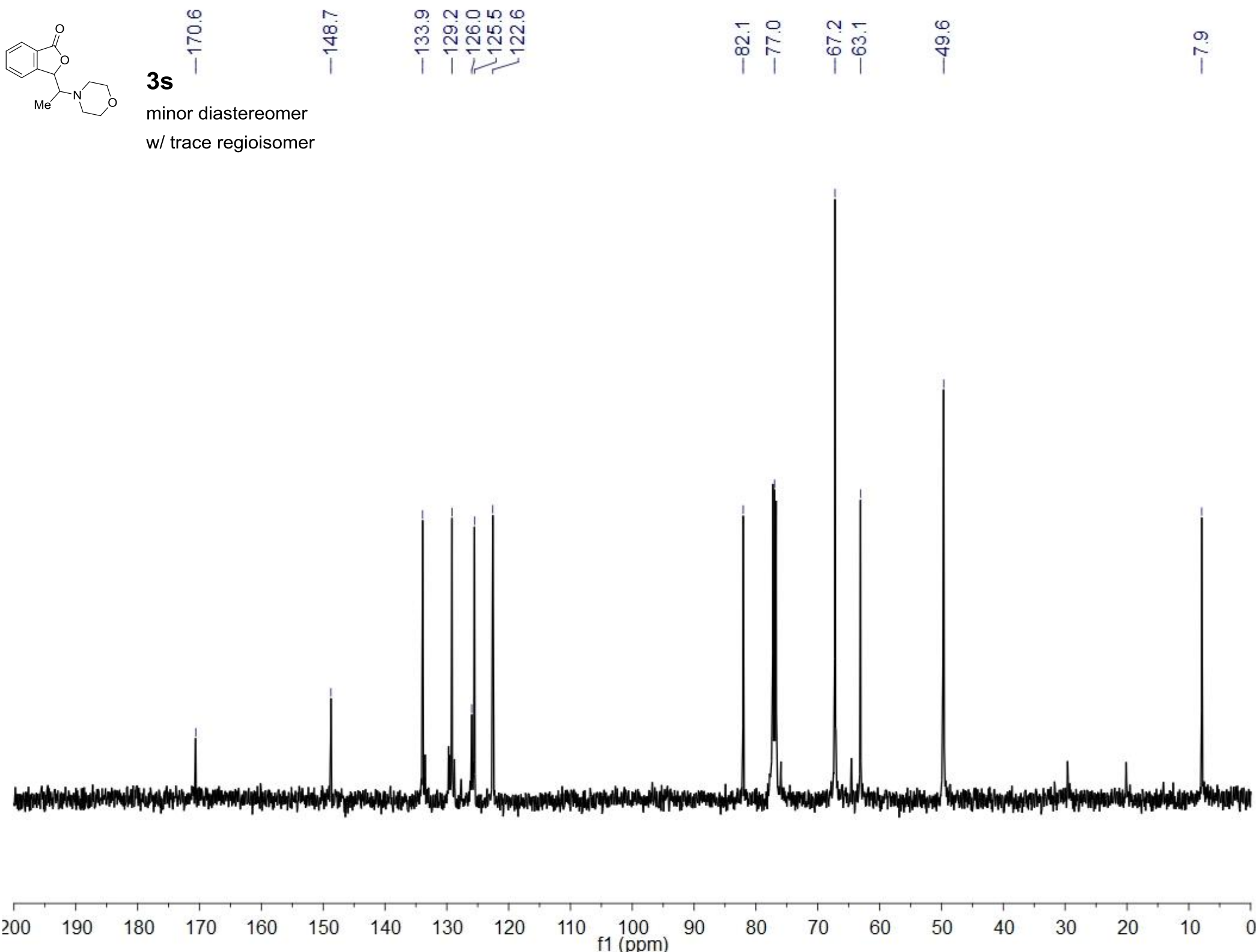


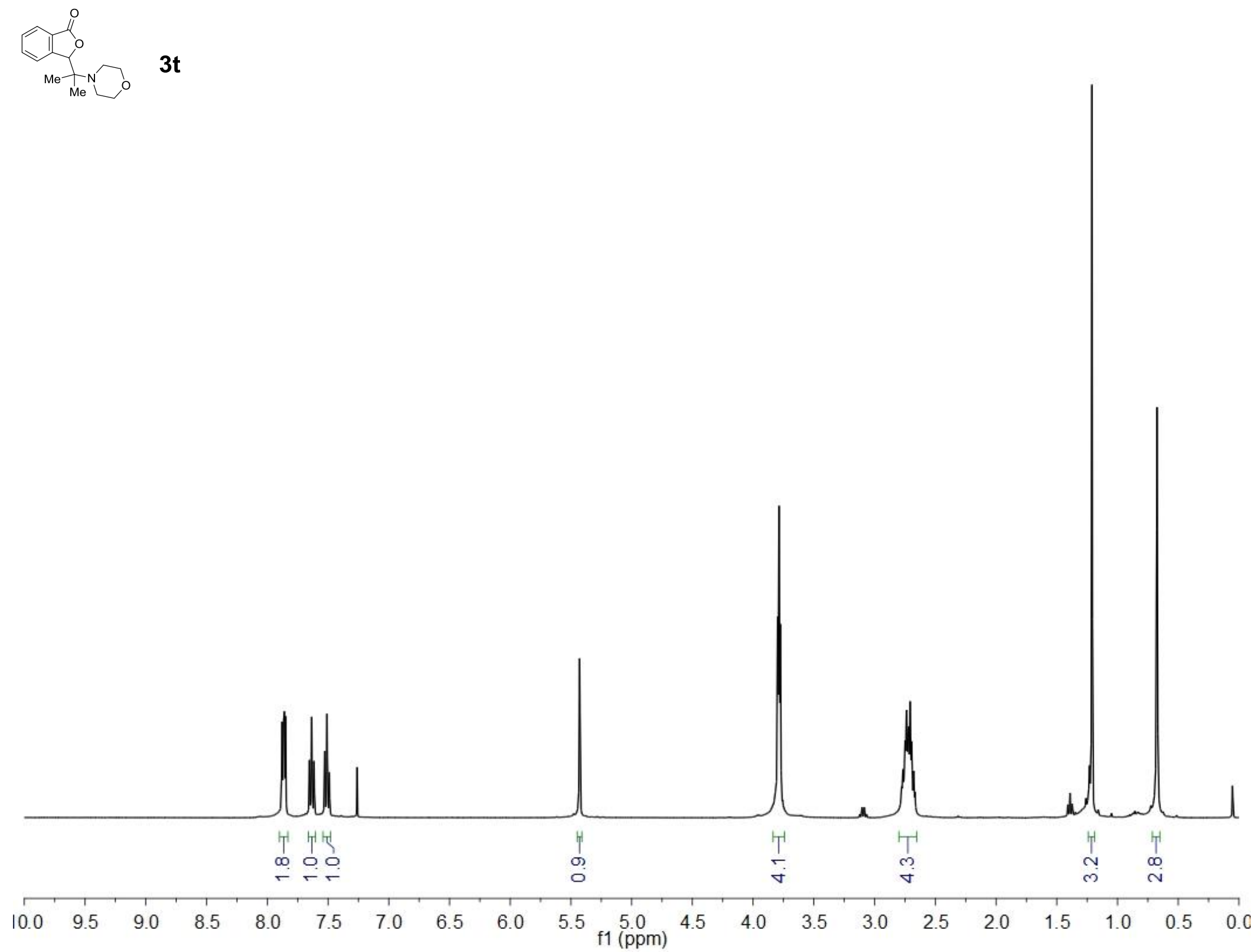




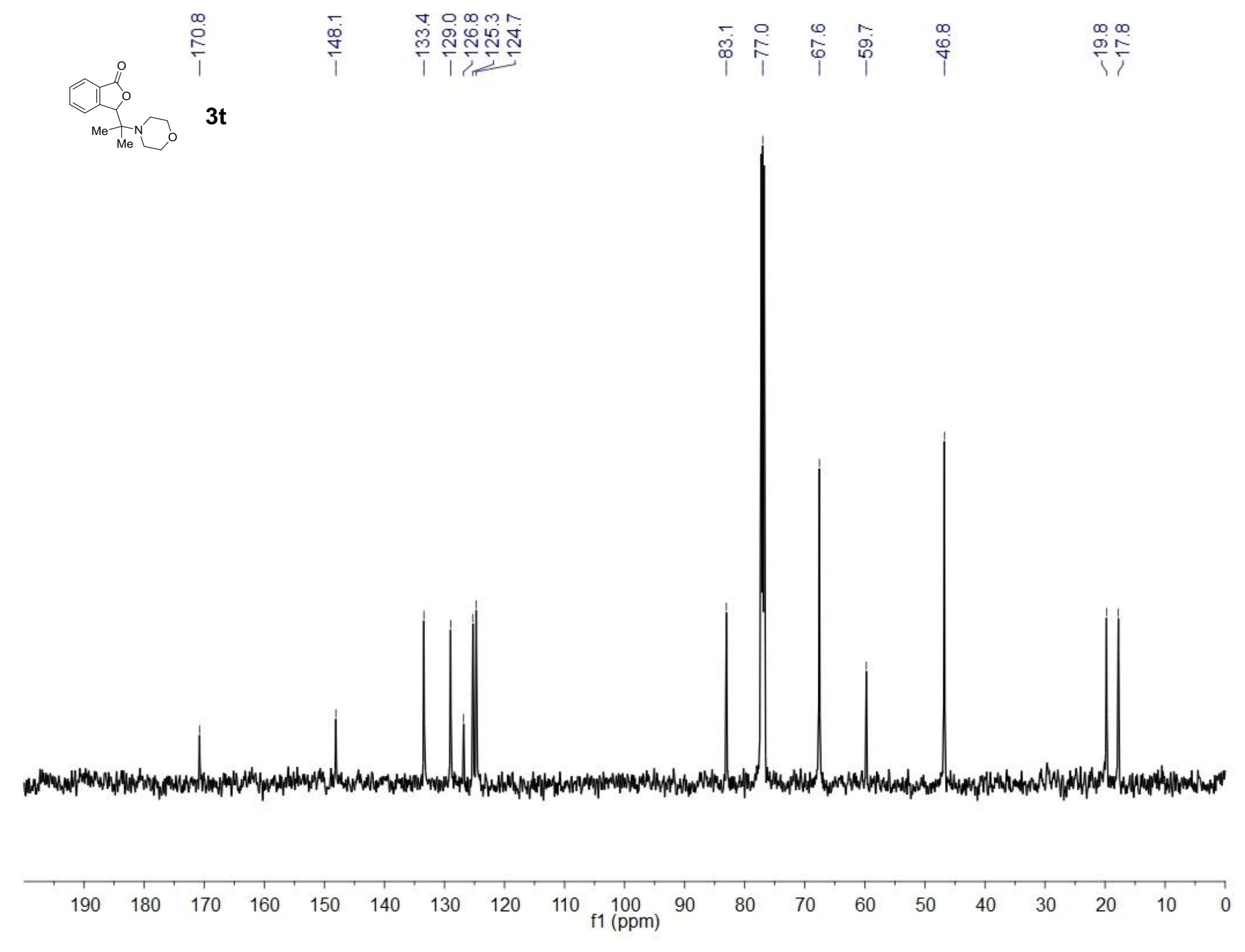



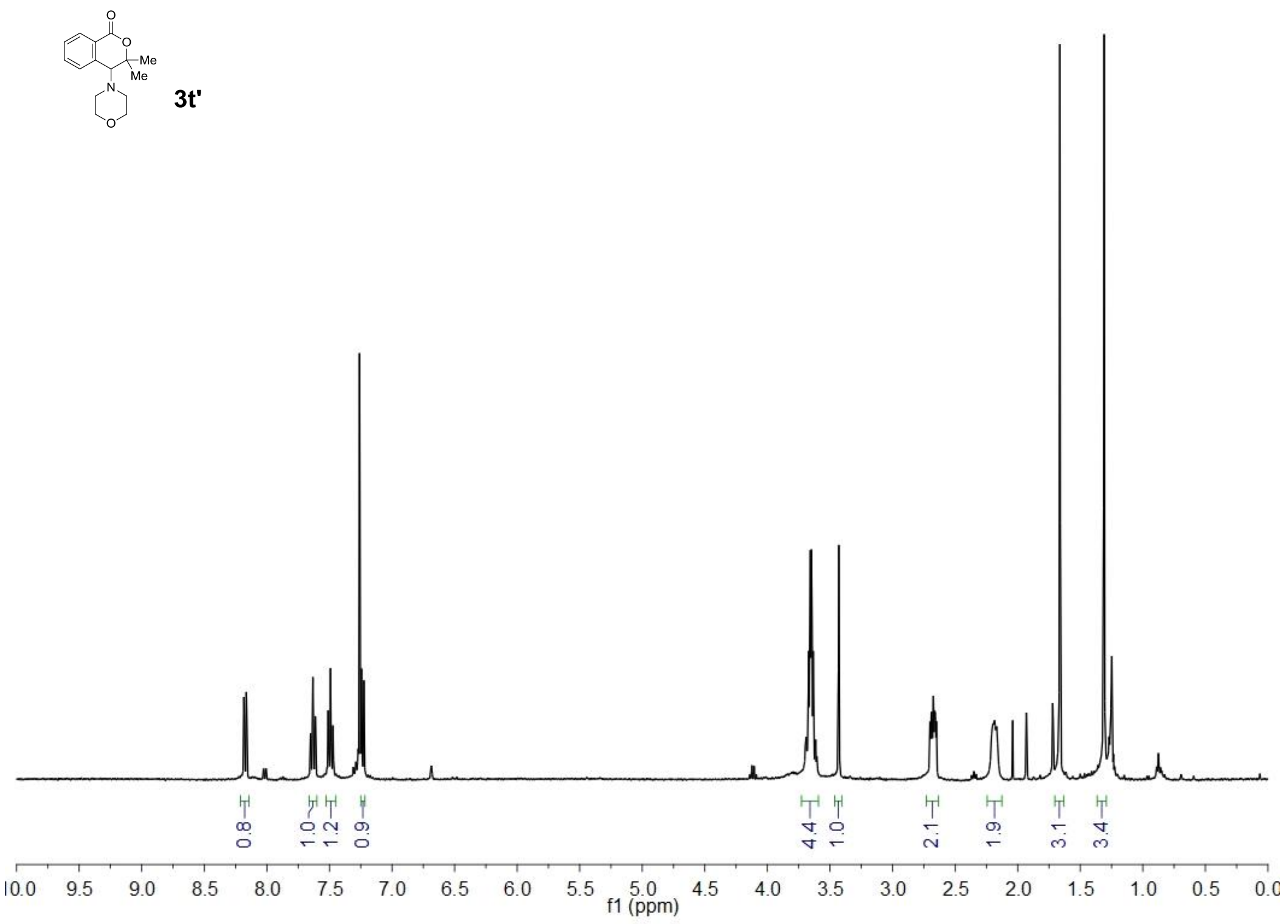


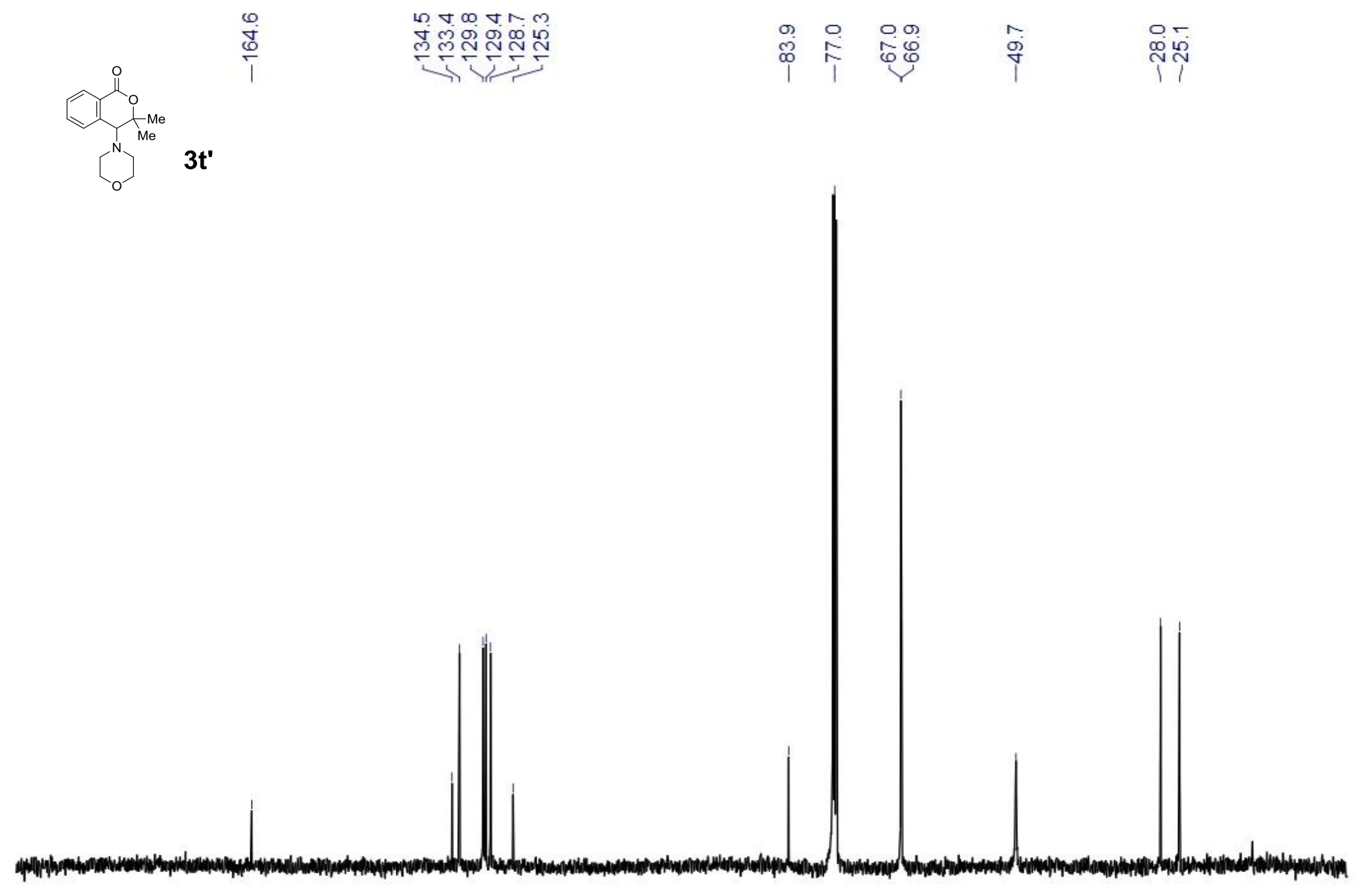

00

120

$110 \quad 100$

80

70

60

50

$40 \quad 30$

$20 \quad 10 \quad 0$ 


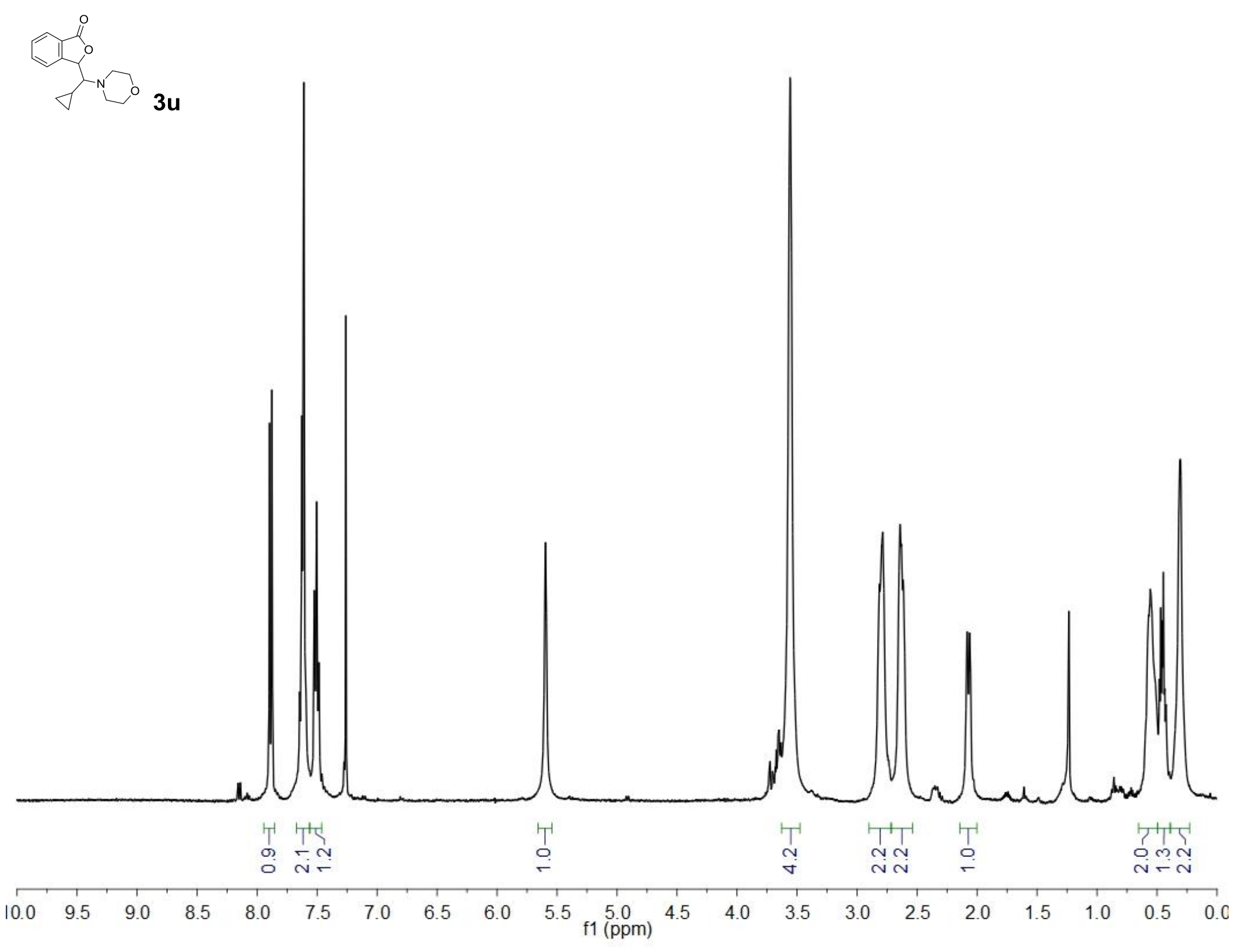




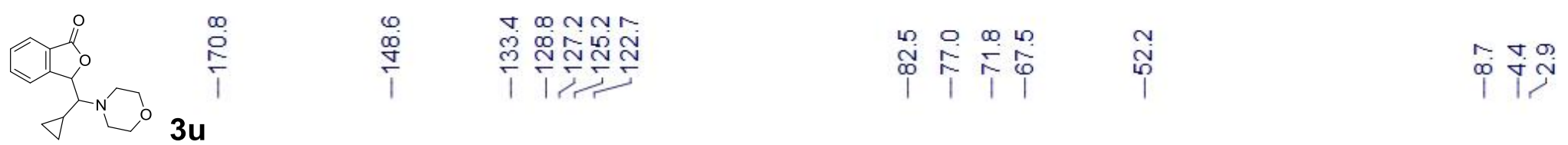

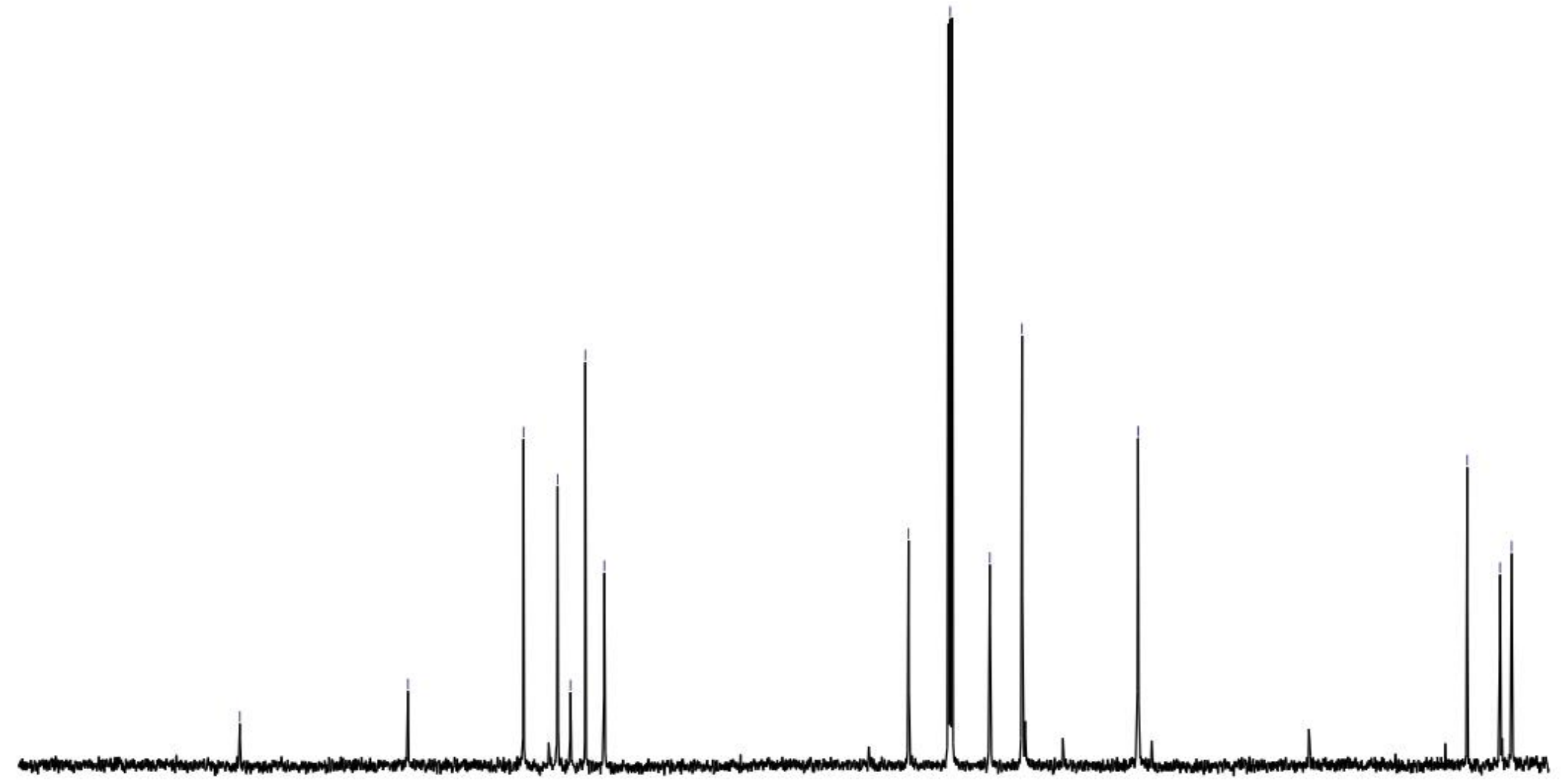




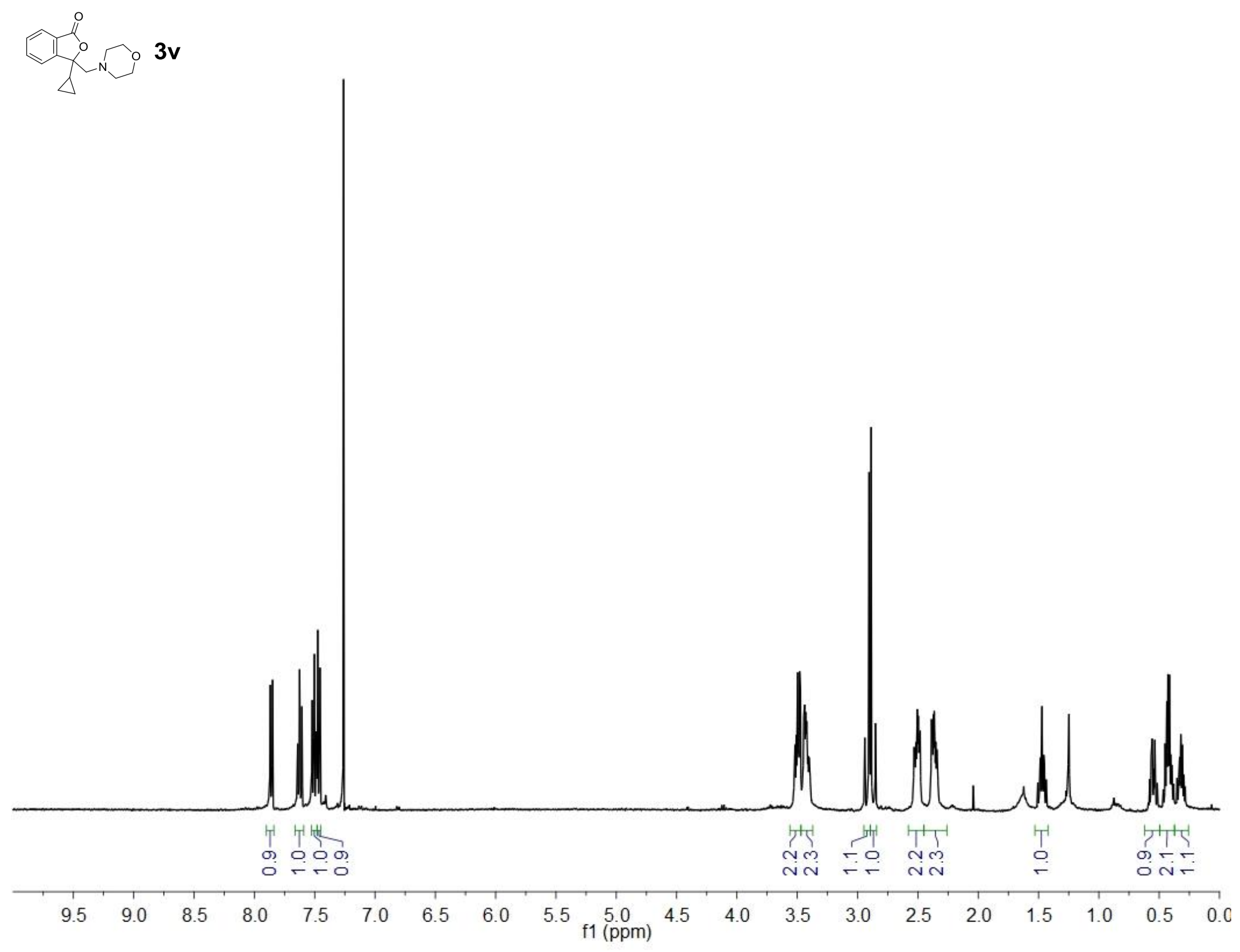



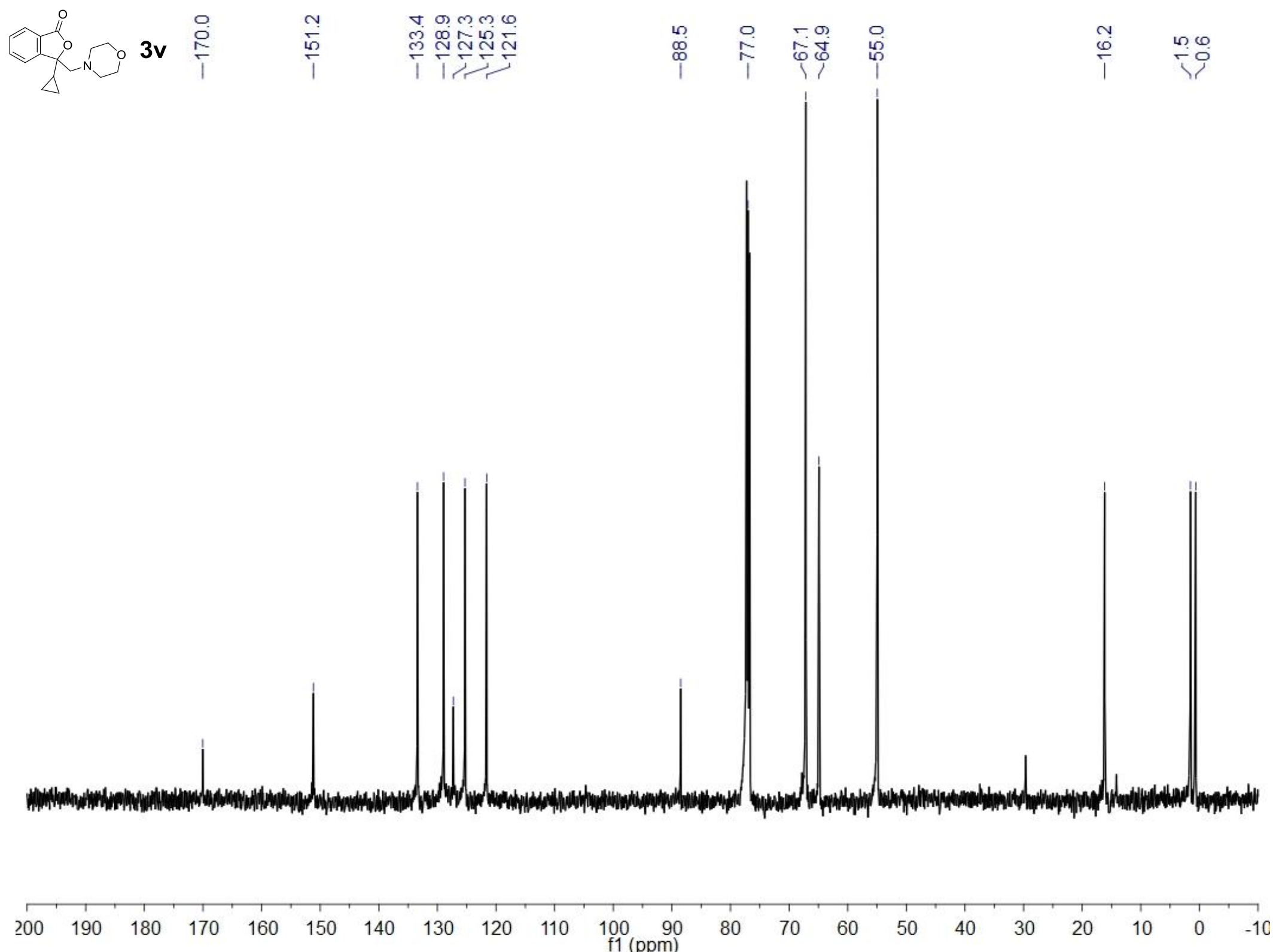


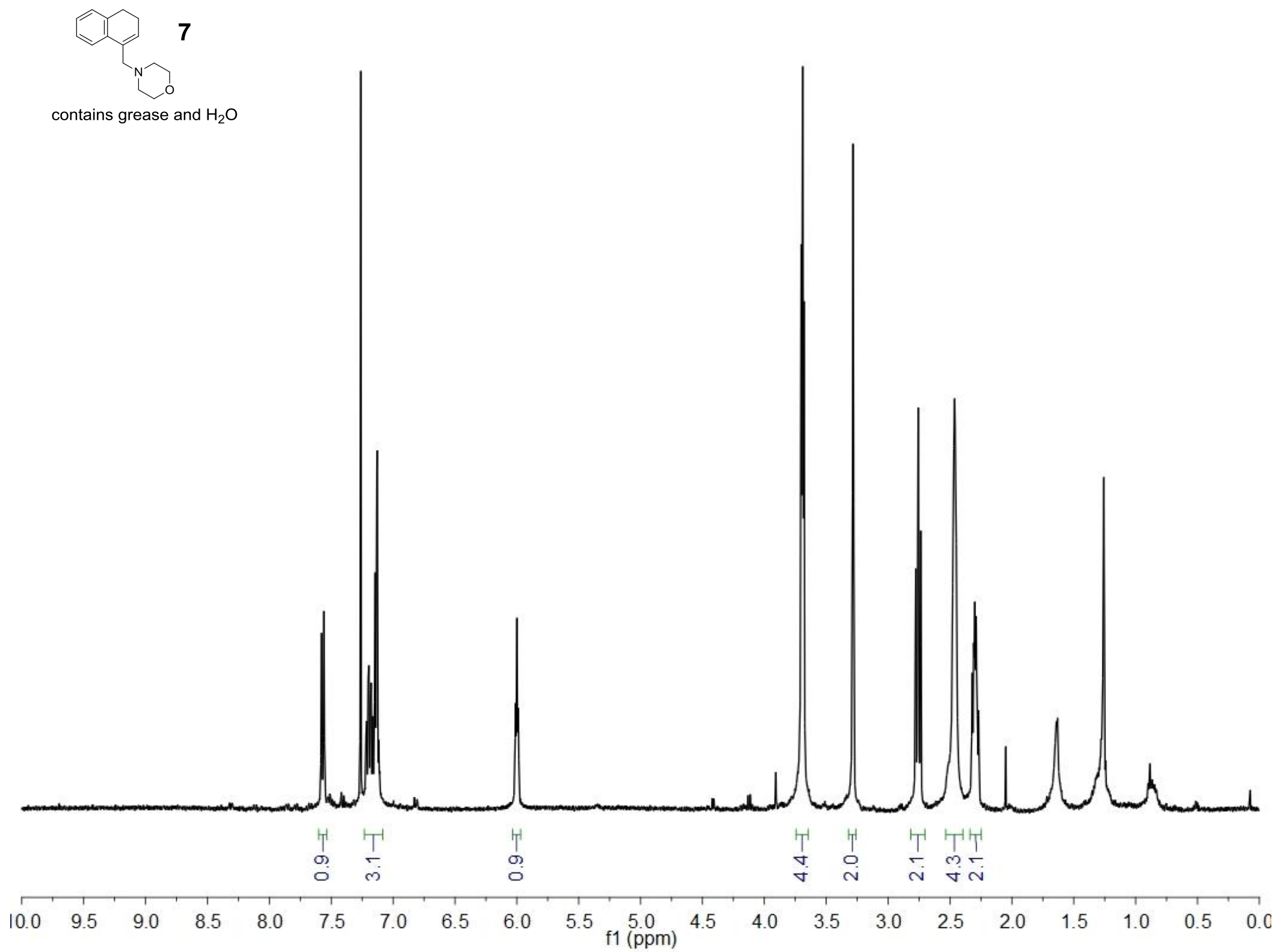

S199 


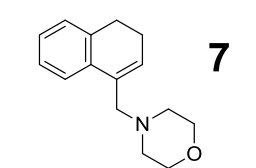

contains grease and $\mathrm{H}_{2} \mathrm{O}$

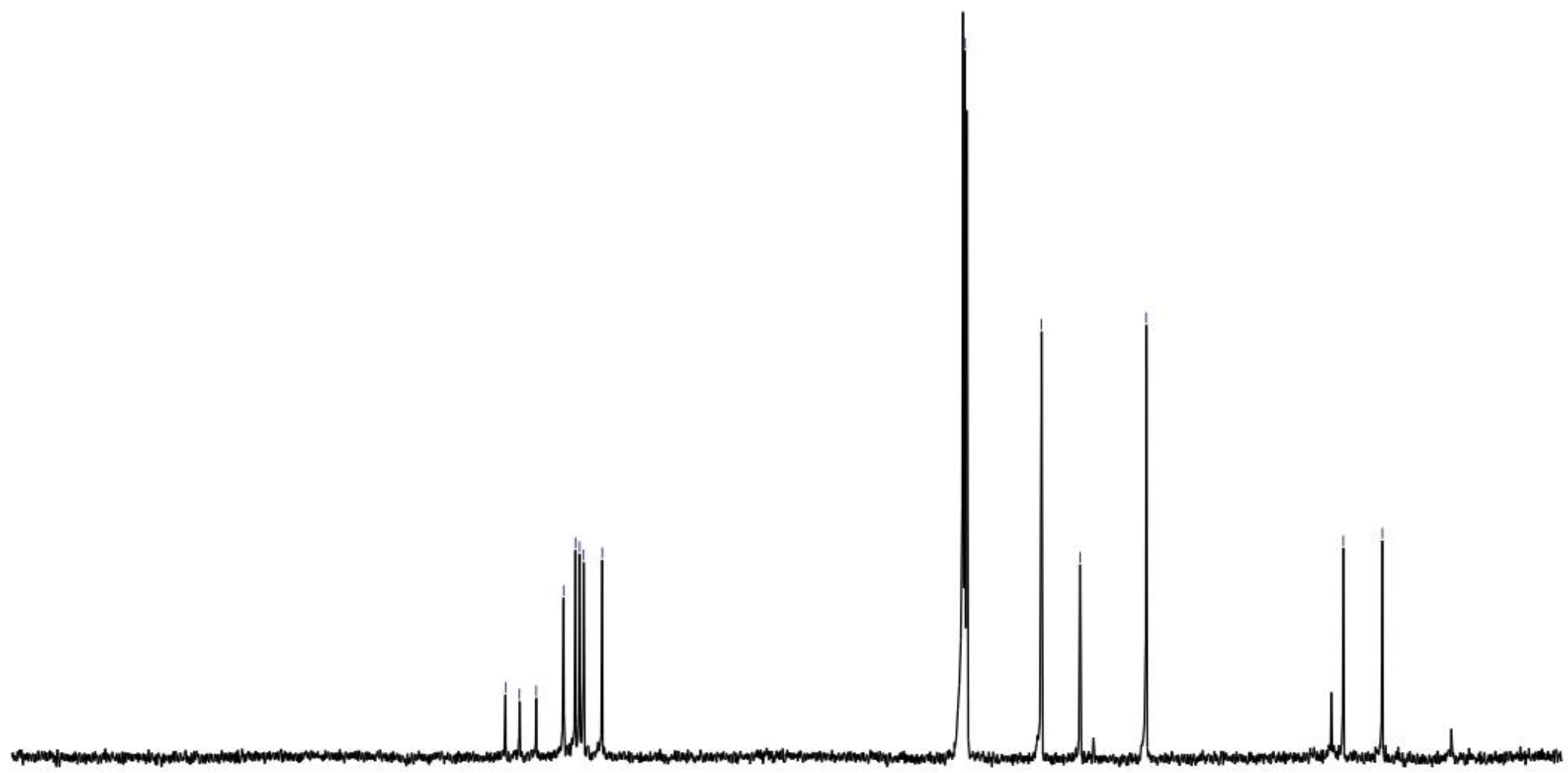




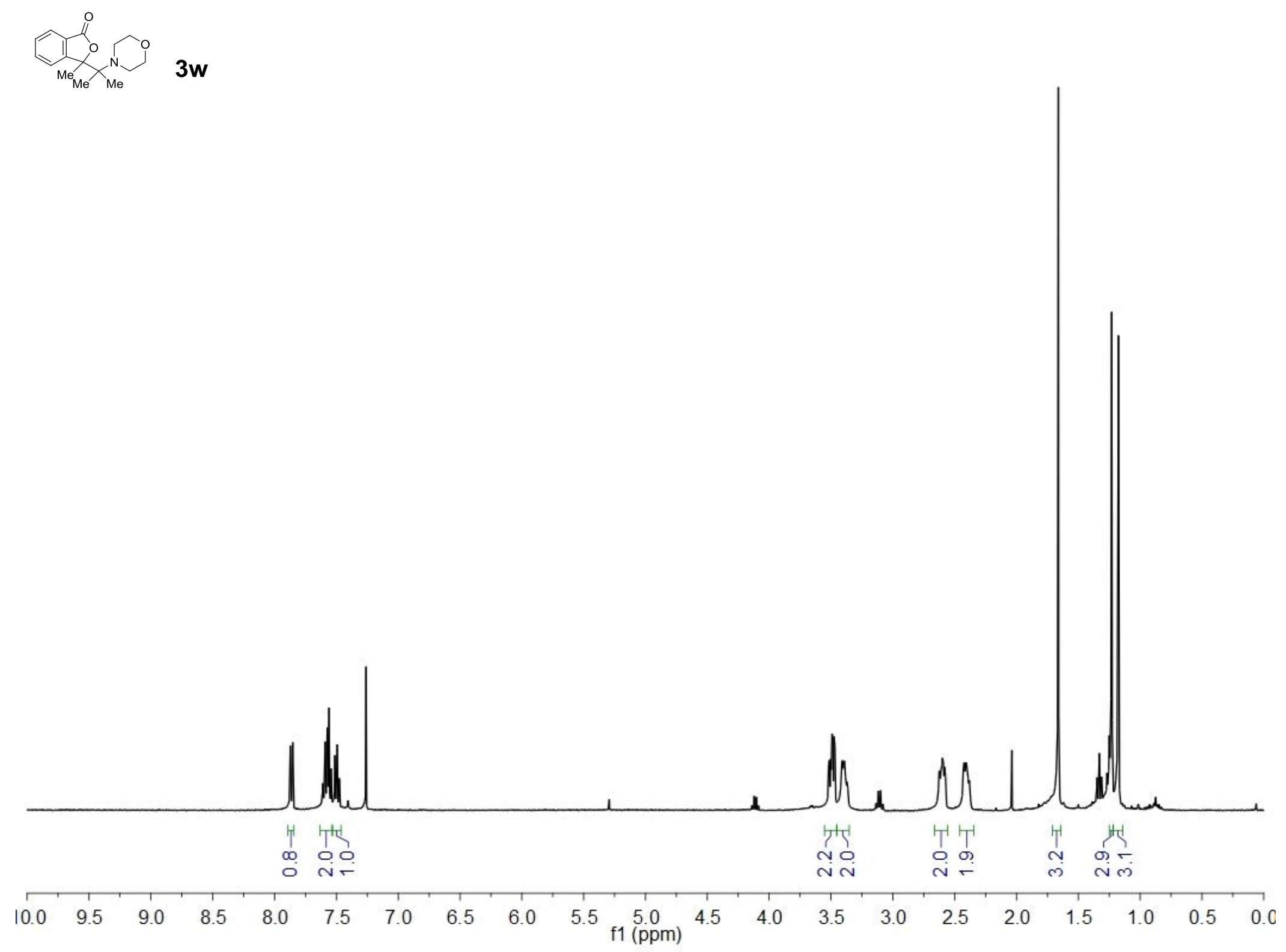




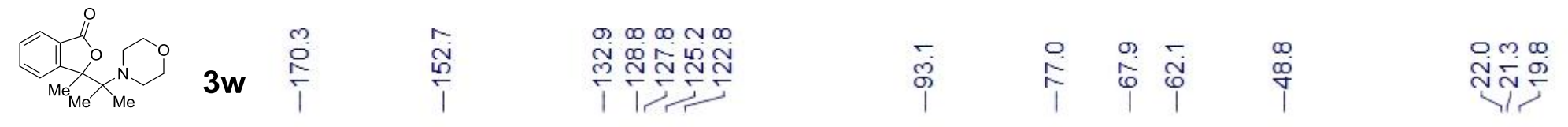

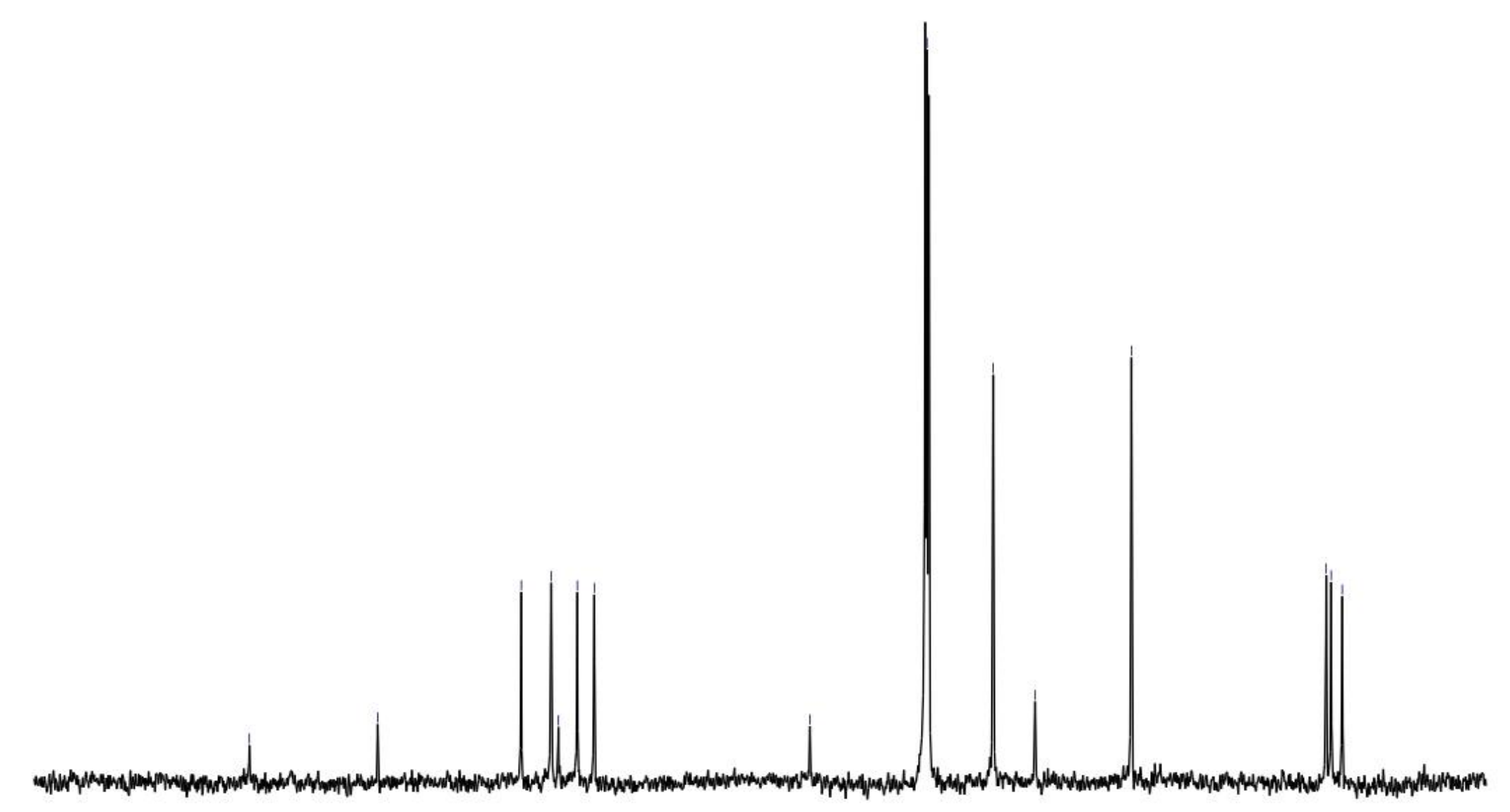



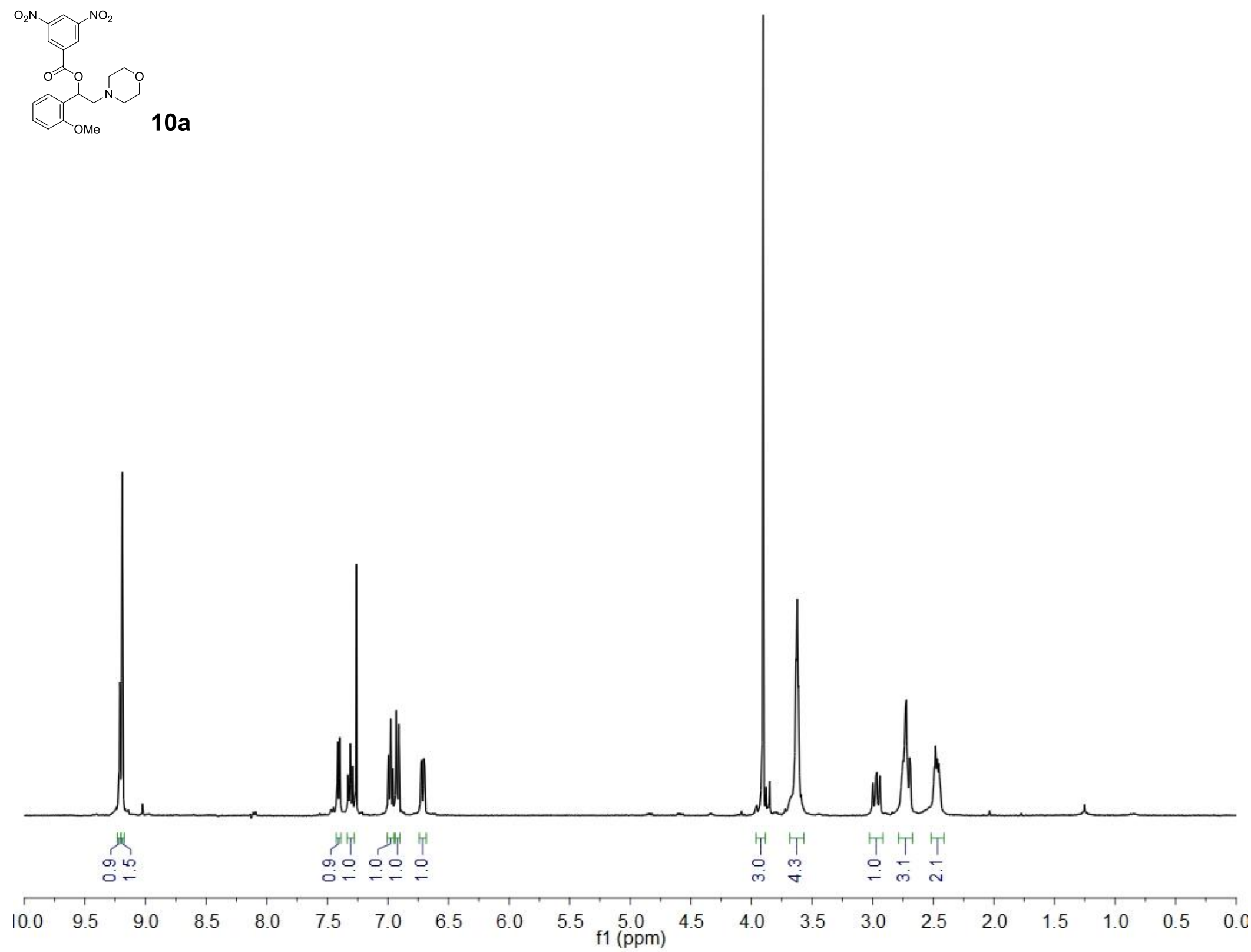


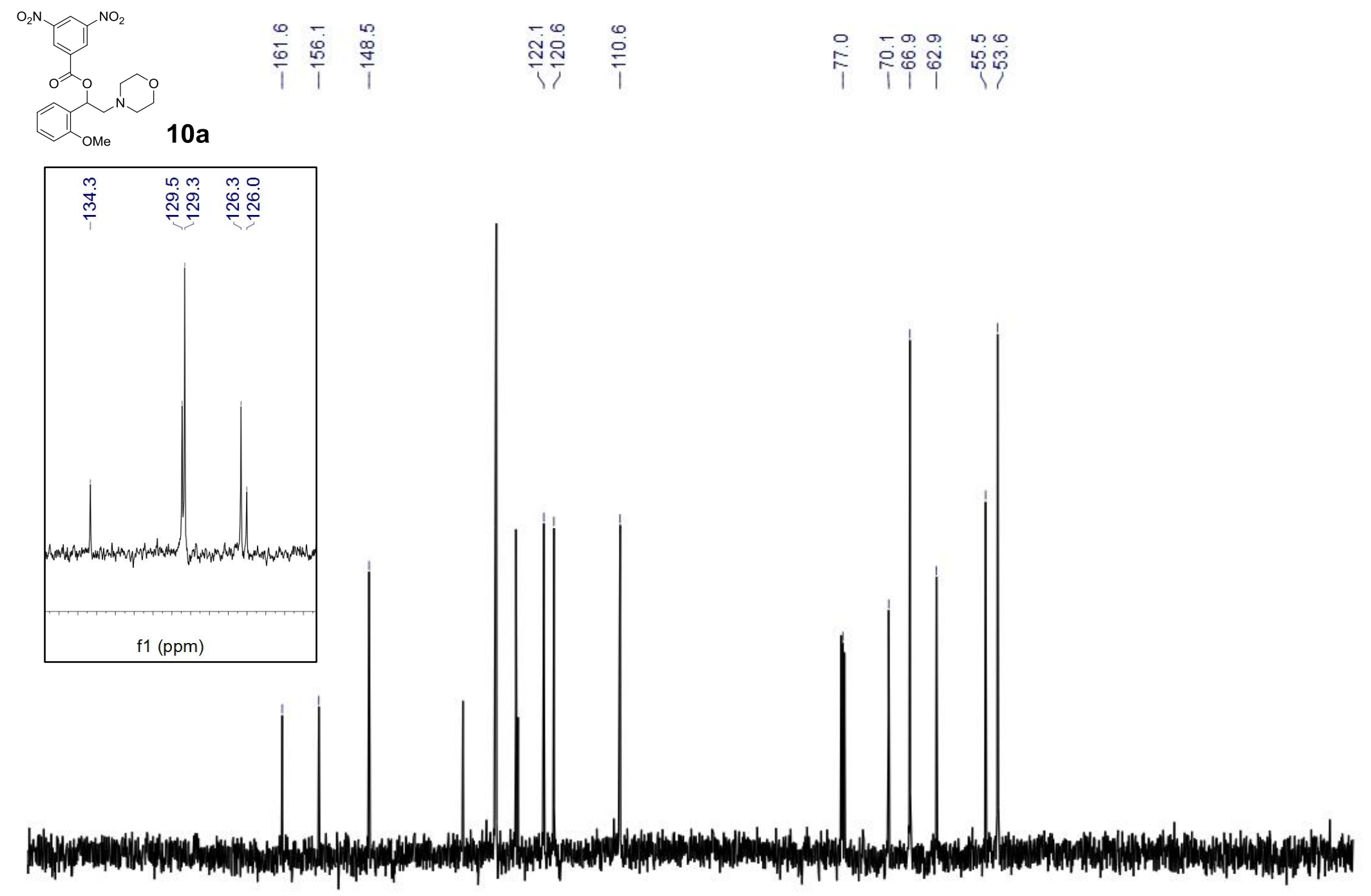

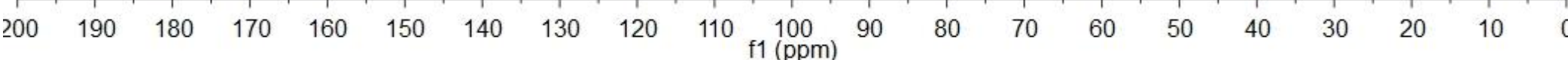



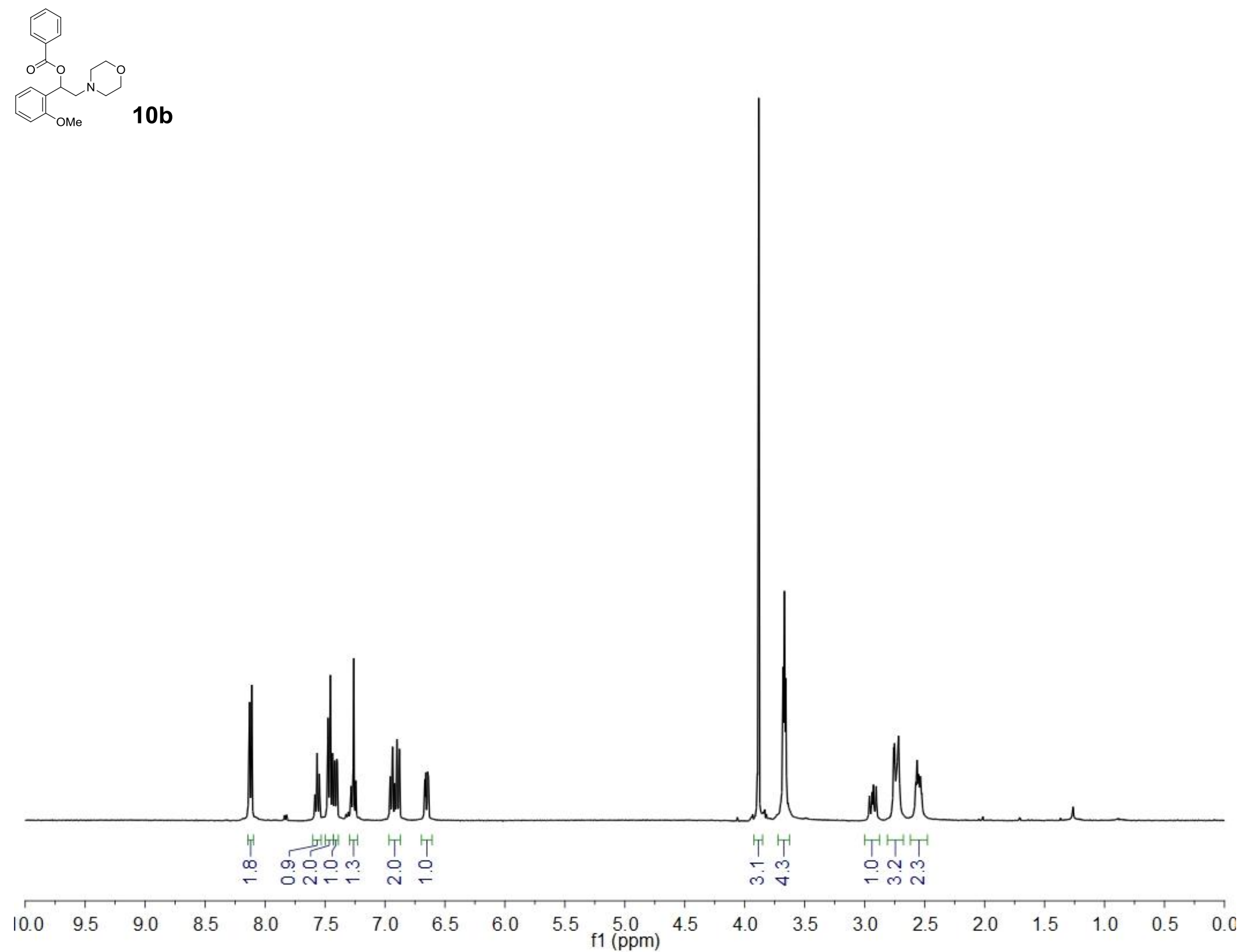

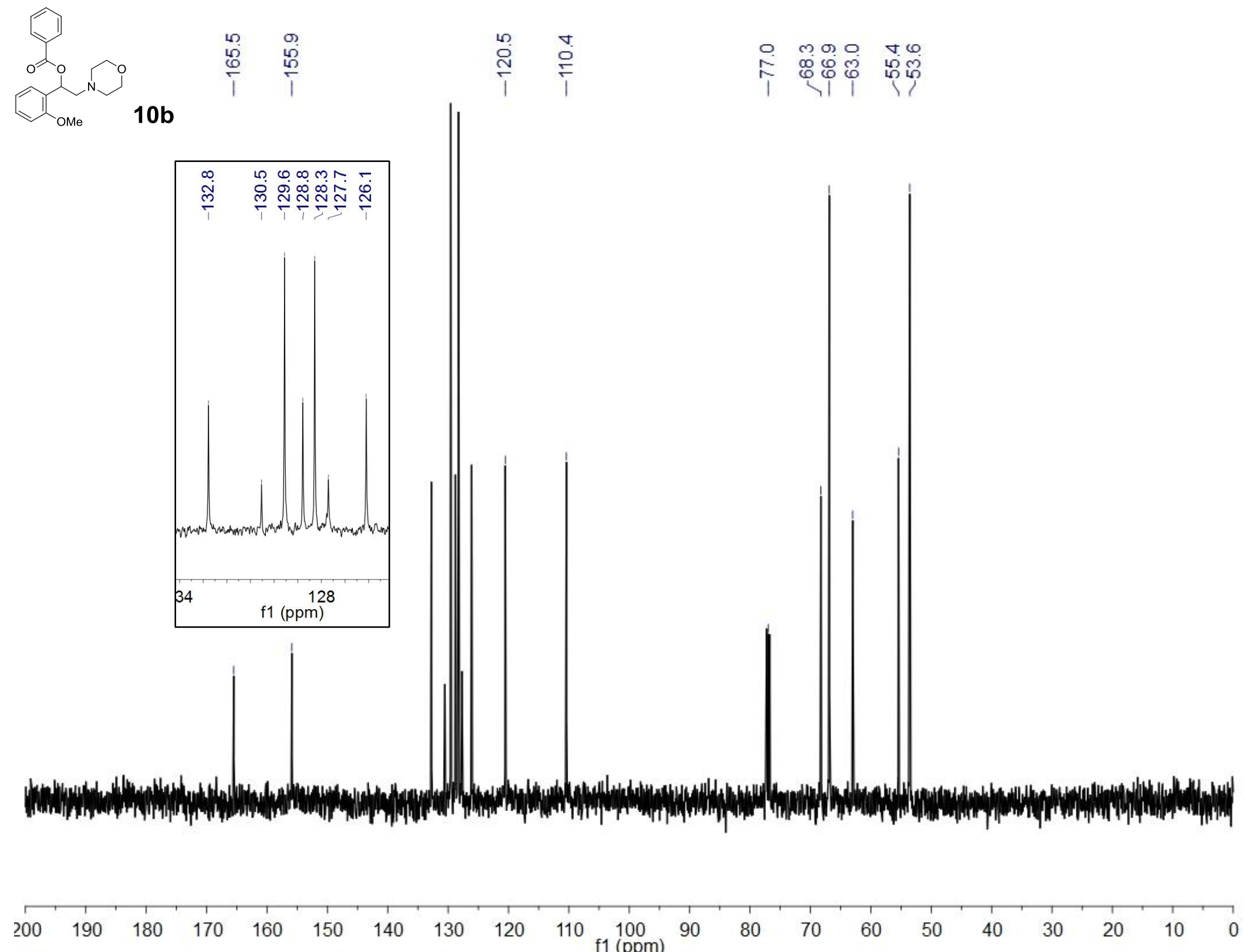


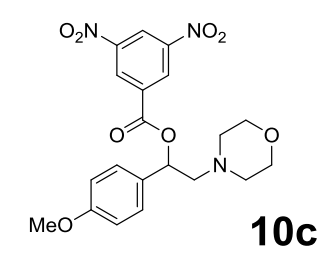

10c

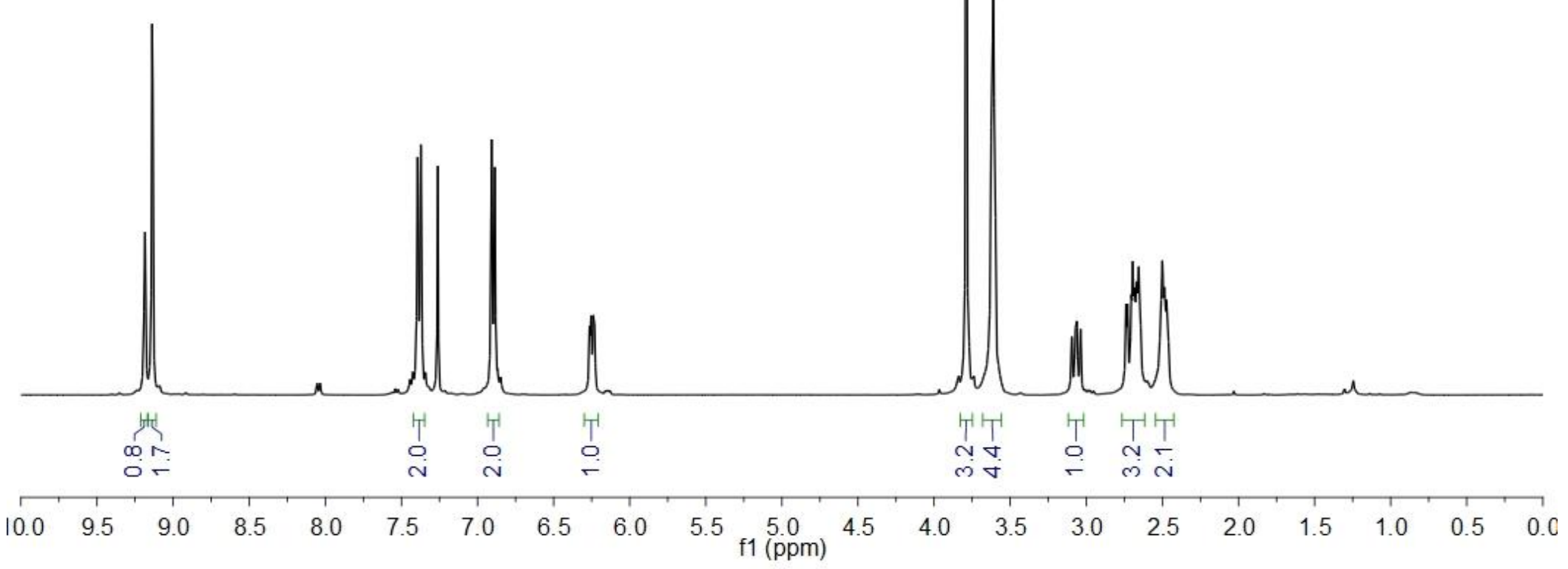



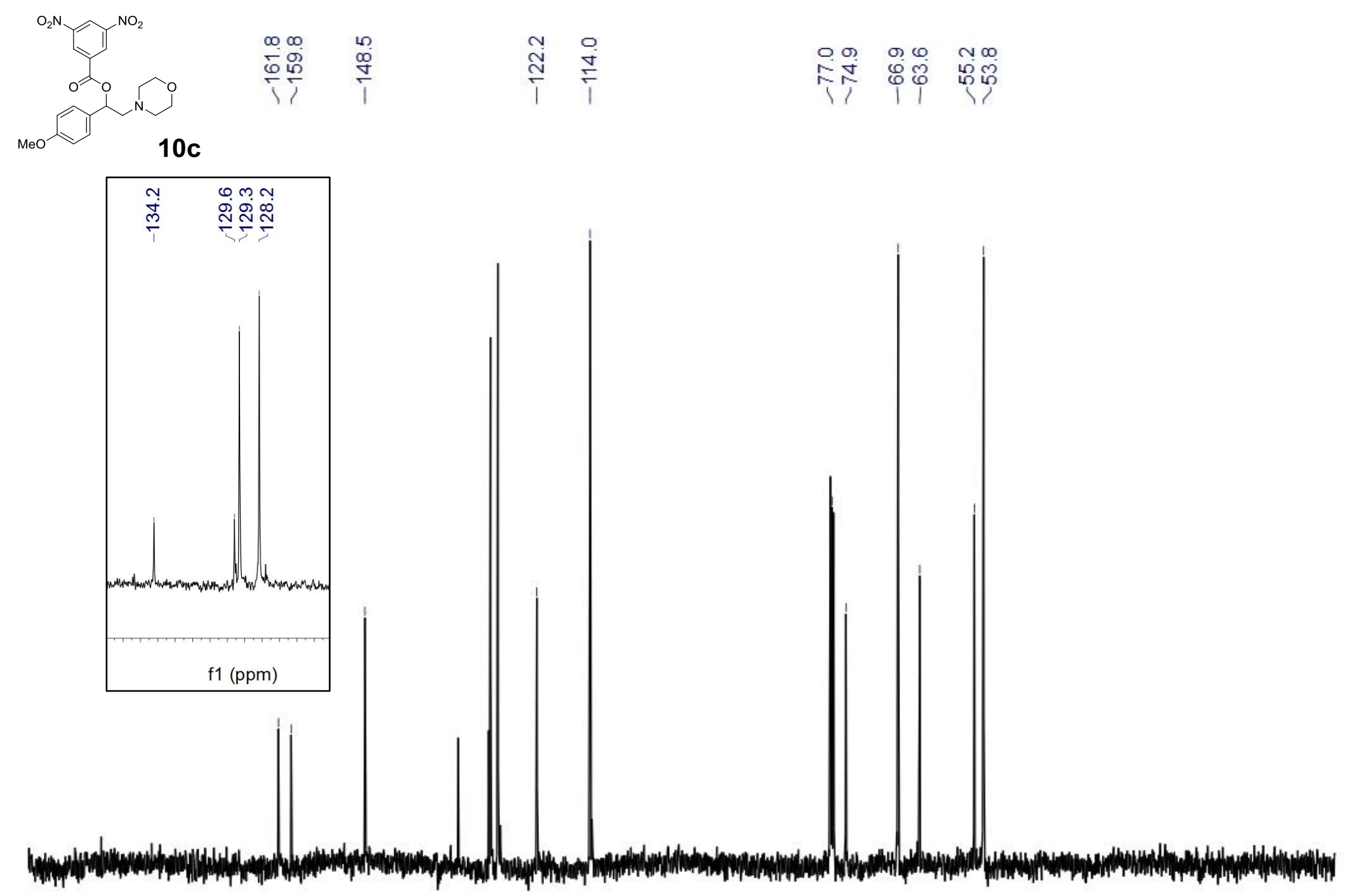


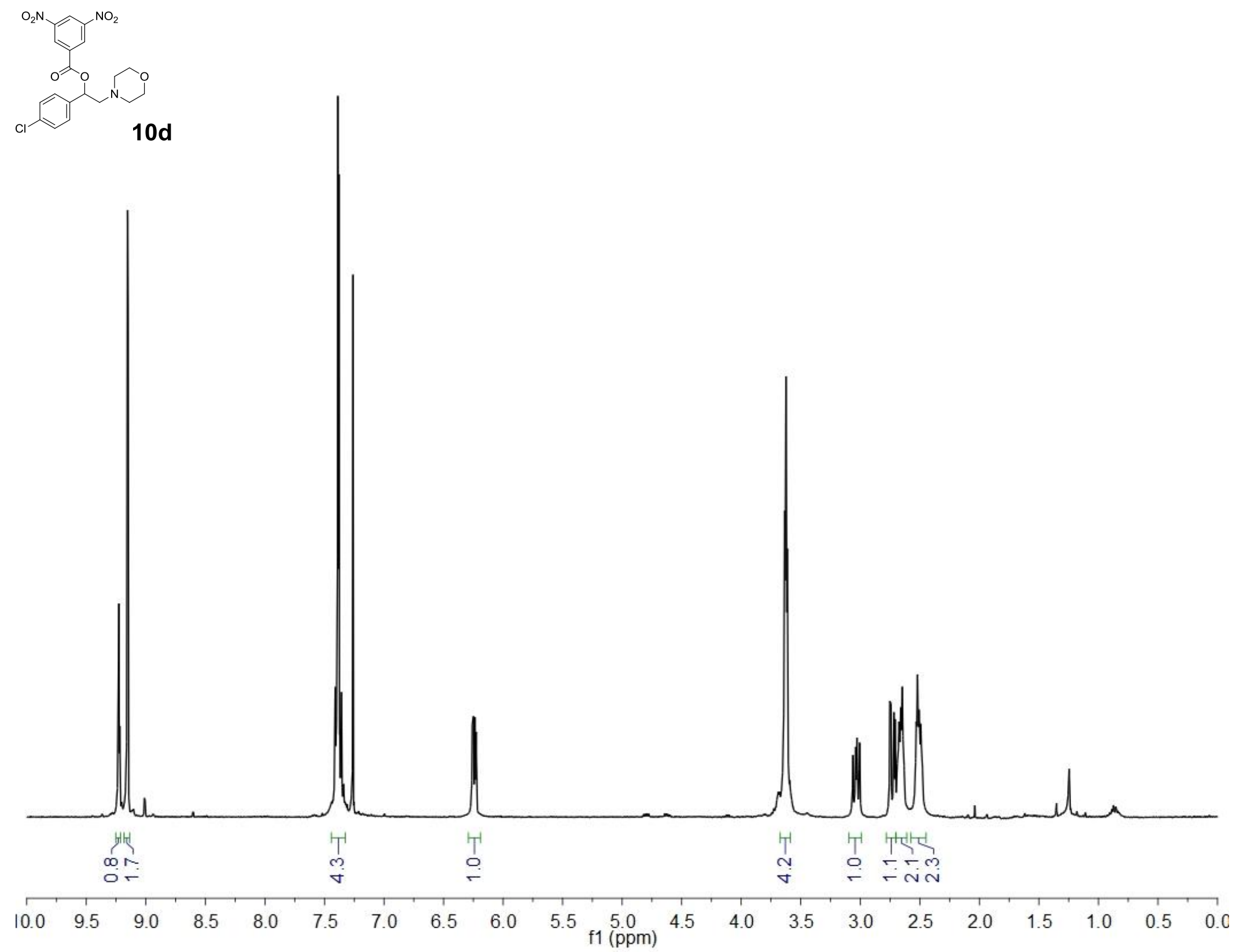



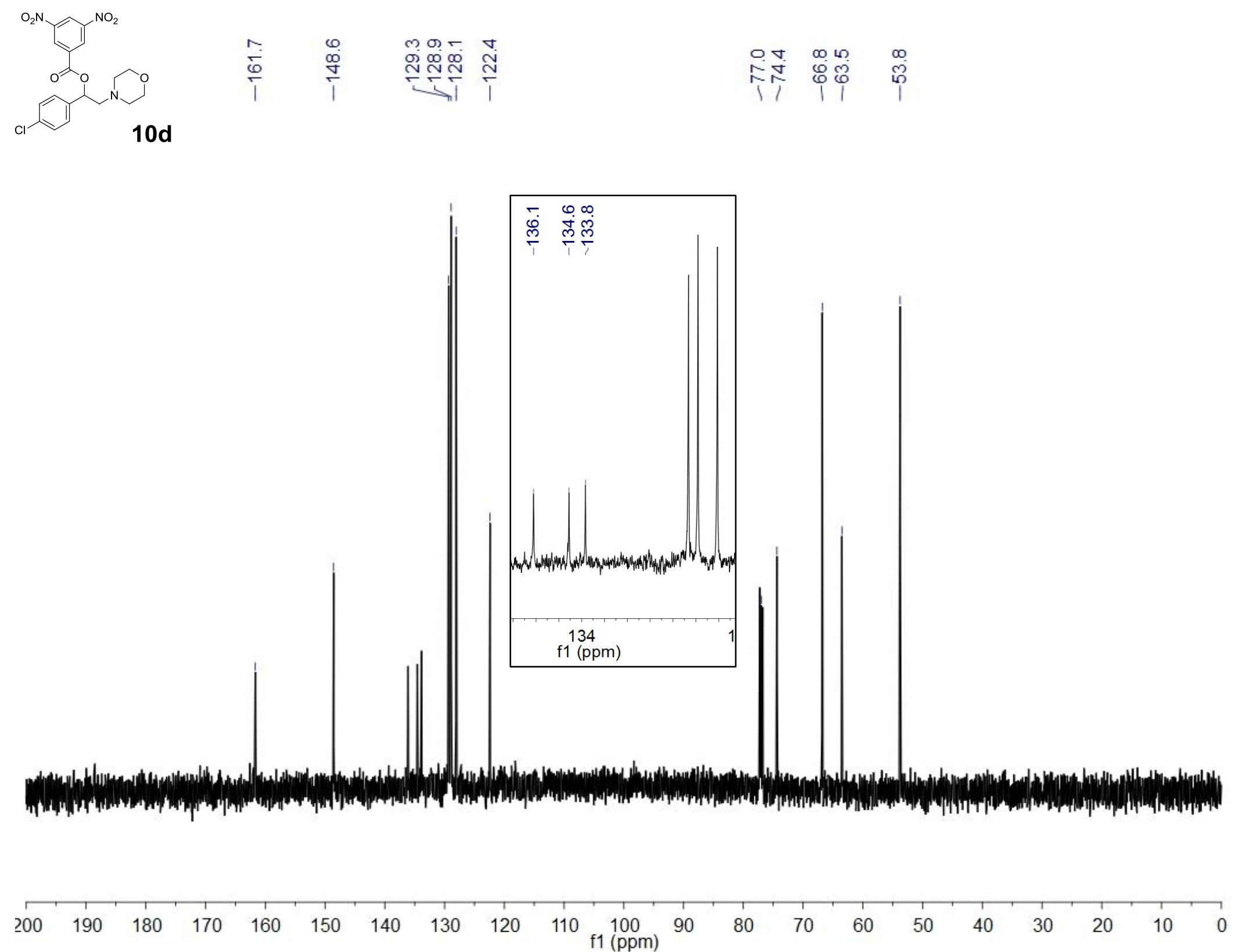

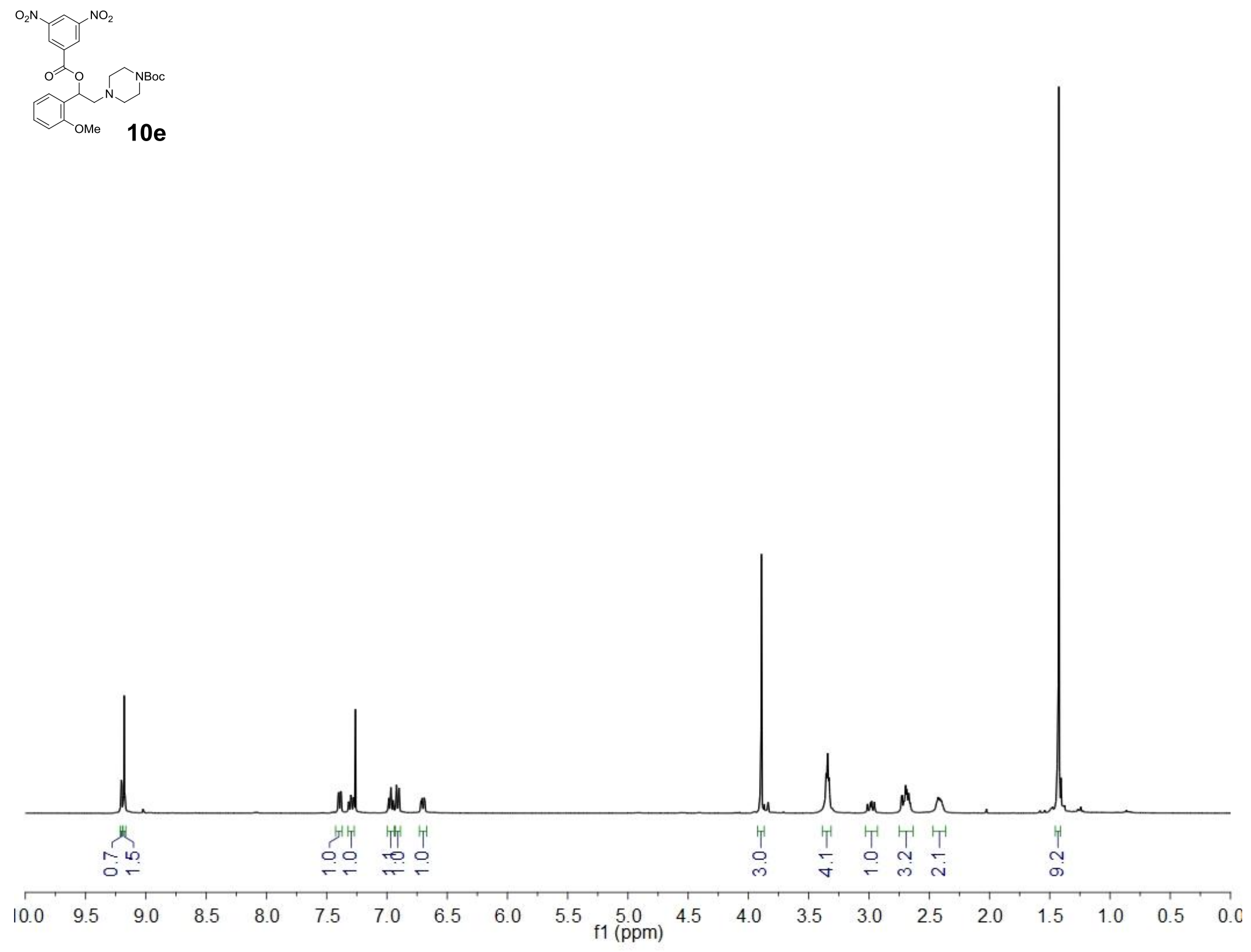

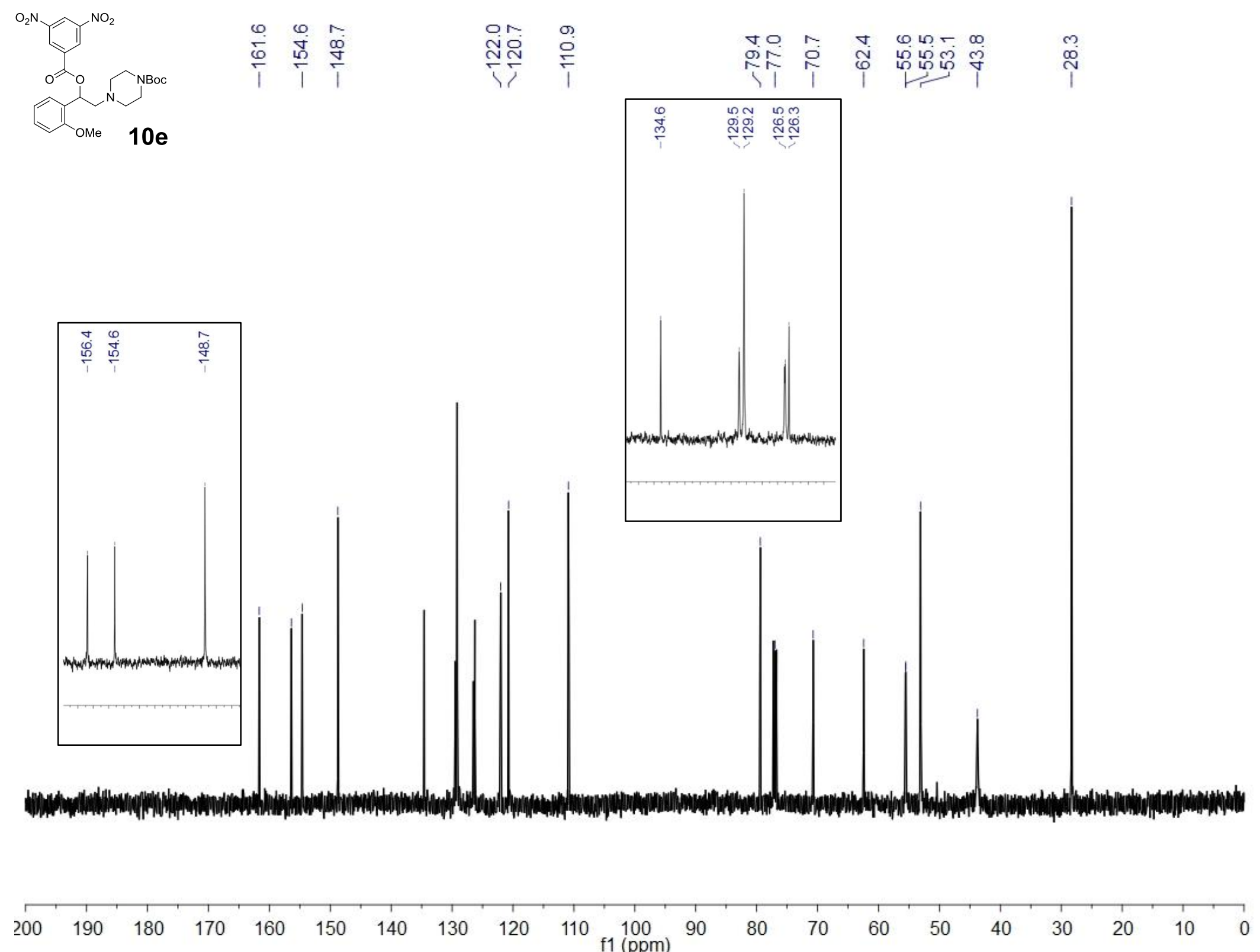


\subsection{X-Ray Crystallography Information}

Crystal Structure Report for rds461 (3r - single diastereomer)
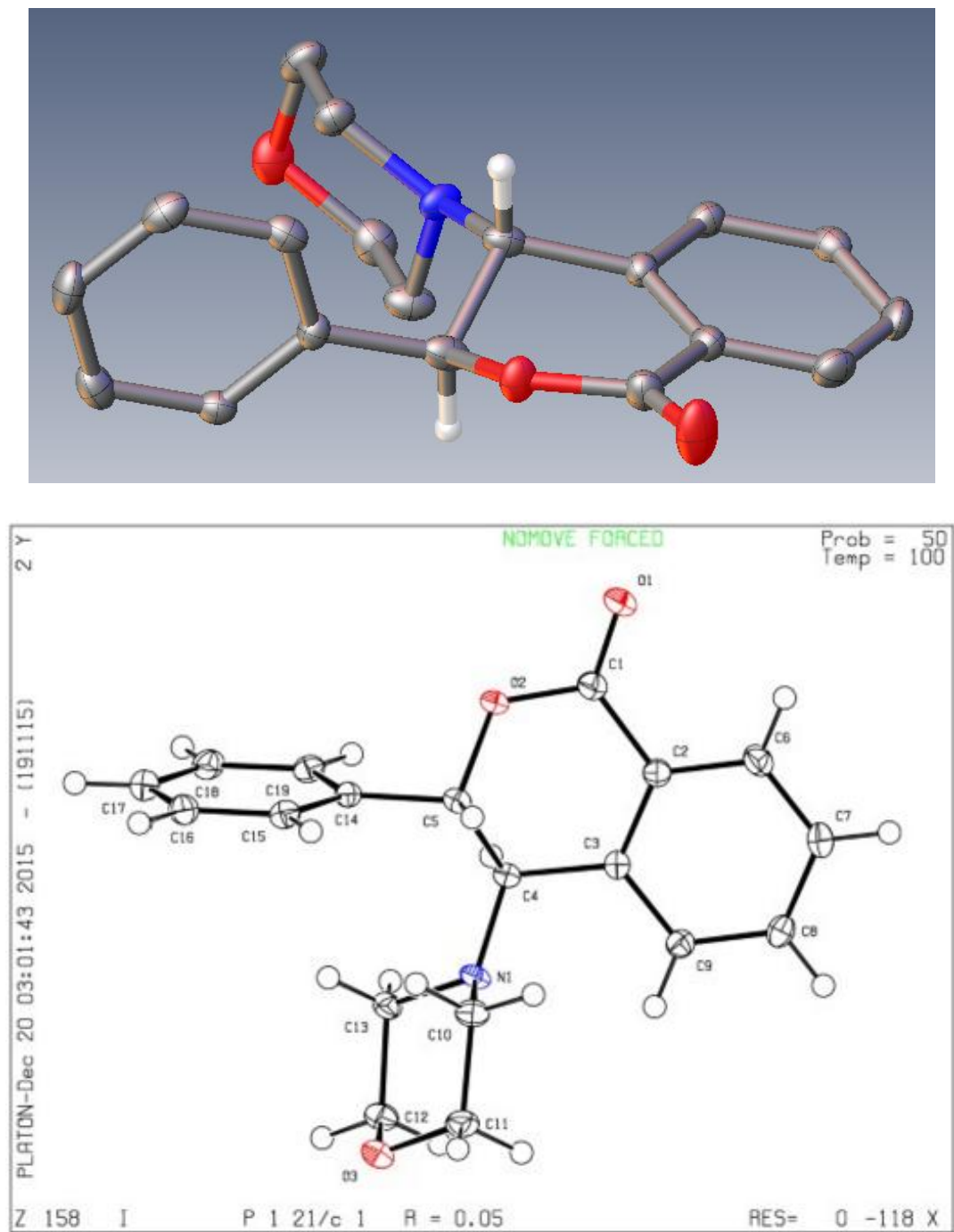

A colorless block-like specimen of $\mathrm{C}_{19} \mathrm{H}_{19} \mathrm{NO}_{3}$, approximate dimensions $0.304 \mathrm{~mm}$ x $0.404 \mathrm{~mm}$ x $0.410 \mathrm{~mm}$, was used for the $\mathrm{X}$-ray crystallographic analysis. The $\mathrm{X}$-ray intensity data were measured.

The total exposure time was 2.95 hours. The frames were integrated with the Bruker SAINT software package using a narrow-frame algorithm. The integration of the data using a monoclinic unit cell yielded a total of 38035 reflections to a maximum $\theta$ angle of $32.03^{\circ}(0.67 \AA$ resolution), of which 5408 were independent (average redundancy 7.033 , completeness $\left.=100.0 \%, \mathrm{R}_{\mathrm{int}}=3.29 \%, \mathrm{R}_{\mathrm{sig}}=2.10 \%\right)$ and $4483(82.90 \%)$ were greater than $2 \sigma\left(\mathrm{F}^{2}\right)$. The final cell constants of $\underline{a}=10.3580(3) \AA, \underline{b}=18.6766(5) \AA, \underline{c}=8.4950(2) \AA, \beta=109.1250(10)^{\circ}$, volume $=1552.67(7) \AA^{3}$, are based upon the refinement of the XYZ-centroids of 308 reflections above $20 \sigma(\mathrm{I})$ with $4.191^{\circ}<2 \theta<62.99^{\circ}$. Data were corrected for absorption effects using the multi-scan method (SADABS). The ratio of minimum to maximum apparent transmission was 0.952 . The calculated minimum and maximum transmission coefficients (based on crystal size) are 0.9640 and 0.9730 .

The final anisotropic full-matrix least-squares refinement on $\mathrm{F}^{2}$ with 208 variables converged at $\mathrm{R} 1=5.11 \%$, for the observed data and $\mathrm{wR} 2=14.39 \%$ for all data. The goodness-of-fit was 1.028 . The largest peak in the final difference 
electron density synthesis was $0.586 \mathrm{e}^{-} / \AA^{3}$ and the largest hole was $-0.263 \mathrm{e}^{-} / \AA^{3}$ with an RMS deviation of $0.063 \mathrm{e}^{-} / \AA^{3}$. On the basis of the final model, the calculated density was $1.323 \mathrm{~g} / \mathrm{cm}^{3}$ and $\mathrm{F}(000), 656 \mathrm{e}^{-}$.

\begin{tabular}{|c|c|c|}
\hline Identification code & \multicolumn{2}{|l|}{ rds461 } \\
\hline Chemical formula & \multicolumn{2}{|l|}{$\mathrm{C}_{19} \mathrm{H}_{19} \mathrm{NO}_{3}$} \\
\hline Formula weight & \multicolumn{2}{|l|}{$309.35 \mathrm{~g} / \mathrm{mol}$} \\
\hline Temperature & \multicolumn{2}{|l|}{$100(2) \mathrm{K}$} \\
\hline Wavelength & \multicolumn{2}{|l|}{$0.71073 \AA$} \\
\hline Crystal size & \multicolumn{2}{|c|}{$0.304 \times 0.404 \times 0.410 \mathrm{~mm}$} \\
\hline Crystal habit & \multicolumn{2}{|l|}{ colorless block } \\
\hline Crystal system & \multicolumn{2}{|l|}{ monoclinic } \\
\hline Space group & \multicolumn{2}{|l|}{ P $121 / c 1$} \\
\hline \multirow[t]{3}{*}{ Unit cell dimensions } & $\mathrm{a}=10.3580(3) \AA$ & $\alpha=90^{\circ}$ \\
\hline & $\mathrm{b}=18.6766(5) \AA$ & $\beta=109.1250(10)^{\circ}$ \\
\hline & $\mathrm{c}=8.4950(2) \AA$ & $\gamma=90^{\circ}$ \\
\hline Volume & \multicolumn{2}{|l|}{$1552.67(7) \AA^{3}$} \\
\hline $\mathbf{Z}$ & \multicolumn{2}{|l|}{4} \\
\hline Density (calculated) & \multicolumn{2}{|l|}{$1.323 \mathrm{~g} / \mathrm{cm}^{3}$} \\
\hline Absorption coefficient & \multicolumn{2}{|l|}{$0.090 \mathrm{~mm}^{-1}$} \\
\hline $\mathbf{F}(\mathbf{0 0 0})$ & \multicolumn{2}{|l|}{656} \\
\hline
\end{tabular}

Data collection and structure refinement for rds461.

\begin{tabular}{|c|c|}
\hline Theta range for data collection & 2.08 to $32.03^{\circ}$ \\
\hline Index ranges & $-15<=\mathrm{h}<=15,-27<=\mathrm{k}<=27,-12<=\mathrm{l}<=12$ \\
\hline Reflections collected & 38035 \\
\hline Independent reflections & $5408[\mathrm{R}(\mathrm{int})=0.0329]$ \\
\hline Coverage of independent reflections & $100.0 \%$ \\
\hline Absorption correction & multi-scan \\
\hline Max. and min. transmission & 0.9730 and 0.9640 \\
\hline Refinement method & Full-matrix least-squares on $\mathrm{F}^{2}$ \\
\hline Refinement program & SHELXL-2013 (Sheldrick, 2013) \\
\hline Function minimized & $\sum \mathrm{w}\left(\mathrm{F}_{\mathrm{o}}^{2}-\mathrm{F}_{\mathrm{c}}^{2}\right)^{2}$ \\
\hline Data / restraints / parameters & $5408 / 0 / 208$ \\
\hline Goodness-of-fit on $\mathbf{F}^{2}$ & 1.028 \\
\hline$\Delta / \sigma_{\max }$ & 0.001 \\
\hline Final $\mathbf{R}$ indices & 4483 data; $\mathrm{I}>2 \sigma(\mathrm{I}) \quad \mathrm{R} 1=0.0511, \mathrm{wR} 2=0.1357$ \\
\hline & all data $\quad \mathrm{R} 1=0.0618, \mathrm{wR} 2=0.1439$ \\
\hline Weighting scheme & $\begin{array}{l}\mathrm{w}=1 /\left[\sigma^{2}\left(\mathrm{~F}_{\mathrm{o}}^{2}\right)+(0.0761 \mathrm{P})^{2}+0.7707 \mathrm{P}\right] \\
\text { where } \mathrm{P}=\left(\mathrm{F}_{\mathrm{o}}^{2}+2 \mathrm{~F}_{\mathrm{c}}^{2}\right) / 3\end{array}$ \\
\hline Largest diff. peak and hole & 0.586 and $-0.263 \mathrm{e}^{-3}$ \\
\hline R.M.S. deviation from mean & $0.063 \mathrm{e}^{-3}$ \\
\hline
\end{tabular}

Atomic coordinates and equivalent isotropic atomic displacement parameters $\left(\AA^{2}\right)$ for rds461.

$\mathrm{U}(\mathrm{eq})$ is defined as one third of the trace of the orthogonalized 


\begin{tabular}{|c|c|c|c|c|}
\hline & $\mathbf{x} / \mathbf{a}$ & $\mathbf{y} / \mathbf{b}$ & $\mathbf{z} / \mathbf{c}$ & $\mathbf{U}(\mathbf{e q})$ \\
\hline $\mathrm{O} 1$ & $0.36298(10)$ & $0.70396(6)$ & $0.72129(13)$ & $0.0323(2)$ \\
\hline $\mathrm{O} 2$ & $0.54714(8)$ & $0.68841(4)$ & $0.65125(10)$ & $0.01713(17)$ \\
\hline $\mathrm{O} 3$ & $0.76079(9)$ & $0.55834(5)$ & $0.08812(11)$ & $0.02272(19)$ \\
\hline N1 & $0.61262(9)$ & $0.57792(5)$ & $0.31176(11)$ & $0.01451(18)$ \\
\hline $\mathrm{C} 1$ & $0.41129(12)$ & $0.68026(6)$ & $0.62065(15)$ & $0.0190(2)$ \\
\hline $\mathrm{C} 2$ & $0.33400(11)$ & $0.63956(6)$ & $0.46913(14)$ & $0.01549(19)$ \\
\hline $\mathrm{C} 3$ & $0.40359(11)$ & $0.59960(6)$ & $0.38291(13)$ & $0.01380(19)$ \\
\hline $\mathrm{C} 4$ & $0.55878(11)$ & $0.60018(6)$ & $0.44283(13)$ & $0.01336(18)$ \\
\hline $\mathrm{C} 5$ & $0.60660(11)$ & $0.67412(6)$ & $0.51970(13)$ & $0.01431(19)$ \\
\hline C6 & $0.19141(12)$ & $0.63851(6)$ & $0.41973(15)$ & $0.0194(2)$ \\
\hline $\mathrm{C} 7$ & $0.11751(11)$ & $0.59724(6)$ & $0.28398(15)$ & $0.0193(2)$ \\
\hline $\mathrm{C} 8$ & $0.18688(11)$ & $0.55686(6)$ & $0.20006(13)$ & $0.0168(2)$ \\
\hline C9 & $0.32835(11)$ & $0.55795(6)$ & $0.24839(13)$ & $0.0156(2)$ \\
\hline $\mathrm{C} 10$ & $0.60378(12)$ & $0.63068(6)$ & $0.18212(14)$ & $0.0174(2)$ \\
\hline $\mathrm{C} 11$ & $0.63159(12)$ & $0.59350(7)$ & $0.03763(14)$ & $0.0198(2)$ \\
\hline $\mathrm{C} 12$ & $0.76796(13)$ & $0.50758(7)$ & $0.21649(15)$ & $0.0213(2)$ \\
\hline $\mathrm{C} 13$ & $0.74673(11)$ & $0.54349(6)$ & $0.36648(14)$ & $0.0162(2)$ \\
\hline $\mathrm{C} 14$ & $0.75884(11)$ & $0.67918(6)$ & $0.60280(13)$ & $0.01360(19)$ \\
\hline $\mathrm{C} 15$ & $0.83474(11)$ & $0.72925(6)$ & $0.54957(13)$ & $0.0158(2)$ \\
\hline C16 & $0.97591(12)$ & $0.73308(6)$ & $0.62617(15)$ & $0.0197(2)$ \\
\hline $\mathrm{C} 17$ & $0.04133(12)$ & $0.68689(7)$ & $0.75528(15)$ & $0.0216(2)$ \\
\hline $\mathrm{C} 18$ & $0.96537(12)$ & $0.63740(6)$ & $0.80959(14)$ & $0.0194(2)$ \\
\hline C19 & $0.82499(12)$ & $0.63356(6)$ & $0.73435(13)$ & $0.0157(2)$ \\
\hline
\end{tabular}

Bond lengths ( $(\AA)$ for rds461.

\begin{tabular}{llll}
\hline O1-C1 & $1.2072(14)$ & O2-C1 & $1.3532(14)$ \\
O2-C5 & $1.4664(13)$ & O3-C11 & $1.4248(15)$ \\
O3-C12 & $1.4285(16)$ & N1-C10 & $1.4582(14)$ \\
N1-C4 & $1.4597(13)$ & N1-C13 & $1.4615(14)$ \\
C1-C2 & $1.4833(16)$ & C2-C6 & $1.3970(15)$ \\
C2-C3 & $1.3997(15)$ & C3-C9 & $1.3910(15)$ \\
C3-C4 & $1.5189(15)$ & C4-C5 & $1.5384(15)$ \\
C4-H4 & 1.0 & C5-C14 & $1.5048(15)$ \\
C5-H5 & 1.0 & C6-C7 & $1.3897(17)$ \\
C6-H6 & 0.95 & C7-C8 & $1.3889(16)$ \\
C7-H7 & 0.95 & C8-C9 & $1.3863(15)$ \\
C8-H8 & 0.95 & C9-H9 & 0.95 \\
C10-C11 & $1.5177(15)$ & C10-H10A & 0.99 \\
C10-H10B & 0.99 & C11-H11A & 0.99 \\
C11-H11B & 0.99 & C12-C13 & $1.5183(15)$ \\
C12-H12A & 0.99 & C12-H12B & 0.99 \\
C13-H13A & 0.99 & C13-H13B & 0.99 \\
C14-C15 & $1.3893(14)$ & C14-C19 & $1.3937(14)$ \\
C15-C16 & $1.3940(16)$ & C15-H15 & 0.95 \\
C16-C17 & $1.3854(18)$ & C16-H16 & 0.95 \\
C17-C18 & $1.3871(17)$ & C17-H17 & 0.95 \\
C18-C19 & $1.3853(16)$ & C18-H18 & 0.95 \\
C19-H19 & 0.95 & &
\end{tabular}


Bond angles $\left({ }^{\circ}\right)$ for rds461.

\begin{tabular}{|c|c|c|c|}
\hline $\mathrm{C} 1-\mathrm{O} 2-\mathrm{C} 5$ & $120.20(9)$ & $\mathrm{C} 11-\mathrm{O} 3-\mathrm{C} 12$ & $110.15(9)$ \\
\hline C10-N1-C4 & $116.00(8)$ & C10-N1-C13 & $110.82(9)$ \\
\hline C4-N1-C13 & 116.33(8) & $\mathrm{O} 1-\mathrm{C} 1-\mathrm{O} 2$ & $117.96(11)$ \\
\hline $\mathrm{O} 1-\mathrm{C} 1-\mathrm{C} 2$ & $124.84(11)$ & $\mathrm{O} 2-\mathrm{C} 1-\mathrm{C} 2$ & 117.13(9) \\
\hline C6-C2-C3 & $120.68(10)$ & $\mathrm{C} 6-\mathrm{C} 2-\mathrm{C} 1$ & $119.00(10)$ \\
\hline $\mathrm{C} 3-\mathrm{C} 2-\mathrm{C} 1$ & $120.22(10)$ & $\mathrm{C} 9-\mathrm{C} 3-\mathrm{C} 2$ & $118.82(10)$ \\
\hline C9-C3-C4 & 121.73(9) & $\mathrm{C} 2-\mathrm{C} 3-\mathrm{C} 4$ & 119.39(9) \\
\hline N1-C4-C3 & $111.66(8)$ & N1-C4-C5 & $115.42(9)$ \\
\hline C3-C4-C5 & $107.87(8)$ & N1-C4-H4 & 107.2 \\
\hline C3-C4-H4 & 107.2 & C5-C4-H4 & 107.2 \\
\hline $\mathrm{O} 2-\mathrm{C} 5-\mathrm{C} 14$ & $105.56(8)$ & $\mathrm{O} 2-\mathrm{C} 5-\mathrm{C} 4$ & $109.25(8)$ \\
\hline C14-C5-C4 & $113.43(8)$ & $\mathrm{O} 2-\mathrm{C} 5-\mathrm{H} 5$ & 109.5 \\
\hline C14-C5-H5 & 109.5 & C4-C5-H5 & 109.5 \\
\hline C7-C6-C2 & $119.85(10)$ & C7-C6-H6 & 120.1 \\
\hline C2-C6-H6 & 120.1 & C8-C7-C6 & $119.36(10)$ \\
\hline C8-C7-H7 & 120.3 & C6-C7-H7 & 120.3 \\
\hline C9-C8-C7 & $120.95(10)$ & C9-C8-H8 & 119.5 \\
\hline C7-C8-H8 & 119.5 & $\mathrm{C} 8-\mathrm{C} 9-\mathrm{C} 3$ & $120.33(10)$ \\
\hline C8-C9-H9 & 119.8 & C3-C9-H9 & 119.8 \\
\hline N1-C10-C11 & $108.66(9)$ & N1-C10-H10A & 110.0 \\
\hline C11-C10-H10A & 110.0 & N1-C10-H10B & 110.0 \\
\hline C11-C10-H10B & 110.0 & H10A-C10-H10B & 108.3 \\
\hline O3-C11-C10 & 112.07(9) & O3-C11-H11A & 109.2 \\
\hline C10-C11-H11A & 109.2 & O3-C11-H11B & 109.2 \\
\hline C10-C11-H11B & 109.2 & H11A-C11-H11B & 107.9 \\
\hline $\mathrm{O} 3-\mathrm{C} 12-\mathrm{C} 13$ & 111.21(9) & O3-C12-H12A & 109.4 \\
\hline C13-C12-H12A & 109.4 & O3-C12-H12B & 109.4 \\
\hline C13-C12-H12B & 109.4 & H12A-C12-H12B & 108.0 \\
\hline N1-C13-C12 & $108.14(9)$ & N1-C13-H13A & 110.1 \\
\hline C12-C13-H13A & 110.1 & N1-C13-H13B & 110.1 \\
\hline C12-C13-H13B & 110.1 & H13A-C13-H13B & 108.4 \\
\hline C15-C14-C19 & $119.37(10)$ & C15-C14-C5 & $120.49(9)$ \\
\hline C19-C14-C5 & 120.14(9) & C14-C15-C16 & $120.10(10)$ \\
\hline C14-C15-H15 & 119.9 & C16-C15-H15 & 119.9 \\
\hline C17-C16-C15 & $120.25(10)$ & C17-C16-H16 & 119.9 \\
\hline C15-C16-H16 & 119.9 & C16-C17-C18 & $119.64(11)$ \\
\hline C16-C17-H17 & 120.2 & C18-C17-H17 & 120.2 \\
\hline C19-C18-C17 & $120.34(11)$ & C19-C18-H18 & 119.8 \\
\hline C17-C18-H18 & 119.8 & C18-C19-C14 & $120.28(10)$ \\
\hline C18-C19-H19 & 119.9 & C14-C19-H19 & 119.9 \\
\hline
\end{tabular}

Torsion angles $\left({ }^{\circ}\right)$ for rds461.

\begin{tabular}{llll}
\hline C5-O2-C1-O1 & $-167.59(11)$ & C5-O2-C1-C2 & $15.29(15)$ \\
O1-C1-C2-C6 & $12.16(19)$ & O2-C1-C2-C6 & $-170.94(10)$ \\
O1-C1-C2-C3 & $-164.24(13)$ & O2-C1-C2-C3 & $12.66(16)$ \\
C6-C2-C3-C9 & $-0.89(16)$ & C1-C2-C3-C9 & $175.44(10)$ \\
C6-C2-C3-C4 & $-178.22(10)$ & C1-C2-C3-C4 & $-1.89(15)$ \\
C10-N1-C4-C3 & $75.59(11)$ & C13-N1-C4-C3 & $-151.35(9)$ \\
C10-N1-C4-C5 & $-48.07(13)$ & C13-N1-C4-C5 & $84.98(11)$ \\
C9-C3-C4-N1 & $22.76(14)$ & C2-C3-C4-N1 & $-160.00(9)$
\end{tabular}




$\begin{array}{llll}\text { C9-C3-C4-C5 } & 150.58(10) & \text { C2-C3-C4-C5 } & -32.17(12) \\ \text { C1-O2-C5-C14 } & -172.87(9) & \text { C1-O2-C5-C4 } & -50.56(13) \\ \text { N1-C4-C5-O2 } & -178.72(8) & \text { C3-C4-C5-O2 } & 55.65(10) \\ \text { N1-C4-C5-C14 } & -61.26(12) & \text { C3-C4-C5-C14 } & 173.10(8) \\ \text { C3-C2-C6-C7 } & 0.21(17) & \text { C1-C2-C6-C7 } & -176.16(11) \\ \text { C2-C6-C7-C8 } & 0.68(18) & \text { C6-C7-C8-C9 } & -0.89(17) \\ \text { C7-C8-C9-C3 } & 0.21(17) & \text { C2-C3-C9-C8 } & 0.68(16) \\ \text { C4-C3-C9-C8 } & 177.95(10) & \text { C4-N1-C10-C11 } & -166.55(9) \\ \text { C13-N1-C10-C11 } & 57.92(12) & \text { C12-O3-C11-C10 } & 57.53(12) \\ \text { N1-C10-C11-O3 } & -56.90(12) & \text { C11-O3-C12-C13 } & -58.63(12) \\ \text { C10-N1-C13-C12 } & -59.16(12) & \text { C4-N1-C13-C12 } & 165.48(9) \\ \text { O3-C12-C13-N1 } & 59.20(12) & \text { O2-C5-C14-C15 } & -119.85(10) \\ \text { C4-C5-C14-C15 } & 120.57(11) & \text { O2-C5-C14-C19 } & 60.08(12) \\ \text { C4-C5-C14-C19 } & -59.50(13) & \text { C19-C14-C15-C16 } & 0.72(16) \\ \text { C5-C14-C15-C16 } & -179.36(10) & \text { C14-C15-C16-C17 } & 0.21(17) \\ \text { C15-C16-C17-C18 } & -0.89(18) & \text { C16-C17-C18-C19 } & 0.63(18) \\ \text { C17-C18-C19-C14 } & 0.31(17) & \text { C15-C14-C19-C18 } & -0.98(16) \\ \text { C5-C14-C19-C18 } & 179.10(10) & & \end{array}$

\section{Anisotropic atomic displacement parameters $\left(\AA^{2}\right)$ for rds461.}

The anisotropic atomic displacement factor exponent takes the form: $-2 \pi^{2}\left[\mathrm{~h}^{2} \mathrm{a}^{* 2} \mathrm{U}_{11}+\ldots\right.$ $\left.+2 \mathrm{~h} \mathrm{k} \mathrm{a}^{*} \mathrm{~b}^{*} \mathrm{U}_{12}\right]$

$\begin{array}{lrlllll} & \mathbf{U}_{11} & \mathbf{U}_{22} & \mathbf{U}_{33} & \mathbf{U}_{23} & \mathbf{U}_{13} & \mathbf{U}_{12} \\ \mathrm{O} 1 & 0.0217(4) & 0.0459(6) & 0.0347(5) & -0.0218(5) & 0.0164(4) & -0.0063(4) \\ \mathrm{O} 2 & 0.0155(4) & 0.0216(4) & 0.0165(4) & -0.0063(3) & 0.0082(3) & -0.0008(3) \\ \mathrm{O} 3 & 0.0203(4) & 0.0315(5) & 0.0202(4) & -0.0049(3) & 0.0119(3) & -0.0018(3) \\ \mathrm{N} 1 & 0.0163(4) & 0.0167(4) & 0.0126(4) & 0.0010(3) & 0.0076(3) & 0.0048(3) \\ \mathrm{C} 1 & 0.0170(5) & 0.0202(5) & 0.0212(5) & -0.0055(4) & 0.0082(4) & -0.0012(4) \\ \mathrm{C} 2 & 0.0150(5) & 0.0146(4) & 0.0167(4) & -0.0014(3) & 0.0051(4) & 0.0001(3) \\ \mathrm{C} 3 & 0.0127(4) & 0.0136(4) & 0.0148(4) & 0.0008(3) & 0.0040(3) & -0.0001(3) \\ \mathrm{C} 4 & 0.0145(4) & 0.0139(4) & 0.0128(4) & -0.0002(3) & 0.0059(3) & 0.0005(3) \\ \mathrm{C} 5 & 0.0151(4) & 0.0148(4) & 0.0145(4) & -0.0008(3) & 0.0070(4) & 0.0008(3) \\ \mathrm{C} 6 & 0.0160(5) & 0.0198(5) & 0.0235(5) & -0.0014(4) & 0.0079(4) & 0.0027(4) \\ \mathrm{C} 7 & 0.0127(4) & 0.0216(5) & 0.0223(5) & 0.0019(4) & 0.0041(4) & 0.0009(4) \\ \mathrm{C} 8 & 0.0156(5) & 0.0167(5) & 0.0153(4) & 0.0020(4) & 0.0012(4) & -0.0002(4) \\ \text { C9 } & 0.0157(5) & 0.0155(4) & 0.0150(4) & -0.0003(3) & 0.0043(4) & 0.0002(4) \\ \text { C10 } & 0.0225(5) & 0.0166(5) & 0.0143(4) & 0.0012(4) & 0.0079(4) & 0.0012(4) \\ \text { C11 } & 0.0227(5) & 0.0244(5) & 0.0145(5) & -0.0024(4) & 0.0090(4) & -0.0034(4) \\ \text { C12 } & 0.0210(5) & 0.0216(5) & 0.0223(5) & -0.0061(4) & 0.0086(4) & 0.0040(4) \\ \text { C13 } & 0.0150(5) & 0.0174(5) & 0.0166(4) & -0.0012(4) & 0.0060(4) & 0.0036(4) \\ \text { C14 } & 0.0141(4) & 0.0141(4) & 0.0130(4) & -0.0011(3) & 0.0050(3) & -0.0007(3) \\ \text { C15 } & 0.0186(5) & 0.0136(4) & 0.0153(4) & 0.0000(3) & 0.0059(4) & -0.0016(4) \\ \text { C16 } & 0.0188(5) & 0.0219(5) & 0.0206(5) & -0.0037(4) & 0.0096(4) & -0.0061(4) \\ \text { C17 } & 0.0145(5) & 0.0302(6) & 0.0194(5) & -0.0053(4) & 0.0044(4) & -0.0008(4) \\ \text { C18 } & 0.0187(5) & 0.0226(5) & 0.0146(5) & -0.0002(4) & 0.0022(4) & 0.0045(4) \\ \text { C19 } & 0.0192(5) & 0.0151(4) & 0.0127(4) & 0.0006(3) & 0.0050(4) & -0.0006(4)\end{array}$

\section{Hydrogen atomic coordinates and isotropic atomic displacement parameters $\left(\AA^{2}\right)$ for rds 461 .

$\begin{array}{rcccc} & \mathbf{x} / \mathbf{a} & \mathbf{y} / \mathbf{b} & \mathbf{z} / \mathbf{c} & \mathbf{U}(\mathbf{e q}) \\ \mathrm{H} 4 & 0.5902 & 0.5642 & 0.5346 & 0.016\end{array}$




$\begin{array}{lllll}\text { H5 } & 0.5760 & 0.7117 & 0.4315 & 0.017 \\ \text { H6 } & 0.1451 & 0.6659 & 0.4788 & 0.023 \\ \text { H7 } & 0.0205 & 0.5967 & 0.2489 & 0.023 \\ \text { H8 } & 0.1367 & 0.5281 & 0.1082 & 0.02 \\ \text { H9 } & 0.3741 & 0.5301 & 0.1893 & 0.019 \\ \text { H10A } & 0.6717 & 0.6692 & 0.2266 & 0.021 \\ \text { H10B } & 0.5116 & 0.6525 & 0.1439 & 0.021 \\ \text { H11A } & 0.5587 & 0.5579 & -0.0117 & 0.024 \\ \text { H11B } & 0.6290 & 0.6293 & -0.0493 & 0.024 \\ \text { H12A } & 0.8584 & 0.4838 & 0.2511 & 0.026 \\ \text { H12B } & 0.6971 & 0.4704 & 0.1727 & 0.026 \\ \text { H13A } & 0.7512 & 0.5075 & 0.4536 & 0.019 \\ \text { H13B } & 0.8190 & 0.5796 & 0.4138 & 0.019 \\ \text { H15 } & 0.7904 & 0.7609 & 0.4608 & 0.019 \\ \text { H16 } & 1.0275 & 0.7675 & 0.5897 & 0.024 \\ \text { H17 } & 1.1377 & 0.6891 & 0.8064 & 0.026 \\ \text { H18 } & 1.0098 & 0.6060 & 0.8988 & 0.023 \\ \text { H19 } & 0.7736 & 0.5997 & 0.7726 & 0.019\end{array}$

